\title{
World checklist of threatened birds
}

\begin{abstract}
Sama 3 का
\end{abstract}

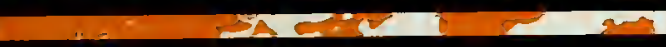
$x$ पा य

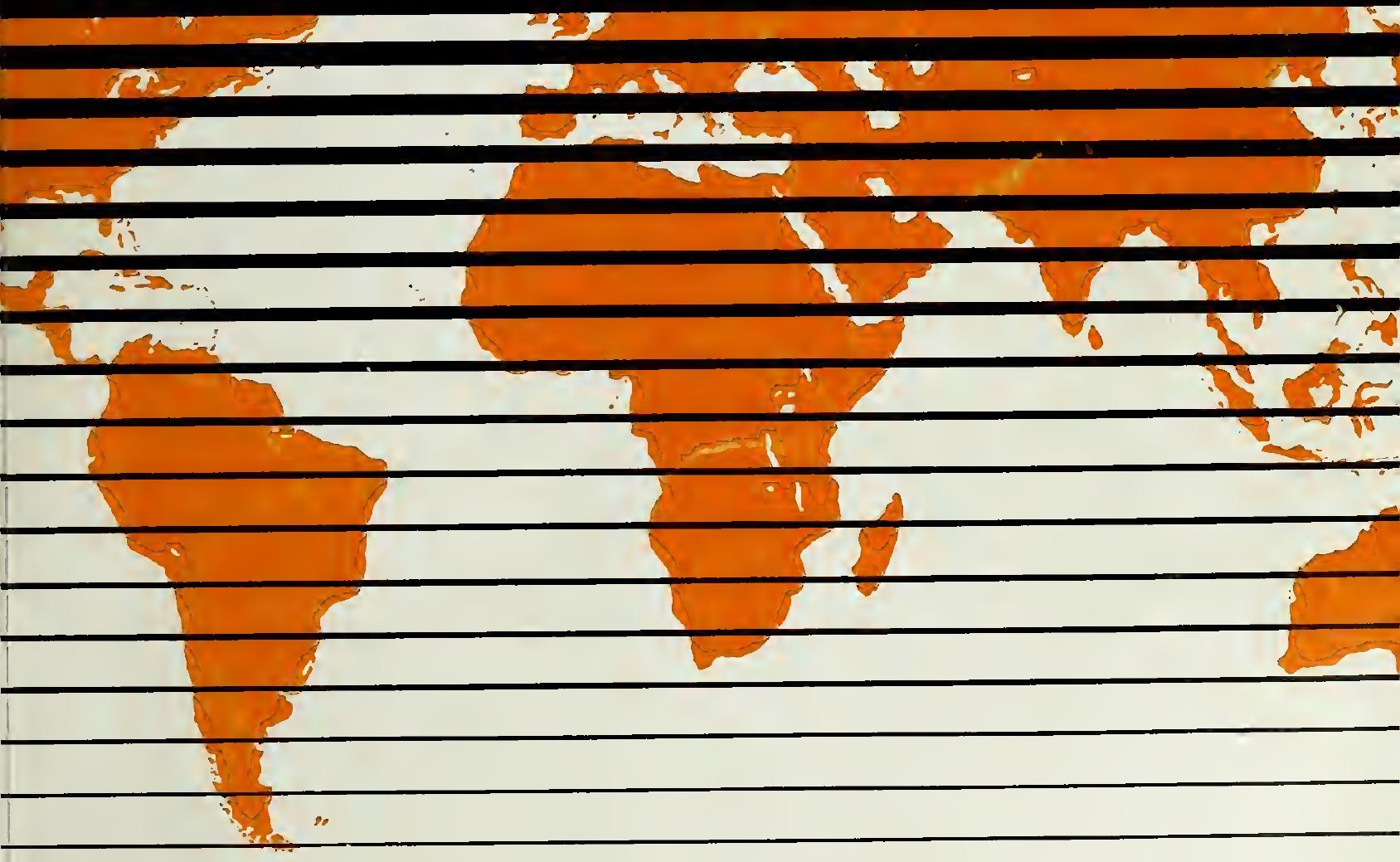


WOPJE CCNGERVATICN

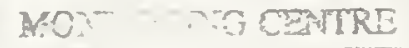

26 AUG 1993

siccus/Anumals 


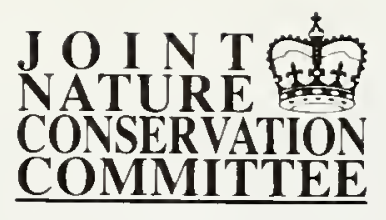

\title{
World checklist of threatened birds
}

\author{
compiled by the
}

\section{World Conservation Monitoring Centre}

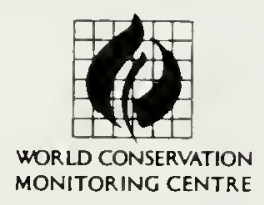

on behalf of the

United Kingdom Scientific Authority for Animals 
Prepared under contract from the Joint Nature Conservation Committee by the World Conservation Monitoring Centre, Cambridge, UK. Established in 1988 as a company limited by guarantee with charitable status, WCMC is managed as a joint-venture between the three partners in the World Conservation Strategy and its successor Caring For The Earth: IUCN - The World Conservation Union, UNEP - United Nations Environment Programme, and WWF - World Wide Fund for Nature. Its mission is to provide information on the status, security, management and utilisation of the world's biological diversity to support conservation and sustainable development.

PubJished by: Joint Nature Conservation Committee

Copyright: 1993 Joint Nature Conservation Committee

ISBN: $\quad 1$ st edition published 1986 ISBN 0-86139-356-2

2nd edition published 1990 ISBN 0-86139-60 I-4

3rd edition published 1993 ISBN 1-873701-45-4

Citation: World Conservation Monitoring Centre (1993). World checklist of threatened hirds. Joint Nature Conservation Committee, Peterborough.

Available from: $\quad$ Further copies and companion volumes for other animal species available from:

Natural History Book Service Ltd

2 Wills Road

Totnes

Devon TQ9 5XN

United Kingdom

Telephone (from UK): $\quad 0803865913$

Telephone (from outside UK): +448038659I3

The designations of geographical entities in this book, and the presentation of the material, do not imply the expression of any opinion whatsoever on the part of JNCC or WCMC concerning the legal status of any country, territory, or area, or of its authorities, or concerning the delimitation of its frontiers or boundaries. 


\section{Contents}

Preface

Acknowledgements

Introduction

Explanatory notes

Introductory references

Taxonomic list

\section{STRUTHIONIFORMES}

Struthionidae

\section{RHEIFORMES}

Rheidae

\section{APTERYGIFORMIES}

Apterygidae

\section{TINAMIFORMES}

Tinamidae

\section{SPHENISCIFORMES}

Spheniscidae

PODICIPEDIFORMES

Podicipedidae

PROCELLARIIFORMES

Diomedeidae

Procellariidae

Hydrohatidae

Pelecanoididae

\section{PELECANIFORMES}

Pelecanidae

Sulidae

Phalacrocoracíá

Fregatidae

\section{CICONIIFORMES}

Ardeidae

Balaenicipitidae

Ciconiidae

Threskiomithidae

Phoenicopteridae

\section{ANSERIFORMES}

Anatidae

\section{FALCONIFORMES}

Cathartidae

Pandionidae

Accipitridae

Sagittariidae

Falconidae

\section{GALLIFORMES}

Megapodiidae

Cracidae

Phasianidae

\section{GRUIFORMES}

Mesitomithidae $\quad 72$

Turnicidae $\quad 72$

Pedionomidae $\quad 73$

Gruidae 73

Rallidae $\quad 75$

Heliomithidae $\quad 78$

Rhynochetidae $\quad 78$

Otididae

\section{CHARADRIIFORMES}

Haematopodidae 81

Recurvirostridae $\quad 81$

Burhinidae $\quad 81$

Glareolidae $\quad 82$

Charadriidae $\quad 82$

Scolopacidae $\quad 83$

Laridae $\quad 84$

Alcidae $\quad 85$

\section{COLUMBIFORMES}

Columbidae

PSITT ACIFORMES

Psittacidae

\section{CUCULIFORMES}

Musophagidae $\quad 121$

Cuculidae

\section{STRIGIFORMES}

Tytonidae

Strigidae

\section{CAPRIMULGIFORMES}

Podargidae

Caprimulgidae

APODIFORMES

Apodidae

Trochilidae

\section{TROGONIFORMES}

Trogonidae

\section{CORACIIFORMES}

Alcedinidae

Momotidae

Brachypteraciidae 
PICIFORMES

Galbulidae

Capitonidae

Indicatoridae

Ramphastidae

Picidae

REFERENCES

\section{PASSERIFORMES}

Eurylaimidae $\quad 177$

Dendrocolaptidae $\quad 177$

Furnariidae $\quad 177$

Formacariidae $\quad 179$

Rhinocryptidae 182

Cotingidae 182

Pipridae 183

Tyrannidae 183

Phytotomidae $\quad 185$

Pittidae 185

Xenicidae 186

Philepittidae 186

Atrichornithidae $\quad 186$

Alaudidae 186

Hirundinidae $\quad 187$

Motacillidae 187

Campephagidae $\quad 187$

Pycnonotidae 188

Laniidae 189

Vangidae $\quad 189$

Cinclidae 190

Troglodytidae $\quad 190$

Mimidae 190

Muscicapidae 190

Paridae 202

Sittidae 203

Rhabdomithidae 203

Dicaeidae 203

Nectariniidae 204

Zosteropidae 204

Meliphagidae 206

Emberizidae 207

Parulidae 211

Drepanididae 211

Vireonidae 213

Icteridae 213

Fringillidae $\quad 214$

Estrildidae 215

Ploceidae 219

Sturnidae 226

Oriolidae 226

Dicruridae 227

Callaeidae 227

Ptilorhynchidae 227

Paradisaeidae 227

Corvidae 231 


\section{Preface}

This checklist is one of a series prepared for the United Kingdom's Scientific Authority for Animals to assist in implementing the Convention on International Trade in Endangered Species of Wild Fauna and Flora (CITES). Similar checklists are available for other animal taxa.

The aim of this volume is to provide a summary of basic information on all species of birds in CITES or appearing in the IUCN Red List of Threatened Animals, compiled by The World Conservation Monitoring Centre. The inclusion of scientific synonyms, English vernacular names and a full list of the countries in which each species occurs will provide a valuable quick source of reference for those assessing applications for CITES permits. The inclusion of many references in the text will permit access to more detailed information on the status of species in particular countries of origin.

Together these features combine to make this volume of interest to all those concerned with the distribution and status of threatened or potentially threatened species whether from a professional or an amateur point of view.

The Joint Nature Conservation Committee is most grateful to the World Conservation Monitoring Centre for preparing this work on its behalf.

Lord Selborne

Chairman, Joint Nature Conservation Committee

Monkstone House

City Road

Peterborough

PE1 1JY

United Kingdom

\section{Acknowledgements}

This checklist was compiled by the World Conservation Monitoring Centre under contract F71-12-56 from the Joint Nature Conservation Committee. The authors were Tim Inskipp and Lorraine Collins.

The volume builds on two earlier editions produced by the International Council for Bird Preservation (ICBP), and the Nature Conservation Bureau. The numerous contributors to those editions are gratefully acknowledged, particularly the authors: John Norton, Simon Stuart and Timothy Johnson. Adrian Long, David Wege and Graham Tucker of ICBP are thanked for providing updated information on the status and distribution of threatened birds of the Americas and Europe. David Denison provicied a program to renumber the extensive reference list to this volume.

For their assistance in producing the current edition, the following WCMC staff are also thanked: Duncan Bennett, Esther Byford, Mary Cordiner, Helen Corrigan, John Easy, Veronica Greenwood, Mandy Haywood, Vanessa Heywood, Richard Luxmoore, Chris Magin, Julie Reay, Lindsay Simpson.

David Morgan of the JNCC International Policy Branch is thanked for providing expert advice and guidance throughout.

Timothy Johnson

Head, Species Unit

World Conservation Monitoring Centre

219 Huntingdon Road

Cambridge

CB3 0DL

United Kingdom 


\section{Introduction}

In April 1991, the Nature Conservancy Council for England, Countryside Council for Wales and Scottish Natural Heritage acting together through the Joint Nature Conservation Committee were appointed by the Secretary of State for the Environment as the United Kingdom's Scientific Authority for Animals under the Convention on International Trade in Endangered Species of Wild Fauna and Flora (CITES). CITES regulates international trade in wild animals and plants and in products derived from them, to help to ensure their conservation on a worldwide scale.

The purpose of this work is to provide a list of bird species appearing in Appendices 1, 11 and 111 of CITES and of those included in the IUCN Red List and the ICBP/IUCN Red Data Book. This revised third edition is larger than the second, having incorporated additions and amendments to the CITES appendices up to and including those made at the 8th Conference of the Parties in March 1992 (effective from 11 June 1992). For each of the 2,246 species and 44 subspecies concerned, a list of range states is given, together with general or specific references relating to its distribution and status. In addition, 45 extra taxa are incorporated, which were newly listed in Threatened Birds of the Americas, published by ICBP in 1992. For background information on the rationale behind the IUCN Red List categories readers are referred to Collar and Stuart (1985), Collar and Andrew (1988), IUCN (1990) and Collar et. al. (1992). For the workings of CITES, Favre (1989) and Wijnstekers (1992) are recommended. For information by country on the diversity and status of birds and other taxonomic groups, and for a general review of biodiversity, readers are referred to WCMC (1992).
In a publication of this nature, it is inevitable that users will discover entries that need correcting or updating. The publishers would be grateful if their attention could be drawn to these entries, and the World Conservation Monitoring Centre would be grateful to receive details of such changes so that they can be incorporated in the database from which this publication is produced. 


\section{Explanatory Notes}

\section{Scientific names}

Following CITES practice, nomenclature of genera and species follows Sibley and Monroe (1990) with genera and species arranged alphabetically within families. The comenclature and sequence of families and higher taxa follows Morony, Bock and Farrand (1975). Scientific names of species in Morony et al. which differ from those in Sibley and Monroe (1990) have been inserted as synonyms. The comprehensive index to families, genera, species and English names should enable entries for taxa to be located quickly.

\section{English names}

These are primarily taken from Sibley and Monroe (1990), but some other commonly used names are given as synonyms. Well-defined subspecies treated by some authors as separate species, are given in parentheses following the species name in this list. Corresponding English names are also given in parentheses to denote that the name refers only to a particular form of the species.

\section{Other information}

The three columns headed CITES, RL, and Ref, list the following information for each taxon.

\section{CITES}

I, Il or III in this column refers to the appendix in which the taxon is listed under the Convention on International Trade in Endangered Species of Wild Fauna and Flora. The two-letter abbreviations following Appendix III entries denote the countries whose governments have placed the taxon in this appendix, as follows:

$\begin{array}{ll}\text { CO } & \text { Colombia } \\ \text { CR } & \text { Costa Rica } \\ \text { GH } & \text { Ghana } \\ \text { GT } & \text { Guatemala } \\ \text { HN } & \text { Honduras } \\ \text { MU } & \text { Mauritius } \\ \text { MY } & \text { Malaysia } \\ \text { NP } & \text { Nepal } \\ \text { TH } & \text { Thailand } \\ \text { UY } & \text { Uruguay }\end{array}$

The CITES appendices include many species which are not threatened but which are considered 'look-alikes' for the purposes of international trade. Thus all Falconiformes and Strigiformes and virtually all Psittaciformes are included, together with the Otididae and Trochilidae. The situation is further complicated because Ghana has included in Appendix III several widespread and common species, such as Bubulcus ibis, Streptopelia turtur and Ploceus cucullatus.

RL

Threat categories follow those given in the 1990 IUCN Red List and additional species in Collar $e t$ Cantian!
los not
Whorl al (1992), and are as follows (see Collar and Stuart (1985) for a full explanation):

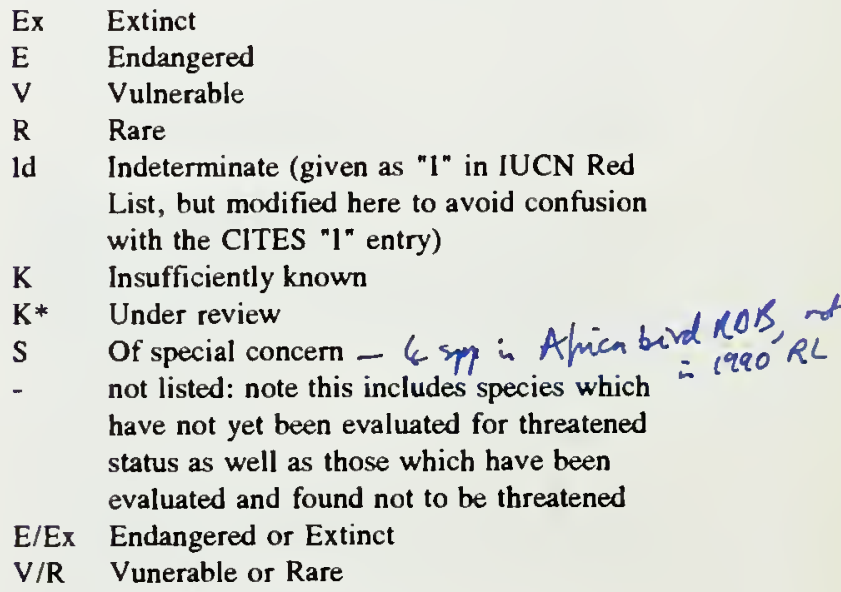

The category $\mathrm{K}^{*}$ applies only to the taxa listed for the first time by Collar and Andrew (1988) and not subsequently revised.

\section{References}

The numbers in the right-hand column refer to entries in the reference list at the end of the document. Many of these general works relevant to more than one country or concerned with particular groups of species. Single country avifaunas and more specific references have generally been inserted in brackets after the appropriate country in the listings for geographical range. Standard reference works for particular countries are lisied in the references at the end of this section. 


\section{Geographical range}

The geographical range of each taxon is given in terms of political units arranged alphabetically. Small island dependencies are also listed alphabetically. Where appropriate islands within groups are listed after a colon, e.g. 'Indonesia: Java'. The same principle applies whenever the distribution of a taxon is given in terms of smaller units than the country concerned.

Place-names and names of countries follow the Times Atlas (1990) and United Nations (1991).

It should be noted that, when a species is listed as occurring within a country, it may not occur throughout the country, and may be confined to only one or a few localities. This is particularly true for migratory species.

A question mark '?' has been used in front of a country to indicate that there is some uncertainty in the status of the species in that country.

A country where the species is known to be extinct is denoted by '(ex)', or by '(ex?)' where there is still a small possibility that the species survives, or where recent searches have been unsuccessful.

'[ ]' denotes a range state where the species has been introduced.

'?[ ]' denotes a range state where there is a possiblity that the species has been introduced.
Each taxon is listed under three headings, as follows:

\section{Breeding}

Countries in which a species is known or presumed to breed. Much of the information on tropical species is based on sight evidence only, but such species are presumed to be breeding by virtue of their sedentary nature.

\section{Non-breeding}

Countries in which a species occurs as a regular non-breeding visitor or passage migrant.

\section{Vagrant}

Countries in which a species has been recorded, but only as a rare or irregular visitor. 


\section{Names of countries and dependent territories}

This checklist has been made as compact as possible by listing some names of countries and dependent territories in the shorter forms given by United Nations (1991). Within the text, Czechoslovakia and Yugoslavia are used to denote the geographic areas as recognised by United Nations (1991). However, changes to the political boundaries within the former USSR have been incorporated in the geographic descriptions.

Countries for which shorter forms of names are used and their shortened form:

\section{Brunei Darussalam}

Democratic People's Republic of Korea

Falkland Islands and Dependencies

Islamic Republic of Iran

Lao People's Democratic Republic

Libyan Arab Jamahiriya

Northern Mariana Islands

Republic of Korea

Saint Vincent and the Grenadines

Syrian Arab Republic

United Republic of Tanzania

United Kingdom of Great Britain and Northem Ireland

United States of America

\author{
Brunei \\ D.P.R. Korea \\ Falkland Islands \\ Iran \\ Lao P.D.R. \\ Libya \\ Northem Marianas \\ Korea Republic \\ Saint Vincent \\ Syria \\ Tanzania \\ United Kingdom \\ USA
}

In the text, parent countries of dependencies are omitted. These are listed below:

American Samoa, USA

Anguilla, United Kingdom

Aruba, Netherlands

Azores, Portugal

Bermuda, United Kingdom

Bouvet Island, Norway

British Antarctic Territory, United Kingdom

British Indian Ocean Territory, United Kingdom

British Virgin Islands, United Kingdom

Canary Islands, Spain

Canton and Enderbury Islands, Kiribati

Cayman Islands, United Kingdom

Channel Islands, United Kingdom

Christmas Island, Australia

Cocos Keeling Islands, Australia

Cook Islands, New Zealand

Falkland Islands, United Kingdom

Faeroe Islands, Denmark

Federated States of Micronesia, USA

French Guiana, France

French Polynesia, France

French Southern and Antarctic Territories, France

Galapagos Islands, Ecuador

Gibraltar, United Kingdom

Greenland, Denmark

Guadeloupe, France

Guam, USA

Guernsey, United Kingdom

Hawaiian Islands, USA

Heard and Macdonald Islands, Australia

Hong Kong, United Kingdom

Isle of Man, United Kingdom

Jersey, United Kingdom
Johnston Atoll, USA

Macao, Portugal

Macquarie Island, Australia

Madeira, Portugal

Marshall Islands, USA

Martinique, France

Mayotte, France

Montserrat, United Kingdom

Netherlands Antilles, Netherlands

New Caledonia, France

Niue, New Zealand

Norfolk Island, Australia

Northern Marianas, USA

Palau, USA

Panama Canal Zone, USA

Pitcairn Islands, USA

Puerto Rico, USA

Queen Maud Land, Norway

Réunion, France

Saint Helena, United Kingdom

Saint Pierre and Miquelon, France

Saint Vincent, United Kingdom

South Orkney lslands, United Kingdom

South Sandwich Islands, United Kingdom

South Georgia, United Kingdom

South Shetland Islands, United Kingdom

Svalbard and Jan Mayen Islands, Norway

Tokelau, New Zealand

Tristan da Cunha Islands, United Kingdom

Turks and Caicos Islands, United Kingdom

Virgin Islands of the United States, USA

Wallis and Futuna, France 


\section{Introductory References}

Collar, N.J. and Andrew, P. 1988. Birds to watch: the ICBP world check-list of threatened birds. International Council for Bird Preservation, Technical Publication No. 8. ICBP, Cambridge, U.K.

Collar, N.J., Gonzaga, L.P, Krabbe, N., Madroño Nieto, A. Naranjo, L.G., Parker, T.A. III and Wege, D.C. 1992. Threatened birds of the Americas: the ICBP/IUCN Red Data Book, 2. Third Edition. Cambridge, U.K.

Collar, N.J. and Stuart, S.N. 1985. Threatened birds of Africa and related islands: the ICBP/IUCN Red Data Book, 1. Third edition. Cambridge, U.K.

Favre, D.S. 1989. Intemational trade in endangered species: a guide to CITES. Martinus Nijhoff, Dordrecht.

IUCN 1990. 1990 IUCN Red List of Threatened Animists. IUCN - The World Conservation Union, Gland, Switzerland and Cambridge, U.K.

Morony, J.J., Bock, W.J. and Farrand, J. 1975. Reference list of the birds of the world. American Museum of Natural History (Department of Ornithology).

\section{Standard country and dependency references}

\section{Afghanistan}

455 FAO 1981 Checklist of the birds of Afghanistan. In Afghanistan, a contribution to a conservation strategy.

Albania

818 Lamani, F. and Puzanov, V. 1962-1963 (Checklist of the birds of Albania.] Bull. U. Sh. Tirane Ser.

Algeria

841 Ledant, J.-P. Jacob, J.-P., Jacobs, P., Malher, F..

Ochando, B. and Roché, J. 1981 Mise à jour de

Andorra

l'avifaune algérienne. Gerfnut 71: 295-398.

998 Muntaner, J., Ferteri, X. and Martinez-Vilalta, A 1983 Atlas dels acells nidificants de Catalunya y Andorra. Ketrés Editora, Barcelona.

Angola

1108 Pinto, A.A. de R. 1983 Omitologia de Angola. Instituto de Investigacao Cientifica Tropical, Lisbon.

Anguilla

1087 Peters, J.L. 1929 Birds of the island of Anguilla, W. Indies. Auk 44: 532-538.

Antigua and Barbuda

357 Danforh, S.T. 1934 The birds of Antigua. Auk 51: 350-364

660 Holland. C.S. and Williams. J.M. 1978 Observations on the birds of Antigua. American Birds 32: 1095-1105 Argentina

1034 Olrog, C.C. 1984 Las aves Argentinas. Administracion de Parques Nacionales. Buenos Aires. Australia

167 Blakers, M., Davies, S.J.J.F. and Reilly, P.N. 1984 The allas of Australian birds. Royal Australian Ornithologists ${ }^{*}$ Union and Melbourne University Press, Melbourne

Australian Antarctic Territory

753 Johnstone, G.W. and Kerry. K.R. 1976 Ornithological observations in the Australian sector of the Southern Ocean. Pp. 725-738 in Proceedings of the 16th Omithologicnl Congress. Australian Academy of Science, Canberra.
Paxton, J., ed. 1988. The statesman's year-book World Gazetteer. 125th edition 1988-89. Macmillan, London.

Sibley, C.G. and Monroe, B.L. 1990. Distribution and taxonamy of birds of the world. Yale University Press, New Haven.

The Times Atlas of the World 1990. Comprehensive (eighth) edition. Times Books, London UK

United Nations, 1991. Terminnlogy Bulletin No. 342. United Nations, New York.

Wijnstekers, W. (1992). The evolution of CITES. A reference to the Convention on Intemational Trade in Endangered Species of Wild Fauna and Flora. Third revised edition. CITES Secretariat, Lausanne, Switzerland.

World Conservation Monitoring Centre. 1992. Global Biodiversity. Stalus of the Earth's living Resources. Chapman \& Hall, London. UK

Austria

1186 Rokitansky, G. 1964 Catalogus Faunae Austriae. XXIb Aves.

Azores

113 Bannerman, D.A. and Bannerman. W.M. 1966 Birds of the Atlantic Islands, 2. A histony of the birds of the Azores. Oliver and Boyd, Edinhurgh.

Bahamas

232 Brudenell-Bruce, P.G.C. 1975 The hirds of New Providence and the Bahama Islands. Collins, London.

236 Buden. D.W. 1987 The birds of the southem Bahamas. British Ornithologists' Union Check-list No. 8. BOU, London.

Bahrain

1019 Nightingale. T. and Hill, M. 1992 The birds of Bahrain. Immel.

Baker, Ilowland, Jarvis, Palmyra lslands

1118 Pratt, J.D., Bruner, P.L. and Berrelt, D.G. 1987 A field guide to the birds of Hawaii and the tropical Pacific. Princeton University Press, Princeton, New Jersey.

Bangladesh

621 Harvey, W.G. 1990 Birds in Bangladesh. University Press Lid., Dhaka.

Barbados

178 Bond, J. 1956 Check-list of birds of the West Indies. The Academy of Natural Sciences. Philadelphia.

Belize

1202 Russell, S.M. 1964 A distributional study of the birds of British Honduras. Omithological Monegraphs 1: 195

1532 Wood, D.S., Leberman, R.C. and Weyer, D. 1986 Checklist of the birds of Belize. Carnegie Museum of Natural History, Pittsburgh.

Belgium

329 Commission pour l'avifaune belge 1967 Avifaune de Belgique. Gerfaut : $273-465$

Benin

667 Holyoak, D.T. and Seddon, M.B. 1990 Distrihutional notes on the birds of Benin. Malimbus 11: 128-134 


\section{Bermuda}

18 Amos, E.J.R. 1991 A guide to the birds of Bermuda. Eric J.R. Amos, Warwick, Bermuda.

Bhutan

873 Ludlow, F. 1937 The birds of Bhutan and adjacent territories of Sikkim and Tibet. Ibis (14) 1: 1-46, 249 . 293. 467-505.

Bolivia

1140 Remsen, J.V. Jr. and Traylor, M.A. 1989 An annataled list of the birds of Bolivio. Buteo Books, Vermillion, South Dakota.

Botswana

1016 Newman, K. 1989 Birds of Botswana. Southern Botswana.

Bonvet Island

1466 Watson, G.E. 1975 Birds of the Antarctic and SubAntarctic. American Geophysical Union. Washingotn D.C.

Brazil

492 Frisch, J.D. and Frisch, S. 1981 Aves Brosileiras Vol. 1. Dalgas-Ecoltec Ecologica Tecnica, Sao Paulo.

1107 Pinto, O. 1978 Nova catalogo dos aves do Brasil. Departmento de Zoologia, Sao Paulo.

1270 Sick. H. 1984 Omilologia Brasileira. Volume 1 Editora Universidade de Brasilia. Brasilia

127) Sick, H. 1985 Omitologia brasileira, uma introducao Editora Universidade de Brasilia, Brasilia.

British Antarctic Territory

1466 Watson, G.E. 1975 Birds of the Antarctic and SuhAntarctic. American Geophysical Union, Washington D.C

British Iodian Ocean Terrilory

189 Bourne, W.R.P. 1971 The hirds of the Chagus group, Indian Ocean. Aroll Res. Bull. 149: 175-207.

Brumei

897 Mann. C.F. 1987 A checklist of the birds of Brunei Darussalam. Brunei Museum Joumal 6: 170-212.

Bulgaria

1074 Pateff, P. 1950 Ptitzits W Bulgarija [The birds of Bulgaria.] Sofia.

Burkina Faso

1388 Thonnerieux, Y. 1988 Etat des connaissances sur la reproduction de l'avifaune du Burkina Faso ex HauteVolta). Oisean Rev. Francaise Omithol. 58: 120-145

Burundi

1230 Schouteden, H. 1966 La faune ornithologique du Burundi. Doc. zool. Mis. Roy. Afr. centr. 11: 1-81.

\section{New Caledonia}

612 Hannecan, F. and Letocart, Y. 1980-1983 Oisearc de Nouvelle Caledonie et des Loyautes. 2 vols. Les Editions Cardinalis, Nouméa, New Caledonia

\section{Cambodia}

1381 Thomas, W.W. 1964 A preliminary list of the birds of Cambodia. Unpublished. [Notes on 394 species, including the first records for Cambodia of Botanis stellaris, Anas penelope, A. poecilorhyncha, A. acula, A. clypeata, Circus cyaneus, Ictinaetus malayensis, Folco tinnunculus, Fulica atra, Treron phoenicoptera, Psinocula finschii, Sirix selopulo, Dendrocopos macei,

\section{Cameroon} Hypsipetes leucacephalus, Ampeliceps comalus].

859 Louette. M. 1981 The birds of Cameroon: an annotated checklist.Verhandelingen Koninklijke Academie. Brusscl kl. Wet 43(163): 1-295.

Canada

539 Godfrey, E. 1986 The birds of Canada. 2nd ed. National Museum of Natural Sciences, Ottawa, Canada.
Canary Islands

111 Bannerman, D.A. 1963 Birds of the Allantic Islands, I A history of the birds of the Canary Islands and of the Salvages. Oliver and Boyd, Edinhurgh.

989 Moreno, J.M. 1988 Gria de las aves de las Islas Canarias. Canaries Inst.

1054 Padron, F.P. 1983 Las aves de Canarias.

Canton and Enderbury Islands

1118 Pratt, J.D., Bruner, P.L. and Berrett, D.G. 1987 A field guide to the birds of Hawaii and the tropical Pacific. Princeton University Press, Princeton, New Jersey.

Cape Verde

114 Bannerman, D.A. and Bannerman, W.M. 1968 Birds of the Allantic Islands, 2. History of the birds of the Cape Verde Islands. Oliver and Boyd, Edinburgh.

Cayman Islauids

194 Bradley, P.E. 1985 A gride to the birds of the Cayman Islands. P.E. Bradley, George Town, Grand Cayman.

Central African Republic

259 Carroll, R.W. 1988 Birds of the Central African Republic. Malimbus 10(2): 177-200

894 Malbrant, R. 1952 Faune du centre africoin français. 2nd ed. Lechevalier, Paris.

Chad

1210 Salvan, J. 1967-1969 Contribution à l'etude des oiseaux du Tchad. Oiseau $37: 255-284 ; 38: 53-85,127-150$, $249-273 ; 39: 38-69$.

1432 Viellard, J. 1971 Données biogeographiques sur 1'avicaune d'Afrique centrale. I. Alaruda 39: 227-248.

Channel Islands

164 Bissor. A.J. 1983 Checklist of the hirds of the Channel Islands.

401 Dobson, R. 1952 The birds of the Channel lslonds. Staples Press, Newton Ahbol, UK.

Chile

82 Araya, L. 1982. Lista patron de las aves Chilenas. Publicaciones ocasionales No.1, Instituto de Oceanologica, Universidad de Valparaiso. Vina del Mar, Chile

747 Johnson, A.W. 1967 The birds of Chile. Volume 2. Platt Estahlecimientos Graficos S.A., Buenos Aires.

China

281 Cheng Tso-hsin 1987 A synopsis of the avifauna of China. Science Press, Beijing.

Christmas Island

1330 Stokes. T. 1988 A review of the birds of Christmas Island, Indian Ocean. Australian National Parks and Wildlife Service, Occasional Paper No. 16

Cocos Keeling Island

525 Gibson-Hill, C.A. 1949 The birds of the CocosKeeling Islands Indian Ocean). Ibis 91 : 221-243

1331 Stokes, T. Sheils, W. and Dunn, K. 1984 Birds of the Colombia Cocos Keeling Islands, Indian Ocean. Emm 84: 23-28

655 Hilty, S. and Brown, W.L. $1986 \mathrm{~A}$ guide to the birds of Colombia. Princeton University Press, Princeton, New Jersey.

Comoros

143 Benson. C.W. 1960 The hirds of the Comorn islands: results of the British Ornitholngists" Uninn Centenary Expedition 1958. Ibis 103b: 5-106.

Congo

895 Malbrant, R. and Maclatchy, A. 1949 Faune de l'equateur africain français. 1. Oiseaux. Lechevalier. Paris

Cook Islands

793 King, W.B. 1974 Conservation status of hirds of central Pacific islands. Elepoio 34: 70-74. 
Costa Rica

1325 Stiles, F.G. Skutch, A.F. and Gardner, D. 1989 A guide to the birds of Costa Rica. Christopher Helm, London.

Côte de Ivoire

1378 Thiollay, J.-M. 1985 Birds of lvory Coast: status and distribution. Malimbus 7(1): 1-59.

Cuba

511 Garrido, O.H. and Montaña Garcia, F. 1975 Catálogo de las aves de Cuba. Academia de Ciencias de Cuba, La Habana.

\section{Cyprus}

480 Flint, P.R. and Stewart, P.F. 1991 The birds of Cyprus. 2nd edition. British Ornithologists' Union Checklisi No. 6. London.

Czechoslovakia

463 Ferianc, O. 1977/79 Vtáky Slovenska 1.2 2nd ed. Veda: Bratislava

682 Hudec, K. 1972-1983 Fauna CSSR, Svazek 24 Placi Aves. 3 vols. Academia Nakladatelstvi Ceskoslovenské Akademie Ved, Praha.

\section{Denmark}

415 Dybbro, T. 1978 Oversigt aver Danntarks fugle. Dansk Ornithologisk Forening, Denmark.

\section{Dominica}

1427 Verrill, A.H. 1892 Notes on the fauna of the island of Dominica: lists of birds obtained and observed, with notes on their habitats, nests and eggs. Trans. Connecticut Acad. Arts Sci. 8: 315-355.

1429 Verrill. A.H. 1905 Additians to the ovifauna of Dominica: notes on species hitherto unrecorded with descriptions of three new species and a list of all birds now known to occur on the island. Privately published. Barbados.

Douninican Republic

1326 Stockton de Dod, A. 1987 Aves de la Republica Dominicana. 2nd edition. Museo Nacional de Historia Natural, Santo Domingo.

1482 Wetmore, A. and Swales, B.H. 1931 The birds of Haiti and the Dominican Republic. Bulletin of the United States National Museum 155.

1498 Wiley, J. W. and Ottenwalder, J. A. 1990 Birds of islas Beata and Alto Velo, Dominican Republic. Studies Djibouti on Neotropical Fauno and Enviromment 25(2): 65-88.

96 Ash, J.S. 1985 Midwinter observations from Djibouti. Ecuador Scopus 9: 43-49

1042 Ortiz-Crespo, F., Greenfield, P.J. and Matheus, J.C. 1990 Birds of Ecuador, locational checklist with English and Spanish common names. Ecuador Ornith. Corp.

Egy pt

551 Goodman, S.M. and Meininger, P.L. 1989 The birls of Egypr. Oxford University Press, Oxford

El Salvador

397 Dickey, D.R. and van Rossem. A.J. 1938 The birds of El Salvador. Publication of the Field Museum Natural

Ethiopia History. Zoology series 23: 1-609.

1410 Urban, E.K. and Brown, L.H. 1971 A checklist of the birds of Ethiopia. Dept. of Biology, Haile Sellassie I University Press, Addis Ababa, Ethiopia.

Equatorial Guinea

422 Eisentrant, M. 1973 Die Wirbeltierfauna von Fernando Poo und Westkamerun. Bonn. zool. Monogr. No.3, 428 pp.

Falkland Islands and Dependencies

919 Matthews, L.H. 1929 The birds of South Georgia.
50 Prince, P.A. and Payne, M.R. 1979 Current status of birds at South Georgia. Brinish Antarctic Survey Bulletin 48: 103-j18.

1535 Woods. R.W. 1988 A guide to the birds of the Falkland Islands. Anthony Nelson, Oswestry. UK

Faeroe Islands

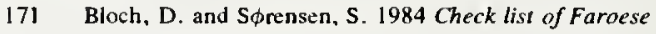
birds. Torshaven.

Federated States of Micronesia

1118 Pratt, J.D., Bruner, P.L. and Berrett, D.G. 1987 A field guide to the birds of Hawaii and the tropical Pacific. Princeton University Press, Princelon, New Jersey.

Finland

1307 Solonen. T. 1985 Suomen linnusto. Lintutielo. Helsinki.

Fiji

1118 Pratt, J.D., Bruner, P.L. and Berrett, D.G. 1987 A field guide to the birds of Hawaii and the tropical Pacific. Princeton University Press, Princeton, New Jersey.

1461 Watling, D. 1982 Birds of Fiji, Tonga and Samoa, Millwood Press, Wellington.

France

924 Mayaud, N. 1953 Liste des oiseaux de France. Alauda 21: 1-63.

French Guiana

152 Berlepsch, H.G. von 1908 On the birds of Cayenne. Novitates Zoologicae 15: 103-164. 261-324.

French Polyuesia

234 Bruner, P.L. 1972 Field guide to the birds of French Polynesia. Pacific Scientific Information Center, Bernice P. Bishop Museum, Honolulu, Hawaii.

1118 Pratt, J.D., Bruner, P.L. and Berrett, D.G. $1987 A$ field guide to the birds of Hawaii and the tropical Pacific. Princeton University Press, Princeton, New Jersey.

Freuch Southern \& Antarctic Territory

384 Derenne, P., Lufbery, J.X. and Tollu, B. 1974 L'avifaune de l'Archipel Kerguelen. CNFRA 40: 107148.

410 Dreux, P. and Milon, P. 1967 Premieres observations sur l'avifaune de l'Ile aux Cochons Archipel Crozet). Alauda 35: 27-32.

1244 Segonzac. M. 1972 Données récentes sur la faune des lles Saint-Paul et Nouvelle Amsterdam. L'Oiseau 42 No. Spec.: 3-68.

Gaboı

895 Malbrant, R, and Maclatchy, A. 1949 Faune de l'equateur aficain franfais. I. Oiseaux. Lechevalier, Paris

Galapagos Islands

614 Harris, M. 1982 A ficld guide to the birds of Galapagos. Revised edition. Collins, London.

Gambia

556 Gore, M.E.J. 1990 The birds of the Gamhia. 2nd ed. B.O.U. Check-list No. 3. British Ornithologists' Union, London.

Germany

1018 Niethammer, G., Kramer, H. and Wolters, H.E. 1964 Die Vägel Deuschlonds, Artenliste. Frankfurt A.M.

893 Makatsch, W. 1981 Verzeichnis der Vogel der Deutschen Demokratischen Republik. Neumann Verlag. Leipzig-Radebeul.

1148 Rheinwald, G., Hill, A. and Ringlehen, H. 1981 Die Vögel der Budestepublik Deutschland und Berlin West). Artenliste. Dachverband Deutscher Avifaunisten, Verlag. 
1014 Neumann, J. and Kolbe, U. 1990 [The birds of East Germany - an annotated species list.] Falke 37(7): 229232.

Ghana

582 Grimes, L. 1987 The birds of Ghana. B.O.U. Checklist No. 9. British Ornithologists' Union, London.

\section{Gibraltar}

334 Cortes, J.E., Finlayson, J.C., Mosquera, M.A. and Garcia, E.F.J. 1980 The birds of Gibraltar, British Ornitholigists' Union, London.

\section{Greece}

644 Helversen, O. von, Hodge, M. and Martens, J. 1969 Catalogus Faunae Graeciae, Pt. 2, Aves. Thessaloniki. Greenland

1207 Salomonsen, F, 1950-1951 Gronlands Fugle. The birds of Greenland. Ejnar Munksgaard, Kobenhavn.

\section{Grenada}

387 Devas, R.P. c. 1940 Birds of Grenada and St Vincent and the Grenadines.

1478 Wells, J.G. 1887 A catalogue of the birds of Grenada. W. Indies, with observations thereon. Proceedings of the United States National Museum 9: 609-633

\section{Guadeloupe}

360 Danforth, S.T. 1939 The birds of Guadeloupe and adjacent islands. J. Agric. Univ. Puerto Rico 23(1): 9. 46.

Guam

731 Jenkins, J.M. 1983 The native forest birds of Guan. American Ornithologists' Union Omithological Monograph 31. AOU, Washington D.C.

783 Kibler. L.F. 1950 Notes on the hirds of Guam. Auk 67 400-403

Guatemala

824 Land, H.C. 1970 Birds of Guatemala. Livingston Publishing, Wynnewood, Pennsylvania

Guinea-Bissau

488 Frade, F. and Bacelar, A. 1955 Catalogo das aves da Guiné Porluguesa - Non passeres. Ansl Jurta lnvest. Ultram.

\section{Guyana}

1303 Snyder, D.E. 1966 The birds of Giyana. Peaboty Museum, Salem.

Ilaiti

1482 Wetmore, A. and Swales, B.H. 1931 The birds of Hait and the Dominican Republic. Bulletin of the United States National Musetum 155

\section{IIawaiian Islands}

1118 Pratt, J.D., Bruner, P.L. and Berrett, D.G. $1987 \mathrm{~A}$ field guide to the birds of Hawaii and the tropical Pacific. Princeton University Press, Princeton. New Jersey.

\section{llonduras}

981 Monroe, B.L. Jr. 1968 A distributional survey of the birds of Honduras. Omithological Monographs 7: 1-4.

Ifong Kong

265 Chalıners, M.L. 1986 Annotaned checklist of the hird. of Hong Kong. Fourth edition. The Hong Kong Bird Watching Society. Hong Kong.

Hungary

457 Farkas, T. 1967 Omilhogeographic Ungams. Duncker \& Humblot, Berlin

757 József, P. 1980 Magyar Mardártani Bibliografia

\section{Iceland}

1390 Timmermann, D. 1938-1949 Dic Vögel Islands. Islendingà Rï. 28:239-524

India

10 Ali, S. and Ripley, S.D. 1987 Compact handbook of the birds of India and Pakistan together with those in

Bangladesh, Nepal, Bhutan and Sri Lanka. Oxford University Press, Oxford.

1159 Ripley, S.D. 1982 A synopsis of the birds of India and Pakistan. Second edition. Bombay Natural History Society, Bombay.

Indonesia

26 Andrew, P. 1992 (The birds of Indonesia: a checklis Peters' sequence). Jakarta: Indonesian Ornithological Sociely.

1486 White, C.M.N. and Bruce, M.D. 1986 (The birds of Wallacea Sulawesi, the Moluccas and Lesser Sunda Islands, Indonesia): an annotated checklist. British Ornithologists' Union Checklist No. 7. BOU, London.

Iran

1241 Scott, D.A., Hamadani, H.M. and Mirhosseyni, A.A. 1975 The birds of Iran. Department of the Environment, Tehran, Iran.

Iraq

12 Allouse, B.E. 1953 The uvifauna of Iraq. Iraq Natural History Museum Puhlication No. 3

Ireland

687 Hutchinson, C.D. 1989 Birds in Ireland. Poyser, Carlton, U.K.

Israel

674 Hovel, H. 1987 Check-list of the hirds of Israel with Sinai: an annotated check-list. SPN1. Tel-Aviv.

Italy

210 Brichetti, P. and Massa, B. 1984 Check-list degli uecelli italiani. Riv. ital. Omithal. 54: 3-37.

Jauaica

406 Downer. A. and Sutton, R. 1990 Birds of Jamaica: a photographic fieldguide. Cambridge University Press. Cambridge.

Japan

205 Brazil, M. 1991 The birds of Japan. Christopher Helm, London

Juhuston Island

1118 Pratt, J.D., Bruner, P.L. and Berrett, D.G. $1987 A$ field guide to the birds of Hawaii and the tropical Pacific. Princeton University Press, Princeton, New Jersey

Jurdau

3 Abu Yaman, 1.K. et al. 1985 Fauna of Jordan. II. Aves: a check list of the birds of Jardan. Amman.

Jordan

400 Disi. A.M. and Bouran. A.H. 1987 A check-list of the Hashemite Kingdom of Jordan: an ecological outlook. Publication of the University of Jordan.

Kenya

212 Britton, P.L. ed. 1980 Birds of East Africa. East Africa Natural History Society, Nairobi.

Kirihati

1118 Pratt, J.D., Bruner, P.L. and Berret, D.G. 1987 A field guide to the birds of Hawaii and the tropical Pacific. Princelon University Press, Princeton, New Jersey.

Korea Repuhlic

I.531 Won Pyong-ob 1976 Checklist of the hirds of the Republic of Karca

D.P.R. Korea

558 Gore, M.E.J and Won, P-O. 1971 The hirds of Korea. Royal Asiatic Society, Seoul

Kuwait

$2+3$ Bundy. G. and Warr, E. 1980 A checklist of the birds of the Arabian Gulf States. Sandgrouse 1: 4-49.

Laos

380 Delacour, J. and Jabouille, P. 1940 Listes des oiseaux de l'Indochine française, completée et mise a jour. L'Oiseau et R.f.O. 10: 89-220. 
Latvia

1436 Viksne, J., ed. 1983 Birds af Latvia: territorial distribution and number. Zinatne, Riga.

Lebanon

808 Kumerloeve, H. 1962 Notes on the birds of the Lebanese Republic. Iraq Natural History Museum Publications 20 and 21 .

1382 Thomé. G. and Thomé, H. 1986 [The birds of Lebanon.] Publ. Univ. libanaise. (In Arabic.)

Lesotho

181 Bonde, K. 1981 An annolated checklist to the birds of Lesotho preliminary edition). Privately published, Maseru.

1045 Osborne, P.E. and Tigar, B.J. 1990 Distribution and status of rare birds in Lesotho. Br. Ecol. Soc. Bulletin 21: $121-128$

Liberia

11 Allen, G.M. 1965. The birds of Liberia. Contr. Dep. trop. Med. \& Inst. trop. Biol. Med. 5: 636-748.

I,ibya

241 Bundy, G. 1976 The birds of Libya. British Ornitholigists' Union, London.

Lichtenstein

1503 Willi. G and Broggi, M.F. 1983-86 Die Vogelwelt des fürstentumus unter Berücksichtigung der benachnarten Gebiet. Ber. Bot,-Zool. Ges. Liechtenstein-Sargans-

Luxembourg Werdenberg. 12: 61-117, 14: 103-143, 15: 37-82.

40 Hulten, M. and Wassenich, V. 1960-1961 Die Vögelfauna Luxembaurgs. Victor Buck, Luxemhourg.

\section{Madagascar}

826 Langrand, O. 1991 Gride to the birds of Madagascar. Yale University Press, New Haven and London.

Madeira

112 Bannerman. D.A. and Bannerman. W.M. 1965 Birds of the Atlanic Islands, 2. A history of the birds of Madcira, the Desertas, and the Porto Santo Islands.

Malawi Oliver and Boyd, Edinburgh.

1017 Newman, K., Johnston-Stewar, N., Medland, B. (1992) The birds of Malawi. A supplement to Newman's Birds of Southern A frica. Southern Book Publishers (Pty).

Malaysia: Peninsular Malaysia

939 Medway, Lord and Wells, D.R. 1976 The birds of the Malay Peninsula. Volume 5: conclusions and survey of every species. H.F. and G. Witherby and Penerbit Universiti Malaya, London and Kuala Lumpur.

Malaysia: Sabah

555 Gore, M.E.J. 1968 A checklist of the birds of Sabah, Borneo. Ibis 110: 165-196.

Malaysia: Sarawak

1295 Smythies, B.E. 1981). The birds of Barneo. 3rd edition. The Sabah Society and the Malayan Nature Society, Malaysia.

Maldives

1093 Phillips, W.W.A. 1963 The birds of the Maldive Islands. Indian Ocean. Jaumal of the Bombay Naurrol History Socicty 60: 546-584.

Mali

819 Lamarche, B. 1980-1981 Liste commentée des oiseaux du Mali. Malimbus 2: 121-158: 3(2): 73-102.

Malta

1340 Sultana, J. and Gauci. C. 1982 A new guside to the birds of Malta. The Ornitholngical Society. Valletta.

Marsball Islauds

1118 Pratt, J.D., Bruner, P.L. and Berrett, D.G. 1987 A field guide to the birds of Hawaii and the tropical
Pocific. Princeton University Press, Princeton, New Jersey.

Martinique

178 Bond, J. 1956 Check-list of birds of the West Indies. The Academy of Natural Sciences of Philadelphia.

Manritani:

820 Lamarche. B. 1987 Liste cammentée des oiseaur de Mauritanie. Privately puhlished, Nouakchntt.

Mauritius

1314 Staub, F. 1976 Birds of the Mascarenes and Saint Brandan. Organisation Normale des Enterprises Ltée, Labama House, Port Louis, Mauritius.

\section{Mayotte}

143 Benson, C.W. 1960 The birds of the Comoro lslands: results of the British Ornithologists' Union Centenary Expedition 1958. Ibis 103b: 5-106.

Mexico

1089 Peterson, R.T and Chalif, E.L 1973 A field guide to Mexican birds. Houghton Miftlin Company, Boston

Mongolia

1319 Stepanyan, L.S., Bold, A. and Fomin, V.E. 1988 [Taxonomic list of birds of Mongolia.] Ominologiya 23 26-35. In Russian.)

1419 Vaurie, C. 1964 A survey of the birds of Mnngolia. Bulletin of the American Musesum of Nalural History 127: $103-143$

Montserrat

1274 Siegel, A. 1983 Birds of Montserrat. Montserrat National Trust, Montserrat.

Morocco

151 Bergier, P. and Bergier, F. $1990 \mathrm{~A}$ birdwotcher's guide to Morocco. Prior Lid., Huntingdon, U.K.

639 Heim de Balsac, H. and Mayaud, N. 1962 Les oiseanx du nord ouest de l'Afrique, distrinbution geographique, ecologies, migrations, reproduction. Ecyclopedie ornithologique vol. 10. Paris, P. Lechevalice, 1962. France.

Mozaubique

487 Frade, F. 1951 Catálogo das aves de Mnçamhique. Ministerio das Colonias, Lishon.

Myanmar

1296 Smythies, B.E. 1986 The birds of Buma. 3rd edition. Nimrod Press, Liss, Hampshire, UK and Silvio Mattacchione and Co., Pickering, Ontario.

Namibia

1505 Williams, A.J. n.d. 1987? Papulor checklist af the birds of South West Africa/Namibia. Department of Agriculture and Nature Conservation, Windhoek. Nauru

1118 Pratt, J.D., Bruner, P.L. and Berrett, D.G. 1987 A field guide to the birds of Hawail and the tropical Pacific. Princeton University Press, Princeton, New Jersey.

Nepal

700 Inskipp. C. and Inskipp, T. 1991 A guide to the birds of Nepol. 2 nd ed. Christopher Helm.

Netherlands

328 Commission voor de Nederlandse Avifauna 1970 Avifauna van Nederland. Brill, Leiden.

Netherlands Antilles

1445 Voous, K.H. 1983 Birds of the Netherlands Antilles. De Walhurg Pers, Zutphen.

New Caledoni:

1329 Stokes. T. 1980 Notes on the landbirds of New Caledonia. Enu 80:81-86

New Zealand

1369 Turbott, E.G. (Ed). 1990 Checklist of the binds of New Zealand and the Rass Dependency, Antarcica. $3 \mathrm{rd}$ ed. 


\section{Nicaragua}

1153 Ridgely, R.S. 1989 A guide to the birds of Panama. with Costa Rica, Nicaragua and Honduras. Princeton University Press. Princeton. New Jersey.

Niger

535 Giraudoux, P., Degauquier, R., Jones, P.J., Weigel, J. and lsenmann. P. 1988 Avifaune du Niger: etat des conaissances en 1986. Malimbus 10: $1-140$.

\section{Nigeria}

424 Elgood, J.H. 1982 The birds of Nigeria. B.O.U. Checklist No.4, British Ornithologists' Union. London.

Nive

1118 Pratt, J.D., Bruner. P.L. and Berrett, D.G. $1987 \mathrm{~A}$ field guide to the birds of Hawaii and the tropical Pacific. Princeton University Press, Princeton, New Jersey.

1527 Wodzicki, K. 1971 The birds of Niue Island. South Pacific: an annotated checklist. Notomis 18: 291-304.

\section{Norfolk Island}

649 Hermes, N. 1985 Birds of Norfolk lslond. Wonderland Publications, Norfolk lsland.

\section{Northern Marianas}

1118 Pratt, J.D., Bruner, P.L. and Berrett, D.G. $1987 \mathrm{~A}$ field guide to the birds of Hawaii and the tropical Pacific. Princeton University Press. Princeton. New Jersey.

\section{Norway}

594 Haftorn, S. 1971 Norges fugler. Universitcteforlaget, Oslo. Norway

\section{Oman}

53 Anon. 1988 The official list of the birds of the Sultanate of Oman. 2nd ed.

504 Gallagher, M. and Woodcock, M.W. 1980 The birds of Oman. Quartet Books, London.

\section{Pakistan}

1170 Roberts, T. 1991-1992 The birds of Pakistan. 2 vols. Oxford University Press, Karachi.

Palau

1118 Pratt, J.D., Bruner, P.L. and Berrelt, D.G. $1987 \mathrm{~A}$ field guide to the birds of Hawaii and the tropical Pacific. Princeton University Press, Princeton, New Jersey.

Pananua

1153 Ridgely, R.S. 1989 A guide to the birds of Panama, with Costa Rica. Nicaragua and Honduras. Princeton University Press, Princeton. New Jersey.

Papau New Guinea

299 Coates, B.J. 1985-1990 Birds of Papua New Guinea. 2 vols. Dove Publications, Alderley. Australia.

\section{Paraguay}

837 Laubmann, A. 1939-1940 Die Vögel von Paraguay. Wissenschaftliche Ergehnisse der Deutschen GranChaco Expedition. 2 vols. Strecker and Schroder. Stuttgar.

Pery

1064 Parker, T.A., Parker, S.A. and Plenge, M.A. 1982 An annotated checklist of Pertuvian birds. Buteo Books. Vermillion, Snuth Dakota.

\section{Peter I Island}

1466 Watson, G.E. 1975 Birds of the Antarctic and SubAntarctic. American Geophysical Union. Wahington D.C.

\section{Philippines}

399 Dickinson, E.C.. Kennedy, R.S. and Parkes, K.C 1991 The birds of the Philippines. B.O.U. Check-list No.12. British Ornithologists' Union. Tring, Herts.
Pitcairn Islands

1118 Pratt, J.D. Bruner, P.L. and Berrett, D.G. 1987 A ficld guide to the birds of Hawaii and the tropical Pacific. Princeton University Press, Princeton. New Jersey

1506 Williams, G.R. 1960 The birds of the Pitcairn Islands, central South Pacific Ocean. Ibis 102: 58-70.

Poland

1394 Tomialojc, L. 1990 Ptaki Polski rozmieszczenie i liczebnosc. Pantstwowe Wydawnictwo Naukowe, Warszawa.

Portugal

1371 Themido, A.A. 1952 Aves de Portugal. Memoriase Esuudas do Museu Zonlogico de Universidade de Coimbra, no. 213.

\section{Puerto Rico}

1129 Raffaele. H.A. 1989 A guide to the birds of Puerto Rico and the Virgin Islands. Revised edition. Princeton University Press, Princeton, New Jersey.

Qatar

243 Bundy, G. and Warr, E. $1980 \mathrm{~A}$ checklist of the birds of the Arabian Gulf States. Sanlgrouse 1: 4-49.

1459 Warr, F.E. 1986 A list of Qatar's hirds. Qatar Natural Histony Group Joumal 1983-86: 38-51.

Queen Maud Land

1466 Watson, G E. 1975 Birds of the Antorctic and SubAntarctic. American Geophysical Union, Washington D.C.

Réunion

1314 Stauh, F, 1976 Birds of the Mascarenes and Saint Brandon. Organisation Normale des Entreprises Lfée. Lahama House, Por Louis, Mauritius.

Rowania

263 Cataneaunu, I. 1978 Aves. Fauna Respuhlicii Socialiste Romania. Vol. XV. Fasc. 1. Editura Academiei RSR. Bucarest.

Kussia

383 Dementiev, G.P. and Gladkov, N.A. 1951 Birds of the Snviet Union. 6 vols. Israel Program for Scientific Translation, Jerusalem.

481 Flint. V.E., Boehme. R.L., Kostin, Y.V. and Kuznetzov. A.A. 1984 A field guide to birds of the USSR. Princeton University Press, Princeton. New Jersey.

Kwanda

1229 Sihouteden, H. 1966 La faune ornithologique du Rwanda. Doc. zool. Mis. Roy. Afr. centr. 10: 1-130.

Wextern Sahara

1412 Valverde, J.A. 1957 Aves del Sahara Español. Instituto de estudion africanos, Madrid.

Saint Lucia

358 Danforth. S.T. 1935 The birds of Saint Lucia. Monographs of the University of Puerto Rico. Physical and Biological Sciences, series B, number 3

Auerican Samoa

1461 Watling, D. 1982 Birds of Fiji, Tonga and Somoa. Miliwood Press, Wellington.

Western Samna

1461 Watling, D. 1982 Birds of Fiji, Tonga and Samoa. Millwood Press, Wellington.

Sao Toure and Principe

1297 Snow. D. 1950 The hirds of Sào Tome and Principe in the Gulf of Guinea. Ibis 92: 579-59.5.

Sudi Arabia

733 Jennings, M.C. 1981 The hirds of Soudi Arabia: a check-list. Privately published. Wittlesford. UK. 
Senegal

986 Morel, G.J. and Morel, M-Y. 1990 Les oiseaur de Senegambie, notices et cantes de distribution. Editions de l'Orstom, Paris.

Seychelles

240 Bullock, 1. 1990 Birds of the Republic of Seychelles. Seychelles Ministry of Edncation, and the International Council for Bird Presevation, Cambridge, U.K.

1080 Pensy, M. 1974 The birds of Seychelles and the autlying islands. Collins, London.

Sicily

689 lapichino, C. and Massa, B. 1989 The birds of Sicily: an annotated checklist. B.O.U. Check-list No.11. British Ornithologists' Union, Tring, Herts.

\section{Sierra Leove}

110 Bannerman, D.A. 1921 A complete list of the birds known from Sierra Leone. lbis 11(3): 289-302.

\section{Singapore}

526 Gibson-Hill, C.A. 1950 A checklist of the birds of Singapore Island. Bull. Rafles Mus. 21: 132-183.

597 Hails, C.J. and Jarvis, F. 1987 Birds of Singapore. Times Editions, Singapore.

Solomon lslands

593 Hadden. D. 1981 Birds of the North Solomons. Papua New Guinea: Wau Ecology Institute Handbook No. 8)

929 Mayr, E. 1945 Birds of the Southwest Pacific. Macmillan, New York.

\section{Somalia}

101 Ash, J.S. and Miskell, J.E. 1983 Birds of Somalia, their habitat, status and distribution. Scopus special suppl. 1: ]-97

\section{South Africa}

285 Clancey, P.A. ed. 1980 Checklist of southem African birds. Southern African Ornithological Society, Pretoria.

Spain

464 Fernandez-Cruz, M. and Araujo, J. eds. 1985 Simacion de la avifauna de la Peninsula lberica. Baleares y Macaronesia. Sociedad Espanola de Ornitologia, Madrid.

926 Mayol, J. 1978 Els Aucells de Ls Balears. Manuals d'Introdučio a les Natutalesa, 2. Edit. Moll. Mallorca. 150 pp.

Sri I,anka

1094 Phillips, W.W.A. 1978 Annotated checklist of the birds of Ceylon. Wldlife and nature protection socicty, Colombo.

\section{Saint Ilelena}

633 Haydock. G.L. 1954 A survey of the birds of St Helena. Ostrich 25: 62-75.

1346 Swales, M.K. 1965 The sea-birds of Gough Island. Ibis 107: 17-42, 215-229.

Saint Kitts and Nevis

359 Danforth, S.T. 1936 The birds of St Kitts and Nevis. Tropical Agriculure 13(8): 213-217.

Saint Lucia

358 Danforth, S.T. 1935 The birds of Saint Lucia. Monographs of the University of Puerto Rico, Physical and Biological Sciences, series $B$, number 3.

Saint Pierre and Miquelon

438 Etcheherry, R. 1982 Les oiseaux de Saint-Pierre et Miquelon. ONC, Paris.

Saint Vincent and the Grenadines

387 Devas, R.P. 1970 Birds of Grenada and Si Vincent and the Grenadines. [3rd ed.] St. George's Grenada, Carenaga Press XV, 88 pp. Grenada.

Sudan

264 Cave. F.O. and Macdonald, J.D. 1955 The birds of Stulan. Edinghurgh. Oliver and Boyd, Scotland.
1020 Nikolaus, G. 1987 Distributional atlas of Sudan's birds, with notes on habitat and status. Bonner Zoologische Monographien 25.

Suriname

402 Donahue, P.K. and Pierson J.E. 1982 Birds of Suriname: an annatated checklist. Privately published.

Svalbard and Jan Mayen Islands

865 Lovenskold, H.L. 1964. Avifauna Svalbardiensis. Norsk Polarinstitutut Shrifter 129:1-460.

Swaziland

144 Parker, V. 1992 Swoziland bird checklist. The Conservation Trust of Swaziland.

Sweden

1162 Risberg, L. 1990 Sveriges Faglar. Sveriges Ornit. For., Stockholm.

Switzerland

1524 Winkler, R. 1984-1987 Avifauna der Schweiz, eine kommentierte Artenliste. Der Om. Beobachter 81 (5-6). Ala, Schweizerische Gesellschaft fur Vogelkinde und Vogelschulz. Swizzerland.

Syria

809 Kumerloeve, H. 1967-1969 Recherches sur l'avifaune de la République arabe syrienne. Alauda 35: 243-266; $36: 1-26,190-207 ; 37: 43-58,114-134,188-205$.

Taiwan

$1539 W_{u}$ Sen-Hsiong and Yang Hsiouying 1991 A guide to the will birds of Taiwan. Tawian Wild Bird Information Centre and Wild Bird Society of Japan. $274 p p$.

Tajikistan

2 Abdusalyamov, I.A. 1977. [Fauna of the Tadjik Saviet Socialistic Republic. Vol.19 Part 3: birds.] Donish, Dushanbe. (In Russian).

Tanzania

212 Britton, P.L. ed. 1980 Birds of East Africa. East Africa Natural History Society, Nairohi.

Thailand

182 Boonsong Lekagul and Round, P.D. 1991 A guide to the birds of Thailand. Sahe Karn Bhaet Co. Lid. Bangkok.

1118 Pratl, J.D., Bruner, P.L. and Berrett, D.G. $1987 \mathrm{~A}$ field guide to the birds of Hawaii and the tropical Pacific. Princeton University Press, Princeton, New Jersey.

Touga

1461 Watling, D. 1982 Birds of Fiji, Tonga and Sanıa. Millwood Press, Wellington.

Togo

275 Cheke, R.A. 1982 More bird records from the Republic of Togo. Malimbus 4: 55-56

277 Cheke, R.A. and Walsh, J.F. 1980 Bird records from the Republic of Togo. Malimbus 2(2): 112-120.

278 Cheke, R.A. and Walsh. J.F. 1984 Further bird record from the Republic of Togo. Malimbus 6(1/2): 15-22.

280 Cheke, R.A.. Walsh. J.F. and Sowah, S.A. 1986 Records of birds seen in the Republic of Togo during 1984-1986. Malimbus 8(2): 51-72.

1455 Walsh, J.F., Cheke, R.A. and Sowah, S.A. 1990 Additional species and breeding records of birds in the Republic of Togo. Malimbus 12: 2-18.

Trinidad \& Tobago

465 ffrench, R.P. 1991 A guide to the birds of Trinidad and Tobago. 4th edition. Livingston Publishing, Wynnewood, Pennsylvania

Tumisia

1387 Thomsen, P. and Jacohsen, P. 1979 The birds of Tunisia. Peder Jacobsen, Copenhagen. 
Turkey

807 Kumerloeve, H. 1962 Zur Kenntnis der Avifauna Kleinasiens. Bonner Zoologische Beiträge 12: 1-318.

Turks and Caicos Jslands

236 Buden, D.W. 1987 The birds of the southem Bahamas. British Ornithologists' Union Check-list No. 8. BOU, London.

1024 Norton, R.L. and Clarke, N.V. 1989 Additions to the birds of the Turks and Caicos Islands. Florida Field Nat. 17: 32-39.

1211 Sanderson, J. 1982 Birds of the Turks and Caicos Islands. Turks and Caicos Current Nov./Dec. 1982.

Tuvalu

929 Mayr, E. 1945 Birds of the Southwest Pacific. Macmillan, New York.

Uganda

212 Britton, P.L. ed. 1980 Birds of East Africa. East African Natural History Society, Nairobi.

260 Carswell, M. 1984 Checklist of the birds of Uganda. Compiled from Britton, P.L. (ed.) and Backhurst, G.C. Birds of East Africa: their habitat, status and distrbution. East African Natural History Society, Nairobi.

United Arab Emirates

1150 Richardson, C. 1990 The birds of the United Arab Emirares. Hobby Publications, Dubai and Werrington, U.K.

United Kingdom of Great Britain and Northern Ireland

211 British Ornithologists' Union 1971. The stallus of birds in Britain and Ireland. British Ornithologists' Union, London.

Uruguay

354 Cuello, J.P. 1985 Lista de referencia y bibliografia de las oves Uniguayas. Musco Damaso Antonio

Larranaga.

United States of America

16 American Ornithologists' Union 1983 Checklist of Narth Americon birds. 6 th edition. American Ornithologists' Union, New York.

Vanuatu

208 Bregulla, H.L. c1992 Birds of Vanualu. Anthory Nelson, Oswestry, Shropshire, UK.

Venezuela

969 Meyer de Schauensee, R. and Phelps, W.H. 1978 A guide to the birds of Venezuela. Princeton University Press, Princeton, New Jersey.

Vietnam

1442 Vo Quy 1983 [A catalogue of the birds of Vietnam.] Pp. 12-43 in L.N. Medvedev, ed. [Fauna and ecology of the animals of Vietnam.] Nauka, Moskva.

British Virgin Islands

1129 Raffaele, H.A. 1989 A guide to the birds of Puerto Rico and the Virgin Islands. Revised edition. Princeton University Press, Princeton, New Jersey.

Virgin Islands of the United States

1129 Raffaele, H.A. 1989 A guide to the birds of Puerto Rico and the Virgin Islands. Revised edition. Princeton University Press, Princeton, New Jersey.

Wake Island

793 King, W.B. 1974 Conservation status of birds of central Pacific islands. Elepaio $34: 70-74$.

Wallis and Futuna Islands

1118 Pratt, J.D., Bruner, P.L. and Berrett, D.G. $1987 \mathrm{~A}$ field guide to the birds of Hawaii and the tropical Pacific. Princeton University Press, Princeton, New Jersey.
Yemen

220 Brooks, D.J., Evans, M.I., Martins, R.P. and Porter, R.F. 1987 The status of birds in North Yemen and the records of the OSME Expedition in autumn 1985 Sandgrouse 9: 4-66.

1136 Rands, M., Rands, G. and Porter, R. 1988 Birds in the Yemen Arab Republic. Repart of the expedition of the Omithological Saciery of the Middle East. OSME, Sandy, UK.

1160 Ripley, S.D. and Bond, G.M. 1966 The birds of Socotra and Abd-el-Kuri. Smithsonian Miscelloneous Collections 151(7): 1-37

Yugoslavia

921 Matvejev, S.D. and Vasic, V.F. 1973 Caralogus Faunae Jugoslaviae, IV/3, Aves. Yugoslavia.

Zaire

854 Lippens, L. and Wille, H. 1976 Les oiseaux du Zaire. Lannoo, Tielt. Belgium.

Zambia

147 Benson, C.W., Brooke, R.K., Dowset, R.J. and Irwin, M.P.S. 1971 The birds of Zambia. Collins, London.

Zimbabwe

701 Irwin, M.P.S. 1982 The birds of Zimbabwe. Quest Publishing, Harare. 



\section{Class: AVES}

\section{Order STRUTHIONIFORMES}

Ostriches

Family STRUTHION1DAE

Ostriches

Struthio camelus Linnaeus 1758

Ostrich

Breeding:

Algeria (ex); ?Burkina Faso; Cameroon; Central African Republic; Chad; Mali; Mauritania; Morocco (ex); Niger; Nigeria; Senegal; Sudan [1520]

Non-CITES populations:

Angola; Bahrain (ex); Botswana; ?Djibouti; Egypt; Ethiopia, Kenya; Kuwait (ex); Libya (ex); Mozambique; Namibia; Saudi Arabia (ex); Somalia; South Africa; Swaziland;

Tanzania; Tunisia (ex); Uganda; Western Sahara; Zambia; Zimbabwe

\section{Order RHEIFORMES}

Rheas

Family RHEIDAE

Rheas

Rhea americana (Linnaeus 1758)

$11 \quad-\quad 166,930,967$

Greater Rhea, Common Rhea

Breeding:

Argentina [154]; Bolivia; Brazil; Paraguay; Uruguay

Rhea pennata (d'Orbigny 1834)

I

$166,794,930,967$

(= Pterocnemia pennata)

Lesser Rhea, Darwin's Rhea

Breeding:

Argentina [154]; Bolivia; Chile [536]; Peru

\section{Order APTERYGIFORMES}

Kiwis

Family APTERYGIDAE

Kiwis

Apteryx owenii Gould 1847

$\mathrm{K}^{*} \quad 318,976$

Little Spotted Kiwi

Breeding:

New Zealand 


\section{Order TINAMIFORMES}

Tinamous

Family TINAMIDAE

Tinamous

Crypturellus erythropus saltuarius Wetmore 1950

(=C. saltuarius)

Magdalena Tinamou

Breeding:

Colombia

Crypturellus kerriae (Chapman 1915)

ld

$166,321,930,967$

Choco Tinamou:

Breeding:

Colombia; Panama

Nothoprocta kalinowskii Berlepsch \& Stolzmann 1901

Kalinowski's Tinamou

$\mathrm{E} / \mathrm{Ex} \quad 166,318,321$

Breeding:

Peru

Nothoprocta taczanowskii Sclater \& Salvin 1875

V

$166,318,321$

Taczanowski's Tinamou

Breeding: Peru

Nothura minor (Spix 1825)

Id

$318,321,890$

Lesser Nothura

Breeding:

Brazil

Rhynchotus rufescens maculicollis Gray 1867

11

930

Bolivian Red-winged Tinamou

Breeding:

Argentina; Bolivia

Rhynchotus rufescens pallescens Kothe 1907

II

930

Argentinian Red-winged Tinamou

Breeding:

Argentina

Rhynchotus rufescens rufescens (Temminck 1815)

Brazilian Red-winged Tinamou

930

Breeding:

Argentina; Bolivia; Brazil; Paraguay; Peru; Unuguay

Taoniscus nanus (Temminck 1815)

Id

$318,321,1364,1365$

Dwarf Tinamou

Breeding:

Argentina (ex?) [154]; Brazil [1273]

Tinamus osgoodi Conover 1949

K

318,321

Black Tinamou

Colombia; Peru

Tinamus solitarius (Vieillot 1819)

I

$318,794,930,967$

Solitary Tinamou

Breeding:

Argentina [154]; Brazil [1273]; Paraguay 


\section{Order SPHENISCIFORMES}

Penguins

\section{Family SPHENISCIDAE}

Penguins

Megadyptes antipodes (Hombron \& Jacquinot 1841) - $\quad$ K* $318,363,618$

Yellow-eyed Penguin

Breeding: New Zealand: Auckland Islands, Campbell Island, South Island, Stewart Island

Spheniscus demersus (Linnaeus 1758)

\section{Jackass Penguin}

Breeding: Namibia; South Africa

Non-breeding: Angola; Mozambique

Vagrant:
Congo; Gabon

Spheniscus humboldti Meyen 1834

II S

I

Humboldt Penguin, Peruvian Penguin

$214,227,285,318,324,333,413$ $618,780,815,816,1000,1135$, 1518,1519

Breeding:

Vagrant:

Chile [83,536]; Peru [636,637]

Colombia [1132]; Ecuador

\section{Order PODICIPEDIFORMES}

\section{Grebes}

Family PODICIPEDIDAE

Grebes

Podiceps andinus (Meyer de Schauensee 1959)

(= Podiceps nigricollis andinus)

Colombian Grebe

Breeding:

Colombia (ex)

Podiceps taczanowskii Berlepsch \& Stolzmann 1894 -

Puna Grebe, Junin Grebe

Breeding:

Pens [33]

Podilymbus gigas Griscom 1929

I Ex

$318,618,685,794,813$

Atitlan Grebe, Giant Grebe

Breeding:

Guatemala (ex)

Tachybaptus pelzelnii (Hartlaub 1861)

K $\quad 318,324,618$

Madagascar Grebe, Madagascar Little Grebe, Madagascar Dabchick

Breeding: Madagasear [373]

Tachybaptus ruficollis rufolavatus

Delacour 1932

E

$318,324,618$

(= T. rufolavatus)

Alaotra Grebe, Delacour's Little Grebe, Alaotra Dabchick

Breeding:

Madagascar [373] 


\section{Order PROCELLARIIFORMES}

Tube-noses

\section{Family DIOMEDEIDAE}

Albatrosses

Diomedea albatrus Pallas 1769

1 E

$318,618,622,623,624,794$

Short-tailed Albatross, Steller's Albatross

Breeding: Japan: Izu Islands

Non-breeding: North Pacific inc. China; Hawaiian lslands [1118]; Russia [799]; Taiwan

Vagrant:

USA: Alaska

Diomedea amsterdamensis Roux, Jouventin, Mougin, Stahl \& Weimerskirch 1983

Amsterdam Albatross $\quad-\quad$ E $\quad 318,324$

Breeding: $\quad$ French Southern and Antarctic Territories: Amsterdam lsland

Family PROCELLARIIDAE

Petrels and Shearwaters

Procellaria parkinsoni Gray 1862

$\mathrm{E}$

$318,618,691,794,1422$

Black Petrel, Parkinson's Petrel

Breeding: New Zealand [1176]

Nor -breeding: $\quad$ south Pacific north to Costa Rica; Ecuador; Peru

Vagrant: Australia; Mexico

$\begin{array}{lllll}\text { Procellaria westlandica Falla } 1946 & \text { - } & \text { V } & 318,618,794,976\end{array}$

Westland Petrel, Westland Black Petrel

Breeding: New Zealand [1176]

Non-breeding: Australia

Pseudobulweria aterrima (Bonaparte 1857)

E $\quad 318,618,794$

(= Pterodroma aterrima)

Mascarene Petrel, Réunion Petrel, Mascarene Black Petrel

Breeding: $\quad$ Mauritius: Rodrigues (ex); Réunion

Pseudobulweria becki (Murphy 1928)

1d $\quad 318,794$

(= Pterodroma becki, Pterodroma rostrata becki)

Beck's Petrel

Breeding: $\quad$ ?Papua New Guinea (ex?); ?Solomon Islands (ex?)

Pseudobulweria macgillivrayi (Gray 1860)

Id

$318,618,794,1118,1465$

(= Pterodroma macgillivrayi)

MacGillivray's Petrel, Fiji Petrel

Breeding:

Fiji: Gau Island [1464]

Pterodroma axillaris (Salvin 1893)

E $\quad 318,618,794,976$

(= P. hypoleuca axillaris)

Chatham Petrel

Breeding: New Zealand: Chatham Islands [1507]

Pterodroma cahow (Nichols \& Mowbray 1916)

Bermuda Petrel, Cahow

Breeding: Bermuda 
Pterodroma cookii (Gray 1843)

$\mathrm{K}^{*} \quad 318,618,1422$

Cook's Petrel

Breeding:

New Zealand [1175, 1176]

Non-breeding:

Vagrant:

seas off South America; Chile; Peru

Hawaiian Islands; Kiribati; ?USA [1167], USA: Aleutian Islands;

Pterodroma defilippiana (Giglioli \& Salvadori 1869) -

V

$318,321,618$

(= $P$. cookii defilippiana)

Defilippe's Petrel, Mas Atierra Petrel

Breeding: Chile: Desventuradas Islands; Juan Femández Islands [218,1224]

Pterodroma feae (Salvadori 1899)

$\mathbf{R}$

$318,324,618$

(= P. mollis feae)

Cape Verde Petrel, Gon-gon

Breeding: Cape Verde; Madeira

Non-breeding: Tropical and subtropical Allantic

Vagrant: ?Israel

Pterodroma hasitata (Kuhl 1820)

Id

$16,179,237,318,321,609,618$, 794,1458

(excluding subspecies caribbaea)

Black-capped Petrel

Breeding:

Cuba [509]; Dominica (ex); Dominican Republic; Guadeloupe (ex); Haiti; Martinique (ex)

Non-breeding:

Caribbean and western North Atlantic

Vagrant:

Aruba; Brazil; Colombia; Costa Rica; Netherlands Antilles; Puerto Rico; Virgin Islands of the United States; United Kingdom

Pterodroma hasitata caribbaea Carte 1866

$\mathrm{E} / \mathrm{Ex}$

$16,179,321,692$

(= $P$. caribbaea)

Jamaica Petrel

Breeding:

Jamaica (ex?)

Pterodroma madeira Mathews 1934

E

$46,318,324,618,1556$

(= P. mollis madeira)

Madeira Petrel, Freira

Breeding:

Madeira

Pterodroma magentae (Giglioli \& Salvadori 1869)

E

$56,318,352,618,976,994$

Magenta Petrel, Chatham Island Taiko

Breeding: New Zealand: Chatham lslands [55,351,1507]

Non-breeding: South Pacific

Pterodroma phaeopygia (Salvin 1876)

E $\quad 16,318,321,618,794,1458$

Dark-rumped Petrel, Hawaiian Petrel

Breeding: Galapagos Islands [337,353,693,1111]; Hawaiian Islands: Hawaii, ?Kauai, Lanai, Maui, ?Molokai [1118, 1281,1332]

Non-breeding: Chile; Colombia: Malpelo Island; Costa Rica; Ecuador; French Polynesia: Clipperton Island; Marquesas Islands; Kiribati: Line Islands; Mexico; Panama; Peru

Vagrant: Indonesia: Ternate; Japan; Philippines

Pterodroma pycrofti Falla 1933

$K^{*} \quad 318,618$

(= P. longirostris pycrofti)

Pycroft's Petrel

Breeding:

New Zealand [1176, 1507] 
(= P. puffinus auricularis)

(excluding $P$. a. newelli)

Townsend's Shearwater

Breeding: $\quad$ Mexico: Revillagigedos lslands

Puffinus auricularis new'elli Henshaw 1900

$\mathrm{K} *$

$318,617,618,1118$

(= Puffinus newelli)

Newell's Shearwater

Breeding: Hawaiian Islands: Hawaii, Kauai

Vagrant: Northem Marianas

Puffinus creatopus Coues 1864

$\mathrm{V} / \mathrm{R}$

\section{Pink-footed Shearwater}

Breeding: $\quad$ Chile: Isla Mocha, Juan Fernández Islands [218, 1224]

Non-breeding: Cocos (Keeling) Islands; Costa Rica; Ecuador; Peru; USA

Vagrant: New Zealand

Puffinus heinrothi Reichenow 1919

ld

$318,593,618,794,1226$

(=P. lherminieri heinrothi)

Heinroth's Shearwater

Breeding:

Papua New Guinea: Bougainville, New Britain

Family HYDROBATIDAE

Storm-Petrels

Oceanodroma macrodactyla Bryant 1887

$\mathrm{E} / \mathrm{Ex}$

318,321

Guadalupe Storm-Petrel

Breeding:

Mexico: Guadalupe Island (ex?)

Oceanodroma tristrami Salvin 1896

$\mathrm{K} *$

Tristram's Storm-Petrel

Breeding: Hawaiian Islands [1137]; Japan: Izu Islands, Volcano Islands

Family PELECANOIDIDAE

Diving-Petrels

Pelecanoides garnotii (Lesson 1828)

V

$318,321,618,713$

Peruvian Diving-Petrel

Breeding: $\quad$ Chile [1224, 1438]; Peru [412]

\section{Order PELECANIFORMES}

Pelicans, Cormorants and allies

Family PELECANIDAE

Pelicans

Pelecanus crispus Bruch 1832

Dalmatian Pelican

Breeding:

Albania; Armenia; Azerbaijan; Bulgaria; China; Greece; Iran; Kazakhstan; Mongolia; Romania; Russia [799]; Turkey; Turkmenia; Ukraine; Uzbekistan [1223]; Yugoslavia 
Non-breeding: Afghanistan; Bangladesh; Egypt; Hong Kong; lndia; 1raq; Lebanon; Pakistan; Syria

Vagrant: Algeria; Cyprus; Czechoslovakia; Hungary; Israel; Italy; Korea Republic; Kuwait; Latvia; ?Norway; Taiwan; Tajikistan; United Arab Emirates; Western Sahara

Pelecanus philippensis Gmelin 1789

$\mathrm{K}^{*}$ $10,318,349,618,792$

Spot-billed Pelican, Grey Pelican

Breeding:

China (ex); India; Malaysia: Peninsular Malaysia (ex); Myanmar (ex); Philippines (ex); Sri Lanka

Non-breeding: $\quad$ Bangladesh; Cambodia; China; Indonesia: Java, Sumatra; Lao P.D.R; Malaysia: Peninsular Malaysia; Myanmar; Nepal; Thailand; Viet Nam

Family SULIDAE

Gannets and Boobies

Papasula abbotti Ridgway 1893

(= Sula abbotti)

I E

$190,318,618,794,1012,1013,1330$

Abbott's Booby

Breeding:

Christmas Island [1147]; ?Costa Rica: Cocos (Keeling) Islands [1012]; Seychelles: Assumption (ex) [1327]

Non-breeding: Indonesia: Java [128]

Vagrant: Indonesia: Moluccas [1287], Sumatra

\section{Family PHALACROCORACIDAE}

Cormorants

Phalacrocorax carunculatus (Gmelin 1789)

K* $\quad 318,618,976$

(= Leucocarbo carunculatus)

Rough-faced Shag

Breeding:

New Zealand [1176]: Cook Strait

Phalacrocorax chalconotus (Gray 1845)

$\mathrm{K}^{*}$

318,1176

Bronze Shag

Breeding:

New Zealand: South Island, Stewart Island

Phalacrocorax harrisi (Rothschild 1898)

$\mathbf{R}$

$318,321,615,618,794,1190,1191$

(= Nannopterum harrisi)

Flightless Cormorant, Galapagos Cormorant

Breeding: Galapagos Islands: Fernandina, Isabela

Phalacrocorax onslowi Forbes 1893

$\mathrm{K}^{*} \quad 318,1176$

Chatham Shag

Breeding: $\quad$ New Zealand: Chatham Islands

Phalacrocorax pygmaeus (Pallas 1773)

$K^{*} \quad 318,344,618,661$

(= Halietor pygmaeus)

Pygmy Cormorant

Breeding:

Albania; Algeria (ex); Azerbaijan; Bulgaria; Greece; Hungary; Iran; Iraq; lsrael (ex); ltaly [148]; Kazakhstan; Moldova; Romania; Russia [799]; Tajikistan; Turkey; Turkmenia; Uzbekistan; Yugoslavia

Non-breeding: Afghanistan; Austria; Georgia; lsrael

Vagrant:

Czechoslovakia; France; Germany; Lebanon; Pakistan; Poland; Sweden; Switzerland; Tunisia; Ukraine 
Family FREGATIDAE

Frigatebirds

Fregata andrewsi Mathews 1914

I $\quad \mathrm{V} \quad 318,618,794,934,1330,1486$

\section{Christmas Frigatebird, Andrews' Frigatebird}

\section{Breeding: Christmas Island}

Non-breeding: Indonesia: Bali, Java, Sumatra; Malaysia: Peninsular Malaysia, Sabah, Sarawak [1295]; Singapore; Thailand; Viet Nam

Vagrant: $\quad$ Australia; Brunei; Cocos (Keeling) 1slands; Indonesia: Kalimantan [445], Timor [934]; Japan; Kenya [896]

Fregata aquila (Linnaeus 1758)

$\mathbf{R}$

$318,324,618$

\section{Ascension Frigatebird}

Breeding: Ascension: Saint Helena

Non-breeding: tropical Atlantic

\section{Order CICONIIFORMES}

Storks and allies

Family ARDEIDAE

Herons and Bitterns

Ardea goliath Cretzschmar 1827

III GH

$212,227,285,606,661,930,1247$, 1298

Goliath Heron

Breeding:

Angola; Benin; Botswana; Burkina Faso [748,1388]; Burundi; Cameroon; Central African Republic; Chad; Congo; Côte d'Ivoire; Djibouti; Equatorial Guinea; Ethiopia [748]; Gabon; Gambia; Ghana; Guinea; Guinea-Bissau [1299]; Iran; Iraq; Kenya; ?Liberia; Malawi; ?Mali; Mozambique; Namibia; Niger; ?Nigeria; Rwanda; ?Saudi Arabia; Senegal [1299]; Sierra Leone; ?Somalia; South Africa; Sudan; Swaziland; Tanzania; Togo [280]; Uganda; ?Yemen; Zaire; Zambia; Zimbabwe

Non-breeding: Saudi Arabia; Yemen

Vagrant: Bangladesh; Egypt; India; Israel; Lesotho; Oman; Pakistan; Sri Lanka; Syria

Ardea humbloti Milne-Edwards \& Grandidier 1885 -

K $\quad 318,324,606$

Madagascar Heron

Breeding: ?Comoros [409]; Madagascar [373]

Non-breeding: Comoros [1299]; Mayotte [1299]

Ardea insignis Hume 1878

$K^{*} \quad 10,318,606$

(= Ardea imperialis Baker 1929)

White-bellied Heron, Imperial Heron

Breeding:

Bangladesh; Bhutan; India; Myanmar; Nepal (ex)

Bubulcus ibis (Linnaeus 1758)

$1 \mathrm{II} \mathrm{GH}-$

$10,16,179,212,227,285,344,606$, $661,792,1118,1247,1298$

(= Ardeola ibis)

Cattle Egret

Breeding:

Algeria; Angola; ?Anguilla; Antigua and Barbuda; Argentina; Armenia; Aruba; [Australia] [167]; Azerbaijan; Bangladesh; Barbados; Belize; Benin [667]; ?Bhutan; Bolivia; Botswana; Brazil; British Virgin Islands; Burkina Faso; Burundi; Cambodia; Cameroon; Canada; Cape Verde; Cayman Islands; Central African Republic; Chad; Chile; 
Non-breeding: Australia; Bahamas; Bahrain; Bermuda; Brunei; Cocos (Keeling) Islands [1331]; Côte

China; Colombia; Comoros; Congo; Costa Rica; Cuba; Dominica; Dominican Republic; Ecuador; Egypt; El Salvador; Ethiopia; France; French Guiana; Gambia; Ghana; Grenada; Guadeloupe; Guatemala; Guinea-Bissau; Guyana; Haiti; [Hawaiian Islands]; Honduras; Hong Kong; India: including Andaman and Nicobar Islands; Indonesia; Iran; Israel; Italy; Jamaica; Japan; Kazakhstan; Kenya; D.P.R. Korea; Korea Republic; Lao P.D.R; Lesotho; Madagascar [373]; Malawi; ?Maldives; Mali; Martinique; Mauritania; Mauritius; Mayotte; Mexico; Montserrat; Morocco; Mozambique; Myanmar; Namibia; Nepal; Netherlands Antilles; Nicaragua; Niger; Nigeria; Pakistan; Panama; Peru; Philippines; Portugal; Puerto Rico; ?Réunion; Russia [799]; Rwanda; Sao Tome and Principe: Sao Tome; Saudi Arabia; Senegal; [Seychelles?]; South Africa; Spain; Sri Lanka; Saint Kitts and Nevis; Saint Lucia; Saint Vincent; Sudan; Suriname; Swaziland; Taiwan; Tanzania; Thailand; Trinidad and Tobago; ?Tunisia; Turkey; Turkmenia; ?Turks and Caicos Islands; USA; Uganda; Uruguay; Venezuela; Viet Nam; Virgin Islands of the United States; Yemen; Zaire; Zimbabwe d'lvoire; Cyprus; Djibouti; Equatorial Guinea; Falkland Islands; Federated States of Micronesia; Gabon; Gibraltar; Guinea; Iraq; Jordan [1451]; Kuwait; Lebanon; Liberia; Libya; Malaysia: Peninsular Malaysia, Sabah, Sarawak [1295]; Maldives; New Zealand; Northern Marianas; Oman; Palau; Papua New Guinea; Sierra Leone; Singapore; Somalia; Syria; Togo; Tunisia; United Arab Emirates; Westem Sahara; Zambia

Vagrant: $\quad$ Austria; Belarus; Belgium; Bulgaria; Canary Islands; Christmas Island; Denmark; Germany; Greece; Guam; Guemsey; Hawaiian Islands; Hungary; Iceland; Ireland; Johnston Atoll; Liechtenstein; Lithuania; Luxembourg; Malta; Marshall Islands; Netherlands; Norfolk Island; Paraguay [1334]; Qatar; Romania; Saint Helena; South Shetland Islands; Sweden [52]; Switzerland; United Kingdom; Uzbekistan; Yugoslavia

Casmerodius albus (Linnaeus 1758)

III $\mathrm{GH}-$

$10,16,179,212,227,285,344,606$, $661,792,967,1247,1298$

(= Egretta alba)

Great Egret, Great White Egret

Breeding:

?Albania; Angola; Antigua and Barbuda; Argentina; Armenia; Australia [167]; Austria; Bahamas; Bangladesh; Belize; Benin [667]; ?Bhutan; Bolivia; Botswana; Brazil; Bulgaria; Cambodia; ?Cameroon; Canada; ?Central African Republic; ?Chad; Chile; China; Colombia; Comoros: Moheli; Congo; Costa Rica; Côte d'Ivoire; Cuba; Czechoslovakia; Dominican Republic; Ecuador; El Salvador; Ethiopia [748]; French Guiana; Gambia; Georgia; Ghana; Greece; Guatemala; Guinea; Guinea-Bissau; Guyana; Haiti; Honduras; Hong Kong; Hungary; India; ?Indonesia; Jamaica; Japan; Kazakhstan; Kenya; D.P.R. Korea [922]; Korea Republic; Lao P.D.R; ?Latvia; ?Lesotho; Madagascar [373]; Malawi; Malaysia: Peninsular Malaysia, ?Sabah; Mali; Mauritania; Mexico; Mongolia; Mozambique; Myanmar; Namibia; Nepal; Netherlands; Nicaragua; Niger; Nigeria; Pakistan; Panama; Papua New Guinea; Paraguay; Peru; Puerto Rico; Romania; Russia; Rwanda; Senegal; Sierra Leone; ?Somalia; South Africa; Sri Lanka; Sudan; Suriname; Swaziland; Tajikistan; Tanzania; Thailand; Togo; Trinidad and Tobago; Turkey; Turkmenia; Uganda; Ukraine; USA; Uruguay; Uzbekistan; Venezuela; Viet Nam; Virgin Islands of the United States; Yugoslavia; Zaire; Zambia

Non-breeding: Afghanistan; Albania; Algeria; Anguilla; Aruba; Bahrain; Barbados; Bermuda; Bhutan; British Virgin Islands; Brunei; Burundi; Cameroon; Cape Verde; Cayman Islands; Central African Republic; Chad; Christmas Island [1330]; Cyprus; Dominica; Egypt; Equatorial Guinea; France; Gabon; Grenada; Guadeloupe; Indonesia; Iran; Iraq; Israel; Italy; Jordan [1451]; Kuwait; Latvia; Lebanon; Lesotho; Liberia; Malawi; Malaysia: Sabah, Sarawak [1295]; Maldives; Martinique; Mayotte; Netherlands Antilles; Oman; Philippines; Qatar; Saudi Arabia; Singapore; Somalia; Saint Kitts and Nevis; Saint Lucia; Saint Vincent; Switzerland; Syria; Taiwan; Tunisia; Turks and Caicos Islands; United Arab Emirates; Yugoslavia 
Vagrant: $\quad$ Belgium; Canary Islands; Denmark; Falkland Islands; Finland; Germany; Hawaiian Islands; India: Andaman Islands; Ireland; Libya; Liechtenstein; Malta; Mauritania; Morocco; Norway; Poland; Portugal; Saint Lucia; Seychelles [461]; South Georgia; Spain; Sweden; United Kingdom; Yemen

Egretta eulophotes (Swinhoe 1860) - V V $\quad 318,606,794,834$

Chinese Egret

Breeding:

Non-breeding:

Vagrant:

Egretta garzetta (Linnaeus 1758)

Little Egret

Breeding:

Non-breeding:

Vagrant:
China; ?Hong Kong; D.P.R. Korea [1310]; Korea Republic

Brunei; Japan; Malaysia: Peninsular Malaysja, Sabah, Sarawak [681]; Philippines: Basilan, Batan, Bohol, Cebu, Luzon, Mindoro, Palawan, Panay, Samar [513,1113]; Russia [799]; Singapore; Taiwan [944]

Indonesia: Java [26], Kalimantan [445], Manterawu [1486], Sulawesi [1486], Sumatra; Malaysia: Sabah; USA: Aleutian Islands

III GH -

$10,16,212,227,285,344,606,661$, $792,967,1247,1298$

?Albania; Algeria; Angola; Armenia; Azerbaijan; Australia [167]; Austria; Bangladesh; Benin [667]; ?Bhutan; Botswana; Brunei; Bulgaria; ?Burkina Faso; Cambodia; Cameroon; Cape Verde; Central African Republic; ?Chad; China; Côte d'Ivoire; Cyprus; Czechoslovakia; Egypt; Ethiopia; France; Gambia; Georgia; Ghana; Greece; Guinea; Guinea-Bissau; Hong Kong; Hungary; India: including Andaman and Nicobar Islands; Indonesia; Iran; Israel; Italy; Japan; Kenya; Korea Republic; Lao P.D.R; ?Lesotho; Liberia; Mali; Malawi; Mauritania; Morocco; Mozambique; Myanmar; Namibia; Nepal; Netherlands; Niger; Nigeria; Pakistan; ?Papua New Guinea; Portugal; Romania; Russia; Senegal; Sierra Leone; South Africa; Spain; Sri Lanka; Swaziland; Syria; Taiwan; Tanzania; Thailand; Togo; Tunisia; Turkey; Turkmenia; Uganda; Ukraine; ?Uzbekistan; Viet Nam; Yugoslavia; Zambia; Zimbabwe

Afghanistan; Albania; Bahrain; Burundi; Canary lslands; Chad; Christmas Island; Congo; Cyprus; Czechoslovakia; Djibouti; Equatorial Guinea; Federated States of Micronesia: Yap; ?Gabon; Germany; Gibraltar; lraq; Jordan [1451]; D.P.R. Korea; Kuwait; Lebanon; Lesotho; Libya; Malaysia: Peninsular Malaysia, Sabah, Sarawak [1295]; Malta; Morocco; Northern Marianas: Saipan; Oman; Palau; Philippines; Qatar; Rwanda; Sao Tome and Principe; Saudi Arabia; Seychelles [460]; Singapore; Somalia; Sudan; Switzerland; United Arab Emirates; Western Sahara; Yemen; Zaire

Barbados [179]; Belgium; Canada; Cocos (Keeling) lslands; Comoros [483]; Denmark; Finland; Guernsey; lreland; Jersey; Latvia; Liechtenstein; Luxembourg; Maldives; Martinique [179]; Netherlands; Norway; Poland; Saint Lucia [179]; Suriname [629]; Sweden; Trinidad and Tobago: Trinidad [179]; United Kingdom

Egretta vinaceigula (Sharpe 1895)

(= Hydranassa vinaceigula)

Slaty Egret

Breeding:

Botswana; Namibia; ?Zambia [407]

Non-breeding:

Vagrant:

?Malawi; South Africa; Zimbabwe

Gorsachius goisagi (Temminck 1835)

\section{Japanese Night-Heron}

Breeding: Japan [1494]

Non-breeding: China; Philippines; Taiwan

Vagrant:

Palau 
Gorsachius magnificus (Ogilvie Grant 1899)

X*

318,606

White-eared Night-Heron

Breeding:

China: Hainan

Vagrant:

Viet Nam [1442]

\section{Family BALAEN1CIPITIDAE}

Shoebills

Balaeniceps rex Gould 1850

$11 \mathrm{~S}$

$212,227,285,318,324,607,1298$

Shoehill, Whale-headed Stork

Breeding:

?Burundi; Central African Republic; Ethiopia [411]; Rwanda; Sudan; Tanzania; Uganda; Zaire; Zambia

Vagrant: $\quad$ ?Angola; Botswana; ?Cameroon [855]; Malawi [610]

\section{Family CICONIIDAE}

Storks

Ciconia boyciana Swinhoe 1873

$1 \quad \mathrm{E}$

$318,607,794,876$

(= C. ciconia boyciana)

Oriental Stork, Oriental White Stnrk

Breeding: $\quad$ China [462,1509]; Japan (ex); Korea Republic (ex) [40]; Russia [799]

Non-breeding: Japan; D.P.R. Korea [1310]; Korea Republic

Vagrant: Bangladesh; Hong Kong; India; Mongolia; Taiwan

Ciconia nigra (Linnaeus 1758)

II

$10,212,227,285,344,607,661,1298$

Black Stork

Breeding:

?Afghanistan; Albania; Armenia; Austria; Azerbaijan; Belarus; Belgium; ?Botswana; Bulgaria; China; Czechoslovakia; Estonia; France; Georgia; Germany; Greece; Hungary; Iran; Kazakhstan; ?D.P.R. Korea; Kyrgyzstan; Latvia; Lesotho; Lithuania; Luxembourg; Malawi; Moldova; Mongolia; Mozambique; Namibia [1389]; Poland; Portugal; Romania; Russia [799]; South Africa; Spain; Swaziland; Tajikistan; Turkey; Ukraine; Uzbekistan; Yugoslavia; Zambia; Zimbabwe

Non-breeding: Afghanistan; Algeria; Angola; Bhutan; Botswana; ?Cameroon; Central African Republic; Chad; Cyprus; Denmark; Egypt; Ethiopia; Gibraltar; Hong Kong; India; Iraq; 1srael; 1taly; Jordan [1451]; Kenya; D.P.R. Korea; Korea Republic; Lebanon; Malta; Mauritania; Morocco; Myanmar; Nepal; Netherlands; ?Niger; Nigeria; Pakistan; Saudi Arabia; Senegal; Sudan; Sweden; Switzerland; Syria; Tanzania; Tunisia; Turkmenia; Uganda; Viet Nam; Yemen; ?Zaire

Vagrant: $\quad$ Finland; Gambia; Ghana; Guernsey; Guinea-Bissau; Ireland [749]; Japan; Kuwait; Lao P.D.R; Libya; Liechtenstein; Mali; Norway; Rwanda; Sri Lanka; Taiwan; Thailand; United Arab Emirates; United Kingdom

Ciconia stormi (Blasius 1896)

Id

$318,607,876$

(= C. episcopus stormi)

Storm's Stork

Breeding:

Indonesia: Kalimantan [664], ?Sumatra [57,663]; Malaysia: Sabah, Sarawak [1295]; Thailand [1001]

Non-breeding: Brunei; Malaysia: Peninsular 
Saddle-billed Stork, Saddlebill Stork

Breeding:

Angola; Benin; Botswana; Burundi; Cameroon; Central African Republic; Chad; Congo; Côte d'lvoire [1453]; Equatorial Guinea; Ethiopia; Gabon; Ghana; Guinea-Bissau; Kenya; Malawi; Mali; Mauritanja; Mozambique; ?Namibia; Niger; ?Nigeria; Rwanda; Senegal;

Non-breeding: Somalia

Vagrant: Gambia
Jabiru mycteria (Lichtenstein 1819)
1
$16,607,967$

Jabiru

Breeding:

Argentina; Belize; Bolivia; Brazil [1268]; Colombia; Costa Rica; Ecuador; El Salvador; French Guiana; Guatemala [127,836]; Guyana; Honduras; Mexico; Nicaragua; Paraguay; Peru; Suriname; Uruguay; Venezuela

Vagrant:

Panama; Trinidad and Tobago [1189]

Leptoptilos crumeniferus (Lesson 1831)

III GH -

$212,227,285,607,1247,1298$

Marabou Stork

Breeding:

Angola; Benin; Botswana; Burkina Faso; Burundi; Cameroon; Central African Republic; Chad; Congo; Ethiopia; ?Gabon; Gambia; Ghana; Guinea; Guinea-Bissau; Kenya; Malawi; Mali; Mauritania; Mozambique; Namibia; Niger; Nigeria; Rwanda; Senegal; Sierra Leone; South Africa [216,774]; Sudan; Swaziland [1068]; Tanzania; Togo; Uganda; Zaire; Zambia; Zimbabwe

Non-breeding: Côte d'1voire; Somalia

Vagrant:

Israel; Spain

Leptoptilos dubius (Gmelin 1789)

$\mathrm{K}^{*}$

$10,318,607,876$

Greater Adjutant

Breeding:

Bangladesh (ex); Cambodia (ex?); India; Lao P.D.R (ex?); Myanmar (ex?); Pakistan (ex); Thailand (ex); Viet Nam (ex?)

Non-breeding: Nepal [700]; Thailand

$\begin{array}{llll}\text { Leptoptilos javanicus (Horsfield 1821) } & \text { - } & K^{*} & 10,318,607,876\end{array}$

Lesser Adjutant

Breeding:

Bangladesh; ?Cambodia; China; India; Indonesia: Bali [231j, Kalimantan [664], Sumatra; Malaysia: Peninsular Malaysia, Sabah, Sarawak [1295]; ?Lao P.D.R; Myanmar; Nepal; Sri Lanka; Thailand; Viet Nam

Non-breeding: Bhutan; Brunei; Singapore

Mycteria cinerea (Raffles 1822)

Milky Stork

I V $\quad 318,607,792,794,1424$

Breeding:

Cambodia; Indonesia: Java, Sumatra [1279, 1280], Sulawesi; Malaysia: Peninsular Malaysia; Viet Nam

Family THRESKIORNITHIDAE

Ibises and Spoonbills

Bostrychia hagedash (Latham 1790)

(= Hagedashia hagedash)

III GH - $\quad 212,227,285,607,1247,1298$

Hadada lbis

Breeding:

Angola; Benin; Botswana; Burkina Faso; Burundi; Cameroon; Central African Republic; Chad; Congo; Côte d'Ivoire; Equatorial Guinea: Bioko; Ethiopia; Gabon; ?Gambia; Ghana; Guinea; Guinea-Bissau; Kenya; Lesotho; Liberia; Malawi; ?Mali; ?Mauritania; Mozambique; ?Namibia; Niger; Nigeria; Rwanda; ?Senegal; Sierra Leone; South Africa; Sudan; Swaziland; Tanzania; Togo; Uganda; Zaire; Zambia; Zimbabwe

Non-breeding: Somalia 
Bostrychia olivacea bocagei (Chapin 1923)

Bostrychia rara (Rothschild, Hartert \& Kleinschmidt 1897)

(= Lampribis rara)

$111 \mathrm{GH}$

$227,607,1247,1298$

Spot-breasted lbis

Breeding:

Angola; Central African Republic; Congo; Côte d'lvoire; ?Equatorial Guinea [1454]; Gabon; Ghana; Guinea; Liberia; ?Sierra Leone

$\begin{array}{lll}\begin{array}{l}\text { Eudocimus ruber } \\ \text { Scarlet Ibis }\end{array} & \text { (Linnaeus 1758) } \\ \text { Breeding: } & \text { Brazil; ?Colombia; Suriname; Venezuela } \\ \text { Non-breeding: } & \text { Colombia; French Guiana; Guyana; Trinidad and Tobago [35] } \\ \text { Vagrant: } & \text { Argentina; Ecuador; Grenada; Jamaica; USA }\end{array}$

Geronticus calvus (Boddaert 1783)

$11 \mathrm{R}$

$216,227,285,318,324,607,899,900$

Bald Ibis, Southern Bald lbis

Breeding:

Lesotho; South Africa; Swaziland

Geronticus eremita (Linnaeus 1758)

$1 \quad \mathrm{E}$

E $\quad 227,318,324,344,607,810$

Waldrapp, Northern Bald Ibis, Hermit Ibis

Breeding: $\quad$ Algeria (ex); Greece (ex) [386]; Morocco [75]; Turkey (ex) [5]

Non-breeding: Ethiopia; Syria; Yemen [220]

Vagrant: $\quad$ Algeria; Austria; Azores; Cape Verde; Egypt [812]; Germany; 1raq; Israel; ?Jordan; Mali; Mauritania; Saudi Arabia; Senegal; Somalia; Spain; Sudan; Westem Sahara; Yugoslavia

Nipponia nippon (Temminck 1835)

$1 \quad$ E

$318,607,794,875,876,1257,1258$

Crested Ibis, Japanese Crested Ibis

Breeding: $\quad$ China; Japan (ex); D.P.R. Korea (ex); Russia (ex) [799]

Vagrant: $\quad$ Japan; D.P.R. Korea; Korea Republic; Russia; Taiwan

Platalea leucorodia Linnaeus $1758 \quad 11 \quad$ - $\quad 10,227,344,607,661$

Eurasian Spoonbill, White Spoonbill

Breeding: $\quad$ Albania; Austria (ex?); Azerbaijan; Bangladesh; Bulgaria; China; Czechoslovakia; Djibouti; Egypt; Ethiopia; France [904]; Greece; Hungary; India; Iran; Iraq; Italy; Kazakhstan; Mauritania; Moldova; Mongolia; Netherlands; Pakistan; Portugal; Romania; Russia [799]; Saudi Arabia; Somalia; Spain; Sri Lanka; Sudan; Turkey; Turkmenia; Ukraine; Uzbekistan; Yemen; Yugoslavia

Non-breeding: Afghanistan; Algeria; Belgium; Canary lslands; Central African Republic; Chad; Cyprus; Denmark; Germany; Hong Kong; Ireland; Israel; Japan; D.P.R. Korea; Korea Republic; Kuwait; Lebanon; Libya; Mali; Malta; Morocco; Myanmar; Nepal; Oman; Saudi Arabia; Senegal; Sudan; Switzerland; Syria; Taiwan; Tajikistan; Tunisia; United Arab Emirates; United Kingdom; Western Sahara

Vagrant: $\quad$ Bahrain; Burkina Faso; ?Cameroon; Cape Verde; Faeroe Islands; Finland; Gambia; Guernsey; Guinea-Bissau; Iceland; Jersey; Jordan; Kenya; Latvia; Luxembourg; Niger; Nigeria; Norway; Poland; Sweden; Tanzania; Thailand; Uganda; Zaire

Platalea minor Temminck \& Schlegel $1849 \quad$ - $\quad K^{*} \quad 318,607$

Black-faced Spoonbill

Breeding: $\quad$ China [778]; D.P.R. Korea [1310]

Non-breeding: $\quad$ Cambodia; China [778]; Hong Kong; Japan; Taiwan; Viet Nam

Vagrant: $\quad$ Brunei; Philippines; Thailand 
Pseudibis davisoni (Hume 1875)

Id

$318,607,792,794,1295$

(= P. papillosa davisoni)

White-shouldered Ibis

Breeding:

?Cambodia; China; Indonesia: Kalimantan [664]; ?Lao P.D.R; ?Myanmar; Thailand (ex) [1194]; Viet Nam

Non-breeding: Malaysia: Peninsular Malaysia, Sarawak [1295]

Pseudibis gigantea (Oustalet 1877)

R $\quad 318,607,792,794$

(= Thaumatibis gigantea)

Giant Ibis

Breeding: $\quad$ Cambodia (ex?); ?Lao P.D.R; Thailand (ex) [1194]; Viet Nam (ex)

Threskiornis aethiopicus (Latham 1790) III GH - 212,227,285,344,607,661,794, 1247,1298

Sacred Ihis

Breeding:

Angola; Botswana; Bunundi; Cameroon; Central African Republic; Chad; Egypt (ex); Equatorial Guinea; Ethiopia; Gambia; Ghana; Guinea-Bissau; Iran; Iraq; Kenya; ?Lesotho; Malawi; Mali; Mauritania; Mozambique; Namibia; Niger; Nigeria; Rwanda; Senegal; South Africa; Sudan; Swaziland; Tanzania; Uganda; Zaire; Zambia; Zimbabwe

Non-breeding: ?Benin; Congo; Djibouti; Gabon; Guinea; Lesotho; Somalia; Togo

Vagrant: Azerbaijan; Bahrain; Côte d'lvoire; Egypt; Kuwait; Oman; Saudi Arabia. Sierra Leone; Yemen

Family PHOENICOPTERIDAE

Flamingos

Phoenicopterus andinus Philippi 1854

II

$166,318,930,967,1032$

(= Phoenicoparrus andinus)

Andean Flamingo

Breeding:

Vagrant:

Argentina [154]; Bolivia; Chile; Penu

Brazil [132]

Phoenicopterus chilensis Molina 1782

II

$930,967,1032$

Chilean Flamingo

Breeding:

Argentina; Bolivia; Brazil; Chile; Paraguay; Peru; Unuguay

Vagrant: Ecuador; Falkland Islands

Phoenicopterus jamesi Sclater 1886

II

$166,318,930,967,1032$

(= Phoenicoparrus jamesi)

Puna Flamingo, James' Flamingo

Breeding:

Argentina [154]; Bolivia; Chile; Peru

Phoenicopterus minor Geoffroy Saint-Hilaire $1798 \quad$ II $\quad-\quad 10,212,227,285,661,1032$

(= Phoeniconaias minor)

Lesser Flamingo

Breeding:

Botswana; Ethiopia; India; Kenya; Madagascar; Mauritania; Namibia; South Africa [215]; Tanzania; ?Uganda; ?Zaire; ?Zambia

Non-breeding: Angola; ?Burundi; Chad; Lesotho; Malawi; Mozambique; Pakistan; Rwanda; Senegal [1089]; Sudan; Uganda; Yemen; Zaire; Zambia; Zimbabwe

Vagrant: $\quad$ Afghanistan; Cameroon; Comoros [1401]; Congo; Gambia; Guinea-Bissau; 1ran; Mauritius; Morocco; Niger; Nigeria; Oman; Réunion; Sao Tome and Principe: Sao Tome; Seychelles; Somalia; Spain; Swaziland 
(includes $P$. roseus Pallas 1811)

Greater Flamingo, Caribbean Flarningo, American Flamingo

Breeding:

Afghanistan; Bahamas; Botswana; Cuba; Dominican Republic; ?Egypt; Ethiopia [770]; France; Galapagos Islands; Haiti [1049]; India; Iran; ltaly; Kazakhstan; Kenya; Madagascar; Mauritania; Mauritius: Rodrigues; Mexico; Morocco; ?Mozambique; Namibia; Netherlands Antilles; Pakistan; Senegal; South Africa; Spain; Tanzania; Tunisia; Turkey

Non-breeding: Algeria; Angola; Aruba; Bahrain; Brazil; Burundi; Cape Verde; Colombia; Cyprus; Djibouti; Egypt; French Guiana; Gambia; Gibraltar; Greece; Guyana; Iraq; Israel; Jamaica; Kuwait; Lebanon; Libya; Malawi; Oman; Portugal; Qatar; Saudi Arabia; Seychelles: Aldabra, Mahé [460]; Sierra Leone; Somalia; Sri Lanka; Sudan; Suriname; Syria; Turkmenia; Turks and Caicos Islands; Uganda; United Arab Emirates; USA; Uzhekistan; Venezuela; Western Sahara; Yemen; Zaire; Zambia; Zimbabwe

Vagrant: $\quad$ Antigua and Barbuda; Austria; Bangladesh; ?Belgium; Belize; Bermuda; British Virgin Islands; Bulgaria [1418]; Cambodia; Canada; Canary Islands; Cocos (Keeling) lslands [979]; Comoros [1401]; Congo; Czechoslovakia; Denmark; Equatorial Guinea; Finland; Gabon; ?Germany; Guadeloupe; Guinea; Hungary; Jamaica; Jordan [1451]; Latvia; Liberia; Malta; Mauritius; Nepal; Norway; Poland; Puerto Rico; Réunion; Romania; Saint Lucia; Swaziland; ?Sweden; ?Switzerland; Virgin Islands of the United States; Yugoslavia

\title{
Order ANSERIFORMES \\ Waterfowl
}

\section{Family ANATIDAE \\ Waterfowl}

\author{
Aix galericulata (Linnaeus 1758) - $\quad \mathrm{K}^{*} \quad 44,889,1313$ \\ Mandarin Duck \\ Breeding: \\ [Austria]; [Belgium]; China; Japan; D.P.R. Korea; Korea Republic; [Netherlands]; Russia \\ [799]; [United Kingdom] \\ Non-breeding: Taiwan \\ Vagrant: $\quad$ Hong Kong; lndia; Myanmar; Nepal; Thailand; Viet Nam
}

$\begin{array}{lll}\text { Alopochen aegyptiacus (Linnaeus 1766) } & 111 \mathrm{GH} \quad-\quad 212,227,285,344,661,889,1247,\end{array}$

\section{Egyptian Goose}

Breeding:

Angola; [Belgium]; Benin; Botswana; Burundi; Cameroon; Central African Republic; Chad; Congo; ?Djibouti; Egypt; Equatorial Guinea; Ethiopia; Gabon; Guinea; Guinea-Bissau; Israel (ex); Kenya; Lesotho; Liberia; Malawi; Mali; Mauritania; Mozambique; Namibia; [Netherlands]; Niger; Nigeria; Rwanda; Senegal; Sierra Leone; Somalia; South Africa; Sudan; Swaziland; Tanzania; Uganda; [United Arab Emirates]; [United Kingdom]; Zaire; Zambia; Zimbabwe

Non-breeding: Algeria; ?Cyprus; Tunisia

Vagrant: Côte d'Ivoire; France; Gambia; Ghana; Hungary; lsrael; Malta; Spain; Syria 


\title{
Northern Pintail, Common Pintail
}

Breeding: $\quad$ Afghanistan; Austria; Belgium; Bulgaria; Canada; China; Czechoslovakia; Denmark; Estonia; Faeroe Islands; Finland; France: [Saint Paul, Amsterdam Island]; Germany; Greenland; Hungary; Iceland; ?Ireland; Kazakhstan; Latvia; Lithuania; Mongolia; Morocco; Netherlands; Norway; Poland; Romania; Russia; Spain; Sweden; Tunisia; Turkey; USA; United Kingdom

Non-breeding: $\quad$ Albania; Algeria; Bahamas; Bahrain; Bangladesh; Belize [433]; ?Benin; Bermuda; Bhutan; Burkina Faso; ?Burundi; Cambodia; Cameroon; Canton and Enderbury Islands; Central African Republic; Chad; Costa Rica; Cuba; Cyprus; ?Djibouti; Dominican Republic; Egypt; Ethiopia; Federated States of Micronesia [1118]; Gambia; Ghana; Greece; Guatemala; Guernsey; Guinea [1454]; ?Guinea-Bissau; Haiti; Hawaiian Islands; Honduras; Hong Kong; India; Iran; Iraq; Ireland; Israel; Italy; Jamaica; Japan; Jersey; Jordan [1451]; Kenya; Kiribati: Gilbert lslands; D.P.R. Korea; Korea Republic; Kuwait; Lao P.D.R; Lebanon; Libya; Liechtenstein; Luxembourg; Malawi; Mali; Malta; Marshall Islands; Mauritania; Mexico; Myanmar; Nepal; Nicaragua; Niger; Nigeria; Northern Marianas; Oman; Pakistan; Palau; Panama; Philippines; Portugal; Qatar; Rwanda; Saudi Arabia; Senegal; Singapore; Somalia; Sri Lanka; Sudan; Switzerland; Syria; Taiwan; Tajikistan; Tanzania; Thailand; Turkey; Uganda; United Arab Emirates; Uzbekistan; Viet Nam; Western Sahara; Yemen; Yugoslavia; Zaire

Vagrant: $\quad$ ?Anguilla; Azores; Brunei [898]; Canary Islands; Colombia; Cook Islands; Côte d'lvoire [104]; French Polynesia: Marquesas Islands, Society Islands; Guadeloupe; Guyana; Indonesia: Java [942]; Malaysia: Peninsular Malaysia, Sabah [1295], Sarawak [1295]; Madeira; Maldives; ?Montserrat; Netherlands Antilles; Papua New Guinea; Puerto Rico; Seychelles [416]; South Africa [288]; Saint Kitts and Nevis; Guadeloupe: Saint Martin; Suriname; Svalbard and Jan Mayen Islands; Togo; Tonga; Turks and Caicos 1slands [236]; Virgin Islands of the United States; Venezuela; Zambia [288]; Zimbabwe [288]

\author{
Anas aucklandica aucklandica (Gray 1844) \\ II $\quad$ V \\ $318,794,889,1511$ \\ Auckland Island Brown Teal \\ Breeding: New Zealand: Auckland 1slands \\ Anas aucklandica chlorotis Gray 1845 \\ Il V \\ $318,794,889,976$ \\ New Zealand Brown Teal \\ Breeding: New Zealand \\ Anas aucklandica nesiotis (Fleming 1935) \\ I \\ V $\quad 318,794,889$ \\ Campbell Island Brown Teal \\ Breeding: New Zealand: Campbell Island \\ Anas bernieri (Hartlaub 1860) \\ II V \\ $318,324,889$ \\ Madagascar Teal \\ Breeding: \\ Madagascar [373] \\ Anas capensis Gmelin 1789 \\ III $\mathrm{GH}$ - \\ $212,227,285,889,1247$ \\ Cape Teal, Cape Wigeon \\ Breeding: \\ Angola; Botswana; ?Cameroon; Chad; Ethiopia; Kenya; Lesotho; ?Libya [66I]; \\ Mozambique; Namibia; Niger; ?Nigeria; South Africa; Sudan; Tanzania; Zaire; Zambia; \\ Zimbabwe \\ Vagrant: $\quad$ Ghana; Israel; Libya; Malawi; Swaziland
}




\section{Northern Shoveler}

Breeding:

Afghanistan; ?Albania; Armenia; Austria; Azerbaijan; Belarus; Belgium; Bulgaria; Canada; China; Czechoslovakia; Denmark; Estonia; Finland; France; Georgia; Germany; Greece; Hungary; Iceland; Jreland; Jsle of Man; Italy; Japan; Jersey; Kazakhstan; Kyrgyzstan; Latvia; Lithuania; Moldova; Mongolia; Netherlands; Norway; Poland; Portugal; Romania; Russia; Spain; Sweden; Switzerland; Turkey; Turkmenia; Ukraine; USA; United Kingdom; Uzbekistan; Yugoslavia

Non-breeding: $\quad$ Albania; Algeria; Antigua and Barbuda; Bahamas; Bahrain; Bangladesh; Belize; ?Bhutan; ?Burundi; Cambodia; ?Cameroon; Canary Islands; Cayman Islands; ?Central African Republic; Chad; Colombia; Costa Rica; Cuba; Cyprus; Dominica; Dominican Republic; Egypt; El Salvador; Ethiopia; Federated States of Micronesia; Ghana; Grenada; Guadeloupe; Guam; Guatemala; Guemsey; Haiti; Hawaiian Islands; Honduras; Hong Kong; India; Iran; Iraq; Israel; Jamaica; Jordan [145I]; Kenya; Kiribati; D.P.R. Korea; Korea Republic; Kuwait; Lao P.D.R; Lebanon; Libya; Liechtenstein; Luxembourg; Malawi [288]; Maldives; Mali; Malta; Marshall Islands; Martinique; Mauritania; Mexico; ?Montserrat; Morocco; Myanmar; Nepal; Nicaragua; Niger; Nigeria; Northem Marianas; Oman; Pakistan; Panama; Philippines; Puerto Rico; Qatar; Saudi Arabia; Senegal; Singapore; Sri Lanka; Saint Kitts and Nevis; Saint Lucia; Saint Vincent; Sudan; Syria; Taiwan; Tajikistan; Tanzania; Thailand; Tunisia; Uganda; United Arab Emirates; Viet Nam; Virgin Islands of the United States; Wake Island; Western Sahara; Yemen; Zaire; Zambia [288]

Vagrant: Aruba; Australia [195]; Bermuda; ?Botswana; Brunei [898]; Burkina Faso; Côte d'lvoire; Faeroe Islands; French Polynesia: Tahiti, Tuamotu Archipelago; Gambia; Guinea; Malaysia: Peninsular Malaysia, Sabah, Sarawak [1295]; ?Mozambique [288]; Namibia; Netherlands Antilles; New Zealand; Rwanda; Somalia; South Africa [288]; Svalbard and Jan Mayen Islands; Trinidad and Tobago: Trinidad; Turks and Caicos Islands; Venezuela; Zimbabwe [288]

\section{Common Teal, Green-winged Teal}

Breeding:

Non-breeding:

Vagrant:
Afghanistan; Armenia; Austria; Azerbaijan; Belarus; Belgium; Bulgaria; Canada; China; Czechoslovakia; Denmark; Estonia; Faeroe Islands; Finland; France; Georgie; Germany; Greece; Greenland; Hungary; Iceland; Ireland; Isle of Man; Italy; Japan; Kazakhstan; Latvia; Lithuania; Luxembourg; Moldova; Mongolia; Netherlands; Norway; Poland; Romania; Russia; Spain; Sweden; Switzerland; Turkey; Ukraine; USA; United Kingdom; Uzbekistan

Albania; Algeria; Antigua and Barbuda; Bahamas; Bahrain; Bangladesh; Belize; Bermuda; Bhutan; Cambodia; Cameroon; Canary Islands; Central African Republic; Chad; Cuba [179]; Cyprus; Dominican Republic; Egypt; Ethiopia; Ghana; Guatemala; Guemsey; Haiti; Hawaiian Islands; Honduras; Hong Kong; India; Iran; Iraq; Israel; Jamaica; Jersey; Jordan [145I]; Kenya; D.P.R. Korea; Korea Republic; Kuwait; Kyrgyzstan; Lao P.D.R; Lebanon; Libya; Liechtenstein; Maldives; Mali; Malta; Mauritania; Mexico; Morocco; Myanmar; Nepal; Niger; Nigeria; Northern Marianas; Oman; Pakistan; Philippines; Portugal; Puerto Rico; Qatar; Saudi Arabia; Senegal; Singapore; Somalia; Sri Lanka; Sudan; Syria; Taiwan; Tajikistan; Thailand; Tunisia; Turkmenia; Uganda; United Arab Emirates; Viet Nam; Western Sahara; Yemen; Yugoslavia

?Anguilla; Barbados; Brunei [898]; Burundi; Cape Verde; Colombia; Costa Rica; Dominica; Gambia; Greenland; Grenada; ?Guadeloupe; Malaysia: Peninsular Malaysia, Sabah; Marshall Islands; ?Martinique; Montserrat; Palau [1118]; Saint Kitts and Nevis; Saint Lucia; Saint Vincent; Svalbard and Jan Mayen Islands; Tanzania; Trinidad and Tobago: Tobago; Virgin Islands of the United States; Zaire 


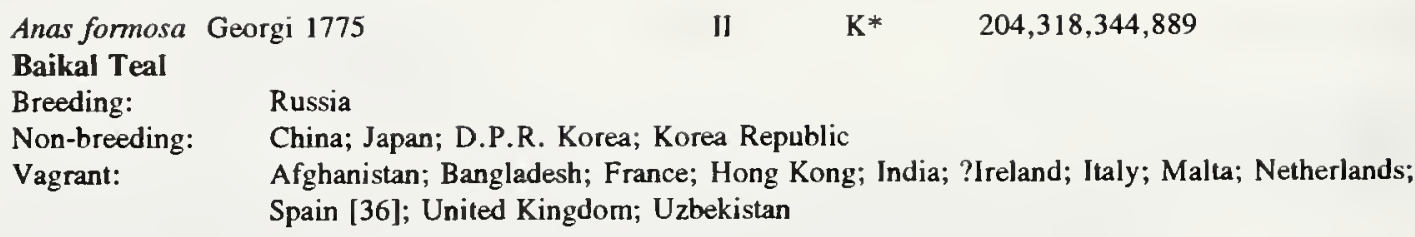

Anas laysanensis Rothschild 1892

I

$16,318,794,889$

(= A. platyrhynchos laysanensis)

Laysan Duck, Laysan Teal

Breeding: Hawaiian Islands: Laysan [1118]

Anas oustaleti Salvadori 1894

(= A. platyrhychos "oustaleti")

Marianas Mallard

Breeding:

Northern Marianas: ?Rota, Tinian (ex?), Saipan (ex?); Guam (ex)

Anas penelope Linnaeus 1758

III GH -

$10,16,212,227,344,661,792,889$, 1247

\section{Eurasian Wigeon}

Breeding:

Armenia; Azerbaijan; Belgium; China; Czechoslovakia; Denmark; Estonia; Finland; Georgia; Germany; Iceland; Kazakhstan; Latvia; Mongolia; Netherlands; Norway; Poland; Russia; Spain; Sweden; Turkey; United Kingdom

Non-breeding: Afghanistan; Albania; Algeria; Austria; Bahrain; Bangladesh; ?Benin; ?Bhutan; Bulgaria; ?Burkina Faso; Cambodia; Cameroon; Canada; Canary Islands; Chad; Cyprus; Egypt; Ethiopia; Faeroe Islands; France; Ghana; Greece; Greenland; Guam; Guernsey; Hong Kong; Hungary; India; Iran; Iraq; Ireland; Israel; Italy; Japan; Jersey; Jordan [1451]; Kenya; D.P.R. Korea; Korea Republic; Kuwait; Lao P.D.R; Lebanon; Libya; Liechtenstein; Luxembourg; Mali; Malta; Marshall Islands; Mauritania; Morocco; Myanmar; Nepal; Niger; Nigeria; Oman; Pakistan; Palau; Philippines; Portugal; Qatar; Romania; Saudi Arabia; Senegal; Sri Lanka; Sudan; Switzerland; Syria; Taiwan; Tanzania; Thailand; Tunisia; United Arab Emirates; USA; Uzbekistan; Viet Nam; Western Sahara; Yemen; Yugoslavia

Vagrant: $\quad$ Antigua and Barbuda: Barbuda [179]; Barbados [179]; Bermuda; ?Djibouti; Dominican Republic [179]; Federated States of Micronesia; Haiti [179]; Hawaiian Islands [1118]; Indonesia: Irian Jaya, Sulawesi [1486]; Malaysia: Peninsular Malaysia, Sabah, Sarawak [1295]; Mexico; Northern Marianas; Papua New Guinea; Puerto Rico [179]; Singapore; Somalia; Svalbard and Jan Mayen Islands; Uganda

Anas querquedula Linnaeus 1758

IIJ $\mathrm{GH}$

$10,16,212,227,344,661,792,889$, 930,1247

Garganey, Garganey Teal

Breeding: $\quad$ Albania; Armenia; Austria; Azerbaijan; Belarus; Belgium; Bulgaria; China; Czechoslovakia; Denmark; Estonia; Finland; France; Georgia; Germany; Greece; Hungary; Iran; ?Ireland; Israel; Italy; Japan; Kazakhstan; Latvia; Lithuania; Luxembourg; Moldova; Mongolia; Netherlands; Norway; Poland; Portugal; Romania; Russia; Spain; Sweden; Switzerland; Turkey; Ukraine; United Kingdom; Uzbekistan; Yugoslavia

Non-breeding: Afghanistan; Algeria; Bahrain; Bangladesh; ?Benin; ?Bhutan; Burkina Faso, Burundi; Cambodia; Cameroon; Canary Islands; Central African Republic; Chad; Côte d'lvoire; Cyprus; ?Djibouti; Egypt; Equatorial Guinea; Ethiopia; Gambia; Ghana; Guernsey; Guinea-Bissau; Hong Kong; India; Indonesia: Java; lraq; Ireland; Jersey; Jordan [1451]; Kenya; D.P.R. Korea; Korea Republic; Kuwait; Kyrgyzstan; Lao P.D.R; Lebanon; Libya; Liechtenstein; Malawi [288]; Malaysia: Peninsular Malaysia, Sabah, Sarawak [1295]; Maldives; Mali; Malta; Mauritania; Morocco; Myanmar; Nepal; Niger; Nigeria; Norway; Oman; Pakistan; Papua New Guinea; Philippines; Qatar; Rwanda; Saudi Arabia; Senegal; Singapore; Somalia; Sri Lanka; Sudan; Syria; Taiwan; Tajikistan; Tanzania; Thailand; 
Vagrant:

Togo; Tunisia; Uganda; United Arab Emirates; Viet Nam; Western Sahara; Yemen; Zaire; Zambia [288]

Australia; Barbados [179]; Botswana [288]; Brunei; Canada; Christmas Island; Faerow Islands; Gabon; Guam; Hawaiian Islands; Iceland; Indonesia: Irian Jaya, Mangole, Seram, Sulawesi, Sumatra, ?Ternate, Timor; Liberia; Mauritius: Rodrigues; Northem Marianas; Palau; Puerto Rico; Seychelles: Amirantes [460]; South Africa [288]; USA; Zimbabwe [288]

Anas wyvilliana Sclater 1878

$\mathrm{K}^{*}$

$16,318,794,889$

(= A. platyrhynchos wyvilliana)

Hawaiian Duck, Koloa

Breeding:

Hawaiian Islands: [Hawaii [1118], Kauai, [Oahu]

Anser erythropus (Linnaeus 1758)

$\mathrm{K}^{*}$

$10,318,344,661,889,1021,1284$

Lesser White-fronted Goose

Breeding: $\quad$ Finland; Norway; Sweden; Russia [799]

Non-breeding: Albania [498]; Bulgaria; China [817]; Greece; Hungary [1320]; India; Iran; Iraq; Japan; Latvia; Pakistan; Romania; Turkey; Turkmenia; Yugoslavia

Vagrant: $\quad$ Austria; Belgium; Czechoslovaskia; Denmark; Egypt; France; Ireland; Kuwait; Netherlands; Switzerland; Turkey; United Kingdom; Uzbekistan

$\begin{array}{lllll}\text { Aythya baeri (Radde 1863) } & \text { - } & \mathrm{K}^{*} & 10,318,889\end{array}$

\section{Baer's Pochard}

Breeding:

Non-breeding:

China; ?D.P.R. Korea; Russia [799]

Vagrant:

Bangladesh; Hong Kong; India; Japan; Myanmar; Nepal; Korea Republic; Thailand; Viet Nam

Aythya innotata (Salvadori 1894)

E

$318,324,889$

Madagascar Pochard

Breeding:

Madagascar [373, 1545]

Aythya nyroca (Güldenstädt 1770)

III GH -

$10,227,344,661,889,1247$

(= Nyraca nyroca)

Ferruginous Pochard, Ferruginous Duck, White-eyed Pochard

Breeding:

Afghanistan; Albania; Armenia; Austria; Azerbaijan; Bulgaria; China; Czechoslovakia; France: Georgia; Germany; Greece; Hungary; India; Iran; Israel; Italy; Kazakhstan; Latvia; Lithuania; Moldova; Mongolia [1097]; Morocco; Poland; Romania; Russia; Saudi Arabia; Spain; Tajikistan; Turkey; Turkmenia; Ukraine; Uzbekistan; Yugoslavia

Non-breeding: Algeria; Bangladesh; Belgium; Bhutan; Cameroon; Central African Republic; Chad; Cyprus; Egypt; Ethiopia; Iraq; Jordan [145I]; Lebanon; Libya; Mali; Malta; ?Mauritania; Myanmar; Nepal; Netherlands; Niger; Nigeria; Oman; Pakistan; Senegal; Sudan; Switzerland; Thailand; Tunisia; United Arab Emirates; Viet Nam; ?Western Sahara; Yemen

Vagrant: $\quad$ Bahrain; ?Benin; Burkina Faso; Canary Islands; Cape Verde; Denmark; Finland; Gambia; Ghana; Ireland; Japan; Kenya; Liechtenstein; Luxembourg; Maldives; Nonway; Portugal; Qatar; Sierra Leone; Sweden; Syria; Uganda; United Kingdom

Branta canadensis leucopareia (Brandt 1836)

\section{Aleutian Goose}

Breeding:

Russia: Kurile Islands; USA: Aleutian Islands

Non-breeding:

Vagrant: Japan; Mexico

Canada 
Red-breasted Goose

Breeding:

Russia [1 15, 185,799]

Non-breeding:

Bulgaria [1284]; Greece; Hungary [1320]; Iran; Iraq; Kazakhstan; Romania; Turkey [124,769]; Turkmenia

Vagrant: Austria; Belgium; China; Czechoslovakia; Denmark; ?Egypt; Finland; France; Germany; India; Ireland; Israel; Italy; Latvia; Netherlands; Norway; Poland; Spain; Sweden; United Kingdom; Uzbekistan; Yugoslavia

Branta sandvicensis (Vigors I834)

(= Nesochen sandvicensis)

Nene, Hawaiian Goose

Breeding:

Hawaiian Islands: Hawaii, Maui [1118]

Cairina moschata (Linnaeus 1758)

III HN -

889,967

Muscovy Duck

Breeding:

Argentina; Belize [338]; Bolivia; Brazil; Chile; Colombia; Costa Rica; Ecuador; El Salvador; French Guiana; Guatemala; Guyana; Honduras; Mexico; Nicaragua; Panama; Paraguay; Peru; Suriname; Uruguay; Venezuela

Vagrant:

Trinidad and Tobago; ?USA

Cairina scutulata (Müller 1842)

I V

$10,3[8,792,794,889$

White-winged Duck, White-winged Wood Duck

Breeding:

Bangladesh [782]; ?Cambodia; India; Indonesia: Java (ex), Sumatra [663, 1005]; Lao P.D.R; Myanmar; Thailand [583,1194]; Viet Nam

Vagrant: Malaysia: Peninsular Malaysia

$\begin{array}{llll}\text { Coscoroba coscoroba (Molina I782) } & \text { II } & - & 889,930,967\end{array}$

Coscoroba Swan

Breeding: $\quad$ Argentina; Brazil; Chile; ?Falkland Islands; Paraguay; Uruguay

$\begin{array}{llll}\text { Cygnus melanocorypha (Molina 1782) II } & \text { - } & 889,930,967\end{array}$

Black-necked Swan

Breeding: $\quad$ Argentina; Brazil; Chile; Falkland Islands; Paraguay; Uruguay

Vagrant: $\quad$ South Shetland Islands; Antarctic Peninsula: including Palmer Archipelago

Dendrocygna arborea (Linnaeus 1758) II $\quad$ V $\quad 179,318,321,427,889,930,967$

West Indian Whistling-Duck, West Indian Tree-Duck, Cuban Tree-Duck, Black-billed Wood-Duck

Breeding: $\quad$ Antigua and Barbuda [45I]; Bahamas [237]; British Virgin Islands; Cayman Islands; Cuba [509,510]; Dominican Republic; Haiti; Jamaica; Puerto Rico; Turks and Caicos Islands; Virgin Islands of the United States

Vagrant: $\quad$ Bermuda; Netherlands Antilles; Saint Kitts and Nevis

$\begin{array}{lll}\text { Dendrocygna autumnalis (Linnaeus 1758) III HN } \quad \text { - } & \text { 179,889,967 }\end{array}$

Black-bellied Whistling-Duck, Red-billed Whistling-Duck

Breeding: $\quad$ Argentina; Belize; Bolivia; Brazil; Colombia; Costa Rica; Ecuador; El Salvador; French Guiana; Guatemala; Guyana; Honduras; Mexico; Nicaragua; Panama; Paraguay; Peru; Suriname; Trinidad and Tobago; USA; Uruguay; Venezuela

Vagrant: $\quad$ Aruba; Barbados; Cuba; Grenada; Guadeloupe; Martinique; Netherlands Antilles; Puerto Rico; Saint Lucia; Saint Vincent; Virgin Islands of the United States 
Fulvous Whistling-Duck, Fulvous Tree-Duck

Breeding:

Angola; Antigua and Barbuda; Argentina; Bangladesh; ?Benin; Bolivia; Botswana; Brazil; Cameroon; Central African Republic; Chad; Colombia; Congo; Costa Rica; Cuba; Dominican Republic; Ecuador; Ethiopia; French Guiana; Gabon; Ghana; Guyana; Haiti; Hawaiian Islands; Honduras; India; Kenya; Madagascar; Malawi; Mali; Mauritania; Mexico; Mozambique; Myanmar; Namibia; Niger; Nigeria; Pakistan; Paraguay; Peru; Rwanda; Senegal; South Africa; Sudan; Suriname; Tanzania; Togo; USA; Uruguay; Venezuela; Zaire; Zambia; Zimbabwe

Non-breeding: Guatemala; Lesotho; Somalia; Swaziland; Uganda

Vagrant:

?Anguilla; Aruba; Bahamas; Barbados; Bermuda; ?British Virgin lslands; Canada; Chile; Côte d'Ivoire; Dominica; France; Gambia; ?Guadeloupe; Martinique; ?Montserrat; Morocco; Nepal; Netherlands Antilles: Bonaire; Panama; Puerto Rico; Spain; Sri Lanka; Saint Kjtts and Nevis; Saint Lucia; Saint Vincent; Trinidad and Tobago: Trinidad; Turks and Caicos 1slands; Virgin Islands of the United States

Dendrocygna viduata (Linnaeus 1766)

$111 \mathrm{GH}-$

$16,212,227,285,889,967,1247$, 1298

White-faced Whistling-Duck, White-faced Tree-Duck

Breeding:

Angola; Argentina; Benin; Bolivia; Botswana; Brazil; Burkina Faso; Burundi; Cameroon; Central African Republic; Chad; Colombia; Congo; Costa Rica; Côte d'lvoire; Equatorial Guinea; Ethiopia; Gabon; Gambia; Ghana; Guinea [1454]; Guinea-Bissau; Guyana; Kenya; Lesotho; Liberia; Madagascar; Malawi; Mali; Mauritania; [Mauritius (ex)]; Mozambique; Namibia; Niger; Nigeria; Panama (ex?); Paraguay; Peru; Puerto Rico (ex); Rwanda; Senegal; Sierra Leone; ?Somalia; South Africa; Sudan; Suriname; Swaziland; Tanzania; Togo; Uganda; Uruguay; Venezuela; Zaire; Zambia; Zimbabwe

Non-breeding: Comoros: Moheli; Mayotte; Seychelles: Aldabra Islands

Vagrant: Anuba; Barbados; Canary Islands; Cuba; Dominican Republic; Netherlands Antilles: Curaçao; Spain; Trinidad and Tobago: Trinidad

Marmaronetta angustirostris (Ménétriés 1832) - - $\quad$ K* $\quad 10,318,344,661,889$

Marbled Teal, Marbled Duck

Breeding:

Afghanistan; Algeria; Armenia; Azerbaijan; Canary lslands (ex); ?China [620]; Cyprus (ex); ?Egypt; France (ex); Georgia; Iran; Iraq [i240]; Israel; Kazakhstan; Morocco; ?Pakistan; Russia [799]; Senegal; Spain; Tajikistan; Tunisia (ex?); Turkey; Turkmenia; Uzbekistan

Non-breeding: $\quad$ Chad; Egypt; India; Malj; Morocco [36]; Pakistan [1171]; Turkey

Vagrant:

Albania; Cyprus; Germany; Italy; Czechoslovakia; Greece [36]; Hungary; Lebanon; Madeira; Malta; Nigeria; Oman; Portugal; Romania

Mergus octosetaceus Vieillot $1817 \quad$ - $\quad$ E $\quad 318,321,427,746,794,889,967$

Brazilian Merganser

Breeding: $\quad$ Argentina [154]; Brazil [1543]; Paraguay

Mergus squamatus Gould $1864 \quad$ - $\quad$ ld $\quad 318,792,794,889$

Scaly-sided Merganser, Chinese Merganser

Breeding: $\quad$ China; D.P.R. Korea [1310]; Russia [799]

Vagrant: $\quad$ Japan; Myanmar; Korea Republic; Taiwan; Vjet Nam 
African Pygmy-goose

Breeding:

Angola; Benin; Botswana; Burkina Faso; Burundi; Cameroon; Central African Republic; Chad; Congo; Côte d'Ivoire; Equatorial Guinea; Ethiopia [748]; Gabon; Gambia; Ghana; Guinea; Guinea-Bissau; Kenya; Liberia; Madagascar; Malawi; Mali; ?Mauritania; Mozambique; Namibia; Niger; Nigeria; Rwanda; Senegal; Sierra Leone; Somalia; South Africa; Sudan; Tanzania; Togo; Uganda; Zaire; Zambia; Zimbabwe

Non-breeding: Swaziland

Vagrant: Lesotho

Oxyura leucocephala (Scopoli 1769)

Il $\quad \mathrm{K}^{*}$

$10,185,227,318,344,66 \mathrm{I}, 889,9] 8$

White-headed Duck

Breeding:

Afghanistan; Algeria; France: Corsica (ex?); Hungary (ex); Iran; Italy: Sardinia (ex); Kazakhstan; Romania; Russia [799]; Spain; Tunisia; Turkey [124]; Turkmenia; Uzbekistan; Yugoslavia (ex)

Non-breeding: $\quad$ Albania; Bulgaria; China; Cyprus; Egypt; Greece; India; Iraq; Italy; Morocco; Pakistan; Syria; Tajikistan

Vagrant: $\quad$ Austria; Belgium; Czechoslovakia; ?Denmark; France; Germany; Israel; Libya; Malta; Mongolia; Netherlands; Poland; Portugal; Saudi Arabia; Switzerland; Yugoslavia

Plectropterus gambensis (Linnaeus 1766)

Ill GH -

$212,227,285,889,1247,1298$

Spur-winged Goose

Breuding:

Angola; Benin; Botswana; Burkina Faso; Burundi; Cameroon; Central African Republic; Chad; Congo; Ethiopia; Gabon; Gambia; Ghana; Guinea [1454]; Guinea-Bissau; Kenya; Liberia; Malawi; Mali; Mauritania; Mozambique; Namibia; Niger; Nigeria; Rwanda; Senegal; Sierra Leone; South Africa; Sudan; Swaziland; Tanzania; Togo; Uganda; Zaire; Zambia; Zimbabwe

Non-breeding: Côte d'Ivoire; Lesotho

Vagrant:

Egypt; Morocco; ?Oman

$\begin{array}{lll}\text { Pteronetta hartlaubii (Cassin 1859) } & \text { III GH } & -\end{array}$

(= Cairina hartlaubii)

Hartlaub's Duck

Breeding:

?Angola; Cameroon; Central African Republic; Congo; Côte d'Ivoire; Equatorial Guinea; Gabon; Ghana; Guinea; Liberia; ?Mali; ?Nigeria; Sierra Leone; Sudan; Zaire
Rhodonessa caryophyllacea (Latham 1790)
I Ex
$10,3 \mathrm{~J} 8,576,577,794,889$
Pink-headed Duck
Breeding:
India (ex); Myanmar (ex); Nepal (ex)
Sarkidiornis melanotos (Pennant 1769)
II
$10,212,227,285,792,889,930$, $967,1247,1298$

Comb Duck, Knob-billed Goose

Breeding:

Angola; Argentina [154]; Bangladesh; Benin; Bolivia; Botswana; Brazil; Burkina Faso; Burundi; Cambodia; Cameroon; Central African Republic; Chad; ?China; Colombia; Congo; Ecuador; Equatorial Guinea; Ethiopia; ?French Guiana; Gabon; Ghana; Guinea; Guinea-Bissau; Guyana; India; Kenya; Lao P.D.R; Liberia; Madagascar; Malawi; Mali; Mauritania; Mexico [332]; Mozambique; Myanmar; Namibia; Nepal; Niger; Nigeria; Pakistan; Panama; Paraguay; Peru; Rwanda; ?Senegal; Sierra Leone; South Africa; Sri Lanka (ex?); Sudan; Tanzania; Thailand; Togo; Trinidad and Tobago: Trinidad; Uganda; Uruguay [4]; Venezuela; Viet Nam; Zaire; Zambia; Zimbabwe

Non-breeding: Gambia; Senegal; Somalia; Swaziland

Vagrant: Côte d'Jvoire; ?Japan; Lesotho; Netherlands Antilles; Oman 
$\begin{array}{lllll}\text { Stictonetta naevosa (Gould 1841) } & - & \text { K* } & 306,318,339,889,912\end{array}$

Freckled Duck

Breeding:

Australia [167]

Tadorna cristata (Kuroda 1917)

Crested Shelduck

Breeding:

Non-breeding:

Vagrant:
?China; ?D.P.R. Korea [1029]; ?Russia [799]

?China; Korea Republic Japan

\section{Order FALCONIFORMES}

Birds of Prey

Family CATHARTIDAE

New World Vultures

Gymnogyps californianus (Shaw 1898)

I $\quad \mathrm{E}$

$15,16,226,318,321,420,765,784$, 794,957

\section{California Condor}

Breeding: Mexico (ex) [1491]; USA.

$\begin{array}{lll}\text { Sarcoramphus papa (Linnaeus 1758) } & \text { III HN } \quad-\quad 15,226,967\end{array}$

King Vulture

Breeding:

Argentina; Bolivia; Belize; Brazil; Colombia; Costa Rica; Ecuador; El Salvador; French Guiana; Guatemala; Guyana; Honduras; Mexico; Nicaragua; Panama; Paraguay; Suriname; Trinidad and Tobago; Uruguay; Venezuela

Vultur gryphus Linnaeus 1758

I

$15,226,967,1367$

Andean Condor

Breeding:

Argentina; Bolivia; Brazil [1268]; Chile [715]; Colombia; Ecuador; Peru; Venezuela [1558]

Family PANDIONIDAE

Osprey

Pandion haliaetus (Linnaeus 1758)

II

$10,15,16,179,212,226,227,285$, $345,661,792,967,1114,1247,1298$

Osprey

Breeding:

Non-breeding: Afghanistan; Albania; Angola; ?Anguilla; Antigua and Barbuda; Argentina; Aruba; Austria;

Algeria; Algeria; Armenia; Australia [167]; Azerbaijan; Bahamas; Bahrain; Belarus; Belize; Bulgaria; Canada; Canary Islands; Cape Verde; ?Chile [715]; China; Cuba; ?Czechoslovakia; Denmark; ?Djibouti; Egypt; Estonia; Ethiopia; Finland; France: Corsica; Georgia; Germany; Guatemala; India; Indonesia; Iran; Italy (ex); Japan; Kazakhstan; Latvia; Lithuania; ?Mauritania; Mexico; Moldova; Mongolia; Morocco; ?Nepal; New Caledonia; Norway; Oman; Papua New Guinea; Poland; Portugal; Qatar; Romania; Russia [799]; Saudi Arabia; Solomon Islands; Somalia; South Africa [286,370]; Spain; Sudan; Sweden; ?Tajikistan; ?Tunisia; Turkey; ?Turks and Caicos Islands; Ukraine; USA; United Arab Emirates; United Kingdom; Yemen Bangladesh; Barbados; Belgium; Benin; Bermuda; Bhutan; Bolivia; Botswana; Brazil; British Virgin Islands; Brunei; Bulgaria; Burkina Faso; Burundi; Cambodia; Cameroon; Cayman Islands; Central African Republic; Chad; Chile [715]: Colombia; Congo; Costa Rica; Côte d'lvoire; Cyprus; Czechoslovakia; Denmark; Djibouti; Dominica; Dominican Republic; Ecuador; El Salvador; Equatorial Guinea; France; French Guiana; Gabon; 
Galapagos Islands; Gambia; Ghana; Gibraltar; Greece; Grenada; ?Guadeloupe; Guinea [1454]; Guinea-Bissau; Guyana; Haiti; Hawaiian Islands [1118]; Honduras; Hong Kong; Hungary; Iraq; Ireland; Israel; Jamaica; Jordan [1451]; Kenya; D.P.R. Korea; Korea Republic; Kuwait; Lao P.D.R; Lesotho; Liberia; Libya; Liechtenstein; Luxembourg; Malawi; Malaysia: Peninsular Malaysia, Sabah, Sarawak [1295]; Mali; Malta; ?Martinique; Mauritania; ?Montserrat; Mozambique; Myanmar; Namibia; Nepal; Netherlands; Netherlands Antilles; Nicaragua; Niger; Nigeria; Pakistan; Panama; Paraguay; Peru; Philippines; Puerto Rico; Rwanda; Sao Tome and Principe; Senegal; Sierra Leone; Singapore; South Africa; Spain; Sri Lanka; Saint Kitts and Nevis; Saint Lucia; Saint Pierre and Miquelon; Saint Vincent; Suriname; Swaziland; Switzerland; Taiwan; Tajikistan; Tanzania; Thailand; Togo; Trinidad and Tobago; Tunisia; Turkey; Uganda; Uruguay; Uzbekistan; Venezuela; Viet Nam; Virgin Islands of the United States; Western Sahara; Yugoslavia; Zaire; Zambia; Zimbabwe

Vagrant: $\quad$ Faeroe Islands; Guam; Guernsey; Iceland; India: Andaman Islands; Jersey; Lebanon; Maldives; Palau; Syria

Family ACCIPITRIDAE

Kites, Eagles, Hawks and allies

Accipiter albogularis Gray 1870

11

$15,226,930,1226$

Pied Goshawk, Pied Sparrowhawk

Breeding:

Papua New Guinea: Bougainville, Feni Islands; Solomon Islands

Accipiter badius (Gmelin 1788)

Il

$10,15,212,226,227,285,345,661$, $792,1247,1298$

Shikra, Little Banded Sparrowhawk

Breeding: Afghanistan; Angola; Azerbaijan (ex?); Bangladesh; Benin [667]; Bhutan; Botswana; Burkina Faso [1388]; Burundi; Cambodia; Cameroon; Central African Republic; Chad; China; Congo; Côte d'lvoire; Djibouti; Ethiopia; Gambia; Ghana; Guinea [1454]; Guinea-Bissau; India; Iran; Kazakhstan; Kenya; Kyrgyzstan; Lao P.D.R; Liberia; Malawi; Mali; Mauritania; Mozambique; Myanmar; Namibia; Nepal; Niger; Nigeria; Pakistan; Rwanda; Saudi Arabia [292]; Senegal; Sierra Leone; Somalia; South Africa; Sri Lanka; Sudan; Swaziland; Tajikistan; Tanzania; Thailand; Togo; Turkmenia; Uganda; Uzbekistan; Viet Nam; ?Yemen; Zaire; Zambia; Zimbabwe

Non-breeding: $\quad$ Malaysia: Peninsular Malaysia; Singapore

Vagrant: $\quad$ Indonesia: Sumatra; 1srael [814]; Kuwait; Lesotho

Accipiter bicolor (Vieillot 1817)

11

$15,16,226,967$

Bicolored Hawk, Bicolored Sparrowhawk

Breeding:

Argentina; Belize; Bolivia; Brazil; Chile [715]; Colombia; Costa Rica; Ecuador; El Salvador; French Guiana; Guatemala; Guyana; Honduras; Mexico; Nicaragua; Panama; Paraguay; Peru; Suriname; Venezuela

Vagrant: Uruguay

Accipiter brachyurus (Ramsay 1879)

Il $\quad \mathrm{K}^{*}$

$15,226,318,930,1226$

New Britain Sparrowhawk

Breeding:

Papua New Guinea: New Britain

Accipiter brevipes (Severtsov 1850)

Il

$15,226,227,345,661$

Levant Sparrowhawk

Breeding:

Albania; Armenia; Azerbaijan; Bulgaria; Georgia; Greece; Hungary; Iran; Kazakhstan; Moldova; Romania; Russia [799]; Turkey; Ukraine; Yugoslavia

Non-breeding:

Vagrant: Cyprus; Egypt; Israel; Jordan [1451]; Lebanon; Sudan; Syria; Yemen Bahrain; Czechoslovakia; Ethiopia; Iraq; Italy; Kenya; Kuwait [1101]; Niger; Poland; Saudi Arabia; Tanzania; Tunisia; United Arab Emirates; Uzbekistan; Zaire 


\section{Chestnut-flanked Sparrowhawk, Chestnut-bellied Sparrowhawk}

Breeding: $\quad$ Angola; Cameroon; Central African Republic; Congo; ?Côte d'Ivoire; ?Equatorial Guinea; Gabon; ?Liberia; ?Nigeria; ?Uganda; Zaire

\section{Accipiter chionogaster (Kaup 1852) \\ 11 \\ White-breasted Hawk \\ Breeding: $\quad$ El Salvador; Guatemala [127]; Honduras; Mexico; Nicaragua}

Accipiter cirrocephalus (Vieillot 1817) II $\quad$ -
Collared Sparrowhawk

Breeding:

Australia [167]; Indonesia: Aru, Irian Jaya, Salawati, Waigeo, Yapen; Papua New Guinea

Accipiter collaris Sclater 1860

II

$15,166,226,318,967$

Semi-collared Hawk, Serni-collared Sparrowhawk
Breeding:
Colombia; Ecuador
Vagrant:
Peru; ?Venezuela
Accipiser cooperii (Bonaparte 1828)
Cooper's Hawk
Breeding:
Canada; Mexico; USA
Non-breeding: Belize; Costa Rica; Guatemala [127];
Vagrant:
Bermuda; Colombia; Honduras

Accipiter erythrauchen Gray 1861

Il $\quad-\quad 15,226$

Rufous-necked Sparrowhawk, Moluccan Sparrowhawk

Breeding: Inconesia: Ambon, Bacan, Buru, Halmahera, Morotai, Obi, Seram [1486]

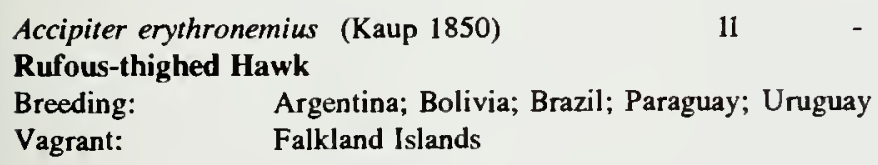

$\begin{array}{llll}\text { Accipiter erythropus (Hartlaub 1855) } & \text { II } & -15,212,226,227,1247,1298\end{array}$

Red-thighed Sparrowhawk

Breeding: Angola; Benin; Cameroon; Central African Republic [259]; Congo; Côte d'lvoire; Equatorial Guinea; Gabon; Gambia; Ghana; Guinea-Bissau; Liberia; ?Mali; ?Nigeria; Senegal; Sierra Leone; Togo [280]; Uganda; Zaire

Accipiter fasciatus (Vigors \& Horsfield)

II $\quad-\quad 15,226,794,930$

Brown Goshawk, Australasian Goshawk

Breeding:

Australia [167]; Indonesia: Damar, Flores, Leti, Lombok, Moa, Sermata, Sumba, Sumbawa, Wetar; New Caledonia; Norfolk Island; Papua New Guinea; Solomon 1slands:

Bellona, Rennell; Vanuatu: Aneityum

Accipiter francesii Smith 1834

II $\quad-\quad 15,226,794,1298$

Frances's Goshawk

Breeding: Comoros; Madagascar [373]; Mayotte 
Northern Goshawk

Breeding:

Non-breeding:

Vagrant:
Albania; ?Andorra; Armenia; Austria; Azerbaijan; Belarus; Belgjum; Bulgaria [1168]; Canada; China; Cyprus; Czechoslovakia; Denmark; Estonia; Finland; France; Georgia; Germany; Greece; Hungary; India; Iran; Italy; Japan; Latvia; Liechtenstein; Lithuania; Luxembourg [1470]; Moldova; Mongolia; Morocco; Nepal; Netherlands; Norway; Poland; Portugal; Romania; Russia; Saint Pierre and Miquelon; Spain; Sweden; Switzerland; ?Syria; Turkey; Ukraine; USA; United Kingdom [906]; Yugoslavia

Afghanistan; Bhutan; Gibraltar; Hong Kong; Iran; Israel; D.P.R. Korea; Korea Republic; ?Lao P.D.R; Lebanon; Morocco; Myanmar; Saudi Arabia; Thailand; Uzbekistan; Viet Nam Algeria; Bangladesh; Bermuda; Egypt; Guernsey; Ireland; Jersey; Jordan [1451]; Libya; Mexico; Oman; Pakistan; Taiwan; Tunisia; United Arab Emirates

Accipiter griseiceps (Schlegel 1862)

II

15,226

Sulawesi Goshawk

Breeding:

Indonesia: Butung [1486], Muna, Sulawesi, Togian
$15,226,792,930$

Accipiter gularis (Temminck \& Schlegel 1844)

II

(= A. virgatus gularis)

Japanese Sparrowhawk

Breeding:

China; Japan; D.P.R. Korea; Korea Republic; Mongolia; Russia; Taiwan

Non-breeding:

?Bangladesh; Brunei; Cambodia; Hong Kong; India; Indonesia: Bali, Java, Kalimantan, Sumatra; Lao P.D.R; Macao; Malaysia: Peninsular Malaysia, Sabah, Sarawak [1295]; Myanmar; Philippines; Singapore; Thailand [1194]; Viet Nam

Vagrant:

Guam [1118]; India: Andaman and Nicobar Islands [945]; Indonesia: Flores, Sulawesi, Tanahjampea, Timor; Northern Marianas

Accipiter gundlachi Lawrence 1860

11

$V / R$

$15,16,226,318,321,1536,1537$, 1538

Gundlach's Hawk

Breeding:

Cuba $[179,509]$

Accipiter haplochrous Sclater 1859

II

$15,226,930$

White-bellied Goshawk, New Caledonia Goshawk

Breeding:

New Caledonia

Accipiser henicogrammus (Gray 1860)

Il

15,226

Moluccan Goshawk, Moluccan Barred Sparrowhawk, Gray's Goshawk

Breeding:

Indonesia: Bacan [1486], Halmahera, Morotai

Accipiter henstii (Schlegel 1873)

Il

Henst's Goshawk

Breeding:

Madagascar [373]

Accipiter imitator Hartert 1926

II $\quad \mathrm{K}^{*}$

$15,226,318,930,1226$

Imitator Sparrowhawk

Breeding:

Papua New Guinea: Bougainville; Solomon 1slands: Choiseul, Santa Isabel [593]

Accipiter luteoschistaceus Rothschild \& Hartert 1926 II

Slaty-mantled Sparrowhawk, Slaty-backed Goshawk

Breeding:

Papua New Guinea: New Britain

Accipiter madagascariensis Smith 1834

Madagascar Sparrowhawk

Breeding:

Madagascar [373] 
Accipiter melanochlamys (Salvadori 1875)

II

$15,131,226,930$

Black-mantled Goshawk

Breeding: Indonesia: Irian Jaya; Papua New Guinea

Accipiter melanoleucus Smith 1830

II

$15,212,226,227,285,1247,1298$

Black Goshawk, Great Sparrowhawk, Black Sparrowhawk

Breeding:

Angola; ?Benin; ?Botswana; Burundi; Cameroon; Central African Republic; Congo; Côte d'lvoire; Equatorial Guinea; Ethiopia; Gabon; Ghana; ?Guinea; Kenya; Malawi; Mozambique; ?Namibia; ?Niger; Nigeria; Rwanda; ?Sierra Leone; South Africa; Sudan; Swaziland; Tanzania; Togo [278]; Uganda; Zaire; Zambia; Zimbabwe

Vagrant: $\quad$ Cape Verde; Gambia; ?Guinea-Bissau; Lesotho; Liberia; Mali; Senegal; Somalia

Accipiter meyerianus (Sharpe 1878)

II

$15,226,930$

Meyer's Goshawk, Papuan Goshawk

Breeding:

Indonesia: Boano [1486], Irian Jaya, Seram, Seram Laut, Yapen; Papua New Guinea: New Britain, Uatom; Solomon Islands: Guadalcanal, Kulomnabgra

Accipiter minullus (Daudin 1800)

II

$15,212,226,227,285,1298$

Little Sparrowhawk, African Little Sparrowhawk

Breeding:

Angola; Botswana; Burundi; Ethiopia [748]; Kenya; Malawi; Mozambique; Namibia; Rwanda; Somalia; South Africa; Sudan; Swaziland; Tanzania; Uganda; Zaire; Zambia; Zimbabwe

Vagrant: $\quad$ Lesotho

Accipiter nanus (Blasius 1897)

II

$\mathrm{K}^{*}$

$15,226,318,1486$

Small Sparrowhawk

Breeding:

Indonesia: Sulawesi [881]

Accipiter nisus (Linnaeus 1758)

II

$10,15,226,227,345$

Eurasian Sparrowhawk

Breeding:

Afghanistan; Albania; Algeria; Andorra; Armenia; Austria; Azerbaijan; Belarus; Belgium; Bhutan [873]; Bulgaria [1168]; Canary 1slands; China; Czechoslovakia; Denmark; Estonia; Finland; France; Georgia; Germany; Greece; Guemsey; Hungary; India; Iran; ?Iraq; Ireland; Israel [494]; Italy; Japan; Kazakhstan; D.P.R. Korea; Korea Republic; Kyrgyzstan; Latvia; Liechtenstein; Lithuania; Luxembourg; Madeira; Moldova; Mongolia; Morocco; Nepal; Netherlands; Norway; Pakistan; Poland; Portugal; Romania; Russia; Spain; Sweden; Switzerland; ?Syria; Tajikistan; Tunisia; Turkey; Ukraine; United Kingdom; Yugoslavia

Non-breeding: $\quad$ Bahrain; Bangladesh; Chad; Channel Islands; Cyprus; Djibouti; Egypt; Ethiopia; Gibraltar; Hong Kong; Iraq; Jersey; Jordan [1451]; Kuwait; Lao P.D.R; Lebanon; Libya; Mali; Malta; ?Mauritania; Myanmar; ?Niger; Oman; Saudi Arabia; Sudan; Syria; Thailand [1194]; United Arab Emirates; Uzbekistan; Viet Nam; Yemen

Vagrant: $\quad$ ?Brunei; Faeroe Islands; Gambia; Iceland; India: Andaman Islands [945]; Kenya; Malaysia: Sarawak [1295]; Somalia; Taiwan; Tanzania; Westem Sahara

Accipiter novaehollandiae (Gmelin 1788)

II

$15,226,930$

(includes A. griseogularis)

Grey Goshawk, Variable Goshawk, White Goshawk, Grey-throated Goshawk

Breeding:

Australia [167]; Indonesia: Alor, Babar [1486], Bacan, Banda, Damar, Flores, Gebe, Halmahera, Komodo, Morotai, Obi, Pantar, Rinca, Sumbawa, Ternate, Tidore; Papua New Guinea; Solomon Islands 
Ovampo Sparrowhawk

Breeding:

Angola; ?Benin; Botswana; Burundi; Cameroon; Central African Republic [168]; Chad; Ethiopia; Kenya; Malawi; Mozambique; Namibia; Rwanda; South Africa; Togo [280]; Zaire; Zambia; Zimbabwe

Non-breeding: Côte d'Ivoire; Ghana; Mali; Nigeria; Tanzania; Uganda

Vagrant: Senegal; Swaziland

Accipiter poliocephalus Gray 1858

II

$15,226,930$

Grey-headed Goshawk, New Guinea Grey-headed Goshawk

Breeding: $\quad$ Indonesia: Aru, Batanta, Irian Jaya, Misool, Salawati, Waigeo, Yapen; Papua New Guinea

Accipiter poliogaster (Temminck 1824)

II

$15,226,318,967$

Grey-bellied Goshawk, Grey-bellied Hawk

Breeding:

Argentina; Bolivia; Brazil; ?Colombia; Ecuador; French Guiana [1380]; Guyana; Paraguay; Peru; Suriname [626]; Venezuela

Non-breeding: Colombia

Accipiter princeps Mayr 1934

II

$15,226,930,1226$

New Britain Goshawk

Breeding:

Papua New Guinea: New Britain

$\begin{array}{llll}\text { Accıpiter rhodogaster (Schlegel 1862) } & \text { II } & - & \text { 15,226 }\end{array}$

Vinous-breasted Sparrowhawk

Breeding: $\quad$ Indonesia: Banggai, Butung, Muna, Peleng, Sula Islands [1486], Sulawesi

$\begin{array}{llll}\text { Accipiter rufitorques (Peale 1848) } & \text { II } & - & \text { I5,226,930 }\end{array}$

Fiji Goshawk

Breeding: $\quad$ Fiji [I1]8]

Accipiter rufiventris Smith 1830

II

$15,212,226,227,285,1298$

Rufous-chested Sparrowhawk

Breeding: $\quad$ Angola; Burundi; Ethiopia; Kenya; ?Lesotho; Malawi; Mozambique; Rwanda; South Africa; Sudan; Swaziland; Tanzania; Uganda; Zaire; Zambia; Zimbabwe

Vagrant: Mali

Accipiter soloensis (Horsfield 1821)

II

$10,15,226,792$

Chinese Groshawk, Grey Frog Hawk, Chinese Sparrowhawk

Breeding:

China; D.P.R. Korea; Korea Republic; Russia [799]; Viet Nam

Non-breeding:

Hong Kong; Indonesia; Japan: Ryukyu Islands; Lao P.D.R; Malaysia: Peninsular Malaysia, Sabah, Sarawak [1295]; Myanmar; Nicobar Islands; ?Papua New Guinea; Philippines; Singapore; Taiwan; Thailand

Vagrant: $\quad$ Brunei; Federated States of Micronesia: [1118]; Guam; Northem Marianas; Palau; Russia

Accipiter striatus Vieillot 1807

II

$15,16,226,794,967$

Sharp-shinned Hawk

Breeding:

Canada; Cuba; Dominican Republic; Haiti; Mexico; Puerto Rico; USA

Non-breeding:

Bahamas; Belize; Costa Rica; El Salvador; Guatemala [127]; Honduras; Jamaica; Nicaragua; Panama; Saint Pierre and Miquelon

Vagrant: Bermuda; Turks and Caicos Islands

Accipiter superciliosus (Linnaeus I758)

II

$15,16,226,967$

Tiny Hawk, Tiny Sparrowhawk

Breeding:

Argentina [154]; Bolivia; Brazil; Colombia; Costa Rica; Ecuador; French Guiana; Guyana; Nicaragua; Panama; Paraguay; Peru; Suriname; Venezuela 
African Goshawk

Breeding:

Angola; Botswana; Burundi; Kenya; Malawi; Mozambique; ?Namibia; Rwanda; Somalia: South Africa; Sudan; Swaziland; Tanzania; Uganda; Zaire; Zambia; Zimbabwe

Vagrant: Lesotho

Accipiter toussenelii (Verreaux \& Verreaux 1855) II

\section{Red-chested Goshawk}

Breeding:

?Benin; Cameroon; Central African Republic; Congo; Côte d'Ivoire; Equatorial Guinea; Ethiopia; Gabon; Gambia; Ghana; Guinea; ?Guinea-Bissau; Liberia; ?Niger; Nigeria; Senegal; Sierra Leone; Sudan; Togo [280]; Zaire

Vagrant: Mali

Accipiter trinotatus Bonaparte 1850

II

Spot-tailed Goshawk, Spot-tailed Sparrowhawk

Breeding:

Indonesia: Butung [1486], Muna, Sulawesi
Accipiter trivirgatus (Temminck 1824)
11
$10,15,226,792$

Crested Goshawk, Asian Crested Goshawk

Breeding:

Bhutan [874]; Brunei; ?Cambodia; China; ?Hong Kong; India; Indonesia: Bali, Java, Kalimantan, Natuna Islands, Nias, Sumatra; Lao P.D.R; Malaysia: Peninsular Malaysia, Sabah, Sarawak [347]; Myanmar; Nepal; Philippines; Sri Lanka; Taiwan; Thailand [1194]; Viet Nam

Non-breeding: Bangladesh

Accipiter ventralis Sclater 1866

11

Plain-breasted Hawk

Breeding: $\quad$ Bolivia; Colombia; Ecuador; Peru; Venezuela

Accipiter virgatus (Temminck 1822) $\quad 11 \quad$ - $\quad 10,15,226,792$

Besra, Besra Sparrowhawk

Breeding: Bhutan; China; India: including Andaman and Nicobar Islands [945]; Indonesia: Bali, Flores, Java, Kalimantan [993], Sumatra; Malaysia: Sabah, Sarawak [1295]; Myanmar; Nepal; Pakistan; Philippines; Sri Lanka; Taiwan; Thailand; Viet Nam

Non-breeding: Bangladesh; Cambodia; Lao P.D.R
Aegypius monachus (Linnaeus 1766)
II
$\mathrm{K}^{*}$
$10,15,226,318,345,661,965$

Cinereous Vulture, Black Vulture

Breeding:

Afghanistan; Albania (ex?); Armenia; Azerbajjan; ?Bulgaria; China; ?Cyprus; Georgia; Greece; Iran; lsrael (ex); Kazakhstan; Kyrgyzstan; Balearic Islands: Mallorca; Mongolia; Pakistan; Portugal; Russia [799]; Spain; ?Syria; Tajikistan; Turkey; Turkmenia; Uzbekistan; ?Yugoslavia

Non-breeding: $\quad$ Bangladesh; ?Bhutan; Egypt; Hong Kong; India; Italy; D.P.R. Korea; Korea Republic; Morocco; Myanmar; Nepal; Oman; Saudi Arabia; Thailand

Vagrant: $\quad$ Austria; Bulgaria; Czechoslovakia; Denmark; France; Germany; Gibraltar; Hungary; Iraq; Israel; Japan; Jordan [1452]; Kuwait; Latvia; Lebanon; Netherlands; Poland; Romania; Sudan; Switzerland; Taiwan; Viet Nam; Yemen; Yugoslavia

Aquila adalberti Brehm 1861

I E

$15,226,318,382,546,661,960$, 962,963

(= A. heliaca adalberti)

Adalbert's Eagle, Spanish Imperial Eagle

Breeding:

Algeria (ex); Morocco (ex); Portugal; Srain [549,647]

Vagrant:

Gibraltar; France 
Breeding: $\quad$ Australia [167]; Indonesia: Irian Jaya [131]; Papua New Guinea

Aquila chrysaetos
Golden Eagle

Breeding:

Non-breeding:

Vagrant:

\section{(Linnaeus 1758)}

Il

?Afghanistan; ?Albania; Algeria; ?Andorra; Armenia; Austria; Azerbaijan; Belarus; Bhutan; Bulgaria; Canada; China; Czechoslovakia; ?Egypt; Estonia; Finland; France; Georgia; Germany; Greece; Hungary; India; Iran; ?Iraq; Ireland (ex); Israel; Italy; Japan; Kazakhstan; D.P.R. Korea; Korea Republic; Kyrgyzstan; Latvia; ?Lebanon; Libya; Liechtenstein; Lithuania (ex); Mauritania; Mexico; Mongolia; Morocco; Nepal; Norway; Oman [502]; Pakistan; Poland; Portugal; Romania; Russia [799]; Saudi Arabia; Spain; Sweden; Switzerland; ?Syria; Tajikistan; Tunisia; Turkey; Turkmenia; Ukraine; USA; United Kingdom; Uzbekistan; Westem Sahara; ?Yemen; Yugoslavia

Afghanistan; Denmark; Iraq; Lebanon; Syria; United Arab Emirates Belgium; Cyprus; Gibraltar; Hawaiian Islands: Kauai [1]18]; ]reland; Jersey; Jordan [1451]; Kuwait [1101]; Malta; Netherlands

Aquila clanga Pallas 1811

11

$10,15,212,226,227,345,661,792$

\section{Greater Spotted Eagle, Spotted Eagle}

Breeding:

Nor-breeding:

Vagrant:

Aquila gurneyi Gray 1860

Belarus; China; Estonia; Finland; India; Iran; Israel (ex); Kazakhstan; Latvia; Lithuania (ex); Moldova; Mongolia; Pakistan; Romania; Russia; Ukraine; Yugoslavia

Afghanistan; ?Albania; Bangladesh; ?Bhutan; Bulgaria; Cambodia; Czechoslovakia; Egypt; Ethiopia; France; Germany; Greece; Hong Kong; Hungary; Iraq; Israel; Italy; Jordan; Kenya; Kuwait; Lao P.D.R; Lebanon; Malaysia: Peninsular Malaysia; Myanmar; Nepal; Oman; Poland; Saudi Arabia; Singapore; Sudan; Sweden; Thailand; Turkey; United Arab Emirates; Viet Nam; Yemen

Austria; Bahrain [656]; Belgium; Chad; Cypnus; Denmark; Gibraltar; Ireland; Japan; D.P.R. Korea; Korea Republic; Libya; Luxembourg; Morocco; Netherlands; Portugal; Spain; Switzerland; Syria; Taiwan; Tunisia; Uganda; United Kingdom

\section{Gurney's Eagle}

Breeding:

Indonesia: Ambon, Aru, Bacan, Halmahera, Morotai, Ternate, Waigeo, Yapen; Papua New Guinea

Vagrant:

Australia

Aquila heliaca Savigny 1809

I K*

$10,15,185,226,227,318,345,523$ 661,794

\section{Imperial Eagle}

Breeding:

Non-breeding:

Vagrant:

Austria; Cameroon; Denmark; Finland; France; Germany; Italy; Japan; Kenya; D.P.R.
?Albania; Azerbaijan; Bulgaria; China; Cyprus; Czechoslovakia; Georgia; Greece; Hungary; lran; Kazakhstan; Moldova; Romania; Russia [541,799]; Turkey [125]; Turkmenia; Ukraine; Uzbekistan; Yugoslavia

Afghanistan; Bangladesh; Djibouti; Egypt; Ethiopia; Hong Kong; India; Iraq; lsrael; Jordan [1451]; Kuwait; Lao P.D.R; Lebanon; Nepal; Oman; Pakistan; Saudi Arabia; Sudan; United Arab Emirates; Viet Nam; Yemen Korea; Korea Republic; Libya; Malaysia: Peninsular Malaysia; Malta; Mongolia; Morocco; Poland; Singapore; Sweden; Syria; Taiwan; Tanzania 
Aquila nipalensis Hodgson 1833

Steppe Eagle

Breeding:

Non-breeding:

Vagrant:

Aquila pomarina Brehm 1831

China; Kazakhstan; Mongolia; Russia [799]

Afghanistan; India; Nepal; Pakistan

D.P.R. Korea; Malaysia: Peninsular Malaysia [642]; Singapore; Turkmenia

\section{Lesser Spotted Eagle}

$11 \quad-\quad 10,15,212,226,227,284,285,345$ $661,792,968$

Breeding:

Non-breeding:

Vagrant:
11

\section{African Tawny-Eagle}

Breeding:

Non-breeding:

Vagrant:

Aquila verreauxii Lesson 1830

Verreaux's Eagle, Black Eagle, African Black Eagle

Breeding:

Angola; Botswana; Central African Republic; Chad; Djibouti; Egypt; Ethiopia [748]; ?Israel; Kenya; ?Lesotho; Malawi; ?Mali; Mozambique; Namibia; Niger [1015]; Oman; Rwanda; Saudi Arabia [42] ]; Somalia; South Africa: Sudan; Swaziland; Tanzania; Uganda; Yemen; Zaire; Zambia [301]; Zimbabwe

Non-breeding: Israel

Aquila vindhiana Franklin 1831

Il

$15,212,226,227,285,345,506$, $661,1247,1298$

$10,15,212,226,227,285,345,661$, 792

(=A. rapax vindhiana)

(includes A. orientalis Cabanis 1854)

Eurasian Tawny-Eagle

Breeding:

Non-breeding:

India; Iran; Myanmar; Nepal; Pakistan; Russia [799]; Turkmenia; Ukraine

Bahrain; Djibouti; Egypt; Ethiopia; lraq; Israel; Jordan [1451]; Kuwait; Lebanon; Malawi; Namibia; Oman; Romania; Saudi Arabia; Sudan; Swaziland; Turkey; United Arab Emirates; Viet Nam; Yemen; Zambia [407]

Vagrant:
Belgium; Bulgaria; Czechoslovakia; Denmark; Finland; France; Germany; Greece; Hungary; Italy; Netherlands; Norway; Poland; Qatar; Singapore; Sweden; Thailand; Yugoslavia 
Wahlberg's Eagle

Breeding:

Angola; Benin; Botswana; Burkina Faso [1388]; Burundi; Cameroon; Central African Republic; Cbad; Ethiopia [748]; Gambia; Ghana; Guinea-Bissau; Kenya; Malawi; Mali; Mozambique; Namibia; Niger; Nigeria; Rwanda; ?Senegal; ?Somalia; South Africa; Sudan; Swaziland; Tanzania; ?Togo; Uganda; Zaire; Zambia; Zimbabwe

Non-breeding: $\quad$ Côte d'Ivoire; Guinea; ?Ljberia; Mauritania; Sierra Leone; Somalia; Togo
Asturina nitida (Latham 1790)
II
$15,16,226,967$

(= Buteo nitidus)

Grey-lined Hawk

Breeding:

Argentina; Bolivia; Brazil; Colombia; Costa Rica; Ecuador; French Guiana; Guyana; Panama; Paraguay; Peru; Suriname; Trinidad and Tobago; Venezuela

Asturina plagiata Schlegel I862

II

(= Buteo nitidus plagiatus)

Grey Hawk

Breeding: $\quad$ Belize; Costa Rica; El Salvador; Guatemala; Honduras; Mexico; Nicaragua; USA

Aviceda cuculoides Swainson 1837

II -

$15,2 \mathrm{I} 2,226,227,285, \mathrm{I} 247, \mathrm{I} 298$

African Baza, African Cuckoo-hawk, African Cuckoo-falcon

Breeding:

Angola; Benin; ?Botswana; Burkina Faso; Burundi; Cameroon; Central African Republic; ?Chad; Congo; Côte d'Ivoire; Equatorial Guinea; Ethiopia; Gabon; Ghana; Guinea-Bissau; Kenya; Liberia; Malawi; ?Mali; Mozambique; ?Namibia; Nigeria; Rwanda; Sierra Leone; ?Somalia; South Africa; ?Sudan; Swaziland; Tanzania; Togo; ?Uganda; Zaire; Zambia; Zimbabwe

Vagrant: Gambia; Senegal

Aviceda jerdoni (Blyth I842)

II

$10,15,226,792,967$

Jerdon's Baza, Asian Baza

Breeding: $\quad$ Bangladesh; ?Bhutan; Brunei; China; India; Indonesia; Lao P.D.R; Malaysia: Peninsular Malaysia, Sabah, Sarawak [1295]; Myanmar; Philippines; ?Singapore; Sri Lanka; Thailand [1194]

Vagrant: Viet Nam [1317]

Aviceda leuphotes (Dumont I820)

II $\quad$ - $\quad 10,15,226,792,967$

Black Baza

Breeding:

Bangladesh; ?Bhutan; China; ?Hong Kong; India; ?Lao P.D.R; Myanmar; Nepal; Thailand [1194]; Viet Nam

Non-breeding: Cambodia; Indonesia: Sumatra; Malaysia: Peninsular Malaysia; Singapore; Sri Lanka

Vagrant: Indonesia: Java [105]

Aviceda madagascariensis (Smith 1834) II - $\quad 15,226$

Madagascar Baza, Madagascar Cuckoo-hawk, Madagascar Cuckoo-falcon

Breeding:

Madagascar [373]

Aviceda subcristata (Gould I838)

II $\quad$ - I5, $226,930,1226$

Pacific Baza, Crested Baza

Breeding:

Australia [167]; Indonesia: Irian Jaya, Wallacea; Papua New Guinea; Solomon Islands

Busarellus nigricollis (Latham I790)

II

$15,16,226,967$

Black-collared Hawk, Collared Fishing Hawk

Breeding:

Argentina; Belize; Bolivia; Brazil; Colombia; Costa Rica; Ecuador; El Salvador; French Guiana; Guatemala; Guyana; Honduras; Mexico; Nicaragua; Panama; Paraguay; Peru; Suriname; Trinidad and Tobago: Trinidad; Uruguay [4]; Venezuela 
Butastur indicus (Gmelin 1788)

II

$15,226,792$

Grey-faced Buzzard, Grey-faced Buzzard-Eagle

Breeding:

China; Japan; D.P.R. Korea; Korea Republic; Russia [799]

Non-breeding:

Brunei; Cambodia; Hong Kong; Indonesia: Halmahera, Morotai, Sangihe, Siau, Sulawesi, Talaud, Ternate; Lao P.D.R; Malaysia: Peninsular Malaysia, Sabah, Sarawak [1295]; Myanmar; Philippines; Singapore; Taiwan; Thailand; Viet Nam

Vagrant: Indonesia: Java [26], Sumatra

Butastur liventer (Temminck 1827)

II

$15,226,792$

Rufous-winged Buzzard, Rufous-winged Buzzard-Eagle

Breeding:

Cambodia; China; Indonesia: Sulawesi, ?Timor [1486]; Lao P.D.R; Myanmar; Thailand [1194]; Viet Nam

Butastur rufipennis (Sundevall 1851)

I1

$15,212,226,227,1247,1298$

Grasshopper Buzzard, Grasshopper Buzzard-Eagle

Breeding: $\quad$ Benin; Burkina Faso; Cameroon; Chad; Ethiopia; Mali; Mauritania; Niger; Nigeria; Sudan

Non-breeding: Central African Republic; Côte d'lvoire; Gambia; Ghana; Guinea [1454]; ?Guinea-Bissau;

Kenya; Liberia; Rwanda; Senegal; Sierra Leone; Somalia; Tanzania; Togo; Uganda; Zaire

Butastur teesa (Franklin 1831)

11

$10,15,226,661,792$

White-eyed Buzzard

Breeding: $\quad$ Bangladesh; ?Bhutan; China; India; Iran; Myanmar; Nepal; Pakistan

Vagrant: Afghanistan; ?Australia

$\begin{array}{llll}\text { Buteo albicaudatus Vieillot } 1816 \quad 11 & - & 15,16,226,967\end{array}$

White-tailed Hawk

Breeding: $\quad$ Argentina; Aruba; Belize [338]; Bolivia; Brazil; Colombia; Costa Rica; ?Ecuador; El Salvador; French Guiana; Guatemala; Guyana; Honduras; Mexico; Netherlands Antilles; Nicaragua; Panama; Paraguay; Peru; Suriname; Trinidad and Tobago: Trinidad; USA; Uruguay; Venezuela

Vagrant: $\quad$ Saint Vincent

Buteo albigula Philippi 1899

II

(= B. brachyurus albigula)

White-throated Hawk

Breeding: $\quad$ Argentina; Bolivia; Chile [1507]; Colombia; Ecuador; Peru; Venezuela
Buteo albonotatus Kaup 1847
II
$15,16,226,967$

Zone-tailed Hawk

Breeding:

?Belize; Bolivia; Brazil; Colombia; Costa Rica; Ecuador; El Salvador; French Guiana; Guatemala [127]; Guyana; Honduras; Mexico; Nicaragua; ?Panama; Paraguay; Peru; Suriname; Trinidad and Tobago: Trinidad; USA; Venezuela

Buteo archeri Sclater 1918

II

(=B. augur archeri)

Archer's Burzard

Breeding:

Somalia

Buteo augur (Rüppell 1836)

II

Augur Buzzard

Breeding:

Angola; Botswana; Burundi; Chad; Ethiopia; Kenya; Malawi; Namibia; Rwanda; Sudan; Tanzania; Uganda; Zaire; Zambia; Zimbabwe 
Red-necked Buzzard, Red-tailed Buzzard, African Red-tailed Buzzard

Breeding:

Angola; Benin [667]; Burkina Faso; Cameroon; Central African Republic; Chad; Congo; Côte d'Ivoire; Equatorial Guinea [1454]; Ethiopia; Gabon; Ghana; Guinea; Liberia; ?Malj; ?Niger; Nigeria; Rwanda; Sierra Leone; Sudan; Togo; Zaire

Non-breeding: Niger; Uganda

Vagrant:

Gambia; ?Guinea-Bissau; ?Mauritania; Senegal

Buteo brachypterus Hartlaub 1860

II

Madagascar Buzzard

Breeding:

Madagascar [373]

Buteo brachyurus Vieillot 1816

Short-tailed Hawk

Breeding:

Argentina; Belize; Bolivia; Brazil; Colombia; Costa Rica; Ecuador; El Salvador; French Guiana; Guatemala [127]; Guyana; Honduras; Mexico; Nicaragua; Panama; Paraguay; Peru; Suriname; USA; Venezuela

Buteo buteo (Linnaeus 1758)

Il

$10,15,212,226,227,285,345$, 661,792

\section{Common Buzzard, Eurasian Buzzard}

Breeding:

Albania; Andorra; Armenia; Austria; Azerbaijan; Azores; Belarus; Belgium; Bulgaria; Canary Islands; Cape Verde; China; Czechoslovakia; Denmark; Estonia; Finland; France; Georgia; Germany; Greece; Hungary; India; Iran; Ireland; Italy; Japan; Kazakhstan; Kyrgyzstan; Latvia; Liechtenstein; Lithuania; Luxembourg; Madeira; Moldova; Mongolia; ?Nepal; Netherlands; Norway; ?Pakistan; Poland; Portugal; Romania; Russia; Spain; Sweden; Switzerland; Turkey; Ukraine; United Kingdom; Yugoslavia

Non-breeding: $\quad$ Afghanistan; Angola; Bahrain; Bangladesh; Bhutan; Botswana; Burundi; Cambodia; Central African Republic; Chad; Côte d'Ivoire; Cyprus; Djibouti; Egypt; Ethiopia; Gibraltar; Guernsey; ?Guinea-Bissau; Hong Kong; Iraq; Israel; Jersey; Jordan [1451]; Kenya; D.P.R. Korea; Korea Republic; Kuwait; Lao P.D.R; Lebanon; Lesotho; Liberia; Libya; Malawi; Malta; Mauritania; Morocco; Mozambique; Myanmar; Namibia; Nepal; Oman; Pakistan; Qatar; Rwanda; Saudi Arabia; ?Sierra Leone; South Africa; Sri Lanka; Sudan; Swaziland; Syria; Taiwan; Tanzania; Thailand; Tunisia; Turkey; Uganda; United Arab Emirates; Uzbekistan; Viet Nam; ?Western Sahara; Yemen; Zaire; Zambia; Zimbabwe

Vagrant: Algeria; ?Cameroon; Faeroe Islands; ?Gabon; Gambia; Ghana; Iceland; Indonesia: Bali, Java [942]; Malaysia: Peninsular Malaysia; Maldives; Mali; Marianas Islands; ?Nigeria; Philippines; Senegal; Singapore; Somalia

Buteo galapagoensis (Gould 1837)

\section{Galapagos Hawk}

Breeding:

Buteo hemilasius Temminck \& Schlegel 1844 Upland Burzard

Breeding:

Non-breeding:

Vagrant:
Il $\quad \mathbf{R}$

$15,226,318,321,450,452,794$, 1446,1447

Galapagos Islands 
Red-tailed Hawk Breeding:

Non-breeding:

Buteo lagopus (Pontoppidan 1763)

Rough-legged Hawk, Rough-legged Buward

Breeding:

Canada; Finland; Latvia (ex); Norway; Russia; Saint Pierre and Miquelon; Sweden; USA: Alaska, Aleutian Islands

Non-breeding: $\quad$ ?Albania; Austria; Belgium; Bulgaria; China; Czechoslovakia; Denmark; France; Germany; Hungary; Iran; Italy; Japan; Kazakhstan; D.P.R. Korea; Korea Republic; Latvia; Luxembourg; Mexico; Mongolia; Netherlands; Poland; Romania; Switzerland; Taiwan; Turkey; Ukraine; United Kingdom; Uzbekistan; Yugoslavia

Vagrant: Afghanistan; Bermuda; Cyprus; Faeroe Islands; Greece; Guernsey; Iceland; Iran; Ireland; Israel; Jersey; Jordan [1451]; Lebanon; Libya; Liechtenstein; Malta; Spain; Tunisia

Buteo leucorrhous (Quoy \& Gaimard 1824)

II

$15,226,967$

White-rumped Hawk, Rufous-thighed Hawk

Breeding:

Argentina; Bolivia; Brazil; Colombia; Ecuador; Paraguay; Peru; Venezuela

Buteo lineatus (Gmelin 1788)

I1

$15,16,226$

Red-shouldered Hawk

Breeding:

Canada; Mexico; USA

Buteo magnirostris (Gmelin 1788)

JI

$15,16,226,967$

Roadside Hawk

Breeding:

Argentina; Belize; Bolivia; Brazil; Colombia; Costa Rica; Ecuador; El Salvador; French Guiana; Guatemala; Guyana; Honduras; Mexico; Nicaragua; Panama; Paraguay; Peru; Suriname; Uruguay; Venezuela

Vagrant: USA

Buteo oreophilus Hartert \& Neumann 1914

$15,212,226,227,285,286,911,1298$

(=B. tachardus (Daudin 1800))

Mountain Buzzard, Forest Buzzard

Breeding:

Burundi; Ethiopia; Kenya; Malawi; Rwanda; South Africa; Sudan; Tanzania; Uganda; Zaire; Zambia

Buteo platypterus (Vieillot 1823)

II

$15,16,179,226,794,967$

Broad-winged Hawk

Breeding: $\quad$ Antigua and Barbuda; Belize; Canada; Cuba; Dominica; Grenada; Martinique; Puerto Rico; Saint Lucia; Saint Vincent; Trinidad and Tobago: Tobago, Little Tobago; USA

Non-breeding: $\quad$ Bolivia; Brazil; Colombia; Costa Rica; Ecuador; El Salvador; French Guiana; Guatemala; Honduras; Mexico; Nicaragua; Panama; Peru; Venezuela

Vagrant:

Barbados; Suriname

Buseo poecilochrous Gurney 1879

II

$15,226,967$

Puna Hawk, Variable Hawk, Gurney's Hawk

Breeding:

Argentina; Bolivia; ?Chile [715]; ?Colombia; Ecuador; Peru 
Red-backed Hawk

Breeding:

Argentina; Bolivia; Chile [715]; ?Colombia; Ecuador; Falkland lslands; Peru

Vagrant: ?Brazil; Uruguay

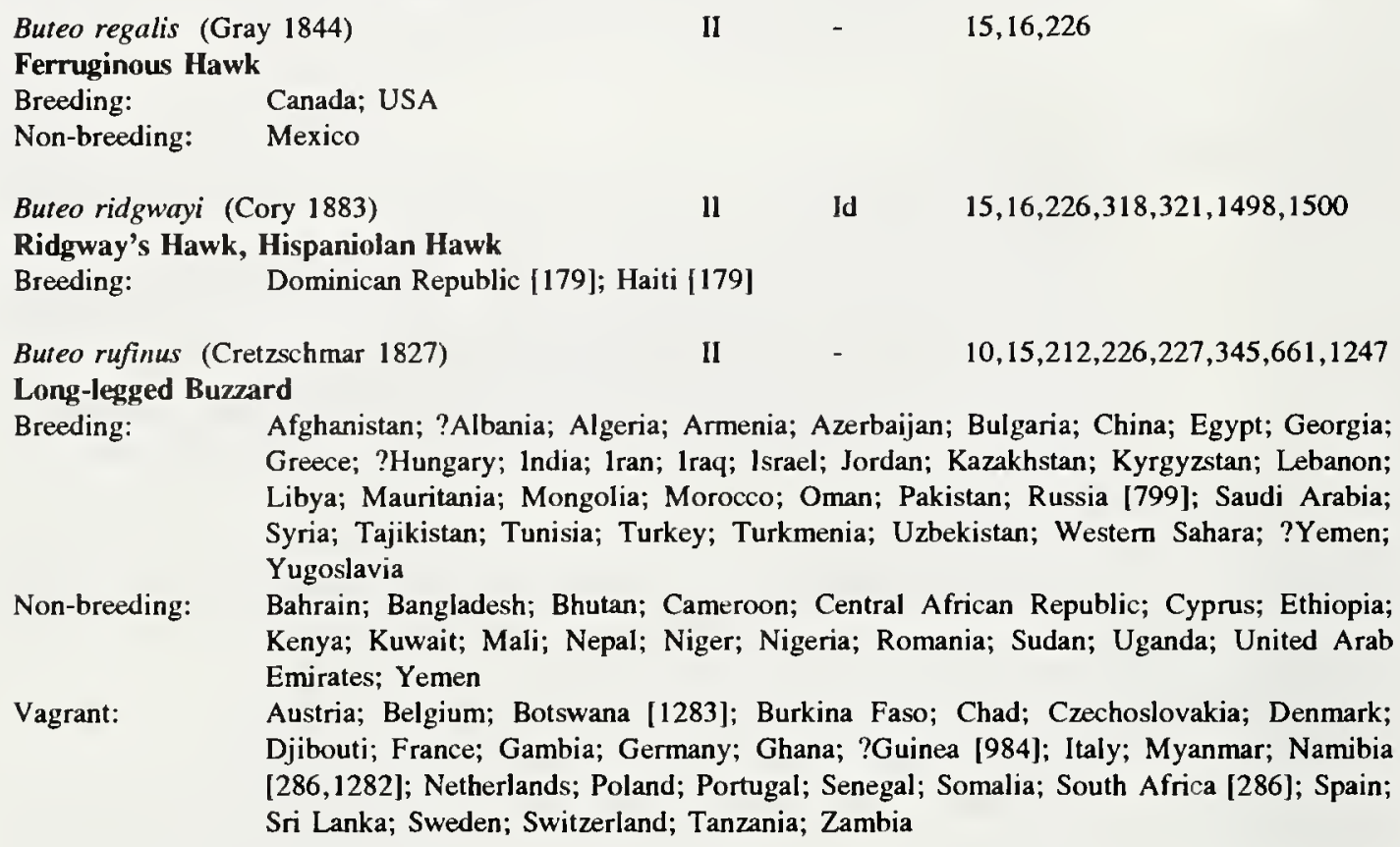

Long-legged Buzzard

Breeding:

Non-breeding:

Vagrant:

Afghanistan; ?Albania; Algeria; Armenia; Azerbaijan; Bulgaria; China; Egypt; Georgia; Greece; ?Hungary; India; Iran; lraq; Israel; Jordan; Kazakhstan; Kyrgyzstan; Lebanon; Libya; Mauritania; Mongolia; Morocco; Oman; Pakistan; Russia [799]; Saudi Arabia; Syria; Tajikistan; Tunisia; Turkey; Turkmenia; Uzbekistan; Western Sahara; ?Yemen; Yugoslavia

Bahrain; Bangladesh; Bhutan; Cameroon; Central African Republic; Cyprus; Ethiopia; Kenya; Kuwait; Mali; Nepal; Niger; Nigeria; Romania; Sudan; Uganda; United Arab Emirates; Yemen

Austria; Belgium; Botswana [1283]; Burkina Faso; Chad; Czechoslovakia; Denmark; Djibouti; France; Gambia; Germany; Ghana; ?Guinea [984]; Italy; Myanmar; Namibia [286,1282]; Netherlands; Poland; Portugal; Senegal; Somalia; South Africa [286]; Spain; Sri Lanka; Sweden; Switzerland; Tanzania; Zambia

\section{Jackal Buzzard \\ Breeding:}

Buteo rufofuscus (Forster 1798)

11

$15,212,226,227,285,1298$

Botswana; Lesotho; Mozambique; Namibia; South Africa; Swaziland
Buteo solitarius Peale 1848
$11 \quad \mathrm{R} \quad 15,16,226,318,794$

Hawaiian Islands: Hawaii
II $\quad-\quad 15,16,226,967$
Swainson's Hawk

Breeding:

Non-breeding:

Vagrant:
Canada; Mexico; USA

Argentina; Belize; Bolivia; Brazil [1268]; Colombia; Costa Rica; Ecuador; El Salvador; Guatemala; Honduras; Nicaragua; Panama; Paraguay; Peru; Uruguay; Venezuela Trinidad and Tobago [22]
Buteo ventralis Gould 1837

Rufous-tailed Hawk, Red-tailed Hawk

Breeding: Argentina [154]; Chile [715]

Buteogallus aequinoctialis (Gmelin 1788)

II

$15,226,291,318,967$

Rufous Crab-Hawk

Breeding:

Brazil; French Guiana; Guyana; Suriname; Venezuela 
Buteogallus anthracinus (Deppe 1830)

\section{Common Black-Hawk, Lesser Black-Hawk}

Breeding: Belize; Colombia; Costa Rica; Cuba; El Salvador; French Guiana; Guatemala; Guyana, Honduras; Mexico; Nicaragua; Panama; Saint Vincent; Trinidad and Tobago: Trinidad; USA; Venezuela

Vagrant: Grenada; Puerto Rico; Saint Lucia

Buteogallus meridionalis (Latham 1790)

II $15,16,226,967$

(= Heterospizias meridionalis)

Savanna Hawk

Breeding:

Argentina; Bolivia; Brazil; Colombia; Costa Rica; Ecuador; French Guiana; Guyana;

Panama; Paraguay; Peru; Suriname; Trinidad and Tobago: Trinidad; Uruguay; Venezuela

Buteogallus subtilis (Thayer \& Bangs 1905)

11

Mangrove Black-Hawk

Breeding: $\quad$ Colombia; Costa Rica; Ecuador; Honduras; Mexico; Panama; Peru

Buteogallus urubitunga (Gmelin 1788) II - $\quad 15,16,226,967$

Great Black Hawk

Breeding: $\quad$ Argentina; Belize; Bolivia; Brazil; Colombia; Costa Rica; Ecuador; El Salvador; French Guiana; Guatemala; Guyana; Honduras; Mexico; Nicaragua; Panama; Paraguay; Peru; Suriname; Trinidad and Tobago: Trinidad; Uruguay; Venezuela

Chelictinia riocourii (Vieillot 1822)

11

$15,212,226,227,1247,1298$

(= Elanus riocourii)

Scissor-tailed Kite, African Swallow-tailed Kite

Breeding: $\quad$ Chad; Ethiopia; Kenya; Mali; ?Mauritania; Niger; ?Somalia; Sudan; ?Uganda

Non-breeding: Benin; Burkina Faso; Cameroon; ?Central African Republic; Gambia; Ghana; ?Guinea; ?Guinea-Bissau; Liberia; Nigeria; Senegal; ?Sierra Leone; Somalia; Togo; Uganda

Vagrant:

Côte d'lvoire; Yemen [661]

Chondrohierax uncinatus (Temminck 1822) II - $\quad 15,16,226,794$

(excluding subspecies wilsonii)

Hook-billed Kite

Breeding:

Argentina; Belize; Bolivia; Colombia; Costa Rica; Ecuador; El Salvador; French Guiana; Grenada; Guatemala; Guyana; Honduras; Mexico; Nicaragua; Panama; Paraguay; Peru; Suriname; Trinidad and Tobago: Trinidad; Venezuela

Vagrant:

USA

$\begin{array}{llll}\text { Chondrohierax uncinatus wilsonii (Cassin 1847) } & 1 & - & 15,16,226,794\end{array}$

(= Chondrohierax wilsonii)

Cuban Hook-billed Kite

Breeding: Cuba

Circaetus cinerascens von Müller 1851

II

$15,212,226,227,285,1247,1298$

Banded Snake-Eagle, Smaller Banded Snake-Eagle

Breeding: Angola; Benin; Botswana; Cameroon; Central African Republic; Chad; Congo; Côte d'Ivoire; Equatorial Guinea; Ethiopia; ?Gabon; Gambia; Ghana; Guinea [1454]; Guinea-Bissau; Kenya; Malawi; ?Mali; Mozambique; ?Namibia [224]; Niger; ?Nigeria; Rwanda; Senegal; ?Sierra Leone; Sudan; Tanzania; Togo; Uganda; Zaire; Zambia; Zimbabwe 
Brown Snake-Eagle, Brown Harrier-Eagle

Breeding:

Angola; Benin; Botswana; ?Burundi; Cameroon; Central African Republic; Chad; Côte d'Ivoire; Equatorial Guinea; Ethiopia; Gambia; Ghana; ?Guinea [984]; Guinea-Bissau; Kenya; Malawi; ?Mali; Mauritania; Mozambique; Namibia; Niger; ?Nigeria; Rwanda; Senegal; Sierra Leone; Somalia; South Africa; Sudan; Swaziland; Tanzania; Togo [278]; Uganda; Zaire; Zambia; Zimbabwe

\section{Circaetus fasciolatus Kaup 1850 \\ $1 \mathrm{I}$ \\ $15,212,226,227,285,1298$}

Fasciated Snake-Eagle, Southern Banded Snake-Eagle

Breeding: $\quad$ Kenya; Malawi; Mozambique; Somalia; South Africa; Tanzania; Zimbabwe

Circaetus gallicus (Gmelin 1788)

II

$10,15,212,226,227,285,345,661$, 1247,1298

(includes $C$. beaudouini Verreaux \& Des Murs 1862)

Short-toed Snake-Eagle, Short-toed Eagle, (Beaudouin's Snake-Eagle)

Breeding: $\quad$ ?Albania; Algeria; Andorra; Angola; Armenia; Azerbaijan; Bangladesh; Belarus; ?Bhutan; Bulgaria; Cameroon; Central African Republic; Chad; China; Côte d'Ivoire; Czechoslovakia; ?Egypt; Estonia; France; Gambia; Georgia; Greece; Guinea [984]; Guinea-Bissau; Hungary; India; Indonesia: Flores, Lombok, Roti, Sumbawa, Timor; Iran; ?Iraq; Israel; Italy; Jordan; Kazakhstan; Kenya; Kyrgyzstan; Latvia; Lebanon; ?Libya; Lithuania; ?Mali; Mauritania; Moldova; Mongolia; Morocco; ?Nepal; ?Niger; Nigeria; ?Oman; Pakistan; Poland; Portugal; Romania; Russia [799]; Rwanda; Saudi Arabia; Senegal; ?Sierra Leone; Spain; ?Switzerland; Syria; Tajikistan; ?Togo; Tunisia; Turkey; Turkmenia; Uganda; Ukraine; Uzbekistan; ?Western Sahara; Yugoslavia

Non-breeding: Afghanistan; Bahrain; Benin; Burkina Faso; Djibouti; Egypt; Ghana; Gibraltar; Iraq; Kuwait; Myanmar; Niger; Oman; Sierra Leone; Singapore; Switzerland; Thailand; United Arab Emirates; Viet Nam; Yemen

Vagrant: $\quad$ Austria; Belgium; ?Congo; Cyprus; Denmark; Finland; Germany; Indonesia: Java [106]; Liberia; Luxembourg; Malaysia: Peninsular Malaysia; Malta; Netherlands; Sweden

Circus pectoralis Smith 1829

II

Black-chested Snake-Eagle

Breeding: $\quad$ Angola; Botswana; Ethiopia; Kenya; Malawi; Mozambique; Namibia; Somalia; South Africa; Sudan; Swaziland; Tanzania; Zaire; Zambia; Zimbahwe

Vagrant: Lesotho

Circus aeruginosus (Linnaeus 1758) II $\quad$ I $\quad 10,15,212,226,227,285,345,661$, Western Marsh-Harrier

Breeding:

Afghanistan; Albania; Algeria; Armenia; Austria; Azerbaijan; Belarus; Belgium; Bulgaria; China; Czechoslovakia; Denmark; Estonia; Finland; France; Georgia; Germany; Greece; Hungary; Iran; Iraq; Ireland; Israel (ex); Italy; Japan; Kazakhstan; Kyrgyzstan; Latvia; Lebanon (ex); Lithuania; Moldova; Morocco; Netherlands; Norway; Poland; Portugal; Romania; Russia; Spain; Sweden; ?Syria; Tajikistan; Tunisia; Turkey; Turkmenia; Ukraine; United Kingdom; Uzbekistan; Yugoslavia

Non-breeding: Angola; Bahrain; Bangladesh; Benin; ?Botswana; Burkina Faso; Burundi; Cambodia; Cameroon; Central African Republic; Chad; Côte d'Ivoire; Cyprus; Djibouti; Egypt; Ethiopia; Gambia; Ghana; Gibraltar; Guernsey; Guinea [1454]; Hong Kong; India: including Andaman Islands; Israel; Jersey; Jordan [1451]; Kenya; Kuwait; Lao P.D.R; Lebanon; Liberia; Libya; Liechtenstein; Luxembourg; Malawi; Malaysia: ?Peninsular Malaysia; Maldives; Mali; Malta; Mauritania; Mozambique; Myanmar; Nepal; Niger; Nigeria; Norway; Oman; Pakistan; Qatar; Rwanda; Saudi Arabia; Senegal; Sierra Leone; Singapore; Somalia; Sri Lanka; Sudan; Switzerland; Syria; Tanzania; Togo [280]; Uganda; United Arah Emirates; Viet Nam; Westem Sahara; Yemen; Zaire; Zambia

Vagrant: Canary lslands; Cape Verde; Faeroe Islands; Iceland; Indonesia: Sumatra; Mauritius; Seychelles; South Africa; ?Thailand; Zimbabwe 


\section{Swamp Harrier}

Breeding:

Vagrant:

Australia [167]; Fiji [1118]; [French Polynesia: Society lslands] [1118]; ?Indonesia: Irian Jaya; New Caledonia; New Zealand; ?Papua New Guinea; Tonga [1118]; Vanuatu; Wallis and Futuna Islands

Circus assimilis Jardine \& Selby 1828

Spotted Harrier

Breeding:

Australia [167]; Indonesia: Sulawesi, Sumba [881], Taliabu, Timor

Circus buffoni (Gmelin 1788)

11

$15,226,967$

Long-winged Harrier

Breeding:

Argentina; Bolivia; Brazil; Chile; Colombia; French Guiana; Guyana; Paraguay; Suriname; Trinidad and Tobago; Uruguay; Venezuela

Vagrant: Chile [715]

Circus cinereus Vieillot 1816 II $15,226,967$

\section{Cinereous Harrier}

Breeding:

Vagrant:

Argentina; Bolivia; Brazil; ?Chile; Colombia; Ecuador; Paraguay; Peru; Uruguay Falkland 1slands

Circus cyaneus (Linnaeus 1766)

Northern Harrier, Hen Harrier, Marsh Hawk

Breeding:

Non-breeding:

Vagrant:
Armenia; Azerbaijan; Belarus; Belgium; Canada; China; Czechoslovakia; Denmark; Estonia; Finland; France; Georgia; Germany; ?Iran; Ireland; Isle of Man; Latvia; Lithuania; Luxembourg; Mexico; Moldova; Mongolia; Netherlands; Norway; Poland; Portugal; Russia; Spain; Sweden; ?Turkey; Ukraine; USA; United Kingdom

Afghanistan; Albania; Algeria; ?Anguilla; Antigua and Barbuda; Austria; Bahamas; Brngladesh; Barbados; Belgium; Belize; Bhutan; ?British Virgin Islands; ?Brunei; Bulgaria; Cambodia; Cayman Islands; Costa Rica; Cuba; Cyprus; Denmark; Dominica; Dominican Republic; Egypt; El Salvador; Gibraltar; Greece; Grenada; Guadeloupe; Guatemala; Guernsey; Haiti; Honduras; Hong Kong; Hungary; India; Iran; Iraq; 1srael; Italy; Jamaica; Japan; Jersey; Jordan [1451]; Kazakhstan; D.P.R. Korea; Korea Republiz; Kuwait; Kyrgyzstan; ?Lao P.D.R; Lebanon; Libya; Liechtenstein; Malaysia: Peninsular Malaysia, Sabah, Sarawak [1295]; Malta; ?Martinique; Mauritania; ?Montserrat; Morocco; Myanmar; Nepal; Netherlands Antilles; Nicaragua; Oman; Pakistan; Panama; Puerto Rico; Romania; Saint Kitts and Nevis; Saint Lucia; Saint Pierre and Miquelon; Saint Vincent; Saudi Arabia; Switzerland; Tajikistan; Thailand; Taiwan; Tunisia; Turkey; Turkmenia; United Arab Emirates; Uzbekistan; ?Viet Nam; Virgin Islands of the United States; Yemen; Yugoslavia Bermuda; Colombia; Faeroe 1slands; Hawaiian 1slands [1118]; 1celand; Qatar; Senegal; Singapore; Syria; Turks and Caicos Islands; Venezuela

Circus macrourus (Gmelin 1770)

$10,15,212,226,227,285,345,661$, 792

\section{Pallid Harrier, Pale Harrier}

Breeding:

Armenia; Azerbaijan; Belarus; China; Georgia; ?Iran; Kazakhstan; Latvia (ex); Moldova; Romania; Russia; Ukraine; Uzbekistan

Non-breeding:
Afghanistan; Albania; Algeria; Angola; Bahrain; Bangladesh; Benin; Bhutan; Botswana; Bulgaria; Burkina Faso; Burundi; Cameroon; Central African Republic; Chad; Côte d'Ivoire; Djibouti; Egypt; Ethiopia; France; Gambia; Ghana; Greece; Guinea [1454]; Guinea-Bissau; India: including Andaman Islands; Iran; 1raq; Israel; Italy; Jordan [1451]; Kenya; Kuwait; Lebanon; Libya; Malawi; Maldives; Mali; Malta; Mauritania; ?Mongolja; Morocco; Mozambique; Myanmar; Namibia; Nepal; Niger; Nigeria; Oman; Pakistan;Qatar; Rwanda; Saudi Arabia; Senegal; Sierra Leone; Somalia; South Africa; Sri Lanka; Sudan; 
?Swaziland; Syria; Tanzania; Togo; Tunisia; Turkey; Uganda; United Arab Emirates; Western Sahara; Yemen; Yugoslavia; Zaire; Zambia; Zimbabwe

Vagrant: $\quad$ Austria; Belgium; Czechoslovakia; Denmark; Finland; Germany; Hungary; Iceland; Japan; Latvia; Lesotho; Liberia; Liechtenstein; Luxembourg; Netherlands; Norway; Spain; Sweden; Switzerland; United Kingdom

Circus maillardi Verreaux 1862
(= C. aeruginosus maillardi)
Madagascar Marsh-Harrier
Breeding: $\quad$ Comoros; Madagascar; Mayotte; Réunion

Circus maurus (Temminck I828) II - $\quad 15,226,227,285,959,1298$

Black Harrier

Breeding:

?Namibia; South Africa

Non-breeding: Botswana; Lesotho; Namibia

Circus melanoleucos (Pennant 1769) $\quad 11 \quad$ - $\quad 10,15,226,792$

Pied Harrier

Breeding:

Non-breeding:

China; India; D.P.R. Korea; Mongolia; Myanmar; Russia

Bangladesh; ?Bhutan; Cambodia; Hong Kong; Indonesia: Kalimantan [1295]; Korea Republic; Lao P.D.R; Malaysia: Peninsular Malaysia, Sabah, Sarawak [1295]; Nepal; Pakistan; Philippines; Singapore; Sri Lanka; Taiwan; Thailand; Viet Nam

Vagrant:

Brunei [898]; Japan

Circus pygargus (Linnaeus 1758)

II

$10,15,212,226,227,285,345,661$

Montagu's Harrier

Breeding:

Algeria; Armenia; Austria; Azerbaijan; Belarus; Belgium; Bulgaria; China; Czechoslovakia; Denmark; Estonia; Finland; France; Georgia; Germany; Greece; Hungary; Iran; Italy; Kazakhstan; Kyrgyzstan; Latvia; Lithuania; Luxembourg; Moldova; Morocco; Netherlands; Poland; Portugal; Romania; Russia; Spain; Sweden; ?Switzerland; Tunisia; Turkey; Ukraine; United Kingdom; Uzbekistan; Yugoslavia

Non-breeding: $\quad$ Afghanistan; Albania; Bahrain; Bangladesh; Benin; ?Bhutan; Botswana; Bulgaria; Burkina Faso; ?Burundi; Cameroon; Central African Republic; Chad; Côte d'Ivoire; Cyprus; Djibouti; Egypt; Ethiopia; Gambia; ?Ghana; Gibraltar; Guinea [984]; Guinea-Bissau; India: including Andaman lslands; Iraq; Israel; Jordan [ I45 I]; Kenya; Kuwait; Laccadive Islands; Lebanon; ?Lesotho; Libya; Liechtenstein; Malawi; Maldives; Mali; Malta; Mauritania; Mozambique; Namibia; Nepal; Niger; Nigeria; Oman; Pakistan; Rwanda; Saudi Arabia; Senegal; ?Sierra Leone; Somalia; South Africa; Sri Lanka; Sudan; Swaziland; Switzerland; Syria; Tajikistan; Tanzania; Togo; Turkmenia; Uganda; United Arab Emirates; Westem Sahara; Yemen; Zaire; Zambia; Zimbabwe

Vagrant: $\quad$ Canary Islands; Cape Verde; Guernsey; Iceland; Ireland; Norway

Circus ranivorus (Daudin 1800)

II

$15,212,226,227,285,1298$

African Marsh-Harrier

Breeding: $\quad$ Angola; Botswana; Burundi; Central African Republic [259]; Kenya; ?Lesotho; Malawi; Mozambique; Namibia; Rwanda; ?Somalia; South Africa; Sudan; Swaziland; Tanzania; Uganda; Zaire; Zambia; Zimbabwe

Circus spilonotus Kaup 1847

Il

$15,226,792,930$

(=C. aeruginosus spilonotus)

\section{Eastern Marsh-Harrier}

Breeding: $\quad$ China; Indonesia: Irian Jaya; ?D.P.R. Korea; Mongolia; Papua New Guinea; Russia

Non-breeding: Brunei; Japan; Malaysia: Peninsular Malaysia, Sabah, Sarawak [1295]; Myanmar; Philippines; 'Thailand; Viet Nam

Vagrant: Indonesia: Sumatra 
Dryotriorchis spectabilis (Schlegel 1863)

Congo Serpent-Eagle, African Serpent-Eagle

Breeding:

Angola; Cameroon; Central African Republic; Congo; Côte d'lvoire; Equatorial Guinez; Gabon; Ghana; Guinea; Liberia; ?Nigeria; Sierra Leone; Togo; Zaire

Elanoides forficatus (Linnaeus 1758)

Il

$15,16,226,967$

Swallow-tailed Kite, American Swallow-tailed Kite

Breeding:

Argentina; Belize; Bolivia; Brazil; Colombia; Costa Rica; Ecuador; French Guiana; Guatemala; Guyana; Honduras; Mexico; Nicaragua; Panama; Paraguay; Peru; Suriname; Trinidad and Tobago: Trinidad; USA; Uruguay; Venezuela

Non-breeding: Cuba; Jamaica

Vagrant: $\quad$ Bahamas; Bermuda; Canada; Cayman Islands

Elanus axillaris (Latham 1801)

II

15,226

( $=E$. notatus Gould 1838)

Black-shouldered Kite, Australian Black-shouldered Kite

Breeding:

Australia [167]

Elanus caeruleus (Desfontaines 1789)

$11 \quad-$

$10,15,212,226,227,285,345,661$, $792,1247,1298$

Black-winged Kite, Black-shouldered Kite

Breeding:

Algeria; Angola; Bangladesh; Benin [667]; ?Bhutan; Botswana; Burkina Faso [1388]; Burundi; Cambodia; Cameroon; Central African Republic; Chad; China; Congo; Côte d'Ivoire; Egypt; Equatorial Guinea; Ethiopia; ?Gabon; Gambia; Ghana; Guinea [1454]; Guinea-Bissau; India; Indonesia; Kenya; Lao P.D.R; Lesotho; Liberia; Malawi; Malaysia: Peninsular Malaysia; Mali; ?Mauritania; Morocco; Mozambique; Myanmar; Namibia; Nepal; Niger; Nigeria; Pakistan; Papua New Guinea; Philippines; Portugal; Rwanda; Senegal; Sierra Leone; Singapore; Somalia; South Africa; Spain; Sri Lanka; Sudan; Swaziland; Tanzania; Thailand [1194]; Togo; Tunisia; Uganda; Viet Nam; Yemen; Zaire; Zambia; Zimbabwe

Vagrant: $\quad$ Belgium; Brunei; Bulgaria; ?Cyprus; Czechoslovakia; France; Germany; Gibraltar; Hong Kong; Israel; Italy; Lebanon; Madagascar; Malaysia: Sabah [1295]; Netherlands; Poland; Romania; Saudi Arabia; Turkey; United Arab Emirates; Uzbekistan

Elanus leucurus (Vieillot 1818) I1 $\quad$ - $\quad 15,16,226,967,1125$

(=E. caeruleus leucurus)

White-tailed Kite

Breeding: $\quad$ Argentina; Belize; Bolivia; Brazil; Chile [715]; Colombia; Costa Rica; El Salvador; Guatemala; Guyana; Honduras; Mexico; Nicaragua; Panama; Paraguay; Suriname; Trinidad and Tobago: Trinidad; USA; Uruguay; Venezuela

Vagrant: Aruba; French Guiana

Elanus scriptus Gould 184

II

15,226

Letter-winged Kite

Breeding:

Australia [167]

Erythrotriorchis buergersi (Reichenow 1914)

II $\quad-\quad 15,226,930,1226$

(= Accipiter buergersi)

Chestnut-shouldered Hawk, Chestnut-mantled Goshawk, Burger's Sparrowhawk

Breeding:

Indonesia: Irian Jaya [393]; Papua New Guinea

Erythrotriorchis radiatus (Latham 1801)

I1 $\quad \mathrm{K}^{*}$

$15,226,318$

(= Accipiter radiatus)

Red Goshawk

Breeding:

Australia [167] 
Eutriorchis astur (Sharpe 1875)

Madagascar Serpent-Eagle

Breeding:

Madagascar $[54,373,1255]$

Gampsonyx swainsonii Vigors 1825

II

$\mathrm{E}$

$15,226,318,324,418$

Pearl Kite

Breeding:

Argentina; Bolivia; Brazil; Colombia; Ecuador; Guyana; Nicaragua; Paraguay; Peru; Suriname; Trinidad and Tobago: Trinidad; Venezuela

Vagrant: French Guiana

Geranoaetus melanoleucus (Vieillot 1819) II - I $\quad 15,226,967$

Black-chested Buzzard-Eagle, Black-chested Eagle, Grey Buzzard-Eagle

Breeding: $\quad$ Argentina; Bolivia; Brazil [1268]; Chile [715]; Colombia; Ecuador; Paraguay; Peru; Uruguay; Venezuela

$\begin{array}{llll}\text { Geranospiza caerulescens (Vieillot 1817) } \quad l 1 & - & 15,16,226,967\end{array}$

Crane Hawk

Breeding: $\quad$ Argentina; Belize; Bolivia; Brazil; Colombia; Costa Rica; Ecuador; El Salvador; French Guiana; Guatemala; Guyana; Honduras; Mexico; Nicaragua; Panama; Paraguay; Peru; Suriname; Uruguay; Venezuela

Gypaetus barbatus (Linnaeus 1758)

11

$10,15,212,226,227,285,345,661$, 1298

\section{Lammergeier, Bearded Vulture}

Breeding: Afghanistan; ?Albania; Algeria; Armenia; Azerbaijan; Bhutan; China; ?Egypt; Ethiopia [748]; France; Georgia; Greece; India; 1ran; ?]raq; 1srael; Jordan; Kazakhstan; Kenya; Kyrgyzstan; Lesotho; Mongolia; Morocco; Nepal; Pakistan; Russia [799]; Sardinia; Saudi Arabia; South Africa; Spain; Sudan; Tajikistan; Tanzania; Tunisia (ex?); Turkey; Turkmenia; Uganda; Uzbekistan; Yemen; Yugoslavia

Non-breeding: Andorra

Vagrant: $\quad$ Austria; Bulgaria; Cyprus; Czechoslovakia; Germany; D.P.R. Korea; Lebanon; Mozambique; Namibia; Portugal; Romania; Switzerland; Syria; ?Zaire

Gypohierax angolensis (Gmelin 1788)

II

$15,212,226,227,285,1247,1298$

Palm-nut Vulture, Vulturine Fish-Eagle

Breeding:

Angola; Benin; Botswana; Burkina Faso [1388]; Burundi; Cameroon; Central African Republic; Chad; Congo; Côte d'lvoire; Equatorial Guinea; Gabon; Gambia; Ghana; Guinea [1454]; Guinea-Bissau; Kenya; Liberia; Malawi; Mali; Mozambique; Niger; Nigeria; Rwanda; Senegal; Sierra Leone; South Africa; Sudan; ?Swaziland; Tanzania; Togo; Uganda; Zaire; Zambia

Non-breeding: Namibia; Zimbabwe

Vagrant: Lesotho

Gyps africanus Salvadori 1865

11

$15,212,226,227,285,1247,1298$

White-backed Vulture, African White-hacked Vulture

Breeding:

Angola; Benin; Botswana; Burkina Faso [1388]; ?Burundi; Cameroon; Central African Republic; Chad; Côte d'Ivoire; ?Djibouti; Ethiopia; Gambia; Ghana; Guinea [1454]; Guinea-Bissau; Kenya; Malawi; Mali; Mauritania; Mozambique; Namibia; Niger; Nigeria; Rwanda; Senegal; Sierra Leone; Somalia: South Africa; Sudan; Swaziland; Tanzania; Togo; Uganda; Zaire; Zambia; Zimbabwe

Vagrant: Equatorial Guinea: Bioko; Gabon [221] 
Vagrant:

Brunei; Russia

Gyps coprotheres (Forster 1798)

1I R $\quad$ R $15,216,226,227,285,318,324$,

$1173,1174,1298$

\section{Cape Griffon, Cape Vulture}

Breeding: $\quad$ Botswana; Lesotho; Mozambique; Namibia [223]; South Africa $[187,188,1109,1172,1426]$;

Zimbabwe (ex) [701]

Non-breeding: Swaziland

Vagrant: $\quad$ Zaire; Zambia [407]

Gyps fulvus (Hablizl 1783)

11

$10,15,226,227,345,661,930$

Eurasian Griffon, Eurasian Griffon Vulture

Breeding:

Afghanistan; ?Albania; Algeria; Armenia; Austria; Azerhaijan; Bulgaria; Cyprus; Egypt; France; Georgia; Greece; India; Iran; ?Iraq; Israel; Italy: Sardinia; Jordan; Kazakhstan; Kyrgyzstan; ?Mongolia; Morocco; Nepal; Pakistan; Portugal [1j06]; Romania (ex); Russia [799]; Saudi Arabia; Spain [1306]; Syria; Tajikistan; ?Tunisia; Turkey; Turkmenia; Uzbekistan; Yemen; Yugoslavia

Non-breeding: Ethiopia; Gibraltar; Lebanon; Mali; Mauritania; Oman; Sudan; United Arab Emirates

Vagrant: Bahrain; Belgium; Central African Republic; ?Chad; Czechoslovakia; Denmark; Djibouti [1474]; Finland; Germany; Hungary; Ireland; Kuwait; Latvia; Malta; Netherlands; Niger; Poland; Senegal; Switzerland; United Kingdom; Western Sahara

Gyps himalayensis Hume 1869

II

$10,15,226$

Himalayan Griffon, Himalayan Griffon Vulture

Breeding: $\quad$ Afghanistan; Bhutan; China; India; Kazakhstan; Kyrgyzstan; Mongolia; Nepal; Pakistan; Tajikistan; ?Uzbekistan

Gyps indicus (Scopoli 1786)

$10,15,226,792$

Long-billed Vulture, Long-billed Griffon

Breeding: $\quad$ Bangladesh; ?Bhutan; Cambodia; India; Lao P.D.R; Myanmar; Nepal; Pakistan; Thailand (ex?) [1194]; Viet Nam

Vagrant: Afghanistan; Malaysia: Peninsular Malaysia

Gyps rueppellii (Brehm 1852)

II

$15,212,226,227,661,1247,1298$

Rueppell's Griffon, Rueppell's Vulture, Rueppell's Griffon Vulture

Breeding:

Benin; Burkina Faso [1388]; Cameroon; Central African Republic; Chad; Djibouti; Ethiopia; Gambia; ?Guinea [984]; Kenya; Mali; Mauritania; Niger; Nigeria; Senegal; Sudan; Tanzania; Uganda; ?Yemen

Non-breeding: Côte d'lvoire; Guinea; Guinea-Bissau

Vagrant: $\quad$ Egypt; Ghana; Saudi Arabia; Somalia; Togo; ?Zaire

$\begin{array}{lll}\text { Haliaeerus albicilla (Linnaeus 1758) } & \text { I V } & \begin{array}{l}15,185,226,318,345,523,661 \text {, } \\ 794,863,930\end{array}\end{array}$

White-tailed Eagle, Grey Sea Eagle

Breeding: Albania (ex?); Algeria (ex); Armenia; Austria; Azerbaijan; Belarus; Bulgaria; China; Corsica (ex); Czechoslovakia; Denmark; Estonia; Finland; Georgia; Germany; Greece; Greenland; Hungary; lceland; Iran; 1srael; Japan; Kazakhstan; Latvia; Lithuania; Moldova; Mongolia [1098]; Nepal; Norway [482, i502]; Poland; Romania; Russia [799]; Sweden [640,641]; Turkey; Turkmenia; Ukraine; [United Kingdom]; Yugoslavia

Non-breeding: $\quad$ Afghanistan; France; Faeroe Islands; India; Israel; Japan; D.P.R. Korea; Korea Republic; Nepal; Pakistan; Taiwan; Tajikistan; USA; Uzbekistan 
Vagrant:

Belgium; Canary Islands; Cyprus; Egypt; Guernsey; Iraq; Ireland; Italy; Jersey; Lebanon; Luxembourg; Malta; Netherlands; Portugal; Saudi Arabia; Spain; Switzerland; Syria; Taiwan; Tunisia

Haliaeetus leucocephalus (Linnaeus 1766)

I

$15,16,226,794$

Bald Eagle, White-headed Eagle

Breeding:

Canada; Mexico; Saint Pierre and Miquelon; USA

Vagrant:

?Belize [338]; Bermuda; Puerto Rico; Virgin Islands of the United States: Saint John

Haliaeetus leucogaster (Gmelin 1788)

11

$10,15,226,792,930$

White-bellied Fish-Eagle, White-bellied Sea-Eagle

Breeding:

Australia [167]; Bangladesh; Brunei; Cambodia; China; Hong Kong; India: including Andaman and Nicobar Islands; Indonesia; Lao P.D.R; Malaysia: Peninsular Malaysia, Sabah, Sarawak [1295]; Myanmar; Papua New Guinea; Philippines; Singapore; Sri Lanka; Thailand; Viet Nam

Vagrant: $\quad$ Christmas Island

Haliaeetus leucoryphus (Pallas 1771) II $\quad \mathrm{K}^{*} \quad 10,15,185,226,318,661,792,930$

Pallas's Sea-Eagle, Pallas's Fish-Eagle, Band-tailed Fish-Eagle

Breeding: $\quad$ Bangladesh [1217]; Bhutan; China; India; Kazakhstan; Mongolia [923, 1098]; Myanmar [1296]; Nepal; Pakistan; ?Russia [799]; Tajikistan; Uzbekistan

Non-breeding: Afghanistan

Vagrant:

Cambodia; Finland; Iran; Iraq; Israel; Norway; Oman; Poland; Saudi Arabia; Thailand; United Arab Emirates; Viet Nam

Haliaeetus pelagicus (Pallas 1811) $\quad 11 \quad$ K* $\quad 15,226,318,930,1259$

Steller's Sea-Eagle

Breeding: Russia [481,799,856]

Non-breeding: $\quad$ Japan [203,626]; ?D.P.R. Korea; Korea Republic [626]

Vagrant: Alaska; China; Hawaiian Islands [1118]

Haliaeetus sanfordi Mayr $1935 \quad$ II $\quad$ - 15,226,318,930,1226

Solomon Fish-Eagle, Solomon Sea-Eagle, Sanford's Sea-Eagle

Breeding: $\quad$ Papua New Guinea: Bougainville; Solomon Islands

Haliaeetus vocifer (Daudin 1800)

II

$15,212,226,227,285,1247,1298$

African Fish-Eagle, River Eagle

Breeding:

Angola; Benin; Botswana; Burkina Faso [1388]; Burundi; Cameroon; Central African Republic; Chad; Congo; Côte d'Ivoire; ?Djibouti; Equatorial Guinea; Ethiopia [748]; Gabon; Gambia; Ghana; Guinea [1454]; Guinea-Bissau; Kenya; Lesotho; Liberia; Malawi; Mali; Mauritania; Mozambique; Namibia; Niger; Nigeria; Rwanda; Senegal; Sierra Leone; Somalia; South Africa; Sudan; Swaziland; Tanzania; Togo; Uganda; Zaire; Zambia; Zimbabwe

Vagrant:

Egypt [661]

Haliaeetus vociferoides Des Murs 1845

II

E

$15,226,318,324,828$

Madagascar Fish-Eagle

Breeding: Madagascar [373,825]

Vagrant: Mauritius 


\begin{abstract}
Haliastur indus (Boddaert 1783)
Il

$10,15,226,930$

Brahminy Kite

Breeding:

Australia [167]; Bangladesh; ?Bhutan; Brunei; Cambodia; China; India; Indonesia; ?Lac P.D.R; Malaysia: Peninsular Malaysia, Sabah, Sarawak [1295]; Myanmar; Nepal; Pakistan; Papua New Guinea; Philippines; Singapore; Solomon Islands; Sri Lanka; Thailand; Viet Nam

Vagrant: $\quad$ Hong Kong; Palau [1118]; United Arab Emirates
\end{abstract}
Haliastur sphenurus (Viejllot 1818)
II
$15,226,930$

Whistling Kite

Breeding:

Australia [167]; Indonesia: Irian Jaya; New Caledonia; Papua New Guinea

Hamirostra melanosternon (Gould 1841)

Il

15,226

Black-breasted Burzard, Black-breasted Kite

Breeding:

Australia [167]
Harpagus bidentatus (Latham 1790)
Il
$15,16,226,967$

Double-toothed Kite

Breeding: $\quad$ Belize; Bolivia; Brazil; Colombia; Costa Rica; Ecuador; El Salvador; French Guiana; Guatemala; Guyana; Honduras; Mexico; Nicaragua; Panama; Peru; Suriname; Venezuela
Harpagus diodon (Temminck 1823)
II
$15,226,967$

Rufous-thighed Kite

Breeding:

Argentina; Bolivia; Brazil; Ecuador [967]; French Guiana; Guyana; Paraguay

Vagrant:

Suriname

Harpia harpyja (Linnaeus 1758)

Harpy Eagle, American Harpy Eagle

Breeding:

Argentina [154]; Belize; Bolivia; Brazil; Colombia; Costa Rica; Ecuador; French Guiana; Guatemala; Guyana; Honduras; Mexico; Nicaragua; Panama; Paraguay; Peru; Suriname; Venezuela
Harpyhaliaetus coronatus (Vieillot 1817)
II V
$15,226,318,321,967$
Crowned Eagle, Crowned Solitary Eagle
Breeding:
Argentina [154,1035]; Bolivia; Brazil [7]; Paraguay; Uruguay

Harpyhaliaetus solitarius (Tschudi 1844)

II

$15,16,166,226,318,967$

Solitary Eagle, Black Solitary Eagle

Breeding:

Argentina [154,872]; Belize; Bolivia; Colombia; Costa Rica; Ecuador; French Guiana [1377]; Guatemala [127]; Honduras; Mexico; ?Nicaragua; ?Panama; Peru; Venezuela

Harpyopsis novaeguineae Salvadori 1875

II $\quad \mathrm{K}^{*} \quad 15,130,226,318,930$

New Guinea Eagle, New Guinea Harpy Eagle

Breeding: Indonesia: Irian Jaya; Papua New Guinea
Henicopernis infuscatus Gurney 1882
II $\mathrm{K}^{*}$
$15,226,318,930,1226$

Black Honey-buzzard, New Britain Honey-buzzard

Breeding: Papua New Guinea: New Britain [163]

Henicopernis longicauda (Gamot 1828)

$15,226,930$

Long-tailed Honey-buzzard, Papuan Honey-buzzard

Breeding:

Indonesia: Aru Islands, Biak, Irian Jaya, Misool, Yapen; Papua New Guinea 
(= H. dubius (Smith 1830) misapplied)

Ayres's Hawk-Eagle, Ayres's Eagle

Breeding:

Angola; ?Benin; Botswana; ?Cameroon; Central African Republic; Chad; ?Congo; Côte d'Ivoire; ?Equatorial Guinea; Ethiopia [94]; Ghana; Guinea; Guinea-Bissau; Kenya; Liberia; Malawi; ?Mali; Mozambique; Niger; ?Namibia; Rwanda; Sierra Leone [94]; Somalia [94]; ?Sudan; Tanzania; Togo; Uganda; Zaire; Zambia; Zimbabwe

Non-breeding: $\quad$ South Africa [773]

Vagrant: $\quad$ ?Nigeria; Senegal

Hieraaetus fasciatus (Vieillot 1822)

II

$10,15,212,226,227,286,345,661$,

Bonelli's Eagle

Breeding:

?Albania; Algeria; China; Cyprus; Egypt; France; Greece; Hong Kong; India; Indonesia: Luang, Sumbawa, Timor, Wetar; Iran; Iraq; Israel; Italy: Sicily; ?Jordan; ?Lao P.D.R; ?Lebanon; Libya; Morocco; Myanmar; Nepal; Oman; Pakistan; Portugal; Saudi Arabia; Spain; Syria; Tajikistan; ?Thailand [1194]; Tunisia; Turkey; Turkmenia; United Arab Emirates; ?Uzbekistan; ?Viet Nam; Yemen

Non-breeding: Afghanistan; Bangladesh; Gibraltar

Vagrant:

Austria; Belgium; Bulgaria; Canary Islands; Czechoslovakia; Denmark; Germany; Hungary; Kuwait; Luxembourg; Netherlands; Romania; Sri Lanka; Sweden; Uzbekistan; Viet Nam; Yugoslavia

Hieraaetus kienerii (Geoffroy Saint-Hilaire 1835) II $\quad-\quad 10,15,226,792$

Rufous-bellied Eagle, Chestnut-bellied Hawk-Eagle

Breeding: ?Bhutan; Brunei; Cambodia; ?China: Hainan [968]; India; Indonesia: Bali, Java, ?Kalimantan [233], ?Satonda, Sulawesi, ?Sumatra, ?Ternate; ?Lao P.D.R; ?Malaysia: Peninsular Malaysia, Sabah, Sarawak [1295]; Myanmar; Nepal; Philippines; Sri Lanka; Thailand [1194]; Viet Nam

Vagrant: $\quad$ Bangladesh; Singapore

Hieraaetus morphnoides (Gould 1841) IJ $\quad$ - $\quad$ 15,226,930

Little Eagle

Breeding: $\quad$ Australia [167]; Indonesia: Irian Jaya; Papua New Guinea

Vagrant: $\quad$ Indonesia: Halmahera [79]]

Hieraaetus pennatus (Gmelin 1788) $\quad$ II $\quad 10,15,212,226,227,285,345,661$,

Booted Eagle

$792,1247,1298$

Breeding:

Non-breeding:

Vagrant:
Afghanistan; ?Albania; Algeria; Armenia; Azerbaijan; Bangladesh; Belarus; Bhutan; Bulgaria; China; Czechoslovakia; France; Georgia; Greece; Hungary; India; Iran; Kakakhstan; Kyrgyzstan; Moldova; Mongolia; Morocco; Namibia; Nepal; Pakistan; Poland; Portugal; Romania; Russia; South Africa; Spain; Tajikistan; Tunisia; Turkey; Turkmenia; Ukraine; Uzbekistan; Yugoslavia

Angola; Austria; Bahrain; ?Benin; Botswana; Burkina Faso; Burundi; Cameroon; Central African Republic; Chad; Côte d'Ivoire; Cyprus; Djibouti; Egypt; Ethiopia; Gambia; Ghana; Gibraltar; ?Guinea-Bissau; Israel; Jordan [1451]; Kenya; Kuwait; Lebanon; ?Lesotho; Libya; Malawi; Mali; Mauritania; Mozambique; Myanmar; Niger; Nigeria; Oman; Saudi Arabia; Senegal; ?Sierra Leone; Somalia; Sri Lanka; Sudan; Swaziland; Syria; Tanzania; Togo [280]; Uganda; United Arab Emirates; Western Sahara; Yemen; Zaire; Zambia [407]; Zimbabwe

Belgium; Finland; Germany; Indonesia: Bali [95]; Iraq; 1taly; Liberia; Malaysia: Peninsular Malaysia; Malta; Rwanda; Singapore; Sweden; Switzerland; Tanzania 
Hieraaetus spilogaster (Bonaparte 1850)

II

(= H. fasciatus spilogaster)

African Hawk-Eagle

Breeding: Angola; Benin; Botswana; Cameroon; Central African Republic; Cbad; Côte d'Ivoire; Djibouti; Ethiopia [748]; Gambia; Ghana; Guinea [1454]; Guinea-Bissau; Kenya; Malawi; ?Mali; Mauritania; Mozambique; Namibia; Niger; Nigeria; Rwanda; Senegal; ?Sierra Leone; Somalia; South Africa; Sudan; Swaziland; Tanzania; Togo; Uganda; Zaire; Zambia; Zimbabwe

Non-breeding: ?Lesotho

Ichthyophaga humilis (Müller \& Schlegel 1841) II $\quad$ - $\quad 10,15,226,792$

(= I. nana (Blyth 1842))

Lesser Fishing Eagle

Breeding: $\quad$ Bangladesh; ?Bhutan; Brunei; ?Cambodia; China: Hainan [968]; India; Indonesia; Lao P.D.R; Malaysia: Peninsular Malaysia, Sabah, Sarawak [1295]; Myanmar; Nepal; ?Singapore; Thailand [1194]; Viet Nam

Ichthyophaga ichthyaetus (Horsfield 1821) II - $\quad 10,15,226,792$

Grey-headed Fishing Eagle

Breeding: $\quad$ Bangladesh; ?Bhutan; Brunei; Cambodia; India; Indonesia; Lao P.D.R; Malaysia: Peninsular Malaysia, Sabah, Sarawak [1295]; Myanmar; Nepal; Philippines; Sri Lanka; Thailand [1194]; Viet Nam

Vagrant: $\quad$ Singapore

Ictinaetus malayensis (Temminck 1822) $\quad$ II - 10,15,226,792

Black Eagle, Asian Black Eagle, Indian Black Eagle

Breeding: $\quad$ Bhutan; Brunei; Cambodia; China; India; Indonesia; Lao P.D.R; Malaysia: Peninsular Malaysia, Sabah, Sarawak [1295]; Myanmar; Nepal; Pakistan; Sri Lanka; Taiwan; Thailand [1194]; Viet Nam

Non-breeding: Bangladesh

$\begin{array}{llll}\text { Ictinia mississippiensis (Wilson 1811) II } & - & 15,16,226,967\end{array}$

Mississippi Kite

Breeding: USA

Non-breeding: $\quad$ Argentina; Bolivia [1254]; Colombia; Costa Rica; Guatemala; Honduras; Mexicu; Panama; Paraguay; Peru

Ictinia plumbea (Gmelin 1788)

II $\quad-\quad 15,16,226,967$

Plumbeous Kite

Breeding:

Argentina; Belize; Bolivia; Brazil; Colombia; Costa Rica; Ecuador; El Salvador; French Guiana; Guatemala; Guyana; Honduras; Mexico; Nicaragua; Panama; Paraguay; Peru; Suriname; Trinidad and Tobago: Trinidad; Venezuela

Kaupifalco monogrammicus (Temminck 1824) II $\quad$ II $\quad 15,212,226,227,285,1247,1298$

Lizard Buzzard

Breeding:

Angola; Benin; Botswana; Burundi; Cameroon; Central African Republic; Chad; Congo; Côte d'Ivoire; Equatorial Guinea; Ethiopia; Gabon; Gambia; Ghana; Guinea [1454]; Guinea-Bissau; Kenya; Liberia; Malawi; Mali; ?Mauritania; Mozambique; Namibia; Niger; Nigeria; Rwanda; Senegal; Sierra Leone; Somalia; South Africa; Sudan; Swaziland; Tanzania; Togo; Uganda; Zaire; Zambia; Zimbabwe 

Peru; Suriname; Trinidad and Tobago: Trinidad; Venezuela

Leptodon forbesi (Swann 1922)

White-collared Kite

Breeding:

Brazil
11

$$
11 \quad-\quad 15,16,226,967
$$

Leucopternis albicollis (Latham 1790)

Belize; Bolivia; Brazil; Colombia; Costa Rica; Ecuador; French Guiana; Guatemala; Guyana; Honduras; Mexico; Nicaragua; Panama; Peru; Suriname; Trinidad and Tobago: Trinidad; Venezuela

Leucopternis kuhli Bonaparte 1850

White-browed Hawk

Breeding:

Bolivia [1066]; Brazil; Peru

\begin{tabular}{|c|c|c|c|}
\hline $\begin{array}{l}\text { Leucopternis lacernulata (Temminck 1827) } \\
\text { White-necked Hawk }\end{array}$ & II & $\mathrm{V} / \mathrm{R}$ & $7,15,226,318,321,914,967$ \\
\hline Brazil [1271] & & & \\
\hline ucopternis melanops (Latham 1790) & 11 & - & $15,226,967$ \\
\hline
\end{tabular}

Black-faced Hawk, Masked Hawk

Breeding: $\quad$ Brazil; Colombia; Ecuador; French Guiana; Guyana; Peru; Suriname; Venezuela

Leucopternis occidentalis Salvin 1876

II E

$15,226,318,321,967$

Grey-backed Hawk

Breeding: $\quad$ Ecuador [1166]; Peru [1063,1490]

Leucopternis plumbea Salvin 1872

Plumbeous Hawk

Breeding:

Colombia; Ecuador; Panama; Peru

Leucopternis polionota (Kaup 1847)

Mantled Hawk

Breeding:

Argentina [154]; Brazil; Paraguay

Leucopternis princeps Sclater 1865

11

$15,16,226,967$

Barred Hawk, Black-chested Hawk

Breeding:

Colombia; Costa Rica; Ecuador; Panama

Leucopternis schistacea (Sundevall 1851)

II

$15,226,967$

Slate-colored Hawk

Breeding:

Bolivia; Brazil; Colombia; Ecuador; French Guiana; Peru; Venezuela

Leucopternis semiplumbea Lawrence 1861

11

$15,16,226,967$

Semiplumbeous Hawk

Breeding:

Colombia; Costa Rica; Ecuador; Honduras [903]; Nicaragua; Panama 
Angola; Benin; Botswana; Burundi; Cameroon; Central African Republic; Chad; Congo; Côte d'Ivoire; Equatorial Guinea; Ethiopia; Gabon; Gambia; Ghana; Guinea; Guinea-Bissau; Kenya; Liberia; Malawi; Mali;?Mauritania; Mozambique; ?Namibia; Niger; Nigeria; Rwanda; Senegal; Sierra Leone; South Africa; Sudan; Swaziland; Tanzania; Togo; Uganda; Zaire; Zambia; Zimbabwe

Vagrant: Somalia

Lophoictinia isura (Gould 1838)

Square-tailed Kite

Breeding:

Australia [167]

Macheiramphus alcinus Westermann 1851

II

$15,212,226,227,285,792,1226$, 1247,1298

(= Machaerhamphus alcinus)

\section{Bat Hawk, Bat Kite}

Breeding:

Angola; Benin; ?Botswana; Brunei; Burundi; Cameroon; Central African Republic; Chad; Congo; Côte d'Ivoire; ?Equatorial Guinea; Ethiopia; Gabon; Ghana; Guinea [I454]; Indonesia: Kalimantan [169], Sulawesi [796], Sumatra; Kenya; Liberia; Madagascar;Malawi; Malaysia: Peninsular Malaysia, Sabah, Sarawak [1295]; Mozambique; Myanmar; ?Namibia; Niger; Nigeria; Papua New Guinea [88I]; Rwanda; South Africa; Sudan; Tanzania; Thailand; Togo; Uganda; Zaire; Zambia; Zimbabwe

Vagrant: Gambia; Senegal; Singapore; Somalia

Megatriorchis doriae Salvadori \& D'Albertis 1875 II $15,226,930,1226$

(= Accipiter doriae)

Doria's Goshawk

Breeding:

Indonesia: Irian Jaya; Papua New Guinea

Melierax canorus (Rislachi 1799)

II

$15,226,227,285,1298$

\section{Pale Chanting-Goshawk}

Breeding:

Vagrant:

Angola; Botswana; Namibia; South Africa; Zimbabwe

Lesotho

Melierax gabar (Daudin 1800)

(= Micronisus gabar)

Gabar Goshawk

Breeding:

Non-breeding:

Vagrant:

Melierax metabates Heuglin $186 \mathrm{I}$

\section{Dark Chanting-Goshawk}

Angola; Benin; Botswana; Burkina Faso [1388]; Burundi; Cameroon; Central African Republic; Chad; Congo; ?Côte d'Ivoire; ?Djibouti; Ethiopia; Gambia; Ghana; Guinea [984]; ?Guinea-Bissau; Kenya; Malawi; Mali; Mauritania; Mozambique; Namibia; Niger; Nigeria; Rwanda; Saudi Arabia; Senegal; ?Sierra Leone; Somalia; South Africa; Sudan; Swaziland; Tanzania; Togo; Uganda; ?Yemen; Zaire; Zambia; Zimbabwe

Breeding:

Vagrant:
$15,212,226,227,285,661,1247$, 1298
Lesotho

II

$15,2 \mathrm{~J} 2,226,227,285,66 \mathrm{I}, \mathrm{I} 247$, 1298

Angola; Benin; Botswana; Burkina Faso [1388]; ?Burundi; Cameroon; Central African Republic; Chad; Côte d'Ivoire; ?Djibcuti; Ethiopia; Gambia; Ghana; Guinea [1454]; Guinea-Bissau; Kenya; Malawi; Mali; Mauritania; Morocco; Mozambique; Namibia; Niger; Nigeria; ?Rwanda; Saudi Arabia; Senegal; ?Sierra Leone; South Africa; Sudan; Tanzania; Togo; Uganda; Yemen; Zaire; Zambia; Zimbabwe Israel; Somalia; Spain; Swaziland; United Arab Emirates 


\section{Black-eared Kite}

Breeding:

?Bhutan; China; Hongkong; India; Japan; D.P.R. Korea; Korea Republic; Nepal; Pakistan; Russia; Taiwan

Non-breeding:

Afghanistan; Bangladesh; Cambodia; Thailand

Vagrant:

Brunei; Indonesia: ?Sumatra; Malaysia: Peninsular Malaysia, Sabah, Sarawak [1295]; Philippines: Palawan

Milvus migrans (Boddaert 1783) 792,1298

\section{Black Kite, Pariah Kite, Yellow-billed Kite}

Breeding:

Non-breeding:

Vagrant:

Milvus milvus (Linnaeus 1758)
Afghanistan; Albania; Algeria; Angola; Armenia; Australia [167]; Austria; Azerbaijan; Bangladesh; Belarus; Belgium [589]; Benin; Botswana; Bulgaria; Burkina Faso [1388]; Burundi; Cambodia; Cameroon; Cape Verde; Central African Republic; Chad; China; Comoros; Congo; Côte d'lvoire; Czechoslovakia; Djibouti; Egypt; Equatorial Guinea; Estonia; Ethiopia; Finland; France; Gabon; Gambia; Georgia; Germany; Ghana; Greece; Guinea [1454]; Guinea-Bissau; Hungary; India; Indonesia; Irian Jaya, Sulawesi; Iran; Iraq; Israel; Italy; Japan; Kazakhstan; Kenya; Korea Republic; Kyrgyzstan; Lao P.D.R; Latvia; Lesotho; Liberia; Liechtenstein; Lithuania; Luxembourg; Madagascar [373]; Malawi; Mali; Mauritania; Mayotte; Moldova; Mongolia; Morocco; Mozambique; Myanmar; Namibia; Nepal; Niger; Nigeria; Pakistan; Papua New Guinea [881]; Poland; Portugal; Romania; Russia; Rwanda; Sao Tome and Principe [1299]; Saudi Arabia; Senegal; Sierra Leone; Somalia; South Africa; Spain; Sri Lanka; Sudan; Sweden; Switzerland; Syria; Tajikistan; Tanzania; Thailand; Togo; Tunisia; Turkey; Turkmenia; Uganda; Ukraine; Uzbekistan; Vjet Nam; Western Sahara; Yemen; Yugoslavia; Zaire; Zambia; Zimbabwe

Bahrain; Cyprus; Gibraltar; Jordan [1451]; Kuwait; Lebanon; Libya; Malaysia: Peninsular Malaysia; Malta; Oman; Qatar; Seychelles: Aldabra Islands; Singapore; Swaziland; United Arab Emirates

Denmark; Guernsey; Iceland; Indonesia: Buru, Lombok, Sumba, Timor [1486]; Ireland; Jersey; Netherlands; Norway; United Kingdom

\section{Red Kite}

Breeding:

Non-breeding:

Vagrant:
II $\quad \mathrm{K}^{*}$
$15,185,226,227,318,345,440$, 523,661
Algeria (ex?); ?Armenia; Austria; Belarus; Belgium; Cape Verde; Czechoslovakia; Denmark; France; Georgia; Germany; Hungary; Iran; 1taly; ?Latvia; Lithuania; Luxembourg; Moldova; Morocco; Netherlands; Poland; Portugal; Romania; Russia; Spain; Sweden; Switzerland [992]; Tunisia (ex?); Ukraine; United Kingdom; Yugoslavia Austria; Bulgaria; Gibraltar; Greece; Liechtenstein; Turkey

Albania; Cyprus; Egypt; Finland; Gambia [521]; Guernsey; lceland; India; Iraq; lreland; Israel; Jersey; Jordan; Lebanon; Libya; Malta; Nepal; Norway; South Africa; Sudan; Syria

Morphnus guianensis (Daudin 1800)

II

$15,16,162,166,226,318,491,794$ 967

\section{Crested Eagle, Guianan Crested Eagle}

Breeding:

Argentina [154,1035]; Bolivia; Brazil; Colombia; Costa Rica; Ecuador; French Guiana [1376]; Guatemala [127]; Guyana; Honduras; ?Nicaragua; Panama; Paraguay; Peru [1064]; Suriname [625]; Venezuela [1092] 
Necrosyrtes monachus (Temminck 1823)

\section{Hooded Vulture}

Breeding:

\section{Non-breeding:}

Vagrant:

Neophron percnopterus (Linnaeus 1758)

Swaziland

Morocco [661]
Angola; Benin; Botswana; Burkina Faso [1388]; Burundi; Cameroon; Central African Republic; Chad; Côte d'Ivoire; Djibouti; Ethiopia; Gambia; Ghana; Guinea [1454]; Guinea-Bissau; Kenya; Malawi; Mali; Mauritania; Mozambique; Namibia; Niger; Nigeria; Rwanda; Senegal; Sierra Leone; Somalia; South Africa; Sudan; Tanzania; Togo; Uganda; Zaire; Zambia; Zimbabwe

\section{Egyptian Vulture, Scavenger Vulture}

$10,15,212,226,227,285,345,661$, $930,1247,1298$

Breeding:

Afghanistan; Albania; Algeria; Armenia; Azerbaijan; ?Bhutan; Bulgaria; Canary Islands; Cape Verde; Chad; Djibouti; Egypt; Ethiopia; France; Georgia; Greece; Guinea; India; Iran; Iraq; Israel; Italy; Jordan; Kazakhstan; Kenya; Kuwait; Kyrgyzstan; ?Lebanon; Libya; Mali; Mauritania; Morocco; Nepal; Niger; Nigeria; Oman; Pakistan; Portugal; Romania; Russia [799]; Saudi Arabia; Senegal; Somalia; South Africa (ex) [842]; Spain; Sudan; Syria; Tajikistan; Tanzania; Tunisia; Turkey; Turkmenia; Uganda; United Arab Emirates; Uzbekistan; Western Sahara; Yemen; Yugoslavia

Non-breeding: Cameroon; Central African Republic; Gibraltar; Mozambique; South Africa

Vagrant:

Angola; Austria; Cyprus; Czechoslovakia; Denmark; Finland; Gambia; Federal Republic of Germany; Ghana; Hungary; Lesotho; Malta; Myanmar; Namibia; ?Norway; Poland; Qatar; Rwanda; Sri Lanka; Svalbard and Jan Mayen Islands; Sweden; Switzerland; United Kingdom; Zaire; Zambia; Zimbahwe

Oroaetus isidori (Des Murs 1845)

Il

$15,226,967$

(= Spizaetus isidori)

Black-and-chestnut Eagle, Isidor's Eagle

Breeding:

Argentina [154]; Bolivia; Colombia; Ecuador; Peru; Venezuela

Parabuteo unicinctus (Temminck 1824)

II

$15,16,226,967$

Harris's Hawk, Bay-winged Hawk

Breeding:

Argentina; Bolivia; Brazil; Chile [715]; Colombia; Costa Rica; Ecuador; El Salvador; Guatemala; Mexico; Nicaragua; Paraguay; Peru; Suriname; USA; Uruguay; Venezuela

Vagrant: Panama

Pernis apivorus (Linnaeus I758)

II

$10,15,212,226,227,285,345,661$, 792,1247

\section{European Honey-buzzard}

Breeding:

Armenia; Austria; Azerbaijan; Belarus; Belgium; Bulgaria; Czechoslovakia; Denmark; Estonia; Finland; France; Georgia; Germany; Greece; Hungary; Iran; Italy; Latvia; Liechtenstein; Lithuania; Luxembourg; Moldova; Netherlands; Norway; Poland; Portugal; Romania; Russia; Spain; Sweden; Switzerland; Turkey; Ukraine; United Kingdom; Yugoslavia

Non-breeding: Albania; Algeria; Angola; Benin; Botswana; Burkina Faso; Burundi; Cameroon; Central African Republic; Chad; ?Congo; Cyprus; Côte d'Ivoire; Egypt; Equatorial Guinea; Ethiopia; Gabon; Gambia; Ghana; Gibraltar; Greece; Guinea; ?Guinea-Bissau; Iraq; Israel; Jordan [1451]; Kenya; Kuwait; Lebanon; Liberia; Malawi; Mali; Malta; Mauritania; Morocco; Mozambique; Namibia; Niger; Nigeria; Rwanda; Saudi Arabia; Senegal; ?Sierra Leone; South Africa; Sudan; Syria; Tanzania; Togo; Tunisia; Uganda; Western Sahara; Yemen; Zaire; Zambia; Zimbabwe

Vagrant: Afghanistan; Bahrain; Canary Islands; Faeroe Islands; Guernsey: Alderney; Iceland; Ireland; Libya; Oman; Seychelles [1402]; ?Swaziland; United Arah Emirates; Uzbekistan 
Barred Honey-buzzard

Breeding: $\quad$ Indonesia: Muna, Peleng, Sulawesi [1486]; Philippines

Pernis ptilorhyncus (Temminck 182I)

II

Oriental Honey-buzzard

Breeding:

Bangladesh; ?Bhutan; ?Brunei; Cambodia; China; India; Indonesia: Java, Kalimantan, Sumatra; Japan; ?D.P.R. Korea; Lao P.D.R; Malaysia: Peninsular Malaysia, Sabah, Sarawak [1295]; Mongolia; Myanmar; Nepal; [700]; Pakistan; Philippines; Sri Lanka; Thailand; Viet Nam

Non-breeding: Brunei; Indonesia: Bali; D.P.R. Korea; Korea Republic; Maldives; Singapore; Taiwan

Vagrant: Hong Kong; Indonesia: Kisar, Salayar [1486]; Macao; Uzbekistan

Pithecophaga jefferyi Ogilvie-Grant $1897 \quad$ I $\quad$ E

$15,226,318,737,794,805,846, \mathrm{~J} 479$

Great Philippine Eagle, Philippine Eagle, Monkey-eating Eagle

Breeding:

Philippines: Leyte, Luzon, Mindanao, Samar

Polemaetus bellicosus (Daudin 1800)

II

$15,212,226,226,285,1247,1298$

(= Hieraaetus bellicosus)

Martial Eagle

Breeding:

Angola; Benin; Botswana; ?Burundi; Cameroon; Central African Republic; Chad; Côte d'lvoire; Ethiopia; ?Gambia; Ghana; ?Guinea [984]; Guinea-Bissau; Kenya; Malawi; Mali; Mauritania; Mozambique; Namibia; Niger; Nigeria; Rwanda; Senegal; Sierra Leone; Somalia; South Africa; Sudan; Swaziland; Tanzania; Togo; Uganda; Zaire; Zambia; Zimbabwe

Polyboroides radiatus (Scopoli 1786) Il
Madagascar Harrier-Hawk, Madagascar Gymnogene

Breeding: Madagascar [373]

Polyboroides typus Smith 1829

II

$15,212,226,227,1247,1298$

African Harrier-Hawk, African Gymnogene

Breeding:

Angola; Benin; Botswana; Burkina Faso [1388]; Burundi; Cameroon; Central African Republic; Chad; Congo; Côte d'Ivoire; ?Equatorial Guinea; Ethiopia; Gabon; Gambia; Ghana; Guinea [1454]; Guinea-Bissau; Kenya; ?Lesotho; Liberia; Malawi; Mali; Mauritania; Mozambique; ?Namibia; Niger; Nigeria; Rwanda; Senegal; Sierra Leone; South Africa; Sudan; Swaziland; Tanzania; Togo; Uganda; Zaire; Zambia; Zimbabwe

Non-breeding: Somalia

$\begin{array}{llll}\text { Rostrhamus hamatus (Temminck 1821) } & \text { Il } & - & 15,226,967\end{array}$

( = Helicolestes hamatus)

Slender-billed Kite

Breeding: $\quad$ Bolivia; Brazil; Colombia; Ecuador; Peru; Suriname; Venezuela

Vagrant: Panama

$\begin{array}{llll}\text { Rostrhamus sociabilis (Vieillot 1817) II } & \text { II } & \text { 15, I6,226,794,967 }\end{array}$

Snail Kite, Everglade Kite

Breeding:

Argentina; Belize; Bolivia; Brazil; Colombia; Costa Rica; Cuba; Ecuador; French Guiana; Guatemala [127]; Guyana; Honduras; Mexico; Nicaragua; Panama; Paraguay; Peru; Suriname; USA; Uruguay; Venezuela

Vagrant: $\quad$ Trinidad and Tobago: Trinidad 
Sarcogyps calvus (Scopoli 1786)

II

$10,15,226,792$

(= Aegypius calvus, Torgos calvus)

Red-headed Vulture, Indian Black Vulture, Pondicherry Vulture

Breeding: $\quad$ Bangladesh; ?Bhutan; Cambodia; China; India; Lao P.D.R; Malaysia: Peninsular Malaysia; Myanmar; Nepal; Pakistan; Thailand [1194]; Viet Nam

Vagrant: Singapore

Spilornis cheela (Latham 1790)

Il

$10,15,226,792,881,930$

\section{Crested Serpent-Eagle}

Breeding:

Bangladesh; Bhutan; Brunei; Cambodia; China; India: including Andaman and Nicobar Islands; Indonesia: Bali, Bawean, Java, Kalimantan, Sumatra; Japan: Ryukyu Islands; Lao P.D.R; Malaysia: Peninsular Malaysia, Sabah, Sarawak [1295]; Myanmar; Nepal; Pakistan; Philippines: Balabac, Busuanga, Palawan; Sri Lanka; Taiwan; Thailand [1194]; Viet Nam

Non-breeding:

Vagrant: Hong Kong Singapore

Spilornis elgini (Blyth 1863)

Andaman Serpent-Eagle, Dark Serpent-Eagle

II

$\mathrm{K}^{*}$

$15,226,318$

Breeding:

India: Andaman Islands [10]

Spilornis holospilus (Vigors 1831)

Il

226

(= S. cheela holospilus)

Philippine Serpent-Eagle

Breeding: Philippines

Spilornis kinabaluensis Sclater 1919

$11 \quad \mathrm{~K}^{*}$

15,318

(= S. cheela kinabaluensis)

Mountain Serpent-Eagle, Kinahalu Serpent-Eagle

Breeding: Brunei; Indonesia: Kalimantan [1091]; Malaysia: Sabah [1295], Sarawak [1295]

Spilornis minimus Hume 1873

II

$10,15,226$

Nicobar Serpent-Eagle

Breeding: India: Nicobar Islands

Spilornis rufipectus Sclater 1919

II

226

Sulawesi Serpent-Eagle

Breeding: Indonesia: Sulawesi and surrounding islands, Sula Islands [1486]

Spizaetus africanus (Cassin 1865)

II

$15,212,226,227,1247,1298$

Cassin's Hawk-Eagle

Breeding: Angola; Burundi; Cameroon; ?Central African Republic; Congo; Côte d'Ivoire; Gabon; Ghana; Guinea; Liberia; Niger; ?Nigeria; Rwanda; Sierra Leone; Togo; Uganda; Zaire

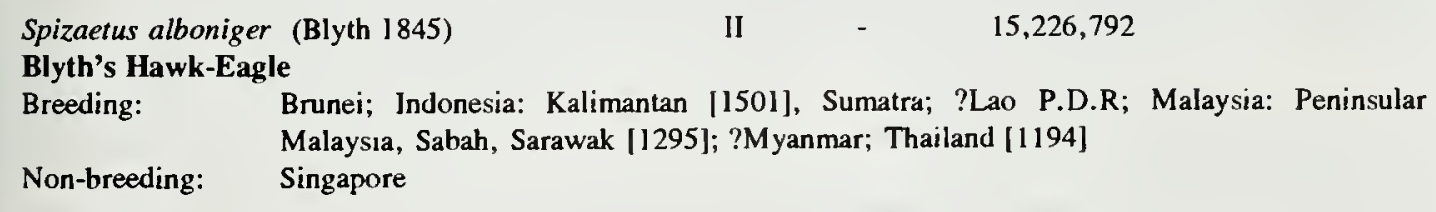

Indonesia: Java [881] 
Changeable Hawk-Eagle, Crested Hawk-Eagle

Breeding:

Bangladesh; ?Bhutan; Brunei; Cambodia; India: Andaman Islands; Indonesia: Flores [1486], Java, Kalimantan [993], Paloe [1486], Sumatra, Sumbawa [1486]; ?Lao P.D.R; Malaysia: Peninsular Malaysia, Sabah, Sarawak [1295]; Myanmar; Nepal; Philippines: Busuanga, Culion, Lubang, Mindanao, Mindoro, Palawan; Singapore; Sri Lanka; Thailand [1194]; Viet Nam

Spizaetus lanceolatus Temminck \& Schlegel $1844 \quad$ II $\quad-\quad 15,226$

Sulawesi Hawk-Eagle

Breeding: Indonesia: Sulawesi, Sula Islands [1486]

Spizaetus nanus Wallace $1868 \quad$ II $\quad K^{*} \quad 15,226,318,792,1477$

Wallace's Hawk-Eagle

Breeding: $\quad$ Brunei; Indonesia: Kalimantan [1295], Sumatra; Malaysia: Peninsular Malaysia, Sabah, Sarawak [1295]; Myanmar; Thailand [1194]

Spizaetus nipalensis (Hodgson 1836)

\section{$1 \mathrm{I}$}

$10,15,226,792$

Mountain Hawk-Eagle, Hodgson's Hawk-Eagle, Feather-toed Hawk-Eagle

Breeding: $\quad$ Bhutan; ?Cambodia; China; India; Japan; Lao P.D.R; Myanmar; Nepal; Pakistan; ?Russia [799]; Sri Lanka; Taiwan; ?Viet Nam

Non-breeding: Thailand [1194]

Vagrant: $\quad$ Korea Republic

$\begin{array}{llll}\text { Spizaetus ornatus (Daudin 1800) } & 11 & - & 15,16,226,967\end{array}$

Ornate Hawk-Eagle

Breeding: $\quad$ Argentina; Belize; Bolivia; Brazil; Colombia; Costa Rica; Ecuador; E1 Salvador; French Guiana; Guatemala; Guyana; Honduras; Mexico; Nicaragua; Panama; Paraguay; Peru; Suriname; Trinidad and Tobago: Trinidad; Venezuela

Spizaetus philippensis Gould $1863 \quad$ II
Philippine Hawk-Eagle

Spizaetus tyrannus (Wied 1820)

Il $\quad-\quad 15,16,226,967$

Black Hawk-Eagle, Tyrant Hawk-Eagle

Breeding: $\quad$ Argentina; Belize [433]; Bolivia; Brazil; Colombia; Costa Rica; Ecuador; French Guiana; Guatemala; Guyana; Honduras; Mexico; Nicaragua; Panama; Paraguay; Peru; Suriname; Trinidad and Tobago: Trinidad; Venezuela

Spizastur melanoleucus (Vieillot 1816)

II

$15,16,226,967$

Black-and-white Hawk-Eagle

Breeding: $\quad$ Argentina; Belize; Bolivia; Brazil; Colombia; Costa Rica; Ecuador; French Guiana; Guatemala [127]; Guyana; Honduras; Mexico; Nicaragua; Panama; Paraguay; Peru; Suriname; Venezuela

Stephanoaetus coronatus (Linnaeus 1766)

II

$15,212,226,227,285,1247,1298$

(= Spizaetus coronatus)

Crowned Hawk-Eagle, African Crowned Eagle, Crowned Eagle

Breeding:

Angola; ?Benin; Burundi; Cameroon; Central African Republic; Congo; Côte d'lvoire; Equatorial Guinea; Ethiopia; Gabon; Ghana; Guinea; Kenya; Liberia; Malawi; Mozambique; ?Namibia; Nigeria; Rwanda; Sierra Leone; South Africa; Sudan; Swaziland; Tanzania; Togo; Uganda; Zaire; Zambia; Zimbabwe

Non-breeding: Guinea-Bissau; Mali

Vagrant: Senegal 


\section{Bateleur, Bateleur Eagle}

Breeding: $\quad$ Angola; Benin; Botswana; Burkina Faso; Burundi; Cameroon; Central African Republic; Chad; Côte d'lvoire; Egypt; Ethiopia; Gambia; Ghana; Guinea [1454]; Guinea-Bissau; Kenya; Malawi; Mali; Mauritania; Mozambique; Namibia; Niger; Nigeria; Rwanda; Saudi Arabia; Senegal; Sierra Leone; Somalia; South Africa; Sudan; Swaziland; Tanzania; Togo; Uganda; Yemen; Zaire; Zambia; Zimbabwe

Vagrant: $\quad$ Congo; Djibouti; Gabon; lraq; lsrael; Lesotho; Liberia

$$
\begin{array}{lll}
\text { Torgos tracheliotus (Forster 1791) } & 11 \quad-\quad 15,212,226,227,285,345,661, \\
\text { (= Aegypius tracheliotus) } & & 1247,1298
\end{array}
$$

Lappet-faced Vulture, Nubian Vulture

Breeding: $\quad$ Angola; Botswana; Burkina Faso [1388]; Burundi; Cameroon; Central African Republic; Chad; ?Djibouti; Egypt; Ethiopia; lsrael; Kenya; Malawi; Mali; Mauritania; Mozambique; Namibia; Niger; Nigeria; Oman [501]; Rwanda; Saudi Arabia; Senegal; Somalia; South Africa; Sudan; Swaziland; Tanzania; Uganda; United Arab Emirates; ?Western Sahara; Zaire; Zambia; Zimbabwe

Non-breeding: Benin; Equatorial Guinea Vagrant: $\quad$ Algeria; Côte d'Ivoire; Egypt; ?France; Gambia; Jordan [1451]; Libya; Morocco; Yemen

Trigonoceps occipitalis (Burchell 1824) 11 $15,212,226,227,285,1247,1298$

(= Aegypius occipitalis)

White-headed Vulture

Breeding: $\quad$ Angola; Benin; Botswana; Burkina Faso [1388]; Burundi; Cameroon; Central African Republic; Chad; Côte d'Ivoire; Ethiopia; Gabon; Gambia; Ghana; Guinea; Guinea-Bissau; Kenya; Malawi; Mali; ?Mauritania; Mozambique; Namibia; Niger; Nigeria; Rwanda; Senegal; ?Sierra Leone; Somalia; South Africa; Sudan; Swaziland; Tanzania; Togo; Uganda; Zaire; Zambia; Zimbabwe

Urocriorchis macrourus (Hartlaub 1855)

Il

$15,212,226,227,1247,1298$

Long-tailed Hawk, African Long-tailed Hawk

Breeding:

Cameroon; Central African Republic; Congo; Côte d'Ivoire; Equatorial Guinea; Gabon; Ghana; Liberia; ?Nigeria; ?Sierra Leone; Sudan; Togo [280]; ?Uganda; Zaire

\section{Family SAGITTARIIDAE}

Secretary-bird
Sagittarius serpentarius (Miller 1779)
Il
$15,212,226,227,1247,1298$

Secretary-bird

Breeding:

Non-breeding:

Vagrant:
Angola; Benin; Botswana; ?Burundi; Cameroon; Central African Republic; Chad; Djibouti; Ethiopia; Guinea-Bissau; Kenya; Malawi; Mali (ex); Mauritania; Mozambique; Namibia; Niger; ?Nigeria; Rwanda; Somalia; South Africa; Sudan; Swaziland; Tanzania; Uganda; Zaire; Zambia; Zimbabwe

Côte d'lvoire; Ghana; Lesotho; Togo

Gambia; Senegal

\section{Family FALCONIDAE}

Falcons
Daptrius americanus (Boddaert 1783)
II
$15,16,226,967$

\section{Red-throated Caracara}

Breeding:

Bolivia; Brazil; Colombia; Costa Rica; Ecuador; French Guiana; Guatemala; Guyana; Honduras; Mexico; Nicaragua; Panama; Peru; Suriname; Venezuela 
Black Caracara, Yellow-throated Caracara

Breeding:

Bolivia; Brazil; Colombia; Ecuador; French Guiana; Guyana; Peru; Suriname; Venezuela

Falco alopex (Heuglin 1861)

11

$15,212,226,227,1247,1298$

\section{Fox Kestrel}

Breeding:

Vagrant:

Falco altaicus (Menzbier 1891)

China; Mongolia; Russia

Altai Falcon

Breeding:
Falco amurensis Radde 1863

Amur Falcon, Manchurian Red-footed Falcon, Eastern Red-footed Falcon

Breeding: China; D.P.R. Korea; Mongolia; Russia

Non-breeding:

Afghanistan; Angola; Bangladesh; Bhutan; Botswana; India; Kenya; Korea Republic; Lesotho; Malawi; Maldives; Mozambique; Myanmar; Nepal; Pakistan; South Africa; Swaziland; Thailand; Uganda; Zaire; Zambia; Zimbabwe

Vagrant:

Falco araea (Oberholser 1917)

1

Seychelles Kestrel

Breeding:

Seychelles

Falco ardosiaceus Vieillot 1823

II

$15,212,226,227,285,1247,1298$

Grey Kestrel

Breeding:

Non-breeding:

Vagrant:
Angola; Benin; Burkina Faso [1388]; Burundi; Cameroon; Central African Republic; Chad; Congo; Côte d'lvoire; Ethiopia; Equatorial Guinea; Gambia; Ghana; Guinea [1454]; Guinea-Bissau; Kenya; Mali; ?Namibia; Niger; Nigeria; Rwanda; Senegal; Sierra Leone; Sudan; Tanzania; Togo; Uganda; Zaire; Zambia

Mauritania

Malawi

Falco berigora Vigors \& Horsfield 1827

II

$15,226,930$

Brown Falcon

Breeding:

Australia [167]; Indonesia: Jrian Jaya [881]; Papua New Guinea [881]

Falco biarmicus Temminck 1825

11

$15,212,226,227,285,661,1247$

Lanner Falcon, Lanner

Breeding:

?Albania; Algeria; Angola; ?Armenia; ?Azerbaijan; Benin; Botswana; Burkina Faso [1388]; Burundi; Cameroon; Central African Republic; Chad; Côte d'Ivoire; Djibouti; Egypt; Ethiopia; Ghana; Greece; ?Guinea; ?Guinea-Bissau; ?Iraq; Israel; Italy; Jordan; Kenya; ?Lebanon; Lesotho; Liberia; Libya; Malawi; Mauritania; Morocco; Mozambique; Namibia; Niger; Nigeria; ?Oman; ?Qatar; Rwanda; Saudi Arabia; Senegal; ?Sierra Leone; Somalia; South Africa; Sudan; Swaziland; ?Syria; Tanzania; Togo; Tunisia; Turkey; Uganda; Western Sahara; Yemen; Yugoslavia; Zaire; Zambia; Zimbabwe

Non-breeding: Gambia

Vagrant:

Bulgaria; ?Cyprus; Czechoslovakia; France; Gibraltar; Iraq; Kuwait; Malta; Portugal; Qatar; Romania; Spain; ?United Arab Emirates 
Falco cenchroides Vigors \& Horsfield 1827

Australian Kestrel, Nankeen Kestrel

Breeding:

Australia [167]; Christmas Island; Indonesia: Irian Jaya; Lord Howe Island; Norfolk Island; Papua New Guinea

Vagrant:

New Zealand

Falco cherrug Gray 1844

II

$10,15,212,226,227,345,661,1247$

\section{Saker Falcon, Saker}

Breeding:

?Afghanistan; Austria; Belarus; Bulgaria; China; Czechoslovakia; Hungary; Iraq; Kazakhstan; Kyrgyzstan; Moldova; Romania; Russia [799]; Tajikistan; Turkey; Turkmenia; Ukraine; Uzbekistan; Yugoslavia

Non-breeding: Bahrain; Cameroon; Chad; Cyprus; Egypt; Ethiopia; India; Israel; Iran; Iraq; Italy; Kenya; Kuwait; Libya; Malta; Mauritania; Nepal; Oman; Pakistan; Saudi Arabia; Sudan; Tunisia; Turkey; United Arab Emirates; Yemen

Vagrant: $\quad$ Albania; Burundi; France; Germany; Greece; Jordan [1451]; Korea Republic; Lehanon; Mali; Morocco; Poland; Qatar; Senegal; Sweden; Syria; Tanzania

Falco chicquera Daudin 1800

I]

$15,212,226,227,285,661,1247$, 1298

Red-necked Falcon, Red-headed Falcon, Red-headed Merlin

Breeding: $\quad$ Angola; Bangladesh; Benin; Botswana; Burundi; Cameroon; Centsal African Republic; Chad; ?Côte d'lvoire; Ethiopia; Gambia; Ghana; Guinea; Guinea-Bissau; India; Isan; Kenya; Malawi; Mali; Mauritania; Mozambique; Namihia; Nepal; Niger; Nigeria; Pakistan; Rwanda; Senegal; ?Sierra Leone; South Africa; Sudan; Tanzania; Togo; Uganda; Zaire; Zambia [411]; Zimbabwe

Vagrant: $\quad$ Somalia

Falco columbarius Linnaeus 1758

$15,16,179,226,227,345,661$

Merlin, Pigeon Hawk

Breeding:

Non-breeding:

Vagrant:
Canada; China; Estonia; Faeroe Islands; Finland; France; Iceland; Ireland; Italy; Kazakhstan; Latvia; Lithuania; Mongolia; Norway; Russia; Sweden; USA; United Kingdom Afghanistan; Albania; Algeria; ?Anguilla; Antigua and Barbuda; Aruba; Austria; Bahamas; Barbados; Belgium; Belize; Bermuda; ?British Virgin lslands; Bulgaria; Cayman lslands; Colombia; Costa Rica; Cuba; Cyprus; Czechoslovakia; Denmark; Dominica; Dominican Republic; Ecuador; Egypt; France; Germany; Gibraltar; Greece; Grenada; ?Guadeloupe; Guatemala; Haiti; Honduras; Hong Kong; Hungary; India; Iran; Iray; Israel; Italy; Jamaica; Japan; Jordan [1451]; D.P.R. Korea; Korea Republic; Lao P.D.R; Luxembourg; ?Martinique; Mexico; ?Montserrat; Morocco; Nepal; Netherlands Antilles; Nicaragua; Pakistan; Panama; Peru; Poland; Portugal; Puerto Rico; Romania; Saudi Arabia; Spain; Saint Kitts and Nevis; Saint Lucia; Saint Vincent; Switzerland; Syria; Tajikistan; Trinidad and Tobago; Tunisia; Turkey; Turkmenia; Turks and Caicos Islands; Venezuela; Viet Nam; Virgin Islands of the United States; Yugoslavia

Bahrain; Kuwait; Lebanon; Libya; Liechtenstein; Malta; Oman; Philippines; Senegal; Sudan; Yemen

Falco concolor Temminck 1825

II

$15,212,226,227,345,661,1247$, 1298

Sooty Falcon

Breeding:

Bahrain; ?Chad; Egypt; Ethiopia: Dahlak Archipelago; Israel; Libya; Oman; Saudi Arabia; Sudan; United Arab Emirates

Non-breeding: Chad; Djibouti; Kenya; Madagascar [373]; Malawi; Mozambique; Réunion; Seychelles;

Somalia; South Africa; Tanzania; Uganda

Vagrant:
Cyprus; Iran; Mali; Malta; Mauritius; Namibia; Pakistan; Qatar; Turkey; Yemen; Zambia [1081] 
African Hobby Breeding:
Angola; Benin; Botswana; Burkina Faso; Burundi; Cameroon; Central African Republic; Chad; Côte d'Ivoire; Ethiopia; Gambia; Ghana; Guinea; Guinea-Bissau; Kenya; Malawi; Mali; Mozambique; ?Namibia; ?Niger; Nigeria; Rwanda; Senegal; Sierra Leone; South Africa; Sudan; Swaziland; Tanzania; ?Togo; Uganda; Zaire; Zambia; Zimbabwe

Falco deiroleucus Temminck 1825 ]I

$15,16,226,318,735,736,967,1158$

\section{Orange-breasted Falcon}

Breeding:

Argentina [154,1035]; Belize [608]; Bolivia [1144]; Brazil; Colombia; Costa Rica (ex?); Ecuador; French Guiana; Guatemala; Guyana; Honduras; Mexico; Nicaragua; Paraguay; Peru; Suriname [628]; Venezuela

Vagrant:

Falco dickinsoni Sclater 1864

Dickinson's Kestrel, White-rumped Kestrel

Breeding:

Vagrant: Malawi; Mozambique; ?Namibia; South Africa; Tanzania; Zaire; Zambia; Zimbabwe Kenya

Falco eleonorae Géné 1839

\section{Eleonora's Falcon}

Breeding:

Algeria; Canary 1slands; Cyprus; France; Greece; Italy; Morocco; Spain [927]; Tunisia; Turkey; Yugoslavia

Non-breeding: Ethiopia; Gibraltar; Israel; Kenya; Madagascar [373]; Malta; ?Mozambique; Portugal; Somalia; ?Sudan; Tanzania; Tunisia

Vagrant: Bahrain; Bulgaria; Egypt; Hungary; Lebanon; Libya; Mauritius; Mauritius; Poland; Réunion; Rwanda; Seychelles [493]; South Africa; Sweden; Syria; Uganda; United Kingdom; Zimbabwe

Falco fasciinucha Reichenow \& Neumann 1895 II

$15,212,226,227,285,1298$

Teita Falcon

Breeding:

Botswana; Ethiopia; Kenya; Malawi; Mozambique; Tanzania; Uganda; Zambia; Zimbabwe

Falco femoralis Temminck 1822

Aplomado Falcon

Breeding:

Il $\quad-\quad 15,16,226,967$

Argentina; Belize; Bolivia; Brazil; Chile [715]; Colombia; Ecuador; ?French Guiana; Guyana; Honduras; Mexico; Nicaragua; Panama; Paraguay; Peru; Suriname; Trinidad and Tobago: Trinidad; Uruguay; Venezuela

Vagrant:

Costa Rica; Falkland Islands; Guatemala; USA

Falco hypoleucos Gould 1841

Il $\quad \mathrm{K}^{*}$

$15,226,253,318,648$

Grey Falcon

Breeding: Australia [167,1037]

Vagrant: Papua New Guinea

Falco jugger Gray 1834

Laggar Falcon

Breeding:

Vagrant:

Afghanistan; Bangladesh; ?Bhutan; India; Iran; Myanmar; Nepal; Pakistan Turkmenia; Viet Nam

Falco longipennis Swainson 1837

II $\quad-\quad 15,226$

Australian Hobby, Little Falcon

Breeding:

Australia [167]; Indonesia: Flores, Lombok, ?Paloe, Pantar, ?Rinca, ?Roti, Sumbawa,

Non-breeding: $\quad$ Papua New Guinea

Timor, Wetar [1486] 
Falco mexicanus Schlegel 1850

\section{Prairie Falcon}

Breeding:
Canada; Mexico; USA

Falco moluccensis (Bonaparte 1850)

Spotted Kestrel, Moluccan Kestrel

Breeding:

Indonesia

Falco naumanni Fleischer 1818

II

$\mathrm{K}^{*}$

$15,16,226$

II

$-$

$15,16,226$

Lesser Kestrel

Breeding:

Non-breeding:

Afghanistan; ?Albania; Algeria; Armenia; Austria; Azerbaijan; Bulgaria; China; ?Czechoslovakia; France; Georgia; Gibraltar; Greece [643]; Hungary; Iran; ?Iraq; Israel; Italy; Jordan; Kazakhstan; Kyrgyzstan; ?Lebanon; Libya; Moldova; Mongolia [1098]; Morocco; ?Poland; Portugal; Romania; Russia; Saudi Arabia; Spain; ?Switzerland; Syria; Tajikistan; Tunisia; Turkey; Turkmenja; Ukraine; Uzbekistan; Yugoslavia

$10,15,161,212,226,227,285,318$, $345,523,661,1247$ Republic; Chad; Congo; Côte d'Ivoire; Cyprus; Djibouti. Egypt; Ethiopia; Ghana; ?Guinea-Bissau; India; Iraq; Kenya; Kuwait; Lao P.D.R; Lebanon; Lesotho; Malawi; Maldives; Mali; Malta; Mauritania; Mozambique; Myanmar; Namibia; Nepal; Niger; Nigeria; Oman; Pakistan; Poland; Qatar; Rwanda; Senegal; ?Sierra Leone; South Africa; Sudan; Tanzania; ?Togo; Turkey; Uganda; United Arab Emirates; ?Western Sahara; Yemen; Zaire; Zambia; Zimbabwe

Vagrant: $\quad$ Belgium; Cameroon; Czechoslovakia; Denmark; Gambia; Germany; Guinea; Ireland; Japan; Liberia; Liechtenstein; Sweden; Somalia; Swaziland; Switzerland; United Kingdom

Falco ncwtoni (Gurney 1863)

(excluding subspecies aldabranus)

Newion's Kestrel, Madagascar Kestrel

Breeding:

Madagascar [373]
11

15,226

Falcu newtoni aldabranus Grote 1928

Aldahra Kestrel

Breeling:

Vagrant:

Aldabra Islands (Seychelles)

Comoros: Anjouan
Falco novaezeelandiae Gmelin 1788

New Zealand Falcon

Breeding:

New Zealand

Falco pelegrinoides Temminck 1829

Barhary Falcon

Breeding:

Non-breeding:

Vagrant:
Afghanistan; Algeria; Canary Islands; China; Egypt; Ethiopia; Iran; Israel; ?Italy; Kenya; Kyrgyzstan; ?Libya; ?Mauritania; Morocco; Niger; Saudi Arabia; Somalia; Sudan; Syria; Tajikistan; Tunisia; Turkmenia; Uzbekistan; Yemen

Ethiopia; India; Iran; Iraq; Libya; Pakistan; Sudan

Greece; Italy; Jordan [1451]; Kenya; Madeira; Mali; Malta; Nepal; Niger; Senegal; Somalia; Spain 
(includes $F$. kreyenborgi)

Peregrine Falcon, Peregrine, Duck Hawk

Breeding:

Albania; Algeria; Andorra; Angola; Argentina; Armenia; Australia [167]; Austria; Azerbaijan; Belarus; Belgium; ?Benin; ?Bhutan; Botswana; Bulgaria; ?Burundi; Cameroon; Canada; Cape Verde; ?Central African Republic; Chad; Chile [715]; China; Comoros; Congo; Côte d'lvoire; Cyprus; Czechoslovakia; ?Djibouti; ?Ecuador; Egypt; Estonia; Ethiopia; Falkland Islands; Fiji; Finland; France; ?Gambia; Georgia; Germany; Ghana; Gibraltar; Greece; Greenland; Hong Kong; ?Hungary; India; Indonesia: Aru Islands, Babar, Bali, Irian Jaya, Java, Kalaotoa, Lombok, Sulawesi, Sumatra, Sumba, Timor, Wetar, Yapen; Iran; Ireland; Israel; Italy; Japan; ?Jordan; Kazakhstan; Kenya; D.P.R. Korea; ?Korea Republic; Kyrgyzstan; Latvia; ?Lebanon; ?Lesotho; ?Libya; Liechtenstein; Lithuania; Madagascar [373]; Malawi; Malaysia: Peninsular Malaysia, Sarawak [1295]; Malta; Mauritania; Mayotte; Mexico; Moldova; ?Mongolia; Morocco; Mozambique; Myanmar; Namibia; Nepal; ?Netherlands; New Caledonia; Niger [1015]; ?Nigeria; Norway; Oman; Pakistan; Papua New Guinea; ?Peru; Philippines; Poland; Portugal; Romania; Russia [799]; Rwanda; Saudi Arabia; ?Senegal; Solomon Islands; Somalia; South Africa; Spain; Sri Lanka; Swaziland; Sweden; Switzerland; Tajikistan; Tanzania; Thailand [1194]; ?Togo; Tunisia; Turkey; Turkmenia; Ukraine; USA: Uganda; United Kingdom; Uzbekistan; Vanuatu; ?Viet Nam; ?Western Sahara; Yemen; Yugoslavia; Zaire; Zambia; Zimbabwe

Non-breeding: $\quad$ ?Anguilla; Antigua and Barbuda; Aruba; Bahamas; Bahrain; Bangladesh; Barbados; Belize; Benin; Bermuda; Bolivia; Brazil; ?British Virgin Islands; Brunei; Burkina Faso; Cambodia; Cayman Islands; Central African Republic; Colombia; Costa Rica; Cuba; Denmark; Dominica; Dominican Republic; Ecuador; El Salvador; Equatorial Guinea; ?French Guiana; Gambia; Grenada; ?Guadeloupe; Guam; Guatemala; Guernsey; Guinea; ?Guinea-Bissau; Guyana; Haiti; Honduras; Hungary; Iraq; Jamaica; Jersey; Jordan [1451]; Kuwait; Lao P.D.R; Lebanon; Liberia; Libya; Luxembourg; Macao; Malaysia: Sabah; Mali; ?Martinique; Montserrat; Netherlands Antilles; Nicaragua; Niger; Nigeria; Palau; Panama; ?Paraguay; Puerto Rico; Senegal; ?Sierra Leone; Singapore; Saint Kitts and Nevis; Saint Lucia; Saint Pierre and Miquelon; Saint Vincent; Suriname [627]; Syria; Taiwan; Togo; Trinidad and Tobago; United Arab Emirates; Uruguay; Venezuela; Virgin Islands of the United States; Western Samoa

Vagrant: $\quad$ Christmas Island; Faeroe 1slands; Federated States of Micronesia [11 18]; Fiji [1118]; Guam [1118]; Hawaiian Islands: Hawaii [1118]; Iceland; India: Andaman Islands; Indonesia: Kalimantan [993], ?Seram, Ternate; Maldives; Mauritius; Qatar; Samoa: Savaii; Seychelles: Aldabra [493]

Falco punctatus Temminck 1821

I $\quad E$

$15,226,274,318,324,754$

Mauritius Kestrel

Breeding: Mauritius

Falco rufigularis Daudin 1800

II

$15,16,226,967$

Bat Falcon

Breeding: $\quad$ Argentina; Belize; Bolivia; Brazil; Colombia; Costa Rica; Ecuador; El Salvador; French Gujana; Guatemala; Guyana; Honduras; Mexico; Nicaragua; Panama; Paraguay; Peru; Suriname; Trinidad and Tobago; Venezuela

Falco rupicoloides Smith 1829

II

$15,212,226,227,285,1298$

Greater Kestrel, White-eyed Kestrel

Breeding:

Angola; Botswana; Ethiopia; Kenya; ?Lesotho; Mozambique; Namibia; Somalia; South Africa; Swaziland; Tanzania; Uganda; Zambia; Zimbabwe 
Falco rusticolus Linnaeus 1758

\section{Gyrfalcon, Gyr Falcon}

Breeding:

Non-breeding:

Vagrant:
Canada; Finland; Greenland; Iceland; Norway; Russia [799]; Sweden; USA (Alaska)

France; Denmark; Japan

Austria; Belgium; Brazil; China; Czechoslovakia; Denmark; France; Germany; Guemsey; Ireland; Jersey; Latvia; Netherlands; Pakistan; Poland; Portugal; Spain; Switzerland; United Kingdom

Falco severus Horsfield 1821

II

$10,15,226,792$

\section{Oriental Hobby}

Breeding:

Vagrant:
Bangladesh; Bhutan; Cambodia; China; India; Indonesia: Ambon, Bali, Buru, Halmahera, Irian Jaya, Java, Kalaotoa, Salawati, Sulawesi, ?Sumatra, Ternate, Yapen; Lao P.D.R; Myanmar; Nepal; Papua New Guinea; Philippines; Thailand [1194]; Viet Nam Brunei; Indonesia: Kalimantan; Malaysia: Peninsular Malaysia, Sabah; Sri Lanka

Falco sparverius Linnaeus 1758

II

$15,16,179,226,967$

\section{American Kestrel}

Breeding:

?Anguilla; Argentina; Aruba; Bahamas; Bolivia; Brazil; British Virgin Islands Canada; Chile [715]; Colombia; Costa Rica; Cuba; Dominica; Dominican Republic; Ecuador; French Guiana; Grenada; ?Guadeloupe; Guatemala; Guyana; Haiti; Honduras; Jamaica; Chile: Juan Fernandez; ?Martinique; Mexico; ?Montserrat; Netherlands Antilles; Nicaragua; Paraguay; Peru; Puerto Rico; Saint Kitts and Nevis; Saint Lucia; Saint Vincent; ?Turks and Caicos Islands; USA; Uruguay; Venezuela; Virgin Islands of the United States

Non-breeding: $\quad$ Antigua and Barbuda; Belize; Bermuda; Costa Rica; El Salvador; Panama; Saint Pierre and Miquelon

Vagrant: $\quad$ Azores; ?Barbados; Cayman Islands; Denmark; Estonia; Falkland Islands; Malta; Trinidad and Tobago: Trinidad; United Kingdom

Falco subbuteo Linnaeus 1758

\section{Eurasian Hobby, Northern Hobby}

Breeding:

Afghanistan; Albania; ?Algeria; Armenia; Austria; Azerbaijan; Belarus; Belgium; Bulgaria; China; Cyprus (ex); Czechoslovakia; Denmark; Estonia; Finland; France; Georgia; Germany; Greece; Hungary; India; Iran; Israel; Italy; Japan; Kazakhstan; ?D.P.R. Korea; Kyrgyzstan; Latvia; Lebanon; Libya (ex); Liechtenstein; Lithuania; Luxembourg; Moldova; Mongolia; Morocco; Myanmar; Nepal; Netherlands; Norway; Pakistan; Poland; Portugal; Romania; Russia; Spain; Sweden; Switzerland; ?Syria; Tajikistan; Tunisia; Turkey; Turkmenia; Ukraine; United Kingdom; Uzhekistan; Viet Nam; Yugoslavia

Non-breeding: Algeria; Angola; Bahrain; ?Benin; Bhutan; Botswana; ?Burkina Faso; ?Burundi; Cameroon; ?Central African Republic; Chad; Côte d'lvoire; Cyprus; ?Djibouti; Egypt; Ethiopia; Gambia; Gibraltar; Guinea [1454]; ?Guinea-Bissau; Hong Kong; Iraq; Ireland; Jordan [145I]; Kenya; D.P.R. Korea; Korea Republic; Kuwait; Libya; Malawi; Maldives; Mali; Malta; ?Mauritania; Mozambique; Namibia; Niger; Nigeria; Oman; Qatar; Rwanda; Saudi Arabia; ?Sierra Leone; Somalia; South Africa; Sudan; Swaziland; Syria; Taiwan; Tanzania; Thailand; Togo; Uganda; United Arab Emirates; Yemen; Zaire; Zambia; Zimbabwe

Vagrant: Bangladesh; Canary Islands; Faeroe Islands; Ghana; Guemsey; Iceland; Indonesia: Java [942], Timor [1486]; Jersey; Lesotho; Liberia; Malaysia: Peninsular Malaysia; Senegal; Seychelles [459]; USA

Falco subniger Gray 1843

\section{Black Falcon}

Breeding:

Australia [167] 


\section{Common Kestrel, Eurasian Kestrel}

Breeding:

Non-breeding:

Vagrant:
Afghanistan; Albania; Algeria; Andorra; Angola; Armenia; Austria; Azerbaijan; ?Bahrain; Belarus; Belgium; Benin; Bhutan; Botswana; Bulgaria; Burkina Faso [1388]; Burundi; Cameroon; Canary Islands; Cape Verde; ?Central African Republic; Chad; China; Congo; Côte d'Jvoire; Cyprus; Czechoslovakia; Denmark; Djibouti; Egypt; Estonia; Ethiopia; Faeroe Islands; Finland; France; Georgia; Germany; Ghana; Gibraltar; Greece; Guernsey; Guinea [984]; ?Guinea-Bissau; Hungary; India; Iran; Iraq; Ireland; Israel; Italy; Japan; Jersey; Jordan [1451]; Kazakhstan; Kenya; D.P.R. Korea; Korea Republic; Kuwait; Kyrgyzstan; ?Lao P.D.R; Latvia; Lebanon; Lesotho; Liberia; Libya; Liechtenstein; Lithuania; Luxembourg; Madeira; Malawi; Mali; ?Malta; ?Mauritania; Moldova; Mongolia; Morocco; Mozambique; Namibia; Nepal; Netherlands; Niger; Nigeria; Norway; Oman; Pakistan; Poland; Portugal; ?Qatar; Romania; Russia; Rwanda; Saudi Arabia; ?Senegal; Sierra Leone; Somalia; South Africa; Spain; Sri Lanka; Sudan; Swaziland; Sweden; Switzerland; Syria; Tajikistan; Tanzania; ?Togo; Tunisia; Turkey; Turkmenia; Uganda; Ukraine; United Arab Emirates; United Kingdom; Uzbekistan; Viet Nam; Western Sahara; Yemen; Yugoslavia; Zaire; Zambia; Zimbabwe

Bahrain; Bangladesh; Cambodia; Central African Republic; Gambia; Guinea [1454]; Hong Kong; Lao P.D.R; Malaysia: Peninsular Malaysia, Sabah; Maldives; Malta; Mauritania; Myanmar; Philippines; Senegal; Singapore; Taiwan; Thailand; Togo

Azores; Bermuda; Brunei; Canada; Greenland; Iceland; Indonesia: Nias [905]; Malaysia: Sarawak [1295]; Martinique [179]; Northern Marianas: Saipan; Svalbard and Jan Mayen Islands; USA

Falco vespertinus Linnaeus 1766

$10,15,212,226,345,661,1247$

Red-footed Falcon, Western Red-footed Falcon

Breeding:

Austria; Belarus; Bulgaria; Canary Islands; Czechoslovakia; Estonia; Hungary; Kazakhstan; Moldova; ?Poland; Romania; Russia; Turkey; Ukraine; Yugoslavia

Non-breeding: $\quad$ ?Albania; Algeria; Angola; Armenia; Azerbaijan; Botswana; Burundi; Cameroon; ?Central African Republic; Chad; Cyprus; Egypt; Ethiopia; Finland; France; Georgia; Germany; Greece; Iran; ?Iraq; Israel; 1taly; Jordan [1451]; Kyrgyzstan; Lao P.D.R; Latvia; ?Lesotho; Libya; Liechtenstein; Malawi; Mali; Malta; Mozambique; Namibia; Nigeria; Oman; Somalia; South Africa; Sudan; Sweden; Switzerland; Syria; Tajikistan; Tunisia; Tukmenia; United Kingdom; Uzbekistan; Zambia; Zimbabwe

Vagrant: $\quad$ Bahrain; Belgium; Côte d'Ivoire; Denmark; Djibouti; ?Ghana; Gibraltar; Ireland; Lebanon; Luxembourg; Morocco; Netherlands; Niger; Norway; Portugal; Rwanda; Saudi Arabia; Senegal; Spain; Swaziland; Togo; Yemen; Zaire

Falco zoniventris Peters 1854

Barred Kestrel, Banded Kestrel

Breeding:

Madagascar [373]
Herpetotheres cachinnans (Linnaeus 1758)

II

15,226

Laughing Falcon

Breeding:
Argentina; Belize; Bolivia; Brazil; Chile; Colombia; Costa Rica; Ecuador; El Salvador; French Guiana; Guatemala; Guyana; Honduras; Mexico; Nicaragua; Panama; Paraguay; Peru; Suriname; Venezuela

Micrastur buckleyi Swann 1919

Buckley's Forest-Falcon, Traylor's Forest-Falcon, Lesser Collared Forest-Falcon 
Micrastur gilvicollis (Vieillot 1817)

II

$15,226,967$

(=M. ruficollis gilvicollis)

Lined Forest-Falcon

Breeding:

Bolivia; Brazil; Colombia; Ecuador; French Guiana [1380]; Guyana; Peru; Suriname; Venezuela

Micrastur mirandollei (Schlegel 1862)

II

$15,16,226,967$

Slaty-backed Forest-Falcon

Breeding:

Bolivia; Brazil; Colombia; Costa Rica; Ecuador; French Guiana; Guyana; Panama; Peru; Suriname; Venezuela

Micrastur plumbeus Sclater 1918

II

$\mathrm{V}$

$15,166,226,318,321,967$

Plumbeous Forest-Falcon

Breeding:

Colombia; Ecuador

Micrastur ruficollis (Vieillot 1817)

11

$15,16,226,967$

Barred Forest-Falcon

Breeding:

Argentina; Belize; Bolivia; Brazil; Colombia; Costa Rica; Ecuador; El Salvador; French Guiana [1380]; Guatemala; Honduras; Mexico; Nicaragua; Panama; Paraguay; Peru; Suriname; Venezuela

Micrastur semitorquatus (Vieillot 1817)

11

$15,16,226,967$

Collared Forest-Falcon

Breeding:

Argentina; Belize; Bolivia; Brazil; Colombia; Costa Rica; Ecuador; El Salvador; French Guiana; Guatemala; Guyana; Honduras; Mexico; Nicaragua; Panama; Paraguay; Peru; Suriname; Venezuela

Microhierax caerulescens (Linnaeus 1758) II - $\quad 15,226$

Collared Falconet, Red-legged Falconet, Red-thighed Falconet

Breeding: $\quad$ Bangladesh; Bhutan; Cambodia; China; India; Lao P.D.R; Myanmar; Nepal; Thailand; Vict Nam

Microhierax erythrogenys (Vigors 1831) I1 $\quad$ - $\quad 15,226$

Philippine Falconet

Breeding: $\quad$ Philippines [1113]

Microhierax fringillarius (Drapiez 1824) II $\quad$ - $\quad 15,226,792$

Black-thighed Falconet, Black-legged Falconet, Black-sided Falconet

Breeding: Brunei; Indonesia: Bali, Java, Kalimantan, Sumatra; Malaysia: Peninsular Malaysia, Sarawak [1295]; Myanmar; Thailand [1194]

Vagrant: $\quad$ Singapore

Microhierax latifrons Sharpe 1879

II $\quad$ - $\quad 15,226,930$

White-fronted Falconet, Bornean Falconet

Breeding: Malaysia: Sabah, Sarawak [1295]

Microhierax melanoleucus (Blyth 1843) II $\quad$ II $\quad 10,15,226,792$

Pied Falconet

Breeding:

Vagrant:

Bangladesh; China; India; Lao P.D.R; Viet Nam

Hong Kong

Milvago chimachima (Vieillot 1816)

11

$15,16,226,967$

Yellow-headed Caracara

Breeding:

Argentina; Bolivia; Brazil; Colombia; Costa Rica; Ecuador; French Guiana; Guyana; Panama; Paraguay; Peru; Suriname; Uruguay; Venezuela

Vagrant:

Netherlands Antilles: Curaçao; Trinidad and Tobago: Trinidad 
White-throated Caracara

Breeding: Argentina; Chile [715]

Phalcoboenus australis (Gmelin 1788)

Striated Caracara, Forster's Caracara

Breeding:

Argentina: Tierra del Fuego, Staten Island; Chile: Tierra del Fuego, islands south of Beagle Channel [715]; Falkland 1slands

Phalcoboenus carunculatus Des Murs $1853 \quad 11 \quad-\quad 15,226,967$
Carunculated Caracara
Breeding: $\quad$ Colombia; Ecuador

$\begin{array}{llll}\text { Phalcoboenus megalopterus (Meyen 1834) } & \text { II } & - & 15,226,967\end{array}$

Mountain Caracara

Breeding: $\quad$ Argentina; Bolivia; Chile [715]; Penu

$\begin{array}{llll}\text { Polihierax insignis Walden } 1872 & \text { I1 } & - & 10,15,226,792\end{array}$

White-rumped Falcon, White-rumped Falconet, Fielden's Falconet

Breeding: $\quad$ Cambodia; Lao P.D.R; Myanmar; Thailand [1194]; Viet Nam

Polihierax semitorquatus (Smith 1836)

Pygmy Falcon, African Pygmy Falcon

Breeding: $\quad$ ?Angola; Botswana; Central African Republic [259]; Ethiopia; Kenya; Mozambique; Namibia; Rwanda; Somalia; South Africa; Sudan; Tanzania; Uganda

Polyborus lutosus Ridgway 1876

Il Ex $\quad 930$

Guadalupe Caracara

Breeding: Mexico: Guadalupe Island (ex)

Polyborus plancus (Miller 1777)

Crested Caracara, Common Caracara

Breeding: Argentina; Aruba; Bolivia; Brazil; Chile [715]; Colombia; Costa Rica; Cuba; Ecuador; El Salvador; Falkland Islands; French Guiana; Guatemala; Guyana; Honduras; Mexico; Netherlands Antilles; Nicaragua; Panama; Paraguay; Peru; Suriname; Trinidad and Tobago: Trinidad; USA; Uruguay; Venezuela

Vagrant: Belize; Jamaica

Spiziapteryx circumcinctus (Kaup 1852)

Spot-winged Falconet, Spot-winged Falcon

Breeding:

Vagrant:

Argentina; Bolivia

Paraguay 


\section{Order GALLIFORMES \\ Game-birds \\ Family MEGAPODIIDAE \\ Megapodes}

Aepypodius bruijnii (Oustalet 1880)

$\mathrm{K}^{*} \quad 318,1226$

Bruijn's Brush-turkey, Waigeo Brush-turkey

Breeding: Indonesia: Waigeo Island

Leipoa ocellata Gould 1840

$K^{*} \quad 183,318$

Malleefowl

Breeding: Australia [167]

Macrocephalon maleo Mũller 1846

$1 \quad \mathrm{~V}$

$88,318,374,375,794,879,1462$, 1486

Maleo, Gray's Brush-turkey

Breeding: Indonesia: Lembeh, Sulawesi

Megapodius bernsteinii Schlegel 1866

$K^{*} \quad 318,1486$

Sula Scrubfowl

Breeding: Indonesia: Banggai (ex?), Sula

Megapodius laperouse Gaimard 1823

R $\quad 318,794$

Micronesian Scrubfowl, Marianas Scrubfowl

Breeding: $\quad$ Guam (ex); Northern Marianas [1118]; Palau [1118]

$\begin{array}{llll}\text { Megapodius nicobariensis Blyth } 1846 & - & \mathrm{K}^{*} & 10,318\end{array}$

(=M. freycinet nicobariensis)

Nicobar Scrubfowl

Breeding: India: North Nicobar lslands

Megapodius pritchardii Gray 1864

$\mathrm{K}^{*} \quad 318$

Polynesian Scrubfowl, Nevafou Megapode

Breeding: $\quad$ Tonga: Niuafo'ou [1118,1391]

Megapodius wallacei Gray $1860 \quad-\quad K^{*} \quad 318,1486$

(= Eulipoa wallacei)

Moluccan Scrubfowl

Breeding:

Indonesia: Ambon, Bacan, Buru, Halmahera, Haruku, Misool, Seram, Ternate

Family CRACIDAE

Guans and Curassows

Crax alberti Fraser 1852

III CO E $\quad 318,321,378,794,967$

Blue-knobbed Curassow, Blue-billed Curassow

Breeding: Colombia

Crax blumenbachii Spix 1825

I R

$318,321,378,794,967,1277,1366$

Red-billed Curassow

Breeding:

Brazil

Crax daubentoni Gray 1867

1II $\mathrm{CO}$

378,967

Yellow-knobbed Curassow

Breeding: Colombia; Venezuela 
Crax globulosa Spix 1825

III CO Id

$166,318,321$

Wattled Curassow

Breeding:

Bolivia; Brazil; Colombia; Ecuador; Peru

Crax rubra Linnaeus 1758

III $\mathrm{CR}$

$16,378,794,967$

Great Curassow

GT HN NI

Breeding:

Belize; Colombia; Costa Rica; Ecuador; El Salvador; Guatemala; Honduras; Mexico; Nicaragua; Panama

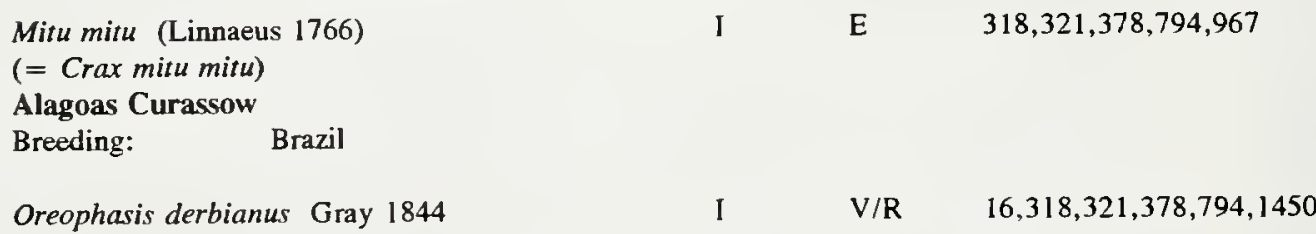

Horned Guan

Breeding:

Guatemala $[61,677]$; Mexico $[547,548]$

Ortalis vetula (Wagler 1830)

$\begin{array}{ll}111 \mathrm{GT} & -\quad 16,378,794 \\ \mathrm{HN} & \end{array}$

Plain Chachalaca

Breeding:

Belize; Costa Rica; El Salvador; Guatemala; Honduras; Mexico; Nicaragua; USA

Pauxi pauxi (Linnaeus 1766)

III CO E

$166,318,321$

(= Crax pauxi)

Helmeted Curassow

Breeding: Colombia; Venezuela

Pauxi unicornis Bond \& Meyer de Schauensee 1939

(= Crax unicornis)

Horned Curassow

Breeding:

Peru; Bolivia

Penelope albipennis Taczanowski 1878

l E

$16,318,321,395,423,794,967,1043$

White-winged Guan

Breeding:

Peru [1031]

Penelope barbata Chapman 1921

$\mathrm{V} / \mathrm{R} \quad 166,318,321$

Bearded Guan

Breeding: $\quad$ Ecuador; Peru

Penelope ochrogaster Pelzeln 1870

$\mathrm{V} / \mathrm{R}$

$166,318,321,341$

Chestnut-bellied Guan

Breeding:

Brazil

Penelope perspicax Bangs 1911

$\mathbf{R}$

$318,321,378,794,967,1010$

Cauca Guan

Breeding:

Colombia

Penelope purpurascens Wagler 1830

$111 \mathrm{HN}$

$166,378,967$

Crested Guan

Breeding:

Belize; Colombia; Costa Rica; Ecuador; El Salvador; Guatemala; Honduras; Mexico; Nicaragua; Panama; Venezuela

Penelopina nigra (Fraser 1852)

$111 \mathrm{GT}-$

$16,61,166,318,378$

Highland Guan

HN

Breeding:

El Salvador (ex?); Guatemala; Honḋuras; Mexico; Nicaragua 
Pipile jacutinga (Spix 1825)

Breeding: $\quad$ Argentina [154]; Brazil; Paraguay

Pipile pipile (Jacquin 1784)

(= Aburria pipile pipile)

Trinidad Piping-Guan

Breeding:

Trinidad and Tobago: Trinidad

Family PHASIANIDAE

Pheasants, Turkeys, Grouse, Guinea-fowl and allies

Afropavo congensis Chapin 1936

Congo Peafowl

Breeding:

Zaire

Agelastes meleagrides Bonaparte 1850

III GH E

$318,324,1247,1298,141]$

White-breasted Guineafowl

Breeding:

Côte d'Ivoire [55]; Ghana (ex?); Liberia; Sierra Leone [364]

Agriocharis ocellata (Cuvier 1820)

III GT -

$16,318,1316$

Ocellated Turkey

Breeding:

Belize; Guatemala; Mexico

Arborophila ardens (Styan 1892)

$K^{*} \quad 318,369$

Hainan Partridge, White-eared Partridge, White-eared Hill-Partridge

Breeding: China $[55,968]$

Arborophila cambodiana Delacour \& Jabouille 1928 - $\quad \mathrm{K}^{*} \quad 318,369$

Chestnut-headed Partridge

Breeding: $\quad$ Cambodia [792]; Thailand

Arborophila charltonii (Eyton 1845)

(= Tropicoperdix charltonii)

Chestnut-necklaced Partridge, Chestnut-breasted Tree-Partridge

Breeding:

Indonesia: Sumatra; Malaysia: Peninsular Malaysia, Sabah; Thailand; Viet Nam

Arborophila davidi Delacour 1927

$K^{*} \quad 318,369$

Orange-necked Partridge

Breeding: Viet Nam [376]

Arborophila gingica (Gmelin 1789)

$\mathrm{K}^{*}$

318,369

White-necklaced Partridge, Rickett's Hill-Partridge

Breeding:

China [968]

Arborophila orientalis (Horsfield 1821)

III MY

369,792

Grey-breasted Partridge, Bar-hacked Partridge

Breeding: $\quad$ China; Indonesia: Java, Sumatra; Lao P.D.R: Malaysia: Peninsular Malaysia; Myanmar; Thailand; Viet Nam

Arborophila rufipectus Boulton 1932

$\mathrm{K}^{*}$

318,369

Sichuan Partridge

Breeding:

China [968] 
Argusianus argus (Linnaeus 1766)

11

377,742

Great Argus, Argus Pheasant

Breeding:

Brunei; Indonesia: Kalimantan [664], Sumatra; Malaysia: Peninsular Malaysia, Sabah, Sarawak [1295]; Myanmar; Thailand [1194]

Caloperdix oculea (Temminck 1815)

II1 MY -

792

Ferruginous Partridge, Ferruginous Wood-Partridge

Breeding: $\quad$ Indonesia: Kalimantan [1091], Sumatra; Malaysia: Peninsular Malaysia, Sarawak [1295]; Myanmar; Thailand

Catreus wallichii (Hardwicke 1827)

$\mathrm{E}$

$10,318,377,742,794,1546,1547$

Cheer Pheasant, Chir Pheasant, Wallich's Pheasant

Breeding: India [512,518]; Nepal [844]; Pakistan

Colinus virginianus ridgwayi I $\quad-\quad 16,794$

Masked Bobwhite

Breeding:

Mexico; USA (ex)

Crossoptilon crossoptilon (Hodgson 1838)

White Eared-Pheasant

I $\quad$ V $\quad 10,318,377,742,794$

Breeding: China [968]

Crossoptilon harmani Elwes 1881

I

$318,377,742,794$

(=C. crossoptilon harmani)

Tibetan Eared-Pheasant

Breeding: China

Crossoptilon mantchuricum Swinhoe 1863

E

$318,377,742,794$

Brown Eared-Pheasant, Manchurian Eared-Pheasant

Breeding:

China [968]

Dendrortyx barbatus Gould 1846

E

Bearded Wood-Partridge

Breeding:

Mexico

Francolinus camerunensis Alexander 1909

$\mathbf{R}$

$318,324,1247,1298,1411$

Cameroon Francolin, Mount Cameroon Francolin

Breeding:

Cameroon $[1245,1246]$

Francolinus gularis (Temminck 1815)

$K^{*} \quad 10,318$

Swamp Francolin

Breeding:

Bangladesh; India; Nepal

Francolinus nahani Dubois 1905

R

$212,318,324,1298,1411$

Nahan's Francolin

Breeding:

Uganda; Zaire

Francolinus ochropectus Dorst \& Jouanin 1952

E

$176,318,324,1298,1411$

Ochre-breasted Francolin, Djibouti Francolin

Breeding:

Djibouti [1471]

Francolinus swierstrai (Roberts 1929)

Id

$318,324,599,1298,1411$

Swierstra's Francolin

Breeding:

Angola 
Gallus sonnerasii Temminck 1813

Grey Junglefowl, Sonnerat's Junglefowl

Breeding:

India

\section{Ithaginis cruentus \\ Blood Pheasant \\ Breeding:}

(Hardwicke 1821)

Bhutan; China; India; Myanmar; Nepal [700]

Lophophorus impejanus (Latham 1790)

Himalayan Monal, Impeyan Pheasant

Breeding:

Afghanistan; Bhutan; China; India; Myanmar; Nepal; Pakistan

Lophophorus lhuysii Geoffroy Saint-Hilaire 1866

Chinese Monal, Chinese Impeyan

E

$318,377,742,794,871$

Breeding:

China [968]

Lophophorus sclateri Jerdon 1870

l R

$10,318,377,742,794$

Sclater's Monal, Crestless Monal

Breeding:

China; India; Myanmar

Lophura bulweri (Sharpe 1874)

V

$10,377,742$

Bulwer's Pheasant, White-tailed Wattled Pheasant

Breeding:

Brunei; Indonesia: Kalimantan [1295]; Malaysia: Sabah, Sarawak [54,1295]

Lophura diardi (Bonaparte 1856)

$\mathrm{K}^{*}$

$318,377,742,792$

Siamese Fireback, Diard's Fireback

Breeding: $\quad$ Cambodia; Lao P.D.R; Thailand [1194]; Viet Nam

Lophura edwardsi (Oustalet 1896)

Edwards's Pheasant

Breeding:

Viet Nam

Lophura erythrophthalma (Raffles 1822)

$111 \mathrm{MY}$ -

$377,742,792$

Crestless Fireback, Rufous-tailed Pheasant

Breeding:

Brunei; Indonesia: Kalimantan [1295], Sumatra; Malaysia: Peninsular Malaysia, Sarawak [1295]

Lophura hatinhensis Vo Quy \& Do Ngoc Quang 1965

Vietnamese Pheasant, Vo Quy's Pheasant

$\mathrm{K}^{*} \quad 318$

Breeding:

Viet Nam

Lophura ignita (Shaw 1797)

III MY K*

$318,377,742,792$

Crested Fireback, Vieillot's Fireback

Breeding:

Brunei; Indonesia: Kalimantan [1295], Sumatra; Malaysia: Peninsular Malaysia, Sabah, Sarawak [1295]; Myanmar; Thailand

Lophura imperialis (Delacour \& Jabouille 1924)

$\mathrm{V}$

$318,377,742,792,794$

Imperial Pheasant

Breeding:

?Lao P.D.R; Viet Nam

Lophura inornata (Salvadori 1879)

$\mathrm{K}^{*}$

$318,377,742$

Salvadori's Pheasant

Breeding:

Indonesia: Sumatra 
Swinhoe's Pheasant

Breeding: Taiwan [1253]

Melanoperdix nigra (Vigors 1829)

III MY -

792

Black Partridge, Black Wood-Partridge

Breeding:

Indonesia: Kalimantan [1295], Sumatra; Malaysia: Peninsular Malaysia, Sarawak [1295]

Odontophorus strophiutn (Gould 1844)

V/R

$201,318,321,794,967,1187$

Gorgeted Wood-Quail

Breeding: Colombia

Ophrysia superciliosa (Gray 1846)

(n)

Himalayan Quail

Breeding: India (ex) [10]

Pavo muticus Linnaeus 1766

II V

$10,318,377,653,742,792,794$

Green Peafowl, Green-necked Peafowl

Breeding: $\quad$ Bangladesh (ex?); Cambodia; China; India; Indonesia: Java; Lao P.D.R; Malaysia: Peninsular Malaysia; Myanmar; Thailand; Viet Nam

Perdicula manipurensis (Hume 1880)

$\mathrm{K}^{*} \quad 10,318$

Ma:ipur Bush-Quail

Breeding: Bangladesh; India

Polyplectron bicalcaratum (Linnaeus 1758)

II

$10,377,742,792$

Grey Peacock-Pheasant, Iris Peacock-Pheasant

Breeding:

Bangladesh; Bhutan; China; India; Lao P.D.R; Myanmar; Thailand [1194]; Viet Nam

Polyplectron emphanum Temminck 1831

I V

V $254,318,377,742,794$

Palawan Peacock-Pheasant, Napoleon's Peacock-Pheasant

Breeding:

Philippines: Palawan

Polyplectron germaini Elliot 1866

II $\quad \mathrm{K}^{*}$

$318,377,742,792$

Germain's Peacock-Pheasant

Breeding:

?Lao P.D.R; Viet Nam

Polyplectron inopinatum (Rothschild 1903)

III MY

$377,742,792$

Mountain Peacock-Pheasant, Rothschild's Peacock-Pheasant

Breeding:

Malaysia: Peninsular

Polyplectron inalacense (Scopoli 1786)

II

K*

$318,377,742,792$

Malayan Peacock-Pheasant, Crested Peacock-Pheasant

Breeding:

Malaysia: Peninsular Malaysia; Myanmar; Thailand [I 194]

$\begin{array}{llll}\text { Polyplectron schleiermacheri Brüggemann } 1877 \text { II } & \text { K* } & 742,1295\end{array}$

(=P. malacense schleiermacheri)

Bornean Peacock-Pheasant

Breeding: Indonesia: Kalimantan [664]; Malaysia: Sabah, Sarawak [1295]

Rheinardia ocellata (Elliot 1871)

I R

$318,377,742,792,794$

(includes $R$. nigrescens (Rothschild 1902))

Crested Argus, Ocellated Pheasant

Breeding:

Lao P.D.R; Malaysia: Peninsular Malaysia; Viet Nam 

Rhizothera longirostris (Temminck 1807) 111 MY
$\begin{array}{ll}\text { Long-billed Partridge } & 792 \\ \text { Breeding: } & \text { Indonesia: Kalimantan [1295], Sumatra; Malaysia: Peninsular Malaysia, Sabah, Sarawak } \\ & \text { [1295]; Myanmar; Thailand }\end{array}$

Rollulus rouloul (Scopoli 1786) $\quad$ III MY - $\quad 792$

Crested Partridge, Roulroul

Breeding: $\quad$ Brunei; Indonesia: Kalimantan [1295], Sumatra; Malaysia: Peninsular Malaysia, Sabah, Sarawak [1295]; Myanmar; Thailand

Syrmaticus ellioti (Swinhoe 1872)

$1 \quad \mathrm{E}$

$318,377,742,794,797$

Elliot's Pheasant, Chinese Barred-backed Pheasant

Breeding:

China [968]

Syrmaticus humiae (Hume 1881)

I $\quad \mathrm{R}$

$10,318,377,742,792,794$

Hume's Pheasant, Hume's Bar-tailed Pheasant

Breeding: China; India; Myanmar [785]; Thailand [1194]

Syrmaticus mikado (Ogilvie-Grant 1906) V I $\quad$ V $\quad 318,377,742,794,1251,1252$

Mikado Pheasant

Breeding: Taiwan

Syrmaticus reevesii (Gray 1829) - - $\quad K^{*} \quad 318,377,742$

Reeves's Pheasant

Breeding: China [968, 1540]; [Czechoslovakia] [1112]

$\begin{array}{lllll}\text { Tetrao mlokosiewiczi Taczanowski } 1875 & \text { K* } & 318\end{array}$

Caucasian Grouse

Breeding: $\quad$ Armenia; Azerbaijan; Georgia; Iran; Russia [799]; Turkey

Tetraogallus caspius (Gmelin 1784) $\quad 1 \quad 345$

Caspian Snowcock

Breeding: $\quad$ Armenia; Azerbaijan; Georgia; Iran; Iraq; Turkey; Turkmenia

$\begin{array}{llll}\text { Tetraogallus tibetanus Gould } 1854 & \text { I } & - & 10\end{array}$

Tibetan Snowcock

Breeding: $\quad$ Bhutan; China; India; Nepal; Tajikistan

$\begin{array}{llll}\text { Tragopan blythii (Jerdon 1870) } & \text { I } & \text { R } & 10,318,377,742,794\end{array}$

Blyth's Tragopan, Grey-bellied Tragopan

Breeding: $\quad$ Bhutan; China; India; Myanmar

Tragopan caboti (Gould 1857) I I E $318,377,742,788,794,1549,1550$,

Cabot's Tragopan, Yellow-billed Tragopan 1551

Breeding: China [968]

Tragopan melanocephalus (Giay 1829)

I E $10,318,377,516,742,794$

Western Tragopan, Western Horned-Pheasant, Black-headed Tragopan

Breeding: $\quad$ China; India [1002]; ?Nepal; Pakistan [61,704,705]

Tragopan satyra (Linnaeus 1758) III NP -

$10,377,742$

Satyr Tragopan, Indian Tragopan, Crimson Horned-Pheasant

Breeding:

Bhutan; China; India; Nepal [700] 
Tympanuchus cupido attwateri Bendire 1893

\section{Order GRUIFORMES}

Cranes, Rails and allies

\section{Family MESITORNITHIDAE}

\section{Mesites}

Mesitornis unicolor (Des Murs 1845)

Brown Roatelo, Brown Mesite

Breeding:

Mesitornis variegata (Geoffroy Saint -Hilaire 1838)

White-breasted Roatelo, White-breasted Mesite

Breeding: Madagascar [373]

Monias benschi Oustalet \& Grandidier 1903

$\mathrm{R}$

Monias, Subdesert Mesite

Breeding:

Madagascar [373]

\section{Family TURNICIDAE}

Buttonquails

Turnix everetti Hartert 1898

$$
\mathrm{K}^{*} \quad 318,1486
$$

( $=$ T. sylvatica everetti)

Sumba Buttonquail

Breeding: Indonesia: Sumba [1486]

Turnix melanogaster (Gould 1837)

Black-breasted Buttonquail

Breeding:

Turnix olivii Robinson 1900

( $=T$. castanota olivii)

Buff-breasted Buttonquail

Breeding: Australia [167]

Turnix worcesteri McGregor 1904

Worcester's Buttonquail

Breeding: Philippines: Luzon 
Family PEDIONOMIDAE

Plains-wanderer

Pedionomus torquatus Gould 1841

Il

$\mathrm{K}^{*}$

318

Plains-wanderer

Breeding:

Australia $[42,167,245]$

Family GRUIDAE

Cranes

Balearica pavonina (Linnaeus 1758)

II

$226,227,741,1247,1298,1411,1449$

Black Crowned Crane

Breeding:

Benin; Burkina Faso [1388]; Cameroon; Central African Republic; Chad; Ethiopia; Guinea; Guinea-Bissau; ?Kenya [707,1051]; Mali; Mauritania; Niger; Nigeria; Senegal; Sierra Leone; Sudan; Togo; ?Uganda [707]; Zaire

Non-breeding: Gambia; Ghana; Kenya; Uganda

Vagrant: Côte d'lvoire

Balearica regulorum (Bennett 1834) II $\quad$ - $\quad 212,227,285,741,1298,1411,1449$

Grey Crowned Crane

Breeding: $\quad$ Angola; Botswana; Burundi; Kenya [1051]; Malawi; Mozambique; Namibia; Rwanda; South Africa; Tanzania; Uganda; Zaire; Zambia; Zimbabwe

Non-breeding: Swaziland

Vagrant: Lesotho

Grus americana (Linnaeus 1758) $\quad 1 \quad$ E $\quad 16,86,318,321,405,420,741,794$,

Whooping Crane

Breeding: Canada; USA (ex)

Non-breeding: USA

Vagrant: Mexico

Grus antigone (Linnaeus 1758)

$11 \quad-\quad 10,741,792,1449$

Sarus Crane

Breeding:

Australia [167]; Bangladesh; ?Cambodia; China; India; ?Lao P.D.R; ?Myanmar; Nepal;

Vagrant:

Pakistan; Philippines: Luzon (ex?); Thailand (ex); Viet Nam

Malaysia: Peninsular

Grus canadensis (Linnaeus 1758)

11

$16,741,794,1449$

(excluding subspecies pulla and nesiotes)

Sandhill Crane

Breeding:

Canada; USA; Russia

Non-breeding:

Mexico; Japan

Vagrant:

Faeroe Islands; Ireland; Netherlands; United Kingdom

Grus canadensis nesiotes Bangs \& Zappey 1905

$16,741,794,1449$

Cuban Sandhill Crane

Breeding: Cuba [179]

Grus canadensis pulla Aldrich 1972

$16,741,794,1449$

Mississippi Sandhill Crane

Breeding:

USA 
Angola; Botswana; Ethiopia; Malawi; Mozambique; Namibia [1508]; South Africa [1350]; Swaziland (ex); Tanzania; Zaire; Zambia; Zimbabwe

Vagrant: ?Guinea-Bissau; Lesotho; Swaziland

Grus grus (Linnaeus 1758)

Common Crane

Breeding:

Armenia; Austria (ex); Azerbaijan; Belarus; Bulgaria (ex); China; Czechoslovakia; Denmark; Estonia; Finland; France [982]; Georgia; Germany; Hungary (ex); Italy (ex); Kazakhstan; Latvia; Lithuania; Mongolia; Norway; Poland; Romania; Russia; Spain (ex); Sweden; Turkey; Ukraine; United Kingdom; Yugoslavia (ex)

Non-breeding: Afghanistan; Albania; Algeria; Austria; Belgium; Bangladesh; Bulgaria; Cyprus; Egypt; Ethiopia; Greece; Hungary; India; Iran; Iraq; Israel; Italy; Japan; Jordan; Korea Republic; ?Lao P.D.R; Lebanon; Ljbya; Luxembourg; Malta; Morocco; Myanmar; Nepal; Netherlands; Pakistan; Portugal; Saudi Arabia; Spain; Sudan; Switzerland; Syria; Tunisia; Turkey; Viet Nam; Yemen; Yugoslavia

Vagrant: $\quad$ Azores; Djibouti; Faeroe Islands; Gibraltar; Guernsey; Hong Kong; Iceland; Ireland; Jersey; D.P.R. Korea; ?Kuwait; Liechtenstein; Niger: Oman; USA; United Arab Emirates

Grus japonensis (Müller 1776)

I V

$318,741,794,1449$

Red-crowned Crane

Breeding: $\quad$ China [1510]; Japan; Russia [799,1115]; Mongolia

Non-breeding: $\quad$ D.P.R. Korea; Korea Republic

Vagrant:

Taiwan

Grus leucogeranus Pallas 1773

I V

$10,318,741,794,916,1449$

Siberian Crane, Siberian White Crane

Breeding:

China [778,968,1510]; ?Mongolia; Russia [799,1115]

Non-breeding:

Afghanistan; India; Iran [661]; Pakistan

Vagrant:

Japan; Mongolia

Grus monacha Temminck 1835

I $\quad \mathrm{V}$

$318,741,794,1449$

Hooded Crane

Breeding:

Russia [799,1115]

Non-breeding:

Vagrant:

Japan; D.P.R. Korea; Korea Republic [1310]; Taiwan

India; Mongolia

Grus nigricollis Przevalski 1876

I $\quad$ V

$10,318,741,794,1449$

Black-necked Crane

Breeding:

China $[968,1178]$; India

Non-breeding:

Vagrant:

Bhutan [295]; Myanmar; Viet Nam

Nepal [700]

Grus paradisea (Lichtenstein 1793)

(= Anthropoides paradisea)

Blue Crane, Stanley Crane

Breeding: Namibia; South Africa; Swaziland

Vagrant: Botswana; Lesotho; Zimbabwe

Grus rubicunda (Perry 1810)

Australia [167]; Indonesia: Irian Jaya; Papua New Guinea

Vagrant:

New Zealand 
Grus virgo (Linnaeus 1758)

(= Anthropoides virgo)

Demoiselle Crane

Breeding:

Non-breeding:

Vagrant:
Algeria (ex); China; Kazakhstan; Moldova; Mongolia; ?Morocco; Russia [799]; Tunisia (ex); Turkey; Ukraine

Afghanistan; Bangladesh; Bhutan; Chad; Cyprus; Egypt; Ethiopia; India; Iran; Kyrgyzstan; Myanmar; Nepal; Niger; Nigeria; Pakistan; Saudi Arabia; Sudan; Tajikistan; Turkmenia; Uzbekistan

Algeria; Bulgaria; Czechoslovakia; Denmark; France; ?Germany; Greece; Hungary; Iraq; Israel; ?1taly; Japan; Korea Republic; Kuwait [196]; Lebanon; Malta; Norway; Oman; Portugal; Romania; Spain; Sweden; Tunisia; United Arab Emirates; Yemen; Yugoslavia

Family RALL1DAE

Rails, Crakes, Coots

Amaurornis olivieri (Grandidier \& Berlioz 1929) - $\quad$ K $\quad 318,324,1158$

(= Porana olivieri)

Olivier's Crake, Sakalava Rail

Breeding: Madagascar [373]

Aramidopsis plateni (Blasius 1886)

$\mathrm{K}^{*} \quad 318,1158,1486$

Snoring Rail, Platen's Rail

Breeding: Indonesia: Sulawesi [1486]

$\begin{array}{llll}\text { Atlantisia rogersi Lowe } 1923 & - & \mathrm{R} & 318,324,1158\end{array}$

Inaccessible Rail

Breeding: $\quad$ Saint Helena: Inaccessible Island

Coturnicops exquisitus (Swinhoe 1873)

$\mathrm{K}^{*} \quad 318,1158$

Swinhoe's Rail, Siberian Crake, Asian Yellow Rail

Breeding: China; Russia [481,799]

Non-breeding: $\quad$ Japan; D.P.R. Korea; Korea Republic

Coturnicops notatus (Gould 1841)

Id $\quad 321,967,1158$

Speckled Rail, Speckled Crake

Breeding:

Argentina [154,1009]; Brazil; Colombia; Guyana; Paraguay; Uruguay [84]; Venezuela

Crex crex (Linnaeus 1758)

$\mathrm{K}^{*} \quad 318,345,661,1158,1411$

Corn Crake

Breeding:

Afghanistan; Albania; ?Armenia; Austria; Azerbaijan (ex?); Belarus; Belgium; Bulgaria; China; Czechoslovakia; Denmark; Estonia; Faeroe Islands (ex); Finland; France; Georgia; Germany; Greece; Hungary; Iran; Ireland; Italy [458]; Kazakhstan; Kyrgyzstan; Latvia; Liechtenstein; Lithuania; Luxembourg; Moldova; Netherlands; Norway; Poland; Romania; Russia; Spain; Sweden; Switzerland; Tajikistan; ?Turkey; Ukraine; United Kingdom; Yugoslavia

Non-breeding: Afghanistan; Algeria; Azerbaijan; Botswana; Egypt; Ethiopia; Ghana; Guernsey; Iraq; Israel; Jersey; Kenya; Malawi; Mali; Mauritania; Morocco; Mozambique; Namibia; Nigeria; Portugal; Saudi Arabia; South Africa; Sudan; Swaziland; Tanzania; Tunisia; Turkmenia; Uganda; Uzbekistan; Zaire; Zambia; Zimbabwe

Vagrant: $\quad$ Angola; Azores; Cameroon; Canary Islands; Côte d'Ivoire; Iceland; Lesotho; Libya; Madeira; Mongolia; Seychelles [460] 
Cyanolimnas cerverai Barbour \& Peters 1927

Zapata Rail

Breeding:

Cuba $[179,509]$

Fulica cornuta Bonaparte 1853

K

$318,321,794,967,1158,1431$

Horned Coot

Breeding:

Argentina [154,1431]; Bolivia; Chile [1052, 1053]

Gallinula nesiotis comeri (Allen 1892)

$\mathbf{R}$

$318,324,1158,1460$

(= Gallinula comeri)

Gough Moorhen, Gough Island Coot

Breeding:

Saint Helena: Gough 1sland; [Tristan da Cunha 1slands]

Gallinula silvestris (Mayr 1933)

Id

$318,794,1158,1226$

(= Pareudiastes silvestris, Edithornis silvestris)

San Cristobal Moorhen, San Cristobal Mountain Rail

Breeding:

Solomon 1slands: San Cristobal

Gallirallus australis hectori (Hutton 1874)

11

Buff Weka

Breeding:

New Zealand: South Island, [Chatham Islands]

Gal'irallus lafresnayanus Verreaux \& Des Murs $1860 \quad-\quad$ K*

318,794

(= Tricholimnas lafresnayanus)

New Caledonian Rail, New Caledonian Wood Rail

Breeding: New Caledonia (ex?) [1328]

Gallirallus okinawae (Yamashina \& Mano 1981)

$K^{*}$

$198,199,200,202,318,1375$

(= Rallus okinawae)

Okinawa Rail

Breeding:

Japan: Okinawa [1541]

Gallirallus owstoni (Rothschild 1895)

V

$318,794,1158$

(= Rallus owstoni)

Guam Rail

Breeding:

Guam [1 1118$]$

Gallirallus sylvestris (Sclater 1869)

I

E

$318,794,1158$

(= Tricholimnas sylvestris)

Lord Howe Rail, Lord Howe Island Woodhen

Breeding:

Lord Howe Island [500]

Gymnocrex rosenbergii (Schlegel 1866)

$\mathrm{K}^{*}$

$318,1158,1486$

Bald-faced Rail, Schlegel's Rail

Breeding:

1ndonesia: Peleng, Sulawesi [1486]

Habroptila wallacii Gray 1860

K*

$318,1158,1486$

Invisible Rail, Wallace's Rail

Breeding:

Indonesia: Halmahera [1486]

Laterallus levraudi (Sclater \& Salvin 1868)

V

$166,318,321,1158$

Rusty-flanked Crake

Breeding:

Venezuela 
Laterallus tuerosi

E

$318,321,1158$

(= L. jamaicensis tuerosi)

Junin Rail

Breeding:

Peru [473]

Laterallus xenopterus Conover 1934

$\mathrm{V} / \mathrm{R}$

$3] 8,321,999,1158,1333$

Rufous-faced Crake

Breeding:

Brazil [1268]; Paraguay

Lewinia mirificus (Parkes \& Amadon 1959)

$\mathrm{K}^{*} \quad 318,1158$

(= Rallus mirificus, Dryolimnas mirificus)

Brown-banded Rail

Breeding:

Philippines: Luzon

Nesoclopeus poecilopterus (Hartlaub 1866)

E

$318,794,1158$

Bar-winged Rail

Breeding:

Fiji: Viti Levu, Oualau | 1118 |

Nesoclopeus noodfordi (Ogilvie-Grant 1889)

$K^{*}$

$318,1158,1226$

Woodford's Rail

Breeding:

Papua New Guinea (ex?); ?Solomon lslands: Guadalcanal, Santa lsabel (ex?)

Porphyrio mantelli (Owen 1848)

E

$318,794,838,975,976,1158$

(= Notormis mantelli)

Takahe

Breeding:

New Zealand: South lstand [1507]

Porzana atra North 1980

$\mathrm{K}^{*} \quad 318,1158$

(= Nesophylax ater)

Henderson Crake, Henderson Island Rail

Breeding:

Pitcairn lslands: Henderson lsland [191]

Porzana spiloptera Durnford 1877

ld

$166,318,321,1158$

Dot-winged Crake

Breeding:

Argentina; Uruguay [1165]

Rallus antarcticus King 1828

E/Ex

318,321

Austral Rail

Breeding:

Argentina (ex?) |154\}; Chile

Rallus semiplumbeus: Sclater 1856

$\mathrm{V} / \mathrm{R}$

$3[8,321,794,967,1158$

Bogotá Rail

Breeding:

Colombia; ?Ecuador

Rallus wetmorei Zimmer \& Phelps 1944

E

318,321

Plain-llanked Rail

Breeding:

Venezuela [969]

Sarothrura ayresi (Gumey 1877)

Id

$285,318,324,1158,1298,1411$

White-winged Fluffail, White-winged Crake

Breeding:

Ethiopia (ex?) [590]; South Africa $[1529,1530]$

Vagrant:

Zambia; Zimbabwe $[672,673]$ 
Sarothrura watersi (Bartlett 1880)

ld

$318,324,1158$

Waters's Flufftail, Slender-hilled Flufftail, Waters's Crake

Breeding:

Madagascar [373]

Family HELIORNITHIDAE

Finfoots, Sun-grebes

Heliopais personata (Gray 1849)

$K^{*} \quad 10,318$

Masked Finfoot, Asian Finfoot

Breeding:

Bangladesh; ?Cambodia; India; Myanmar [1296]; ?Thailand; ?Viet Nam

Non-breeding:

Cambodia; Indonesia: Java [977], Sumatra; Malaysia: Peninsular Malaysia; Thailand; Viet Nam

Family RHYNOCHETIDAE

Kagu

Rhynochetos jubatus Verreaux \& Des Murs 1860 I $\quad$ E $\quad 207,318,794$

Kagu

Breeding: New Caledonia

Family OTIDIDAE

Bustards

Ardeotis arabs (Linnaeus 1758)

II

$345,661,882,1399,1411$

(= Choriotis arabs)

Arabian Bustard

Breeding:

Burkina Faso; Cameroon; Chad; Djibouti [1475]; Ethiopia; Ghana; Mali; Mauritania; Morocco (ex?); Niger [279]; Saudi Arabia [1260]; Senegal; Sudan; Yemen [1136]

Non-breeding:

Nigeria

Vagrant:

Algeria; Côte d'Ivoire; Gambia; ?Kenya [1399]; Somalia

Ardeotis australis (Gray 1829)

II

581,1487

(= Choriotis australis)

Australian Bustard

Breeding:

Australia [167]; Indonesia: Irian Jaya; Papua New Guinea

Ardeotis kori (Burchell 1822)

II $\quad$ - $\quad 882,883,885,1411$

(= Choriotis kori)

Kori Bustard

Breeding:

Angola; Botswana; Ethiopia; Kenya; Mozambique; Namibia; Somalia [101]; South Africa [216]; Sudan; Swaziland (ex?); Tanzania [990]; Uganda; Zambia; Zimbabwe [1180]

Ardeotis nigriceps (Vigors 1831)

I V

$10,318,794,806,1130,1131,1414$

(= Choriotis nigriceps)

Indian Bustard, Great Indian Bustard

Breeding:

India; ?Pakistan

Non-breeding:

Pakistan

Vagrant:

?Nepal [700]

Chlamydotis undulata (Jacquin 1784)

$1 \quad K^{*}$

$10,303,318,661,1411$

Houbara Bustard

Breeding:

?Afghanistan; Algeria [1288]; Armenia (ex); Canary 1slands [261,322,1044]; China; Egypt [1206]; Iran [901]; Iraq; Israel; Jordan; Kazakhstan; Kuwait (ex); Kyrgyzstan; Libya; Mauritania; Mongolia; Morocco [559]; Oman; Pakistan [971]; Russia [799]; Saudi Arabia; Sudan; Syria; Tajikistan; Tunisia; Turkmenia; Uzbekistan; Western Sahara; Yemen

Non-breeding: Afghanistan; Bahrain; India; Kuwait; Qatar; United Arab Emirates 
Vagrant: $\quad$ Belgium; Cyprus; Czechoslovakia; Denmark; Finland; France; Germany; Greece; India; Italy; Latvia; Lebanon; Malta; Netherlands; Poland; Portugal; Romania; Sweden; Switzerland; United Kingdom; Yugoslavia
Eupodotis afra (Linnaeus 1766)
II
$883,885,1411$
(= Afrotis atra)
Black Bustard, Black Korhaan
Breeding:
South Africa
Eupodotis afraoides (Smith 1831)
11
$138,883,885,1411$
(=E. afra afraoides)

White-quilled Bustard

Breeding:

Angola; Botswana; Lesotho; Namibia; South Africa

Eupodotis bengalensis (Gmelin 1789) $\quad 1 \quad$ E $\quad 9,10,318,696,697$

(= Houbaropsis bengalensis)

Bengal Florican

Breeding:

Bangladesh (ex) [766]; ?Bhutan; ?Cambodia; India [698]; Nepal [698,700]; Viet Nam

Eupodotis caerulescens (Vieillot 1820)

11

$883,885,1411$

Blue Bustard, Blue Korhaan

Breeding: Lesotho; South Africa

Eupodotis gindiana Oustalet 1881

11

$882,1298,1411$

$(=E$. ruficrista gindiana)

Buff-crested Bustard

Breeding: $\quad$ Djibouti; Ethiopia; Kenya; Somalia; Sudan; Tanzania

Eupodotis hartlaubii (Heuglin 1863) II $\quad-\quad 882,1411$

(= Lissotis hartlaubii)

Hartlaub's Bustard

Breeding:

Ethiopia; Kenya; Somalia; Sudan; Tanzania; Uganda

Eupodotis humilis (Blyth 1856)

II

882,1411

Little Brown Bustard

Breeding:

Ethiopia [93]; Somalia [85]

Eupodotis indica (Miller 1782)

Il E

$9,10,318,560,1214,1215$

( $=$ Sypheotides indica)

Lesser Florican, Likh

Breeding: $\quad$ India [892,1218]; ?Nepal [700]

Non-breeding: Bangladesh; Nepal [700]; Pakistan

Eupodotis melanogaster (Rüppell 1835)

II

$882,883,885,1411$

(= Lissotis melanogaster)

Black-bellied Bustard, Black-hellied Korhaan

Breeding:

Angola; Benin; ?Botswana; Burkina Faso [1388]; Burundi; Cameroon; Central African Republic; Chad; Congo; Côte d'Ivoire; Ethiopia; ?Gabon; Gambia; Ghana; Guinea [1454]; Guinea-Bissau; Kenya; Malawi; Mali; Mozambique; ?Namibia; Niger [279]; Nigeria; Rwanda; Senegal; ?Sierra Leone; Somalia; South Africa; Sudan; Swaziland; Tanzania; Togo; Uganda; Zaire; Zambia; Zimbabwe 
Angola; Botswana; Mozambique; Namibia; South Africa; Swaziland; Zambia; Zimbabwe

Eupodotis savilei (Lynes 1920)

II

1411

(= Lophotis savilei)

Savile's Bustard

Breeding:

Chad; Mali [819]; Mauritania; Niger; ?Nigeria; Senegal; Sudan

\section{Eupodotis senegalensis (Vieillot 1820) \\ 11 \\ $882,885,1411$}

White-bellied Bustard, Senegal Bustard, White-bellied Korhaan

Breeding:

Angola; Benin; Botswana; Burkina Faso; Cameroon; Central African Republic [259]; Chad; Congo; Côte d'lvoire; Djibouti; Ethiopia; Gabon; ?Gambia [556]; ?Ghana; Guinea [984]; Kenya; Lesotho; Mali; Mauritania; ?Niger; Nigeria; Senegal; Somalia; South Africa; Sudan; Swaziland; Tanzania; Togo; ?Uganda; Zaire; Zambia

Eupodotis vigorsii (Smith 1831)

I1

$883,885,1411$

Karoo Bustard, Vigors's Bustard, Karoo Korhaan

Breeding:

Lesotho; Namibia [1439]; South Africa

Neotis denhami (Children 1826)

11

$345,882,883,885,1411$

(= Neotis cafra)

(includes N. burchellii (Heuglin 1867))

Stanley Bustard, Denham's Bustard

Breeding:

Angola; Benin; Botswana; Burkina Faso [1388]; Burundi; Cameroon; Central African Republic; Chad; Congo; Côte d'Ivoire; Ethiopia; Ghana; Guinea [984]; ?Guinea-Bissau; Kenya [1400,1403]; Malawi [1521]; Mali; ?Mauritania; Mozambique; ?Namibia; Niger; Nigeria; Rwanda; ?Senegal; ?Sierra Leone; South Africa; Sudan; Swaziland; Tanzania; Togo; Uganda; Zaire; Zambia; Zimbabwe [678]

Non-breeding: Lesotho; Senegal

Vagrant: Gambia

Neotis heuglinii (Hartlaub 1859)

II

$85,882,1411$

Heuglin's Bustard

Breeding:

?Djibouti; Ethiopia; Kenya; Somalia

Neotis ludwigii (Rüppell 1837)

II

$883,885,1411$

Ludwig's Bustard

Breeding: $\quad$ Angola; Namibia; South Africa [216]

Vagrant: Lesotho

Neoris nuba (Cretzschmar 1826)

I1

$345,882,1411$

Nubian Bustard

Breeding:

Chad; Mali; Mauritania; Niger [1015]; Sudan

Vagrant:

?Nigeria

Otis tarda Linnaeus 1758

$11 \quad \mathrm{R}$

$10,307,315,318,345,661,1411$

Great Bustard

Breeding:

Algeria (ex); Austria; Bulgaria (ex); China [798]; Czechoslovakia; Denmark (ex); France (ex); Germany; Greece (ex); Hungary [456]; Iran; Iraq (ex); Kazakhstan; Kyrgyzstan; Moldova; Mongolia [798]; Morocco; Poland (ex) [149]; Portugal [238]; Romania; Russia [798,799]; Spain [456,1048]; Sweden (ex); Switzerland (ex); ?Syria; Tajikistan; Turkey [562,768]; Ukraine; United Kingdom (ex); Uzbekistan; ?Yugoslavia

Non-breeding: Afghanistan; Azerbaijan; Bulgaria; 1raq; Syria; Turkmenia; Yugoslavia 
Vagrant: $\quad$ Albania; Algeria; Belgium; Cyprus; Denmark; Finland; France; Gibraltar; Greece; Ireland; Israel; Italy; Japan; Jersey; D.P.R. Korea; Korea Republic; Latvia; Lebanon; Luxembourg; Malta; Myanmar; Netherlands; Pakistan; Saudi Arabia; Sweden; Switzerland; Tunisia; United Kingdom
Tetrax tetrax (Linnaeus 1758)
II $\quad$ R
$10,318,345,1238,14 \mathrm{I} 1$

(= Otis tetrax)

Little Bustard

Breeding:

?Albania; Algeria; Bulgaria (ex); China; Czechoslovakia (ex); France [21]; Greece (ex); Iran; ?Iraq; Italy [1090,1220]; Kazakhstan; Kyrgyzstan; Morocco; Portugal; Romania (ex); Russia [799]; Spain; Tunisia (ex); Turkey [768]; Ukraine; ?Uzbekistan; Yugoslavia (ex)

Non-breeding: Azerbaijan; Egypt; Georgia; Israel; Libya; Pakistan; Romania; Tajikistan; Tunisia; Turkmenia; Yugoslavia

Vagrant: $\quad$ Afghanistan; Austria; Belgium; Cyprus; Czechoslovakia; Denmark; Finland; Germany; Greece; Guernsey; Hungary; India; Iraq; Ireland; Japan; Jersey; Latvia; Lebanon; Luxembourg; Malta; Netherlands; Norway; Poland; Sweden; Switzerland; Syria; United Kingdom

\section{Order CHARADRIIFORMES \\ Waders, Gulls and allies \\ Family HAEMATOPODIDAE \\ Oystercatchers}

Haematopus moquini meadewaldoi Bannerman 1913 - Ex

$318,324,967$

(= $\mathrm{H}$. meadewaldoi)

Canary Oystercatcher, Canarian Black Oystercatcher

Breeding: Canary Islands (ex) [658]

Haematopus unicolor chathamensis Hartert 1927

E

$318,794,976$

(= H. chathamensis)

Chatham Oystercatcher

Breeding: New Zealand: Chatham Islands [1507]

\section{Family RECURVIROSTRIDAE}

Arocets and Stilts

Himantopus novaezelandiae Gould $1841 \quad$ - $\quad$ E $\quad 319,794,976,1099$

Black Stilt

Breeding: New Zealand: South Island [1507]

Non-breeding: New Zealand: North Island

Family BURHINIDAE

Thick-knees

Burhinus bistriatus (Wagler 1829)

III GT - $\quad 16,967$

Double-striped Thick-knee

Breeding: $\quad$ Brazil; Colombia; Costa Rica; Dominican Republic; El Salvador; Guatemala; Guyana; Haiti; Honduras; Mexico; Nicaragua; Venezuela

Vagrant: $\quad$ Barbados; Netherlands Antilles: Curaçao; USA 
Family GLAREOLIDAE

Pratincoles, Coursers

Rhinoptilus bitorquatus (Blyth 1848)

$\mathrm{K}^{*}$

$158,159,318,794$

( $=$ Cursorius bitorquatus)

Jerdon's Courser

Breeding:

India [157]

Family CHARADRIIDAE

Plovers

Charadrius melodus Ord 1824

V/R

$16,179,318,321,420,595,596,868$

Piping Plover

Breeding:

Canada [937]; USA

Non-breeding:

Bahamas; Barbados; Bermuda; British Virgin 1slands; Cuba; Dominican Republic; Haiti; Mexico; Puerto Rico; Virgin Islands of the United States

$\begin{array}{llll}\text { Charadrius novaeseelandiae Gmelin } 1789 & \text { - } & \text { E } & 318,794,976\end{array}$

(= Thinornis novaeseelandiae)

Shore Plover, New Zealand Shore Plover

Breeding: $\quad$ New Zealand (ex): Rangatira lsland [1507]

$\begin{array}{llll}\text { Charadrius rubricollis } \text { Gmelin } 1789 & - & K^{*} & 318\end{array}$

Hooded Plover

Breeding: Australia [167]

Charadrius sanctaehelenae (Harting 1873)

$\mathbf{R}$

$8,318,324$

Saint Helena Plover, Wirebird

Breeding: $\quad$ Saint Helena

Charadrius thoracicus (Richmond 1896)

R $\quad 318,324$

Black-banded Plover, Madagascar Plover

Breeding: Madagascar [373]

Vanellus gregarius (Pallas 1771)

$\mathrm{K}^{*}$

$318,346,661,1411$

(= Chettusia gregaria)

Sociable Lapwing, Sociable Plover

Breeding:

Kazakhstan [185]; Russia [185,799]

Non-breeding:

Ethiopia; India; Iran; Iraq; Israel; Kyrgyzstan; Oman; Pakistan; Saudi Arabia; Sudan; Tajikistan; Turkey; Turkmenia; Uzbekistan; Yemen

Vagrant: $\quad$ Afghanistan; Belgium; Czechoslovakia; Egypt [1263]; France; Germany; Greece; Hungary; lreland; Israel; Italy; Jordan; Kuwait; Lebanon; Malta; Morocco [534]; Netherlands; Poland; Portugal; Romania; Somalia; Spain; Switzerland; United Arab Emirates; United Kingdom

Vanellus macropterus (Wagler 1827)

ld

318,794

(= Hoplopterus macropterus, Xiphidiopterus tricolor (Horsfield 1821))

Javanese Lapwing, Sunda Plover

Breeding:

Indonesia: Java (ex?)

Vagrant:

Indonesia: ?Sumatra, ?Timor 
Family SCOLOPACIDAE

Sandpipers and allies

Calidris paramelanotos S.A. Parker 1982

$\mathrm{K}^{*}$

$318,635,1318$

(= ?bybrid)

Cox's Sandpiper

Breeding:

Vagrant:

not known, possibly Russia

(The specific status of these birds is currently under debate $[235,1235])$

Coenocorypha aucklandica (Gray 1845)

$\mathrm{R}$

$318,794,976$

(includes C. pusilla (Buller 1869))

Subantarctic Snipe, New Zealand Snipe, Chatham Islands Snipe

Breeding: New Zealand: Antipodes Island, Auckland 1slands, Chatham 1slands, Jacky Lee 1sland (ex), Snares 1sland, South Cape Islands (ex)

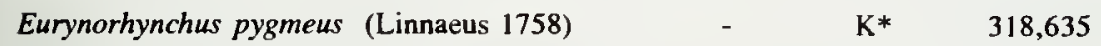

Spoonbill Sandpiper

Breeding: Russia [799]

Non-breeding: Bangladesh; China; Hong Kong; India; Japan; D.P.R. Korea; Korea Republic; Singapore Vagrant: $\quad$ Canada; Sri Lanka; Thailand [1348]; USA

$\begin{array}{llll}\text { Gallinago nemoricola Hodgson } 1836 & - & K^{*} & 318,635\end{array}$

Wood Snipe

Breeding: Bhutan; China; lndia; Nepal

Non-breeding: Pakistan

Vagrant: $\quad$ Bangladesh; Lao P.D.R; Myanmar; Singapore [527]; Thailand; Viet Nam

Limnodromus semipalmatus (Blyth 1848) $\quad$ - $\quad$ R $\quad 10,120,318,661,792,794,848$,

Asian Dowitcher, Asiatic Dowitcher

Breeding: China; Mongolia [466]; Russia [799, 1548]

Non-breeding: Australia [827]; Bangladesh; Brunei; Cambodia; Hong Kong; India; Indonesia: Java, Kalimantan [1295], Sumatra [1279]; Malaysia: Peninsular Malaysia, Sabah [1295], Sarawak [1295]; Myanmar; Philippines: Cebu, Luzon, Olargo, Simunul; Singapore; Thailand [952,1193]; Viet Nam

Vagrant: Indonesia: Sulawesi [108], Timor [25]; Japan; Kenya; Sri Lanka; Taiwan; United Arab Emirates; ?Yemen

Numenius borealis (Forster 1772)

$1 \quad \mathrm{E} / \mathrm{Ex} \quad 16,179,304,318,321,420,453$,

Eskimo Curlew

Breeding:

Non-breeding:

Vagrant:

?Alaska; Canada

Brazil; French Guiana; Guyana; Paraguay; Suriname

Argentina [154]; Barbados; Bermuda; Brazil; Chile; Falkland 1slands; Greenland; Grenada; Guadeloupe; Guatemala; Iceland; 1reland; Mexico; Paraguay; Puerto Rico; Saint Vincent; Trinidad and Tobago; USA; Russia; United Kingdom; Uruguay

Numenius tahitiensis (Gmelin 1789)

$\mathrm{V} / \mathrm{R} \quad 16,318,321,529,1118$

Bristle-thighed Curlew

Breeding: Alaska [530]

Non-breeding: $\quad$ American Samoa; Fiji; French Polynesia: Marquesas lslands, Tuamotu Archipelago [531];

Tonga; Hawaiian Islands; Marshall Islands; Western Samoa

Vagrant: Japan; New Zealand: Kermadec Islands; Philippines 
Slender-billed Curlew

Breeding:

Russia [799]

Non-breeding:

Greece; Hungary; Italy; Morocco [175]; Romania; Tunisia; Turkey; Yugoslavia

Vagrant:

Algeria; Austria; Azores; Belgium; Bulgaria; Canada; Cyprus; Czechoslovakia; Egypt; France; Germany; Iran; Iraq; Israel; Italy; ?Japan; Jordan; Kuwait; Latvia; Libya; Malta; Netherlands; Oman; Poland; Seychelles: Aldabra; Spain; Switzerland; Yemen

Prosobonia cancellatus (Gmelin 1789)

V

$318,631,669,794$

Tuamotu Sandpiper, Sharp-billed Sandpiper

Breeding: $\quad$ Kiribati: Kiritimati (ex) [1118]; French Polynesia: Tuamoto Archipelago [1118]

Scolopax celebensis Riley 1921

K* $\quad 318,1486$

Sulawesi Woodcock

Breeding:

lndonesia: Sulawesi [1486]

Scolopax rochussenii Schlegel 1866

$K^{*} \quad 318,1486$

Moluccan Woodcock, Obi Woodcock

Breeding:

Indonesia: Bacan, ?Halmahera, Obi, ?Ternate [1486]

Tringa guttifer (Nordmann 1835)

I Id

$10,318,680,792,794$

Nordmann's Greenshank, Spotted Greenshank

Brecding:

Russia [ ] 85,679,799]

Non-breeding:

Bangladesh; ?Cambodia; China; Japan; D.P.R. Korea; Korea Republic; ?Lao P.D.R; Malaysia: Peninsular Malaysia; Myanmar; Philippines: Cebu, Luzon; Thailand

Vagrant: Australia; Guam; Hong Kong [779]; India; Indonesia: Sumatra; Malaysia: Sabah, Sarawak [1295]; Singapore; Taiwan

Family LARIDAE

Gulls and Terns

Chlidonias albostriatus (Gray 1845)

K* $\quad 283,318,618,1176$

(= Chlidonias hybridus albostriatus, Sterna albostriata)

Black-fronted Tern

Breeding: New Zealand: South Island

Larus atlanticus Olrog 1958

K

$318,321,388$

(= L. belcheri atlanticus)

Olrog's Gull

Breeding:

Argentina [154]

Non-breeding:

Brazil [1443]; Unuguay

Larus audouinii Payraudeau 1826

R

$227,318,346,618,661,670,716$, $758,794,1411$

Audouin's Gull

Breeding:

Non-breeding:

Vagrant:

Algeria [711]; Corsica; Cyprus; Greece; Morocco [126]; Sardinia; Spain; Tunisia; Turkey Gibraltar; Libya; Western Sahara

Canary Islands; Egypt; Gambia; Georgia; Israel; Jordan; Lebanon; MaIta; Mauritania; Portugal; Senegal; Switzerland

Larus leucophthalmus Temminck 1825 

Larus relictus Lōnnberg 1931
l $\quad \mathrm{R}$
$318,618,794,795,1559$

\section{Relict Gull}

Breeding:

Non-breeding:

China; Kazakhstan [115]; Mongolia [467]; Russia [799]

Vagrant:

Korea Republic

Hong Kong; Japan; Viet Nam

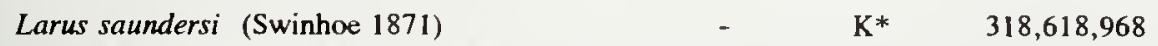

Saunders's Gull, Chinese Black-headed Gull

Breeding: China

Non-breeding: $\quad$ Hong Kong; Japan; ?D.P.R. Korea; ?Korea Republic; Taiwan; Viet Nam

Sterna balaenarum (Strickland 1852) $\quad$ - $\quad$ R $\quad 285,318,324,618,1247$

Damara Tern

Breeding: $\quad$ ?Angola; Namibia; South Africa

Non-breeding: Angola; Cameroon; Gabon; Ghana; Nigeria; ?Zaire

Sterna bernsteini Schlegel 1863

Id $\quad 318,618,792,794$

(= Thalasseus zimmermanni (Reichenow 1903))

Chinese Crested Tern

Breeding:

?China [968]

Vagrant:

Indonesia: Halmahera [1486]; Malaysia: Sarawak [1295]; Philippines [398]; Thailand [598]

Sterna virgata Cabanis 1875

K* $\quad 318,618,1504$

Kerguelen Tern

Breeding:

French Southern and Antarctic Territories: Crozet Islands, Kerguelen Islands; Prince Edward Island (South Africa)

Family ALCIDAE

Auks, Murres, Puffins and allies

Synthliboramphus wumizusume (Termminck 1835) - $\quad K^{*} \quad 318,383,618$

Japanese Murrelet, Crested Murrelet

Breeding: Japan: mainland, lzu Islands

Non-breeding: D.P.R. Korea; Korea Republic; Russia [799]

\section{Order COLUMBIFORMES}

Pigeons and Sandgrouse

Family COLUMBIDAE

Pigeons and Doves

Caloenas nicobarica (Linnaeus 1758)

Nicobar Pigeon

Breeding:

Cambodia; India: Andaman and Nicohar Islands: South Sentinal; Indonesia; Malaysia: Peninsular Malaysia, Sabah [1295], Sarawak [1295]; Myanmar; Palau; Papua New Guinea; Philippines; Solomon Islands; Thailand; Viet Nam 
Silvery Wood-Pigeon, Grey Wood-Pigeon

Breeding:

Indonesia: Anamba Islands (ex?), Kalimantan (ex?) [1295], Karimata Islands (ex?) [1295], Lingga Archipelago (ex?) [905], Mentawai 1slands (ex?) [905], Natuna Islands (ex?) [1295], Riau Archipelago (ex?) [905], Simeulue (ex?) [905], Sumatra; Malaysia: Burong Island (ex) [1295]

Columba bollii Godman 1872

$\mathbf{R}$

$318,324,342,554,909$

Bolle's Pigeon, Dark-tailed Laurel Pigeon, Bolle's Laurel Pigeon

Breeding:

Canary lslands [683,910,1088]

Columba caribaea Jacquin 1784

V

$318,321,365,554,1415$

Ring-tailed Pigeon

Breeding:

Jamaica [179]

Columba elphinstonii (Sykes 1833)

$-\quad K^{*}$

K* $\quad 318,554$

Nilgiri Wood-Pigeon

Breeding:

India [10]

Columba guinea Linnaeus 1758

Ill $\mathrm{GH}$

$212,285,554,1196,1247,1298,1411$

Speckled Pigeon, Triangular-spotted Pigeon, Speckled Rock Pigeon

Breeding:

Angola; Benin; Botswana; Burkina Faso [1388]; Burundi; Cameroon; Central African Republic; Chad; Côte d'lvoire; Djibouti; Ethiopia [748]; Gambia; Ghana; Guinea [984]; Guinea-Bissau; Kenya; Lesotho; Mali; Malawi; Mauritania; Namibia; Niger; Nigeria; Rwanda; Senegal; Sierra Leone; Somalia; South Africa; Sudan; Swaziland; Tanzania; Togo [275]; Uganda; Zaire; Zimbabwe

Columba inornata Vigors 1827

ld

321,1085

Plain Pigeon

Breeding:

Cuba; Dominican Republic; Haiti; Jamaica [1415]; Puerto Rico [1086]

Columba iriditorques Cassin 1856

$111 \mathrm{GH}$

$554,1247,1298,1411$

Western Bronze-naped Pigeon

Breeding:

Angola; Benin; Cameroon; ?Central African Republic; Congo; Côte d'1voire; Equatorial Guinea; Gabon; Ghana; Guinea [1454]; Liberia; ?Nigeria; Rwanda; Sierra Leone; Sudan; Togo [280]; Uganda; Zaire; Zambia

Columba junoniae Hartert 1916

Laurel Pigeon, White-tailed Laurel Pigeon

Breeding:

Canary 1slands [910]
Columba livia Gmelin 1789

III $\mathrm{GH}$

$318,324,342,554,683$

Rock Pigeon, Rock Dove

Breeding:
Afghanistan; Albania; Algeria; [Angola] [216]; Armenia; [Austria]; Azerbaijan; Azores; Bangladesh; Belarus; Bhutan; [Brunei]; Bulgaria; Canary Islands; Cape Verde; Chad; China; [Costa Rica]; Côte d'Ivoire; Cyprus; Egypt; Estonia; Ethiopia; Faeroe Islands; Federated States of Micronesia [1118]; Fiji [1118]; France; [French Polynesia]; Gambia; Georgia; Ghana; [Gibraltar]; [Guatemala] [127]; Greece; [Guernsey]; Guinea; Hawaiian Islands; Iceland; India; [Indonesia: Bali] [915], Irian Jaya, Kalimantan [664], ?Sumatra [26]; 1ran; Iraq; 1reland; Israel; Italy; Jordan; Kazakhstan; Kyrgyzstan; Latvia; Lebanon; [Lesotho]; Libya; [Liechtenstein]; Lithuania; Madeira; [Malaysia: Peninsular]; Mali; Malta; Mauritania; Moldova; Mongolia; Morocco; [Mozambique] [216]; [Namibia]; Nepal; Niger; Nigeria; Norway; Oman; Pakistan; Portugal; Romania; Russia; Sao Tome and Principe: Sao Tome [417]; Saudi Arabia; Senegal; [Seychelles: Aldabra] [493]; [Singapore]; [South Africa] [216,286]; Spain; Sri Lanka; Sudan; [Swaziland]; Sweden; [Switzerland]; Syria; 
Tajikistan; Togo; Tunisia; Turkey; Turkmenia; Ukraine; United Arab Emirates; United Kingdom; Uzbekistan; Western Sahara; Yemen; Yugoslavia; [Zambia] [216]; [Zimbabwe]

Vagrant: [216]

Jersey

Columba mayeri Prévost 1843

III MU E

$318,324,554$

$(=$ Nesoenas mayeri)

Pink Pigeon

Breeding:

Mauritius

Columba oenops Salvin 1895

V

321

Peruvian Pigeon

Breeding:

Peru

Columba oliviae Clarke 1918

R

$318,324,554,1298,1411$

Somali Pigeon, Somali Stock Dove

Breeding: Somalia [85]

Columba pallidiceps (Ramsay 1877)

$\mathrm{K}^{*} \quad 318,554$

Yellow-legged Pigeon

Breeding:

Papua New Guinea: Bismarck Archipelago; Solomon Islands (ex?)

Columba punicea Blyth 1842

$K^{*} \quad 318,554,792$

Pale-capped Pigeon, Purple Wood-Pigeon

Breeding: $\quad$ China; India; Lao P.D.R; Myanmar; Thailand; Viet Nam

Columba thomensis Barboza du Bocage $1888 \quad-\quad K^{*} \quad 318,324,554,1298,1411$

Maroon Pigeon, Sao Tome Olive Pigeon

Breeding: Sao Tome and Principe: Sao Tome [755]

Columba torringtoni Ponaparte 1854

$\mathrm{K}^{*} \quad 318,554$

Ceylon Wood-Pigeon

Breeding: $\quad$ Sri Lanka $[10,659]$

Columba trocaz Heineken 1829

- $\quad$ R $\quad 318,324,342,554,1557$

Trocaz Pigeon, Madeira Laurel Pigeon

Breeding: Madeira

Columba unicincta Cassin 1860

$111 \mathrm{GH}$

$212,554,1247,1298,1411$

Afep Pigeon, African Wood-Pigeon, Grey Wood-Pigeon

Breeding:

Angola; Cameroon; Central African Republic; Congo; Côte d'Ivoire; Equatorial Guinea;

Gabon; Ghana; Guinea [984]; Liberia; Sudan; Tanzania; Uganda; Zaire; Zambia

Columbina cyanopis (Pelzeln 1870)

$\mathrm{E}$

$318,321,554,1103$

Blue-eyed Ground-Dove

Breeding:

Brazil

Didunculus strigirostris (Jardine 1845)

V

$134,135,318,554,794$

Tooth-billed Pigeon

Breeding:

Western Samoa: Savaii, Upolu [1118]

Drepanoptila holosericea (Temminck 1810)

V

$318,554,794$

Cloven-feathered Dove

Breeding:

New Caledonia 
Ducula aurorae (Peale 1848)

V

$318,554,669,794$

Polynesian Imperial-Pigeon, Society Islands Pigeon

Breeding:

French Polynesia: Tahiti, Makatea [1 118 ]

Ducula galeata (Bonaparte 1855)

E

$318,554,669,794$

Marquesan Imperial-Pigeon, Nukuhiva Imperial-Pigeon

Breeding:

French Polynesia: Nukuhiva [1118]

Ducula goliath (Gray 1859)

V

$318,554,794$

New Caledonian Imperial-Pigeon, Giant Imperial-Pigeon

Breeding:

New Caledonia

Ducula mindorensis (Whitehead 1896)

1

K*

318,554

Mindoro Imperial-Pigeon

Breeding:

Philippines: Mindoro

Ducula pickeringii (Cassin 1854)

K* $\quad 318,554,1486$

Grey Imperial-Pigeon

Breeding:

Indonesia: Miangas, Talaud [1486]; Malaysia: Sabah [1295]; Philippines

Ducula whartoni (Sharpe 1887)

V

$318,554,652,794$

Christmas Imperial-Pigeon

Breeding: $\quad$ Christmas Island; [Cocos (Keeling) Islands (ex)]

Gallicolumba erythroptera (Gmelin 1789)

ld $\quad 318,554,669,794$

Polynesian Ground-Dove, Society Islands Ground-Dove, White-collared Ground-Dove

Breeding:

French Polynesia: Society lslands (Moorea (ex), Tahiti (ex), Tuamoto Archipelago [1118]

Gallicolumba hoedtii (Schlegel 1871)

$\mathrm{K}^{*} \quad 318,554,1486$

Wetar Ground-Dove

Breeding:

Indonesia: Timor, Wetar [1486]

Gallicolumba keayi (Clarke 1900)

$\mathrm{K}^{*} \quad 318,554$

Negros Bleeding-heart

Breeding: Philippines: Negros

Gallicolumba luzonica (Scopoli 1786)

11

554

Luzon Bleeding-heart

Breeding:

Philippines: Catanduanes, Luzon, Polillo [1113]

Gallicolumba menagei (Boums \& Worcester 1894)

K* $\quad 318,554$

Sulu Bleeding-heart, Tawitawi Bleeding-heart

Breeding: Philippines: Sulu Archipelago, Tawitawi

Gallicolumba platenae (Salvadori 1891)

K* $\quad 318,554$

Mindoro Bleeding-heart

Breeding:

Philippines: Mindoro

Gallicolumba rubescens (Vieillot 1818)

Id

$318,554,669,794$

Marquesan Ground-Dove

Breeding:

French Polynesia: Nukuhiva (ex), Hatutu, Fatuhuku [1 118 ]

Gallicolumba salamonis (Ramsay 1882)

$K^{*}$

$318,554,1226$

Thick-billed Ground-Dove

Breeding: Solomon Islands: Malaita, San Cristobal 
Gallicolumba santaecrucis Mayr 1935

$\mathrm{K}^{*} \quad 318,554$

Santa Cruz Ground-Dove

Breeding:

Solomon Islands: Tinakula, Utupua; Vanuatu: Espiritu Santo

Goura cristata (Pallas 1764)

II $\quad \mathrm{K}^{*}$

$318,554,1226$

Western Crowned-Pigeon, Blue Crowned-Pigeon

Breeding:

Indonesia: Batanta, Irian Jaya, Misool, Salawati, Waigeo [131]

Goura scheepmakeri Finsch 1876

II $\quad \mathrm{K}^{*}$

$131,318,554,1226$

Southern Crowned-Pigeon, Maroon-breasted Crowned-Pigeon, Scheepmaker's Crowned-Pigeon

Breeding:

Indonesia: Irian Jaya; Papua New Guinea
Goura victoria (Fraser 1844)
$11 \quad \mathrm{~K}^{*}$
$131,318,554,1226$

Victoria Crowned-Pigeon

Breeding:

Indonesia: Biak, 1rian Jaya, Yapen; Papua New Guinea

Leptotila conoveri Bond \& Meyer de Schauensee 1943

Tolima Dove

Breeding:

Colombia

$\mathrm{V} / \mathrm{R} \quad 318,321,554,794,967$

Leptotila ochraceiventris Chapman 1914

E

$318,321,554,967$

Ochre-bellied Dove

Breeding:

Ecuador [1166]; Peru

Leptotila wellsi (Lawrence 1884)

E

$172,174,318,321,554,794$

Grenada Dove

Breeding:

Grenada $[173,179]$

Microgoura meeki Rothschild 1904

Choiseul Pigeon, Solomon Islands Crowned-Pigeon

Ex $\quad 318,554,1226$

Breeding:

So'omon Islands: Choiseul (ex)

Oena capensis (Linnaeus 1766)

$111 \mathrm{GH}-$

$212,285,345,554,1196,1247$, 1298,1411

Namaqua Dove, Long-tailed Dove, Masked Dove

Breeding:

Algeria; Angola; Botswana; Burkina Faso [1388]; Burundi; Cameroon; Central African Republic; Chad; Djibouti; Ethiopia; ?Gambia; ?Ghana; 1srael; Jordan; Kenya; Lesotho; Madagascar; Malawi; Mali; Mauritania; Morocco; Mozambique; Namibia; Niger; Nigeria; Oman; Rwanda; Saudi Arabia; Senegal; Somalia; South Africa; Sudan; Swaziland; Tanzania; Uganda; Western Sahara; Yemen; Zaire; Zambia; Zimbabwe

Non-breeding: Bahrain; Benin; Congo; Côte d'lvoire; Gabon; Gambia; Ghana; Guinea [1454]; Guinea-Bissau; ?Sierra Leone; Togo

Vagrant: $\quad$ Egypt; Iraq; Kuwait [1101]; Qatar; United Arab Emirates

Ptilinopus arcanus Ripley \& Rabor 1955

$\mathrm{K}^{*} \quad 318,554$

Negros Fruit-Dove

Breeding:

Philippines: Negros

Ptilinopus dohertyi (Rothschild 1896)

$K^{*} \quad 318,554,1486$

Red-naped Fruit-Dove

Breeding:

Indonesia: Sumba [1486]

Prilinopus granulifrons Hartert 1898

$\mathrm{K}^{*} \quad 318,554,1486$

Carunculated Fruit-Dove

Breeding: Indonesia: Obi [1486] 
Ptilinopus huttoni Finsch 1874

R

$318,554,669,794$

Rapa Fruit-Dove

Breeding:

French Polynesia: Tubuai 1slands (Rapa)

Ptilinopus mercierii (Des Murs \& Prévost 1849)

$\mathrm{K}^{*}$

$318,554,669$

Red-moustached Fruit-Dove

Breeding: $\quad$ French Polynesia: ?FatuhivaHivaoa (ex?), Nukuhiva (ex), ?Tahuata

Ptilinopus roseicapilla (Lesson 1831)

V

$318,554,794$

Marianas Fruit-Dove

Breeding:

Guam [731]; Northem Marianas

Starnoenas cyanocephala (Linnaeus 1758)

Id

$318,321,554$

Blue-headed Quail-Dove

Breeding:

Cuba $[179,510,545,1183]$

Streptopelia decipiens (Hartlaub \& Finsch 1870) $\quad 111$ GH -

$212,285,554,1196,1247,1298,1411$

Mourning Collared-Dove, African Mourning Dove

Breeding:

Angola; Benin; Botswana; Burkina Faso [1388]; Burundi; Cameroon; Central African Republic; Chad; ?Djibouti; Ethiopia; Gambia; Ghana; Kenya; Malawi; Mali; Mauritania; Mozambique; Namibia; Niger; Nigeria; Rwanda; Senegal; Somalia; South Africa; Sudan; Tanzania; Togo; Uganda; Zaire; Zambia; Zimbabwe

Streptopelia roseogrisea (Sundevall 1857)

111 GH -

$554,661,1247,1298,1411$

African Collared-Dove, Pink-headed Dove, Rose-grey Dove

Breeding:

Benin; Cameroon; Chad; Djibouti; Egypt; Ethiopia; ?Gambia; Mali; Mauritania; Niger; ?Nigeria; Saudi Arabia; Senegal; Somalia; Sudan; Yemen

Vagrant: Ghana

Streptopelia semitorquata (Rüppell 1837)

$111 \mathrm{GH} \quad-$

$212,285,554,661,1196,1247$, 1298,1411

Red-eyed Dove

Breeding:

Angola; Benin; Botswana; Burkina Faso [1388]; Burundi; Cameroon; Central African Republic; Chad; Congo; Côte d'lvoire; Equatorial Guinea; Ethiopia; Gabon; Gambia; Ghana; Guinea [1454]; Guinea-Bissau; Kenya; Lesotho; Liberia; Malawi; Mali; Mauritania; Mozambique; Namibia; Niger; Nigeria; Rwanda; Saudi Arabia; Senegal; Sierra Leone; Somalia; South Africa; Sudan; Swaziland; Tanzania; Togo; Uganda; Yemen; Zaire; Zambia; Zimbabwe

Streptopelia senegalensis (Linnaeus 1766)

111 GH

$10,212,285,345,554,661,1196$, $1247,1298,1411$

Laughing Dove, Senegal Dove, Palm Dove

Breeding: $\quad$ Afghanistan; Algeria; Angola; [Australia); Bangladesh; Benin; Bhutan; Botswana; Burkina Faso [1388]; Burundi; Cameroon; Central African Republic; Chad; China; Côte d'Ivoire; Djibouti; Egypt; Equatorial Guinea; Ethiopia; ?Gabon; Gambia; Ghana; Guinea [1454]; Guinea-Bissau; India: including Andaman 1slands; 1ran; Israel; Jordan; Kazakhstan; Kenya; Kyrgyzstan; Lebanon; Lesotho; Liberia; Libya [925]; Malawi; Mali; Mauritania; Mozambique; Namibia; Nepal; Niger; Nigeria; Oman; Pakistan; Rwanda; Sao Tome and Principe: Sao Tome; Saudi Arabia; Senegal; Sierra Leone; Somalia; South Africa; Sudan; Swaziland; Syria; Tajikistan; Tanzania; Togo; Tunisia; Turkey; Turkmenia; Uganda; United Arab Emirates; Yemen; Zaire; Zambia; Zimbabwe

Vagrant: $\quad$ Bahrain; Cyprus; Finland; Greece; Iraq; Italy; Kuwait [1101]; Malta; Morocco 
Streptopelia turtur (Linnaeus 1758)

$111 \mathrm{GH}$

$212,345,554,661,987,1411$

European Turtle-Dove, Western Turtle-Dove, Turtle Dove

Breeding:

Afghanistan; Albania; Algeria; Andorra; Armenia; Austria; Azerbaijan; Bahrain; Belarus;

Belgium; Bulgaria; Canary Islands; Chad; China; Cyprus; Czechoslovakia; Denmark; Egypt; Estonia; Finland; France; Georgia; Germany; Greece; Guernsey; Hungary; 1ran; Iraq; Ireland; Israel; Italy; Jersey; Jordan; Kazakhstan; Kuwait; Kyrgyzstan; Latvia; Lebanon; Libya; Liechtenstein; Lithuania; Luxembourg; Madeira; Malta; Moldova; Mongolia; Morocco; Netherlands; Niger; Oman; Poland; Portugal; Qatar; Romania; Russia; Saudi Arabia; Spain; Sudan; Switzerland; Syria; Tajikistan; Tunisia; Turkey; Turkmenia; Ukraine; ?United Arab Emirates; United Kingdom; Uzbekistan; Yugoslavia

Non-breeding: Burkina Faso; Cameroon; Chad; Côte d'Ivoire; Ethiopia; Faeroes; Gambia; Ghana; Gibraltar; Guinea [985]; Guinea-Bissau; Libya; Mali; Mauritania; Niger; Nigeria; Senegal; Sweden; Togo; Western Sahara; Yemen

Vagrant: $\quad$ Azores; Cape Verde; Gabon; lceland; Kenya [592]; Maldives; Namibia [1526]; Norway; Pakistan; Seychelles: Aldabra [493]; Somalia; South Africa; Uganda; Zaire

Streptopelia vinacea (Gmelin 1789)

III GH

$212,554,1247,1298,1411$

Vinaceous Dove

Breeding:

Benin; Burkina Faso [1388]; Cameroon; Central African Repuolic; Chad; Côte d'Ivoire; Ethiopia; Equatorial Guinea; Gambia; Ghana; Guinea [1454]; Guinea-Bissau; Liberia; Mali; Mauritania; Niger; Nigeria; Senegal; Sierra Leone; Sudan; Togo; Uganda; Zaire

Treron calva (Temminck 1808)

III GH -

$212,285,554,1196,1247,1298,1411$

$(=$ Treron australis calva)

African Green-Pigeon

Breeding:

Angola; Benin; Botswana; Burkina Faso; Burundi; Cameroon; Central African Republic; Chad; Congo; Côte d'lvoire; Ethiopia; Equatorial Guinea: continental, Bioko; Ethiopia; Gahon; Gambia; Ghana; Guinea [1454]; Guinea-Bissau; Kenya; Liberia; Malawi; Mali; Mozambique; Namibia; Niger; Nigeria; Rwanda; Sao Tome and Principe: Principe; Senegal; Sierra Leone; South Africa; Sudan; Swaziland; Tanzania; Togo; Uganda; Zaire; Zambia; Zimbabwe

Treron capellei (Temminck 1823)

$\mathrm{K}^{*}$

318,554

Large Green-Pigeon

Breeding:

Brunei; Indonesia: Java, Kalimantan [664], Sumatra; Malaysia: Peninsular, Sabah, Sarawak [1295]; Myanmar; Thailand [1194]

Treron psittacea (Temminck 1808)

$\mathrm{K}^{*}$

$318,554,1486$

Timor Green-Pigeon

Breeding:

Indonesia: Roti, Seniau, Timor [1486]

Treron teysmannii Schlegel 1879

$K^{*} \quad 318,554,1486$

Sumba Green-Pigeon

Breeding:

Indonesia: Sumba [1486]

Treron waalia (Meyer 1793)

$111 \mathrm{GH}$

$212,554,1247,1298,1411$

Bruce's Green-Pigeon, Yellow-hellied Green-Pigeon

Breeding:

Benin; Burkina Faso [1388]; Cameroon; Central African Republic; Chad; Côte d'Ivoire; Djibouti; Ethiopia; Gambia; Ghana; Guinea [1454]; Guinea-Bissau; Kenya; Mali; ?Mauritania; Niger; Nigeria; ?Oman; Saudi Arabia; Senegal; Somalia; Sudan; Togo; Uganda; Yemen; Zaire 
Black-hilled Wood-Dove

Breeding:

Benin; Burkina Faso [1388]; Cameroon; Central African Republic; Chad; Côte d'Ivoire;

Djibouti; Ethiopia; Gambia; Ghana; Guinea [1454]; Guinea-Bissau; ?Kenya; Mali; Mauritania; Niger; Nigeria; Senegal; Sudan; Togo; Uganda

Vagrant: Liberia

Turtur afer (Linnaeus 1766)

III GH

$212,285,554,1247,1298,1411$

Blue-spotted Wood-Dove, Red-billed Wood-Dove

Breeding:

Angola; Benin; Burkina Faso [1388]; Burundi; Cameroon; ?Central African Republic; Chad; Congo; Côte d'lvoire; Equatorial Guinea; Ethiopia [748]; Gabon; Gambia; Ghana; Guinea; Guinea-Bissau; Kenya; Liberia; Malawi; Mali; Mozambique; Niger; Nigeria; Rwanda; Senegal; Sierra Leone; South Africa; Sudan; Tanzania; Togo; Uganda; Zaire; Zambia; Zimbabwe

Turtur brehmeri (Hartlaub 1875)

$111 \mathrm{GH}$

$554,1247,1298,1411$

Blue-headed Wood-Dove, Blue-headed Dove

Breeding:

Angola; Cameroon; Central African Republic; Congo; Côte d'Ivoire; Equatorial Guinea; Gabon; Ghana; Guinea; Liberia; Nigeria; Sierra Leone; Togo [280]; Zaire

Turtur tympanistria (Temminck 1810)

$111 \mathrm{GH}-$

$212,285,554,1196,1247,1298,1411$

Tambourine Dove

Bresding:

Angola; Benin; Burundi; Cameroon; Central African Republic; Comoros; Congo; Côte d'Ivoire; Ethiopia; Equatorial Guinea; Ethiopia; Gabon; Ghana; Guinea; Kenya; Liberia; Malawi; Mozambique; Nigeria; Rwanda; Sierra Leone; Somalia; South Africa; Sudan; Swaziland; Tanzania; Togo [280]; Uganda; Zaire; Zambia; Zimbabwe

Vagrant:

Senegal

Zenaida graysoni Lawrence 1871

E

$116,318,321,554,726,1072$

Socorro Dove

Breeding:

Mexico: Revillagigedos 1slands [729]

\section{Order PSITTACIFORMES}

Parrots and allies

Family PSITTACIDAE

Parrots, Lories, Cockatoos

Agapornis canus (Gmelin 1788)

$11 \quad-\quad 485,1298$

Grey-headed Lovebird, Madagascar Lovebird

Breeding:

[Comoros]; Madagascar [373]; [Mauritius (ex)]; [Mayotte]; [Réunion]; [Seychelles: Amirantes]; [South Africa (ex)]; [Tanzania (ex)]

Agapornis fischeri Reichenow 1887

Il

$212,485,496,1298$

Fischer's Lovebird

Breeding:

Burundi; [Kenya]; Rwanda; Tanzania

Agapornis lilianae Shelley 1894

II -

$285,485,496,1298$

$(=$ A. l. lilianae $)$

Lilian's Lovebird, Nyasa Lovebird

Breeding: Malawi; ?Mozambique; Tanzania: Zambia; Zimbabwe 
Agapornis nigrigenis Sclater 1906

Il $\quad$ R

$285,318,324,485,1298$

$(=A$. lilianae nigrigenis)

Black-cheeked Lovebird

Breeding: $\quad$ Botswana; ?Namibia; Zambia; Zimbabwe (ex?)

Agapornis personatus Reichenow 1887

II

$212,485,496,1298$

Yellow-collared Lovebird, Masked Lovebird, Black-masked Lovebird

Breeding:

Vagrant:

Tanzania

Kenya

Agapornis pullarius (Linnaeus 1758)

II

$212,485,496,1247,1298$

Red-headed Lovebird, Red-faced Lovebird

Breeding:

Angola; Benin; Burundi; Cameroon; Central African Republic; Chad; Congo; ?Côte d'lvoire; Equatorial Guinea: Bioko; Ethiopia; Gabon; Ghana; Guinea [984]; Kenya; Nigeria; Rwanda; Sao Tome and Principe: Sao Tome; Sierra Leone; Sudan; Tanzania; Togo; Uganda; Zaire

Agapornis roseicollis (Vieillot 1818)

II

$285,485,496,1247$

Rosy-faced Lovebird, Peach-faced Lovehird

Breeding:

Angola; ?Botswana; Namibia; South Africa

Agapornis swindernianus (Kuhl 1820)

II

$212,485,496,1247,1298$

Black-collared Lovebird

Breeding:

Cameroon; Central African Republic; Congo; ?Côte d'Ivoire; Gabon; Ghana; Liberia; Uganda; Zaire

Agapornis taranta (Stanley 1814)

II $\quad-\quad 485,496,1298$

Black-winged Lovebird, Ahyssinian Lovehird

Breeding: Ethiopia

Alisterus amboinensis (Linnaeus 1766)

II

485

Moluccan King-Parrot, Ambon King-Parrot

Breeding:

Indonesia: Ambon, Buru, Halmahera, Irian Jaya, Peleng, Seram, Sula Islands, Western Papuan Islands [1486]

Alisterus chloropterus (Ramsay 1879)

11

485

Papuan King-Parrot, Green-winged King-Parrot

Breeding: Indonesia: Irian Jaya [1486]; Papua New Guinea [1486]

Alisterus scapularis (Lichtenstein 1818)

II

485,1292

Australian King-Parrot

Breeding:

Australia [167]

Amazona aestiva (Linnaeus 1758)

11

$485,967,1152$

Blu-fronted Parrot, Turquoise-fronted Parrot, Blue-fronted Amazon

Breeding: Argentina [154]; Bolivia; Brazil; Paraguay

Amazona agilis (Linnaeus 1758)

I1

16,485

Black-billed Parrot, Black-billed Amazon

Breeding:

Jamaica [179]

Amazona albifrons (Sparrman 1788)

II

$16,485,1152$

White-fronted Parrot, White-fronted Amazon

Breeding:

Belize; Costa Rica; El Salvador; Guatemala; Honduras; Mexico; Nicaragua 
Amazona amazonica (Linnaeus 1766)

11

$485,967,1152$

Orange-winged Parrot, Orange-winged Amazon

Breeding:

Bolivia; Brazil; Colombia; Ecuador; French Guiana; Guyana; Peru; [Puerto Rico]; Suriname; Trinidad and Tobago; Venezuela

Amazona arausiaca (Mūller 1776)

I R

$16,318,321,444,485,794$

Red-necked Parrot, Red-necked Amazon

Breeding:

Dominica [179]

Amazona auropalliata (Lesson 1842)

II

$16,485,1073$

Yellow-naped Parrot, Yellow-naped Amazon

Breeding: $\quad$ Costa Rica; El Salvador; Guatemala; Honduras; Nicaragua

Amazona autumnalis (Linnaeus 1758)

$11 \quad-\quad 16,485,967,1152$

Red-lored Parrot, Red-lored Amazon

Breeding:

Belize; Brazil; Colombia; Costa Rica; Ecuador; Guatemala; Honduras; Mexico; Nicaragua; Panama; Venezuela

Amazona barbadensis (Gmelin 1788)

$1 \mathrm{~K}$

$318,321,485,967,1152$

Yellow-shouldered Parrot, Yellow-shouldered Amazon

Breeding:

Aruba (ex?); Netherlands Antilles: Bonaire; Venezuela $[1185,1435]$

Amazona brasiliensis (Linnaeus 1758)

1

E

$318,321,485,794,967,1073,1152$, 1222

Red-tailed Parrot, Red-tailed Amazon

Breeding: Brazil

Amazona collaria (Linnaeus 1758)

11

16,485

Yellow-billed Parrot, Yellow-hilled Amazon

Breeding: Jamaica [179]

Amazona dufresniana (Shaw 1812)

11

$485,967,1152$

Blue-cheeked Parrot, Blue-cheeked Amazon

Breeding: $\quad$ French Guiana; Guyana; Suriname; Venezuela

Amazona farinosa (Boddaert 1783)

$11 \quad-\quad 16,485,967,1152$

Mealy Parrot, Mealy Amazon

Breeding: Belize; Bolivia; Brazil; Colombia; Costa Rica; Ecuador; French Guiana; Guatemala; Guyana; Honduras; Mexico; Nicaragua; Panama; Peru; Suriname; Venezuela

Amazona fesriva (Linnaeus 1758)

11

$485,967,1152$

Festive Parrot, Festive Amazon

Breeding: $\quad$ Brazil; Colombia; ?Ecuador; Guyana; Peru; Venezuela

Amazona finschi (Sclater 1864)

I1 $\quad-\quad 16,485$

Lilac-crowned Parrot, Lilac-crowned Amazon

Breeding: Mexico

Amazona guildingii (Vigors 1837)

1

$\mathbf{R}$

$16,250,318,321,485,721,794$, 822,823

Saint Vincent Parrot, Saint Vincent Amazon

Breeding: $\quad$ Saint Vincent [179]

Amazona imperialis Richmond 1899

I E

$16,318,321,444,485,794$

Imperial Parrot, Imperial Amazon

Breeding:

Dominica [179] 
Amazona kawalli Grantsau \& Camargo 1989

Kawall's Parrot, Kawall's Amazon

Breeding: Brazil

Amazona leucocephala (Linnaeus 1758)

I

$16,179,485,794$

Cuban Parrot, Cuban Amazon, Caribbean Amazon

Breeding:

Bahamas: Great Inagua, Abaco, Long (ex), Crooked (ex), Fortune (ex), Acklins (ex) [1304]; Cayman Islands; Cuba

Amazona mercenaria (Tschudi 1844) $\quad$ II $\quad$ - $\quad 485,967,1152$

Scaly-naped Parrot, Scaly-naped Amazon

Breeding: $\quad$ Bolivia; Colombia; Ecuador; Peru; Venezuela

Amazona ochrocephala (Gmelin 1788) II - $\quad 16,485,967,1073$

Yellow-crowned Parrot, Yellow-crowned Amazon, Yellow-fronted Amazon

Breeding: $\quad$ Bolivia; Brazil; Colombia; French Guiana; Guyana; Honduras; Peru; Suriname; ?Trinidad and Tobago: Trinidad; Venezǔela $P_{\text {es arma }}$

Amazona oratrix Ridgway 1887

II V

$16,186,297,321,485,1073$

Yellow-headed Parrot, Yellow-headed Amazon

Breeding: Belize; ?Guatemala; Mexićo [1421]; [Puerto Rico]

Amazona pretrei (Temminck 1830) $\quad 1 \quad$ V/R $\quad 318,321,485,794,967,1073,1152$, 1276

Red-spectacled Parrot, Red-spectacled Amazon

Breeding: $\quad$ Argentina [154]; Brazil [1271]; ?Paraguay; Uruguay

Amazona rhodocorytha (Salvadori 1890) $\quad 1 \quad$ V/R $\quad 318,321,485,967,1152$

(= A. rhodocorytha)

Red-browed Parrot, Red-topped Parrot, Red-hrowed Amazon, Red-topped Amazon

Breeding: Brizil [1239]

Amazona tucumana (Cabanis 1885) $\quad 1 \quad-\quad 485,967,1073$

Tucuman Parrot, Alder Parrot, Tucuman Amazon

Breeding: $\quad$ Argentina [154]; Bolivia (ex?)

Amazona ventralis (Müller 1776)

Il

$16,179,485,1073$

Hispaniolan Parrot, Hispaniolan Amazon

Breeding: $\quad$ Dominican Republic; Haiti; [Puerto Rico]

Amazona versicolor (Müller 1776)

I R

$16,249,251,252,318,321,485$, $720,721,723,794,1073,7075$

Saint Lucia Parrot, Saint Lucia Amazon

Breeding: $\quad$ Saint Lucia [179]

Amazona vinacea (Kuhl 1820)

I V/R $318,321,485,967,1073,1152$

Vinaceous Parrot, Vinaceous Amazon

Breeding: Argentina [154]; Brazil; Paraguay

Amazona viridigenalis (Cassin 1853)

II

$\mathrm{V}$

$16,318,321,485,1073,1152$

Red-crowned Parrot, Green-cheeked Amazon, Red-crowned Amazon

Breeding:

Mexico [296]; [Puerto Rico] [1129]; [USA] 


\section{Puerto Rican Parrot, Puerto Rican Amazon}

Breeding: $\quad$ Puerto Rico [179]

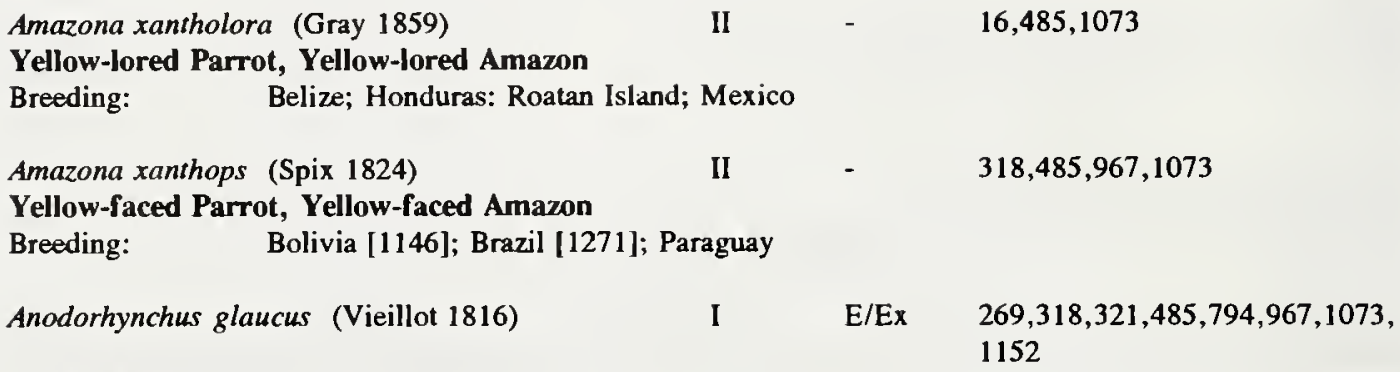

Glaucous Macaw

Breeding:

Argentina (ex?) [154]; Brazil (ex?); Paraguay (ex?); Unuguay (ex)

Anodorhynchus hyacinthinus (Latham 1790)

I $\quad \mathrm{V} / \mathrm{R} \quad 290,318,321,485,967,997,1073$, 1152,1275

Hyacinth Macaw

Breeding:

Bolivia; Brazil [587,588,996,1271]; Paraguay [634]

Anodorhynchus leari Bonaparte 1856

1

E

$196,318,321,485,619,794,878$, 967,1073

Indigo Macaw, Lear's Macaw

Breeding: $\quad$ Brazil [1269,1542]

Aprosmictus erythropterus (Gmelin 1788) $\quad$ II $\quad$ - $\quad 485,1292$

Red-winged Parrot

Breeding: $\quad$ Australia [167]; Indonesia: Irian Jaya; Papua New Guinea

Aprosmictus jonquillaceus (Vieillot 1818) II $\quad$ - 485

Olive-shouldered Parrot, Timor Red-winged Parrot

Breeding: $\quad$ Indonesia: Roti, Timor, Wetar [1486]

Ara ambigua (Bechstein 1811)

$1 \quad-\quad 16,485,794,967,1073$

Great Green Macaw, Buffon's Macaw

Breeding: $\quad$ Colombia; Costa Rica; Ecuador; Honduras [903]; Nicaragua; Panama

Ara ararauna (Linnaeus 1758)

II

$485,967,1073$

(includes $A$. caninde (Wagler 1832))

Blue-and-yellow Macaw, Blue-and-gold Macaw

Breeding: $\quad$ Bolivia; Brazil; Colombia; Ecuador; French Guiana; Guyana; Panama; Peru; Suriname; Trinidad and Tobago: Trinidad; Venezuela

Ara auricollis Cassin 1853

II

$485,967,1073$

Yellow-collared Macaw, Golden-collared Macaw

Breeding:

Argentina [154]; Bolivia; Brazil; Paraguay

Ara chloropterus Gray 1859

II $16,485,967,1073$

Red-and-green Macaw, Green-winged Macaw

Breeding: Argentina (ex) [154]; Bolivia; Brazil; Colombia; Ecuador; French Guiana; Guyana; Panama; Paraguay; Peru; Suriname; Venezuela 
Ara couloni Sclater 1876

II

$485,967,1073$

Blue-headed Macaw

Breeding:

Bolivia [1066]; ?Brazil; Peru

Ara glaucogularis Dabbene 1921

I E

$78,318,321,485,794,830,967,1073$

(=A. caninde misapplied)

Blue-throated Macaw

Breeding:

?Argentina [154]; Bolivia [1146]; ?Paraguay

Ara macao (Linnaeus 1758)

1

$16,485,967,1073$

Scarlet Macaw

Breeding:
Belize; Bolivia; Brazil; Colombia; Costa Rica; Ecuador; El Salvador (ex); French Guiana; Guatemala; Guyana; Honduras; Mexico; Nicaragua; Panama; Peru; Suriname; ?Trinidad and Tobago: Trinidad; Venezuela
Ara manilata (Boddaert 1783)
Il
$485,967,1073$
Red-bellied Macaw
Breeding:
Bolivia; Brazil; Colombia; Ecuador; French Guiana; Guyana; Peru; Suriname; Trinidad and Tobago: Trinidad; Venezuela

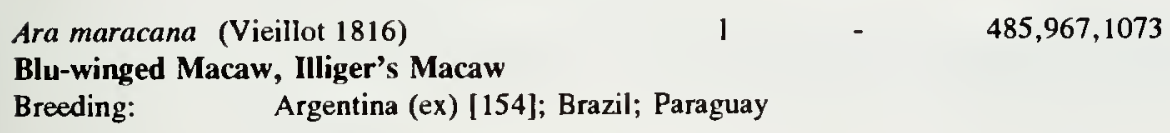

Ara nobilis (Linnaeus 1758)

Red-shouldered Macaw, Nohle Macaw

Breeding:

Bolivia; Brazil; ?French Guiana; Guyana; Pen; Suriname; Venezuela

Ara rubrogenys Lafresnaye 1847

I V

$192,293,318,321,485,830,831$, $967,995,1073,1152$

Red-fronted Macaw

Breeding:

Bolivia

Ara severa (Linnaeus 1758)

11

$485,967,1073$

Chestnut-fronted Macaw, Severe Macaw

Breeding: $\quad$ Bolivia; Brazil; Colombia; Ecuador; French Guiana; Guyana; Panama; Peru; Suriname; [USA] [845]; Venezuela

Aratinga acuticaudata (Vieillot 1818)

Blue-crowned Parakeet, Blue-crowned Conure

Breeding:

Argentina; Bolivia; Brazil; Colombia; Paraguay; Unguay; Venezuela

Aratinga aurea (Gmelin 1788)

II

$485,967,1073$

Peach-fronted Parakeet, Peach-fronted Conure, Golden-crowned Conure

Breeding:

Argentina [154]; Bolivia; Brazil; Paraguay; Peru; Suriname

Aratinga auricapilla (Kuhi 1820)

Il V

$318,321,485,967,1073,1152$

Golden-capped Parakeet, Golden-capped Conure

Breeding:

Brazil 


\section{Hispaniolan Parakeet, Hispaniolan Conure}

Breeding: $\quad$ Dominican Republic; Haiti; [Puerto Rico]

Aratinga erythrogenys (Lesson 1844)

II

$485,967,1073$

Red-masked Parakeet, Red-masked Conure

Breeding:

Ecuador; Peru

$\begin{aligned} & \text { Aratinga euops (Wagler 1832) } \\ & \text { Cuban Parakeet, Cuban Conure } \\ & \text { Breeding: }\end{aligned}$
Cuba [179]

Crimson-fronted Parakeet, Crimson-fronted Conure, Finsch's Conure

Breeding: $\quad$ Costa Rica; Nicaragua; Panama

Aratinga guarouba (Gmelin 1788) I $\quad$ V $\quad 318,321,485,794,967,1073,1272$

(= Guaruba guarouba)

Golden Parakeet, Golden Conure, Queen of Bavaria's Conure

Breeding: Brazil [1039]

Aratinga holochlora (Sclater 1859) II $\quad$ - $\quad 16,485,1073$

(excluding subspecies brevipes)

Green Parakeet, Green Conure

Breeding: $\quad$ El Salvador; Guatemala; Honduras; Mexico; Nicaragua

Aratinga holochlora brevipes (Lawrence 187I) $\quad$ Il $\quad K \quad K \quad 318,321,1184$

(= A. brevipes)

Socorro Parakeet

Breeding: Mexico: Socorro lsland

Aratinga jandaya (Gmelin 1788)

11

$485,967,1073$

Jandaya Parakeet, Yellow-headed Parakeet, Jandaya Conure

Breeding: Brazil

Aratinga leucophthalmus (Müller 1776)

II $\quad-\quad 485,967,1073$

White-eyed Parakeet, White-eyed Conure

Breeding: $\quad$ Argentina; Bolivia; Brazil; Colombia; Ecuador; French Guiana; Guyana; Paraguay; Penu; Suriname; Uruguay; Venezuela

Aratinga mitrata (Tschudi 1844)

II

$485,967,1073$

Mitred Parakeet, Mitred Conure

Breeding: Argentina; Bolivia; Peru

Aratinga nana (Vigors 1830) 
Aratinga pertinax (Linnaeus 1758)

II

$16,485,967,1073$

Brown-throated Parakeet, Brown-throated Conure

Breeding: $\quad$ Aruba; Brazil; Colombia; French Guiana; Guyana; Netherlands Antilles; Panama; [Puerto Rico]; Suriname [628]; Venezuela; [Virgin Islands of the United States: Saint Thomas]

Aratinga solstitialis (Linnaeus 1758)

II

$485,967,1073$

Sun Parakeet, Sun Conure

Breeding:

Brazil; French Guiana; Guyana; Suriname; Venezuela
Aratinga strenua (Ridgway 1915)
II
$16,485,1073$

Pacific Parakeet

Breeding:

El Salvador; Guatemala; Honduras; Mexico; Nicaragua

Aratinga wagleri (Gray 1845)

II

$485,967,1073$

Scarlet-fronted Parakeet, Red-fronted Conure

Breeding:

Colombia; Ecuador; Peru; Venezuela

Aratinga weddellii (Deville 1851) II

$485,967.1073$

Dusky-headed Parakeet, Dusky-headed Conure

Breeding: Bolivia; Brazil; Colombia; Ecuador; Peru

Bolbopsittacus lunulatus (Scopoli 1786)

$11 \quad-\quad 485$

Guaiabero

Breeding: Philippines

Bolborhynchus aurifrons (Lesson 1830)

11

$485,967,1073$

Mountain Parakeet, Golden-fronted Parakeet

Breeding: $\quad$ Argentina; Bolivia; Chile; Peru

Bolborhynchus aymara (d'Orbigny 1839)

$485,967,1073$

Grey-hooded Parakeet, Sierra Parakeet

Breeding:

Argentina; Bolivia; ?Chile

Bolborhynchus ferrugineifrons (Lawrence 1880)

I]

V/R

$318,321,485,571,794,967,1073$, 1152

Rufous-fronted Parakeet

Breeding:

Colombia

Bolborhynchus lineola (Cassin 1853)

11

$16,485,967,1073$

Barred Parakeet

Breeding:

Colombia; Costa Rica; Ecuador; El Salvador; Guatemala; Honduras; Mexico; Nicaragua; Panama; Peru; Venezuela

Bolborhynchus orbygnesius (Souancẻ 1856)

11

$485,967,1073$

Andean Parakeet

Breeding:

Bolivia; Peru

Brotogeris chiriri (Vieillot 1818)

II

$485,967,1073$

( $=B$. versicolurus chiriri)

Yellow-chevroned Parakeet

Breeding: Argentina [154]; Bolivia; Brazil

Brotogeris chrysopterus (Linnaeus 1766) II $\quad$ - $\quad 485,967,1073$

Golden-winged Parakeet

Breeding: $\quad$ Brazil; French Guiana; Guyana; Suriname; Venezuela 
Brotogeris cyanoptera (Salvadori 1891)

11

$485,967,1073$

Cobalt-winged Parakeet

Breeding: $\quad$ Bolivia; Brazil; Colombia; Ecuador; Peru; Venezuela
Brotogeris jugularis (Müller 1776)
11
$485,967,1073$
Orange-chinned Parakeet

Breeding:

Colombia; Costa Rica; El Salvador; Guatemala; Honduras; Mexico; Nicaragua; Panama; Venezuela

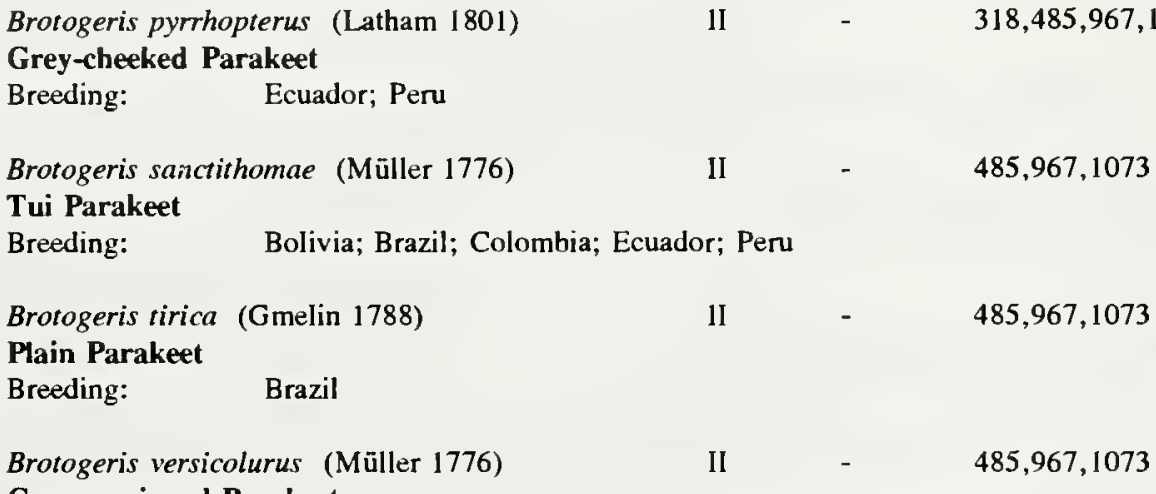

Carary-winged Parakeet

Breeding: $\quad$ Brazil; Colombia; ?Ecuador; French Guiana; Peru; [Puerto Rico]; ?Suriname; [USA]

Cacatua alba (Müller 1776) II $\quad K^{*} \quad 313,318,485,1486$

White Cockatoo, Umbrella Cockatoo

Breeding: $\quad$ lndonesia: Bacan, Halmahera, Obi, Ternate, Tidore

Cacatua ducorpsii Pucheran I853 II $\quad$ - $\quad 313,485$

Ducorps's Cockatoo

Breeding: $\quad$ Papua New Guinea: Bougainville; Solomon 1slands

Cacatua galerita (Latham 1790)

II -

485,1292

Sulphur-crested Cocatoo, Greater Sulphur-crested Cockatoo

Breeding: $\quad$ Australia [167]; Indonesia: Irian Jaya, [Kai Kecil] [1486], [Seram Laut], Western Papuan Islands [Palau] [1118]; Papua New Guinea; [New Zealand] [845]; [Singapore].

Cacatua goffini (Finsch 1863) II $\quad \mathrm{K}^{*} \quad 313,318,485,1486$

Tanimbar Cockatoo, Tanimbar Corella, Goffin's Cockatoo

Breeding: $\quad$ Indonesia: Tanimbar Islands, ?[Tual]

$\begin{array}{llll}\text { Cacatua haematuropygia (Müller 1776) } & \text { II } & \mathrm{K}^{*} & \mathbf{3 1 3 , 3 1 8 , 4 8 5}\end{array}$

Philippine Cockatoo, Red-vented Cockatoo

Breeding: Philippines

Cacatua leadbeateri (Vigors 1831)

II $\quad$ - $\quad 485,1292$

Pink Cockatoo, Major Mitchell's Cockatoo, Leadbeater's Cockatoo

Breeding: Australia [167]

Cacatua moluccensis (Gmelin 1788)

I $\quad K^{*} \quad 313,318,485,1486$

Salmon-crested Cockatoo, Moluccan Cockatoo

Breeding: Indonesia: ?[Ambon], Haruku, Seram, Saparua; [Singapore] 


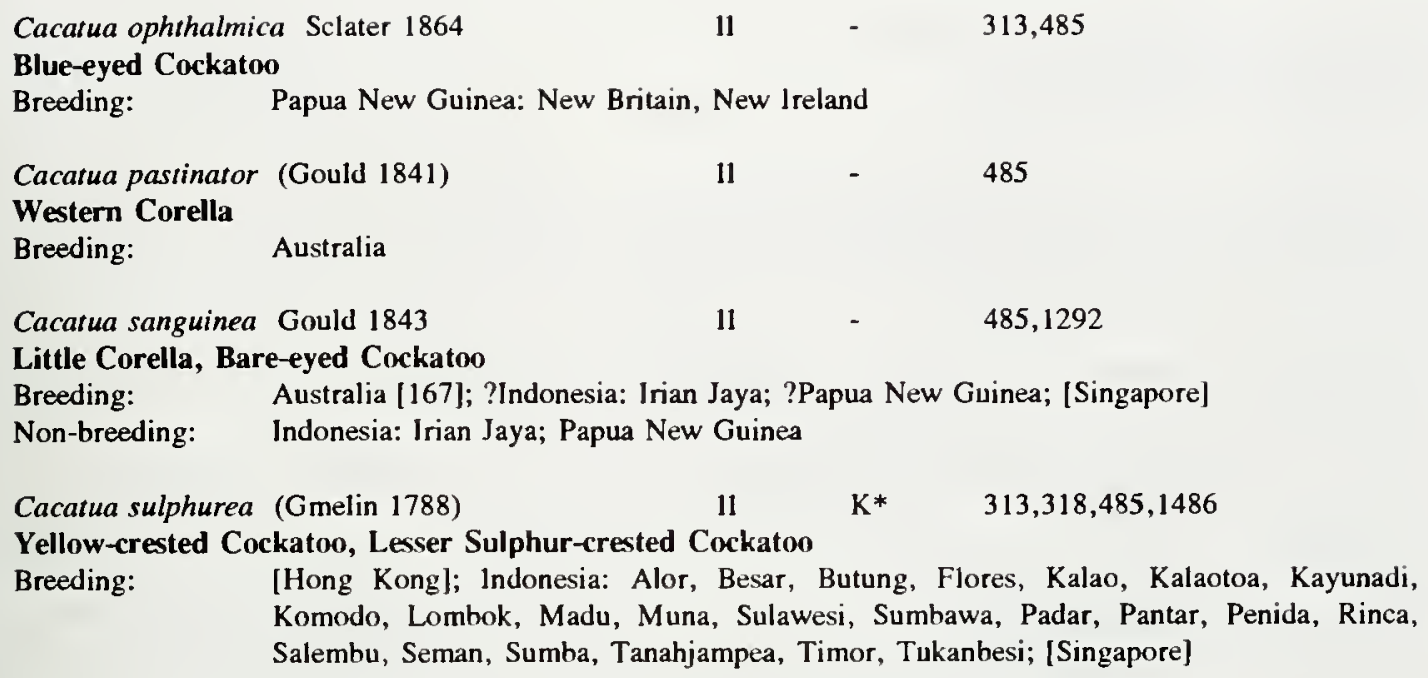


Brown Lory, Duyvenbode's Lory

Breeding:

Indonesia: Irian Jaya; Papua New Guinea

Chalcopsitta sintillata Temminck 1835

Yellow-streaked Lory, Greater Streaked Lory

Breeding: Indonesia: Aru lslands, Irian Jaya; Papua New Guinea

Charmosyna amabilis (Ramsay 1875)

Red-throated Lorikeet

Breeding:

Fiji: Viti Levu, Ovalau, Taveuni [1]18]

Charmosyna diadema (Verreaux \& Des Murs 1860) II $\quad K^{*} \quad 318,485$

New Caledonian Lorikeet

Breeding: New Caledonia (ex?)

Charmosyna josefinae (Finsch 1873)

Josephine's Lorikeet, Josephine's Lory

Breeding: $\quad$ Indonesia: Irian Jaya; Papua New Guinea

$\begin{array}{llll}\text { Charmosyna margarethae } & \text { Tristram } 1879 & \text { II } & -\end{array}$

Duchess Lorikeet

Breeding:

Papua New Guinea: Bougainville; Solomon Islands

Charmosyna meeki (Rothschild \& Hartert 1901) II

Meek's Lorikeet

Breeding:

Papua New Guinea: Bougainville; Solomon Islands: Guadalcanal, Kulambangra, Malaita

Charmosyna multistriata (Rothschild 1911)

II

485,1226

Striated Lorikeet, Streaked Lorikeet

Breeding: Indonesia: Irian Jaya; Papua New Guinea

$\begin{array}{llll}\text { Charmosyna palmarum (Gmelin 1788) } & \text { II } & \text { - } & 485\end{array}$

Palm Lorikeet

Breeding:

Solomon lslands: Banks, Santa Cruz; Vanuatu

Charmosyna papou (Scopoli 1786)

II

485,1226

Papuan Lorikeet

Breeding:

Indonesia: Irian Jaya; Papua New Guinea

Charmosyna placentis (Temminck 1834)

II

485

Red-flanked Lorikeet

Breeding:

Indonesia: Ambelau, Ambon, Aru Islands, Biak, Irian Jaya, Kai Islands, Moluccas, Panjang, Seram, Seram Laut, Tayandu, Western Papuan Islands; Papua New Guinea

Charmosyna pulchella Gray 1859

II

485

Little Red Lorikeet, Fairy Lorikeet

Breeding:

Indonesia: Irian Jaya; Papua New Guinea

Charmosyna rubrigularis (Sclater 1881)

11

485,1226

Red-chinned Lorikeet

Breeding:

Papua New Guinea: Karkar Island, New Britain, New Jreland

Charmosyna rubronotata (Wallace 1862)

II

485,1226

Red-fronted Lorikeet, Red-spotted Lorikeet

Breeding:

Indonesia: Biak, Irian Jaya, Salawati; Papua New Guinea 
Charmosyna wilhelminae (Meyer 1874)

\section{Black Parrot}

Breeding: $\quad$ Comoros; Madagascar [373]; Seychelles: Praslin Island
Coracopsis vasa (Shaw 1812)
II
485

\section{Vasa Parrot}

Breeding:

Comoros; Madagascar [373]

Cyanoliseus patagonus (Vieillot 1818)

Burrowing Parakeet, Patagonian Conure

$\begin{array}{ll}\text { Breeding: } & \text { Argentina [154]; Chile; Uruguay } \\ \text { Vagrant: } & \text { Falkland Islands }\end{array}$

Cyanopsitta spixii (Wagler 1832)

Little Blue Macaw, Spix's Macaw

Breeding: Brazil

Cyanoramphus auriceps (Kuhl 1820)

Yellow-fronted Parakcet, Yellow-crowned Parakeet, Yellow-fronted Kakariki

Breeding: New Zealand

Cyanoramphus auriceps forbesi Rothschild 1893 I - $\quad 485,794,976,1352$

Chatham Island Yellow-fronted Parakeet

Breeding: New Zealand: Chatham Islands

Cyanoramphus cookii (Gray 1859)

I $\quad-\quad 485,794$

(= C. novaezelandiae cookii)

Norfolk Parakeet, Norfolk Island Parakeet

Breeding: Norfolk Island

Cyanoramphus novaezelandiae (Sparrman 1787) I $\quad$ - $\quad 485,794,1352$

Red-fronted Parakeet

Breeding:

Australia: Lord Howe Island (ex); Macquarie Island (ex); New Caledonia; New Zealand

Cyanoramphus unicolor (Lear 1831)

II $\quad \mathrm{K}^{*} \quad 318,485,1352$

Antipodes Parakeet, Antipodes Green Parakeet

Breeding: New Zealand: Antipodes Islands [1507]

Cyclopsitta diophthalma (Hombron \& Jacquinot 1841) II

485,1292

(= Opopsitta diophthalma)

(excluding subspecies coxeni)

Double-eyed Fig-Parrot, Dwarf Fig-Parrot

Breeding:

Australia [167]; Indonesia: Aru Islands, Irian Jaya, Western Papuan Islands; Papua New Guinea 


\section{Orange-breasted Fig-Parrot}

Breeding: Indonesia: Aru Islands, Irian Jaya, Salawati in Westem Papuan Islands; Papua New Guinea

Deroptyus accipitrinus (Linnaeus 1758)

$485,967,1073$

Red-fan Parrot, Hawk-headed Parrot

Breeding:

Brazil; Colombia; Ecuador; French Guiana; Guyana; Peru; Suriname; Venezuela

$\begin{array}{llll}\text { Eclectus roratus (Müller 1776) } & \text { II } & - & 485,1292\end{array}$

Eclectus Parrot

Breeding:

Australia [167]; Indonesia: Ambon, Aru Islands, Bacan, Buru, Damar, Irian Jaya, islands in Geelvink Bay, Halmahera, Haruku, Kai lslands, Kayoa, Laut, Mare, Morotai, Obi, Saparua, Seram, Sumba, Tanimbar Islands, Tatandu, Ternate, Western Papuan Islands; [Palau] [1118]; Papua New Guinea; Solomon Islands

Enicognathus ferrugineus (Müller 1776)

II

$485,967,1073$

Austral Parakeet, Austral Conure

Breeding:

Argentina; Chile

Vagrant:

Falkland lslands

Enicognathus leptorhynchus (King 1831)

11

$485,967,1073$

Slender-billed Parakeet, Slender-billed Conure

Breeding: Chile

Eolophus roseicapillus (Vieillot 1817)

Il $\quad-$

485,1292

(= Cacatua roseicapilla)

Galah, Roseate Cockatoo

Breeding:

Australia [167]

Eos bornea (Linnaeus 1758)

II

Red Lory, Moluccan Lory

Breeding:

Indonesia: Ambon, Buru, Haruku, Kai lslands, Laut [1486], Saparua, Seram, Tayandu, Watubela

Eos cyanogenia Bonaparte 1850

II $\quad \mathrm{K}^{*}$

$318,485,1226$

Black-winged Lory, Biak Red Lory

Breeding: $\quad$ Indonesia: Biak, Manim, Meos Num [131], Numfor

Eos histrio (Müller 1776)

II $\quad \mathrm{K}^{*}$

$318,485,1486$

Red-and-blue Lory

Breeding:

Indonesia: Miangas, Ruang, Sangihe, Siau, Talaud

Eos reticulata (Müller 1841)

II $\quad \mathrm{K}^{*}$

$318,485,1486$

Blue-streaked Lory

Breeding: Indonesia: Babar, [Damar], [Kai Islands], Tanimbar

Eos semilarvasa Bonaparte 1850

II

Blue-eared Lory

Breeding:

Indonesia: Seram [1486] 
Eos squamata (Boddaert 1783)

II

485,1226

Violet-necked Lory, Moluccan Red Lory

Breeding: $\quad$ Indonesia: Moluccas, Schildpad, Western Papuan Islands, Widi
Eunymphicus cornutus (Gmelin 1788)
II $\quad-\quad 485,794$
Horned Parakeet
Breeding:
New Caledonia
Forpus coelestis (Lesson I847)
II
$485,967,1073$
Pacific Parrotlet, Celestial Parrotlet
Breeding:
Ecuador; Peru
Forpus conspicillatus (Lafresnaye 1848)
II
$485,967,1073$
Spectacled Parrotlet
Breeding:
Colombia; Panama; Venezuela
Forpus cyanopygius (Souancé 1856)
II
$16,485,1073$
Mexican Parrotlet, Blue-rumped Parrotlet
Breeding: Mexico
Forpus passerinus (Linnaeus 1758)
II
$485,967,1073$

Green-rumped Parrotlet

Breeding: [Barbados] [179]; Brazil; Colombia; French Guiana; Guyana; [Jamaica] [179]; [Netherlands Antilles: Curaçao]; Suriname [628]; Trinidad and Tobago: Trinidad; Venezuela

Forpus sclateri (Gray I859)

II

485,967

Dusky-billed Parrotlet, Sclater's Parrotlet

Breeding: $\quad$ Bolivia; Brazil; Colombia; Ecuador; French Guiana; Guyana; Peru; Venezuela

Forpus xanthops (Salvin I895)

II $\quad$ V

$32 I, 485,967,1164$

Yellow-faced Parrotlet

Breeding: Peru

Forpus xanthopterygius (Spix 1824)

II $\quad-\quad 485,967,1073$

Blue-winged Parrotlet

Breeding: $\quad$ Argentina; Bolivia; Brazil; Colombia; Ecuador; Paraguay; Peru

$\begin{array}{llll}\text { Geoffroyus geoffroyi (Bechstein 1811) II } & - & 485,1226,1292\end{array}$

Red-cheeked Parrot

Breeding:

Australia [167]; Indonesia: Ambon, Aru Islands, Bacan, Boana, Flores, Halmahera, Hanku, Irain Jaya, Kai Islands, Lombok, Morotai, Obi, Saparua, Semau, Seram, Seram Laut, Sumba, Sumbawa, Tanimbar, Timor, Western Papuan Islands, Wetar [1486]; Papua New Guinea [88I]

Geoffroyus heteroclitus (Hombron \& Jacquinot I84I) II

485,1226

Singing Parrot

Breeding:

Papua New Guinea: Bismarck Archipelago; Solomon Islands

Geoffroyus simplex (Meyer 1874)

II

485,1226

Blue-collared Parrot

Breeding:

Indonesia: Irian Jaya; Papua New Guinea

Glossopsitta concinna (Shaw 179I)

Musk Lorikeet

Breeding:

Australia [167] 
Glossopsitta porphyrocephala (Dietrichsen 1837)

II

485,1292

Purple-crowned Lorikeet

Breeding:

Australia [167]

Glossopsinta pusilla (Shaw 1790)

II

485,1292

Little Lorikeet

Breeding:

Australia [167]

Graydidascalus brachyurus (Kuhl 1820)

II

$485,967,1073$

Short-tailed Parrot

Breeding:

Brazil; Colombia; Ecuador; Peru

Gypopsitta vulturina (Kuhl 1820)

II

$485,967,1073$

Vulturine Parrot

Breeding:

Brazil

Hapalopsittaca amazonina (Des Murs 1845)

II

$\mathrm{V} / \mathrm{R}$

$318,321,485,794,967,1073,1152$

Rusty-faced Parrot

Breeding:

Colombia; Venezuela

Hapalopsittaca fuertesi (Chapman 1912)

II

E

321,485

$(=H$. amazonina fuertesi)

Indigo-winged Parrot, Fuertes's Parrot

Breeding:

Colombia

Hapalopsittaca melanotis (Lafresnaye 1847)

11

$485,967,1073$

Black-winged Parrot, Black-eared Parrot

Breeding:

Bolivia; Peru

Hapalopsittaca pyrrhops (Salvin 1876)

II

E

321,485

(=H. amazonina pyrrhops)

Red-faced Parrot

Breeding:

Ecuador; Peru

Lathamus discolor (White 1790)

Il

485,1292

Swift Parrot

Breeding:

Australia [167]

Leptosittaca branickii Berlepsch \& Stolzmann 1894

Golden-plumed Parakeet, Golden-plumed Conure

Il $\quad \mathrm{V} / \mathrm{R}$

$318,321,485,967,1073,1152$

Breeding:

Colombia [1011]; Ecuador; Peru

Loriculus amabilis Wallace 1862

Il

485

Moluccan Hanging-Parrot

Breeding: $\quad$ Indonesia: Bacan, Banggai [1486], Halmahera, Peleng, Sula Islands

Loriculus aurantiifrons Schlegel 1873

Il

485,1226

Orange-fronted Hanging-Parrot, Papuan Hanging-Parrot

Breeding:

Indonesia: Irian Jaya, Misool, Waigeo; Papua New Guinea

Loriculus beryllinus (Forster 1781)

II

10,485

Ceylon Hanging-Parrot

Breeding:

Sri Lanka 
Loriculus catamene Schlegel 1873

(= L. amabilis catamene)

\section{Sangihe Hanging-Parrot}

Breeding:

Indonesia: Sangihe

Loriculus exilis Schlegel 1866

Red-billed Hanging-Parrot, Green Hanging-Parrot

Breeding: Indonesia: Sulawesi [1486]

Loriculus flosculus Wallace 1864

Wallace's Hanging-Parrot

Breeding: Indonesia: Flores [ 1486]

Loriculus galgulus (Linnaeus 1758)

II

485,792

Blue-crowned Hanging-Parrot

Breeding:

Brunei; Indonesia: Anamba Islands, Bangka, Belitung, Enggano, Kalimantan and some offshore islands, Nias, Riau Archipelago, Siberut, Sipora, Sumatra; Malaysia: Peninsular Malaysia, Sabah, Sarawak [1295]; Singapore; Thailand [1 194]

Loriculus philippensis (Müller 1776)
Colasisi, Philippine Hanging-Parrot
Breeding: $\quad$ Philippines

Loriculus pusillus Gray 1859

Yellow-throated Hanging-Parrot

Breeding:

Indonesia: Bali, Java
I]

II

II

Loriculus stigmatus (Mūller 1843)

Sulawesi Hanging-Parrot

Breeding:

Indonesia: Butung, Mangka, Muna [1486], Lembeh, Monterawu, Sulawesi, Togian Islands

Loriculus tener Sclater 1877

Il

485

(= L. stigmatus tener),

Green-fronted Hanging-Parrot

Breeding: $\quad$ Papua New Guinea: Bismark Archipelago

Loriculus vernalis (Sparrman 1787) $\quad$ II $\quad-\quad$ 10,485,792

Vernal Hanging-Parrot

Breeding:

Bangladesh; Bhutan; Cambodia; China; India: including Andaman and Nicobar Islands; Lao P.D.R; Myanmar; Nepal; Thailand [1194]; Viet Nam

Lorius albidinuchus (Rothschild \& Hartert 1924) 11

485,1226

White-naped Lory

Breeding:

Papua New Guinea: New Ireland

Lorius chlorocercus Gould 1856

II

485

Yellow-bibbed Lory

Breeding: $\quad$ Solomon Islands: Guadalcanal, Malaita, Rennell, San Cristobal, Savo, Ugi

Lorius domicella (Linnaeus 1758) II $\quad$ K* $\quad 318,485,554,1486$

Purple-naped Lory, Purple-capped Lory

Breeding: Indonesia: Ambon, [Buru], Seram

Lorius garrulus (Linnaeus 1758)

485,1071

485
Chattering Lory, Yellow-hacked Lory
Breeding: $\quad$ Indonesia: Moluccas [1486] 
Breeding: Indonesia: Irian Jaya, Western Papuan Islands, islands in Geelvink Bay; Papua New Guinea

Micropsitta bruijnii (Salvadori 1875)

II

485,1226

Red-breasted Pygmy-Parrot

Breeding: Indonesia: Buru, Irian Jaya, Seram; Papua New Guinea; Solomon Islands: Guadalcanal, Kulambangra

Micropsitta finschii (Ramsay 1881)

II

485,1226

Finsch's Pygmy-Parrot

Breeding: $\quad$ Papua New Guinea: Bougainville, Lihir Islands, New Hanover, New Ireland; Solomon Islands

$\begin{aligned} & \text { Micropsitta geelvinkiana (Schlegel 187I) } \\ & \text { Geelvink Pygmy-Parrot }\end{aligned}$
$\begin{aligned} & \text { Breeding: } \\ & \text { Indonesia: Biak, Numfor }\end{aligned}$
$\begin{aligned} & \text { Mic:opsitta keiensis (Salvadori 1875) } \\ & \text { Yellow-capped Pygmy-Parrot }\end{aligned}$

Breeding: Indonesia: Aru Islands, Irian Jaya, Kai lslands, Western Papuan Islands; Papua New Guinea

Micropsitta meeki Rothschild \& Hartert 1924 II

485,1226

Meek's Pygmy-Parrot

Breeding: $\quad$ Papua New Guinea: Admiralty Islands, Saint Matthias

$\begin{array}{llll}\text { Micropsitta pusio (Sclater I866) } & \text { II } & - & 485,1226\end{array}$

Buff-faced Pygmy-Parrot

Breeding: Indonesia: Irian Jaya; Papua New Guinea

Myiopsitta monachus (Boddaert 1783)

Monk Parakeet, Quaker Parakeet, Grey-breasted Parakeet

Breeding: $\quad$ Argentina; Bolivia; Brazil; Paraguay; [Puerto Rico]; [Spain] [845]; [USA] [845]; Uruguay

Nandayus nenday (Vieillot I823)

II

$485,967,1073$

Nanday Parakeet, Black-headed Conure, Black-headed Parakeet, Nanday Conure

Breeding: Argentina; Brazil; Paraguay; [USA]

Nannopsittaca dachilleae O'Neil, Munn, Franke 1991

\section{Amazonian Parakeet}

Breeding: $\quad$ Bolivia; Peru

Nannopsittaca panychlora (Salvin \& Godman 1883) II

$485,967,1073$

Tepui Parakeet

Breeding:

?Brazil; Guyana; Venezuela

Neophema chrysogaster (Latham 1790)

1

$\mathbf{R}$

$228,229,318,485,794,870,1292$

Orange-bellied Parrot

Breeding:

Australia: Tasmania [167]

Non-breeding:

Australia [738] 
Blue-winged Parrot, Blue-winged Grass-Parakeet

Breeding: Australia [167]

Neophema elegans (Gould 1837)

Elegant Parrot, Elegant Grass-Parakeet

Breeding: Australia [167]

Neophema petrophila (Gould 1841)

Rock Parrot

Breeding:

Australia [167]

Neophema pulchella (Shaw 1792)

Turquoise Parrot, Turquoise Grass-Parakeet

Breeding:

Australia [167]

Neophema splendida (Gould 1841)

II

Scarlet-chested Parrot, Splendid Grass-Parakeet

Breeding:

Australia [167]

Neopsephotus bourkii (Gould 1841)

11

485,1292

(= Neophema bourkii)

Bourke's Parrot

Breeding: Australia [167]

Neopsittacus musschenbroekii (Schlegel 1871)

Yellow-billed Lorikeet, Musschenbroek's Lorikeet

Breeding: Indonesia: Irian Jaya; Papua New Guinea

Neopsittacus pullicauda Hartert 1896

II

485

Orange-billed Lorikeet, Emerald Lorikeet

Breeding:

Indonesia: Irian Jaya; Papua New Guinea

Nestor meridionalis (Gmelin 1788)

11

133,485

Kaka

Breeding: New Zealand

Nestor notabilis Gould 1856

Kea

Breeding: New Zealand: South Island

Nestor productus (Gould 1836)

II

Ex

Norfolk Kaka

Norfolk Island (ex)

Breeding:

Northiella haematogaster (Gould 1838)

II

485,1292

(= Psephotus haematogaster)

\section{Bluebonnet}

Breeding:

Australia [167]

Ognorhynchus icterotis (Massena \& Souancé 1854) 1

Yellow-eared Parrot, Yellow-eared Conure

Breeding:

Colombia; Ecuador 
Plum-faced Lorikeet, Whiskered Lorikeet

Breeding:

Indonesia: Irian Jaya; Papua New Guinea

Pezoporus occidentalis Gould I861

(= Geopsittacus occidentalis)

Night Parrot

Breeding:

Australia (ex?) [167]

Pezoporus wallicus (Kerr 1792)

I

$318,485,794,1292$

Ground Parrot

Breeding:

Australia [167]

Phigys solitarius (Suckow 1800)

Collared Lory

Breeding:

Fiji [I I 18]

Pionites leucogaster (Kuhl 1820)

II

$485,967,1073$

White-bellied Parrot, White-bellied Caique

Breeding: $\quad$ Bolivia; ?Brazil; ?Ecuador; Peru

Pionites melanocephala (Linnaeus 1758)

Black-headed Parrot, Black-headed Caique

Breeding:

Brazil; Colombia; Ecuador; French Guiana; Guyana; Peru; Suriname [627]; Venezuela

Pionopsitta barrabandi (Kuhl 1820)

II

$485,967,1073$

Orange-cheeked Parrot, Barraband's Parrot

Breeding:

Bolivia [ 1066]; Brazil; Colombia; Ecuador; Peru; Venezuela

Pionopsitta caica (Latham 1790)

II

$485,967,1073$

Caica Parrot

Breeding:

Brazil; French Guiana; Guyana; Suriname; Venezuela

Pionopsitta haematotis (Sclater \& Salvin 1860)

II $\quad 16,485,1073$

Brown-hooded Parrot

Breeding:

Belize; Colombia; Costa Rica; Guatemala; Honduras; Mexico; Nicaragua; Panama

Pionopsitta pileata (Scopoli 1769)

$485,967,1073$

Pileated Parrot, Red-capped Parrot

Breeding:

Argentina; Brazil; Paraguay

Pionopsitta pulchra Berlepsch 1897

II

$485,967,1073$

Rose-faced Parrot, Beautiful Parrot

Breeding:

Colombia; Ecuador

Pionopsitta pyrilia (Bonaparte 1853)

II

$16,485,967,1073$

Saffron-headed Parrot

Breeding:

Colombia; ?Panama; Venezuela

Pionus chalcopterus (Fraser 184I)

II

$16,485,1073$

Bronze-winged Parrot

Breeding:

Colombia; Ecuador; Peru; Venezuela

Pionus fuscus (Müller 1776)

II

$485,967,1073$

Dusky Parrot

Breeding:

Brazil; Colombia; French Guiana; Guyana; Suriname; Venezuela 
Pionus maximiliani (Kuhl 1820)

II

$485,967,1073$

Scaly-headed Parrot

Breeding:

Argentina; Bolivia; Brazil; Paraguay

Pionus menstruus (Linnaeus 1766)

11

$16,485,967,1073$

Blue-headed Parrot, Red-vented Parrot

Breeding:

Bolivia; Brazil; Colombia; Costa Rica; Ecuador; French Guiana; Guyana; Panama; Peru; Suriname; Trinidad and Tobago: Trinidad; Venezuela

Pionus senilis (Spix 1824)

11

$16,485,967,1073$

White-crowned Parrot, White-capped Parrot

Breeding: $\quad$ Belize; Costa Rica; Guatemala; Honduras; Mexico; Nicaragua; Panama

Pionus sordidus (Linnaeus 1758)

11

$485,967,1073$

Red-billed Parrot

Breeding:

Bolivia; Colombia; Ecuador; Peru; Venezuela

Pionus tumultuosus (Tschudi 1844)

II

$485,96 ?, 1073$

(includes $P$. seniloides (Massena \& Souancé 1854))

Plum-crowned Parrot

Breeding:

Bolivia; Colombia; Ecuador; Peru; Venezuela

Platycercus adscitus (Latham 1790)

11

485,1292

Pale-headed Rosella, Mealy Rosella

Breeding: Australia [167]; [Hawaiian 1slands: Maui (ex?)]

Platycercus barmardi (Vigors \& Horsfield 1827) II

485,1292

(= Barnardius barnardi)

Mallee Ringneck, Barnard's Parakeet

Breeding:

Australia [167]

Platycercus caledonicus (Gmelin 1788)

II

485,1292

Green Rosella, Tasmanian Rosella

Breeding:

Australia [167]

Platycercus elegans (Gmelin 1788)

1I

485,1292

Crimson Rosella, Pennant's Parakeet

Breeding: Australia [167]; [New Zealand]; Norfolk Islands

Platycercus eximius (Shaw 1792)

Eastern Rosella, Golden-mantled Rosella

Breeding:

Australia [167]; [New Zealand]

Platycercus flaveolus Gould 1837

11

485,1292

Yellow Rosella

Breeding:

Australia [167]

Platycercus icterotis (Kuhl 1820)

Western Rosella, Stanley Parakeet

Breeding:

Australia [167]

Platycercus venustus (Kuhl 1820)

Northern Rosella

Breeding:

Australia [167] 
Platycercus zonarius (Shaw 1805)

II

485,1292

(= Barnardius zonarius)

Port Lincoln Ringneck, Twenty-eight Parakeet

Breeding: Australia [167]

$\begin{array}{llll}\text { Poicephalus crassus (Sharpe 1884) } & \text { IJ } & 485,496,1247,1298\end{array}$

Niam-niam Parrot

Breeding: $\quad$ Central African Republic [259]; Chad; Sudan; Zaire

$\begin{array}{lll}\text { Poicephalus cryptoxanthus (Peters 1854) II } & \text { I } & 212,285,485,496,1298\end{array}$

Brown-headed Parrot

Breeding: $\quad$ Kenya; Malawi; Mozambique; South Africa; Swaziland; Tanzania; Zimbabwe; ?Zambia

Poicephalus flavifrons (Rüppell 1845) $\quad$ Il $\quad-\quad 485,496,1298$

Yellow-fronted Parrot

Breeding: $\quad$ Ethiopia [748]

Poicephalus gulielmi (Jardine 1849)

II

$212,485,496,1247,1298$

Red-fronted Parrot, Jardine's Parrot, Red-crowned Parrot

Breeding: $\quad$ Angola; Cameroon; Central African Republic; Congo; Côte d'Ivoire; Equatorial Guinea; Gabon; Ghana; Kenya; Liberia; Tanzania; Uganda; Zaire

Poicephalus meyeri (Cretzschmar 1827)

Il

$212,285,485,496,1247,1298$

Meyer's Parrot, Brown Parrot

Breeding:

Angola; Botswana; Burundi; Central African Republic; Chad; Ethiopia; Kenya; Malawi; Mozambique; Namibia; Rwanda; South Africa; Sudan; Tanzania; Uganda; Zaire; Zambia; Zimbabwe

Poicephalus robustus (Gmelin 1788)

II

485,496

Brown-necked Parrot, Cape Parrot

Breeding: Angola; Congo; Côte d'lvoire; Equatorial Guinea; Gambia; ?Ghana; Guinea; Guinea-Bissau; Malawi; Mali; Mozambique; ?Namibia; Rwanda; ?Senegal; South Africa; South Africa; Swaziland; Tanzania; Togo; Uganda; Zaire; Zambia; ?Zimbabwe

Vagrant: $\quad$ Nigeria

Poicephalus rueppellii (Gray 1849) II $\quad$ - $\quad 285,485,496,1298$

Rueppell's Parrot

Breeding: $\quad$ Angola; Namibia

Poicephalus rufiventris (Rüppell 1845)

$212,485,496,1298$

Red-bellied Parrot, African Orange-bellied Parrot

Breeding:

Ethiopia; Kenya; Somalia; Tanzania

Poicephalus senegalus (Linnaeus 1766)

II

$485,496,1247,1298$

Senegal Parrot

Breeding:

Benin; Burkina Faso [1388]; Cameroon; Chad; Côte d'lvoire; Gambia; Ghana; Guinea [1454]; Guinea-Bissau; ?Liberia; Mali; Mauritania; Niger; Nigeria; Senegal; ?Sierra Leone; Togo

Polytelis alexandrae Gould $1863 \quad$ II $\quad \mathrm{K}^{*}$

$318,485,1292$

Alexandra's Parrot, Princess Parrot, Princess of Wales Parakeet

Breeding:

Australia [167]

Polytelis anthopeplus (Lear 1831)

II

485,1292

Regent Parrot, Rock Peppler

Breeding: Australia [167] 
Polytelis swainsonii (Desmarest 1826)

11

485,1292

Superb Parrot, Barraband Parakeet, Green Leek Parrot

Breeding:

Australia [167]

Prioniturus discurus (Vieillot 1818)

11

Blue-crowned Racquet-tail, Palawan Racket-tailed Parrot

Breeding:

Philippines

Prioniturus flavicans Cassin 1853

II

485

Yellow-breasted Racquet-tail

Breeding: $\quad$ Indonesia: Bangka, Lembeh, ?Peleng, Sulawesi, Togian Islands [1486]
Prioniturus luconensis Steere 1890
II
$\mathbf{R}$
318,485

Green Racquet-tail, Green-headed Racket-tailed Parrot, Green-crowned Racket-tailed Parrot

Breeding:

Philippines: Luzon, Marinduque

Prioniturus mada Hartert 1900

II

$K^{*}$

$318,485,1486$

Buru Racquet-tail

Breeding:

lndonesia: Buru

Prioniturus montanus Ogilvie-Grant 1895

II

485

Montane Racquet-tail

Breeding: $\quad$ Philippines: Luzon [1113]

Prioniturus platenae Blasius 1888

II

(=P. discurus platenae)

Blue-headed Racquet-tail

Breeding: Philippines: Balabac, Busuanga, Calauit, Culion, Dumaran, Palawan

Prioniturus platurus (Vieillot 1818)

11

485

Golden-mantled Racquet-tail

Breeding: $\quad$ lndonesia: Banggai, Butang, Dodepo, Lembeh, Muna, ?Peleng, ?Sangihe [1486]; Siau, Sulawesi, Talaud, Taliabu, Togian

Prioniturus verticalis Sharpe 1893

11

(=P. discurus vericalis)

Blue-winged Racquet-tail

Breeding: Philippines: Sulu Archipelago

Prioniturus waterstradti Rothschild 1904

II

(= P. montanus waterstradti)

Mindanao Racquet-tail

Breeding: $\quad$ Philippines: Mindanao

Probosciger aterrimus (Gmelin 1788)

$1 \quad-\quad 485,1292$

Palm Cockatoo

Breeding:

Australia [167]; Indonesia: Aru 1slands, Irian Jaya, [Kai Kecil], Western Papuan Islands, Yapen; Papua New Guinea

Prosopeia personata (Gray 1848)

II

485

Masked Shining-Parrot

Breeding:

Fiji: Viti Levu, Ovalau (ex), Mbau (ex) 
Prosepeia splendens (Peale 1848)

11

485

( = P. tabuensis splendens)

Crimson Shining-Parrot

Breeding:

Fiji: Kandavu, [Viti Levu]

Prosopeia tabuensis (Gmelin 1788)

11

485

Red Shining-Parrot

Breeding:

Fiji: Vanua Levu, Kea, Koro, Taveuni, Gamea, Gau, Kandavu [1118]; [Eua,Tonga: Tongatapu (ex?)] [528,1118]

Psephotus chrysopterygius Gould 1858

$1 \quad \mathbf{R}$

$318,485,794,1292,1467$

Golden-shouldered Parrot

Breeding:

Australia [167]

Psephotus dissimilis Collett 1898

$K^{*} \quad 318,485,794$

(=P. chrysopterygius dissimilis)

Hooded Parrot

Breeding:

Australia [167]

Psephotus haematonotus (Gould 1838)

11

485,1292

Red-rumped Parrot

Breeding:

Australia [167]

Psephotus pulcherrimus (Gould 1845)

I

E

$318,485,794,1292$

Paradise Parrot

Breeding:

Australia (ex?) [167]

Psephotus varius Clark 1910

II

485,1292

Mulga Parrot, Many-coloured Parakeet

Breeding:

Australia [167]

Pseudeos fuscata (Blyth 1858)

Dusky Lory

Breeding:

Indonesia: Irian Jaya, Salawati, Yapen; Papua New Guinea.

Psittacella brehmii Schlegel 1873

II

485,1226

Brehm's Tiger-Parrot

Breeding:

Indonesia: Irian Jaya; Papua New Guinea

Psittacella madaraszi Meyer 1886

】

485,1226

Madarasz's Tiger-Parrot

Breeding: Indonesia: Irian Jaya; Papua New Guinea

Psittacella modesta Schlegel 1873

11

485,1226

Modest Tiger-Parrot

Breeding:

Indonesia: Irian Jaya [881]; Papua New Guinea [881]

Psittacella picta Rothschild 1896

I1

485

Painted Tiger-Parrot

Breeding:

Indonesia: Irian Jaya; Papua New Guinea 
Psittacula alexandri (Linnaeus 1758)

II

$10,485,792$

Red-breasted Parakeet, Moustached Parakeet

Breeding: Bangladesh; Bhutan; Cambodia; China; India: including Andaman Islands; Indonesia: Bali, Java, ?[Kalimantan], Kangean Islands; Lao P.D.R; Malaysia: Peninsular Malaysia (ex); Myanmar; Nepal; [Singapore]; Thailand [1194]; Viet Nam

Vagrant: Hong Kong

Psittacula calthropae (Blyth 1849)

II $\quad-\quad 10,485$

Layard's Parakeet, Emerald-collared Parakeet

Breeding: Sri Lanka

Psittacula caniceps (Blyth 1846)

II $\quad \mathrm{K}^{*} \quad \mathrm{I} 0,318,485$

Nicobar Parakeet, Blyth's Parakeet

Breeding: India: Nicobar Islands

Psittacula columboides (Vigors 1830)
$\begin{aligned} & \text { Malabar Parakeet } \\ & \text { Breeding: II }\end{aligned}$
$\begin{aligned} & \text { Psittacula cyanocephala (Linnaeus 1766) } \\ & \text { Plia }\end{aligned}$

Plum-headed Parakeet

Breeding: India; Nepal; Pakistan; Sri Lanka
Psittacula derbiana (Fraser 1852)
II
10,485

Derbyan Parakeet

Breeding:

China; India

Psittacula echo (Newton \& Newton 1876)

I $\quad$ E

$47,318,324,485$

(=P. eques echo)

Mauritius Parakeet

Breeding: $\quad$ Mauritius

Psittacula eupatria (Linnaeus 1766)

Il

$10,485,661,792$

Alexandrine Parakeet

Breeding: $\quad$ Afghanistan; Bangladesh; ?Bhutan; Cambodia; India: including Andaman Islands; [Iran]; [Kuwait]; Lao P.D.R; Myanmar; Nepal; [Oman]; Pakistan; [Saudi Arabia]; Sri Lanka; Thailand [1194]; Viet Nam; [Yemen]
Psittacula exsul (Newton 1872)
II Ex
485
Newton's Parakeet
Breeding:
Mauritius: Rodrigues (ex)
Psittacula finschii (Hume 1874)
II $\quad-$
485
(= P. himalayana finschii)
Grey-headed Parakeet
Breeding:
Bangladesh; Bhutan; Cambodia; China [968]; India; Thailand [1194]; Viet Nam
Psittacula himalayana (Lesson 1832)
II
$10,485,792$
Slaty-headed Parakeet
Breeding: $\quad$ Afghanistan; India; Lao P.D.R; Nepal; Pakistan
Psittacula intermedia (Rothschild 1895)
II $\quad \mathrm{K}^{*}$
$10,318,485$
Intermediate Parakeet, Rothschild's Parakeet
Breeding:
?India [738] 
Rose-ringed Parakeet, Ring-necked Parakeet [Egypt]; Ethiopia; Gambia; [Germany]; Ghana; Guinea; Guinea-Bissau; [Hawaiian Islands] [1118]; [Hong Kong]; India; [Iran]; [Iraq]; [Israel]; [Italy]; [Japan]; [Kenya]; [Kuwait]; ?Lao P.D.R; [Lebanon]; [Macao]; Mali; Mauritania; [Mauritius]; Myanmar; Nepa]; [Netherlands]; Niger; Nigeria; [Oman]; Pakistan; [Portugal]; [Qatar]; [Saudi Arabia]; Senegal; ?Sierra Leone; [Singapore]; [South Africa]; [Spain]; Sri Lanka; Sudan; [Tanzania (Zanzibar (ex?)]; Togo; [USA]; Uganda; [United Arab Emirates]; [United Kingdom]; Viet Nam; [Yemen]

Vagrant:

Cape Verde; Somalia

Psittacula longicauda (Boddaert 1783)

$10,485,792$

Long-tailed Parakeet

Breeding:

Brunei; Coco Islands; India; including Andaman and Nicobar Islands; Indonesia: Anambas Islands, Bangka, Kalimantan, Natuna Islands, Nias, Sumatra; Malaysia: Peninsular Malaysia, Sabah, Sarawak [1295]; Myanmar; Singapore; Viet Nam

Psittacula roseata Biswas 1951

II

$10,485,792$

Blossom-headed Parakeet

Breuding: $\quad$ Bangladesh; Cambodia; ?China; India; Lao P.D.R; Myanmar; Thailand [1194]; Viet Nam

Psittaculirostris desmarestii (Desmarest 1826)

II

Large Fig-Parrot, Golden-headed Fig-Parrot, Desmarest's Fig-Parrot

Breeding: Indonesia: Batanta, Irian Jaya, Misool, Sulawati; Papua New Guinea

\begin{tabular}{|c|c|c|c|}
\hline $\begin{array}{l}\text { Psittaculirostris edwardsii (Oustalet 1885) } \\
\text { Edwards's Fig-Parrot }\end{array}$ & II & - & 485 \\
\hline Indonesia: lrian Jaya; Papua & Guinea & & \\
\hline $\begin{array}{l}\text { Psittaculirostris salvadorii (Oustalet 1880) } \\
\text { Salvadori's Fig-Parrot }\end{array}$ & $\mathrm{Il}$ & $\mathrm{K}^{*}$ & $318,485,1226$ \\
\hline Breeding: Indonesia: Irian Jaya [131] & & & \\
\hline Psittacus erithacus Linnaeus 1758 & Il & - & $212,485,496,1$ \\
\hline
\end{tabular}

(excluding subspecies princeps)

Grey Parrot

Breeding:

Angola; Benin; Burundi; Cameroon; Central African Republic; Congo; Côte d'Ivoire; Equatorial Guinea; Gabon; Ghana; Guinea [1454]; Guinea-Bissau; Kenya; Liberia; Mali; Nigeria; Rwanda; Sierra Leone; Tanzania; Togo; Uganda; Zaire
Psittacus erithacus princeps Alexander 1909
I
$485,1247,1298$
Principe Grey Parrot

Breeding: $\quad$ Equatorial Guinea: Bioko; Sao Tome and Principe: Principe, ?[Sao Tome]

Psitteuteles goldiei Sharpe 1882

II

485

(= Trichoglossus goldiei)

Goldie's Lorikeet

Breeding:

Indonesia: Irian Jaya; Papua New Guinea

(= Trichoglossus iris)

Iris Lorikeet, Iris Lory

Breeding:

Indonesia: Timor, Wetar 
Psitteuteles versicolor Lear 1831

11

485,1292

(= Trichoglossus versicolor)

Varied Lorikeet

Breeding: Australia [167]

Psittinus cyanurus (Forster 1795)

II

485,792

Blue-rumped Parrot

Breeding:

Brunei; Indonesia: Bangka, Kalimantan, Mentawaik, Rhio Archipelago, Simeulue, Siumat, Sumatra; Malaysia: Peninsular Malaysia, Sabah, Sarawak [1295]; Myanmar; Thailand; Viet Nam [1194]

Vagrant: $\quad$ Singapore

Psittrichas fulgidus (Lesson 1830)

$11 \quad-\quad 485,1226$

Pesquet's Parrot, Vulturine Parrot

Breeding:

Indonesia: Irian Jaya [881]; Papua New Guinea [881]

Purpureicephalus spurius (Kuhl 1820)

$11 \quad-\quad 485,1292$

Red-capped Parrot, Pileated Parakeet

Breeding:

Australia [167]

Pyrrhura albipectus Chapman 1914

$11 \quad \mathrm{~K}$

$318,321,485,967,1073,1152$

White-necked Parakeet, White-necked Conure, White-hreasted Parakeet

Breeding:

Ecuador

Pyrrhura calliptera (Massena \& Souancé 1854) $11 \quad$ V/R 318,321,485,967,1073,1152

Brown-breasted Parakeet, Flame-winged Conure, Flame-winged Parakeet

Breeding: Colombia

Pyrrhura cruentata (Wied 1820) I $\quad$ R $\quad 318,321,485,794,967,1073,1152$

Blue-throated Parakeet, Red-eared Conure, Ochre-marked Parakeet, Blue-chested Parakeet

Breeding: Brazil

Pyrrhura devillei (Massena \& Souancé 1854)

I1 $\quad-\quad 485,967,1073$

Blaze-winged Parakeet, Blaze-winged Conure

Breeding: Brazil; Paraguay

Pyrrhura egregia (Sclater 1881) II - $\quad 485,967,1073$

Fiery-shouldered Parakeet, Fiery-shouldered Conure

Breeding: Brazil; Guyana; Venezuela

Pyrrhura frontalis (Vieillot 1818) II - $\quad 485,967,1073$

Maroon-bellied Parakeet, Red-bellied Conure, Reddish-bellied Parakeet

Breeding:

Argentina; Brazil; Paraguay; Uruguay

Pyrrhura hoematotis Souancé 1857

II

$485,967,1073$

Red-eared Parakeet, Red-breasted Conure, Blond-eared Parakeet

Breeding:

Venezuela

Pyrrhura hoffmanni (Cabanis 1861)

II

$16,485,1073$

Sulphur-winged Parakeet, Hoffmann's Conure

Breeding:

Costa Rica; Panama

Pyrrhura leucotis (Kuhl 1820)

11

$485,967,1073$

White-eared Parakeet, White-eared Conure, Maroon-faced Parakeet

Breeding:

Brazil; Venezuela 
Pyrrhura melanura (Spix 1824)

II

$485,967,1073$

Maroon-tailed Parakeet, Maroon-tailed Conure

Breeding:

Brazil; Colombia; Ecuador; Peru; Venezuela

Byrrhura molinae (Massena \& Souancé 1854)

$11 \quad$ - $\quad 485,967,1073$

(includes $P$. hypoxantha Salvadori 1899)

Green-cheeked Parakeet, Green-cheeked Conure

Breeding:

Argentina; Bolivia; Brazil; ?Paraguay

Pyrrhura orcesi Ridgely \& Robbins 198

1I

$\mathrm{V} / \mathrm{R}$

321,485

El Oro Parakeet, El Oro Conure

Breeding:

Ecuador

Pyrrhura perlata (Spix 1824)

II

$318,485,967,1073,1152$

Pearly Parakeet, Pearly Conure

Breeding: Brazil

Pyrrhura picta (Müller 1776)

11

$16,485,967,1073$

Painted Parakeet, Painted Conure

Breeding:

Bolivia; Brazil; Colombia; Ecuador; French Guiana; Guyana; Peru; Suriname; Venezuela

Pyrrhura rhodocephala (Sclater \& Salvin 1871)

11

$485,967,1073$

Rose-headed Parakeet, Rose-crowned Conure, Rose-headed Conure

Breeding:

Venezuela

Pyrrhura rhodogaster (Sclater 1864)

11

$90,485,967,1073$

Crimson-bellied Parakeet, Crimson-bellied Conure

Breeding: Bolivia [121]; Brazil

Pyrrhura rupicola (Tschudi 1844)

11

$485,967,1073$

Black-capped Parakeet, Black-capped Conure, Rock Parakeet

Breeding:

Bolivia; Brazil [1268]; Peru

Pyrrhura viridicata Todd 1913

Il

$485,967,1073$

Santa Marta Parakeet, Santa Marta Conure

Breeding:

Colombia

Rhynchopsitta pachyrhyncha (Swainson 1827)

1

V

$16,318,321,485,794,802,832,833$

(=R.p. pachyrhyncha)

869,1073

Thick-billed Parrot

Breeding:

Mexico; [USA] [752]

Vagrant:

USA

Rhynchopsitta terrisi Moore 1947

1

V

$16,318,321,485,794,839,1073$

(=R. pachyrhyncha terrisi)

Maroon-fronted Parrot

Breeding:

Mexico

Strigops habroptilus Gray 1845

$1 \quad \mathrm{E}$

$34,155,156,318,485,767,958,976$

Kakapo

Breeding:

New Zealand: North Island (ex), South Island, Stewart Island [1507]

Tanygnathus gramineus (Gmelin 1788)

Il

485,1486

Black-lored Parrot

Breeding:

Indonesia: Buru 
Tanygnathus lucionensis (Linnaeus 1766)

II

485

Blue-naped Parrot

Breeding:

Indonesia: Maratua, Talaud Islands [1486]; Malaysia: Mantanani Island, Siamil Island; Philippines

Tanygnathus megalorynchos (Boddaert 1783)

11

485

Great-billed Parrot

Breeding:

Indonesia: Ambon, Babar, Bacan, Batanta, Buru, Flores, Gebe, Halmahera, Kalaotoa, Madu, Mayu, Misool [1486], Moluccas, Morotan, Salawati, Sangihe Islands and other small islands north of Sulawesi, Semau, Seram, Sumba, Talaud Islands, Tanahjampea, Taninibar 1slands, Timor, Tukangbesi, Waigeo; Philippines: Balut

$\begin{array}{llll}\text { Tanygnathus sumatranus (Raffles 1822) } & \text { I1 } & - & 485\end{array}$

(includes T. heterurus Salvadori 1912)

Blue-backed Parrot, Müller's Parrot

Breeding: $\quad$ Indonesia: Banggai, Butung, Muna, Sangihe, Sula Islands [881], Sulawesi and surrounding islands, Talaud Islands, Togian Islands; Philippines

Touit batavica (Boddaert 1783) $\quad$ II $\quad-\quad 485,967,1073$

Lilac-tailed Parrotlet, Seven-colored Parrotlet

Breeding: $\quad$ Guyana; Suriname; Trinidad and [Tobago]; Venezuela

$\begin{array}{llll}\text { Touit costaricensis (Cory 1913) } & \text { II } & \text { - } & 16,485\end{array}$

(= T. dilectissima costaricensis)

Red-fronted Parrotlet

Breeding: Costa Rica; Panama

Touit dilectissima (Sclater \& Salvin 1871)

II

$485,967,1073$

Blue-fronted Parrotlet

Breeding: $\quad$ Colombia; Ecuador; Venezuela

Touit huetii (Temminck 1830)

11

$485,967,1073$

Scarlet-shouldered Parrotlet

Breeding:

Bolivia [1066]; Brazil; Colombia; Ecuador; Guyana; Peru; Trinidad and Tobago (ex); Venezuela

Touit melanonotus (Wied 1820)

II $\quad$ V/R

$318,321,485,794,967,1073,1102$,

Brown-backed Parrotlet, Black-eared Parrotlet

Breeding:

Brazil

Touit purpurata (Gmelin 1788)

II

$485,967,1073$

Sapphire-rumped Parrotlet

Breeding: $\quad$ Brazil; Colombia; Ecuador; French Guiana; Guyana; Peru; Suriname; Venezuela

Touit stictoptera (Sclater 1862)

II $\quad \mathrm{K}$

$318,321,485,967,1073$

Spot-winged Parrotlet

Breeding: Colombia; Ecuador; Peru

Touit surda (Kuhl 1820)

II V/R $318,321,485,794,967,1073,1102$,

Golden-tailed Parrotlet

Breeding: Brazil 
Trichoglossus chlorolepidotus (Kuhl 1820)

Scaly-breasted Lorikeet

Breeding:

Australia [ 167]

Trichoglossus euteles (Temminck 1835)

II

485

Olive-headed Lorikeet, Perfect Lorikeet

Breeding:

Indonesia: Lesser Sunda lslands from Lomblem east to Nila and Babar [1486], Timor

Trichoglossus flavoviridis Wallace 1863

Il

485

Yellow-and-green Lorikeet, Meyer's Lorikeet

Breeding:

Indonesia: Sula Islands [1486], Sulawesi

Trichoglossus haematodus (Linnaeus 177I)

$485,1226,1292$

Rainbow Lorikeet, Green-naped Lorikeet, Rainbow Lory

Breeding:

Australia [167]; [Hong Kong]; Indonesia: Ambon, Aru Islands, Bali, Buru, Flores, Irian Jaya, Kai Islands, Kalatoa, Kisar, Lombok, Romang, Seram, Seram Laut, Sumba, Sumbawa, Tanahjampen, Tayandu, Timor, Watubela Islands, Western Papuan Islands, Wetar; New Caledonia; Papua New Guinea; Solomon Islands; Vanuatu

Trichoglossus johnstoniae Hartert 1903

II

485

Mindanao Lorikeet, Johnstone's Lorikeet

Breeding:

Philippines: Mindanao [1113]

Trichoglossus ornatus (Linnaeus 1758)

II

485

Ornate Lorikeet, Ornate Lory

Breeding: Indonesia: Sulawesi and adjacent islands [1486]

Trichoglossus rubiginosus (Bonaparte 1850) II $\quad$ - 485

Pohnpei Lorikeet, Pohnpei Lory

Breeding: $\quad$ Federated States of Micronesia: Pohnpei [1118]

Trichoglossus rubritorquis Vigors \& Horsfield 1827 II $\quad-\quad 485$

(= T. haematodus rubritorquis)

Red-collared Lorikeet

Breeding:

Australia

Triclaria malachitacea (Spix 1824)

II $\quad$ V/R

$318,321,485,967,1073,1199$

Blue-bellied Parrot, Purple-bellied Parrot

Breeding: $\quad$ Argentina [154]; Brazil [1239, 1273]

Vini australis (Gmelin 1788)

II

485,1118

Blue-crowned Lorikeet

Breeding:

American Samoa: Manua; Fiji: Lau Archipelago; Niue; Tonga; Wallis and Futuna Islands; Western Samoa: Savaii, Upoli

Vini kuhlii (Vigors 1824)

II

$\mathrm{K}^{*}$

$318,485,669,1118$

Kuhl's Lorikeet, Scarlet-breasted Lorikeet

Breeding: [Kiribati: Kiritimati, Tabueran, Teraina]; French Polynesia: Rimitara, ?Tubuai

Vini peruviana (Müller 1776)

Il $\quad \mathbf{R}$

$318,485,669,794,1118$

Tahitian Lorikeet, Blue Lorikeet

Breeding:

Cook Islands: ?[Aitutaki]; French Polynesia: Society lslands, Tuamotu Archipelago 
Vini stepheni (North 1908)

l[

$\mathrm{K}^{*}$

318,485

Stephen's Lorikeet, Henderson Lorikeet

Breeding:

Pitcaim 1slands: Henderson Island

Vini ultramarina (Kuhl 1820)

II

$\mathbf{R}$

$79,318,485,669,794$

Ultramarine Lorikeet, Ultramarine Lory

Breeding: $\quad$ French Polynesia: Nukuhiva, [Uahuka], Uapou [1118]

\section{Order CUCULIFORMES}

Cuckoos and allies

Family MUSOPHAGIDAE

Turacos

Corythaeola cristata (Vieillot 1816) III GH - $\quad 212,496,1247,1298$

Great Blue Turaco, Blue Plaintain-eater

Breeding: $\quad$ Angola; Benin; Burundi; Cameroon; Central African Republic; Congo; Côte d'Ivoire; Equatorial Guinea [1454]; Gabon; Ghana; Guinea; Guinea-Bissau; Kenya; Liberia; Nigeria; Rwanda; Sierra Leone; Sudan; Tanzania; Togo; Uganda; Zaire
Crinifer piscator (Boddaert 1783)
$111 \mathrm{GH}$
$496,1247,1298$

Western Grey Plaintain-eater, Grey Plantain-eater

Breeding: $\quad$ Benin; Burkina Faso [1388]; Cameroon; Central African Republic; Chad; Congo; Côte d'Ivoire; Gambia; Ghana; Guinea [1454]; Guinea-Bissau; ?Liberia; Mali; Mauritania; Niger; Nigeria; Senegal; Sierra Leone; Togo; Zaire
Musophaga porphyreolopha (Vigors 1831)
II
$212,285,496,1298$

(= Tauraco porphyreo'ophus)

Purple-crested Turaco, Violet-crested Turaco

Breeding: $\quad$ ?Burundi; Kenya; Malawı; Mozambique; Rwanda; South Africa; Swaziland; Tanzania; Uganda; Zambia; Zimbabwe

Musophaga violacea Isert 1789

IIl GH - $\quad 1247,1298$

Violet Turaco, Violet Plantain-eater

Breeding: $\quad$ Benin; Burkina Faso; Cameroon; Central African Republic; Chad; Côte d'Ivoire; Gambia; Ghana; Guinea [1454]; Guinea-Bissau; ?Liberia; Mali; Niger; Nigeria; Senegal; Sierra Leone; Togo

Tauraco bannermani (Bates 1923)

$\mathrm{E}$

$318,324,496,888,1247,1298$

Bannerman's Turaco

Breeding:

Carneroon

Tauraco corythaix (Wagler 1827)

(= T. persa corythaix)

Knysna Turaco

Breeding: South Africa; Swaziland

Tauraco fischeri (Reichenow 1878)

Il

496,1298

(= T. corythaix fischeri)

Fischer's Turaco

Breeding:

Kenya; Somalia; Tanzania 
Tauraco livingstonii (Gray 1864)

Il

496,1298

(=T. corythaix livingstonii)

Livingstone's Turaco

Breeding:

Burundi; Malawi; Mozambique; South Africa; Tanzania

Tauraco macrorhynchus (Fraser 1839) IIl GH - 496,1247,1298

Yellow-billed Turaco, Crested Turaco, Verreaux's Turaco, Black-tip Crested Turaco

Breeding: ?Angola; Cameroon; Congo; Côte d'Ivoire; Equatorial Guinea: Bioko; Gabon; Ghana; Guinea; Liberia; ?Nigeria; Sierra Leone; Zaire

Tauraco persa (Linnaeus 1758)

II

$496,1247,1298$

( $=T$. corythaix persa)

Guinea Turaco

Breeding:

Angola; Benin; Cameroon; Congo; Côte d'Ivoire; Equatorial Guinea; Gabon; Gambia; Ghana; Guinea [1454]; Guinea-Bissau; Liberia; Mali; ?Nigeria; Senegal; Sierra Leone; Togo; Zaire

Tauraco ruspolii (Salvadori 1896)

R

$318,324,496,1298$

Ruspoli's Turaco

Breeding:

Ethiopia

Tauraco schalowi (Reichenow 1891)

Il $\quad-\quad 496,1298$

(=T. corythaix schalowi)

Schalow's Turaco

Breeding: Angola; Botswana; Burundi; Kenya; Malawi; ?Namibia; Tanzania; Zaire; Zambia; Zimbabwe

Tauraco schuettii (Cabanis 1879)

I1

496,1298

(= T. corythaix schuettii)

Black-billed Turaco

Breeding:

Angola; Burundi; Central African Republic; Congo; Kenya; Rwanda; Sudan; Tanzania; Uganda; Zaire

Family CUCULIDAE

Cuckoos, Coucals

Carpococcyx radiceus (Temminck 1832) - $\quad \mathrm{K}^{*} \quad 318,1295$

Sunda Ground-Cuckoo, Malay Ground-Cuckoo

Breeding: $\quad$ Brunei; Indonesia: Kalimantan, Sumatra; Malaysia: Sabah, Sarawak [1295]

$\begin{array}{lllll}\text { Centropus chlororhynchus Blyth } 1849 & - & K^{*} & 10,318\end{array}$

Green-billed Coucal

Breeding: $\quad$ Sri Lanka [659]

Centropus nigrorufus (Cuvier 1817) $\quad-\quad K^{*} \quad 318$

Sunda Coucal, Javan Coucal

Breeding: Indonesia: Java, ?Sumatra

$\begin{array}{lllll}\text { Centropus rectunguis } & \text { Strickland } 1847 & - & \mathrm{K}^{*} & 318\end{array}$

Short-toed Coucal

Breeding: $\quad$ Brunei; Indonesia: Kalimantan [664], Sumatra [905]; Malaysia: Peninsular Malaysia, Sabah [1295], Sarawak [1295]

Centropus steerii Bourns \& Worcester 1894

$K^{*} \quad 318$

Black-hooded Coucal, Steere's Coucal

Breeding: Philippines: Mindoro 
Chrysococcyx rufomerus Hartert 1900

K* $\quad 318,1486$

(= Chalcites malayanus rufomerus)

Green-cheeked Bronze-Cuckoo

Breeding: Indonesia: Damar, Romang

Coua delalandei (Temminck 1827)

Ex

318,324

Delalande's Coua, Snaileating Coua

Breeding:

Madagascar (ex) [373]

Hyetornis rufigularis (Hartlaub 1852)

Id

$16,179,321$

Rufous-breasted Cuckoo, Bay-breasted Cuckoo

Breeding:

Dominican Republic, Haiti

Neomorphus radiolosus Sclater \& Salvin 1878

V

318,321

Banded Ground-Cuckoo

Breeding:

Colombia $[55,655,1010]$; Ecuador

Phaenicophaeus pyrrhocephalus (Pennant 1769)

K*

10,318

Red-faced Malkoha

Breeding:

Sri Lanka [659]

Vagrant:

?India

\section{Order STRIGIFORMES \\ Owls}

Family TYTONIDAE

Barn Owls

Phodilus badius (Horsfield 1821)

II $\quad$ - $\quad 10,15,247,792$

Oriental Bay-Owl, Bay Owl

Breeding:

?Bangladesh; ?Bhutan; Brunei; China; India; Indonesia; Lao P.D.R; Malaysia: Peninsular Malaysia, Sabah, Sarawak [1295]; Myanmar; Singapore (ex); Sri Lanka; Thailand; Viet Nam

Vagrant: $\quad$ ?Nepal; Philippines: Samar

Phodilus prigoginei Schouteden 1952

II Id

$15,247,318,324$

Congo Bay-Owl, Itombwe Owl

Breeding:

?Burundi; Zaire

Tyto alba (Scopoli 1769)

II

$10,15,16,179,212,244,247,285$,

$342,496,661,792,967,1247$

\section{Barn Owl, Common Barn-Owl}

Breeding:

Albania; Algeria; American Samoa [1118]; Andorra; Angola; Argentina; Australia [167];

Austria; Bahamas; Bahrain; Bangladesh; Belarus; Belgium; Belize; Benin; Bermuda; ?Bhutan; Bolivia; Botswana; Brazil; Bulgaria; Burkina Faso [1388]; Burundi; Cambodia; Cameroon; Canada [1321\}; Canary Islands; Cape Verde; Cayman Islands; Central African Republic; Chad; Chile 1715]; China; Colombia; Comoros; Congo; Costa Rica; Côte d'Ivoire; Cuba; Cyprus; Czechoslovakia; Denmark; Dominica; Dominican Republic; Ecuador; Egypt; El Salvador; Equatorial Guinea; Ethiopia; ?Falkland Islands; Fiji [1118]; France; French Guiana; Gabon; Galapagos Islands; Gambia; Germany; Ghana; Gibraltar; Greece; Grenada; Guatemala; Guernsey; Guinea [1454]; Guinea-Bissau; Guyana; Haiti; [Hawaiian Islands] [1118]; Honduras; Hungary; India: including Andaman Islands; 
Indonesia; Iran; 1raq; Ireland; 1srael; 1taly; Jamaica; Jersey; Jordan [192]; Kenya; ?Kuwait; Lao P.D.R; Latvia; Lebanon; ?Lesotho; Liberia; ?Libya; Liechtenstein; Lithuania; [Lord Howe Island]; Luxembourg; Madagascar [373]; Madeira; Malawi; Malaysia: Peninsular Malaysia; Mali; Malta; Mauritania; Mayotte; Mexico; Moldova; Morocco; Mozambique; Myanmar; Namibia; Nepal; Netherlands; Netherlands Antilles: Bonaire, Curaçao; Nicaragua; Niger; Nigeria; Niue [1118]; Oman; Pakistan; Panama; Papua New Guinea; Paraguay; Penu; Poland; Portugal; Romania; Rwanda; Sao Tome and Principe; Saudi Arabia; Senegal; [Seychelles] [845]; Sierra Leone; Singapore; Solomon Islands; Somalia; South Africa; Spain; Sri Lanka; Saint Lucia; Saint Vincent; Sudan; Suriname; Swaziland; Sweden; Switzerland; ?Syria; Tanzania; Thailand [1194]; Togo [280]; Tonga [1118]; Trinidad and Tobago; Tunisia; Turks and Caicos Islands; USA [1321]; Uganda; Ukraine; United Arab Emirates; United Kingdom; Uruguay; Vanuatu; Venezuela; Viet Nam; Wallis and Futuna Islands [1118]; Western Sahara; Western Samoa [1118]; Yemen; Yugoslavia; Zaire; Zambia; Zimbabwe

Non-breeding: Libya; Syria

Vagrant:

Azores; Estonia; Finland; Kuwait; New Zealand; Norway; Puerto Rico; Turkey

Tyto aurantia (Salvadori 1881) II $\quad K^{*} \quad 15,247,318,1226$

Bismarck Masked-Owl, New Britain Masked-Owl, Golden Owl

Breeding: $\quad$ Papua New Guinea: New Britain

Tyto capensis (Smith 1834)

11

$15,212,247,285,496,1247$

African Grass-Owl

Breeding:

Angola; Burundi; Cameroon; Congo; Ethiopia [1398]; Kenya; Lesotho; Malawi; Mozambique; Namibia; Rwanda; South Africa; Swaziland; Tanzania; Uganda; Zaire; Zambia; Zimbabwe

Tyto castanops (Gould 1837)

II

( $=T$. novaehollandiae castanops)

Tasmanian Masked-Owl

Breeding: Australia

Tyto glaucops (Kaup 1852)

11

15,16

(=T. alba glaucops)

Ashy-faced Owl

Breeding:

Dominican Republic; Haiti

Tyto inexspectata (Schlegel 1879)

$11 \quad \mathrm{~K}^{*}$

$15,247,318,1486$

Minahassa Masked-Owl, Minahassa Owl, Minahassa Barn-Owl

Breeding:

Indonesia: Sulawesi [881]

Tyto longimembris (Jerdon 1839)

II

$10,15,247,881,1226$

(=T. capensis longimembris)

Eastern Grass-Owl

Breeding:

Australia [167]; Bangladesh; China; Fiji: Viti Levu (ex) [1118]; India; Indonesia: Flores, Irian Jaya, Sulawesi; Myanmar; Nepal; New Caledonia; Papua New Guinea; Philippines; Taiwan; Viet Nam

Vagrant: $\quad$ ?Hong Kong; Japan

Tyto manusi Rothschild \& Hartert 1914

(=T. novaehollandiae manusi),

Manus Masked-Owl

Breeding:

Papua New Guinea: Manus Island 
Tyto multipunctata Mathews 1912

Tyto nigrobrunnea Neumann 1939

Taliabu Masked-Owl, Sula Barn-Owl, Taliabu Ow!

Breeding: Indonesia: Taliabu

Tyto novaehollandiae (Stephens 1826)

Australian Masked-Owl

Breeding:

Australia [167]; Indonesia: Irian Jaya; Papua New Guinea

Tyto rosenbergii (Schlegel 1866)

Sulawesi Owl

Breeding:

Indonesia: Peleng [1486], Sangihe, Sulawesi

Tyto sororcula (Sclater 1883)

II $\quad \mathrm{K}^{*}$

$15,247,318,1486$

(= $T$. novaehollandiae sororcula)

Lesser Masked-Owl, Tanimbar Owl

Breeding: Indonesia: Buru, Tanimbar Islands

Tyto soumagnei (Milne-Edwards 1878)

1 Id

$15,247,318,324$

Soumagne's Owl, Madagascar Red Owl, Madagascar Owl

Breeding:

Madagascar (ex?) [373]

Tyto tenebricosa (Gould 1845)

1I

Greater Sooty-Owl

Breeding:

Australia [167]; Indonesia: Irian Jaya, Yapen; Papua New Guinea

Family STRIGIDAE

Typical owls

Aegolius acadicus (Gmelin 1788)

II

$15,16,247$

Northern Saw-whet Owl, Saw-whet Owl

Breeding:

Canada; Mexico; USA

Vagrant:

Bermuda

Aegolius funereus (Linnaeus 1758)

II

$15,16,247,342$

Boreal Owl, Tengmalm's Owl

Breeding:

Andorra; Austria; Belarus; Belgium; Bulgaria; Canada; China; Czechoslovakia; ?Denmark; Estonia; Finland; France; Germany; Greece; Italy; India; Japan; Kazakhstan; Kyrgyzstan; Latvia; Liechtenstein; Lithuania; Mongolia; Netherlands; Norway; Poland; ?Romania; Russia; Spain; Sweden; Switzerland; ?Tajikistan; ?Turkey [661]; Ukraine; USA

Non-breeding: Japan; Saint Pierre and Miquelon

Vagrant: $\quad$ Hungary; Luxembourg; Turkey; United Kingdom

Aegolius harrisii (Cassin 1849)

II

$15,247,967$

Buff-fronted Owl

Breeding:

Argentina; Bolivia; Brazil; Colombia; Ecuador; Paraguay; Peru; Uruguay; Venezuela

Aegolius ridgwayi (Alfaro 1905)

II

$15,16,247$

Unspotted Saw-whet Owl

Breeding:

Vagrant:

Costa Rica; El Salvador; Guatemala; Mexico

Panama 
(= A. otus abyssinicus)

Abyssinian Owl

Breeding:

Ethiopia; Kenya; Uganda; Zaire

Asio capensis (Smith 1834)

II $\quad-$

$15,212,247,285,496,661,1247$

\section{Marsh Owl, African Marsh Owl}

Breeding: $\quad$ Algeria (ex); Angola; Benin; Botswana; Burkina Faso; Burundi; Cameroon; Chad; Congo; Ethiopia; Kenya; ?Lesotho; Madagascar [373]; Malawi; Mali; Morocco; Mozambique; Namibia; Niger; Nigeria; Rwanda; South Africa; Sudan; Swaziland; Tanzania; Uganda; Zaire; Zambia

Non-breeding: Canary lslands; Gambia

Vagrant: $\quad$ Algeria; Portugal; Senegal; Spain

$\begin{array}{llll}\text { Asio clamaror (Vieillot 1807) } & 11 & - & 15,247,967\end{array}$

(= Rhinoptynx clamator)

Striped Owl

Breeding: $\quad$ Argentina; Belize; Bolivia; Brazil; Colombia; Costa Rica; Ecuador; El Salvador; French Guiana; Guatemala; Guyana; Honduras; Mexico; Nicaragua; Panama; Paraguay; Peru; Suriname; Trinidad and Tobago: Tobago; Uruguay; Venezuela

Asio flammeus (Pontoppidan 1763)

$11 \quad-$

$10,15,16,179,247,342,496,792$, 967,1247

\section{Short-eared Owl}

Breeding:

Argentina [977,1034]; Armenia; Austria; Azerbaijan; Belarus; Belgium; Bolivia [956]; Brazil [139,1107]; Bulgaria; Canada; Chile [82,218]; China; Colombia; Cuba [511]; Czechoslovakia; Denmark; Dominican Republic [1326]; Ecuador [248]; Estonia; Falkland lslands; Federated States of Micronesia: Pohnpei [1118]; Finland; France; French Guiana; Galapagos Islands [248,614]; Georgia; Germany; Greece; Guyana [1303]; Haiti; Hawaiian Islands; Hungary; Iceland; Ireland; Israel; Kazakhstan; Kyrgyzstan; Latvia; Lithuania; ?Madeira; Moldova; Mongolia; Netherlands; Norway; Paraguay; Peru [1064]; Poland; Puerto Rico; Romania; Russia; Saint Pierre and Miquelon; Spain; Suriname [402]; Sweden; Turkey; Turkmenia; Ukraine; USA; United Kingdom; Uruguay [354,557]; Uzbekistan; Venezuela [969]

Non-breeding: $\quad$ Afghanistan; Albania; Algeria; Bahrain [243]; Bangladesh; ?Chad; Cyprus; Egypt; Ethiopia; Faeroe Islands; Guernsey; India; Iran [661]; Iraq; Italy; Japan [1494]; Jersey; D.P.R. Korea; Korea Republic; Kuwait [243]; Lao P.D.R; Lebanon; Libya; Luxembourg; Maldives; Mali [819]; Malta; Mauritania; Mexico [165,1089]; Morocco; Myanmar [1296]; Nepal; Oman; Pakistan; Portugal; Qatar [243]; Saudi Arabia; Senegal; Sudan; Switzerland; Taiwan [1253]; Tajikistan; Tunisia [1387]; United Arab Emirates [243]; Viet Nam; Yugoslavia

Vagrant: $\quad$ Azores; Bermuda; Brunei; Canary Islands; Cape Verde Islands; Costa Rica [1285]; Gibraltar; Greenland; Guam; Guatemala [824]; Guinea [984]; Hong Kong; Jordan [192]; Kenya [212]; Liechtenstein; Malaysia: Peninsular Malaysia, Sarawak [1295]; Marshall Islands; Mauritania; Niger; Northern Marianas [1118]; Philippines [381 , 1113]: Guadeloupe: Saint Barthelemy; Singapore; Somalia [101]; Sri Lanka; Svalbard and Jan Mayen Islands; Syria; Thailand; Turks and Caicos Islands [236]; Uganda; Virgin Islands of the United States [1347]; Yemen 
Long-eared Owl

Breeding:

Non-breeding:

Vagrant:
Afghanistan; Albania; Algeria; Andorra; Armenia; Austria; Azerbaijan; Azores; Belarus; Belgium; Bulgaria; Canada; Canary Islands; China; ?Cyprus; Czechoslovakia; Denmark; Estonia; Ethiopia; Finland; France; Georgia; Germany; Greece; Guemsey; Hungary; India; Iran; Ireland; Israel; Italy; Japan; Kazakhstan; Kenya; Kyrgyzstan; Latvia; Liechtenstein; Lithuania; Luxembourg; Mexico; Moldova; Mongolia; Morocco; Netherlands; Norway; Pakistan; Poland; Portugal; Romania; Russia; Spain; Sweden; Switzerland; Tajikistan; Tunisia; Turkey; Turkmenja; Ukraine; USA; United Kingdom; Uzbekistan; Yugoslavia Afghanistan; Egypt; Iraq; Jersey; Jordan; D.P.R. Korea; Korea Republic; Malta; Taiwan Bermuda; Cuba [179]; Faeroe lslands; Gibraltar; Hong Kong; Iceland; Iraq; Lao P.D.R; Lebanon; Libya; Myanmar; Nepal; Oman; Saudi Arabia; Saint Pierre and Miquelon; Syria; United Arab Emirates
Asio stygius (Wagler 1832)
I1
$15,16,179,247,967$

Stygian Owl

Breeding:

Argentina; Belize; Bolivia [122]; Brazil; Colombia; Cuba; Dominican Republic; Ecuador; Guatemala; Haiti; Mexico; Nicaragua; Paraguay; Venezuela
Athene blewitti (Hume 1873)
$1 \quad$ ld
$10,15,247,318,794$
Forest Owlet, Forest Little Owl
Breeding:
India (ex?)
Athene brama (Temminck 1821)
11
$10,15,247,661,792,1544$

Spotted Owlet, Spotted Little Owl

Breeding:

Bangladesh; Bhutan; Cambodia; India; Iran; Lao P.D.R; Myanmar; Nepal; Pakistan; Thailand [1194]; Viet Nam

Athene noctua (Scopoli 1769)

11

$10,15,247,342,496,522,661,1247$

Little Owl, Northern Little Owl, Tihet Owlet

Breeding:

Afghanistan; Albania; Algeria; Andorra; Armenia; Austria; Azerbaijan; Belarus; Belgium; Bulgaria; Chad; China; Cyprus; Czechoslovakia; Denmark; Djibouti; Egypt; Ethiopia; France; Georgia; Germany; Gibraltar; Greece; Hungary; India; Iran; 1raq; Israel; Italy; Jordan; Kazakhstan; Korea Republic; ?Kuwait; Kyrgyzstan; Latvia; Lebanon; Libya; Liechtenstein (ex?); Lithuania; Luxembourg; Mali; Mauritania; Moldova; Mongolia; Morocco; Nepal; Netherlands; [New Zealand]; ?Nigeı; Oman; Pakistan; Poland; Portugal; Romania; Russia; Saudi Arabia; Somalia; Spain; Sudan; Switzerland; Syria; Tajikistan; Tunisia; Turkey; Turkmenia [1339]; Ukraine; United Arab Emirates; [United Kingdom]; Uzbekistan; Western Sahara; Yemen; Yugoslavia

Vagrant: $\quad$ Finland; Ireland; Jersey; D.P.R. Korea; Malta; Norway; Sweden

Bubo africanus (Temminck 1821)

$15,212,247,285,496,661,1247$

\section{Spotted Eagle-Owl, African Eagle-Owl}

Breeding:

Angola; Benin; Botswana; Burundi; Cameroon; Central African Republic; Chad; Congo; Côte d'Ivoire; ?Djibouti; Equatorial Guinea; Ethiopia; Gabon; Ghana; Guinea; Guinea-Bissau; Kenya; ?Lesotho; Liberia; Malawi; Mali; ?Mauritania; Mozambique; Namibia; Niger; Nigeria; Oman; Rwanda; Saudi Arabia; Senegal; Sierra Leone; Somalia; South Africa; Sudan; Swaziland; Tanzania; Togo; Uganda; Yemen; Zaire; Zambia; Zimbabwe

Non-breeding: Gambia 
(= B. bubo ascalaphus)

Pharaoh Eagle-OwI

Breeding:

Algeria; Chad; Egypt; Ethiopia; Iraq; Israel; Jordan; Kuwait; Libya; Mali; Mauritania; Morocco; Niger; Oman; Sajdi Arabia; Sudan; Tunisia; United Arab Emirates; Western Sahara

Vagrant:

Senegal

Bubo bengalensis (Franklin 1831)

II

$10,15,1149$

(= B. bubo bengalensis)

Rock Eagle-Ow]

Breeding:

Bangladesh; Bhutan; India; Myanmar; Nepal; Pakistan

Bubo bubo (Linnaeus 1758)

II

$10,15,247,342$

Eurasian Eagle-Owl

Breeding:

?Afghanistan; Albania; Andorra; Armenia; Austria; Azerbaijan; Belarus; Belgium; Bulgaria; China; Czechoslovakia; Estonia; Finland; France; Georgia; Germany; Greece; Hong Kong; Hungary; India; Iran; Iraq; Israel; Italy; Japan; Kazakhstan; D.P.R. Korea; Korea Republic; Kyrgyzstan; Latvia; Lebanon; Liechtenstein; Lithuania; Luxembourg; Moldova; Mongolia; Netherlands; Norway; Pakistan; Poland; Portugal; Romania; Russia; Spain; Sweden; Switzerland; Syria; Tajikistan; Turkey; Turkmenia; Ukraine; Uzbekistan; Yugoslavia

Vagrant: Denmark; Gibraltar; United Kingdom

Bubo capensis Smith 1834

\section{II}

$15,212,247,285,496$

Cape Eagle-Owl, Mackinder's Eagle-OwI, Mountain Eagle-Owl

Breeding:

Ethiopia; Kenya; ?Lesotho; Malawi; Mozambique [708]; Namibia [920,1456]; South Africa; Swaziland; Tanzania; Uganda; Zimbabwe

Bubo coromandus (Latham 1790)

$11 \quad-\quad 10,15,247,792$

Dusky Eagle-OwI

Breeding:

Bangladesh; ?Bhutan; India; Myanmar; Nepal; Pakistan; ?Thailand [1 194]

Vagrant:

China; Malaysia: Peninsular

Bubo lacteus (Temminck 1820)

II

$15,212,247,285,496,1247$

Verreaux's Eagle-Owl, Giant Eagle-Owl, Milky Eagle-Owl

Breeding:

Angola; Benin; Botswana; Burkina Faso [1388]; Burundi; Cameroon; Central African Republic; Chad; Côte d'lvoire; Ethiopia; Gambia; Ghana; Guinea; Guinea-Bissau; Kenya; Lesotho (ex); Liberia; Malawi; Mali; Mauritania; Mozambique; Namibia; Niger; Nigeria; Rwanda; Senegal; Somalia; South Africa; Sudan; Swaziland; Tanzania; Togo; Uganda; Zaire; Zambia; Zimbabwe

Bubo leucostictus Hartlaub 1855

II

$15,247,496,1247$

Akun Eagle-Owl, Sooty Eagle-Owl

Breeding:

Angola; Cameroon; Congo; Côte d'Ivoire; Equatorial Guinea; Gabon; Ghana; Guinea; Liberia; ?Nigeria; Sierra Leone; Zaire

Bubo nipalensis Hodgson 1836

Spot-bellied Eagle-Owl, Forest Eagle-OwI

Breeding:

Bangladesh; Bhutan; Cambodia; India; Lao P.D.R; Myanmar; NepaI; Sri Lanka; Thailand [1194; Viet Nam

Vagrant:

China

Bubo philippensis (Kaup 1851)

(= Pseudoptynx philippensis)

Philippine Eagle-Owl

Breeding

Philippines [ 11113 ] 
(includes $B$. vosseleri Reichenow 1908)

Fraser's Eagle-Owl, (Usambara Eagle-Owl)

Breeding: $\quad$ Angola; Cameroon; Central African Republic; Congo; Côte d'lvoire; Equatorial Guinea; Gabon; Ghana; Guinea; Liberia; Nigeria; Sierra Leone; Tanzania; Uganda; Zaire

Bubo shelleyi (Sharpe \& Ussher 1872)

II

$15,247,496,1247$

\section{Shelley's Eagle-Owl, Banded Eagle-Owl}

Breeding: $\quad$ Cameroon; Côte d'Ivoire; Gabon; Ghana; Guinea; Liberia; Zaire
Bubo sumatranus (Raffles 1822)
1]
$15,247,792$

\section{Barred Eagle-Owl}

Breeding:

Brunei; Indonesia: Bali, Java, Kalimantan, Sumatra; Malaysia: Peninsular Malaysia, Sabah, Sarawak [1295]; Myanmar; Singapore (ex); Thailand [1194]

Vagrant: Cocos (Keeling) Islands
Bubo virginianus (Gmelin 1788)
II
$15,16,247,967$

\section{Great Horned Owl}
Breeding:
Argentina; Belize; Bolivia; Brazil; Canada; Chile [715]; Colombia; Costa Rica; Ecuador; El Salvador; ?French Guiana; Guatemala; Guyana; Honduras; Mexico; Nicaragua; Paraguay; Peru; Suriname; USA; Uruguay; Venezuela
Non-breeding: $\quad$ Saint Pierre and Miquleon
Vagrant: $\quad$ Bermuda; Falkland Islands; Panama

Glaucidium albertinum Prigogine 1983

$11 \quad \mathrm{R}$

$15,318,324,496$

Albertine Owlet, Prigogine's Owlet

Breeding:

Rwanda; Zaire

Glaucidium brasilianum (Gmelin 1788)

II

$15,16,247,967$

Ferruginous Pygmy-Owl

Breeding:

Argentina; Belize; Bolivia; Brazil; Chile [715]; Colombia; Costa Rica; Ecuador; El Salvador; French Guiana; Guatemala; Guyana; Honduras; Mexico; Nicaragua; Panama; Paraguay; Peru; Suriname; Trinidad and Tobago: Trinidad; USA; Uruguay; Venezuela

Glaucidium brodiei (Burton 1836)

II

$10,15,247,792$

Collared Owlet, Collared Pygmy-Owl

Breeding:

Afghanistan; Bangladesh; Bhutan; Brunei; China; India; Indonesia: Kalimantan, Sumatra; Lao P.D.R; Malaysia: Peninsular Malaysia, Sabah, Sarawak [1295]; Myanmar; Nepal; Pakistan; Taiwan; Thailand [1194]; Viet Nam

Glaucidium californicum Sclater 1857

II

16

(= G. gnoma californicum),

Northern Pygmy-Owl

Breeding:

Canada; Mexico; USA

Glaucidium capense (Smith I834)

$15,212,247,285,496$

African Barred Owlet, Barred Owlet

Breeding: $\quad$ Angola; Botswana; Malawi; Mozambique; Namibia; South Africa; Swaziland; Tanzania; Zambia; Zimbabwe

Vagrant: Rwanda

Glaucidium castaneum Neumann 1893

Il 496

(= G. capense castaneum)

Chestnut Owlet

Breeding:

Côte d'lvoire; Liberia 
Glaucidium castanonotus (Blyth 1846)

(= G. radiatum castanonotus)

Chestnut-backed Owlet

Breeding:

Sri Lanka

Glaucidium castanopterum (Horsfield 1821)

Javan Owlet

Breeding:

Indonesia: Bali, Java
II

10

Glaucidium cuculoides (Vigors 1831)

Asian Barred Owlet, Cuckoo Owlet

II

$10,15,247,792$

Breeding: $\quad$ Bangladesh; Bhutan; Cambodia; China; Hong Kong (ex); India; Lao P.D.R; Myanmar; NepaI; Pakistan; Thailand [1194]; Viet Nam

$\begin{array}{llll}\text { Glaucidium gnoma Wagler } 1832 & 11 & - & 15,16,247\end{array}$

Mountain Pygmy-OwI

Breeding: $\quad$ Belize [338]; Guatemala; Honduras; Mexico; USA

Glaucidium jardinii (Bonaparte 1855)

II

$15,16,967$

Andean Pygmy-Owl

Breeding: $\quad$ Argentina; Bolivia; Colombia; Costa Rica; Ecuador; Panama; Peru; Venezuela

$\begin{array}{llll}\text { Glaucidium minutissimum (Wied 1821) } & \text { II } & - & 15,16,247.967\end{array}$

Least Pygmy-Owl

Breeding:

Belize; Bolivia [1066]; Brazil; Colombia; Costa Rica; Guatemala [127]; Guyana; Honduras; Mexico; Panama; Paraguay; Peru; Suriname

Glaucidium ngamiense (Roberts 1932) $\quad$ II $\quad-\quad 286,496$

( $=$ G. capense ngamiense)

Breeding: $\quad$ Angola; Botswana; Namibia; Zaire; Zambia

Glaucidium passerinum (Linnaeus 1758)

11

$15,247,342$

Eurasian Pygmy-Owl

Breeding:

Austria; Belarus; China; Czechoslovakia; Estonia; Finland; France; Germany; Italy; Latvia; Liechtenstein; Lithuania; Mongolia; Norway; Poland; Romania; Russia; Sweden; Switzerland; Yugoslavia

Vagrant: $\quad$ Belgium; Bulgaria; Denmark; Spain; Ukraine

Glaucidium perlasum (Vieillot 1818) 은 $\quad$ ll $\quad 15,212,247,285,496,1247$

Pearl-spotted Owlet

Breeding: $\quad$ Angola; Benin; Botswana; ?Burkina Faso; Burundi; Cameroon; Central African Republic; Chad; Côte d'lvoire; ?Djibouti; Ethiopia; Gambia; Ghana; Guinea [1454]; Guinea-Bissau; Kenya; Malawi; Mali; Mozambique; Namibia; Niger; Nigeria; Rwanda; Senegal; Somalia; South Africa; Sudan; Swaziland; Tanzania; Togo; Uganda; Zaire; Zambia; Zimbabwe

Glaucidium peruanum Kōnig 1992

II

Breeding: $\quad$ Ecuador; Peru

Glaucidium radiatum (Tickell 1833 )

II

$10,15,247$

Jungle Owlet, Barred Jungle Owlet

Breeding:

Bangladesh; ?Bhutan; India; Myanmar; Nepal [700] 
Glaucidium siju (d'Orbigny 1839)

Cuban Pygmy-OwI

Breeding:

Cuba

Glaucidium sjostedti Reichenow 1893

Sjostedt's Owlet

Breeding:

Cameroon; Congo; Equatorial Guinea; Gabon; Zaire

Glaucidium tephronotum Sharpe 1875

II

$15,212,247,496,1247$

Red-chested Owlet, Yellow-legged Owlet

Breeding: $\quad$ Cameroon; Congo; Côte d'Ivoire; Ghana; Kenya; Liberia; Uganda; Zaire

Jubula lettii (Būttikofer 1889)

II

$15,247496,1247$

(= Lophostrix lettii)

Maned Owl

Breeding:

Cameroon; Central African Republic; Congo; Côte d'Ivoire; Equatorial Guinea; Gabon [22I]; Ghana; Liberia; ?Nigeria; Sierra Leone; Zaire

$\begin{array}{llll}\text { Ketupa blakistoni (Seebohm 1884) II } & \text { K* } & 15,247,3 \text { I8 }\end{array}$

(= Bubo blakistoni)

Blakiston's Fish-Owl

Breeding:

China; Japan: Hokkaido [201]; Russia [185,481,799]

Ketupa flavipes (Hodgson I836)

II

$10,15,247,792$

(= Bubo flavipes)

Tawny Fish-OwI

Breeding:

Bangladesh; Bhutan; China; India; Lao P.D.R; Myanmar; Nepal; Taiwan; Viet Nam

Ketupa ketupu (Horsfield I82I)

II

$10,15,247,792$

(= Bubo ketupu)

Buffy Fish-Owl, Malay Fish-Owl

Breeding:

Brunei; India (ex); Cambodia; Indonesia; Lao P.D.R; Malaysia: Peninsular Malaysia, Sabah, Sarawak [1295]; Myanmar; ?Singapore (ex); Thailand [1194]; Viet Nam

Vagrant:

Cocos (Keeling) Islands

Ketupa zeylonensis (Gmelin 1788)

II

$\mathrm{I} 0,15,247,342,66 \mathrm{I}, 792$

(= Bubo zeylonensis)

Brown Fish-OwI

Breeding:

Bangladesh; ?Bhutan; Cambodia; China; India; Iran; ?Iraq; Israel; ?Jordan; Lao P.D.R; Malaysia: Peninsular Malaysia; Myanmar; Nepal; Pakistan; Sri Lanka; ?Syria; Thailand [1194]; Turkey [891]; Viet Nam

Non-breeding: Hong Kong

Vagrant: Lebanon

Lophostrix cristata (Daudin I800)

II

$15,16,247,967$

Crested Owl

Breeding:

Bolivia; Brazil; Colombia; Costa Rica; Ecuador; EI Salvador; French Guiana; Guatemala; Guyana; Honduras; Mexico; Nicaragua; Panama; Peru; Suriname; Venezuela 


$\begin{aligned} & \text { Micrathene whitneyi (Cooper 1861) } \\ & \text { EIf Owl } \\ & \text { Breeding: }\end{aligned}$ Mexico; USA
$\begin{aligned} & \text { Mimizuku gurneyi (Tweeddale 1879) } \\ & \text { (= Otus gurneyi) }\end{aligned}$
$\begin{aligned} & \text { Giant Scops-Owl, Lesser Eagle-Ow] } \\ & \text { Breeding: } \quad \text { Philippines: Marinduque [1113], Mindanao }\end{aligned}$
$\begin{aligned} & \text { Nesasio solomonensis (Hartert 1901) } \\ & \text { Il }\end{aligned}$

Fearful OwI

Breeding: $\quad$ Papua New Guinea: Bougainville; Solomon Islands: Choiseul, Santa Isabel

Ninox affinis Beavan 1867

II

$10,15,247$

Andaman Hawk-Owl

Breeding: India: Andaman and Nicobar Islands

Ninox boobook (Latham 1801)

II

(= N. novaeseelandiae boobook)

Southern Boobook

Breeding: Australia

Ninux connivens (Latham 1801)

II $\quad-\quad 15,247$

Barking Owl, Winking Owl

Breeding: $\quad$ Australia [167]; Indonesia: Bacan, Halmahera, Irian Jaya, Morotai, Obi; Papua New Guinea

Ninox jacquinoti (Bonaparte 1850)

II

$15,247,1226$

Solomon Hawk-Owl

Breeding: Papua New Guinea: Bougainville; Solomon Islands

Ninox meeki Rothschild \& Hartert 1914

II $\quad-\quad 15,247,1226$

Manus Hawk-Owl, Admiralty Hawk-OwI

Breeding: $\quad$ Papua New Guinea: Admiralty Islands

$\begin{array}{llll}\text { Ninox novaeseelandiae (Gmelin 1788) } & 11 & - & 15,247\end{array}$

(excluding subspecies undulata)

Morepork

Breeding:

Indonesia: Alor, Babar, Irian Jaya, Kai Islands, Leti [1486], Moa, Romang, Sawu, Sumba, Timor; Lord Howe Island (ex?); New Zealand; Papua New Guinea.

Ninox novaeseelandiae undulata (Latham 1801) I $\quad$ - $\quad 15,247,794,1036$

$(=N$. novaeseelandiae royana $)$

Norfolk Boobook

Breeding:

Norfolk Island $[167,1188]$

Ninox ochracea (Schlegel 1865)

(=N. perversa Stresemann 1938)

Ochre-bellied Hawk-Owl

Breeding: Indonesia: Butung [1486], Sulawesi

Ninox odiosa Sclater 1877

II

$15,247,1226$

Russet Hawk-Owl, New Britain Hawk-Owl

Breeding: $\quad$ Papua New Guinea: New Britain 
Ninox philippensis Bonaparte 1855

Philippine Hawk-Owl

Breeding:

Philippines [1 1113 ]
II

15,247

II

15,247

Ninox punctulata (Quoy \& Gaimard 1830)

Speckled Hawk-Owl

Breeding:

Indonesia: Butung, Kabaena, Muna, Sulawesi [1486]

Ninox rudolfi Meyer 1882

II

15

(= N. novaeseelandiae rudolfi)

Sumba Boobook

Breeding:

Indonesia: Sumba

Ninox rufa (Gould 1846)

II

15,247

Rufous Owl

Breeding:

Australia [167]; Indonesia: Aru Islands, Irian Jaya, Waigeo; Papua New Guinea

Ninox scutulata (Raffles 1822)

II

$10,15,247,792$

Brown Hawk-Owl

Breeding:

Bangladesh; Bhutan; Brunei; Cambodia; China; India: Andaman and Nicobar Islands; Indonesia; Japan; D.P.R. Korea; Korea Republic; Lao P.D.R; Malaysia: Peninsular Malaysia, Sabah, Sarawak [1295]; Myanmar; Nepal; Philippines [1113]; Russia [799]; Singapore; Sri Lanka; Taiwan; Thailand [1194]; Viet Nam

Non-breeding: Hong Kong

Vagrant: Australia

$\begin{array}{llll}\text { Ninox squamipila (Bonaparte 1850) } & \text { II } & - & 15,247\end{array}$

(excluding subspecies natalis)

Moluccan Hawk-Owl

Breeding: $\quad$ Indonesia: Bacan, Buru, Halmahera, Seram, Tanimbar [1486], Ternate

Ninox squamipila natalis Lister 1889

] $\quad-\quad 15,794,1038$

Christmas Hawk-Owl, Christmas Island Hawk-Owl

Breeding:

Christmas Island

Ninox strenua (Gould 1838)

II

Powerful Owl

Breeding:

Australia [167]

Ninox superciliaris (Vieillot 1817)

II

Madagascar Hawk-Owl, White-browed Owl

Breeding:

Madagascar [373]

Ninox theomacha (Bonaparte 1855)

II

$15,247,1226$

(includes $N$. goldii Gumey 1883)

Jungle Hawk-Owl, Sooty-backed Hawk-Owl, Chestnut Hawk-Owl

Breeding: Indonesia: Irian Jaya, Misool, Waigeo; Papua New Guinea

Ninox variegata (Quoy \& Gaimard 1830)

II

15,247

(=N. solomonis Sharpe 1876)

Bismarck Hawk-Owl, New Ireland Hawk-Owl

Breeding:

Papua New Guinea: New Britain, New Hanover, New Ireland 
Snowy Owl

Breeding:

Non-breeding:

Vagrant:
Canada; Finland; Greenland; Iceland; Norway; Russia; Sweden; United Kingdom; USA: Alaska

Faeroe Islands; Kazakhstan; Saint Pierre and Miquelon

Albania; Austria; Azores; Belgium; Bermuda; China; Czechoslovakia; Denmark; France; Germany; Hungary; Iran [661]; Ireland; Japan; D.P.R. Korea; Korea Republic; Kyrgyzstan; Latvia; Luxembourg; Mongolia; Netherlands; Pakistan; Poland; Turkmenia; Ukraine; USA; Yugoslavia

Otus albogularis (Cassin 1850)

II $\quad-\quad 15,247,967$

White-throated Screech-Owl

Breeding: $\quad$ Bolivia; Colombia; Ecuador; Peru; Venezuela

Otus alfredi (Hartert 1897)

II

$\mathrm{K}^{*}$

$15,247,318,1486$

Flores Scops-Owl

Breeding:

Indonesia: Flores

Otus angelinae (Finsch 1912)

II

$\mathrm{K}^{*}$

$15,27,247,318$

$(=O$. spilocephalus angelinae)

Javan Scops-Owl

Breeding:

Indonesia: Java

Otus asio (Linnaeus 1758)

II

$15,16,247$

Eastern Screech-Owl

Breeding:

Canada; Mexico; USA.

Vagrant:

Bermuda

Otus atricapillus (Temminck 1822)

II

$15,16,247,967$

(includes O. guatemalae (Sharpe 1875), O. hoyi König \& Straneck 1989, O. sanctaecatarinae (Salvin I897)) Variable Screech-Owl, Black-capped Screech-Owl, (Long-tufted Screech Owl)

Breeding: $\quad$ Argentina; Belize [338]; Bolivia; Brazil; Colombia; Costa Rica; Ecuador; Guatemala; Honduras; Mexico; Nicaragua; Panama; Paraguay; Peru; Uruguay; Venezuela

Otus bakkamoena Pennant $1769 \quad$ II $\quad-\quad 10,15,247$

Indian Scops-Owl

Breeding:

India; Iran; Nepal; Pakistan; Sri Lanka

Vagrant:

?Oman [661]

Otus balli (Hume 1873)

II

$10,15,247$

Andaman Scops-Owl

Breeding: India: Andaman Islands

Otus barbarus (Sclater \& Salvin 1868)

II

$15,16,247$

Bearded Screech-Owl

Breeding: Guatemala; Mexico

Otus brookii (Sharpe 1892)

II

15,247

Rajah Scops-Owl

Breeding:

Indonesia: Java, Sumatra; Malaysia: Sarawak [1295] 
Pallid Scops-Owl, Striated Scops-Owl

Breeding:

Iran; Iraq; ?Israel; ?Jordan; Kazakhstan; ?Lebanon; Oman; Pakistan; ?Syria; Tajikistan; Turkey; Turkmenia; ?United Arab Emirates; Uzbekistan; ?Yemen

Non-breeding: India; Jordan [1451]; Saudi Arabia; Syria

Vagrant:

Otus choliba (Vieillot 1817)

II

$15,16,247,967$

Tropical Screech-OwI

Breeding: $\quad$ Argentina; Bolivia; Brazil; Colombia; Costa Rica; Ecuador; French Guiana; Guyana; Panama; Paraguay; Peru; Suriname; Trinidad and Tobago: Trinidad; Uruguay; Venezuela

Otus clarkii Kelso \& Kelso 1935

II

$15,16,247,967$

Bare-shanked Screech-Owl

Breeding: $\quad$ Colombia; Costa Rica; Panama

Otus cooperi (Ridgway 1878)

I1

$15,16,247$

Pacific Screech-Owl

Breeding:

Costa Rica; El Salvador; ?Guatemala; ?Honduras; Mexico; Nicaragua

Otus elegans (Cassin 1852)

I1

( $=$ O. scops elegans)

Elegant Scops-Owl

Breeding:

Japan: Daito Islands, islands off Kyushu, Ryukyu Islands; Philippines: Batan, Calayan, Sabtang; Taiwan: Lan Yũ 1sland [1250]

Otus enganensis Riley 1927

II

(=O. umbra enganensis)

Enggano Scops-Owl

Breeding: Indonesia: Enggano [905]

Otus flammeolus (Kaup 1853)

I1

$15,16,247$

Flammulated Owl

Breeding: Canada; Guatemala; Mexico; USA

Non-breeding: El Salvador

Otus fuliginosus (Sharpe 1888)

II

(= O. bakkamoena fuliginosus)

Palawan Scops-Owl

Breeding: Philippines: Palawan

Otus hartlaubi (Giebel 1849)

II $\quad \mathrm{R}$

$15,247,318,324,496,1247$

Sao Tome Scops-Owl

Breeding:

Sao Tome and Principe: Sao Tome, ?Principe [755]

Otus icterorhynchus (Shelley 1873)

11

$15,247,496,1247$

Sandy Scops-Owl

Breeding:

Cameroon; Côte d'lvoire; Ghana; Liberia; Zaire

Otus ingens (Salvin 1897)

II

$15,247,967$

(includes $O$. colombianus Traylor 1952)

Rufescent Screech-Owl, (Colombian Screech-Owl)

Breeding: Bolivia; Colombia; Ecuador; Peru; Venezuela 
Otus ireneae Ripley 1966

Il

E

Sokoke Scops-Owl, Morden's Scops-Owl

Breeding:

Kenya

Otus kennicottii (Elliot 1867)

II

16

$(=0$. asio kennicottii)

Western Screech-Owl

Breeding: Canada; Mexico; USA

Otus koepckeae Hekstra 1982

II

15

Koepcke's Screech-Owl

Breeding: Bolivia; Peru

Otus lawrencii (Sclater \& Salvin 1868)

II

$15,16,247$

(= Gymnoglaux lawrencii)

Bare-legged Owl

Breeding:

Cuba [179]

Otus lempiji (Horsfield 1821)

II

(=O. bakkamoena lempiji)

Collared Scops-Owl

Breeding:

Bangladesh; Bhutan; Brunei; Cambodia; China; Hong Kong; India; Indonesia: Bali, Bangka, Banguran, Belitung, Jaca, Kalimantan, Kangean Islands, Siberut, Sumatra; Japan; D.P.R. Korea; Korea Republic; Lao P.D.R; Malaysia: Peninsular Malaysia, Sabah, Sarawak [1295]; Myanmar; Nepal; Pakistan; Russia; Singapore; Taiwan; Thailand [1194]; Viet Nam

Otus leucotis (Temminck 1820)

II

$15,212,247,285,496,1247$

White-faced Scops-Owl

Breeding:

Angola; Benin; Botswana; Burkina Faso [1388]; Burundi; Cameroon; Central African Republic; Chad; Congo; Côte d'lvoire; ?Djibouti; Equatorial Guinea; Ethiopia; Gabon; Gambia; Ghana; Guinea [1454]; Guinea-Bissau; Kenya; Liberia; Malawi; Mali; Mauritania; Mozambique; Namibia; Niger; Nigeria; Rwanda; Senegal; Sierra Leone; Somalia; South Africa; Sudan; Swaziland; Tanzania; Togo [278]; Uganda; Zaire; Zambia; Zimbabwe

Otus longicornis (Ogilvie-Grant 1894)

11

(= O. scops longicornis)

Luzon Scops-Owl

Breeding: Philippines: Luzon

Otus magicus (Müller 1841)

II

1226

( $=O$. manadensis magicus)

(includes $O$. beccarii (Salvadori 1876))

(excluding subspecies insularis)

Moluccan Scops-Owl, (Papuan Scops-Owl)

Breeding: Indonesia: Ambon, Bacan, Biak, Buru, Flores, Halmahera, Lomblen, Lombok, Morotai, Obi, Seram, Sumbawa, Ternate, Wetar

$\begin{aligned} & \text { Otus magicus insularis (Tristram 1880) } \\ & (=\text { O. insularis) }\end{aligned}$
$\begin{aligned} & \text { Seychelles Scops-Owl } \\ & \text { Breeding: Seychelles: Mahe }\end{aligned}$
$\begin{aligned} & \text { Otus manadensis (Quoy \& Gaimard 1830) } \\ & \text { S Il }\end{aligned}$

Sulawesi Scops-Owl

Breeding: Indonesia: Kaledupa, Mangole, Peleng, Sangihe, Sulawesi, Siau 
RL

Ref

Otus mantananensis (Sharpe 1892)

11

( $=$ O. scops mantananensis)

Mantanani Scops-Owl

Breeding: Malaysia: Mantanani Island; Philippines: Banton, Cuyo, Dicabaito, Linapacan, Palawan, Romblon, Semirana, Sibutu, Sibuyan, Tablas, Tumindao

Otus marshalli Weske \& Terborgh I98I

II

15

Cloud-forest Screech-Owl

Breeding: $\quad$ Peru [148I]

Otus megalotis (Walden 1875)

II $\quad-\quad 15$

(= O. bakkamoena megalotis)

Philippine Scops-Owl

Breeding: $\quad$ Philippines: Basilan, Biliran, Bohol, Catanduanes, Dinagat, Leyte, Luzon, Marinduque, Mindanao, Mindoro, Negros

Otus mentawi Chasen \& Kloss 1926

II

(=O. bakkamoena mentawi)

Mentawai Scops-Ow]

Breeding: Indonesia: Pagai, Siberut, Sipura

Otus mindorensis (Whitehead 1899)

II $\quad \mathrm{K}^{*} \quad \mathrm{I} 5,3 \mathrm{I} 8$

(= O. scops mindorensis)

Mindoro Scops-Owl

Breeding: $\quad$ Philippines: Mindoro

Otus mirus Ripley \& Rabor 1968

(=O. scops mirus)

Mindanao Scops-Owl

Breeding:

Philippines: Mindanao

Otus nudipes (Daudin 1800)

II $\quad-\quad 15,16,179,247$

Puerto Rican Screech-Owl

Breeding: $\quad$ British Virgin Islands: Tortola, Virgin Gorda; Puerto Rico; Virgin Islands of the United States

Otus petersoni Fitzpatrick \& O'Neill 1986

Il

Cinnamon Screech-Owl

Breeding: $\quad$ Ecuador; Peru [469]

Otus podarginus (Hartlaub \& Finsch 1872)

Il

15,247

(= Pyrroglaux podargina)

Palau Scops-Owl

Breeding:

Palau [1118]

Otus roboratus Bangs \& Noble 1918

II $\quad-\quad 15,247,967$

West Peruvian Screech-Owl

Breeding: ?Ecuador; Peru

Otus rufescens (Horsfield 1821)

II $\quad-\quad 15,247,792$

Reddish Scops-Owl

Breeding:

Brunei; Indonesia: Bangka, Java, Kalimantan, Sumatra; Malaysia: Peninsular Malaysia, Sabah, Sarawak [1295]; Philippines: ?Jolo Island; Thailand [I 194] 
Otus rutilus (Pucberan 1849)

(includes O. pembaensis Pakenham 1937, O. capnodes)

(excluding subspecies pauliani)

Madagascar Scops-Owl, (Pemba Scops-Owl)

Breeding:

Comoros: Anjouan; Madagascar [373]; Mayotte; Tanzania: Pemba

Otus rutilus pauliani (Benson 1960)

II

$\mathrm{R}$

$15,318,324,651$

$(=0$. pauliani $)$

Grand Comoro Scops-Owl

Breeding: Comoros: Grand Comoro [862]

Otus sagittatus (Cassin 1848)

$11 \quad \mathrm{~K}^{*}$

$15,247,318,792$

White-fronted Scops-Owl

Breeding: $\quad$ Indonesia: ?Sumatra [905]; Malaysia: Peninsular Malaysia; Myanmar; Thailand [1194]

Otus scops (Linnaeus 1758)

II

$10,15,212,247,342,496,661,792$

Eurasian Scops-Owl

Breeding:

?Afghanistan; Albania; Algeria; Andorra; Armenia; Austria; Azerbaijan; Belarus; Bulgaria; China; Cyprus; Czechoslovakia; France; Georgia; ?Gibraltar; Greece; Hungary; Iran; Iraq; Israel; Italy; ?Jordan; Kazakhstan; Kyrgyzstan; Lebanon; Libya; Mali; Moldova; Morocco; Poland; Portugal; Romania; Russia; Spain; Switzerland; ?Syria; Tajikistan; Tunisia; Turkey; Turkmenia; Ukraine; Uzbekistan; Yugoslavia

Non-breeding: Bahrain; ?Burkina Faso; Cameroon; Chad; Côte d'lvoire; Egypt; Ethiopia; Ghana; Hong Kong; Jordan [1451]; Kenya; Kuwait; Liberia; Mali; Malta; Mauritania; Nigeria; Oman; Saudi Arabia; Senegal; Sudan; Tunisia; Uganda; United Arab Emirates; Western Sahara; Zaire

Vagrant: $\quad$ Afghanistan; Belgium; Canary Islands; Denmark; Faeroe Islands; Germany; Iceland; Ireland; Latvia; Liechtenstein; Luxembourg; Madeira; Netherlands; Niger; Norway; Qatar; Somalia; Sweden; Tanzania; United Kingdom; Yemen

Otus seductus Moore 1941

I1

$(=O$. asio seductus $)$

Balsas Screech-Owl

Breeding:

Mexico

Otus senegalensis (Swainson 1834)

II

$15,212,247,285,661,1247$

African Scops-Owl, Senegal Scops-Owl

Breeding:

Angola; Benin; Botswana; Burundi; Cameroon; Central African Republic; Chad; Congo; Côte d'Ivoire; Djibouti; Equatorial Guinea: Annobon; Ethiopia [748]; ?Gabon; Gambia; Ghana; Guinea [984]; Guinea-Bissau; Kenya; Malawi; Mali; Mauritania; Namibia; Niger; Nigeria; Oman; Rwanda; Saudi Arabia; Senegal; ?Sierra Leone; Somalia; South Africa; Sudan; Swaziland; Tanzania; Togo; Uganda; Yemen; Zaire; Zambia; Zimbabwe

Vagrant: Lesotho

Otus silvicola (Wallace 1864)

Wallace's Scops-Owl, Lesser Sunda Scops-Owl

Breeding:

Indonesia: Flores [1486], Sumbawa

Otus spilocephalus (Blyth 1846)

11

$10,15,247,792$

(excluding subspecies stresemanni)

Mountain Scops-Owl, Spotted Scops-Owl

Breeding:

Bangladesh; Bhutan; China; India; Indonesia: ?Kalimantan; Lao P.D.R; Malaysia: Peninsular Malaysia, Sabah, Sarawak [1295]; Myanmar; Nepal; Pakistan; Taiwan; Thailand [1194]; Viet Nam 


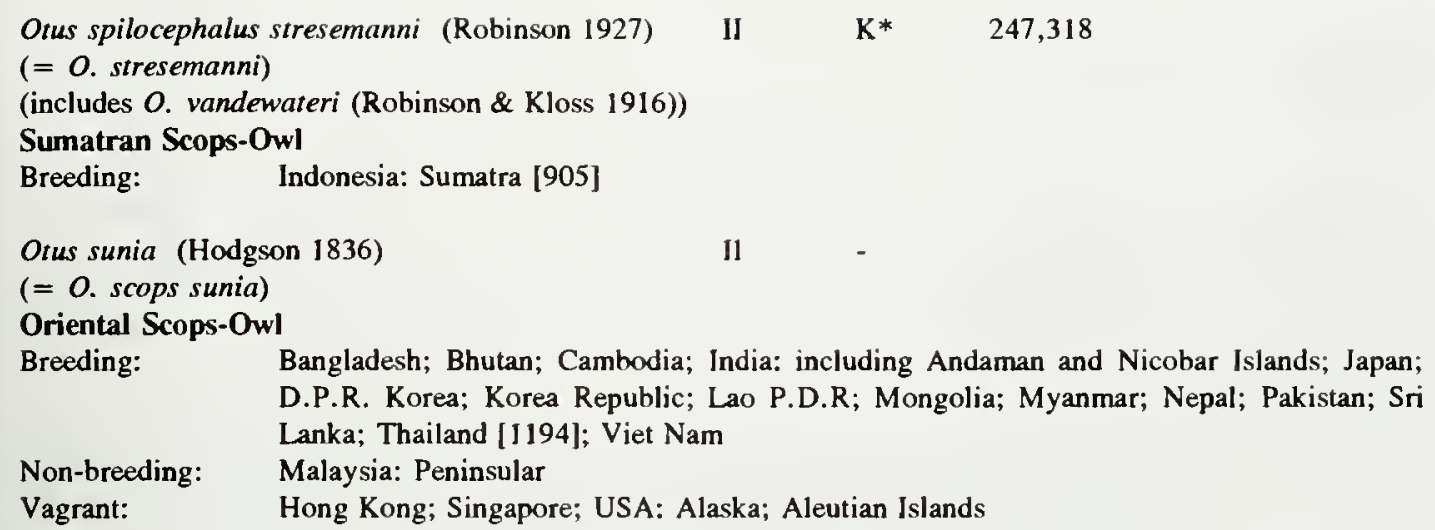

\section{Spectacled Owl}

Breeding:
Argentina; Belize; Bolivia; Brazil; Colombia; Costa Rica; Ecuador; El Salvador; French Guiana; Guatemala [127]; Guyana; Honduras; Mexico; Nicaragua; Panama; Paraguay; Peru; Suriname; Trinidad and Tobago: Trinidad; Venezuela 
Sceloglaux albifacies (Gray 1844)

I1 Ex

$15,247,976$

Laughing Owl, White-faced Owl

Breeding:

New Zealand (ex)

Scotopelia bouvieri Sharpe 1875

11

$15,247,496,1247$

Vermiculated Fishing-Owl

Breeding:

Angola; Cameroon; Central African Republic; Congo; ?Equatorial Guinea; Gabon; Liberia; ?Nigeria; Zaire

Scotopelia peli (Bonaparte 1850)

11

$15,212,247,285,496,1247$

Pel's Fishing-Owl

Breeding:

Angola; Botswana [225]; Cameroon; Central African Republic; Congo; Côte d'lvoire; Equatorial Guinea; Ethiopia [225,748]; Gabon; Gambia; Ghana; ?Guinea; Guinea-Bissau; Kenya; ?Liberia; Malawi; Mozambique; Namibia; Niger; Nigeria; Senegal; Sierra Leone; Somalia; South Africa; Sudan; Swaziland (ex?); Tanzania; Togo; Uganda; Zaire; Zambia; Zimbabwe

Scotopelia ussheri Sharpe 1871

$11 \quad \mathrm{R}$

$15,247,318,324,496,1247$

Rufous Fishing-Owl

Breeding: $\quad$ Côte d'Ivoire; Ghana; Guinea; Liberia; Sierra Leone
Speotyto cunicularia (Molina 1782)
11
$15,16,179,247,967$

(= Athene cunicularia)

Burrowing Owl

Breeding:

Antigua and Barbuda: Antigua and Barbuda (ex); Argentina; Aruba; Bahamas; Bolivia; Brazil; Canada; Chile [715]; Colombia; Cuba; Dominican Republic; Ecuador; Guadeloupe: Marie Galante (ex); Guyana; Haiti; Mexico; Netherlands Antilles: Aruba; Paraguay; Peru; Saint Kitts and Nevis (ex); Suriname; USA; Uruguay; Venezuela

Non-breeding: $\quad$ El Salvador; Guatemala

Vagrant: $\quad$ Costa Rica; Falkland lslands; Honduras; Panama

Strix albitarsus (Bonaparte 1850)

Il

$15,247,967$

(= Ciccaba albitarsus)

Rufous-banded Owl

Breeding:

Bolivia; Colombia; Ecuador; Peru; Venezuela

Strix aluco Linnaeus 1758

II

$10,15,247,342,496,661$

Tawny Owl, Tawny Wood-Owl

Breeding:

Afghanistan; Albania; Algeria; Andorra; Armenia; Austria; Azerbaijan; Belarus; Belgium; Bhutan; Bulgaria; China; Czechoslovakia; Denmark; Estonia; Finland; France; Georgia; Germany; Greece; Hungary; India; Iran; Iraq; Israel; Italy; ?Jordan; Kazakhstan; D.P.R. Korea; Korea Republic; Kyrgyzstan; Latvia; Lebanon; Liechtenstein; Lithuania;Luxembourg; Moldova; Morocco; Myanmar; Nepal; Netherlands; Norway; Pakistan; Poland; Portugal; Romania; Russia; Spain; Sweden; Switzerland; Syria; Taiwan [944]; Tajikistan; Tunisia; Turkey; Turkmenia; Ukraine; United Kingdom; Uzbekistan; Viet Nam; Yugoslavia

Non-breeding: Guernsey; Jersey

Strix butleri (Hume 1878)

Il

$15,247,496,661$

Hume's Owl, Hume's Tawny Owl, Hume's Wood-Owl, Desert Owl

Breeding:

Egypt; ?Iran; Israel [91]; Jordan; ?Oman; Pakistan; Saudi Arabia; ?Yemen

Strix davidi (Sharpe 1875)

11

15

(=S. uralensis davidi)

Sichuan Wood-Owl, David's Owl

Breeding: China [968] 
Strix fulvescens (Sclater \& Salvin 1875)

11

(=S. varia fulvescens)

Fulvous Owl

Breeding:

El Salvador; Guatemala; Honduras; Mexico

Strix huhula Daudin 1800

II

$15,247,967$

(= Ciccaba huhula)

Black-banded Owl

Breeding:

Argentina; Bolivia; Brazil; Colombia; Ecuador; French Guiana; Guyana; Paraguay; Peru; Suriname; Venezuela

Strix hylophila Temminck 1825

11

$15,247,967$

Rusty-barred Owl

Breeding:

Argentina [154]; Brazil; Paraguay
Strix leptogrammica Temminck 1831
11
$10,15,247,792$

Brown Wood-Owl

Breeding:

Bhutan; Brunei; China; India; Indonesia; Lao P.D.R; Malaysia: Peninsular Malaysia, Sabah, Sarawak [1295]; Myanmar; Nepal; Sri Lanka; Taiwan [944]; Thailand [1194]; Viet

Vagrant:

Nam

Strix nebulosa Thunberg 1798

Il $\quad-\quad 15,16,247,342$

Great Grey Owl, Lapp Owl

Breeding: $\quad$ Belarus; Canada; China; Finland; Latvia (ex); Lithuania; Mongolia; Norway; Russia; Sweden; USA

Non-breeding: Latvia

Vagrant: Germany; Poland

$\begin{array}{lllll}\text { Strix nigrolineata Sclater } 1859 & \text { II } & - & 15,16,247,967\end{array}$

( = Ciccaba nigrolineata)

Black-and-white Owl

Breeding: $\quad$ Belize; Colombia; Costa Rica; Ecuador; El Salvador; Guatemala; Honduras; Mexico; Nicaragua; Panama; Peru; Venezuela

Strix occidentalis (Xantus de Vesey 1860) II $\quad$ I $\quad 15,16,247,318,1492$

Spotted Owl

Breeding: $\quad$ Canada; Mexico; USA [591]

Strix ocellata (Lesson 1839) II $\quad$ - $\quad 10,15,247$

Mottled Wood-Owl

Breeding: $\quad$ ?Bangladesh; India; Myanmar; Pakistan

Strix rufipes King 1828

11

$15,247,967$

Rufous-legged OwI

Breeding:

Argentina; Chile [715]; Paraguay

Vagrant:

Falkland Islands

Strix seloputo Horsfield 1878

II

$15,247,792$

Spotted Wood-OwJ

Breeding:

Cambodia; Indonesia: Bawean, Java, ?Sumatra [905]; Malaysia: Peninsular Malaysia; Myanmar; Philippines: Palawan [1113]; Singapore; Thailand [1194]; Viet Nam 
Ural Owl

Breeding:

?Albania; ?Austria; Belarus; Bulgaria; China; Czechoslovakia; Estonia; Finland; Hungary; Japan; Latvia; Lithuania; Mongolia; Norway; Poland; Romania; Russia; Sweden; Yugoslavia

Vagrant:

Germany; Italy; D.P.R. Korea; Korea Republic

Strix varia Barton 1799

II

$15,16,247$

Barred Owl

Breeding:

?Alaska; Canada; Mexico; USA

Vagrant:

Bermuda

Strix virgata (Cassin 1850)

II

$15,16,247,967$

(= Ciccaba virgata)

Mottled Owl

Breeding:

Vagrant:

Argentina [154]; Belize; Bolivia; Brazil; Colombia; Costa Rica; Ecuador; El Salvador; French Guiana; Guatemala; Guyana; Honduras; Mexico; Nicaragua; Panama; Paraguay; Peru; Suriname; Trinidad and Tobago: Trinidad; Venezuela

Strix woodfordii (Smith 1834)

II

$15,212,247,285,496,1247$

(= Ciccaba woodfordii)

African Wood-Owl, Woodford's Owl

Breeding:

Angola; Benin; Botswana; Burundi; Cameroon; Central African Republic; Congo; Côte d'lvoire; Equatorial Guinea: Bioko; Ethiopia; Gabon; Ghana; Guinea; Guinea-Bissau; Kenya; Liberia; Malawi; Mozambique; Namibia; Nigeria; Rwanda; Senegal; Sierra Leone; Somalia; South Africa; Sudan; Swaziland; Tanzania; Uganda; Zaire; Zambia; Zimbabwe

Surnia ulula (Linnaeus 1758)

II

$15,16,247,342$

Northern Hawk Owl, Hawk Owl

Breeding:

Canada; China; Estonia; Finland; D.P.R. Korea; Latvia (ex); Mongolia; Norway; Russia; Sweden; USA

Non-breeding:

Kazakhstan; Latvia

Vagrant:

Austria; Belgium; Bermuda; ?Canary Islands; Czechoslovakia; Denmark; Faeroe Islands; France; Germany; Hungary; Japan; Luxembourg; Netherlands; Poland; Romania; Switzerland; Ukraine; United Kingdom; Yugoslavia

Uroglaux dimorpha (Salvadori 1874)

II

Papuan Hawk-Owl

Breeding:

Indonesia: Irian Jaya, Yapen; Papua New Guinea

Xenoglaux loweryi O'Neill \& Graves 1977

II

K

Long-whiskered Owlet

Breeding: Peru 


\section{Order CAPRIMULGIFORMES}

Nightjars and allies

Family PODARGIDAE

Frogmouths

Basrachostomus harterti Sharpe 1892

$\mathrm{K}^{*} \quad 318$

Dulit Frogmouth

Breeding:

Indonesia: Kalimantan [1295]; Malaysia: Sarawak [1295]

\section{Family CAPRIMULGIDAE \\ Nightjars}

Caprimulgus candicans (Pelzeln 1867)

E

$318,321,957$

White-winged Nightjar

Breeding: Brazil [1139,1271]; ?Paraguay

$\begin{array}{lllll}\text { Caprimulgus centralasicus } & \text { Vaurie } 1960 & \text { K } & & 318\end{array}$

Vaurie's Nightjar

Breeding: China [968]

Caprimulgus maculosus (Todd 1920)

ld

Cayenne Nightjar

Breeding:

French Guiana

Caprimulgus noctitherus (Wetmore 1919)

$\mathrm{R}$

$16,318,321,794,1437$

(= C. vociferus noctitherus)

Puerto Rican Nightjar, Puerto Rican Whip-poor-will

Breeding: $\quad$ Puerto Rico [179,1129]

Caprimulgus pulchellus Salvadori 1879

K* $\quad 318$

Salvadori's Nightjar

Breeding:

Indonesia: Java, Sumatra [905]

Eleorhreptus anomalus (Gould 1838)

K $\quad 318,321,967$

Sickle-winged Nightjar

Breeding: Argentina [154,331]; Brazil [1271,1335]; Paraguay

Eurostopodus diabolicus Stresemann 1931

$\mathrm{K}^{*} \quad 318,1486$

Satanic Eared-Nightjar, Satanic Nightjar

Breeding: Indonesia: Sulawesi

Siphonorhis americanus (Linnaeus 1758)

E/Ex $\quad 318,321,794,1344$

Jamaican Poorwill, Jamaican Paraque

Breeding: Jamaica (ex?) 


\section{Order APODIFORMES}

Swifts and Hummingbirds

\section{Family APODIDAE}

Swifts

Apus acuticauda (Jerdon 1864)

K*

318

Dark-rumped Swift

Breeding:

India [10]

Vagrant: $\quad$ ?Nepal; Thailand

Apus barbatus sladeniae (Ogilvie-Grant 1904) - $\quad$ K $\quad 318,324,496,779$

(= Apus sladeniae)

Fernando Po Swift

Breeding: $\quad$ Angola; Cameroon; Equatorial Guinea: Bioko; ?Nigeria

Collocalia elaphra Oberholser 1906

R $\quad 318,324$

(= Aerodramus elaphrus)

Seychelles Swiftlet

Breeding: $\quad$ Seychelles

Collocalia leucophaeus (Peale 1848) $\quad-\quad K^{*} \quad 318,669,1118$

(= Aerodramus leucophaeus)

Polynesian Swiftlet, Tahiti Swiftlet

Breeding:

French Polynesia: Bora Bora (ex), Huahine (ex), Tahiti

Vagrant:

French Polynesia: Moorea

Collocalia sawtelli Holyoak 1974

K*

$318,666,1118$

(= Aerodramus sawtelli)

Sawtell's Swiftlet, Aitu Swiftlet

Breeding: Cook lslands: Aitu

Cypseloides lemosi Eisenmann \& Lehmann $1962 \quad-\quad$ ld $\quad 318,321,967$

White-chested Swift

Breeding: $\quad$ Colombia; ?Ecuador

Hydrochous gigas (Hartert \& Butler 1901)

$K^{*} \quad 318,789,1308$

(= Collocalia gigas)

Waterfall Swift, Giant Swiftlet

Breeding: $\quad$ Indonesia: Java, ?Kalimantan [1295], Sumatra [905]; Malaysia: Peninsular Malaysia, ?Sabah [1295], ?Sarawak [1295]

Schoutedenapus schoutedeni (Prigogine 1960)

Id $\quad 318,324,496,779$

Schouteden's Swift, Congo Swift

Breeding: Zaire

\section{Family TROCHILIDAE}

Hummingbirds

Abeillia abeillei (Lesson \& DeLattre 1839)

II

16

Emerald-chinned Hummingbird

Breeding:

Belize [1202]; El Salvador [397]; Guatemala [824]; Honduras [981]; Mexico [1089]; Nicaragua 
Acestrura astreans Bangs 1899

Il

967

(= A. heliodor astreans)

Santa Marta Woodstar

Breeding: Colombia

Acestrura berlepschi (Simon 1889)

1I

$\mathrm{E}$

$318,321,967$

Esmeraldas Woodstar

Breeding:

Ecuador [248]

Acestrura bombus (Gould 1871)

$11 \quad$ E

$318,321,967$

Little Woodstar

Breeding: $\quad$ Ecuador [248]; Peru [1064]

Acestrura heliodor (Bourcier 1840)

II

16,967

Gorgeted Woodstar

Breeding:

Colombia; Ecuador [248]; Panama [1165]; Venezuela [969]

Acestrura mulsant (Bourcier 1842)

11

967

White-bellied Woodstar

Breeding:

Bolivia [956]; Colombia; Ecuador [248]; Peru [1064]

Adelomyia melanogenys (Fraser 1840)

11

967

Speckled Humminghird

Breeding:

Argentina [154,1034]; Bolivia [956]; Colombia; Ecuador [248]; Peru [1064]; Venezuela [969]

Aglaeactis aliciae Salvin 1896

$11 \quad$ ld

$318,321,967$

Purple-backed Sunbeam

Breeding: $\quad$ Peru [1064]

Aglaeactis castelnaudii (Borcier \& Mulsant 1848) 11

White-tufted Sunbeam

Breeding:

Peru $[1064]$

Aglaeactis cupripennis (Bourcier 1843)

II

967

Shining Sunbeam

Breeding:

Colombia; Ecuador [248]; Peru [1064]

Aglaeactis pamela (D'Orbigny 1838)

11

967

Black-hooded Sunbeam

Breeding:

Bolivia [956]

Aglaiocercus coelestis (Gould 1861)

II

967

Violet-tailed Sylph

Breeding:

Colombia; Ecuador [248]

Aglaiocercus kingi (Lesson 1832)

II

967

Long-tailed Sylph

Breeding:

Bolivia [956]; Colombia; Ecuador [248]; Peru [1064]; Venezuela [969]

Amazilia amabilis (Gould 1853)

I1

16,967

Blue-chested Hummingbird

Breeding:

Colombia; Costa Rica [1285]; Ecuador [248]; Nicaragua; Panama 
Amazilia amazilia (Lesson 1828)

II

967

Amazilia Hummingbird

Breeding:

Ecuador [248]; Peru [1064]

Amazilia beryllina (Deppe 1830)

II

16

Berylline Hummingbird

Breeding:

El Salvador [397]; Guatemala [127,824]; Honduras [981]; Mexico [165, 1089]

Vagrant: USA [740]

Amazilia boucardi (Mulsant 1877)

II $\quad$ V

$16,318,321,1351$

Mangrove Humminghird

Breeding:

Costa Rica [1285]

Amazilia candida (Bourcier \& Mulsant 1846)

11

16

White-bellied Emerald

Breeding: $\quad$ Belize [1202]; Guatemala [824]; Honduras [981]; Mexico [1089]; Nicaragua Vagrant: Costa Rica

Amazilia castaneiventris Gould 1856

$11 \quad \mathrm{~V}$

$318,321,967$

Chestnut-bellied Hummingbird

Breeding:

Colombia $[654,655]$

Amazilia chionogaster (Tschudi 1844)

Il

967

White-bellied Hummingbird

Breeding:

Argentina [1034]; Bolivia [956]; Brazil [1270]; ?Paraguay; Peru [1064]

Amazilia chionopectus (Gould 1859)

II

967

White-chested Emerald

Breeding:

Brazil [1270]; French Guiana [152]; ?Grenada; Guyana [1303]; Suriname [402]; Trinidad and Tobago [465]; Uruguay [557]

Amazilia cyanifrons (Bourcier 1843)

11

Indigo-capped Humminghird

Breeding: Colombia; ?Costa Rica [1285]

Amazilia cyanocephala (Lesson 1829)

II

16

Azure-crowned Hummingbird

Breeding:

Belize [1202]; El Salvador [397]; Guatemala [127,824]; Honduras [981]; Mexico [165, 1089]; Nicaragua [16]

Amazilia cyanura Gould 1859

11

16

Blue-tailed Hummingbird

Breeding:

El Salvador [397]; Guatemala [824]; Honduras [981]; Mexico [1089]; Nicaragua

Vagrant:

Costa Rica

Amazilia decora (Salvin 1891)

II

16

(= A. amabilis decora)

Charming Hummingbird

Breeding:

Costa Rica; Panama

Amazilia distans Wetmore \& Phelps 1956

11

V

$318,321,967$

Tachira Emerald

Breeding:

Venezuela [969] 


\section{Glittering-throated Emerald}

Breeding:

Boljvia [956]; Brazi] [1239,1270]; Colombia; Ecuador [248]; French Guiana [152]; Guyana [1303]; Paraguay; Peru [1064]; Suriname [402]; Trinidad and Tobago [465]; Venezuela [969]

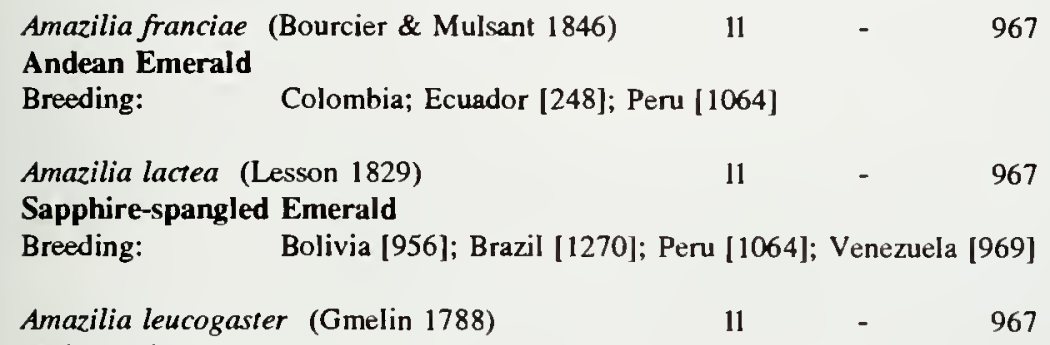


Violed-crowned Hummingbird

Breeding:

Mexico [165, 1089]; USA [740]

Amazilia viridicauda (Berlepsch 1883)

II

Green-and-white Humminghird

Breeding:

Peru [1064]

Amazilia viridifrons (Elliot 1871)

II

16

Green-fronted Hummingbird

Breeding:

Belize [50,1202]; Mexico [1089]

Amazilia viridigaster (Bourcier 1843)

II

967

Green-bellied Hummingbird

Breeding:

Brazil [1270]; Colombia; Guyana [1303]; Venezuela [969]

Amazilia yucatanensis (Cabot 1845)

II

16

Buff-bellied Humminghird, Fawn-breasted Humminghird

Breeding:

Belize [1202]; Guatemala [824]; Mexico [1089]; USA [740]

Vagrant: Honduras [981]

Androdon aequatorialis Gould 1863

II

16,967

Tooth-billed Hummingbird

Breeding:

Colombia; Ecuador [248]; Panama [1153,1165]

Anthocephala floriceps (Gould 1854)

11

967

Blossomcrown

Breeding:

Colombia $[654,655]$

Anthracothorax dominicus (Linnaeus 1766)

II

16,179

Antillean Mango, Dominican Mango

Breeding: $\quad$ British Virgin Islands (ex) [1129]; Dominican Republic; Haiti [1534]; Puerto Rico [1129]; Virgin Islands of the United States [1129]

Anthracothorax mango (Linnaeus 1758)

II

16

Jamaican Mango

Breeding:

Jamaica [179]

Anthracothorax nigricollis (Vieillot 1817)

II

16,967

Black-throated Mango

Breeding:

Argentina [1034]; Bolivia [956]; Brazil [1239,1270]; Colombia; Ecuador [248]; French Guiana [153]; Guyana [1303]; Panama [1153]; Paraguay; Peru [1064]; Suriname [402]; Trinidad and Tobago [465]; Venezuela [969]

Vagrant: Uruguay [557]

Anthracothorax prevostii (Lesson 1832)

11

$16,179,967$

(includes $A$. veraguensis Reichenbach 1855)

Green-breasted Mango, (Veraguas Mango)

Breeding:

Bahamas; Belize [1202]; Colombia; Costa Rica [1285]; Ecuador [248]; El Salvador [397]; Guatemala [824]; Honduras [981]; Mexico [1089]; Nicaragua; Panama [1 153]; Peru [1064]; Venezuela [969]

Anthracothorax viridigula (Boddaert 1783)

II

967

Green-throated Mango

Breeding:

Brazil [1270]; French Guiana [152]; ?Grenada [16]; Guyana [1303]; Suriname [402];

Trinidad and Tobago [465]; Venezuela [969] 
Anthracothorax viridis (Audebert \& Vieillot 1801) 11

Green Mango

Breeding:

Puerto Rico [179, 1129]

Aphantochroa cirrochloris (Vieillot 1818)

11

967

Sombre Hummingbird

Breeding:

Brazil $[1239,1270]$

Archilochus alexandri (Bourcier \& Mulsant 1846)

Black-chinned Hummingbird

$11 \quad-\quad 16,740$

Breeding: $\quad$ Canada; Mexico [1089]; USA

Archilochus colubris (Linnaeus 1758)

$11 \quad-\quad 16,179$

Ruby-throated Hummingbird

Breeding: $\quad$ Canada [740]; USA [740]

Non-breeding: $\quad$ Bahamas [257]; Bermuda [16]; Belize [1202]; Cayman Islands [194]; Costa Rica [1285]; Cuba [511]; El Salvador [397]; Guatemala [127,824]; Honduras [981]; Mexico [1089]; Nicaragua; Panama [1153]

Vagrant: $\quad$ ?Dominican Republic [16]; Haiti [16,1534]; ?Jamaica [16]; ?Puerto Rico [16]

Atthis ellioti Ridgway 1878

II $\quad-\quad 16$

Wine-throated Hummingbird

Breeding: $\quad$ El Salvador [397]; Guatemala [824]; Honduras [981]; Mexico [1089]

Atthis heloisa (Lesson \& DeLattre 1839) $\quad$ II $\quad 16$

Bumblebee Hummingbird

Breeding: Mexico [1089]

Vagrant: $\quad$ USA [740]

Augastes geoffroyi (Bourcier 1843)

II $\quad-\quad 967$

(= Schistes geoffroyi)

Wedge-billed Hummingbird

Breeding: $\quad$ Bolivia [956]; Colombia [654,655]; Ecuador [248]; Peru [1064]; Venezuela [969]

Augastes lumachellus (Lesson 1838)

11

568,967

Hooded Visorbearer

Breeding:

Brazil [1270]

Augastes scutatus (Temminck 1822)

II

$318,568,967$

Hyacinth Visorbearer

Breeding:

Brazil [1270,1271]

Avocettula recurvirostris (Swainson 1822)

II

967

Fiery-tailed Awlbill

Breeding:

Brazil [1270]; Ecuador [248]; ?French Guiana; Guyana [1303]; Suriname [402,1395]; Venezuela [969]

Boissonneaua flavescens (Loddiges 1832)

II

967

Buff-tailed Coronet

Breeding:

Colombia; Ecuador [248]; Venezuela [969]

Boissonneaua jardini (Bourcier 1851)

Il

967

Velvet-purple Coronet

Breeding:

Colombia; Ecuador [248] 
Chestnut-breasted Coronet

Breeding:

Colombia; Ecuador [248]; Peru [1064]

Calliphlox amethystina (Boddaert 1783)

11

967

Amethyst Woodstar

Breeding:

Argentina [154, 1034]; Bolivia [956]; Brazil [1239, 1270]; Colombia; Ecuador [248]; French Guiana [152]; Guyana [1303]; Paraguay; Peru [1064]; Suriname [402,627]; Venezuela [969]

Calliphlox evelynae (Bourcier 1847)

II

16

(= Philodice evelynae)

Bahama Woodstar

$\begin{array}{ll}\text { Breeding: } & \text { Bahamas [179,232] } \\ \text { Vagrant: } & \text { USA [740] }\end{array}$

Calothorax lucifer (Swainson 1827)

II

16

Lucifer Hummingbird

Breeding: $\quad$ Mexico [165,1089]; USA [740]

Calothorax pulcher Gould 1859

1 I

16

Beautiful Humminghird

Breeding:

Mexico [1089]

Calypte anna (Lesson 1829)

II

Anna's Hummingbird

Breeding:

USA [740]

Vagrant:

Canada [740]; Mexico [1089]

Calypte costae (Bourcier 1839)

II $\quad-\quad 16,107$

Costa's Hummingbird

Breeding:

Mexico [1089]; USA [740]

Vagrant:

?Canada [16,740]

Campylopterus curvipennis (Deppe 1830)

II

16

Wedge-tailed Sabrewing

Breeding: $\quad$ Belize [1202]; Guatemala [824]; Honduras [981]; Mexico [1089]

Campylopterus duidae Chapman $1929 \quad$ II $\quad$ - $\quad 967$

Buff-breasted Sabrewing

Breeding: $\quad$ Brazil [967]; Venezuela [969]

Campylopterus ensipennis (Swainson 1822)

II $\quad \mathrm{V} / \mathrm{R}$

$318,321,967$

White-tailed Sabrewing

Breeding: $\quad$ Trinidad and Tobago $[465,1370]$ : Tobago; Venezuela $[61,969]$

Campylopterus excellens (Wetmore 1941)

II

16

(= C. curvipennis excellens),

Long-tailed Sabrewing

Breeding: Mexico

Campylopterus falcatus (Swainson 1821)

11

967

Lazuline Sabrewing

Breeding:

Colombia; Ecuador [248]; Venezuela [969] 
Campylopterus hemileucurus (Deppe 1830)

11

16

Violet Sabrewing

Breeding:

Belize [1202]; Costa Rica [1285]; El Salvador [397]; Guatemala [824]; Honduras [981]; Mexico [1089]; Nicaragua; Panama [1153]

Campylopterus hyperythrus Cabanis 1848

11

967

Rufous-breasted Sabrewing

Breeding:

Brazil [967]; Venezuela [969]

Campylopterus largipennis (Boddaert 1783)

11

967

Grey-breasted Sabrewing

Breeding:

Bolivia [956]; Brazil [1270]; Colombia; Ecuador [248]; French Guiana [396]; Guyana [1303]; Peru [1064]; Suriname [402]; Venezuela [969]

Campylopterus phainopeplus Salvin \& Godman 187911

Santa Marta Sabrewing

Breeding: Colombia

Campylopterus rufus Lesoon 1840

II

16

Rufous Sabrewing

Breeding:

Guatemala [824]; Mexico [165,1089]

Campylopterus villaviscensio (Bourcier 1851)

11

967

Napo Sabrewing

Breeding:

Ecuador [248]

Chaetocercus jourdanii (Bourcier 1839)

II

967

Rufous-shafted Woodstar

Breeding:

Colombia; Trinidad and Tobago [465]; Venezuela [969]

Chalcostigma herrani (DeLattre \& Bourcier 1846) 11

967

Rainbow-bearded Thornbill

Breeding: Colombia; Ecuador [248]; Peru [1064]

Chalcostigma heteropogon (Boissoneau 1839)

II

967

Bronze-tailed Thornbill

Breeding: Colombia; Venezuela [969]

Chalcostigma olivaceum (Lawrence 1864)

11

967

Olivaceous Thornbill

Breeding:

Bolivia [956]; Peru [1064]

Chalcostigma ruficeps (Gould 1846)

11

967

Rufous-capped Thornbill

Breeding:

Bolivia [956]; ?Colombia [655,1232]; Ecuador [248]; Peru [1064]

Chalcostigma stanleyi (Bourcier 1851)

II

967

Blue-mantled Thornbill

Breeding:

Bolivia [956]; Ecuador [248]; Peru [1064]

Chalybura buffonii (Lesson 1832)

11

967

White-vented Plumeleteer

Breeding:

Colombia; Ecuador [248]; Panama [1153]; Peru [1064]; Venezuela [969] 
Chalybura urochrysia (Gould 1861)

11

16,967

Bronze-tailed Plumeleteer

Breeding:

Colombia; Costa Rica [1285]; Ecuador [248]; Honduras [16,903]; Nicaragua; Panama [1153]

Chlorestes notatus (Reichenbach 1795)

II

967

Blue-chinned Sapphire

Breeding:

Brazil [1270]; Colombia; Ecuador [248]; French Guiana [396]; Guyana [1303]; Peru [1064]; Suriname [402]; Trinidad and Tobago [465]; Venezuela [969]

Chlorostilbon alice (Bourcier \& Mulsant 1848)

II

967

Green-tailed Emerald

Breeding:

Venezuela [969]

Chlorostilbon assimilis Lawrence 1861

11 16

$(=C$. canivetii assimilis $)$

Garden Emerald

Breeding: $\quad$ Costa Rica; Panama

Chlorostilbon aureoventris (d'Orbigny \& Lafresnaye 1838)

Glittering-bellied Emerald

11

Breeding: $\quad$ Argentina [1034]; Bolivia [956]; Brazil [1239, 1270]; Paraguay; Uruguay [354,557],967

Chlorostilbon bracei (Lawrence 1877)

II

16

(= C. maugeus bracei)

Brace's Emerald

Breeding: Bahamas (ex?)

Chlorostilbon canivetii (Lesson 1832)

II

16

Fork-tailed Emerald

Breeding: $\quad$ Belize [433,1202]; Costa Rica [1285]; El Salvador [397]; Guatemala [824]; Honduras [981]; Mexico [165,1089]; Nicaragua

Chlorostilbon gibsoni (Fraser 1840)

II

967

Red-billed Emerald

Breeding:

Colombia

Chlorostilbon maugaeus (Audebert \& Vieillot 1801) I1

16

Puerto Rican Emerald

Breeding:

Puerto Rico [179,1129]

Chlorostilbon mellisugus (Linnaeus 1758)

II $\quad-\quad 16,967$

Blue-tailed Emerald

Breeding: $\quad$ Aruba [1445]; Bolivia [956]; Brazil [1270]; Colombia; Ecuador [248]; French Guiana [153]; Guyana [1303]; Netherlands Antilles [1445]; Peru [1064]; Suriname [402,628]; Trinidad and Tobago [465,1370]; Venezuela [969]

Chlorostilbon poortmani (Bourcier 1843)

II $\quad-\quad 967$

(includes C. auratus (Cabanis \& Heine 1860), C. inexpectatus (Berlepsch 1879))

Short-tailed Emerald

Breeding: $\quad$ Colombia [655,1088]; ?Peru [1088]; Venezuela [969]

Chlorostilbon ricordii (Gervais 1935)

Il $\quad-\quad 16$

Cuban Emerald

Breeding:

Bahamas [232]; Cuba [179,511]

Vagrant:

USA [740] 
Chlorostilbon russatus (Salvin \& Godman 1881) $\quad 11 \quad$ - 967

Coppery Emerald

Breeding:

Colombia; Venezuela [969]

Chlorostilbon stenura (Cabanis \& Heine 1860)

11

967

Narrow-tailed Emerald

Breeding:

Colombia; ?Ecuador [248]; Venezuela [969]

Chlorostilbon swainsonii (Lesson 1829)

11

16,179

Hispaniolan Emerald

Breeding:

Dominican Republic; Haiti [1534]

Chrysolampis mosquitus (Linnaeus 1758)

II

16,967

Ruby-topaz Hummingbird

Breeding:

Aruba [1445]; Bolivia [956]; Brazil [1270]; Colombia; French Guiana [153]; Guyana [1303]; Netherlands Antilles [1445]; Suriname [402]; Trinidad and Tobago [465]; Venezuela [969]

Vagrant: Grenada [16,179]; Panama

Chrysuronia oenone (Lesson 1832)

11

967

Golden-tailed Sapphire

Breeding: $\quad$ Bolivia [956]; Brazil [1270]; Colombia; Ecuador [248]; Peru [1064]; ?Trinidad and Tobago [465]; Venezuela [969]

Clytolaema rubricauda (Boddaert 1783)

II

967

Brazilian Ruby

Breeding:

Brazil $[1239,1270]$

Coeligena bonapartei (Boissoneau 1840)

II

967

Golden-bellied Starfrontlet

Breeding:

Colombia [654,655]; Venezuela [969]

Coeligena coeligena (Lesson 1833)

11

967

Bronzy Inca

Breeding:

Bolivia [956]; Colombia; Ecuador [248]; Peru [1064]; Venezuela [969]

Coeligena helianthea (Lesson 1838)

II

967

Blue-throated Starfrontlet

Breeding: Colombia; Venezuela [969]

Coeligena iris (Gould 1854)

11

967

Rainbow Starfrontlet

Breeding:

Ecuador [248]; Peru [1064]

Coeligena lutetiae (DeLattre \& Bourcier 1846)

11

967

Buff-winged Starfrontlet

Breeding:

Colombia; Ecuador [248]; Peru [1064]

Coeligena orina Wetmore 1953

Dusky Starfrontlet

Breeding:

Colombia

Coeligena phalerata (Bangs 1898)

11

White-tailed Starfrontlet

Breeding:

Colombia 


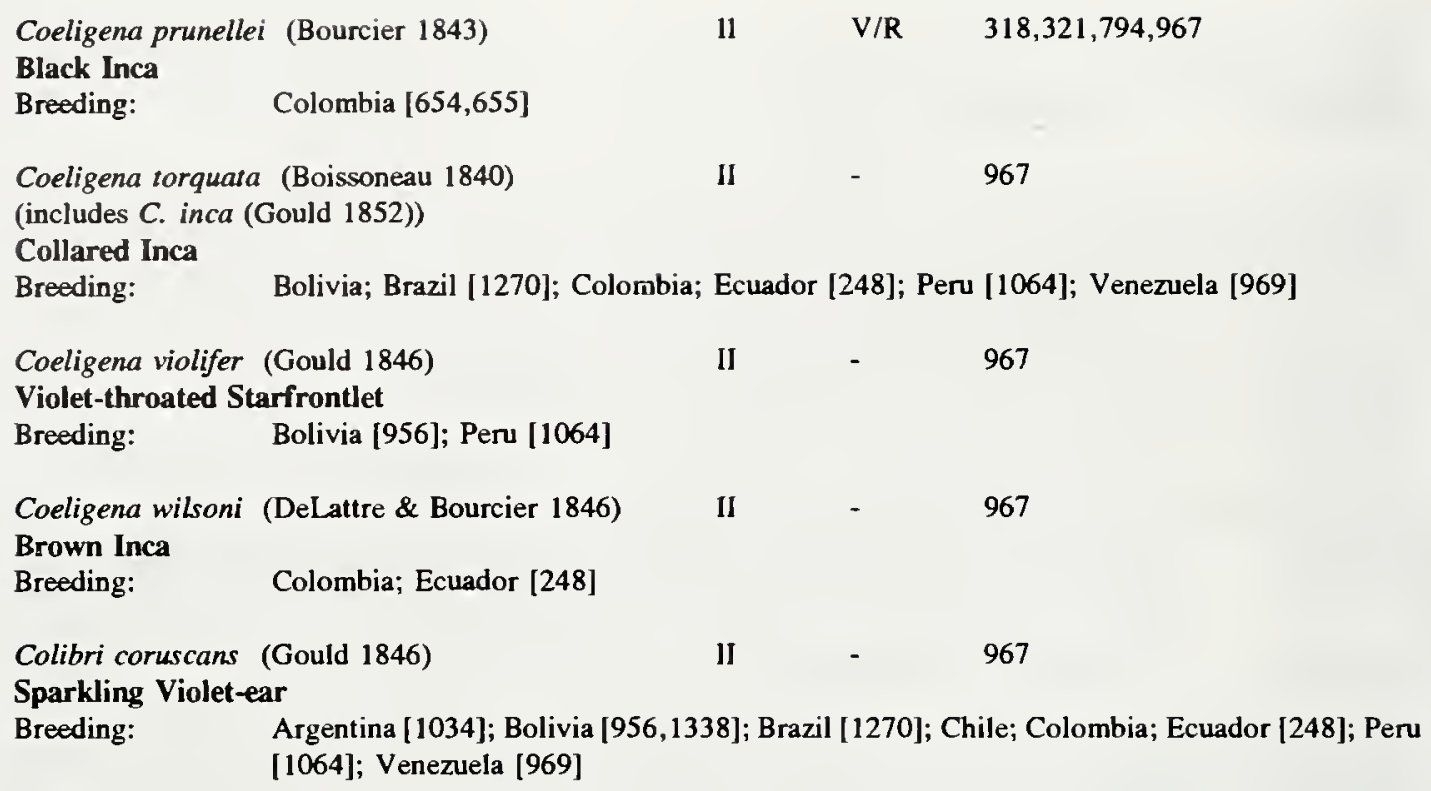

Colibri delphinae (Lesson 1839)

$11 \quad-\quad 16,967$

Brown Violet-ear

Breeding:

Belize [1202]; Bolivia [956]; Brazil [1270]; Colombia; Costa Rica [1285]; Ecuador [248]; ?French Guiana; Guatemala [824]; Guyana [1303]; Honduras [981]; Nicaragua; Panama [1153]; Peru [1064]; Suriname [402]; Trinidad and Tobago [465]; Venezuela [969]

Colibri serrirostris (Vieillot 1816)

II $\quad-\quad 967$

White-vented Violet-ear

Breeding: $\quad$ Argentina [1034]; Bolivia [956]; Brazil [1239, 1270]; ?Paraguay

$\begin{array}{llll}\text { Colibri thalassinus (Swainson 1827) } & \text { Il } & - & 16,967\end{array}$

Green Violet-ear

Breeding: $\quad$ Argentina [154]; Bolivia [956]; Colombia; Costa Rica [1285]; Ecuador [248]; El Salvador [397]; Guatemala [824]; Honduras [981]; Mexico [165,1089]; Nicaragua; Panama [1153]; Peru [1064]; Venezuela [969]

Vagrant: $\quad$ Argentina [1034]; USA [740]

$\begin{array}{llll}\text { Cyanophaia bicolor (Gmelin 1788) II } \quad- & 16,179\end{array}$

Blue-headed Humminghird

Breeding: $\quad$ Dominica [1233]; Martinique [1233]

Cynanthus latirostris Swainson 1827

II $\quad-\quad 16$

Broad-billed Hummingbird

Breeding: $\quad$ Mexico [165, 1089]; USA [740]

Cynanthus sordidus (Gould 1859)

II $\quad 16$

Dusky Hummingbird

Breeding: $\quad$ Mexico [165, 1089]

Damophila julie (Bourcier 1842)

I1

16,967

Violet-bellied Hummingbird

Breeding: $\quad$ Colombia; Ecuador [248]; Panama [1153]; Peru [1064]

Vagrant: ?Costa Rica [1285] 
Racket-tailed Coquette

Breeding:

Brazil [1270]; French Guiana [153]; Guyana [1303]; Suriname [402]; Venezuela [969]

Doricha eliza (Lesson \& DeLattre 1839)

Il

16

Mexican Sheartail

Breeding:

Mexico [419,1089]

Doricha enicura (Vieillot 1818)

11

16

\section{Slender Sheartail}

Breeding:

El Salvador [397]; Guatemala [824]; Honduras [981]; Mexico [1089]

Doryfera johannae (Bourcier 1847)

II

967

Blue-fronted Lancebill

Breeding:

Brazil [1270]; Colombia; Ecuador [248]; Peru [1064]; Venezuela [969]

Doryfera ludovicae (Bourcier \& Mulsant 1847) II $\quad$ - $\quad 16,967$

Green-fronted Lancebill

Breeding: $\quad$ Bolivia [956]; Colombia; Costa Rica [1285]; Ecuador [248]; Panama [1153]; Peru [1064]; Venezuela [969]

$\begin{array}{llll}\text { Elvira chionura (Gould 1851) } & \text { II } & - & 16\end{array}$

White-tailed Emerald

Breeding: $\quad$ Costa Rica [1285]; Panama [1153]

Elvira cupreiceps (Lawrence 1866) $\quad 11 \quad$ - $\quad 16$

Coppery-headed Emerald

Breeding: $\quad$ Costa Rica [1285]

Ensifera ensifera (Boissoneau 1839) $\quad 11 \quad-967$

Sword-billed Humminghird

Breeding: $\quad$ Bolivia [956]; Colombia; Ecuador [248]; Peru [1064]; Venezuela [969]

$\begin{array}{lllll}\text { Eriocnemis alinae (Bourcier 1842) } & 11 & - & 967\end{array}$

Emerald-bellied Puffleg

Breeding: $\quad$ Colombia [654,655]; Ecuador [248]; Peru [1064]

$\begin{array}{llll}\text { Eriocnemis cupreoventris (Fraser 1840) } & \text { II } & - & 967\end{array}$

Coppery-bellied Puffleg

Breeding: Colombia; Venezuela [969]

Eriocnemis derbyi (DeLattre \& Bourcier 1846) $\quad 11 \quad$ - $\quad 318,967$

Black-thighed Pufneg

Breeding: Colombia; Ecuador [248]

Eriocnemis glaucopoides (d'Orbigny \& Lafresnaye 1838) $11 \quad$ - 967

Blue-capped Puffleg

Breeding: $\quad$ Argentina [1034]; Bolivia [956]

Eriocnemis godini (Bourcier 1851) $\quad 11 \quad$ E/Ex $318,321,967$

Turquoise-throated Puffleg

Breeding: Colombia; Ecuador [248]

Eriocnemis luciani (Bourcier 1847)

$11 \quad-\quad 967$

Sapphire-vented Puffleg

Breeding: Colombia; Ecuador [248]; Peru [1064] 
Eriocnemis mirabilis Meyer de Schauensee 1967

$11 \mathrm{R}$

$318,321,967,1010$

Colourful Puffleg

Breeding: Colombia [654,655]

Eriocnemis mosquera (DeLattre \& Bourcier 1846) II

967

Golden-breasted Puffleg

Breeding: Colombia; Ecuador [248]

Eriocnemis nigrivestis (Bourcier \& Mulsant 1852) $\quad$ II $\quad$ E $\quad 318,321,967$

Black-breasted Puffleg

Breeding: $\quad$ Ecuador [170,248]

Eriocnemis vestitus (Lesson 1838)

II

967

Glowing Puffleg

Breeding:

Colombia; Ecuador [248]; Peru [1064]; Venezuela [969]

Eugenes fulgens (Swainson 1827)

II

16

Magnificent Hummingbird, Rivoli's Humminghird

Breeding:

Costa Rica [1285]; El Salvador [397]; Guatemala [824]; Honduras [981]; Mexico [419,1089]; Nicaragua; Panama [1153]; USA [740]

Eulampis holosericeus (Linnaeus 1758)

II

$16,179,1445$

(= Sericotes holosericeus)

Green-throated Carib

Breeding:

Antigua and Barbuda; Barbados; British Virgin Islands [1129]; Dominica; Grenada; Guadeloupe; Martinique; Montserrat; Netherlands Antilles [1445]; Puerto Rico [1 129]; Saint Kitts and Nevis; Saint Lucia; Saint Vincent; Virgin Islands of the United States [1129]

Eulampis jugularis (Linnaeus 1766)

II

$16,179,1445$

Purple-throated Carib

Breeding:

Antigua and Barbuda: Antigua and Barbuda; Dominica; Guadeloupe; Martinique; Montserrat; Netherlands Antilles; Saint Kitts and Nevis; Saint Lucia; Saint Vincent

Vagrant:

Antigua and Barbuda: Barbuda; Barbados; Brazil [1270]; Grenada; Virgin Islands of the United States [1025]

Eulidia yarrellii (Bourcier 1847)

I1 K

$318,321,794,967$

Chilean Woodstar

Breeding:

Chile [82]; Peru [1059]

Eupetomena macroura (Gmelin 1788)

II

967

Swallow-tailed Hummingbird

Breeding:

Bolivia [956]; Brazil [1239,1270]; French Guiana [152]; Guyana [1303]; Paraguay; Peru [1064]; Suriname [402]

Eupherusa cyanophrys Rowley \& Orr 1964

II V

$16,318,321$

Blue-capped Hummingbird, Oaxaca Hummingbird

Breeding:

Mexico [1089, 1197]

Eupherusa eximia (DeLattre 1843)

Il

16

Stripe-tailed Hummingbird

Breeding:

Belize [1202]; Costa Rica [1285]; Guatemala [824]; Honduras [981]; Mexico [165,1089]; Nicaragua; Panama [1153]

Black-bellied Hummingbird

Breeding:

Costa Rica [1285]; Panama [1153] 


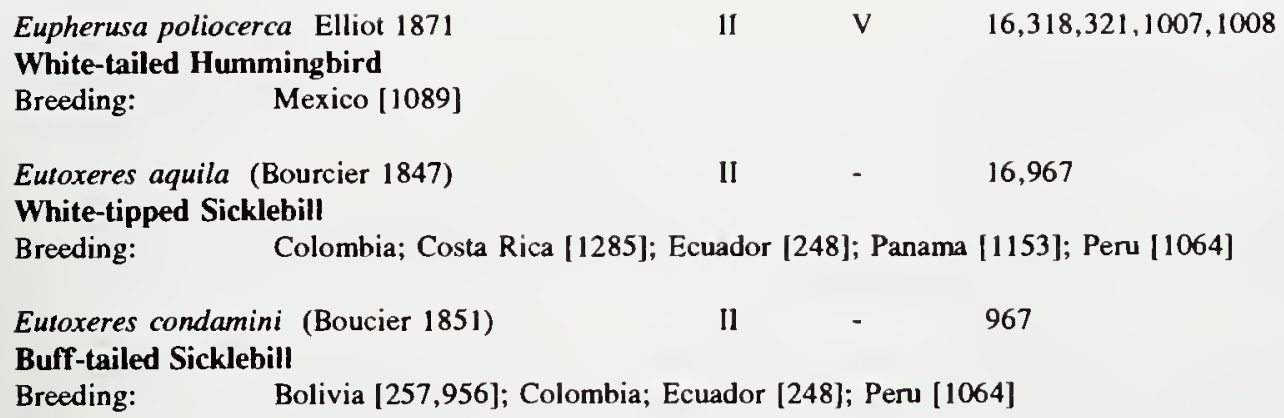
Florisuga mellivora (Linnaeus 1758)
II
$16,179,967$

White-necked Jacobin

Breeding: $\quad$ Aruba [1445]; Belize [1202]; Bolivia [956]; Brazil [1270]; Colombia; Costa Rica [1285]; Ecuador [248]; French Guiana [153]; Guatemala [824]; Guyana [1303]; Honduras [981]; Mexico [165, 1089]; Nicaragua; Panama [1153]; Peru [1064]; Suriname [402,628]; Trinidad and Tobago [465]; Venezuela [969]

Vagrant: Netherlands Antilles [1445]

Glaucis aenea Lawrence 1867

16,967

Bronzy Hermit

Breeding:

Colombia; Costa Rica [1285]; Ecuador [248]; Nicaragua; Panama [1153]

Glaucis hirsuta (Gmelin 1788)

Il

16,967

(includes Threnetes grimeki Ruschi 1973)

Rufous-breasted Hermit

Breeding: $\quad$ Bolivia [956]; Brazil [794,1200,1239,1270]; Colombia; Ecuador [248]; French Guiana [153]; Grenada [179]; Guyana [1303]; Panama [1153]; Peru [1064]; Suriname [402]; Trinidad and Tobago [465]; Venezuela [969]

Vagrant: Netherlands Antilles [1445]

Goethalsia bella Nelson 1912

II

16,967

Rufous-cheeked Humminghird

Breeding:

Colombia; Panama $[1153,1165]$

Goldmania violiceps Nelson 1911

II

16,967

Violet-capped Humminghird

Breeding:

Colombia; Panama [1153]

Haplophaedia aureliae (Bourcier \& Mulsant 1846) II

16,967

Greenish Puffleg

Breeding:

Bolivia; Colombia; Ecuador [248]; Panama [1153]; Peru [1064]
Haplophaedia lugens (Gould 1851)
II
$\mathrm{V} / \mathrm{R}$
$318,321,967$
Hoary Puffleg
Breeding:
Colombia; Ecuador [248]
Heliactin cornuta (Wied 1821)
946,967
(= H. bilophum (Temminck 1820))
Horned Sungem
Breeding:
Bolivia [956]; Brazil [1270]; Suriname [366,402]

Heliangelus amethysticollis (d'Orbigny \& Lafresnaye 1938)

Amethyst-throated Sunangel II - 967

Breeding: $\quad$ Bolivia [956]; Colombia; Ecuador [248]; Peru [1064]; Venezuela [969] 
Orange-throated Sunangel

Breeding:

Colombia; Venezuela [969]

Heliangelus regalis Fitzpatrick, Willard \& Terborgh 1979

Royal Sunangel

Breeding:

Peru $[471,1064]$

Heliangelus spencei (Bourcier 1847)

I1

II K

K

318,321

Merida Sunangel

Breeding:

Venezuela [969]

Heliangelus strophianus (Gould 1846)

II

Gorgeted Sunangel

Breeding:

Colombia; Ecuador [248]

Heliangelus viola (Gould 1853)

1]

Purple-throated Sunangel

Breeding: $\quad$ Ecuador [248]; Peru [1064]

Heliodoxa aurescens (Gould 1846)

J]

(= Polyplancta aurescens)

Gould's Jewelfront

Breeding:

Bolivia [956,1234]; Brazil [1270]; Colombia [470,655]; Ecuador [248]; Peru [1064]; Venezuela [969]

Heliodoxa branickii (Taczanowski 1874)

J] 967

Rufous-webhed Brilliant

Breeding:

Peru $[1064,1236]$

Heliodoxa gularis (Gould 1860)

II

967

Pink-throated Brilliant

Breeding:

Brazil [1270]; Colombia [470,655]; Ecuador [248]; Peru [1064]

Heliodoxa imperatrix (Gould 1856)

II

967

Empress Brilliant

Breeding:

Colombia; Ecuador [248]

Heliodoxa jacula Gould 1850

II

16,967

Green-crowned Brilliant

Breeding:

Colombia; Costa Rica [1285]; Ecuador [248]; Panama [1153]

Heliodoxa leadbeateri (Bourcier 1843)

I1

967

Violet-fronted Brilliant

Breeding:

Bolivia [956]; Colombia; Ecuador [248]; Peru [1064]; Suriname [402]; Venezuela [969]

Heliodoxa rubinoides (Bourcier \& Mulsant 1846) JJ 967

Fawn-breasted Brilliant

Breeding:

Colombia [654,655]; Ecuador [248]; Peru [1064] 
Heliodoxa schreibersii (Bourcier 1847)

11

967

Black-throated Brilliant

Breeding:

Brazil [1270]; Colombia [470,655]; Ecuador [248]; Peru [1064]

Heliodoxa xanthogonys Salvin \& Godman 1882

Velvet-browed Brilliant, Velvet-crowned Brilliant

Breeding:

Brazil [1270]; Guyana [1303]; Venezuela [969]

Heliomaster constantii (DeLattre 1843)

1]

16

Plain-capped Starthroat

Breeding:

Costa Rica [1285]; El Salvador [397]; Guatemala [824]; Honduras [981]; Mexico [1089]; Nicaragua

Vagrant:

USA [740]

Heliomaster furcifer (Shaw 1812)

$11 \quad-\quad 967$

Blue-tufted Starthroat

Breeding:

Argentina [1034]; Bolivia [956]; Brazil [1270]; Colombia; Paraguay; Uruguay [354,557]

Heliomaster longirostris (Audebert \& Vieillot 1801) 11

16,967

Long-billed Starthroat

Breeding: $\quad$ Bolivia [956]; Brazil [1270]; Colombia; Costa Rica [1285]; Ecuador [248]; El Salvador [397]; French Guiana [152]; Guatemala [824]; Guyana [1303]; Honduras [981]; Mexico [1089]; Nicaragua; Panama [1153]; Peru [1064]; [465]; Suriname [366,402]; Trinidad and Tobago; Venezuela [969]

Heliomaster squamosus (Temminck 1823)

II

967

Stripe-breasted Starthroat

Breeding: $\quad$ Argentina [1034]; Brazil [1270]

Heliothryx aurita (Gmelin 1788)

II

967

Black-eared Fairy

Breeding:

Bolivia [956]; Brazil [1239,1270]; Colombia; Ecuador [248]; French Guiana [396]; Guyana [1303]; Peru [1064]; Suriname [402]; Venezuela [969]

Heliothryx barroti (Bourcier 1843)

11

16,967

Purple-crowned Fairy

Breeding:

Belize [1202]; Colombia; Costa Rica [1285]; Ecuador [248]; Guatemala [824]; Honduras [981]; Mexico [1089]; Nicaragua; Panama [1153]

Hylocharis chrysura (Shaw 1812)

II

967

Gilded Hummingbird

Breeding:

Argentina [1034]; Bolivia [956]; Brazil [1270]; Paraguay; Venezuela [969]

Hylocharis cyanus (Vieillot 1818)

II $\quad-\quad 967$

White-chinned Sapphire

Breeding: $\quad$ Argentina [154,1034]; Bolivia [956]; Brazil [1239,1270]; Colombia; Ecuador [248]; French Guiana [152]; Guyana [1303]; Peru [1064]; Suriname [402]; Venezuela [969]

Hylocharis eliciae (Bourcier \& Mulsant 1846)

11

16,967

Blue-throated Goldentail

Breeding:

Belize; Colombia; Costa Rica [1285]; El Salvador [397]; Guatemala [824]; Honduras [981]; Mexico [165,1089]; Nicaragua; Panama [1153]

Hylocharis grayi (DeLattre \& Bourcier 1846)

11

16,967

Blue-headed Sapphire

Breeding:

Colombia; Ecuador [248]; Panama [1153] 
Hylocharis leucotis (Vieillot 1818)

II

16

White-eared Humminghird

Breeding:

El Salvador [397]; Guatemala [824]; Honduras [981]; Mexico [419,1089]; Nicaragua

Hylocharis pyropygia (Salvin \& Godman 1881)

$11 \quad-\quad 967$

Flame-rumped Sapphire

Breeding: ?Brazil $[1088,1270]$

Hylocharis sapphirina (Gmelin 1788) $\quad$ Il $\quad$ - 967

Rufous-throated Sapphire

Breeding: $\quad$ Argentina [1034]; Bolivia [956]; Brazil [1239,1270]; Colombia; Ecuador [248]; French Guiana [152,153]; Guyana [1303]; Paraguay; Peru [1064]; Suriname [402]; Venezuela [969]

Hylocharis xantusii (Lawrence 1860)

II

16

Xantus's Hummingbird, Black-fronted Hummingbird

Breeding:

Mexico [165,1089]

Vagrant:

USA

Hylonympha macrocerca Gould 1873

II

V/R

$318,321,967$

Scissor-tailed Hummingbird

Breeding: Venezuela $[61,969]$

Klais guimeti (Bourcier 1843)

11

$-\quad 16,967$

Violet-headed Hummingbird

Breeding:

Bolivia [956]; Brazil [1270]; Colombia; Costa Rica [1285]; Ecuador [248]; Honduras [981]; Nicaragua; Panama [1153]; Peru [1064]; Venezuela [969]

Lafresnaya lafresnayi (Boissoneau 1840)

Il

967

Mountain Velvetbreast

Breeding:

Colombia; Ecuador [248]; Peru [1064]; Venezuela [969]

Lampornis amethystinus Swainson 1827

II

16

Amethyst-throated Hummingbird

Breeding:

El Salvador [397]; Guatemala [824]; Honduras [981]; Mexico [1089]

Lampornis castaneoventris (Gould 1851) $\quad$ II $\quad-\quad 16$

(includes L. calolaema (Salvin 1865), L. cinereicauda (Lawrence 1867))

Variable Mountaingem, (Purple-throated Mountaingem, White-throated Mountaingem)

Breeding:

Costa Rica [1285]; Nicaragua; Panama [1153]

Lampornis clemenciae (Lesson 1829)

II

16

Blue-throated Humminghird

Breeding: $\quad$ USA [740]; Mexico [1089]

Lampornis hemileucus (Salvin 1865)

II

16

White-bellied Mountain-gem

Breeding:

Costa Rica [1285]; Panama [1153]

Lampornis sybillae (Salvin \& Godman 1892)

II

16

( $=L$. viripallens sybillae)

Green-breasted Mountain-gem

Breeding:

Honduras [981]; Nicaragua

Lampornis viridipallens (Bourcier \& Mulsant 1846)

16

Green-throated Mountain-gem

Breeding:

El Salvador [397]; Guatemala [824]; Honduras [981]; Mexico [1089] 
Lamprolaima rhami (Lesson 1838)

II

16

Garnet-throated Hummingbird

Breeding:

El Salvador [397]; Guatemala [824]; Honduras [981]; Mexico [165,1089]

Lepidopyga coeruleogularis (Gould 1851)

II

16,967

Sapphire-throated Hummingbird

Breeding:

Colombia; Costa Rica [1285]; Panama [1153]

Lepidopyga goudoti (Bourcier 1843)

1I

967

Shining-green Hummingbird

Breeding:

Colombia; Venezuela [969]

Lepidopyga lilliae Stone 1917

II Id

$318,321,967$

Sapphire-bellied Hummingbird

Breeding:

Colombia

Lesbia nuna (Lesson 1832)

II

967

Green-tailed Trainbearer

Breeding:

Bolivia [956]; Colombia; Ecuador [248]; Peru [1064]

Vagrant:

?Venezuela [969]

Lesbia victoriae (Bourcier \& Mulsant 1846)

11

967

Black-tailed Trainbearer

Breeding:

Colombia; Ecuador [248]; Peru [1064]

Leucippus baeri Simon 1901

II

Tumbes Hummingbird

Breeding:

Peru [1064]

Leucippus chlorocercus (Gould 1866)

II

967

Olive-spotted Hummingbird

Breeding: $\quad$ Brazil [1270]; Colombia; Ecuador [248]; Peru [ 1064]

Leucippus fallax (Bourcier 1843)

II

967

Buffy Humming bird

Breeding:

Colombia; ?French Guiana [953]; Venezuela [969]

Leucippus taczanowskii (Sclater 1879)

11

Spot-throated Hummingbird

Breeding:

Peru [1064]

Leucochloris albicollis (Vieillot 1818)

I1

967

White-throated Hummingbird

Breeding: $\quad$ Argentina [1034]; Brazil [1239,1270]; Paraguay; Uruguay [354,557]

Loddigesia mirabilis (Bourcier 1847)

II V

$177,318,321,967,1349$

Marvelous Spatuletail

Breeding:

Peru $[1064]$

Lophornis adorabilis Salvin 1870

II

16

(= Paphosia adorabilis)

White-crested Coquette, Adorable Coquette

Breeding:

Costa Rica [1285]; Panama [1153] 
Lophornis chalybeus (Vieillot 1823)

II

967

Festive Coquette

Breeding:

Argentina [154,1034]; Bolivia [956]; Brazil [1270]; Colombia; Ecuador [248]; Peru [1064];

Venezuela [969]

Lophornis delattrei (Lesson 1839)

$11 \quad-\quad 16,967$

(excluding subspecies brachylopha)

Rufous-crested Coquette

Breeding:

Bolivia [956]; Colombia; ?Costa Rica [1285]; Panama [1153]; Peru [1064].

Lophornis delattrei brachylopha

II $\quad \mathrm{E}$

$109,321,675$

(= L. brachylopha)

Short-crested Coquette

Breeding: $\quad$ Mexico $[61,1089]$

Lophornis gouldii (Lesson 1833)

II

967

Dot-eared Coquette

Breeding: Bolivia [956]; Brazil [1270]

Lophornis helenae (DeLattre 1843)

11

16

(= Paphosia helenae)

Black-crested Coquette

Breeding: $\quad$ Belize [1202]; Costa Rica [1285]; Guatemala [127,824]; Honduras [981]; Mexico [1089]; Nicaragua

Lophornis magnificus (Vieillot 1817)

11

967

Frilled Coquette

Breeding:

Brazil [1270]

Lophornis ornatus (Boddaert 1783)

Il

967

Tufted Coquette

Breeding:

Brazil [1270]; French Guiana [953]; Guyana [1303]; Suriname [402]; Trinidad and Tobago [465]; Venezuela [969]

Lophornis pavoninus Salvin \& Godman 1882

II

967

Peacock Coquette

Breeding:

Brazil [1270]; Guyana [1303]; Venezuela [969]

Lophornis stictolophus Salvin \& Elliot 1873

II

967

Spangled Coquette

Breeding:

Colombia; Ecuador [248]; Peru [1064]; Venezuela [969]

Melanotrochilus fuscus (Vieillot 1817)

II

967

Black Jacobin

Breeding:

Argentina [1034]; Brazil [1270]; Uruguay [557]

Mellisuga helenae (Lembeye 1850)

II

16,318

(= Calypte helenae)

Bee Hummingbird

Breeding:

Cuba $[179,509]$

Mellisuga minima (Linnaeus 1758)

II

16,179

Vervain Hummingbird

Breeding:

Vagrant:

Dominican Republic; Haiti [1534]; Jamaica

Puerto Rico [16,1129] 
Violet-throated Metaltail

Breeding: $\quad$ Ecuador $[248,1041,1151]$

Metallura eupogon (Cabanis 1874)

ll

Fire-throated Metaltail

Breeding: $\quad$ Peru [1064]

Metallura iracunda Wetmore 1947

II

Perija Metaltail

Breeding:

Colombia; Venezuela [969]

Metallura odomae Graves 1980

$11 \quad \mathrm{R}$

318,321

Neblina Metaltail

Breeding:

Peru $[569,1064,1067]$

Metallura phoebe (Lesson \& DeLattre 1839)

II

967

Black Metaltail

Breeding: Peru [1064]

Metallura theresiae Simon 1902

11

Coppery Metaltail

Breeding:

Peru [1064]

Metallura tyrianthina (Loddiges I832)

II $\quad-\quad 967$

Tyrian Metaltail

Breeding:

Bolivia [956]; Colombia; Ecuador [248]; Peru [1064]; Venezuela [969]

Metallura williami (DeLattre \& Bourcier 1846) II

967

Viridian Metaltail

Breeding:

Colombia; Ecuador [248]

Microchera albocoronata (Lawrence 1855)

ll

16

Snowcap

Breeding:

Costa Rica [1285]; Honduras [981]; Nicaragua; Panama [1 153]

Microstilbon burmeisteri (Sclater 1887)

II

967

Slender-tailed Woodstar

Breeding:

Argentina [1034]; Bolivia [956]

Myrmia micrura (Gould 1854)

II

967

Short-tailed Woodstar

Breeding: $\quad$ Ecuador [248]; Peru [1064]

Myrtis fanny (Lesson 1838)

ll

Purple-collared Woodstar

Breeding:

Ecuador [248]; Peru [1064]

Ocreatus underwoodii (Lesson 1832)

Il

967

Booted Racket-tail

Breeding:

Bolivia [956]; Colombia; Ecuador [248]; Peru [1064]; Venezuela [969] 
Opisthoprora euryptera (Loddiges 1832)

Mountain Avocetbill

Breeding:

Colombia [470]; Ecuador [248]; Peru [1064]

Oreonympha nobilis Gould 1869

11

967

Bearded Mountaineer

Breeding: Peru [1064]

Oreotrochilus adela (d'Orbigny \& Lafresnaye 1838) 11

Wedge-tailed Hillstar

Breeding:

Bolivia [956]

Oreotrochilus chimborazo (DeLattre \& Bourcier 1846)

$(=0$. estella chimborazo $)$

Ecuadorian Hillstar

Breeding: $\quad$ Ecuador [248]

Oreotrochilus estella (d'Orbigny \& Lafresnaye 1838) II $\quad-\quad 967$

Andean Hillstar

Breeding: $\quad$ Argentina [1034]; Bolivia [956]; Chile [82]; Peru [1064]

$\begin{array}{llll}\text { Oreotrochilus leucopleurus Gould } 1847 & \text { II } & - & 967\end{array}$

White-sided Hillstar

Breeding: Argentina [1034]; Bolivia [956]; Chile [82,1163]

Oreotrochilus melanogaster Gould 1847

II $\quad-\quad 967$

Black-breasted Hillstar

Breeding: Peru [1064]

Orthorhynchus cristatus (Linnaeus 1758)

Antillean Crested Humminghird

Breeding:

Antigua and Barbuda; Barbados; British Virgin Islands [1129]; Dominica; Grenada; Guadeloupe; Martinique; Montserrat; Netberlands Antilles [1445]; Puerto Rico [1129]; Saint Kitts and Nevis; Saint Lucia; Saint Vincent; Virgin Islands of the United States [1129]

Vagrant: ?USA [740]

Oxypogon guerinii (Boissoneau 1840)

II $\quad-\quad 967$

Bearded Helmetcrest

Breeding: Colombia; Venezuela [969]

$\begin{array}{llll}\text { Panterpe insignis Cabanis \& Heine } 1860 & \text { II } & - & 16,1322\end{array}$

Fiery-throated Hummingbird

Breeding: $\quad$ Costa Rica [1285]; Panama [1153]

Patagona gigas (Vieillot 1824) $\quad$ Il $\quad$ - $\quad 967$

Giant Hummingbird

Breeding: $\quad$ Argentina [1034]; Bolivia [956]; Chile [82]; ?Colombia; Ecuador [248]; Peru [1064]

Phaeochroa cuvierii (DeLattre \& Bourcier 1846) J1 $\quad$ - $\quad 16,967$

Scaly-breasted Hummingbird

Breeding: $\quad$ Belize [1202]; Colombia; Costa Rica [1285]; Guatemala [824]; Honduras [981]; Nicaragua; Panama [1153]

Vagrant: $\quad$ Mexico 
$\begin{array}{llll}\text { Phaethornis anthophilus (Bourcier 1843) } & 11 & - & 16,967\end{array}$

Pale-bellied Hermit

Breeding: $\quad$ Colombia; Panama [1153]; Venezuela [969]

$\begin{array}{llll}\text { Phaethornis augusti (Bourcier 1847) } & \text { II } & - & 967\end{array}$

Sooty-capped Hermit

Breeding: $\quad$ Brazil [1270]; Colombia; Guyana [1303]; Venezuela [969]

$\begin{array}{llll}\text { Phaethornis bourcieri (Lesson 1832) } & \text { II } & - & 967\end{array}$

Straight-billed Hermit

Breeding: $\quad$ Brazil [1270]; Colombia; Ecuador [248]; French Guiana [953]; Guyana [1303]; Peru [1064]; Suriname [402]; Venezuela [969]

$\begin{array}{llll}\text { Phaethornis eurynome (Lesson 1832) } & \text { II } & \text { - } & 794,967\end{array}$

(includes $P$. nigrirostris Ruschi 1973)

Scale-throated Hermit

Breeding: $\quad$ Argentina [1034]; Brazil [1268,1270]; Paraguay

$\begin{array}{llll}\text { Phaethornis gounellei Boucard } 1891 & 11 & - & 967\end{array}$

Broad-tipped Hermit

Breeding: Brazi] [1270]

$\begin{array}{llll}\text { Phaethornis griseogularis Gould 1851 } & 11 & - & 967\end{array}$

(includes $P$. porcullae Carriker 1935)

Grey-chinned Hermit, (Porculla Hermit)

Breeding: $\quad$ Bolivia [956]; Colombia; Ecuador [248]; Peru [1064]; Venezuela [969]

Phaethornis guy (Lesson 1833) II $\quad$ - $\quad 16,967$

Green Hermit

Breeding: $\quad$ Colombia; Costa Rica [1285]; Ecuador [248]; Panama [1153]; Peru [1064]; Trinidad and Tobago [465]; Venezuela [969]

$\begin{array}{llll}\text { Phaethornis hispidus (Gould 1846) } & \text { Il } & - & 967\end{array}$

White-bearded Hermit

Breeding: $\quad$ Bolivia [956]; Brazil [1270]; Colombia; Ecuador [248]; Peru [1064]; Venezuela [969]

$\begin{array}{llll}\text { Phaethornis idaliae (Bourcier \& Mulsant 1856) II } & \text { II } & 967\end{array}$

Minute Hermit

Breeding: $\quad$ Brazil $[1239,1270]$

Phaethornis koepckeae Weske \& Terborgh 1977 II $\quad-\quad 1480$

Koepcke's Hermit

Breeding: $\quad$ Peru [1064,1480]

$\begin{array}{llll}\text { Phaethornis longuemareus (Lesson 1832) } & \text { II } & - & 16,967\end{array}$

Little Hermit

Breeding:

Belize [1202]; Brazil [1270]; Colombia; Costa Rica [1285]; Ecuador [248]; French Guiana [953]; Guatemala [824]; Guyana [1303]; Honduras [981]; Mexico [165, 1089]; Nicaragua; Panama [1153]; Peru [1064]; Suriname [402]; Trinidad and Tobago [465]; Venezuela [969]

Phaethornis malaris (Nordmann 1835)

II

967

(includes $P$. margarettae Ruschi 1972)

Great-billed Hermit, (Margaretta's Hermit)

Breeding:

Brazil [943, 1270]; French Guiana [396]; Suriname [366,402] 
Phaethornis nattereri Berlepsch 1887

I1

967

(includes $P$. maranhaoensis Grantsau 1968)

Cinnamon-throated Hermit

Breeding:

Bolivia [956]; Brazil [1270]

Phaethornis philippii (Bourcier 1847)

I1

967

Needle-billed Hermit

Breeding:

Bolivia [956]; Brazil [1270]; Peru [1064]

Phaethornis pretrei (Lesson \& DeLattre 1839)

11

967

Planalto Hermit

Breeding:

Argentina [1034]; Bolivia [956]; Brazil [1270]; Paraguay

Phaethornis ruber (Linnaeus 1758)

II

967

Reddish Hermit

Breeding:

Bolivia [956]; Brazil [1239,1270]; Colombia; Ecuador [248]; French Guiana [954]; Guyana [1303]; Peru [1064]; Suriname [402]; Venezuela [969]

Phaethornis squalidus (Temminck 1822)

II

967

Dusky-throated Hermit

Breeding:

Brazil [1239, 1270]; Colombia; Guyana [1303]; Venezuela [969]

Phaethornis stuani Hartert 1897

I1

967

White-browed Hermit

Breeding:

Bolivia [956]; Peru [1064]

Phaethornis subochraceus Todd 1915

II

967

Buff-bellied Hermit

Breeding:

Bolivia [956]; Brazil [1270]

Phaethornis superciliosus (Linnaeus 1766)

$11 \quad-\quad 16,967$

Long-tailed Hermit

Breeding:

Belize [1202]; Bolivia [956]; Brazil [1270]; Colombia; Costa Rica [1285]; Ecuador [248];

French Guiana [396]; Guatemala [824]; Guyana [1303]; Honduras [981]; Mexico [1089];

Nicaragua; Panama [1153]; Peru [1064]; Suriname [402]; Venezuela [969]

Phaethornis symatophorus Gould 1851

II

967

Tawny-bellied Hermit

Breeding:

Colombia; Ecuador [248]; Peru [1064]

Phaethornis yaruqui (Bourcier 1851)

II

967

White-whiskered Hermit

Breeding:

Colombia; Ecuador [248]

Philodice bryantae (Lawrence 1867)

II

16

(= Calliphlox bryantae)

Magenta-throated Woodstar

Breeding: Costa Rica [1285]; Panama [1153]

Philodice mitchellii (Bourcier 1847)

II

16,967

(= Calliphlox mitchellii)

Purple-throated Woodstar

Breeding:

Colombia; Ecuador [248]; Panama [1153,1165] 
Phlogophilus harterti Berlepsch \& Stolzmann 1901 II

967

Peruvian Piedtail

Breeding:

Pen [1064]

Phlogophilus hemileucurus Gould 1860

11

967

Ecuadorian Piedtail

Breeding:

Colombia [470; Ecuador [248]; Peru [1064]
Polyonymus caroli (Bourcier 1847)
II
967
Bronze-tailed Comet
Breeding:
Peru [1064]
Polytmus guainumbi (Pallas 1764)
II
967

White-tailed Goldenthroat

Breeding: $\quad$ Argentina [154,1034]; Bolivia [956]; Brazil [1239,1270]; Colombia; French Guiana [152]; Guyana [1303]; Paraguay; Peru [566,1064]; Suriname [402,628]; Trinidad and Tobago [465,1370]; Venezuela [969]

$\begin{array}{llll}\text { Polytmus milleri Chapman } 1929 & \text { II } & - & 967\end{array}$

Tepui Goldenthroat

Breeding: Brazil [1270]; Venezuela [969]

Polytmus theresiae (Da Silva Maia 1843) $\quad$ Il $\quad-\quad 967$

Green-tailed Goldenthroat

Breeding: $\quad$ Brazil [1270]; Colombia; Ecuador [248]; Guyana [1303]; Peru [1064]; Suriname [402,628]; Venezuela [969]

Popelairia conversii (Bourcier \& Mulsant 1846) $11 \quad-\quad 16,967$

(= Discosura conversii)

Green Thorntail

Breeding: $\quad$ Colombia; Costa Rica [1285]; Ecuador [248]; Panama [1153]

$\begin{array}{llll}\text { Popelairia langsdorffi (Temminck 1821) } & \text { II } & - & 967\end{array}$

(= Discosura langsdorffi)

Black-bellied Thorntail

Breeding: $\quad$ Bolivia [1066]; Brazil [1270]; Colombia; Ecuador [248]; Peru [1064]; Venezuela [969]

Popelairia letitiae (Bourcier \& Mulsant 1846) II Id $318,321,967$

(= Discosura letitiae)

Coppery Thorntail

Breeding: ?Bolivia

Popelairia popelairii (DuBus \& Gisignies 1846) II $\quad-\quad 967$

(=Discosura popelairii)

Wire-crested Thorntail

Breeding: $\quad$ Colombia; Ecuador [248]; Peru [1064]

$\begin{array}{lll}\text { Pterophanes cyanopterus (Fraser 1839) } & \text { II } & -\end{array}$

Great Sapphirewing

Breeding:

Bolivia [956]; Colombia; Ecuador [248]; Penu [1064]

Ramphodon dohrnii (Bourcier \& Mulsant 1852) I I/R $\quad 311,318,321,794,967$

(= Glaucis dohrnii)

Hook-billed Hermit

Breeding: Brazil [1270] 
Black-backed Thornbill

Breeding: Colombia

Ramphomicron microrhynchum (Boissoneau 1839) II $\quad$ - 967

Purple-backed Thornbill

Breeding:

Bolivia [956]; Colombja; Ecuador [248]; Venezuela [969]

Rhodopis vesper (Lesson 1829)

IJ

Oasis Humminghird

Breeding:

Chile [82]; Peru [1064]

Sappho sparganura (Shaw 1812)

II

967

Red-tailed Comet

Breeding:

Argentina [1034]; Bolivia [956]; Chile [550]; Peru [1064]

Selasphorus ardens Salvin 1870

II $\quad$ V

$16,318,321$

Glow-throated Humminghird

Breeding:

Panama [1153]

Selasphorus flammula Salvin 1864

I1

16

(includes S. simoni Carriker 1910, S. torridus Salvin 1870),

Volcano Humminghird, (Cerise-throated Hummingbird, Heliotrope-throated Humminghird)

Breeding: Costa Rica [1285]; Panama [1153]

Selasphorus platycercus (Swainson 1827)

II

16

Broad-tailed Hummingbird

Breeding:

Guatemala [824]; Mexico [1089]; USA [740]

Selasphorus rufus (Gmelin 1788)

II

16

Rufous Hummingbird

Breeding:

Canada [740]; Mexico [1089]; USA [740]

Selasphorus sasin (Lesson 1829)

II

16

Allen's Hummingbird

Breeding:

Mexico [1089]; USA [740]

Vagrant:

?Canada [740]

Selasphorus scintilla (Gould 1851)

II

16

Scintillant Hummingbird

Breeding:

Costa Rica [1285]; Panama [1153]

Sephanoides fernandensis (King 1831)

II $\quad$ V/R

$305,318,321$

Juan Fernandez Firecrown

Breeding:

Chile: Juan Fernandez Islands $[218,1323]$

Sephanoides sephaniodes (Lesson 1827)

II

Green-backed Firecrown

Breeding:

Vagrant:

Argentina [1034]; Chile [82]

Falkland lslands 
Stellula calliope (Gould 1847)

11

16

Calliope Hummingbird

Breeding:

USA [740]; Canada [740]; Mexico [1089]

Stephanoxis lalandi (Vieillot 1818)

11

967

Plovercrest

Breeding:

Argentina [1034]; Brazil [1239, 1270]; Paraguay

Sternoclyta cyanopectus (Gould 1846)

II

967

Violet-chested Hummingbird

Breeding: Venezuela [969]

Taphrolesbia griseiventris (Taczanowski 1883)

Il

V

$318,321,967$

Grey-bellied Comet

Breeding: Peru [1064]

Taphrospilus hypostictus (Gould 1862)

11

967

Many-spotted Hummingbird

Breeding: ?Argentina [1034]; Bolivia [956]; Brazil [1270]; Ecuador [248]; Penu [1064]

Thalurania colombica (Bourcier 1843)

II $\quad-\quad 16,967$

(= T. furcata colombica)

(excluding subspecies ridgwayi)

(includes T. fannyi?)

Crowned Woodnymph, (Green-crowned Woodnymph)

Breeding: Belize [1202]; Colombia; Costa Rica; Ecuador; Guatemala [824]; Honduras [981]; Nicaragua; Panama [1153]; Venezuela

Thalurania colombica ridgwayi Nelson $1900 \quad$ ll $\quad$ ld 321,435

(=T. ridgwayi)

Mexican Woodnymph

Breeding: Mexico [165, 1089]

Thalurania furcata (Gmelin 1788)

$11 \quad 967$

Fork-tailed Woodnymph

Breeding: $\quad$ Argentina [154,1034]; Bolivia [956]; Brazil [1270]; Colombia; Ecuador [248]; French Guiana [396]; Guyana [1303]; Paraguay; Peru [1064]; Suriname [402]; ?Trinidad and Tobago [465]; Venezuela [969]

Thalurania glaucopis (Gmelin 1788)

11

967

Violet-capped Woodnymph

Breeding: $\quad$ Argentina [1034]; Brazil [1239, 1270]; Paraguay; Uruguay [557]

Thalurania watertonii (Bourcier 1847)

II

967

Long-tailed Woodnymph

Breeding:

Brazil [1270]; ?Guyana [1088]

Thaumastura cora (Lesson 1827)

II

967

Peruvian Sheartail

Breeding:

Peru [1064]

Vagrant:

Chile [82] 
Threnetes leucurus (Linnaeus 1766)

II

967

(includes T. cristinae Ruschi 1975, T. loehkeni Grantsau 1969)

Pale-tailed Barbthroat, (Bronze-tailed Barbthroat)

Breeding: $\quad$ Bolivia [956]; Brazil [1201,1270]; Colombia; Ecuador [248]; French Guiana [152]; Guyana [1303]; Peru [1064]; Suriname [402]; Venezuela [969]

Threnetes niger (Linnaeus 1758)

II

967

Sooty Barbthroat

Breeding:

Brazil [1270]; French Guiana [152]

Threnetes ruckeri (Bourcier 1847)

I1 $\quad$ - $\quad 16,967$

Band-tailed Barbthroat

Breeding: $\quad$ Belize [1202]; Colombia; Costa Rica [1285]; Ecuador [248]; Guatemala [824]; Honduras [981]; Nicaragua; Panama [1153]; Venezuela [969]

Tilmatura dupontii (Lesson 1832)

II

16

Sparkling-tailed Hummingbird, Sparkling-tailed Barbthroat

Breeding:

?Costa Rica [1285]; El Salvador [397]; Guatemala [824]; Honduras [981]; Mexico [1089]; Nicaragua

Topaza pella (Linnaeus 1758)

II

967

Crimson Topaz

Breeding:

Brazil [1270]; Ecuador [248]; French Guiana [153]; Guyana [1303]; Suriname [402]; Venezuela [969]

Topaza pyra (Gould 1846)

II

967

Fiery Topaz

Breeding:

Colombia; Ecuador [248]; Peru [1064]; Venezuela [969]

Trochilus polytmus Linnaeus 1758

I!

(includes T. scitulus (Brewster \& Bangs 1901))

Streamertail

Breeding:

Jamaica [179,1231]

Urochroa bougueri (Bourcier 1851)

11

967

White-tailed Hillstar

Breeding: $\quad$ Colombia; Ecuador [248]; Peru [1064]

Urosticte benjamini (Bourcier 1851)

II

967

Purple-bibbed Whitetip

Breeding: Colombia [470]; Ecuador [248]

Urosticte ruficrissa Lawrence 1864

II

(= U. benjamini ruficrissa)

Rufous-vented Whitetip

Breeding:

Colombia [470]; Ecuador [248]; Peru [1064] 


\section{Order TROGONIFORMES \\ Trogons}

Family TROGONIDAE

Trogons

Euptilotis neoxenus (Gould 1838)

ld

$16,318,321$

Eared Trogon, Eared Quetzal

Breeding:

Mexico; USA [1555]

Pharomachrus mocinno de la Llave 1832

Resplendent Quetzal

Breeding:

Costa Rica; El Salvador; Guatemala; Honduras [613]; Mexico; Nicaragua; Panama

\section{Order CORACIIFORMES \\ Kingfishers and allies}

\section{Family ALCEDINIDAE \\ Kingfishers}

Actenoides bougainvillei (Rothschild 1904) $\quad-\quad \mathrm{K}^{*} \quad 318,1226$

(= Halcyon bougainvillei, Monachalcyon bougainvillei)

Moustached Kingfisher

Breeding: $\quad$ Papua New Guinea: Bougainville; Solomon Islands: Guadalcanal (ex?)

Actenoides hombroni (Bonaparte 1850)

$\mathrm{K}^{*} \quad 318$

(= Halcyon hombroni, H. lindsayi hombroni)

Blue-capped Kingfisher, Hombron's Kingfisher

Breeding: $\quad$ Philippines: Mindanao [381]

$\begin{array}{llll}\text { Alcedo hercules Laubmann } 1917 & \text { - } & K^{*} & 10,318,792\end{array}$

Blyth's Kingfisher

Breeding:

Vagrant:

Bangladesh; Bhutan; China; Lao P.D.R; India; Myanmar; ?Nepal; Viet Nam Thailand

Tanysiptera riedelii Verreaux 1866

$\mathrm{K}^{*} \quad 318,1226$

Biak Paradise-Kingfisher

Breeding: Indonesia [131]

Todirhamphus australasia (Vieillot 1818) - $\quad \mathrm{K}^{*} \quad 318,1486$

(= Halcyon australasia)

Cinnamon-backed Kingfisher, Lesser Sunda Kingfisher

Breeding: Indonesia: Lombok, Sumba, Timor, Wetar and small islands east to Tanimbar

Todirhamphus gambieri (Oustalet 1895)

$\mathrm{K}^{*} \quad 318$

(= Halcyon gambieri)

Tuamotu Kingfisher, Mangareva Kingfisher

Breeding:

French Polynesia: Tuamotu Archipelago (Mangareva (ex), Niau) $[669,1118]$

Todirhamphus godeffroyi (Finsch 1877)

$\mathrm{K}^{*} \quad \mathbf{3 1 8}$

(= Halcyon godeffroyi)

Marquesan Kingfisher

Breeding: $\quad$ French Polynesia: Marquesas Islands (Hivaoa, Tahuata) $[669,1118]$ 
Todirhamphus ruficollaris (Holyoak 1974)

(= Halcyon ruficollaris)

Mangaia Kingfisher, Cook Islands Kingfisher

Breeding:

Cook Islands: Mangaia $[669,1118]$

Family MOMOTIDAE

Motmots

Electron carinatum (Du Bus \& Gisignies 1847)

K $\quad 16,61,318,321$

Keel-billed Motmot

Breeding:

Belize [973,974]; Costa Rica; Guatemala; Honduras; Mexico; Nicaragua

Family BRACHYPTERACIIDAE

Ground-Rollers

Atelornis crossleyi Sharpe 1875

R

318,324

Crossley's Ground-Roller, Rufous-headed Ground-Roller

Breeding:

Madagascar [373]

Brachypteracias leptosomus (Lesson 1833)

R $\quad 318,324$

Short-legged Ground-Roller

Breeding:

Madagascar [373]

Brachypteracias squamigera Lafresnaye 1838

Scaled Ground-Roller

R $\quad 318,324$

Breeding: $\quad$ Madagascar [373]

Uratelornis chimaera Rothschild 1895

Long-tailed Ground-Roller

Breeding: Madagascar [373]

Family BUCEROTIDAE

Hornbills

Aceros cassidix (Temminck 1823)

11

775

(= Rhyticeros cassidix)

Knobbed Hornbill

Breeding:

Indonesia: Butung, Lembeh, Muna, Sulawesi, Togian 1slands

Aceros comatus (Raffles 1822)

II $\quad-$

775

(= Rhyticeros comatus)

White-crowned Hornbill

Breeding:

Brunei; Indonesia: Kalimantan, Sumatra; Malaysia: Peninsular Malaysia, Sabah, Sarawak [1295]; Myanmar; Thailand; Viet Nam

Aceros corrugatus (Temminck 1832)

II $\quad \mathrm{K}^{*}$

318,775

(= Rhyticeros corrugatus)

Wrinkled Hornbill

Breeding:

Brunei; Indonesia: Batu Islands, Kalimantan, Sumatra; Malaysia: Peninsular Malaysia, Sabah, Sarawak [1295]; Thailand

Aceros everetti Rothschild 1897

$11 \quad \mathrm{~K}^{*}$

$318,775,1486$

(= Rhyticeros everetti)

Sumba Hornbill

Breeding: Indonesia: Sumba 
Aceros leucocephalus (Vieillot 1816)

I1

775

(= Rhyticeros leucocephalus)

Writhed Hornbill

Breeding: Philippines: Camiguin Sur, Mindanao

$\begin{array}{llll}\text { Aceros narcondami Hume } 1873 \quad \text { I1 } & K^{*} \quad 10,318,686,775\end{array}$

(= Rhyticeros narcondami)

Narcondam Hornbill

Breeding: India: Narcondam

$\begin{array}{llll}\text { Aceros nipalensis (Hodgson 1829) } & \text { I } & \text { K* } & 10,318,775,792\end{array}$

Rufous-necked Hornbill, Rufous-cheeked Hornbill

Breeding: $\quad$ Bangladesh; Bhutan; China; Lao P.D.R; India; Myanmar; Nepal (ex?) [700]; Thailand [1194]; Viet Nam

Aceros plicatus (Forster 1781) $\quad$ II $\quad-\quad 775$

(= Rhyticeros plicatus)

Blyth's Hornbill, Papuan Hornbill

Breeding:

Indonesia: Ambon, Bacan, Halmahera, Irian Jaya, Kasiratu, Kelang, Morotai, Obi, Seram; Papua New Guinea; Solomon Islands
Aceros subruficollis (Blyth 1843)
I $\quad \mathrm{K}^{*}$
318,775
(=Aceros plicatus subruficollis, Rhyticeros subruficollis)
Plain-pouched Hornbill
Breeding: India; Indonesia: Sumatra; Myanmar; Thailand
Aceros undulatus (Shaw 1811)
$11 \quad-\quad 775$
(= Rhyticeros undulatus)
Wreathed Hornbill
Breeding:
Bangladesh; Bhutan; Brunei; Cambodia; India; Indonesia: Bali, Batu Islands, Java, Kalimantan, Lingga Archipelago, Sumatra; Lao P.D.R; Malaysia: Peninsular Malaysia, Sabah, Sarawak [1295]; Myanmar; Thailand; Viet Nam

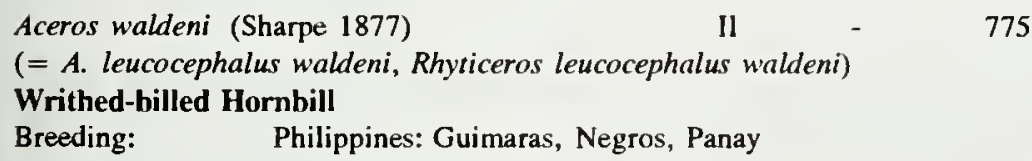

Breeding: $\quad$ Brunei; Indonesia: Kalimantan, Natuna Islands, Sumatra; Malaysia: Peninsular Malaysia, Sabah, Sarawak [1295]; Myanmar; Thailand

Anorrhinus tickelli (Blyth 1855)

(= Ptilolaemus tickelli)

Brown Hornbill

Breeding: $\quad$ China; India; Lao P.D.R; Myanmar; Thailand; Viet Nam

Anthracoceros albirostris (Shaw \& Nodder 1807) II $\quad-\quad 775$

(= A. malabaricus)

(includes $A$. convexus (Temminck 1831))

Oriental Pied-Hornbill, (Malaysian Pied-Hornbill)

Breeding: $\quad$ Bangladesh; Brunei; Cambodia; China; India; Indonesia; Lao P.D.R; Malaysia: Peninsular Malaysia, Sabah, Sarawak [1295]; Myanmar; Nepal; Thailand; Viet Nam

Vagrant: Singapore 
Anthracoceros coronatus

(Boddaert 1783)

II

Malabar Pied-Hornbill

Breeding:

India; Sri Lanka

Anthracoceros malayanus (Raffles 1822)

II

775

Black Hornbill

Breeding:

Brunei; Indonesia: Bangka, Belitung, Kalimantan, Sumatra; Malaysia: Peninsular Malaysia, Sabah, Sarawak [1295]; Thailand

Anthracoceros marchei Oustalet 1885

II

775

Palawan Hornbill

Breeding:

Philippines: Balabac, Busuanga, Calamian Islands, Palawan

Anthracoceros montani (Oustalet 1880)

II $\mathbf{K}^{*}$

318,775

Sulu Hornbill

Breeding:

Philippines: Jolo, Tawitawi

Buceros bicornis Linnaeus 1758

I

$10,775,792,1213$

Great Hornbill

Breeding:

Bangladesh; Bhutan; Cambodia; China; India; Indonesia: Sumatra [905]; Lao P.D.R; Malaysia: Peninsular Malaysia; Myanmar; Nepal [695]; Thailand [1194]; Viet Nam

Vagrant: Singapore

Buceros hydrocorax Linnaeus 1766

Il

775

Rufous Hornbill

Breeding:

Philippines [ 11113 ]

Buceros rhinoceros Linnaeus 1758

Il

775,792

Rhinoceros Hornbill

Breeding:

Brunei; Indonesia: Java, Kalimantan, Sumatra; Malaysia: Peninsular Malaysia, Sabah, Sarawak [1295]; Singapore (ex); Thailand [1194]

Buceros vigil (Forster 1781)

1 Id

$318,775,792$

(= Rhinoplax vigil)

Helmeted Hornbill

Breeding:

Brunei; Indonesia: Kalimantan [664], Sumatra [905]; Malaysia: Peninsular Malaysia, Sabah, Sarawak [1295]; Myanmar; Thailand [1194]

Penelopides affinis Tweeddale 1877

II

775

(= P. panini affinis)

Mindanao Hornbill

Breeding:

Philippines: Basilan, Dinagat, Mindanao, Siargao

Penelopides exarhatus (Temminck 1823)

II

Sulawesi Hornbill

Breeding:

Indonesia: Butung, Lembeh, Muna, Sulawesi

Penelopides manillae (Boddaert 1783)

II

775

(= P. panini manillae)

Luzon Hormbill

Breeding:

Philippines: Catanduanes, Luzon, Marindique, Polillo

Penelopides mindorensis Steere 1890

II

1775 ,

Mindoro Hornbill

Breeding: $\quad$ Philippines: Mindoro 
(= P. panini panini)

Tarictic Hornbill

Breeding:

Philippines: Guimaras, Masbate, Negros, Panay, Ticao

Penelopides samarensis Steere 1890

(= P. panini samarensis)

Samar Hornbill

Breeding:

Philippines: Bohol, Leyte, Samar

\section{Order PICIFORMES \\ Woodpeckers and allies

\author{
Family GALBULIDAE \\ Jacamars
}

Galbula pastazae Taczanowski \& Berlepsch 1885

Coppery-chested Jacamar

Breeding: ?Brazil; Colombia; Ecuador

Jacamaralcyon tridactyla (Vieillot 1817) - $\quad$ E $\quad 318,321$

Three-toed Jacamar

Breeding: $\quad$ Brazil [1271]

\section{Family CAPITONIDAE}

Barbets

Capito hypoleucus Salvin 1897

$\mathrm{V} / \mathrm{R} \quad 318,321$

White-mantled Barbet

Breeding: Colombia

$\begin{array}{lllll}\text { Megalaima javensis (Horsfield 1821) } & \text { - } & \text { R } & 318\end{array}$

Black-banded Barbet

Breeding: Indonesia: Java

Pogoniulus makawai Benson \& Irwin 1965

Id

$318,324,496,779$

(= aberrant example of $P$. bilineatus (Sundevall 1850))

White-chested Tinkerbird

Breeding: Zambia

Semnornis ramphastinus (Jardine 1855)

III $\mathrm{CO}$

$318,321,794,967$

Toucan Barbet

Breeding: Colombia; Ecuador

Family INDICATORIDAE

Honeyguides

Melignomon eisentrauti Louette 1981

K

$318,324,496$

Yellow-footed Honeyguide

Breeding:

Cameroon; ?Côte d'Ivoire; Ghana; ?Guinea; Liberia [327] 
Family RAMPHASTIDAE

Toucans

Aulacorhynchus huallagae

Carriker 1933

K

$318,321,967$

Yellow-browed Toucanet

Breeding:

Peru [1064]

Baillonius bailloni (Vieillot 1819)

II

967

Saffron Toucanet

Breeding:

Argentina [154]; Brazil [1268]

Pteroglossus aracari (Linnaeus 1758)

Il

967

Black-necked Aracari

Breeding: Brazil; French Guiana; Guyana; Suriname; Venezuela

Pteroglossus castanotis Gould 1834

II

967

Chestnut-eared Aracari

Breeding:

Argentina; Bolivia; Brazil; Colombia; Ecuador; Paraguay; Peru

Pteroglossus viridis (Linnaeus 1766)

II

967

Green Aracari

Breeding:

Brazil; French Guiana; Guyana; Suriname; Venezuela

Ramphastos sulfuratus Lesson 1830

II

16,967

Keel-billed Toucan

Breeding:

Belize; Colombia; Costa Rica; Guatemala; Honduras; Mexico; Nicaragua; Panama; Venezuela

Ramphastos toco Müller 1776

II

967

Toco Toucan

Breeding:

Argentina [154]; Bolivia; Brazil; Guyana; Paraguay; Peru; Suriname

Ramphastos tucanus

Linnaeus 1758

II

967

Red-billed Toucan

Breeding:

Brazil; Colombia; French Guiana; Guyana; Suriname; Venezuela

Ramphastos vitellinus Lichtenstein 1823

II

967

Channel-billed Toucan

Breeding:

Brazil; French Guiana; Guyana; Suriname; Venezuela

Selenidera maculirostris (Lichtenstein 1823)

II

967

Spot-billed Toucanet

Breeding:

Argentina [154]; Brazi]

Family PICIDAE

Woodpeckers

Campephilus imperialis (Gould 1832)

Imperial Woodpecker

$1 \quad \mathrm{E} / \mathrm{Ex} \quad 16,318,321,794,1264$

Breeding:

Mexico

Campephilus principalis (Linnaeus 1758)

Ivory-billed Woodpecker

Breeding:

Cuba $[6,37,179,936]$; USA (ex?)

E/Ex $\quad 16,318,321,420,710,794,1264$ 
Dryocopus galearus (Temminck 1822)

Helmeted Woodpecker

Breeding:

Argentina [154]; Brazil; Paraguay

Dryocopus javensis richardsi Tristan 1879

Tristram's Woodpecker

Breeding: $\quad$ Japan: Tsushima (ex); D.P.R. Korea; Korea Republic

Picoides borealis (Vieillot 1809)

Red-cockaded Woodpecker

Breeding:

USA

Picus rabieri (Oustalet 1898)

Red-collared Woodpecker

Breeding:

China; Lao P.D.R; Viet Nam

Sapheopipo noguchii (Seebohm 1887)

E

$318,794,1264$

$16,318,321,420,709,794,850$, $932,1264,1409$

Okinawa Woodpecker, Pryer's Woodpecker, Noguchi's Woodpecker

Breeding: Japan: Okinawa

K* $\quad 318,792.1264$

\section{Order PASSERIFORMES \\ Passerines \\ Family EURYLAIMIDAE \\ Broadbills}

Pseudocalyptomena graueri Rothschild 1909

$\mathbf{R}$

318,324

Grauer's Broadbill, African Green Broadbill

Breeding:

Uganda; Zaire

Family DENDROCOLAPTIDAE

Woodcreepers

Xiphocolaptes falcirostris (Spix 1824)

V

$20,318,321,967$

(includes X. franciscanus Snethlage 1927)

Moustached Woodcreeper, (Snethlage's Woodcreeper)

Breeding:

Brazil

Family FURNARIIDAE

Ovenbirds

Aphrastura masafuerae (Philippi \& Landbeck 1866) -

K

$318,321,1420$

Mas Afuera Rayadito

Breeding:

Chile: Juan Fernandez Islands [218,219] 
Asthenes anthoides (King 1831)

(= Thripophaga anthoides)

Austral Canastero

Breeding:

Argentina [154, 1004]; Chile [980]

Asthenes berlepschi (Hellmayr 1917)

Id

321,967

Berlepsch's Canastero

Breeding:

Bolivia

Asthenes dorbignyi huancavelicae Morrison 1938

V

318,321

(=A. huancavelicae)

(includes A. usheri Morrison 1947)

Pale-tailed Canastero, (White-tailed Canastero)

Breeding:

Peru

Asthenes luizae Vielliard 1990

ld

$321,1079,1434$

Cipó Canastero

Breeding:

Brazil

Automolus ruficollis (Taczanowski 1884)

E

$318,321,967,1420$

(= Syndactyla ruficollis)

Rufous-necked Foliage-gleaner

Breeding: $\quad$ Ecuador; Peru [1064, 1067]

Cinclodes excelsior aricomae (Carriker 1932)

(= Geositta excelsior aricomae, Cinclodes aricomae)

Royal Cinclodes

Breeding:

Bolivia; Peru $[476,479]$

Cinclodes palliatus (Tschudi 1844)

White-bellied Cinclodes

Breeding: Peru

Hylocryptus erythrocephalus Chapman 1919

(= Automolus erythrocephalus)

Henna-hooded Foliage-gleaner

Breeding:

Ecuador; Peru [1064,1490]

Leptasthenura xenothorax Chapman 1921

E

$318,321,476,967$

$(=L$. pileata xenothorax)

White-browed Tit-Spinetail

Breeding:

Peru [1062]

Megaxenops parnaguae Reiser 1905

K

$318,321,967,1420$

Great Xenops

Breeding:

Brazil

Philydor novaesi Teixeira \& Gonzaga 1983

E

318,321

Alagoas Foliage-gleaner

Breeding:

Brazil [1359, 1362]

Premnoplex tatei Chapman 1925

(= Margarornis tasei)

White-throated Barbtail

Breeding:

Venezuela [61,969]

V/R $\quad 318,321,967$ 


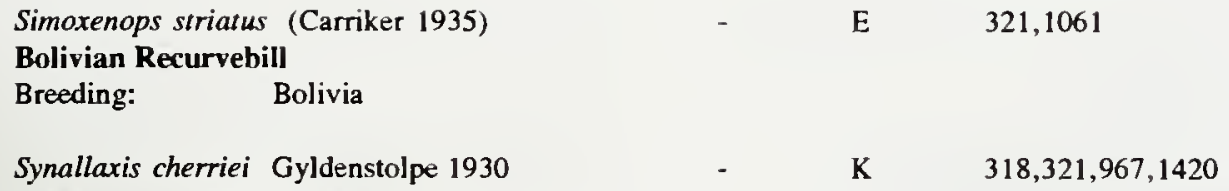


Clytoctantes atrogularis Lanyon, Stotz \&Willard $1990 \quad-\quad$ ld

ld $\quad 321,835$

Rondônia Bushbird

Breeding: Brazil

Dysithamnus occidentalis (Chapman 1923)

$\mathrm{V} / \mathrm{R} \quad 321,1488$

Western Antvireo, Bicolored Antvireo

Breeding:

Colombia; Ecuador

Dysithamnus plumbeus (Wied 1831)

$\mathrm{V} / \mathrm{R} \quad 318,321,967$

(= Thamnomanes plumbeus)

Plumbeous Antvireo, Plumbeous Antshrike

Breeding: Brazil [1239,1273]

Formicarius rufifrons Blake 1957

V

$318,321,967$

Rufous-fronted Antthrush

Breeding:

Peru [1060]

Formicivora erythronotos (Hartlaub 1852)

(= Myrmotherula erythronotos)

Black-hooded Antwren

Breeding:

Brazil $[45,1239]$

Formicivora iheringi Hellmayr 1909

V

$318,321,794,967$

Narrow-billed Antwren

Breeding:

Brazil [1271,1513]

Formicivora littoralis Gonzaga \& Pacheco 1990

E

$321,544,1309$

(=F. serrana littoralis)

Restinga Antwren

Breeding:

Brazil

Grallaria alleni Chapman 1912

$\mathrm{E} / \mathrm{Ex}$

$318,321,794,967$

Moustached Antpitta

Breeding:

Colombia

Grallaria chthonia Wetmore \& Phelps 1956

E

$318,321,967$

Tachira Antpitta

Breeding:

Venezuela [969]

Grallaria gigantea Lawrence 1866

Giant Antpitta

Breeding: Colombia [654]; Ecuador

Grallaria milleri Chapman 1912

Brown-banded Antpitta

Breeding: Colombia

Grallaria rufocinerea Sclater \& Salvin 1879

Bicolored Antpitta

Breeding:

Colombia

Grallaricula cucullata (Sclater 1856)

Hooded Antpitta

Breeding:

Colombia; Venezuela [969]

K $\quad 318,321,967$

$\mathrm{E} / \mathrm{Ex} \quad 318,321,794,967$

ld $\quad 318,321,967$

$\mathrm{V} / \mathrm{R} \quad 318,321,967$ 
Herpsilochmus parkeri Davis \& O'Neill 1986

Ash-throated Antwren

Breeding:

Peru [367]

Herpsilochmus pectoralis Sclater 1857

Pectoral Antwren

Breeding:

Brazil [1271]

Myrmeciza griseiceps (Chapman 1923)

E

$318,321,967$

Grey-headed Antbird

Breeding:

Ecuador; Peru $[1064,1067,1490]$

Myrmeciza ruficauda (Wied 1831)

V/R $\quad 318,321,967$

Scalloped Antbird

Breeding:

Brazil [1245,1270]

Myrmotherula fluminensis Gonzaga 1988

ld

321

Rio de Janeiro Antwren

Breeding:

Brazil

Myrmotherula grisea Carriker 1935

$\mathrm{V} / \mathrm{R} \quad 318,321,967$

Ashy Antwren

Breeding: Bolivia

Myrmotherula snowi Teixeira \& Gonzaga 1983

E

321

Alagoas Antwren

Breeding:

Brazil

Pithys castanea Berlioz 1938

ld

321,967

White-masked Anthird

Breeding:

Peru

Pyriglena atra (Swainson 1825)

E

$318,321,794,967$

Fringe-backed Fire-eye

Breeding: Brazil [1273,1513]

Rhopornis ardesiaca (Wied 1831)

V

$318,321,794,967,1355$

Slender Antbird

Breeding: Brazil [1271,1513]

Terenura sharpei Berlepsch 1901

V

$318,321,967$

Yellow-rumped Antwren

Breeding:

Bolivia; Pen

Terenura sicki Teixeira \& Gonzaga 1983

E $\quad 318,321,1357,1517$

Alagoas Antwren, Orange-bellied Antwren

Breeding:

Brazil [1360]

Xenornis setifrons Chapman 1924

K $\quad 318,321,967$

Speckled Antshrike

Breeding:

Colombia; Panama [1153] 
Family RHINOCRYPTIDAE

Tapaculos

Merulaxis stresemanni Sick 1960

ld

$318,321,794,967$

Stresemann's Bristlefront

Breeding: Brazil

Scytalopus novacapitalis Sick 1958

R $\quad 318,321,794,967$

Brasilia Tapaculo

Breeding:

Brazil [1271]

Scytalopus psychopompus Teixeira \& Camevalli 1989

Bahia Tapaculo

E

321,1358

Breeding:

Brazil

Family COTINGIDAE

Cotingas

Calyptura cristata (Vieillot 1818)

E/Ex $\quad 318,321,794,967,1301$

Kinglet Calyptura, Kinglet Cotinga

$-\quad \mathrm{E} / \mathrm{Ex}$

Breeding:

Brazil (ex?)

Carpodectes antoniae Ridgway 1884

Yellow-billed Cotinga

Breeding:

Costa Rica [1351]; Panama

Carpornis melanocephalus (Wied 1820)

V/R $\quad 318,321,967,1301$

Black-headed Berryeater

Breeding: Brazil [1363]

Cephalopterus glabricollis Gould 1851

Bare-necked Umbrellabird

Breeding:

Costa Rica [1351]; Panama

Cephalopterus ornatus Geoffroy Saint-Hilaire 1809 111 CO

967,1301

Amazonian Umbrellabird, Ornate Umbrellabird

Breeding:

Bolivia; Brazil; Colombia; Ecuador; French Guiana; Guyana; Peru [1249]; Suriname; Venezuela

Cephalopterus penduliger Sclater 1859

Long-wattled Umbrellabird

$111 \mathrm{CO} \quad-\quad 318,794,967,1301$

Breeding:

Colombia; Ecuador

Cotinga maculata (Müller 1776)

Banded Cotinga

Breeding:

Brazil [1239]

Iodopleura pipra (Lesson 1831)

Buff-throated Purpletuft

$\mathrm{V} / \mathrm{R} \quad 16,318,321,1301$

Breeding:

Brazil $[255,1271,1514]$

Laniisoma elegans elegans (Thunberg 1823)

(= L. elegans)

Shrike-like Cotinga

Breeding:

Brazil 
Lipaugus lanioides (Lesson 1844)

V/R $\quad 318,321,967,1301$

Cinnamon-vented Piha

Breeding:

Brazil [1239]

Pachyramphus spodiurus Sclater 1860

E

321,967

Slaty Becard

Breeding:

Ecuador; Peru

Rupicola peruviana (Latham 1790)

II $\quad$ - $\quad 967,1301$

Andean Cock-of-the-rock

Breeding: $\quad$ Bolivia; Colombia; Ecuador; Peru; Venezuela

Rupicola rupicola (Linnaeus 1766)

$11 \quad-\quad 967,1301$

Guianan Cock-of-the-rock

Breeding: $\quad$ Brazil; Colombia; French Guiana; Guyana; Suriname; Venezuela

Tijuca condita Snow 1980

R $\quad 318,321,967,1301$

Grey-winged Cotinga

Breeding: Brazil [1239,1300]

Xipholena atropurpurea (Wied 1820)

I $\quad$ R

$318,321,794,967,1301$

White-winged Cotinga

Breeding:

Brazil

Zaratornis stresemanni (Koepcke 1954)

K

(= Ampelion stresemanni)

White-cheeked Cotinga

Breeding: Peru [473]

Family PIPRIDAE

Manakins

Pipra vilasboasi Sick 1959

Id $318,321,967$

Golden-crowned Manakin

Breeding: Brazil

Piprites pileatus (Temminck 1822)

$\mathrm{V} / \mathrm{R} \quad 318,321,967,1102$

Black-capped Piprites, Black-capped Manakin

Breeding: $\quad$ Argentina [154]; Brazil [1271]

Family TYRANNIDAE

Tyrant-flycatchers

Agriornis andicola Sclater 1860

V/R $\quad 318,321,967$

(= A. albicauda (Philippi \& Landbeck 1863))

White-tailed Shrike-Tyrant

Breeding: $\quad$ Argentina [154]; Bolivia [473]; Chile; Ecuador; Peru [473]

Alectrurus risora (Vieillot 1824)

V $\quad 318,321,967$

(= Yetapa risora)

Strange-tailed Tyrant

Breeding: $\quad$ Argentina [154]; Brazil (ex?) [140,1271]; Paraguay; Uruguay (ex?)

Anairetes alpinus (Carriker 1933)

E

$318,321,967$

Ash-breasted Tit-Tyrant

Breeding:

Bolivia; Peru [473, 1062] 
Attila torridus Sclater 1860

E

321,967

Ochraceous Attila

Breeding:

Colombia; Ecuador [1166]; Peru

Empidonax griseipectus Lawrence 1870

V/R $\quad 318,321,967$

(= Lathrotriccus griseipectus)

Grey-breasted Fycatcher

Breeding:

Ecuador; Peru

Euscarthmus rufomarginatus (Pelzeln 1868)

Id

$122,321,967$

Rufous-sided Pygmy-Tyrant

Breeding: Bolivia; Brazil; Suriname

Hemitriccus cinnamomeipectus Fitzpatrick \& O'Neill 1979

Cinnamon-breasted Tody-Tyrant

Breeding:

Peru

Hemitriccus furcatus (Lafresnaye 1846)

$\mathbf{R}$

$318,321,967$

(= Ceratotriccus furcatus)

Fork-tailed Tody-Tyrant

Breeding: Brazil [1239]

Hemitriccus kaempferi (Zimmer 1953)

Id $\quad 318,321,967$

(= Idioptilon kaempferi)

Kaempfer's Tody-Tyrant

Breeding: Brazil [468]

Hemitriccus mirandae (Snethlage 1925)

V/R $\quad 321,967$

Buff-breasted Tody-Tyrant

Breeding: Brazil

Onychorhynchus coronatus occidentalis (Sclater 1860)

( $=0$. occidentalis)

E

321

Western Royal-Flycatcher, Pacific Royal Flycatcher

Breeding:

Ecuador; Peru

Phylloscartes ceciliae Teixeira 1987

E

318,321

Alagoas Tyrannulet, Long-tailed Tyrannulet

Breeding: Brazil [1356]

Phylloscartes lanyoni Graves 1988

Id

321,1324

Antioquia Bristle-Tyrant

Breeding:

Colombia

Phylloscares paulistus Thering \& Thering 1907

$\mathbf{V} / \mathbf{R}$

$318,321,967,1493$

São Paulo Tyrannulet

Breeding:

?Argentina [154]; Brazil [1239,1271,1513]; Paraguay

Phylloscartes roquettei Snethlage 1928

V

$318,321,967$

Minas Gerais Tyrannulet

Breeding:

Brazil

Platyrinchus leucoryphus Wied 1831

Russet-winged Spadebill

V/R

$318,321,967$

Breeding:

?Argentina [154]; Brazil [140,1239,1271]; Paraguay 


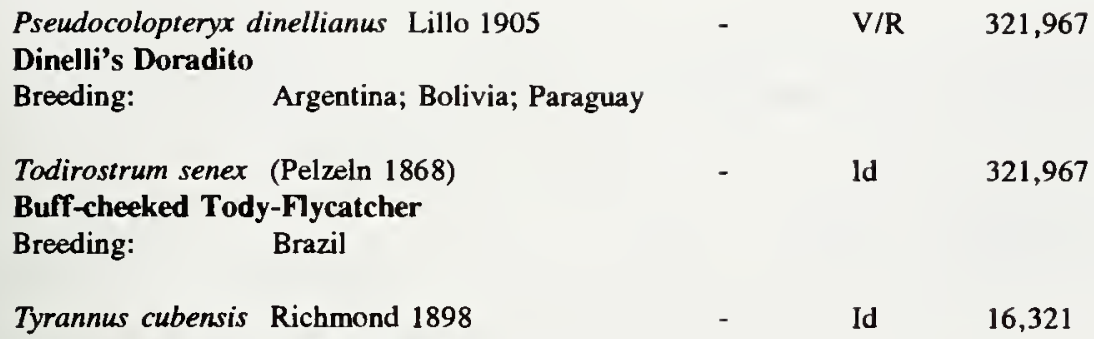

Giant Kingbird, Cuban Flycatcher

Breeding: $\quad$ Bahamas (ex); Cuba; Turks and Caicos Islands

Vagrant: Mexico: Mujeres Island

\section{Family PHYTOTOMIDAE}

Piantcutters

$\begin{array}{lllll}\text { Phytotoma raimondii Taczanowski } 1883 & - & \text { E } & 318,321,967\end{array}$

Peruvian Plantcutter

Breeding: Pen [1064]

Family PITTIDAE

Pittas

Pitta anerythra Rothschild $1901 \quad$ - $\quad \mathrm{K}^{*} \quad 318,1226$

Black-faced Pitta, Solomons Pitta

Breeding: $\quad$ Papua New Guinea: Bougainville (ex?); Solomon Islands: Santa Isabel (ex?)

$\begin{array}{llll}\text { Pitta elliotii Oustalet } 1874 & - & \mathrm{K}^{*} & 318,792\end{array}$

Bar-bellied Pitta

Breeding: $\quad$ Cambodia; Lao P.D.R; Thailand; Viet Nam

Pitta guajana (Müller 1776)

$11 \quad 792$

Banded Pitta, Blue-tailed Pitta

Breeding: $\quad$ Brunei [898]; Indonesia; Malaysia: Peninsular Malaysia, Sabah, Sarawak [1295]; Thailand [1194]

Pitta gurneyi Hume 1875

$1 \quad \mathrm{E}$

$318,323,578,792,794$

Gurney's Pitta

Breeding: $\quad$ Myanmar (ex?); Thailand [1194,1195]

Pitta kochi Brüggemann 1876

I $\quad \mathrm{K}^{*} \quad 318,737$

Whiskered Pitta, Koch's Pitta

Breeding: $\quad$ Philippines: Luzon $[399,1113]$

$\begin{array}{lllll}\text { Pitta nympha Temminck \& Schlegel } 1850 \quad \text { II } & \mathrm{K}^{*} & 318,792\end{array}$

(= P. brachyura nympha)

Fairy Pitta

Breeding:

China [944]; Japan [944, 1494]; Korea Republic: Cheju Do and nearby islands [558,944]; Taiwan [1253]

Non-breeding: Indonesia: Kalimantan [944]; Malaysia: Sarawak [944,1295]; Viet Nam

Vagrant: Brunei; Hong Kong

Pitta schneideri Hartert 1909

K*

Schneider's Pitta

Breeding:

Indonesia: Sumatra $[54,905]$ 
Pitta steerii (Sharpe 1876)

$\mathrm{K}^{*}$

318

Azure-breasted Pitta, Steere's Pitta

Breeding:

Philippines: Bohol, Leyte, Mindanao, Samar [1113]

Pitta superba Rothschild \& Hartert 1914

$\mathrm{K}^{*}$

318,1226

Black-backed Pitta, Superb Pitta

Breeding: Papua New Guinea: Manus

Family XENICIDAE

New Zealand Wrens

Xenicus longipes (Gmelin 1789)

E

$318,794,976$

Bush Wren, New Zealand Bush Wren

Breeding:

New Zealand (ex?) [1507]

Family PHILEPITTIDAE

Asitys

Neodrepanis hypoxanthus Salomonsen 1933

Id

$318,324,858$

Small-billed Asity, Yellow-bellied Sunbird-asity

Breeding:

Madagascar [373]

Family ATRICHORNITHIDAE

Scrub-birds

Atrichornis clamosus (Gould 1844)

E

$318,362,794,1291$

Noisy Scrub-bird

Breeding:

Australia [167]

Atrichornis rufescens (Ramsay 1867)

$\mathbf{R}$

318

Rufous Scrub-bird

Breeding:

Australia [167]

Family ALAUDIDAE

Larks

Spizocorys fringillaris (Sundevall 1850)

(= Calandrella fringillaris)

Botha's Lark

Breeding: South Africa [215]

Alauda razae (Alexander 1898)

(= Calandrella razae)

Raso Lark, Raza Island Lark

Breeding:

Cape Verde: Raso Island

Heteromirafra archeri Clarke 1920

Id

$318,324,600$

( $=H$. ruddi archeri)

Archer's Lark, Somali Long-clawed Lark

Breeding:

Somalia

Heteromirafra ruddi (Grant 1908)

ld

$285,318,324,600$

(= Mirafra ruddi ruddi)

Rudd's Lark, South African Long-clawed Lark

Breeding: Lesotho; South Africa [215]; Swaziland 
(= Mirafra sidamoensis)

Sidamo Lark, Sidamo Long-clawed Lark, Sidamo Bushlark

Breeding:

Ethiopia [102]

Mirafra ashi Colston 1982

K

318,324

Ash's Lark

Breeding:

Somalia

Mirafra degodiensis Erard 1975

K $\quad 318,324$

Degodi Lark

Breeding: Ethiopia

Family HIRUNDINIDAE

Swallows

Hirundo megaensis Benson 1942

$\mathbf{R}$

$318,324,600,1404$

White-tailed Swallow

Breeding:

Ethiopia

Hirundo perdita Fry \& Smith 1985

K* $\quad 318,497,1404$

Red Sea Swallow, Red Sea Cliff-Swallow

Breeding:

?Sudan

Pseudochelidon sirintarae Thonglongya 1968

$1 \quad 1 d$

$318,792,794,1404$

White-eyed River-Martin

Breeding: ?Thailand [1033,1194,1311]

Non-breeding: Thailand

Family MOTACILLIDAE

Wagtails and Pipits

Anthus chloris Lichtenstein 1842

$\mathrm{K}^{*} \quad 318$

Yellow-breasted Pipit

Breeding: Lesotho; South Africa[215]

Anthus nattereri Sclater 1878

ld

$318,321,967$

Ochre-breasted Pipit

Breeding:

Argentina [154]; Brazil [140,1271]; Paraguay

Anthus sokokensis van Someren 1921

V

$212,318,324,600$

Sokoke Pipit

Breeding: Kenya; Tanzania (ex?)

Family CAMPEPHAGIDAE

Cuckoo-shrikes

Campephaga lobata (Temminck 1824)

V

$318,324,600,1247$

Ghana Cuckoo-shrike, Western Wattled Cuckoo-shrike

Breeding:

Côte d'Ivoire [55]; Ghana; Liberia [327]; Sierra Leone

Coracina coerulescens (Blyth 1842)

$\mathrm{K} *$

318

Blackish Cuckoo-shrike, Luzon Graybird

Breeding:

Philippines: Cebu (ex), Luzon [1 1 13] 
RL Ref

Coracina sula (Hartert 1917)

K*

318,1486

(=C. morio sula)

Sula Cicadabird, Sula Cuckoo-shrike, Moluccan Graybird

Breeding:

Indonesia: Sula Islands

Coracina newtoni (Pollen 1866)

V

318,324

Réunion Cuckoo-shrike

Breeding:

Réunion

Coracina ostenta Ripley 1952

$\mathrm{K}^{*} \quad 318$

(=C. panayensis (Steere 1890))

White-winged Cuckoo-shrike, White-winged Graybird

Breeding: $\quad$ Philippines: Guimaras, Negros, Panay

Coracina schistacea (Sharpe 1879)

$K^{*} \quad 318,1486$

Slaty Cuckoo-shrike, Sula Cuckoo-shrike

Breeding: Indonesia: Banggai, Peleng, Sula

Coracina typica (Hartlaub 1865) - N V $\quad 318,324$

Mauritius Cuckoo-shrike

Breeding: Mauritius

Family PYCNONOTIDAE

Bulbuls

Chlorocichla prigoginei De Roo 1967

V $318,324,600$

Prigogine's Greenbul

Breeding: Zaire

Criniger olivaceus (Swainson 1837)

Yellow-bearded Bulbul, Olivaceous Bulbul

Breeding:

Côte d'Ivoire [55]; Ghana; Guinea; Liberia [327]; Senegal; Sierra Leone

Hypsipetes borbonicus olivaceus Jardine \& Selby 1835 - V V

318,324

(= H. olivaceus)

Mauritius Bulbul

Breeding:

Mauritius

Lxos siquijorensis (Steere 1890)

K* $\quad 318$

(= Hypsipetes siquijorensis)

Streak-breasted Bulbul, Mottle-breasted Bulbul

Breeding:

Philippines: Cebu (ex), Romblon, Siquijor, Tablas [381]

Phyllastrephus apperti Colston 1972

$\mathbf{R}$

318,324

Appert's Tetraka, Appert's Greenbul

Breeding: Madagascar [373]

Phyllastrephus cinereiceps (Sharpe 1881)

$\mathbf{R}$

318,324

Grey-crowned Tetraka, Grey-crowned Greenbul

Breeding:

Madagascar [373]

Phyllastrephus leucolepis Gätter 1985

$\mathrm{K}^{*} \quad 318$

Liberian Greenbul, Spot-winged Greenbul

Breeding: Liberia [519] 
Phyllastrephus tenebrosus (Stresemann 1925)

$\mathbf{R}$

318,324

Dusky Tetraka, Dusky Greenbul

Breeding:

Madagascar [373]

Pycnonotus nieuwenhuisii (Finsch 1901)

$\mathrm{K}^{*}$

318

Blue-wattled Bulbul

Breeding:

Indonesia: Kalimantan [1295], Sumatra [905]

Family LANIIDAE

Helmet-shrikes, Bushshrikes and Shrikes

Lanius liberatus Smith, Arctander, Fjeldså and Amir 1991

Bulo Burti Boubou

Breeding: Somalia

K* $\quad 1290$

Lanius newtoni Barboza du Bocage 1891

ld

$318,324,600,1247$

Newton's Fiscal, Sao Tome Fiscal Shrike

Breeding:

Sao Tome and Principe: Sao Tome (ex?)

Malaconotus alius Friedmann 1927

$\mathbf{R}$

$212,318,324,600$

Uluguru Bushshrike

Breeding:

Tanzania

Malaconotus monteiri (Sharpe 1870)

ld

$80,318,324,600$

(= M. blanchoti monteiri)

Monteiro's Bushshrike

Breeding: $\quad$ Angola; Cameroon (ex?); Kenya

Malaconotus gladiator (Reichenow 1892)

$\mathrm{R}$

$80,318,324,600,1247$

Green-breasted Bushshrike, Cameroon Mountain Bushshrike

Breeding:

Cameroon; ?Nigeria

Prionops gabela Rand 1957

Angola Helmetshrike, Gabela Helmetshrike

Breeding:

Angola

Telophorus kupeensis (Serle 1951)

ld

$65,318,324,600,1247$

(= Malaconotus kupeensis)

Serle's Bushshrike, Kupe Bushshrike

Breeding: Cameroon

\section{Family VANGIDAE}

Vangas

Xenopirostris damii Schlegel 1866

R

324,324

Van Dam's Vanga

Breeding:

Madagascar [373]

Xenopirostris polleni (Schlegel 1868)

Pollen's Vanga

Breeding:

Madagascar [373]

R $\quad 318,324,794$ 
Family CINCLIDAE

Dippers

Cinclus schulzi Cabanis 1882

$\mathrm{K}$

$141,318,321,794,967$

Rufous-throated Dipper

Breeding: $\quad$ Argentina [154, 1023, 1209]; Bolivia [1144]

Family TROGLODYTIDAE

Wrens

Catherpes sumichrasti Lawrence 1871

V/R $\quad 16,318,321$

(= Hylorchilus sumichrasti)

Slender-billed Wren, Sumichrast's Wren

Breeding: Mexico

Cistothorus apolinari Chapman 1914

$\mathbf{R}$

$318,321,794,967$

Apolinar's Wren, Apolinar's Marsh-Wren

Breeding:

Colombia

Ferminia cerverai Barbour 1926

$\mathrm{V} / \mathrm{R}$

$16,318,321,794,913$

Zapata Wren

Breeding:

Cuba $[179,509]$

Thryothorus nicefori Meyer de Schauensee 1946

Id

$318,321,967$

Niceforo's Wren

Breeding:

Colombia

Family MIMIDAE

Mockinghirds and Thrashers

Mimodes graysoni (Lawrence 1871)

E

$49,318,321,726,1070,1216$

Socorro Mockingbird, Socorro Thrasher

Breeding:

Mexico: Socorro [729]

Nesomimus trifasciatus (Gould 1837)

E

321,355

Charles Mockingbird, Floreana Mockinghird

Breeding:

Galapagos Islands: Champion, Floreana (ex), Gardner

Ramphocinclus brachyurus (Vieillot 1818)

E

$16,179,318,321,794$

White-breasted Thrasher

Breeding:

Martinique; Saint Lucia [246,690]

Family MUSCICAPIDAE

Thrushes, Babblers, Old World Warblers and Old World Flycatchers

Acrocephalus familiaris (Rothschild 1892)

V

$16,318,794,1118$

(includes $A$. kingi (Wetmore 1924))

Millerbird, (Nihoa Millerbird, Nihoa Reed Warbler)

Breeding:

Hawaiian lslands: Laysan (ex), Nihoa [1118]

Acrocephalus paludicola (Vieillot 1817)

$\mathrm{K}^{*}$

$318,584,661,702,1302$

Aquatic Warbler

Breeding:

Austria; Belarus; Germany; Hungary; Latvia; Lithuania; Moldova; Poland; Russia; Ukraine

Non-breeding:

France; Iran; Italy; Latvia; Mali [356,819,884]; Morocco; Netherlands; Portugal; Senegal [719]; Spain; Switzerland; United Kingdom 
Vagrant: $\quad$ Algeria; Azores; Belgium; Bulgaria; Canary Islands; Cyprus; Czechoslovakia; Denmark; Egypt; Finland; Ghana; Greece; Guemsey; Ireland; Israel; Jersey; Jordan; Luxembourg; Malta; Oman; Norway; Romania; Sweden; Tunisia [1387]; Turkey; Yugoslavia

Acrocephalus rehsei (Finsch 1883)

Nauru Reed-Warbler, Finsch's Reed-Warbler

Breeding:

Nauru [ 11118 ]

Acrocephalus sorghophilus (Swinhoe 1863)

Streaked Reed-Warbler, Speckled Reed-Warbler

Breeding:

China [968]

Non-breeding:

Alcippe variegaticeps Yen 1932

Gold-fronted Fulvetta, Yellow-fronted Fulvetta

K*

318

Breeding:

China [968]

Alethe choloensis Sclater 1927

E

$318,324,600$

Cholo Alethe, Cholo Mountain Alethe

Breeding:

Malawi; Mozambique

Amaurocichla bocagii Sharpe 1892

Id

$318,324,417$

Bocage's Longbill, Sao Tome Short-tail

Breeding:

Sao Tome and Principe: Sao Tome

Amytornis barbatus Favaloro \& McEvey 1968

$K^{*}$

318

Grey Grasswren

Breeding:

Australia [167, 1057]

Amytornis dorotheae (Mathews 1914)

$\mathrm{K}^{*}$

Carpentarian Grasswren, Red-winged Grasswren

Breeding:

Australia [167]

Amytornis goyderi (Gould 1875)

Id

$318,794,1056$

Eyrean Grasswren

Breeding:

Australia [167,1057]

Amytornis textilis (Dumont 1824)

K*

318

Thick-billed Grasswren, Western Grass-wren

Breeding:

Australia [167,245, 1057]

Apalis argentea Moreau 1941

$\mathbf{R}$

$212,318,324,600$

(= A. rufogularis argentea)

Kungwe Apalis

Breeding:

Burundi; Rwanda; Tanzania; Zaire

Apalis chariessa Reichenow 1879

$\mathrm{K}^{*}$

318,324

White-winged Apalis

Breeding:

Kenya (ex?); Malawi; Mozambique; Tanzania

Apalis kaboboensis Prigogine 1955

$\mathbf{R}$

$318,324,600$

(= Apalis porphyrolaema kaboboensis)

Kabobo Apalis

Breeding:

Zaire 
Chestnut-breasted Whiteface

Breeding:

Australia [167]

Arcanator orostruthus (Vincent 1933)

$\mathbf{R}$

(= Modulatrix orostruthus, Phyllastrephus orostruthus)

Dapple-throat, Dappled Mountain Robin

Breeding:

Mozambique; Tanzania

Batis minima (Verreaux \& Verreaux 1855)

K*

318,324

Verreaux's Batis, Gabon Batis, Forest Batis

Breeding:

Cameroon; Gabon [222]

Bebrornis rodericanus (Newton 1865)

(= Acrocephalus rodericanus)

Rodriguez Brush-Warbler, Rodrigues Warbler

Breeding:

Mauritius: Rodrigues

Bebrornis sechellensis (Oustalet 1877)

$\mathbf{R}$

$318,324,372$

(= Acrocephalus sechellensis)

Seychelles Brush-Warbler, Seychelles Warbler

Breeding:

Seychelles: Cousin

Brachypteryx hyperythra Jerdon \& Blyth 1861

$\mathrm{K}^{*} \quad 318$

Rusty-bellied Shortwing, Rusty-breasted Shortwing

Breeding:

China; India [10]

Bradypterus grandis Ogilvie-Grant 1917

$\mathbf{K}$

Ja River Scrub-Warbler, Giant Swamp-Warbler

Breeding:

Cameroon; Gabon

Bradypterus graueri Neumann 1908

Grauer's Scrub-Warbler, Grauer's Swamp-Warbler, Grauer's Rush-Warbler

Breeding:

Burundi; Rwanda; Uganda; Zaire

Bradypterus major (Brooks 1872)

K* $\quad 318$

Long-billed Bush-Warbler

Breeding:

Vagrant:

China; India; Pakistan

Tajikistan

Chaetornis striatus (Jerdon 1841)

$K^{*}$

10,318

Bristled Grassbird, Bristled Grass-Warbler

Breeding:

Bangladesh; India; Nepal; Pakistan

Chloropeta gracilirostris Ogilvie-Grant 1906

$\mathbf{R}$

$212,318,324,600$

Thin-billed Flycatcher-Warbler, Papyrus Yellow Warbler, Yellow Swamp-Warbler

Breeding:

Bunundi; Kenya; Rwanda; Uganda; Zaire; Zambia

Chrysomma altirostre (Jerdon 1862)

$K^{*} \quad 10,318$

(= Moupinia altirostris)

Jerdon's Babbler

Breeding:

India; Myanmar (ex?); Nepal; Pakistan 
Cisticola haesitatus (Sclater \& Hartlaub 1881)

Island Cisticola, Socotra Cisticola

Breeding:

Yemen: Socotra [324]

Cisticola restrictus Traylor 1967

K

Tana River Cisticola

Breeding:

Kenya; ?Somalia

Clytorhynchus hamlini (Mayr 1931)

$\mathrm{K}^{*} \quad 318,1226$

Rennell Shrikebill

Breeding:

Solomon Islands: Rennell

Cochoa azurea (Temminck 1824)

$\mathrm{K}^{*} \quad 317,318$

(=C. a. azurea)

Javan Cochoa

Breeding: Indonesia: Java

Cochoa beccarii Salvadori 1879

$\mathrm{K}^{*} \quad 317,318$

(=C. azurea beccarii)

Sumatran Cochoa

Breeding: Indonesia: Sumatra [905]

Copsychus cebuensis (Steere 1890)

$\mathrm{K}^{*} \quad 318$

(=C. niger cebuensis)

Black Shama

Breeding: Philippines: Cebu

Copsychus sechellarum Newton 1865

E $\quad 318,324,580$

Seychelles Magpie-Robin

Breeding: Seychelles: Aride, Fregate

Cossypha heinrichi Rand 1955

ld $318,324,600$

White-headed Robin-Chat, Angolan Robin-Chat

Breeding:

Angola; Zaire

Crocias langbianis Gyldenstolpe 1939

K* $\quad 318$

Grey-crowned Crocias

Breeding:

Viet Nam [792]

Crossleyia xanthophrys (Sharpe 1875)

Id 318,324

(= Phyllastrephus xanthophrys)

Yellow-browed Oxylabes, Madagascar Yellowhrow

Breeding: Madagascar [373]

Cyornis herioti Ramsay 1886

$\mathrm{K}^{*} \quad 318$

(= Niltava herioti)

Blue-breasted Flycatcher

Breeding:

Philippines: Catanduanes, Luzon

Cyornis ruckii (Oustalet 1881)

11

$\mathrm{K}^{*}$

318

(= Niltava ruecki, Muscicapa ruecki)

Rueck's Blue-Flycatcher, Rueck's Niltava

Breeding: Indonesia: Sumatra (ex?) [905]; ?Malaysia: Peninsular 
Cyornis sanfordi Stresemann 1931

$K^{*} \quad 318,1486$

(= Niltava sanfordi)

Matinan Flycatcher, Sanford's Niltava

Breeding: Indonesia: Sulawesi

Dasyornis brachypterus (Latham 1801)

$\mathrm{K}^{*} \quad 318$

Eastern Bristlebird

Breeding:

Australia [167]

Dasyornis broadbenti litoralis (Milligan 1902)

I

794,1056

Western Rufous Bristlebird

Breeding:

Australia [167]

Dasyornis longirostris Gould 1841

$\mathbf{R}$

$167,318,794$

(=D. brachypterus longirostris)

Western Bristlebird

Breeding: Australia [167,935]

Eremomela turneri van Someren 1920

R

$212,318,324,600$

Turner's Eremomela

Breeding: Kenya; Uganda; Zaire

Eutrichomyias rowleyi (Meyer 1878)

$\mathrm{K}^{*} \quad 318,1486$

Cerulean Paradise-Flycatcher, Rowley's Flycatcher

Breeding: Indonesia: Sangihe (ex?) [1489]

Ficedula bonthaina (Hartert 1896)

$\mathrm{K}^{*} \quad 318,1486$

Lompobattang Flycatcher

Breeding: Indonesia: Sulawesi

Ficedula harterti (Siebers 1928)

$K^{*} \quad 318,1486$

Sumba Flycatcher, Sumba Blue Flycatcher

Breeding: Indonesia: Sumba

Ficedula henrici (Hartert 1899)

$K^{*} \quad 318,1486$

Damar Flycatcher, Damar Blue Flycatcher

Breeding: Indonesia: Damar

Ficedula timorensis (Hellmayr 1919)

K* $\quad 318,1486$

Black-banded Flycatcher, Timor Blue Flycatcher

Breeding: lndonesia: Timor

Garrulax bieti (Oustalet 1897)

$\mathrm{K}^{*} \quad 318$

White-speckled Laughingthrush, Biet's Laughingthrush

Breeding:

China [968]

Garrulax cinereifrons Blyth 1851

$\mathrm{K}^{*} \quad 318$

Ashy-headed Laughingthrush

Breeding: $\quad$ Sri Lanka [10]

Garrulax milleti Robinson \& Kloss 1919

$\mathrm{K}^{*} \quad 318$

Black-hooded Laughingthrush

Breeding: Viet Nam [792] 
Garrulax sukatschewi (Berezowski \& Bianchi 1891) - $\quad$ K* 318

Snowy-cheeked Laughingthrush, Sukatschev's Laughingthrush

Breeding: China [968]

Garrulax yersini (Robinson \& Kloss 1919)

$\mathrm{K}^{*} \quad 318$

Collared Laughingthrush

Breeding: Viet Nam [792]

Gerygone magnirostris hypoxantha Salvadori 1878 - $\quad K^{*} \quad 318,1226$

(=G. hypoxantha)

Biak Gerygone, Salvadori's Gerygone

Breeding: Indonesia: Biak [131]

Humblotia flavirost ris Milne-Edwards \& Oustalet 1885

R $\quad 318,324$

Humblot's Flycatcher, Grand Comoro Flycatcher

Breeding: $\quad$ Comoros: Grand Comoro [862]

Hypothymis coelestis Tweeddale $1877 \quad$ - $\quad K^{*} \quad 318$

Celestial Monarch, Celestial Blue Monarch

Breeding: $\quad$ Philippines: Basilan, Dinagat, Luzon [381], Mindanao, Negros, Samar, Sibuyan

Hypothymis helenae (Steere 1890) - $\quad \mathrm{K}^{*} \quad 318$

Short-crested Monarch, Short-crested Blue Monarch

Breeding: $\quad$ Philippines: Dinagat, Luzon, Mindanao [381], North Camiguin, Polillo Islands, Samar

Jabouilleia danjoui (Robinson \& Kloss 1919) $\quad-\quad K^{*} \quad 318$

Short-tailed Scimitar-Babbler

Breeding: Viet Nam [792]

Kupeornis gilberti Serle $1949 \quad$ - $\quad$ R $\quad 318,324,600,1247$

(= Lioptilus gilberti)

White-throated Mountain-Babbler

Breeding: Cameroon; Nigeria

Liocichla omeiensis Riley 1926

$\mathrm{K}^{*} \quad 318$

Omei Shan Ljocichla, Mount Omei Liocichla

Breeding: China [968]

Luscinia obscura (Berezowski \& Bianchi 1891)

$\mathrm{K}^{*} \quad 318$

(= Erithacus obscurus)

Black-throated Blue Robin, Black-throated Robin

Breeding: China [968]

Vagrant: Thailand

Luscinia ruficeps (Hartert 1907)

$\mathrm{K}^{*} \quad \mathbf{3 1 8}$

(= Erithacus ruficeps)

Rufous-headed Robin

Breeding:

China [968]

Vagrant:

Malaysia: Peninsular

Macrosphenus pulitzeri Boulton 1931

Id

$318,324,600$

Pulitzer's Longbill

Breeding:

Angola 
Malacocincla perspicillata (Bonaparte 1850)

( = Trichastoma perspicillatum)

Black-browed Babbler, Black-browed Jungle-Babbler

Breeding:

Indonesia: Kalimantan [1295]

Malacocincla vanderbilti Meyer de Scbauensee \& Ripley 1940

(= Trichastoma vanderbilti)

Vanderbilt's Babbler

Breeding: Indonesia: Sumatra [905]

Malurus coronatus Gould 1858

Purple-crowned Fairy-wren, Lilac-crowned Wren

Breeding:

Australia $[167,1110]$

Megalurus albolimbatus (d'Albertis \& Salvadori 1879) -

Fly River Grassbird, Fly River Grass Warbler

Breeding: Papua New Guinea [131]

Megalurus pryeri Seebohm 1884

Marsh Grassbird, Japanese Marsh Warbler

Breeding:

China [778,968]; Japan [1494]; ?Mongolia

Melaenornis annamarulae Forbes-Watson 1970

Liberian Black-Flycatcher, Nimba Flycatcher

Breeding:

Côte d'lvoire [55]; Liberia [327]

Metabolus rugensis (Hombron \& Jacquinot 1841)

R

$318,794,1118$

Truk Monarch

Breeding:

Federated States of Micronesia: Chuuk

Monarcha boanensis van Bemmel 1939

$\mathrm{K}^{*} \quad 318,1486$

(=M. trivirgatus boanensis)

Black-chinned Monarch

Breeding: Indonesia: Boano

Monarcha brehmii Schlegel 1871

Biak Monarch

Breeding: Indonesia: Biak [131]

Monarcha everetti Hartert 1896

$(=M$. frater everetti)

White-tipped Monarch

Breeding: Indonesia: Tanahjampea

Muscicapa lendu (Chapin 1932)

Chapin's Alseonax, Chapin's Flycatcher

Breeding: Kenya; Uganda; Zaire

Myadestes lanaiensis (Wilson 1891)

(= Phaeornis obscurus Lanaiensis)

Olomao, Hawaiian Thrush

Breeding:

Hawaiian Islands: Lanai (ex), Maui (ex), Molokai 
Myadestes myadestinus (Stejneger 1887)

(= Phaeornis obscurus myadestinus)

Kamao, Hawaiian Thrush

Breeding:

Hawaiian Islands: Kauai

Myadestes palmeri (Rothschild 1893)

E

$16,318,794,1118$

(= Phaeornis palmeri)

Puaiohi, Small Kauai Thrush

Breeding: Hawaiian Islands: Kauai

Myiagra atra Meyer 1874

$\mathrm{K}^{*}$

Biak Flycatcher, Black Myiagra

Breeding: Indonesia: Biak, Numfor [131]

Myiagra freycineti Oustalet 1881

$\mathrm{K}^{*} \quad 318,1118$

Guam Flycatcher, Guam Broadbill

Breeding:

Guam

Myiophonus blighi (Holdsworth 1872)

$\mathrm{K}^{*} \quad 318$

Ceylon Whistling-Thrush

Breeding:

Sri Lanka $[10,659]$

Napothera rabori Rand 1960

K*

Rabor's Wren-Babbler, Luzon Wren-Babbler

Breeding:

Philippines: Luzon

Nesillas aldabrana Benson \& Penny 1968

$E$

318,324

Aldabra Tsikirity, Aldabra Warbler, Aldabra Brush-warbler

Breeding:

Seychelles: Aldabra (ex) [601,1169]

Newtonia fanovanae Gyldenstolpe 1933

Id

318,324

Fanovana Newtonia, Red-tailed Newtonia

Breeding:

Madagascar (ex?) [373]

Orthotomus moreaui (Sclater 1931)

$\mathbf{R}$

$212,318,324,600$

(= Apalis moreaui)

Long-billed Tailorbird, Long-billed Apalis

Breeding: Mozambique; Tanzania

Pachycephala meyeri Salvadori 1890

$\mathrm{K}^{*} \quad 318,1226$

Vogelkop Whistler

Breeding:

Indonesia: Irian Jaya [131]

Pachycephala rufogularis Gould 1841

$\mathrm{K}^{*} \quad 318$

Red-lored Whistler

Breeding:

Australia [167]

Paradoxornis davidianus (Slater 1897)

$\mathrm{K}^{*} \quad 318$

Short-tailed Parrotbill

Breeding:

China; Lao P.D.R [792]; Myanmar [1296]; Thailand; Viet Nam [792].

Paradoxornis flavirostris Gould 1836

$\mathrm{K}^{*} \quad 10,318$

Black-breasted Parrotbill

Breeding: $\quad$ Bangladesh; India; Myanmar; Nepal (ex?) 
Paradoxornis przewalskii (Berezowski \& Bianchi 1891)

Rusty-throated Parrothill, Przewalski's Parrotbill -

Breeding:

China [968]

Paradoxornis ruficeps Blyth 1842

$\mathrm{K}^{*} \quad 318$

Rufous-headed Parrotbill, Greater Rufous-headed Parrotbill

Breeding:

Bangladesh; Bhutan [10]; China; India [10]; Lao P.D.R [792]; Myanmar; Viet Nam [792]

Paradoxornis zappeyi (Thayer \& Bangs 1912) - $\quad \mathrm{K}^{*} \quad 318$

Grey-hooded Parrothill, Crested Parrotbill, Dusky Parrotbill

Breeding:

China [968]

$\begin{array}{llll}\text { Pellorneum palustre Gould } 1872 & \text { K* } & \text { K* } & 318\end{array}$

Marsh Babbler

Breeding: Bangladesh; India [10]

Petroica traversi (Buller 1872)

E $\quad 318,794,976$

Chatham Robin, Chatham Island Black Robin

Breeding:

New Zealand: Chatham Islands: Chatham (ex), Pitt (ex), Little Mangere (ex), [Mangere], [Rangatira] [1507]

Phylloscopus amoenus (Hartert 1929)

$\mathrm{K}^{*}$

318,1226

Sombre Leaf-Warbler, Kulambangra Warbler

Breeding:

Solomon Islands: Kulambangra

Picathartes gymnocephalus (Temminck 1825)

l V

$276,318,324,600,1247,1383$

White-necked Rockfowl, Yellow-headed Rockfowl, White-necked Picathartes

Breeding: $\quad$ Côte d'Ivoire [55]; Ghana; Guinea; Liberia [327]; Sierra Leone

$\begin{array}{llll}\text { Picathartes oreas Reichenow } 1899 & 1 & R & 318,324,600,1247\end{array}$

Grey-necked Rockfowl, Red-headed Rockfowl, Grey-necked Picathartes

Breeding: $\quad$ Cameroon; Equatorial Guinea: Bioko, ?continental; Gabon [221,222]; Nigeria [98]

Platysteira laticincta Bates 1926

E $\quad 318,324,600,1247$

(= Platysteira peltata laticincta)

Banded Wattle-eye, Black-throated Wattle-eye

Breeding: Cameroon

Pomarea dimidiata (Hartlaub \& Finsch 1871)

V $318,794,1118$

Rarotonga Monarch, Rarotonga Flycatcher, Cook Islands Flycatcher

Breeding: Cook 1slands: Rarotonga

Pomarea iphis Murphy \& Mathews $1928 \quad$ - $\quad$ K* $\quad 318,794,1118$

Iphis Monarch, Ua Huka Flycatcher

Breeding:

French Polynesia: Marquesas Islands (Uahuka, Eiao (ex))

Pomarea mendozae (Hartlaub 1854) - $\quad \mathrm{K}^{*} \quad 318,1118$

Marquesan Monarch, Marquesan Flycatcher

Breeding: $\quad$ French Polynesia: Marquesas Islands (Tahuata (ex?), Nukuhiva (ex?), Mohotani, Uapoa, Hivaoa) 
Pomarea whitneyi Murphy \& Mathews 1928

K*

318,1118

Fatuhiva Monarch, Fatu Hiva Flycatcher

Breeding:

French Polynesia: Fatuhiva [669]

Prinia burnesii (Blyth 1844)

K* $\quad 318$

(includes $P$. cinerascens (Walden 1874))

Rufous-vented Prinia, Long-tailed Prinia

Breeding: India [10]; Pakistan [10]

$\begin{array}{lllll}\text { Prinia fluviatilis Chappuis } 1974 & - & \text { K } & 318,324\end{array}$

River Prinia

Breeding: Cameroon; Chad; Mali; Senegal

Pseudocossyphus bensoni Farkas 1971

K $\quad 318,324,325$

(= Monticola bensoni)

Farkas's Robinchat, Benson's Rock-Thrush

Breeding: Madagascar [373]

Psophodes nigrogularis Gould 1844

Western Whipbird

Breeding:

Australia $[167,935]$

Rhinomyias albigularis Sharpe 1888

K* $\quad 318$

$(=R$. gularis albigularis)

White-throated Jungle-Flycatcher

Breeding:

Philippines: Guimaras, Negros [381]

Rhinomyias brunneata (Slater 1897)

$\mathrm{K}^{*}$

318

Brown-chested Jungle-Flycatcher, Brown-chested Flycatcher

Breeding: $\quad$ China; India: ?Nicobar Islands

Non-breeding: Malaysia: Peninsular Malaysia; India: Nicobar Islands; Thailand

Vagrant: Brunei; Singapore

Rhinomyias colonus Hartert 1898

Henna-tailed Jungle-Flycatcher, Sulawesi Jungle-Flycatcher

$\mathrm{K}^{*} \quad 318,1486$

Breeding:

Indonesia: Mangole, Peleng, Sanana, Sulawesi

Rhipidura malaitae Mayr 1931

K* $\quad 318,1226$

Malaita Fantail

Breeding:

Solomon Islands: Malaita (ex?)

Rhyacornis bicolor (Ogilvie-Grant 1894)

K*

318

Luzon Water-Redstart, Luzon Redstart, Philippine Water-Redstart

Breeding:

Philippines: Luzon [381]

Saxicola dacotiae (Mead-Waldo 1889)

$\mathbf{R}$

$318,324,343$

Canary Chat, Canary Islands Chat, Fuerteventura Stonechat

Breeding: $\quad$ Canary Islands: Fuerteventura [160], ?Alegranza, ?Montaña Clara

Saxicola gutturalis (Vieillot 1818) - $\quad K^{*} \quad 318,1486$

White-bellied Bushchat, Timor Bushchat

Breeding: Indonesia: Roti, Semau, Timor 
White-throated Bushchat, Hodgson's Bushchat

Breeding:

Kazakhstan; Mongolia; ?Russia [799]

Non-breeding: China; India; Nepal [700]

Saxicola macrorhyncha (Stoliczka 1872)

White-browed Bushchat, Stolickka's Bushchat

Breeding: India [10]; Pakistan [10]

Vagrant:

Afghanistan

Scepomycter winifredae (Moreau 1938)

$\mathbf{R}$

$212,318,324,600$

(= Bathmocercus winifredae)

Mrs Moreau's Warbler

Breeding:

Tanzania

Sheppardia gabela (Rand 1957)

Id

$318,324,600$

(= Erithacus gabela)

Gabela Akalat

Breeding:

Angola

Sheppardia gunningi Haagner 1909

R

$212,285,318,324,600$

(= Erithacus gunningi)

East Coast Akalat, Gunning's Akalat

Breeding: Kenya; Malawi; Mozambique; Tanzania

Sheppardia lowei (Grant \& Mackworth-Praed 1941) -

$\mathbf{R}$

$212,318,324,600$

(= Dryocichloides lowei, Alethe lowei)

Iringa Akalat, Iringa Ground Robin

Breeding:

Tanzania

Sheppardia montana (Reichenow 1907)

R

$212,318,324,600$

(= Dryocichloides montanus, Alethe montana)

Usambara Akalat, Usambara Ground Robin

Breeding:

Tanzania

Spelaeornis badeigularis Ripley 1948

$K^{*}$

318

Rusty-throated Wren-Babbler, Mishmi Wren-Babbler

Breeding: India [10]

Spelaeornis caudatus (Blyth 1845)

$\mathrm{K}^{*} \quad 10,318$

Rufous-throated Wren-Babbler, Tailed Wren-Babbler, Short-tailed Wren-babbler Breeding: Bhutan; India; Nepal

Spelaeornis longicaudatus (Moore 1854)

Tawny-breasted Wren-Babbler, Assam Wren-Babbler

$\mathbf{K}^{*}$

Breeding: India [10]

Sphenocichla humei (Mandelli 1873)

$\mathrm{K}^{*}$

Wedge-billed Wren-Babbler

Breeding:

China; India [10]; Myanmar

Stachyris grammiceps (Temminck 1827)

White-breasted Babbler, White-breasted Tree-Babbler

K* 
Stachyris herberti (Baker 1920)

Sooty Babbler

Breeding:

Lao P.D.R [792]

Stachyris nigrorum Rand \& Rabor 1952

$\mathrm{K}^{*}$

Negros Striped-Babbler, Negros Tree-Babbler

Breeding:

Philippines: Negros, Panay [1113]

Stachyris oglei (Godwin-Austen 1877)

$\mathrm{K}^{*} \quad 318$

Snowy-throated Babbler, Austen's Babbler

Breeding: India [10]

Stachyris rodolphei Deignan 1939

$\mathrm{K}^{*} \quad 318$

Deignan's Babbler

Breeding:

Thailand [792]

Stachyris speciosa (Tweeddale 1878)

$\mathrm{K}^{*}$

Flame-templed Babbler, Rough-templed Tree-Babbler

Breeding:

Philippines: Negros, Panay

Swynnertonia swynnertoni (Shelley 1906)

R

(= Pogonocichla swynnertoni)

Swynnerton's Robin, Swynnerton's Forest Robin

Breeding: Mozambique; Tanzania; Zimbabwe

Terpsiphone bourbonnensis (Müller 1776)

III MU -

794

(= Tchitrea bourbonnensis)

Mascarene Paradise-Flycatcher, Coq de Bois

Breeding: Mauritius; Réunion

Terpsiphone corvina (Newton 1867)

$\mathrm{R} \quad 318,324$

Seychelles Paradise-Flycatcher, Seychelles Black Paradise-Flycatcher

Breeding: $\quad$ Seychelles: ?Felicite, La Digue, Praslin

Trichastoma woodi (Mearns 1905)

$\mathrm{K}^{*} \quad 318$

(= Leonardina woodi)

Bagobo Babbler

Breeding:

Philippines: Mindanao [381]

Trichocichla rufa Reichenow 1890

E

318,794

(= Ortygocichla rufa)

Long-legged Thicketbird, Long-legged Warbler

Breeding: $\quad$ Fiji: Viti Levu, Vanua Levu

Turdoides hindei (Sharpe 1900)

V

$212,318,324,600$

Hinde's Pied-Babbler

Breeding:

Kenya

Turdus feae (Salvadori 1887)

$\mathrm{K}^{*} \quad 318$

Grey-sided Thrush

Breeding:

China $[790,968]$

Non-breeding: India [10]; Myanmar; Thailand

Turdus haplochrous Todd 1931

K

Unicolored Thrush

Breeding:

Bolivia 
Turdus menachensis Ogilvie-Grant 1913

Yemen Thrush

Breeding:

Saudi Arabia; Yemen [193]

Turdus olivaceus helleri (Mearns 1913)

E

$212,318,324,600$

(= T. helleri)

Teita Thrush

Breeding:

Kenya [933]

Turdus swalesi (Wetmore 1927)

R

$16,179,321$

La Selle Thrush

Breeding:

Dominican Republic; Haiti

Zoothera everetti (Sharpe 1892)

$K^{*}$

318

Everett's Thrush, Everett's Ground-Thrush

Breeding:

Malaysia: Sabah, Sarawak [1295]

Zoothera guttata (Vigors 1831)

$\mathbf{R}$

$212,285,286,318,324,600$

(includes Turdus fischeri Hellmayr 1901)

Spotted Ground-Thrush, Natal Thrush, Spotted Forest Thrush

Breeding:

Malawi; ?Mozambique; South Africa; Sudan; Zaire [1121]

Non-breeding: Kenya [142]; Tanzania

Zoothera kibalensis Prigogine 1978

ld

$212,318,324$

(= Turdus kibalensis)

Kibale Ground-Thrush

Breeding:

Uganda

Zoothera machiki (Forbes 1883)

Fawn-breasted Thrush

$K^{*} \quad 318,1486$

Breeding:

Indonesia: Larat, Yamdena

Zoothera major Ogawa 1905

$K^{*} \quad 318,703,794$

(= Z. dauma major, Turdus amami)

Amami Thrush

Breeding:

Japan: Amami-o-shima

Zoothera oberlaenderi (Sassi 1914)

$\mathbf{R}$

$212,318,324,600$

(= Turdus oberlaenderi)

Oberlaender's Ground-Thrush, Forest Ground-Thrush

Breeding:

Uganda; Zaire

Zoothera peronii (Vieillot 1818)

$\mathbf{K}^{*}$

318,1486

Orange-banded Thrush, Timor Ground-Thrush

Breeding: Indonesia: Babar, Damar, Romang, Timor, Wetar

Zoothera schistacea (Meyer 1884)

$K^{*} \quad 318,1486$

Slaty-backed Thrush, Tanimbar Thrush

Breeding: Indonesia: Tanimbar Islands

Family PARIDAE

Titmice, Chickadees

Parus holsti Seebohm 1894

$K^{*}$

Yellow Tit

Breeding:

Taiwan [266,1253] 
Parus nuchalis Jerdon 1845

$\mathrm{K}^{*}$

318

White-winged Tit, White-winged Black Tit

Breeding: India [10]

Family SITTIDAE

Nuthatches

Sitta formosa Blyth 1843

$\mathrm{K}^{*} \quad 10,318,792$

Beautiful Nuthatch

Breeding:

China; India; Lao P.D.R; Myanmar; Thailand; Viet Nam

Sitta ledanti Vielliard 1976

R $\quad 318,324,661$

Algerian Nuthatch, Kabylian Nuthatch

Breeding: Algeria

Sitta magna Ramsay 1876

$\mathrm{K}^{*} \quad 318$

Giant Nuthatch

Breeding:

China; Myanmar; Thailand [792]

Sitta solangiae (Delacour \& Jabouille 1930)

$\mathrm{K}^{*} \quad 318$

Yellow-billed Nuthatch

Breeding: China; Viet Nam [792]

Sitta victoriae Rippon 1904

$\mathrm{K}^{*} \quad 318$

White-browed Nuthatch

Breeding: Myanmar [1296]

Sitta yunnanensis Ogilvie-Grant 1900

K* $\quad 318$

Yunnan Nuthatch, Black-masked Nuthatch

Breeding: China [968]

Family RHABDORNITHIDAE

Philippine Creepers

Rhabdornis grandis Salomonsen 1953

$\mathrm{K}^{*} \quad 318$

$(=R$. inornatus grandis)

Long-billed Rhabdornis

Breeding: $\quad$ Philippines: Luzon [399]

Family DICAEIDAE

Flowerpeckers

Dicaeum everetti (Sharpe 1877)

K* $\quad 318$

Brown-backed Flowerpecker

Breeding: Indonesia: Kalimantan, Natuna Islands, Riau Archipelago; Malaysia: Peninsular Malaysia, Sabah, Sarawak [1295]

Pardalotus quadragintus Gould 1838

K*

Forty-spotted Pardalote

Breeding:

Australia: Tasmania [167] 
Family NECTARINIIDAE

Sunbirds

Aethopyga duyvenbodei (Schlegel 1871)

$\mathrm{K}^{*} \quad 318,1486$

Elegant Sunbird, Sanghir Sunbird

Breeding:

Indonesia: Sangihe, Siau

Anthreptes pallidigaster Sclater \& Moreau 1935

$\mathbf{R}$

$212,318,324,600$

Amani Sunbird

Breeding:

Kenya; Tanzania

Anthreptes rubritorques Reichenow 1905

$\mathbf{R}$

$212,318,324,600$

(=A. rectirostris rubritorques)

Banded Sunbird, Grey-chinned Sunbird

Breeding:

Tanzania

Nectarinia buetrikoferi (Hartert 1896)

K* $\quad 318,1486$

Apricot-breasted Sunbird, Sumba Sunbird

Breeding:

Indonesia: Sumba

Nectarinia prigoginei (Macdonald 1958)

E

318,324

(= N. afra prigoginei)

Prigogine's Double-collared Sunbird, Marungu Sunbird

Breeding:

Zaire

Nectarinia rockefelleri (Chapin 1932)

$\mathbf{R}$

$318,324,600$

Rockefeller's Sunbird

Breeding:

Zaire

Nectarinia rufipennis Jensen 1983

$\mathbf{R}$

318,324

Rufous-winged Sunbird

Breeding:

Tanzania

Nectarinia thomensis Barboza du Bocage 1889

$\mathrm{K}^{*}$

318,324

(= Dreptes thomensis)

Sao Tome Sunbird, Giant Sunbird

Breeding: $\quad$ Sao Tome and Principe: Sao Tome [755]

Family ZOSTEROPIDAE

White-eyes

Heleia muelleri Hartlaub 1865

$\mathrm{K}^{*} \quad 318,1486$

Spot-breasted White-eye, Timor White-eye

Breeding: Indonesia: Timor

Madanga ruficollis Rothschild \& Hartert 1923

$\mathrm{K} *$

318,1486

Rufous-throated White-eye, Buru Mountain White-eye

Breeding:

Indonesia: Buru

Rukia longirostra (Takatsukasa \& Yamashina 1931) -

R

$318,794,1118$

Long-billed White-eye, Large Ponhpei White-eye

Breeding: $\quad$ Federated States of Micronesia: Pohnpei

Rukia ruki (Hartert 1897)

Truk White-eye, Great Truk White-eye, Large Truk White-eye

Breeding:

Federated States of Micronesia: Truk (Tol 1sland, ?Polle, ?Onei, ?Pata) 
Fernando Po Speirops

Breeding: $\quad$ Equatorial Guinea: Bioko

$\begin{array}{lllll}\text { Speirops leucophoeus Hartlaub } 1857 & - & K^{*} & 318\end{array}$

Principe Speirops

Breeding: Sao Tome and Principe: Principe [755]

Woodfordia lacertosa (Murphy \& Mathews 1929) - $\quad K^{*} \quad 318$

Sanford's White-eye

Breeding: Solomon lslands: Ndeni Island

Zosterops albogularis Gould 1837

E

White-chested White-eye, White-breasted White-eye

Breeding: $\quad$ Norfolk Island [1188]

Zosterops anomalus Meyer \& Wiglesworth 1896

$K^{*} \quad 318,1486$

Lemon-throated White-eye, Sulawesi White-eye

Breeding:

Indonesia: Sulawesi [1489]

Zosterops chloronothos (Vieillot 1817)

V $\quad 318,324$

Mauritius Olive White-eye

Breeding:

Mauritius

Zosterops ficedulinus Hartlaub 1866

Principe White-eye, Sao Tome White-eye

Breeding: Sao Tome and Principe [755]

Zosterops flavus (Horsfield 1821)

$K^{*} \quad 14,318$

Javan White-eye

Breeding:

Indonesia: Java, Kalimantan [664]; Malaysia: Sarawak [1295]

Zosterops kuehni Hartert 1906

$K^{*} \quad 318,1486$

Ambon Yellow White-eye

Breeding:

Indonesia: Ambon, ?Seram

Zosterops luteirostris Hartert 1904

$K^{*} \quad 318,1226$

Splendid White-eye, Gizo White-eye, Ganonga White-eye

Breeding: $\quad$ Solomon Islands: Gizo, Ranongga

Zosterops meeki Hartert 1898

$\mathrm{K}^{*} \quad 318,1226$

White-throated White-eye, Tagula White-eye, Sudest White-eye

Breeding:

Papua New Guinea: Tagula Island

Zosterops modestus Newton 1867

E

318,324

Seychelles Grey White-eye

Breeding:

Seychelles: Mahé

Zosterops mouroniensis Milne-Edwards \& Oustalet 1885 -

$\mathbf{R}$

318,324

Comoro White-eye, Mount Karthala White-eye

Breeding: Comoros: Grand Comoro [862] 
Zosterops uropygialis Salvadori 1874

$\mathrm{K}^{*}$

318,1486

Golden-bellied White-eye, Little Kai White-eye

Breeding:

Indonesia: Kai Islands

Family MELIPHAGIDAE

Honeyeaters

Apalopteron familiare (Kittlitz 1831)

$\mathrm{K}^{*} \quad 318,794$

Bonin Honeyeater

Breeding:

Japan: Chichijima (ex), Haha-jima and possibly offshore islands [1494], Muko-jima (ex)

Lichenostomus melanops cassidix (Gould 1867) 794

(= Meliphaga cassidix, Lichenostomus cassidix, M. melanops cassidix)

Helmeted Honeyeater

Breeding:

Australia [167]

Lichmera notabilis (Finsch 1898)

$\mathrm{K}^{*} \quad 318,1486$

Black-chested Honeyeater, Wetar Honeyeater

Breeding: Indonesia: Wetar

Manorina flavigula melanotis (Wilson 1911) $\quad-\quad \mathrm{K}^{*} \quad 318$

(= M. melanotis)

Black-eared Miner, Dusky Miner

Breeding: Australia [167]

$\begin{array}{lllll}\text { Melidectes princeps Mayr \& Gilliard } 1951 & \text { K* } & \text { K* } & 318,1226\end{array}$

Long-bearded Melidectes, Long-bearded Honeyeater

Breeding: $\quad$ Papua New Guinea [131]

Meliphaga vicina (Rothschild \& Hartert 1912) - $\quad$ K* $\quad 318,1226$

Tagula Honeyeater, Sudest Meliphaga, Louisiades Meliphaga

Breeding: $\quad$ Papua New Guinea: Tagula 1sland

$\begin{array}{lllll}\text { Moho bishopi (Rothschild 1893) } & \text { - } & \mathrm{K}^{*} & 318,1118\end{array}$

Bishop's Oo, Molokai Oo

Breeding: Hawaiian lslands: Maui [661], Molokai (ex)

Moho braccatus (Cassin 1855)

E

$16,318,794,1118$

Kauai Oo

Breeding:

Hawaiian Islands: Kauai

Myzomela kuehni Rothschild 1903

Crimson-hooded Myzomela, Wetar Myzomela

- $\quad \mathrm{K}^{*} \quad 318,1486$

Breeding:

Indonesia: Wetar (ex?)

Notiomystis cincta (Du Bus \& Gisignies 1839)

Stitchbird

Breeding:

New Zealand: North Island (ex), Great Barrier Island (ex), ?Kapiti Island (ex), Little Barrier Island [1507]

Philemon brassi Rand 1940

$K^{*} \quad 318,1226$

Brass's Friarbird

Breeding:

Indonesia: Irian Jaya [131] 
Philemon fuscicapillus (Wallace 1862)

Dusky Friarbird, Morotai Friarbird

Breeding: Indonesia: Bacan, Halmahera, Morotai

Xanthomyza phrygia

(Shaw 1794)

$\mathrm{K}^{*}$

318,1056

Regent Honeyeater

Breeding:

Australia [167]

Family EMBERIZIDAE

Buntings and tanagers

Atlapetes flaviceps Chapman 1912

Olive-headed Brush-Finch, Yellow-headed Brush-Finch

Breeding:

Colombia

Atlapetes pallidiceps (Sharpe 1900)

E/Ex

$318,321,967$

Pale-headed Brush-Finch

Breeding: Ecuador

Bangsia aureocincta (Hellmayr 1910)

V

$318,321,706,967$

(= Buthraupis aureocincta)

Gold-ringed Tanager

Breeding:

Colombia [654]

Bangsia melanochlamys (Hellmayr 1910)

$\mathrm{V} / \mathrm{R}$

$318,321,706,967$

(= Buthraupis melanochlamys)

Black-and-gold Tanager

Breeding: Colombia [654]

Buthraupis aureodorsalis Blake \& Hocking $1974 \quad-\quad$ K

$318,321,706,967$

(= Bangsia aureodorsalis)

Golden-backed Mountain-Tanager

Breeding: Peru

Buthraupis wetmorei (Moore 1934)

$\mathrm{V} / \mathrm{R} \quad 321,967$

Masked Mountain-Tanager

Breeding: Colombia; Ecuador; Peru

Calyptophilus frugivorus (Cory 1883)

$\mathrm{V} / \mathrm{R} \quad 16,179,321$

Chat Tanager

Breeding: Dominican Republic; Haiti

Camarhynchus heliobates (Snodgrass \& Heller 1901)

Mangrove Finch

Breeding:

Galapagos Islands: Fernandina, Isabela

Id

$318,321,567,794$

Chlorochrysa nitidissima Sclater 1873

$\mathbf{V} / \mathbf{R}$

$318,321,706,967$

Multicolored Tanager

Breeding:

Colombia [654]

Chlorospingus flavovirens (Lawrence 1867)

K $\quad 318,321,706,967$

Yellow-green Bush-Tanager

Breeding:

Colombia; Ecuador 


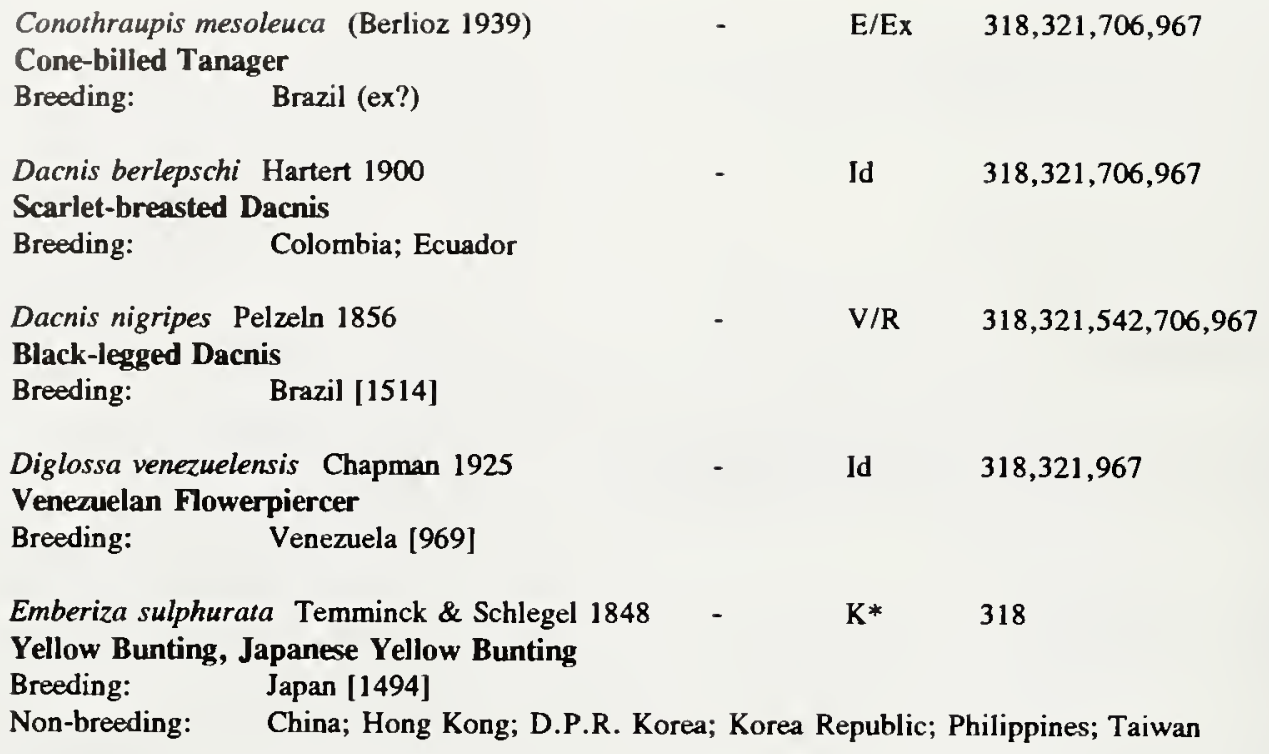

$\begin{aligned} & \text { Gubernatrix cristata (Vieillot 1817) } \\ & \text { Yellow Cardinal }\end{aligned}$

Breeding: $\quad$ Argentina [154]; Brazil; Paraguay (ex?); Uruguay

Hemispingus goeringi (Sclater \& Salvin 1870) - $\quad$ V/R $\quad 321,967$

Slaty-backed Hemispingus

Breeding: Venezuela

Incaspiza ortizi Zimmer 1952

Grey-winged Inca-Finch

V $\quad 318,321,967$

Breeding: Peru [1064,1067]

Junco insularis Ridgway $1876 \quad-\quad$ E $\quad 16,318,321,950$

(=J. hyemalis insularis)

Guadalupe Junco

Breeding: Mexico: Guadalupe Island [728]

Nemosia rourei Cabanis 1870

E/Ex $\quad 318,321,706,794,967$

Cherry-throated Tanager

Breeding: Brazil [1268]

Nesospiza acunhae Cabanis 1873

Nightingale Finch, Tristan Bunting, Tristan Finch

$\mathrm{R} \quad 318,324$

Breeding:

Saint Helena: Inaccessible Island, Nightingale Island, Middle Island, Stoltenhoff Island, Tristan da Cunha

Nesospiza wilkinsi Lowe 1923

R $\quad 318,324$

Wilkins's Finch, Grosbeak Bunting, Big-billed Bunting

Breeding: $\quad$ Tristan da Cunha (Saint Helena - United Kingdom): Inaccessible Island, Nightingale Island

Oreothraupis arremonops (Sclater 1855)

Tanager Finch

Breeding:

Colombia; Ecuador

$\mathrm{V} / \mathrm{R} \quad 318,321,967$ 
Paroaria capitata (d'Orbigny \& Lafresnaye 1837) 11 967

Yellow-billed Cardinal

Breeding:

Argentina; Bolivia; Brazil; [Hawaiian Islands: Hawaii] [1118]; Paraguay; ?Uruguay

Paroaria coronata (Miller 1776)

11

967

Red-crested Cardinal

Breeding:

Argentina; Bolivia; Brazil; [Hawaiian Islands] [1118]; Paraguay; Uruguay

Poospiza alticola Salvin 1895

$\mathrm{V} / \mathrm{R} \quad 318,321,967$

Plain-tailed Warbling-Finch

Breeding: Peru [1064]

Poospiza baeri (Oustalet 1904)

V/R $\quad 318,321,967$

Tucuman Mountain-Finch

Breeding: $\quad$ Argentina [154, 1004]

Poospiza cinerea Bonaparte 1851

Id $\quad 321,967$

Cinereous Warbling-Finch

Breeding: Brazil

Poospiza garleppi (Berlepsch 1893)

E $\quad 318,321,967$

Cochabamba Mountain-Finch

Breeding: Bolivia

Poospiza rubecula Salvin 1895

E $\quad 318,321,967$

Rufous-breasted Warbling-Finch

Breeding: $\quad$ Peru [1064]

Pseudodacnis hartlaubi (Sclater 1855)

(= Dacnis hartlaubi)

Turquoise Dacnis-Tanager, Turquoise Dacnis

Breeding: Colombia

Rowettia goughensis (Clarke 1904)

R $\quad 318,321,324$

Gough Finch, Gough Bunting

Breeding: $\quad$ Saint Helena: Gough Island

Saltator rufiventris d'Orbigny \& Lafresnaye $1837 \quad-\quad$ V/R $\quad 321,967$

Rufous-bellied Saltator

Breeding: Argentina; Bolivia

$\begin{array}{llll}\text { Sporophila falcirostris (Temminck 1820) } & \text { V/R } \quad 318,321,967\end{array}$

Temminck's Seedeater

Breeding: $\quad$ Argentina [154]; Brazil [1271]; Paraguay

$\begin{array}{llll}\text { Sporophila frontalis (Verreaux 1869) } & \text { V/R } & 318,321,967\end{array}$

Buffy-fronted Seedeater, Buffy-throated Seedeater

Breeding: $\quad$ ?Argentina [154]; Brazil [1271]; Paraguay

Sporophila hypochroma Todd 1915

K

$318,321,967$

Grey-and-chestnut Seedeater, Rufous-rumped Seedeater

Breeding:

Argentina [154]; Bolivia; Brazil [1139]; Paraguay

Sporophila insulata Chapman 1921

E/Ex $\quad 318,321,794,967$

Tumaco Seedeater

Breeding:

Colombia: Tumaco Island (ex) 
Sporophila melanops (Pelzeln 1870)

E/Ex

321,967

Hooded Seedeater

Breeding: Brazil (ex?)

Sporophila nigrorufa (d'Orbigny \& Lafresnaye 1837) -

Id

321,967

Black-and-tawny Seedeater

Breeding:

Bolivia [122]; Brazil [1516]

Sporophila palustris (Barrows 1883)

K $\quad 318,321,967$

Marsh Seedeater

Breeding: $\quad$ Argentina [154]; Brazil [140,1271]; Paraguay; Uruguay

Sporophila zelichi Narosky 1977

E

$318,321,967$

Narosky's Seedeater, Entre Rios Seedeater

Breeding: Argentina [154, 1003]

Tangara cabanisi (Sclater 1866)

V/R $\quad 16,318,321,706,794$

Azure-rumped Tanager

Breeding: Guatemala; Mexico [61,638]

Tangara fastuosa (Lesson 1831)

V/R $\quad 318,321,706,794,967$

Seven-colored Tanager

Breeding:

Brazil

Tangara meyerdeschauenseei Schulenberg \& Binford 1985

Green-capped Tanager

K $\quad 318,321$

Breeding:

Peru [1237]

Tangara peruviana (Desmarest 1806)

V/R $\quad 318,321,706,967$

Black-backed Tanager, Black-cheeked Tanager

- V/R $\quad 318,321,706,967$

Breeding: Brazil [1239]

Tangara phillipsi Graves \& Weske 1987

K

Sira Tanager

Breeding:

Peru [572]

Torreornis inexpectara Barbour \& Peters 1927

$\mathrm{V} / \mathrm{R}$

$16,179,318,321,794$

Zapata Sparrow, Cuban Sparrow

Breeding: Cuba [179,509]

Wetmorethraupis sterrhopteron Lowery \& O'Neill 1964

Orange-throated Tanager

V $\quad 321,967$

Breeding:

Ecuador; Peru

Xenospingus concolor (d'Orbigny \& Lafresnaye 1837)

Slender-billed Finch

Breeding:

Chile; Peru

Xenospiza baileyi Bangs 1931

V/R

$16,318,321,448$

(= Ammodramus baileyi)

Sierra Madre Sparrow

Breeding:

Mexico 


\section{Family PARULIDAE}

New World Warblers

Basileuterus griseiceps Sclater \& Salvin $1869 \quad$ - $\quad$ ld $\quad 318,321,967$

Grey-headed Warbler

Breeding: Venezuela [969]

Conirostrum tamaragense Johnson \& Millie 1972 - $\quad$ Id $318,321,931,967$

Tamarugo Conebill

Breeding: Chile; Peru

Dendroica chrysoparia Sclater \& Salvin $1861 \quad$ - $\quad$ V/R $16,318,321,420,714$

Golden-cheeked Warbler

Breeding: USA

Non-breeding: Guatemala; Honduras; Mexico [197]; Nicaragua

Dendroica kirtlandii (Baird 1852)

E

$16,179,318,321,420,772,794$, $1123,1124,1468,1469$

Kirtland's Warbler

Breeding: ?Canada; USA

Non-breeding: Bahamas; Turks and Caicos Islands

Geothlypis speciosa Sclater 1859

V $\quad 16,318,321,1008$

Black-polled Yellowthroat

Breeding: Mexico

Leucopeza semperi Sclater 1877

E/Ex $\quad 16,179,318,321,794$

Semper's Warbler

Breeding:

Saint Lucia (ex?) [179]

Myioborus pariae Phelps \& Phelps 1949

E

$318,321,967$

Yellow-faced Redstart, Paria Redstart

Breeding:

Venezuela $[61,969]$

Vermivora bachmanii (Audubon 1833)

E/Ex

$16,179,318,321,602,794,1141$

Bachman's Warbler

Breeding:

USA $[50,117]$

Non-breeding: ?Cuba [50]

Vagrant: Bahamas

Xenoligea montana (Chapman 1917)

$\mathrm{V} / \mathrm{R} \quad 16,179,318,321$

White-winged Warhler, White-winged Ground-Warbler

Breeding:

Dominican Republic; Haiti

Family DREPANIDIDAE

Hawaiian Honeycreepers

Hemignathus lucidus Lichtenstein 1839

E $\quad 16,318,794,1118$

Nukupuu

Breeding:

Hawaiian Islands; Hawaii, Kauai (ex?), Maui, Oahu (ex)

Hemignathus wilsoni (Rothschild 1893)

E

$16,318,794,1118$

(= H. munroi)

Akiapolaau

Breeding:

Hawaiian Islands: Hawaii 
(includes $H$. procerus)

Akialoa

Breeding:

Hawaiian lslands: Kauai

Loxioides bailleui (Oustalet 1877)

E

$16,318,794,1118,1157,1243$

(= Psittirostra bailleui)

Palila

Breeding:

Hawaiian lslands: Hawaii

Loxops coccineus (Gmelin 1789)

K*

318,1118

Akepa

Breeding:

Hawaiian Islands: Hawaii, Kauai, Lanai (ex), Maui, Molokai, Oahu (ex)

Melamprosops phaeosoma Casey \& Jacobi 1974

$\mathrm{R}$

$16,318,794,1118$

Poo-uli, Black-faced Honeycreeper

Breeding: Hawaiian lslands: Maui

Oreomystis bairdi (Stejneger 1887)

$\mathrm{K}^{*} \quad 318,1118$

Kauai Creeper, Akikiki

Breeding:

Hawaiian lslands: Kauai

Palmeria dolei (Wilson 1891)

V

$16,318,794,1118$

Akohekohe, Crested Honeycreeper

Breeding:

Hawaiian lslands: Maui, Molokai (ex)

Paroreomyza flammea (Wilson 1889)

$\mathrm{K}^{*} \quad 318,1118$

(= Loxops flammea)

Molokai Creeper, Kakawahie

Breeding:

Hawaiian lslands: Molokai (ex?)

Paroreomyza maculata (Cabanis 1850)

$\mathrm{K}^{*} \quad 318,1118$

( = Loxops maculata)

Oahu Creeper

Breeding:

Hawaiian Islands: Oahu

Pseudonestor xanthophrys Rothschild 1893

$\mathrm{V}$

$16,318,794,1118$

Maui Parrotbill

Breeding: Hawaiian lslands: Maui

Psittirostra psittacea (Gmelin 1789)

E $\quad 16,318,794,1118$

Ou

Breeding: Hawaiian 1slands: Hawaii, Kauai, Lanai (ex), Maui (ex), Molokai (ex), Oahu (ex)

Telespiza cantans Wilson 1890

$\mathrm{K}^{*} \quad 318,1118$

(= Psittirostra cantans)

Laysan Finch

Breeding:

Hawaiian lslands: [Hermes Reef], Laysan, [Midway (ex)], [Pearl]

Telespiza ultima Bryan 1917

$\mathrm{K}^{*} \quad 318,1118$

(= Psittirostra ultima)

Nihoa Finch

Breeding:

Hawaiian Islands: [French Frigate Shoals], Nihoa 
Family VIREONIDAE

Vireos

Vireo atricapillus Woodhouse 1852

E

$16,309,318,321,585,1242$

\section{Black-capped Vireo}

Breeding:

Mexico [145, 146]; USA [586]

Vireo caribaeus Bond \& Meyer de Schauensee 1942 -

Id

321,1405

Saint Andrew Vireo, San Andrés Vireo

Breeding:

Colombia: San Andrés Island

\section{Family ICTERIDAE}

Icterids

Agelaius flavus (Gmelin 1788)

III UY V

$318,321,967$

(= Xanthopsar flavus)

Saffron-cowled Blackbird

Breeding: $\quad$ Argentina [154,489]; Brazil; Paraguay; Uruguay

Agelaius xanthomus (Sclater 1862)

E

$16,179,318,321,794,1408,1499$

Yellow-shouldered Blackbird

Breeding:

Puerto Rico $[32,179]$

Cacicus koepckeae Lowery \& O'Neill 1965

K $\quad 318,321,967$

Selva Cacique

Breeding:

Peru [1064]

Curaeus forbesi (Sclater 1886)

E $\quad 318,321,967$

Forbes's Blackbird

Breeding:

Brazil

Gymnostinops cassini Richmond 1898

Id $\quad 318,321,967$

(= Psarocolius cassini)

Chestnut-mantled Oropendola, Baud6 Oropendola

Breeding:

Colombia

Hypopyrrhus pyrohypogaster (de Tarragon 1847)

1d

$318,321,967$

Red-bellied Grackle

Breeding:

Colombia [654]

Icterus bonana (Linnaeus 1766)

E

$16,179,318,321$

Martinique Oriole

Breeding:

Martinique [179,1533]

Sturnella militaris (Linnaeus 1771)

Id

$318,321,538,967$

(=S. defilippii)

Pampas Meadowlark, Lesser Red-breasted Meadowlark

Breeding:

Argentina [154]; ?Brazil [140,1271]; ?Uruguay

Non-breeding:

Brazil; Uruguay 
Family FRINGILLIDAE

Finches

Carduelis cucullata Swainson 1820

1

E

$16,19,316,318,321,490,564,565$,

$794,967,1127$

(= Spinus cucullatus)

Red Siskin

Breeding:

Colombia; [Puerto Rico] [1128, 1129]; ?Trinidad and Tobago (ex); Venezuela [300]

Carduelis johannis (Clarke 1919)

R $\quad 318,324,600$

(= Acanthis johannis)

Warsangli Linnet

Breeding:

Somalia

Carduelis siemiradzkii (Berlepsch \& Taczanowski 1883)

\section{Saffron Siskin}

Breeding:

Ecuador; ?Peru (415]

Carduelis yarrellii (Audubon 1839)

(= Spinus yarrellii)

Yellow-faced Siskin

Breeding:

Brazil [1271]; Venezuela

Fringilla teydea Webb, Berthelot \& Moquin-Tandon 1841

Teydefinch, Blue Chaffinch, Canary Islands Chaffinch

R $\quad 318,324,1006$

Breeding:

Canary lslands: Tenerife, Gran Canaria

Neospiza concolor (Barboza du Bocage 1888) - $\quad$ Id

d $\quad 318,324$

Sao Tome Canary, Sao Tome Grosbeak, Sao Tome Goldfinch

Breeding: $\quad$ Sao Tome and Principe: Sao Tome (ex?) [755]

$\begin{array}{lllll}\text { Serinus ankoberensis } & \text { Ash } 1979 & - & \text { R } & 318,324\end{array}$

Ankober Serin

Breeding: Ethiopia

Serinus canicapillus (Du Bus \& Gisignies 1855) I11 GH - $\quad 212,285,600,1247$

( $=$ S. gularis canicapillus)

West African Seedeater

Breeding: $\quad$ Cameroon; Central African Republic; ?Chad; Côte d'lvoire; Ghana; Guinea [1454]; ?Mali; Niger; Nigeria; Sudan; Togo [280]; Uganda; Zaire

Serinus flavigula Salvadori 1888

ld

318,324

(= aberrant specimens of $S$. xanthopygius Rüppell 1840)

Yellow-throated Seedeater, Yellow-throated Serin

Breeding:

Ethiopia [55]

Serinus leucopygius (Sundevall 1850)

III GH $\quad-\quad 600$

White-rumped Seedeater, Grey Singing Finch

Breeding:

Burkina Faso [1388]; Cameroon; Central African Republic; Chad; Ethiopia; ?Gambia; Ghana; [Hawaiian Islands: Oahu (ex?)]; Mali; Mauritania; Niger; Nigeria; Senegal; Sudan; Togo; Uganda 
Yellow-fronted Canary, Green Singing Finch Republic; Chad; Congo; Côte d'Ivoire; Ethiopia [748]; Gabon; Gambia; Ghana; Guinea [1454]; Guinea-Bissau; [Hawaiian Islands: Hawaii, Oahu] [845,1118]; Kenya; Liberia; Malawi; Mali; Mauritania; [Mauritius] [845]; Mozambique; Namibia; Niger; Nigeria; [Puerto Rico] [845]; Rwanda; Sao Tome and Principe: Sao Tome; Senegal; [Réunion] [845]; [Seychelles]; Sierra Leone; ?Somalia; South Africa; Sudan; Swaziland; Tanzania; ?Togo; Uganda; Zaire; Zambia; Zimbabwe

Vagrant: Lesotho

\section{Family ESTRILDIDAE \\ Waxbills and allies}

Amadina fasciata (Gmelin 1789) $\quad$ III GH $\quad-\quad 212,553,600,1247$

Cut-throat, Ribbon Finch

Breeding:

?Angola; Botswana; Cameroon; Chad; Ethiopia; Ghana; Kenya; Malawi; Mali; Mauritania; Mozambique; Namibia [285]; Niger; Nigeria; Senegal; Somalia; South Africa; Sudan; Tanzania; Uganda; Zambia; Zimbabwe

Non-breeding: Gambia

Vagrant: Côte d'Ivoire

$\begin{array}{lllll}\text { Amandava formosa } & \text { (Latham 1790) } & - & \mathrm{K}^{*} & 10,318,517 \\ \text { (= Estrilda formosa) } & & \\ \text { Green Avadavat } & & & \\ \text { Breeding: } & \text { India } \\ \text { Vagrant: } & \text { Pakistan }\end{array}$

Amandava subflava (Vieillot 1819) IIl GH - 212,553,600,1247

(= Estrilda subflava, Sporaeginthus subflavus)

Zehra Waxbill, Golden-breasted Waxhill, Orange-hreasted Waxbill

Breeding:

Angola; Botswana; Burundi; Cameroon; Central African Republic; Chad; Congo; Côte d'Ivoire; Ethiopia; Equatorial Guinea; Gabon; Gambia; Ghana; Guinea-Bissau; Kenya; ?Lesotho; Liberia; Malawi; Mali; ?Mauritania; Mozambique; ?Namibia; Nigeria; Rwanda; Senegal; Sierra Leone; South Africa [285]; Sudan; Swaziland; Tanzania; Togo [071]; Uganda; Yemen; Zaire; Zambia; Zimbabwe [285]

Chloebia gouldiae (Gould 1844)

K* $\quad 318$

(= Erythrura gouldiae)

Gouldian Finch

Breeding:

Australia [167]

Erythrura coloria Ripley \& Rabor 1961

K*

318,553

Red-eared Parrotfinch, Mindanao Parrotfinch

Breeding:

Philippines: Mindanao [399]

Erythrura kleinschmidti (Finsch 1878)

$\mathrm{R}$

$318,553,794,1118$

Pink-billed Parrotfinch

Breeding:

Fiji: Viti Levu

Erythrura viridifacies Hachisuka \& Delacour 1937

Green-faced Parrotfinch

Breeding:

Philippines: Luzon 
Common Waxbill, Saint Helena Waxbill

Breeding:
Angola; Botswana; [Brazil]; Burundi; Cameroon; [Cape Verde]; Central African Republic; Congo; Côte d'Ivoire [1378]; Equatorial Guinea; Ethiopia; [French Polynesia: Tahiti] [1118]; Gabon; Ghana; Guinea; [Hawaiian Islands: Oahu] [1118]; Kenya; Lesotho; Liberia; Malawi; [Mauritius]; Mozambique; Namibia; [New Caledonia]; Niger; ?Nigeria; [Portugal]; [Puerto Rico]; [Réunion]; Rwanda; [Sao Tome and Principe]; [Seychelles]; Sierra Leone; Somalia; South Africa; Saint Helena: Ascension; Sudan; Swaziland; Tanzania; Uganda; [Vanuatu]; Zaire; Zambia; Zimbabwe

Estrilda caerulescens (Vieillot 1817)

Lavender Waxbill, Lavender Firefinch

Breeding:

Benin; Burkina Faso [1388]; Cameroon; Central African Republic; Chad; Côte d'Ivoire [1378]; Gambia; Ghana; Guinea [1 149]; Guinea-Bissau; [Hawaiian Islands: Hawaii, Oahu] [1118]; Liberia; Mali; Niger [803]; Nigeria; Senegal; Togo

Estrilda melpoda (Vieillot 1817)

III GH -

$553,600,845,1247$

Orange-cheeked Waxbill

Breeding:

Angola; ?Benin; [Bermuda]; Burundi; Cameroon; Central African Republic; Chad; Congo; Côte d'lvoire; Equatorial Guinea; Gabon; Gambia; Ghana; Guinea [1454]; Guinea-Bissau; [Hawaiian Islands] [1118]; Liberia; Mali [819]; Niger [803]; Nigeria; [Puerto Rico]; Rwanda; Senegal; Sierra Leone; Togo; Zaire; Zambia

Estrilda nigriloris Chapin 1928

K

$318,324,553,600$

Black-faced Waxbill, Black-lored Waxbill, Kiabo Waxbill

Breeding:

Zaire

Estrilda poliopareia Reichenow 1902

$\mathrm{K}$

$318,324,553,600,1247$

(= E. paludicola poliopareia, E. anambrae Kemp 1907)

Fawn-breasted Waxbill, Anambra Waxbill

Breeding: Nigeria

Estrilda troglodytes (Lichtenstein 1823)

III GH

$553,575,600,845,1247$

Black-rumped Waxbill, Red-eared Waxbill, Pink-cheeked Waxbill

Breeding:

Benin; Burkina Faso [1388]; Cameroon; Central African Republic; Chad; Côte d'Ivoire; Ethiopia; Gambia; Ghana; [Guadeloupe]; Guinea; [Hawaiian Islands (ex)]; Kenya; Mali; Mauritania [230]; Niger; Nigeria; [Puerto Rico]; Senegal; Sudan; Togo; Uganda; Zaire

Lagonosticta rara (Antinori 1864)

III GH -

$212,553,600,1247$

Black-bellied Firefinch, Black-bellied Waxbill

Breeding: $\quad$ Cameroon; Central African Republic; Chad; Côte d'Ivoire; Ghana; Guinea [984]; Kenya; Liberia; Nigeria; Senegal; Sierra Leone; Sudan; Togo [277]; Uganda; Zaire

Lagonosticta rubricata (Lichtenstein 1823)

III GH -

$212,553,575,600$

African Firefinch, Blue-billed Firefinch, Brown-backed Firefinch

Breeding:

Angola; ?Benin; Burundi; Cameroon; Central African Republic; Congo; Côte d'Ivoire; Ethiopia; Gabon; Ghana; Guinea; Guinea-Bissau; Kenya; Liberia; Malawi; Mali; Mozambique; Nigeria; Rwanda; ?Senegal; Sierra Loone; South Africa; Sudan; Swaziland; Tanzania; Togo [277]; Uganda; Zaire; Zambia; Zimbabwe

Lagonosticta rufopicta (Fraser 1843)

$111 \mathrm{GH}-$

$212,553,600,1247$

Bar-breasted Firefinch, Bar-breasted Waxbill

Breeding:

Benin; Burkina Faso [1388]; Cameroon; Central African Republic; Chad; Côte d'Ivoire; Gambia; Ghana; Guinea [1454]; Guinea-Bissau; Kenya; Mali [819]; Nigeria; ?Rwanda; Senegal; Sierra Leone; Sudan; Togo; Uganda; Zaire 
Red-billed Firefinch, Senegal Firefinch

[Algeria]; Angola; Benin; Botswana; Burkina Faso [1388]; Burundi; Cameroon; Central African Republic; Chad; Congo; Côte d'Ivoire; Djibouti; Ethiopia; Gambia; Ghana; Guinea [1454]; Guinea-Bissau; Kenya; ?Lesotho; Liberia; Malawi; Mali [819]; Mauritania; Mozambique; Namibia; Niger; Nigeria; Rwanda; Senegal; Sierra Leone; Somalia; South Africa; Sudan; Swaziland; Tanzania; Togo; Uganda; Zaire; Zambia; Zimbabwe
Lagonosticta vinacea (Hartlaub 1857)
III GH -
$212,553,600$

(= L. larvata vinacea)

Black-faced Firefinch, Vinaceous Firefinch, Vinaceous Waxbill

Breeding:

Benin; Burkina Faso; Cameroon; Central African Republic; Chad; Congo; Côte d'Ivoire; Ethiopia; Gambia; Ghana; Guinea-Bissau; Mali [819]; Niger; Nigeria; Senegal; Sudan; Togo [071]; Uganda; Zaire

Lonchura bicolor (Fraser I843) III GH - 212,553,600,1247

(= Spermestes bicolor)

Black-and-white Munia, Blue-billed Mannikin, Black-and-white Mannikin

Breeding: $\quad$ Angola; Benin; Burundi; Cameroon; Central African Republic; Congo; Côte d'Ivoire; Equatorial Guinea; Ethiopia; Gabon; Ghana; Guinea; Guinea-Bissau; Kenya; Liberia; Mali; Nigeria; Rwanda; Sierra Leone; Sudan; Swaziland; Tanzania; Togo [278]; Uganda; Zaire

Vagrant: ?Senegal

Lonchura cantans (Gmelin 1789)

III GH

$212,243,553,575,600,661,1247$

(= L. malabarica cantans, Euodice cantans)

African Silverbill

Breeding:

Bahrain; Benin; Burkjna Faso [1388]; Cameroon; Chad; Djibouti [1472]; Egypt; Ethiopia; Gambia; Ghana; [Hawaiian Islands] [845,1118]; Kenya; Mali; Mauritania; Niger; Nigeria; Oman [1448]; [Puerto Rico]; Saudi Arabia; Senegal; Somalia; Sudan; Tanzania; Togo [071]; Uganda; Yemen

Lonchura cucullata (Swainson 1837)

III GH -

$212,553,600,1247$

(= Spermestes cucullatus)

Bronze Munia, Bronze Mannikin, Bronze-winged Mannikin

Breeding:

Angola; Benin; Botswana; Burkina Faso [1388]; Burundi; Cameroon; Central African Republic; Chad; Comoros; Congo; Côte d'Ivoire; Equatorial Guinea; Ethiopia; Gabon; Gambia; Ghana; Guinea [1454]; Guinea-Bissau; Kenya; Liberia; Malawi; Mali [819]; Mozambique; ?Namibia; Niger; Nigeria; [Puerto Rico] [179,845]; Rwanda; Sao Tome and Principe; Senegal; Sierra Leone; South Africa; Sudan; Swaziland; Tanzania; Togo; Uganda; Zaire; Zambia; Zimbabwe

Lonchura fringilloides (Lafresnaye 1835)

III GH $212,553,600,1247$

(= Spermestes fringilloides)

Magpie Munia, Magpie Mannikin, Pied Mannikin

Breeding:

Angola; Cameroon; Central African Republic; Congo; Côte d'Ivoire; Ethiopia [92]; Gabon; Gambia; Ghana; Guinea; ?Guinea-Bissau; Kenya; Liberia; Malawi; ?Mali [819]; Mozambique; Nigeria; Senegal; Sierra Leone; South Africa; Sudan; Swaziland; Tanzania; Togo; Uganda; Zaire; Zambia; Zimbabwe

Vagrant: Namibia 
Mandingoa nitidula (Hartlaub 1865)

IIJ $\mathrm{GH}$

$212,553,600,1247$

(= Hypargos nitidula)

Green-backed Twinspot, Green Twinspot

Breeding:

Angola; Benin; Burundi; Cameroon; Central African Republic; Congo; Côte d'Ivoire; Equatorial Guinea [984]; Ethiopia; Gabon; Ghana; Guinea; Kenya; Liberia; Malawi; Mozambique; Rwanda; Sierra Leone; South Africa; Sudan; Swaziland; Tanzania; Togo; Uganda; Zaire; Zambia; Zimbabwe [701]

Vagrant: Nigeria

Nesocharis capistrata (Hartlaub 1861)

III GH

$212,553,600,1247$

Grey-headed Olive-back, White-cheeked Olive Weaver

Breeding:

Burkina Faso; Cameroon; Central African Republic; Chad; Côte d'Ivoire; Ghana; Guinea-Bissau; Mali; Nigeria; Sierra Leone; Sudan; Togo; Uganda; Zaire

Vagrant: Gambia

Nigrita bicolor (Hartlaub 1844)

III $\mathrm{GH}$

$212,553,600,1247$

Chestnut-breasted Negrofinch

Breeding:

Angola; Benin; Cameroon; Central African Republic; Congo; Côte d'Ivoire; Equatorial Guinea; Gabon; Ghana; Guinea; Guinea-Bissau; Kenya; Liberia; Mali [819]; Nigeria; Sao Tome and Principe; Senegal; Sierra Leone; Togo; Uganda; Zaire

Vagrant: Gambia

Nigrita canicapilla (Strickland 1841)

III GH

$212,553,600,1247$

Grey-headed Negrofinch, Grey-crowned Negrofinch

Breeding: Angola; Benin; Burundi; Cameroon; Central African Republic; Congo; Côte d'Ivoire; Equatorial Guinea; Gabon; Ghana; Guinea [1454]; Kenya; Liberia; ?Mali [819]; Nigeria; Rwanda; Sierra Leone; Sudan; Tanzania; Togo; Uganda; Zaire

Nigrita fusconota Fraser 1843

III $\mathrm{GH} \quad-$

$2 \mathrm{I} 2,553,600,1247$

White-breasted Negrofinch

Breeding:

Angola; Benin; Cameroon; Central African Republic; Congo; ?Côte d'Ivoire [1378]; Equatorial Guinea; Gabon; Ghana; Guinea; Kenya; Liberia; Mali [819]; Nigeria; Uganda; Zaire

Nigrita luteifrons Verreaux \& Verreaux 1851

III GH

$212,553,600,1247$

Pale-fronted Negrofinch

Breeding: $\quad$ Angola; Benin; Cameroon; Central African Republic; Congo; Equatorial Guinea; Gabon; Ghana; ?Nigeria; Uganda; Zaire

Ortygospiza atricollis (Vieillot 1817)

African Quailfinch

Breeding:

Angola; ?Benin; Botswana; Burundi; Cameroon; Chad; Congo; Côte d'Ivoire; Equatorial Guinea; Ethiopia; Gabon; Gambia; Ghana; Guinea [1149,1454]; Guinea-Bissau; Kenya; Lesotho; Liberia; Malawi; Mali; Mauritania [230]; Mozambique; Namibia; Niger; Nigeria; Rwanda; Senegal; Sierra Leone; ?Somalia; South Africa; Sudan; Swaziland; Tanzania; Togo [277]; Uganda; Zambia; Zimbabwe

Parmoptila rubrifrons (Sharpe \& Ussher 1872)

III GH

$553,600,1247$

(= P. woodhousei rubrifrons)

Jameson's Antpecker, Red-fronted Antpecker

Breeding: $\quad$ Côte d'Ivoire; Ghana; Liberia; ?Mali [819]; Zaire

Pholidornis rushiae (Cassin 1855)

III $\mathrm{GH}-$

$212,553,600,1247$

Tit-hylia

Breeding:

Angola; Cameroon; Côte d'Ivoire; Equatorial Guinea; Gabon [221]; Ghana; Guinea; Liberia; Nigeria; Sierra Leone; ?Uganda; Zaire 
(includes $P$. frommi Kothe 1911, P. maximus Chapin 1923, $P$. rothschildi Neumann 1910)

\section{Black-bellied Seedcracker}

Breeding: $\quad$ Angola; Cameroon; Central African Republic; Congo; Côte d'lvoire; Equatorial Guinea; Vagrant: $\quad$ Gabon;

\section{Pytilia hypogrammica Sharpe 1870 \\ III $\mathrm{GH}$ $553,600,1247$}

Red-faced Pytilia, Yellow-winged Pytilia

Breeding:

?Benin; Cameroon; Central African Republic; Chad; Côte d'lvoire; Ghana; Guinea [1454]; Nigeria; Sierra Leone; Togo

Pytilia phoenicoptera Swainson 1837

$111 \mathrm{GH}$

$212,553,600,1247$

\section{Red-winged Pytilia, Aurora Finch, Crimson-winged Pytilia}

Breeding:

?Benin; Cameroon; Central African Republic [574]; Chad; Côte d'lvoire [1378]; Ethiopia [748]; Gambia; Ghana; Guinea-Bissau; Mali; Niger [803]; Nigeria; Senegal; Sudan; Togo; Uganda; Zaire

Spermophaga haematina (Vieillot 1805)

$111 \mathrm{GH}-$

$553,600,1247$

Western Bluebill, Blue-billed Weaver

Breeding:

Angola; Cameroon; Central African Republic; Congo; Côte d'Ivoire; Equatorial Guinea; Gabon; Gambia; Ghana; Guinea; Guinea-Bissau; Liberia; Mali [819]; Nigeria; Senegal; Sierra Leone; Togo; Zaire

Uraeginthus bengalus (Linnaeus 1766)

IIl GH -

$212,553,600,1247$

(= Estrilda bengala)

Red-cheeked Cordonbleu, Cordonbleu

Breeding:

Angola; Benin; Burkina Faso [1388]; Burund; Cameroon; Central African Republic; Chad; Congo; Côte d'Ivoire; Ethiopia; Gambia; Ghana; Guinea [1454]; Guinea-Bissau; [Hawaiian Islands]; Kenya; Mali; Mauritania; Niger; Nigeria; Rwanda; Senegal; Somalia; Sudan; Tanzania; Togo; Uganda; Zaire; Zambia

Family PLOCEIDAE

Sparrows, Weavers and allies

Amblyospiza albifrons (Vigors 1831)

$111 \mathrm{GH}$ -

$212,285,600,1247$

Grosbeak Weaver, White-fronted Grosbeak, Thick-billed Weaver

Breeding: Angola; ?Benin; Botswana; Burundi; Cameroon; Central African Republic; Congo; Côte d'Ivoire; Equatorial Guinea; Ethiopia; Gabon; Ghana; Kenya; Liberia; Malawi; Mozambique; Namibia; Nigeria; Rwanda; Sierra Leone; Somalia; South Africa; Sudan; Swaziland; Tanzania; Togo; Uganda; Zaire; Zambia; Zimbabwe

Vagrant: Senegal

Anaplectes rubriceps (Sundevall 1850) Ill GH -

(= Malimbus rubriceps, Anaplectes melanotis (Lafresnaye 1840))

Red-headed Weaver

Breeding:

Angola; ?Benin; Botswana; Burundi; Cameroon; Central African Republic; ?Chad; Côte d'lvoire; Ethiopia; Ghana; Guinea [1454]; Guinea-Bissau; Kenya; Malawi; ?Mali; Mozambique; Namibia; Nigeria; Rwanda; ?Senegal; Somalia; South Africa; Sudan; Swaziland; Tanzania; Togo; Uganda; Zaire; Zambia; Zimbabwe

Vagrant: Gambia 


\section{Parasitic Weaver, Cuckoo Weaver}

Breeding: $\quad$ Angola; ?Botswana; Cameroon; Congo; Côte d'Ivoire; Ethiopia; Guinea; Kenya; Liberia; Malawi; Mali; Mozambique; ?Namibia; Nigeria; Rwanda; Sierra Leone; South Africa; Swaziland; Tanzania; Togo; Uganda; Zaire; Zambia; Zimbabwe

Vagrant: Gambia; Ghana

\section{Bubalornis albirostris (Vieillot 1817) \\ III GH - \\ $212,600,1247$}

White-billed Buffalo Weaver

Breeding:

?Benin; Burkina Faso [1388]; Cameroon; ?Central African Republic; Chad; Côte d'Ivoire; Ethiopia; Gambia; Ghana; Guinea [984]; Guinea-Bissau; Kenya; Mali; Mauritania; Niger; Nigeria; Rwanda; Senegal; ?Sierra Leone; Sudan; ?Togo; Uganda; Zaire

Euplectes afer (Gmelin 1789)

III GH -

$212,285,600,1247$

Yellow-crowned Bishop, Golden Bishop, Napoleon Weaver

Breeding:

Angola; Benin; Botswana; Burkina Faso [1388]; Cameroon; Central African Republic; Chad; Congo; Côte d'lvoire; Equatorial Guinea; Ethiopia; Gabon; Gambia; Ghana; Guinea [1454]; Guinea-Bissau; Japan; Kenya; Lesotho; Liberia; Malawi; Mali; Mauritania; Mozambique; Namibia; Niger; Nigeria; [Puerto Rico]; Senegal; Sierra Leone; South Africa; Sudan; Swaziland; Tanzania; Togo; Uganda; Zaire; Zambia; Zimbabwe

Euplectes ardens (Boddaert 1783)

III $\mathrm{GH}$

$212,285,600,1247$

(= Coliuspasser ardens)

Red-collared Widowbird, Red-collared Whydah

Breeding:

Angola; Burundi; Cameroon; Central African Republic; ?Congo; Côte d'Ivoire; Ethiopia [748]; Gabon; ?Ghana; Guinea [1454]; Kenya; Lesotho; Liberia; Malawi; Mozambique; Niger; Nigeria; Rwanda; Sierra Leone; South Africa; Sudan; Swaziland; Tanzania; Togo; Uganda; Zaire; Zambia; Zimbabwe

Vagrant: Gambia; ?Guinea-Bissau; Senegal

$\begin{aligned} & \text { Euplectes franciscanus (Isert 1789) } \\ & (=\text { E. orix franciscanus) }\end{aligned}$
OIl GH

Orange Bishop, Orange Weaver

Breeding: $\quad$ Benin; [Bermuda]; Burkina Faso [1388]; Burundi; Cameroon; Central African Republic; Chad; Côte d'Ivoire; Djibouti; Ethiopia; Gambia; Ghana; Guinea [1454]; Guinea-Bissau; Kenya; Mali; [Martinique]; Mauritania; Niger; Nigeria; [Puerto Rico]; Rwanda; Senegal; Sierra Leone; Somalia; Sudan; Togo; Uganda; Zaire

Euplectes hordeaceus (Linnaeus 1758)

IIl GH -

$212,285,600,1247$

Black-winged Bishop, Fire-crowned Bishop

Breeding:

Angola; Benin; Burkina Faso [1388]; Burundi; Cameroon; Central African Republic; Chad; Congo; Côte d'Ivoire; ?Equatorial Guinea; Ethiopia [748]; Gabon; Gambia; Ghana; Guinea [1454]; Guinea-Bissau; Kenya; Liberia; Malawi; Mail; Mozambique; Niger; Nigeria; Rwanda; Sao Tome and Principe: Sao Tome; Senegal; Sierra Leone; Sudan; Tanzania; Togo; Uganda; Zaire; Zambia; Zimbabwe
Euplectes macrourus (Gmelin 1789)
IIl GH -
$212,600,1247$

(= Coliuspasser macrourus)

Yellow-mantled Widowbird, Yellow-backed Whydah, Yellow-mantled Whydah

Breeding:

Angola; Benin; Burkina Faso [1388]; ?Burundi; Cameroon; Central African Republic; Chad; Congo; Côte d'lvoire; Equatorial Guinea; Ethiopia; Gabon; Gambia; Ghana; Guinea [1454]; Guinea-Bissau; Kenya; Liberia; Malawi; Mozambique; Niger; Nigeria; ?Rwanda; Senegal; Sierra Leone; Sudan; Tanzania; Togo; Uganda; Zaire; Zambia; Zimbabwe

Vagrant: Mali 
Foudia flavicans Newton 1865

E

318,324

Yellow Fody, Rodrigues Fody

Breeding:

Mauritius: Rodrigues

Foudia rubra (Gmelin 1789)

E

318,324

Mauritius Fody

Breeding:

Mauritius

Foudia sechellarum Newton 1865

$\mathbf{R}$

$217,318,324$

Seychelles Fody

Breeding:

Seychelles: Cousin, Cousine, Fregate

Malimbus ballmanni Wolters 1974

Id

318,324

Ballmann's Malimbe

Breeding:

?Côte d'Ivoire; Liberia; Sierra Leone

Malimbus cassini (Elliot 1859)

IIl GH

600,1247

Black-throated Malimbe, Cassin's Malimbe

Breeding:

?Angola; Cameroon; Central African Republic; Congo; ?Equatorial Guinea; Gabon; Ghana; Zaire

Malimbus flavipes Chapin 1916

$\begin{array}{lll}- & \mathrm{R} & 318,324,600\end{array}$

(= Ploceus flavipes)

Yellow-legged Malimbe, Yellow-legged Weaver

Breeding:

Zaire

Malimbus ibadanensis Elgood 1958

- $\quad$ E $318,324,600,1247$

Ibadan Malimbe

Breeding:

Nigeria [98]

Malimbus malimbicus (Daudin 1802)

II1 GH

$212,600,1247$

Crested Malimbe

Breeding:

Angola; Cameroon; Central African Republic; Congo; Côte d'Ivoire; ?Equatorial Guinea; Gabon; Ghana; Guinea; Liberia; Mali; Nigeria; Sierra Leone; Togo; Uganda; Zaire

Malimbus nitens (Gray 1831)

III GH -

$212,600,1247$

Gray's Malimbe, Blue-billed Malimbe

Breeding:

?Angola; Benin; Cameroon; Central African Republic; Congo; Côte d'Ivoire; Equatorial Guinea; Gabon; Ghana; Guinea; Guinea-Bissau; Liberia; ?Mali; Niger; Nigeria; Senegal; Sierra Leone; Togo; Uganda; Zaire

Vagrant:

Gambia

Malimbus rubricollis (Swainson 1838)

III $\mathrm{GH}$

$212,600,1247$

Red-headed Malimbe, Red-headed Weaver

Breeding:

Angola; Benin; Cameroon; Central African Republic [259]; Congo; Côte d'Ivoire; Equatorial Guinea: Bioko; Gabon; Ghana; Guinea; Guinea-Bissau; Kenya; Liberia; ?Mali; Nigeria; ?Rwanda; Sierra Leone; Sudan; Tanzania; Togo; Uganda; Zaire

Malimbus scutatus (Cassin 1849)

III $\mathrm{GH} \quad-$

600,1247

Red-vented Malimbe

Breeding:

Benin; Cameroon; Côte d'Ivoire; Ghana; Guinea; Liberia; Nigeria; Sierra Leone 
Pachyphantes superciliosus (Shelley 1873)

III GH -

$212,600,1247$

(= Ploceus superciliosus)

Compact Weaver

Breeding:

Angola; Benin; Burundi; Cameroon; Central African Republic; Congo; Côte d'Ivoire; Equatorial Guinea; Ethiopia [221]; Gabon [221]; Ghana; Guinea [984]; Kenya; Liberia; Niger; Nigeria; Rwanda; Sierra Leone; Sudan; Tanzania; Togo; Uganda; Zaire; Zambia

Vagrant: Senegal

Passer griseus (Vieillot 1817)

III GH -

$2 \mathrm{~J} 2,285,600,1247$

Grey-headed Sparrow

Breeding:

Angola; Benin; Burkina Faso [1388]; Burundi; Cameroon; Central African Republic; Chad; Congo; Côte d'Ivoire; Djibouti; Equatorial Guinea; Ethiopia; Gabon; Gambia; Ghana; Guinea [ 1454]; Guinea-Bissau; Kenya; Liberia; Malawi; Mali; Mauritania; Niger; Nigeria; Rwanda; Senegal; Sierra Leone; Sudan; Tanzania; Togo; Uganda; Zaire; Zambia

Petronia dentata (Sundevall 1850)

III $\mathrm{GH} \quad-$

600,1247

Bush Petronia, Bush Sparrow

Breeding:

Benin; Burkina Faso [1388]; Cameroon; Central African Republic; Chad; Côte d'Ivoire; Ethiopia; Gambia; Ghana; Guinea [1454]; Guinea-Bissau; Mali; Mauritania; Niger; Nigeria; Senegal; Sierra Leone; Sudan; Togo; Yemen

Plocepasser superciliosus (Cretzschmar 1827)

III GH -

$212,600,1247$

Chestnut-crowned Sparrow-Weaver

Breeding:

?Benin; Burkina Faso; Cameroon; Central African Republic; Chad; Côte d'Ivoire; Ethiopia [748]; Gambia; Ghana; Guinea-Bissau; Kenya; Mali; Niger; Nigeria; Senegal; ?Sierra Leone; Togo; Uganda; Zaire

Ploceus albinucha (Barboza du Bocage 1876)

III GH -

$212,600,1247$

Maxwell's Black Weaver, White-naped Weaver

Breeding:

Cameroon; ?Congo; Côte d'Ivoire; Equatorial Guinea: Bioko; Gabon; Ghana; Liberia; ?Nigeria; Sierra Leone; Uganda; Zaire

Ploceus aurantius (Vjeillot 1805) $\quad$ IIl GH $\quad-\quad 212,600,1247$

Orange Weaver

Breeding:

Angola; ?Benin; Burkina Faso; Cameroon; Central African Republic; Congo; Côte d'Ivoire; Equatorial Guinea; Gabon; Ghana; Ljberia; Nigeria; Sierra Leone; Tanzania; Togo; Uganda; Zaire

Vagrant:

Kenya

Ploceus aureonucha Sassi 1920

R

$318,324,600$

Golden-naped Weaver

Breeding: Zaire [38]

Ploceus bannermani Chapin 1932

V

$318,324,600,1247$

Bannerman's Weaver

Breeding: Cameroon; ?Nigeria

Ploceus batesi (Sharpe 1908)

$\mathbf{R}$

$318,324,600,1247$

Bates's Weaver

Breeding:

Cameroon 
(includes $P$. nigriceps (Layard 1867))

Village Weaver, Spot-backed Weaver, (Layard's Black-headed Weaver)

Breeding:

Angola; Benin; Botswana; Burkina Faso [1388]; Burundi; Cameroon; Central African Republic; Chad; Congo; Côte d'Ivoire; [Dominican Republic]; Equatorial Guinea: Bioko; Ethiopia; Gabon; Gambia; Ghana; Guinea; Guinea-Bissau; Kenya; Liberia; Malawi; Mali; [Martinique]; Mauritania; [Mauritius]; Mozambique; Namibia; Niger; Nigeria; [Puerto Rico]; [Réunion]; Rwanda; [Sao Tome and Principe: Sao Tome]; Senegal; Sierra Leone; Somalia; South Africa; Sudan; Swaziland; Tanzania; Togo; Uganda; Zaire; Zambia; Zimbabwe

Ploceus golandi (Clarke 1913)

E

$212,318,324,600$

Clarke's Weaver

Breeding:

Kenya

Ploceus heuglini Reichenow 1886

$111 \mathrm{GH} \quad-$

$212,600,1247$

(= Texiu atrogularis Heuglin 1864)

Heuglin's Masked-Weaver

Breeding: ?Benin; Cameroon; Central African Republic; Côte d'lvoire; Gambia; Ghana; ?Guinea-Bissau; Kenya; ?Liberia; Mali; Niger; Nigeria; Senegal; ?Sierra Leone; Sudan; Togo; Uganda; Zaire

Ploceus luteolus (Lichtenstein 1823)

III GH -

$212,324,600,1247$

Little Weaver

Breeding:

?Benin; Burkina Faso [1388]; Cameroon; Central African Republic; Chad; Côte d'Ivoire; Ethiopia; Gambia; Ghana; Guinea-Bissau; Kenya; Mali; Mauritania; Niger; Nigeria; Senegal; Sudan; Tanzania; Togo; Uganda; Zaire

Ploceus megarhynchus Hume 1869 K* $\quad 318$

Yellow Weaver, Finn's Baya Weaver, Himalayan Weaver

Breeding: India [10]

Ploceus melanocephalus (Linnaeus 1758) $\quad 111 \mathrm{GH} \quad-\quad 212,600,1247$

(= Sitagra melanocephala)

(includes $P$. capitalis (Latham 1790))

Black-headed Weaver, (Yellow-backed Weaver)

Breeding: $\quad$ Benin; Burkina Faso [1388]; Burundi; Cameroon; Central African Republic; Chad; Congo; Ethiopia; Gambia; Ghana; Guinea [1454]; Guinea-Bissau; Kenya; Mali; Mauritania; Niger; Nigeria; Rwanda; Senegal; Sierra Leone; Sudan; Tanzania; Togo; Uganda; Zaire; Zambia

Ploceus nicolli Sclater 1931

R $\quad 212,318,324,600$

(= P. olivaceiceps nicolli)

Usambara Weaver, Tanzanian Mountain Weaver

Breeding:

Tanzania

Ploceus nigerrimus Vieillot 1819

IIl $\mathrm{GH}-$

$212,600,1247$

Vieillot's Black Weaver

Breeding:

Angola; Benin; Burundi; Cameroon; Central African Republic; Congo; Côte d'lvoire; Equatorial Guinea; Gabon; Gambia; Ghana; Guinea [1454]; ?Guinea-Bissau; Kenya; Liberia; Nigeria; Rwanda; Sierra Leone; Tanzania; Togo; Uganda; Zaire 
Black-necked Weaver

Breeding:

Angola; Benin; Burkina Faso [1388]; Burundi; Cameroon; Central African Republic; ?Chad; Congo; Côte d'Ivoire; Equatorial Guinea: Bioko; Ethiopia; Gabon; Gambia; Ghana; Guinea; Guinea-Bissau; Kenya; Liberia; ?Mali; Niger; Nigeria; Rwanda; Senegal; Sierra Leone; Somalia; Sudan; Tanzania; Togo; Uganda; Zaire

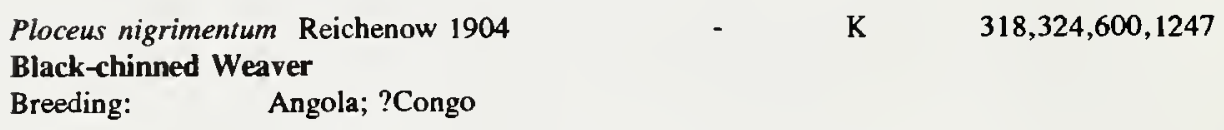

Ploceus pelzelni (Hartlaub 1887)

III GH -

$212,600,1247$

Slender-billed Weaver

Breeding: $\quad$ Angola; ?Benin; Burundi; Cameroon; ?Central African Republic; Congo; Côte d'Ivoire; Gabon; Ghana; ?Guinea-Bissau; Kenya; ?Liberia; Nigeria; Rwanda; ?Sierra Leone; Sudan; Tanzania; Togo; Uganda; Zaire; Zambia

Ploceus preussi (Reichenow 1892)

III GH -

600,1247

Preuss's Weaver, Yellow-capped Weaver, Golden-backed Weaver

Breeding: $\quad$ ?Angola; Cameroon; Central African Republic; Congo; Côte d'Ivoire; Equatorial Guinea; Gabon [221]; Ghana; Guinea [984]; Liberia; ?Nigeria; Sierra Leone; Zaire

Ploceus ruweti Louette \& Benson 1982

Ruwet's Masked-Weaver, Lake Lufira Weaver

Breeding:

Ploceus subpersonatus (Cabanis 1876)

Loango Weaver, Loango Slender-billed Weaver

Breeding:

Angola; Gabon; Zaire
K $\quad 318,324$

K $\quad 318,324,600,1247$

Ploceus tricolor (Hartlaub 1854) $\quad 111 \mathrm{GH} \quad-\quad 212,600,1247$

Yellow-mantled Weaver

Breeding: Angola; Cameroon; ?Congo; Côte d'lvoire; ?Equatorial Guinea; Gabon [221]; Ghana; Guinea; Kenya; Liberia; Nigeria; Sierra Leone; Togo; Uganda; Zaire

Ploceus victoriae Ash 1986

Victoria Masked-Weaver, Entebbe Weaver

Breeding: Uganda [97,99]

Ploceus vitellinus (Lichtenstein 1823)

(= P. velatus vitellinus)

Vitelline Masked-Weaver

Breeding:

Benin; Burkina Faso [1388]; Cameroon; Central African Republic; Chad; Ethiopia; Gambia; Ghana; Kenya; Mali; Mauritania; Niger; Nigeria; Senegal; Somalia; Sudan; Tanzania; Togo; Uganda; Zaire

Quelea erythrops (Hartlaub 1848)

Red-headed Quelea, Red-headed Dioch

Breeding:

Angola; Benin; Burkina Faso [1388]; Burundi; Cameroon; Central African Republic; Chad; Congo; Côte d'lvoire; Equatorial Guinea; Ethiopia; Gabon; ?Gambia; Ghana; Guinea-Bissau; Kenya; Liberia; Malawi; Mali; Mozambique; Niger; Nigeria; Rwanda; Sao Tome é Principe: Sao Tome; ?Senegal; Sierra Leone; South Africa; Sudan; ?Swaziland; Tanzania; Togo; Uganda; Zaire; Zambia

Vagrant:

$$
\text { K* } \quad 318,860
$$

$$
111 \mathrm{GH} \quad-\quad 212,285,600,1247
$$

$111 \mathrm{GH} \quad-\quad 212,285,600,1247$ Somalia 
Sporopipes frontalis (Daudin 1802)

III GH -

$212,600,1247$

Speckle-fronted Weaver, Scaly-fronted Weaver

Breeding:

Burkina Faso [1388]; Cameroon; Central African Republic; Chad; Ethiopia; Ghana; Kenya; Mali; Niger; Nigeria; Senegal; Sudan; Tanzania; Uganda

Vagrant:

Gambia; Mauritania; Somalia

Vidua chalybeata (Müller 1776)

III GH

$212,285,600,1076,1247$

(= Hypochera chalybeata)

(includes V. amauropteryx (Sharpe 1890), V. centralis (Neunzig 1928), V. neumanni (Alexander 1908), V. okavangoensis Payne 1973, V. ultramarina (Gmelin 1789))

Village Indigobird, Green Indigobird, Senegal Combassou

Breeding:

Angola; Benin; Botswana; Burkina Faso [1388]; Burundi; Cameroon; Central African Republic; Chad; Côte d'Ivoire; Ethiopia; Gambia; Ghana; Guinea; Guinea-Bissau; Kenya; ?Lesotho; Malawi; Mali; Mauritania; Mozambique; Namibia; Niger; ?Nigeria; Rwanda; Senegal; Sierra Leone; Somalia; South Africa; Sudan; Swaziland; Tanzania; ?Togo; Uganda; Zaire; Zambia; Zimbabwe

Vidua interjecta (Grote 1922)

III GH -

1077

$(=V$. orientalis interjecta)

Long-tailed Paradise-Whydah, Uelle Paradise-Whydah

Breeding:

Cameroon; Central African Republic; Chad; Ethiopia; Ghana; Guinea; Mali; Nigeria; Sudan; Zaire

Vidua larvaticola Payne $1982 \quad 111 \mathrm{GH} \quad-\quad 1076,1077$

Baka Indigobird

Breeding:

?Benin; ?Burkina Faso; Cameroon; ?Central African Republic; ?Chad; Côte d'Ivoire; Ethiopia; Gambia; ?Ghana; ?Guinea; ?Guinea-Bissau; ?Mali; Nigeria; Sudan; ?Togo; ?Zaire

Vidua macroura (Pallas 1764)

III GH -

$212,285,600,1247$

Pin-tailed Whydah

Breeding:

Angola; Benin; Botswana; Burkina Faso [1388]; Burundi; Cameroon; Central African Republic; Chad; Congo; Côte d'Ivoire; Equatorial Guinea: Bioko; Ethiopia; Gabon; Gambia; Ghana; Guinea [1454]; Guinea-Bissau; [Hawaiian Islands (ex)]; Kenya; Lesotho; Liberia; Malawi; Mali; Mauritania; Mozambique; Namibia; Niger; ?Nigeria; Sao Tome and Principe; [Puerto Rico]; Rwanda; Senegal; Sierra Leone; Somalia; South Africa; Sudan; Swaziland; Tanzania; Togo; Uganda; Zaire; Zambia: Zimbabwe

Vidua orientalis Heuglin 1871

IIl GH -

$212,285,600,1247$

(=V. paradisaea orientalis)

Northern Paradise-Whydah

Breeding: Benin; Burkina Faso [1388]; Cameroon; Central African Republic; Chad; Côte d'Ivoire; Ethiopia; Gambia; Ghana; Guinea; ?Guinea-Bissau; Mali; Mauritania; ?Niger; ?Nigeria; Senegal; Sierra Leone; Sudan; Togo

Vidua raricola Payne 1982

III GH - $\quad 1076,1077$

Jambandu Indigobird

Breeding:

Cameroon; ?Chad; Ghana; Nigeria; Sierra Leone; Sudan; ?Zaire

Vidua togoensis (Grote 1923)

III GH -

1077

$(=V$. orientalis togoensis)

Togo Paradise-Whydah

Breeding:

Cameroon; Chad; Côte d'lvoire; Ghana; Liberia; Nigeria; Sierra Leone; Togo 
Vidua wilsoni (Hartert 1901)

$111 \mathrm{GH}$

$600,1076,1247$

(= Hypochera wilsoni)

(includes V. camerunensis (Grote 1922), V. incognita Nicolai 1972, V. lorenzi Nicolai 1972)

Pale-winged Indigobird, Wilson's Indigobird

Breeding:

?Botswana; Cameroon; Central African Republic; ?Côte d'Ivoire; Gambia; Ghana;

Guinea-Bissau; ?Namibia; Nigeria; Senegal; Sudan; Togo; Zaire; ?Zimbabwe

Family STURNIDAE

Starlings

Aplonis cinerascens Hartlaub \& Finsch $1871 \quad-\quad K^{*} \quad 318$

Rarotonga Starling

Breeding: Cook Islands: Rarotonga

$\begin{array}{llll}\text { Aplonis pelzelni Finsch } 1876 & \text { - } & K^{*} & 318,794,1118\end{array}$

Pohnpei Starling

Breeding: $\quad$ Federated States of Micronesia: Pohnpei (ex?)

Mountain Starling, Santo Mountain Starling

R $\quad 74,318,794$

Breeding: Vanuatu: Espirito Santo [1096]

Basilornis galeatus Meyer $1894 \quad$ - $\quad K^{*} \quad 318,1486$

Helmeted Myna, Sula Myna

Breeding: $\quad$ Indonesia: Banggai Islands, Sula lslands

Cinnyricinclus femoralis (Richmond 1897)

K* $\quad 318,324$

Abbott's Starling

Breeding:

Kenya; Tanzania

Gracula religiosa Linnaeus 1758

III TH

Hill Myna

Breeding:

Bangladesh; Bhutan; Brunei; Cambodia; China; [Christmas Island]; India; Indonesia: Alor, Bali, Flores, Java, Pantar, Sumatra, Sumbawa; Lao P.D.R; Malaysia: Peninsular Sabah, Sarawak [1295]; Myanmar; Nepal; Philippines; [Puerto Rico]; Sri Lanka; Thailand; Viet Nam

Leucopsar rothschildi Stresemann 1912

I $\quad$ E

$72,77,318,794$

Bali Myna, Bali Starling, Rothschild's Mynah

Breeding: Indonesia: Bali [645]

Streptocitta albertinae (Schlegel 1866)

$\mathrm{K}^{*}$

318,1486

Bare-eyed Myna, Sula Starling

Breeding:

Indonesia: Sula Islands

Family ORIOLIDAE

Orioles

Oriolus isabellae Ogilvie-Grant 1894

K* $\quad 318$

Isabela Oriole

Breeding:

Philippines: Mindanao [381], Luzon 
Silver Oriole

Breeding:

China [968]

Non-breeding: Thailand

Vagrant: Cambodia

Family DICRURIDAE

Drongos

Dicrurus fuscipennis (Milne-Edwards \& Oustalet J887)

Comoro Drongo, Grand Comoro Drongo

$\mathbf{R}$

318,324

Breeding:

Comoros: Grand Comoro [862]

Dicrurus waldenii Schlegel 1866

$\mathbf{R}$

318,324

Mayotte Drongo

Breeding:

Mayotte [862]

Family CALLAEIDAE

Wattlebirds

Callaeas cinerea (Gmelin 1788)

E

$294,318,630,794,976,1030$

Kokako

New Zealand [1507]

Creadion carunculatus (Gmelin 1789)

K*

$318,794,976$

(= Philesturnus carunculatus)

Saddleback

Breeding:

New Zealand [1507]

Family PTILONORHYNCHIDAE

Bowerbirds

Amblyornis flavifrons Rothschild 1895

Golden-fronted Bowerbird, Yellow-fronted Bowerhird

Breeding: Indonesia: Irian Jaya [131,391]

Sericulus bakeri (Chapin 1929)

K*

318,1226

Fire-maned Bowerbird, Adelbert Bowerhird, Adelbert Regent Bowerbird

Breeding: Papua New Guinea [131]

Family PARADISAEIDAE

Birds of Paradise

Astrapia mayeri Stonor 1939

II

$\mathbf{K}^{*}$

$318,335,1226$

Ribbon-tailed Astrapia, Ribbon-tailed Bird-of-paradise

Breeding: Papua New Guinea [131]

Astrapia nigra (Gmelin 1788)

I1

335,1226

Arfak Astrapia

Breeding:

Indonesia: Irian Jaya

Astrapia rothschildi Foerster 1906

Huon Astrapia

Breeding:

Papua New Guinea 
Splendid Astrapia

Breeding:

Indonesia: Irian Jaya; Papua New Guinea

Astrapia stephaniae (Finsch 1885)

11 -

Stephanie's Astrapia, Princess Stephanie's Bird-of-paradise

Breeding: Papua New Guinea

Cicinnurus magnificus (Pennant 1781)

(= Diphyllodes magnificus)

Magnificent Bird-of-paradise

Breeding: Indonesia: Irian Jaya, Misool, Salawati, Japen; Papua New Guinea

Cicinnurus regius (Linnaeus 1758)

Il

335,1226

King Bird-of-paradise

Breeding:

Indonesia: Irian Jaya, Misool, Salawati, Batanta, Japen, Aru; Papua New Guinea

Cicinnurus respublica (Bonaparte 1850)

II

335,1226

(= Diphyllodes respublica)

Wilson's Bird-of-paradise

Breeding: Indonesia: Batanta, Waigeo

Cnemophilus loriae (Salvadori 1894)

II

335

(= Loria loriae)

Loria's Bird-of-paradise

Breeding: Indonesia: Irian Jaya; Papua New Guinea

$\begin{array}{lll}\text { Cnemophilus macgregorii De Vis } 1890 \quad 11 & \text { - } & \text { 335, I226 }\end{array}$

Crested Bird-of-paradise

Breeding: $\quad$ Papua New Guinea

Epimachus albertisi (Sclater 1873)

(= Drepanornis albertisi)

Black-billed Sicklebill, Buff-tailed Sicklebill

Breeding: Indonesia: Irian Jaya; Papua New Guinea

Epimachus bruijnii (Oustalet 1880)

I1 $\quad-\quad 335$

(= Drepanornis bruijnii)

Pale-billed Sicklehill, White-billed Sicklebill

Breeding: Indonesia: Irian Jaya; Papua New Guinea

Epimachus fastuosus (Hermmann 1783) II $\quad K^{*} \quad 318,335,1226$

Black Sicklebill

Breeding: Indonesia: Irian Jaya; Papua New Guinea

Epimachus meyeri Finsch 1885

11

Brown Sicklebill

Breeding:

Indonesia: Irian Jaya; Papua New Guinea

Loboparadisea sericea Rothschild I896 II -

Yellow-breasted Bird-of-paradise, Wattle-billed Bird-of-paradise

Breeding: $\quad$ Indonesia: Irian Jaya; Papua New Guinea.

Lophorina superba (Pennant 1781)

Il

335,1226

Superb Bird-of-paradise

Breeding:

Indonesia: Irian Jaya; Papua New Gujnea 
Lycocorax pyrrhopterus (Bonaparte 1851)

Paradise-crow, Silky-crow

Breeding: $\quad$ Indonesia: Bacan, Halmahera, Morotai, Obi, Rau

$\begin{array}{llll}\text { Macgregoria pulchra De Vis } 1897 & \text { II } & - & 335,1226\end{array}$

MacGregor's Bird-of-paradise

Breeding: Indonesia: Irian Jaya; Papua New Guinea

Manucodia atra (Lesson 1830)

II

335,1226

Glossy-mantled Manucode

Breeding:

Indonesia: Aru Islands, Batanta, Gebe, Gemien, Irian Jaya, Misool, Salawati, Waigeo; Papua New Guinea

Manucadia chalybata (Pennant 1781)

II

Crinkle-collared Manucode

Breeding:

Indonesia: Irian Jaya, Misool [88!]; Papua New Guinea

Manucodia comrii Sclater 1876

II

335,1226

Curl-crested Manucode

Breeding: $\quad$ Papua New Guinea: Fergusson, Goodenough, Kaileuna, Kiriwana, Normanby
Manucodia jobiensis Salvadori 1876
II
335,1226

Jobi Manucode

Breeding:

Indonesia: 1rian Jaya, Yapen; Papua New Guinea

Manucodia keraudrenii (Lesson \& Gamot 1826) II

335,1226

(= Phonygammus keraudrenii)

Trumpet Manucode, Trumpetbird

Breeding: $\quad$ Australia [167]; Indonesia: Aru Islands, Irian Jaya; Papua New Guinea

Melampitta gigantea (Rothschild 1899) $\quad$ II $\quad$ - $\quad 1226$

Greater Melampitta

Breeding: Indonesia: lrian Jaya; Papua New Guinea

Melampitta lugubris Schlegel $1873 \quad$ II $\quad$ - $\quad 1226$

Lesser Melampitta

Breeding: Indonesia: Irian Jaya; Papua New Guinea

Paradigalla brevicauda Rothschild \& Hartert 1911 II $\quad$ - 335

Short-tailed Paradigalla

Breeding: $\quad$ Papua New Guinea

Paradigalla carunculata Lesson $1835 \quad$ I1 $\quad \mathrm{K}^{*} \quad 318,335,1226$

Long-tailed Paradigalla

Breeding: $\quad$ Indonesia: Irian Jaya [131]

Paradisaea apoda Linnaeus 1758

II

335,1226

Greater Bird-of-paradise

Breeding: Indonesia: Aru Islands, Irian Jaya; Papua New Guinea; [Trinidad and Tobago: Little Tobago]

Paradisaea decora Salvin \& Godman 1883

II $\quad \mathrm{K}^{*}$

$318,335,1226$

Goldie's Bird-of-paradise

Breeding: $\quad$ Papua New Guinea: Fergusson, Normanby [131] 
Emperor Bird-of-paradise, Emperor of Germany's Bird-of-paradise

Breeding: Papua New Guinea

Paradisaea minor Shaw 1809

II

335,1226

Lesser Bird-of-paradise

Breeding: Indonesia: Irian Jaya, Misool, Yapen; Papua New Guinea

Paradisaea raggiana Sclater $1873 \quad$ II
Raggiana Bird-of-paradise, Count Raggi's Bird-of-paradise

Breeding: Indonesia: Irian Jaya; Papua New Guinea

Paradisaea rubra Daudin 1800

II

335,1226

Red Bird-of-paradise

Breeding:

Indonesia: Batanta, Gemien, Saonek [131], Waigeo

Paradisaea rudolphi (Finsch 1885)

II

335,1226

Blue Bird-of-paradise

Breeding: Papua New Guinea

Parotia carolae Meyer 1894

I1

335,1226

Carola's Parotia, Queen Carola's Parotia

Breeding: Indonesia: 1rian Jaya; Papua New Guinea

Parotia helenae De Vis 1891

II

(= P. lawesii helenae)

Eastern Parotia

Breeding:

Papua New Guinea

Parotia lawesii Ramsay 1885

Lawes's Parotia

Breeding:

Papua New Guinea

Parotia sefilata (Pennant 1781)

Western Parotia, Arfak Parotia, Six-plumed Parotia

Breeding:

Indonesia: Irian Jaya

Parotia wahnesi Rothschild 1906

II $\quad \mathrm{K}^{*}$

$318,335,1226$

Wahnes's Parotia

Breeding:

Papua New Guinea [131]

Pteridophora alberti Meyer 1894

II

King-of-Saxony Bird-of-paradise

Breeding:

Indonesia: Irian Jaya; Papua New Guinea

Ptiloris magnificus (Vieillot 1819)

11

335

Magnificent Riflebird

Breeding: Australia [167]; Indonesia: Irian Jaya; Papua New Guinea

Ptiloris paradiseus Swainson 1825

11

Paradise Riflebird

Breeding:

Australia [167]

Ptiloris victoriae Gould 1850

11

Victoria's Riflebird, Queen Victoria's Riflebird

Breeding:

Australia [167] 
RL Ref

Seleucidis melanoleuca (Daudin 1800)

II

335

Twelve-wired Bird-of-paradise

Breeding:

Indonesia: Irian Jaya, Salawati; Papua New Guinea

Semioptera wallacii Gould 1859

II

335

Standard-wing Bird-of-paradise, Wallace's Standardwing

Standardwing

Breeding: Indonesia: Bacan [1486], Halmahera

Family CORVIDAE

Crows

Corvus florensis Büttikofer 1894

$\mathrm{K}^{*} \quad 318,552,1486$

Flores Crow

Breeding: Indonesia: Flores

Corvus hawaiiensis Peale 1848

E

$16,239,318,552,794,1118,1205$

(= C. tropicus Kerr 1792)

Hawaiian Crow

Breeding:

Hawaiian Islands: Hawaii

Corvus kubaryi Reichenow 1885

E $\quad 318,552,794,972,1118,1393$

Mariana Crow, Guam Crow

Breeding: Guam; Northem Marianas: Rota

Corvus unicolor (Rothschild \& Hartert 1900)

$\mathrm{K}^{*} \quad 318,552,1486$

Banggai Crow

Breeding: Indonesia: Banggai

Crypsirina cucullata Jerdon 1862

$\mathrm{K}^{*} \quad 318,552$

Hooded Treepie, Hooded Racket-tailed Treepie

Breeding: Myanmar [1296]

Cyanolyca mirabilis Nelson 1903

White-throated Jay

Breeding:

Mexico

Cyanolyca nano (Du Bus \& Gisignies 1847)

E

$16,318,321,552$

Dwarf Jay

Breeding:

Mexico

Perisoreus internigrans (Thayer \& Bangs 1912)

$\mathrm{K}^{*} \quad 318,552$

Sichuan Jay, Sichuan Grey Jay, Sooty Jay

Breeding:

China [968]

Urocissa ornata (Wagler 1829)

$\mathrm{K}^{*} \quad 318,552$

Ceylon Magpie, Ceylon Blue Magpie

Breeding: Sri Lanka [10]

Zavattariornis stresemanni Moltoni 1938

R $\quad 318,324,552,600$

Stresemann's Bush-Crow, Ethiopian Bush-Crow Breeding: Ethiopia 



\section{REFERENCES}

1. Abdulali, H. 1965. The birds of the Andaman and Nicobar Islands. J. Bombay Nat. Hist. Soc. 61: 438-571.

2. Abdusalyamov, I.A. 1977. [Fauna of the Tadjik Soviet Socialistic Republic. Vol.19 Part 3: birds.] Donish. Dushanbe. (In Russian).

3. Abu Yaman, I.K. et al. 1985. Fauna of Jordan. II. Aves: a check list of the birds of Jordan. Amman.

4. Achaval, F. 1989. Lista de Especies de Veriebratos del Uriguay Parte 2: Anfibios, repriles aves y mamíferos. Facultad de Humanidades y Ciencias, Universidad de la Republica, Uruguay.

5. Akcakaya, H.R. and Akeakaya, R. 1986. Concern for Turkey's last Bald Thises. WWF Monthly Reports September: 243-245.

6. Alayón, G. and Garrido, O.H. 1991 . Current situation of the lvory-billed Woodpecker (Campephilis principalis) in Cuba. Unpublished.

7. Albuquerque, J.L.B. 1986. Conservation and status of raptors in southern Brazil. Birds of Prey Bull. 3: 88-94.

8. Alexander, C. 1985. The St. Helena Wirebird, its status and distribution. Unpublished.

9. Ali, S., Daniel. J.C. and Rahmani, A.R. 1986. Stıdy of ecology of certoin endangered species of willife and their habitats. The Floricans. Annual Report I Bombay Natural History Society, Bombay.

10. Ali, S. and Ripley, S.D. 1987. Compact handbonk of the birds of Indio and Pakistan together with those in Bangladesh, Nepal, Bhutan and Sri Lanka. Oxford University Press, Oxford

11. Allen, G.M. 1965. The birds of Liberia. Contr. Dep. trop. Med. \& Inst. trop. Biol. Med. 5: 636-748.

12. Allouse, B.E. 1953. The avifauna of Iraq. Iraq Natural History Museum Publication No. 3.

13. Allport, G. 1989. West of the Dahomey Gap. World Birdwatch 11(4): 9

14. Allport, G. and Milton, G.R. 1988. A note on the recent sighting of Znsterops fava Javan White-eye. Kukila 3: 142-149.

15. Amadon, D., Bull, J., Marshall. J.T. and King, B.F. 1988. Hawks and owls of the world: a distributional and taxonomic list. Proceedings of the Western Foundation of Vertebrate Zoology 3(4): 295-357.

16. American Ornitholngists' Union. 1983. Checklist of North Americon birds. 6th edition. American Ornithologists' Uninn, New York.

17. Amerson, A.B. 1969. Ornithology of the Marshall and Gilbert Islands. Atoll Research Bulletin 127

18. Amos, E.J.R. 1991. A guide to the birds of Bermida Eric J.R. Amns, Warwick, Bermuda.

19. Amos, S.H. 1985/86. Efforts to save the endangered Black-hooded Red Siskin. AFA Watchbird 12(6): 19.
20. Andrade, M.A., Freitas, M.V. and Mattos, G.T 1987. Dados preliminares sobre a distribuicao e conservacao de Xiphocolaptes franciscanus Snethlage: Dendrocolaptidae endemico do Brasil. III Congresa de Omitologia Neotropical: Resumenes. Unpublished.

21. Andre, R. 1985. Some aspects of the biological reproduction of the Little Bustard and a contribution to an estimate of its population in France 1978/79. Bustard Studies 2: 153-160.

22. Andres, B., Haag, W. and Andres, S. 1991. Recent records of Swainson's Hawk in Trinidad and Tobago. J. Trinidad and Tobago Field Nat. Club 1991: 45.

23. Andrew, D. 1990. Austrelia - the lucky country? World Birdwatch 12(4): 8-9.

24. Andrew, P. 1985. An annotated checklist of the birds of Cihodas-Gunung Gede Nature Reserve. Kukila 2: $10-28$

25. Andrew, P. 1986. Notes on some hirds of Timor. Kukila 2: 92-95.

26. Andrew, P. 1992. The hirds of Indonesia: a checklist (Peters' sequence). Indonesian Ornitholngical Society, Jakarta.

27. Andrew, P. and Milton, G.R. 1988. A note on the Javan Scops-owl Ous angelinae Finsch. Kukila 3: 79.

28. Andrusaitis, G. (ed.). 1985. [Red data hook of the Latvian SSR: rare and endangered species of animals and plants]. Zinatne, Riga. (In Latvian and Russian.)

29. Angehr, G.R. 1985. Stitchbird. John McIndoe and New Zealand Wildlife Service, Dunedin. New Zealand.

30. Anon. 1972. Birds in Jersey, a systematic list. Société Jersiaise

31. Anon. 1983. European news. British Birds 76: $566-571$.

32. Anon. 1983. Yellow-shouldered Blackhird recovery plon. U.S. Fish and Wildlife Service, Atlanta, Georgia.

33. Anon. 1985. Pair of Flightless Grebes moved to safer lake in Peru. World Birdwatch 7(1): 4

34. Anon. 1985. No Kakapo chicks survive hreeding season in New Zealand. World Birdwatch 7(2): 1-2.

35. Anon. 1985. Conservationists hattle for Caroni Swamp and Scarlet Ibis. World Birdwatch 7(3): 3

36. Anon. 1985. European news. British Birds 78: 638-645

37. Anon. 1986. Ivary-billed Woodpecker found in Cuba. World Birdwatch 8(2): 1-2

38. Anon. 1986. Weaver alive and well. World Birdwatch $8(3): 5$.

39. Anon. 1986. Recent reports. Oriental Bird Club Bull. 3: $33-36.40$

4C. Anon. 1987. Weaver again. World Birlwatch 9(1): 5

41. Anon. 1987. Rare woodpecker sighted. World Birdwatch 9(2): 4 
42. Anon. 1987. More Plains-wanderers located. Worll Birdwatch $9(2): 5$.

43. Anon. 1987. Sao Tomé and Principe birds increase. World Birdwatch 9(4): 1-2

44. Anon. 1987. Mandarin Duck population. World Birdwaich 9(4): 5 .

45. Anon. 1987. World birdwatch exclusive: Black-hooded Antwren re-discovered in Brazil. World Birdwatch 9(4): 4 .

46. Anon. 1987. Freira's first breeding success in years. World Birdwatch 9(4): 4 .

47. Anon. 1988. Parakeets breed in Mauritius. World Birdwatch 10(1): 3

48. Anon. 1988. Gorgeted Wood-Quail for protection World Birdwaich 10(2): 4.

49. Anon. 1988. Socorro Mockingbird still just surviving World Birdwatch 10(2): 9.

50. Anon. 1988. Bachman's Warbler still a mystery. World Birdwatch 10(3/4): 9

51. Anon. 1988. Montserrat: orioles and "mountain chickens'. World Birdwatch 10(3/4): 12

52. Anon. 1988. European news. Brilish Birds $81: 330-340$.

53. Anon. 1988. The official list of the birds of the Sultanate of Oman. 2nd edition.

54. Anon. 1989. Rediscoveries. World Birdwatch 11(1): 4.

55. Anon. 1989. Tai Forest news. World Birdwatch $11(2): 3$.

56. Anon. 1989. Taiko: latest. World Birdwatch 11(2): 3.

57. Anon. 1989. Storm's Stork: Sumatran discovery. World Birdwatch 11(2): 3.

58. Anon. 1989. Threatened birds on Asian islands. World Birdwatch 11(2): 4

59. Anon. 1989. Rediscoveries. World Birdwatch 11(2): 4 .

60. Anon. 1989. Paria Peninsula, Venezuela. World Birdwatch 11(3): 3 .

61. Anon. 1989. Tragopan triumph. Work Birdwatch 11(3): 3 .

62. Anon. 1989. Rarities in Central America. World Birdwarch $\mathrm{N}(3): 3$

63. Anon. 1989. Brazil: the good news. World Birdwatch $11(3): 4$.

64. Anon. 1989. North America's rarest: latest. World Birdwaich 11(3): 4 .

65. Anon. 1989. Kupe Bush-Shrike rediscovered. World Birdwaich 1](4): 3 .

66. Anon. 1989. Ethiopian endemics update. World Birdwatch 11(4): 4

67. Anon. 1989. Atlantic islands update. World Birdwatch 11(4): 4

68. Anon. 1989. Tuamotus yield new data. World Birdwatch 11(4): 4

69. Anon. 1990. Rediscoveries. World Birdwatch $12(1-2): 5$
70. Anon. 1991. Solomons survey. World Birdwatch 13(1): 3 .

71. Anon. 1991. The Cocha Antshrike - rediscovered and real! World Birdwatch 13(1): 4

72. Anon. 1991. Recent news from Bali. World Birdwatch 13(2): 3 .

73. Anon. 1991. Woodstar turn. World Birdwatch 13(2): 3 .

74. Anon. 1991. Santo Starling survives! World Birdwatch 13(4): 5 .

75. Anon. 1992. Morocco last hope. World Birdwatch 14(3): 4 .

76. Anon. 1992. New species of owl. World Birdwatch $14(3): 4$

77. Anon. 1992. Bali Starling increase. World Birdwatch 14(3): 5 .

78. Anon. 1992. Bolivian find. World Birdwatch 14(4): 3 .

79. Anon. 1992. Loriket transfer. World Birdwatch 14(4): 4 .

80. Anon. 1992. Bush-shrike finds. World Birdwaich 14(4): 5 .

81. Appert, O. 1985. Zur Biologie der Mesitomithiformes (Nakas oder "Stelzenrallen") Madagaskars und erste fotografische Dokumente von Vertretem der Ordnung Om. Beoh. 82:31-54

82. Araya, L. 1982. Lista patron de los aves Chilenas. Publicaciones oc asionales No.1, Instituto de Oceanologica. Universidad de Valparaiso, Vina del Mar, Chile.

83. Araya Modinger, B. 1983. A preliminary report on the staus and distribution of the Humholdi Penguin in Chile. Pp. 125-140 In: Proceedings of the Jean Delacour/IFCB Symposium on breeding birds in captivity. North Hollywood, California: International Foundation for the Conservation of Birds.

84. Arhallo, E. 1990. Nuevos registros para avifauna uruguaya. Homero 13: 179-187.

85. Archer, G. and Godman. E.M. 1937-1961. The birds of British Somaliland and the Gulf of Aden. Volumes 1 and 2. Gurney and Jackson, London; Volumes 3 and 4. Oliver and Boyd, Edinhurgh.

86. Archihald, G. 1988. Whooping Crane update, ICF Bugle 14(1): 4-6.

87. Archihald, G. and Pasquier, R.F. 1987. Proceedings of the 1983 Intemational Crane Workshop. Baraboo, Wisconsln: Intermational Crane Foundation.

88. Argeloo, M. 1992. The Maleo-more than a symbol. World Birdwatch 14(1): 8-9.

89. Arndt, T. 1986. Sudamerikanische Sirtiche. 5. Bomlitz, Germany: Horst Müller-Verlag.

90. Amdt, T. and Roth, P. 1986. Der Rotbauchsittich Pyrrhura hodogaster im Vergleich mit den verschiedenen Unterarten des Blausteisssittichs Pyrhhura perlata: Vorschlag für nomenklatorische und systematische Anderungen. Ver. Om. Ges. Bayem 24: 313-317.

91. Aronson, L. and van den Berg. A.B. 1979. Hume's Tawny Owl Strix bustleri in Israel. Dutch Birding 1: $18-19$. 
92. Ash, J. 1973. Six species of hirds new to Ethiopia Bull. Brit. Om. Club 93:3-6.

93. Ash, J.S. 1977. Four species of birds new to Ethiopia and other notes. Bull. Brit. Om. Club 97: 4-9.

94. Ash, J.S. 1981. Ayres' Hawk Eagle Hieraaetus dubius in Ethiopia and Somalia. Scopus 5: 54-56.

95. Ash, J.S. 1984. Bird observations on Bali. Bull. Brit. Om. Club 104: 24-35.

96. Ash, J.S. 1985. Midwinter observations from Djibouti. Scopus 9: 43-49.

97. Ash, J.S. 1986. A Ploceus sp, nov. from Uganda. Ibis 128: $330-336$

98. Ash, J.S. 1987. Surveys of Picalhartes oreas, Malimbus ibadanensis and other species in Nigeria. International Council for Bird Preservation and Nigerian Conservation Foundation. Unpublished.

99. Ash, J.S. 1987. Ploceus victoriae. Ibis 129: 406-407

100. Ash, J.S. 1989. An atlas of the past and present distribution of hustards in Ethiopia and Somalia Bustard Studies 4

101. Ash. J.S. and Miskell. J.E. 1983. Birds of Somalia their habitat. status and distrihution. Scopus special suppl. 1: 1-97.

102. Ash, J.S. and Olson, S.L. 1985. A second specimen of Mirafra (Heteromirafra) sidamoensis Erard. Bull. Bril. Om. Club 105: 141-143.

103. Baker, N. 1989. The distribution and legal status of bustards in Tanzania. Bustard Studies 4: 35-40.

104. Balchin, C.S. 1988. Recent observations of birds from the Ivory Coast. Malimhus 10(2): 201-206.

105. Balen, S. van. 1984. Sight records of the Black Baza Aviceda leuphotes on Java. Ardea 72: 234.

106. Balen, S. van and Compost, A.R. 1989. Overlooked evidence of the Short-lned Eagle Circoetus gallicis on Java. Kukila 4: 44-46.

107. Baltosser, W.H. 1989. Costa's Hummingbird: its distrihution and status. Westem Birds 20(2): 1-62

108. Baltzer, M.C.A. 1990. A report on the wetland avifauna of south Sulawesi. Kukila 5: 27-55

109. Banks, R.C. 1990. Taxonomic status of the coquette hummingbird of Guerrero, Mexico. Auk 107: 191-192.

110. Bannerman, D.A. 1921. A complete list of the hirds known from Sierra Leone. Ihis 11(3): 289-302.

111. Bannerman. D.A. 1963. Birds of the Allantic Islands, 1. A history of the birds of the Canary lslands and of the Salvages. Oliver and Boyd. Edinhurgh.

112. Bannerman, D.A. and Bannerman, W.M. 1965. Birds of the Atlantic Islands, 2. A histony of the birds of Madeira, the Deserias and the Parto Santo Islands. Oliver and Boyd, Edinburgh.

113. Bannerman, D.A. and Bannerman, W.M. 1966. Birds of the Allantic Islonds. 2. A history of thc birds of the Azores. Oliver and Boyd, Edinburgh.

114. Bannerman, D.A. and Bannerman, W.M. 1968. Birds of the Atlantic Islonds, 2. History of the hirls of the Cape Verde Islands. Oliver and Boyd. Edinhurgh.

115. Bannikov, A.S. (ed.). 1978. Red Data book of USSR. Lesnaya Promyshlennost, Moscow.
116. Baptista. L.F. 1991. Socorro Dove project update from International Dove Society Newsletter, Sept. 1991. ASA Avicult. Bull. Novemher: 14-16.

117. Barber. R.D. 1985. A recent record of the Bachman's Warbler in Florida. Florida Field Nat. 13: 64-66.

118. Barlow, K., Beltrán, W., Downing, C., Kingston, T., Payne, T., Salaman, P. and Serrano, V.H. 1992. The survey and conservation of fauna in south-west Narino, Colombia. Unpublished draft report.

119. Bamett, A. and Gretton, A. 1987. Rio Mazan - a people's forest. World Birdwaich 9(1): 3-4.

120. Barthel. P.H. 1988. [The Asiatic Dowitcher (Limnodromus semipalmatus).] Limicola 2: 152-155.

121. Bates, J.M., Garrin, M.C., Schmit, D.C. and Schmilt. C.G. 1989. Notes on bird distributions in northeastern dept, Santa Cruz, Bolivia with 15 species new to Bolivia. Bull. Brit. Om. Club 109(4): 236-244

122. Bates, J.M., Parker, T.A., Capparella, A.P. and Davis, T.J. 1992. Observations on the campo, cerrado and forest avifaunas of eastern Dpio. Santa Cruz, Bolivia, including 21 species new to the country. Bull. Bril. Om. Club 112: 86-98.

123. Bauer, S. and Thieleke, G. 1982. IEndangered breeding hird species in the Federal Repuhlic of Germany and in West Berlin - population trends, reasons and protective measures.] Vogelwarte 31: 183-391

124. Beaman. M. (ed.). 1975. Turkey hird repon 1970 1973. Omithological Snciety of Turkey, Sandy

125. Beaman. M. and Ponter, R.F. 1985. Status of birds of prey in Turkey. Bull. World Working Group on Birds of Prey 2: 52-56.

126. Beauhrun, P.-C. 1983. |Audouin's Gull Larts audowinii Payr. on Mornccan coasts.] Oiseau Rev. Fr. Om. 53: 209-226.

127. Beavers, R.A., Delaney, D.J.. Leahy, C.W. and Oatman. G.F. 1991. New and noteworthy hird records from Petén. Guatemala. including Tikal National Park. Bull. Brit. Om. Club 111: 77-90.

128. Becking, J.H. 1976. Feeding range of Ahbott's Booby Sula ahhotti at the conast of Java. lhis 118: 589-590.

129. Beehler, B.M. 1978. Notes on the mountain hirds of New Ireland. Emu 78: 65-70.

130. Bechler, B.M. 1985. Conservation of New Guinea rainforest hirds. Pp. 233-247 In: Diamnnd A.W. and Lovejoy. T.E. (eds.) Conservation of iropical forest birds. (Techn. Puhl. 4). International Council for Bird Preservation, Cambridge, U.K.

131. Eechler. B.M., Pratt, T.K. and Zimmerman, D.A. 1986. Birds of New Guinea. Princeton University Press, Princeton, New Jersey.

132. Bege. L.A.R. and Pauli. B.T. 1990. Twn new birds to the Brazilian avifauna. Bull. Brit. Om. Club 110: $93-94$

133. Beggs, J. and Wilson. P. 1988. Kala: a threatened species? Forest and Bird 250: 18-19.

134. Beichle, U. 1982. Untersuchungenzur Biologie und Systematik der Zahntauhe Didunculus strigirostris (Jardine 184.5). Kiel: Christian-Alhrechts Universitat doctoral dissertation. 
135. Beichle, U. 1987. Lebensraum, Bestand und Nahrungsaufnahme der Zahntaube, Didunculus strigirostris, J. Om. 128: 75-89.

136. Belarbi, A. 1989. A note on the Little Bustard in Algeria. Bustard Sudies 4.

137. Bell, B.D. 1986. The conservation staus of New Zealand wildlife. Wellington, New Zealand: Wildlife Service Occ. Publ. no. 12.

138. Bell, J. 1970. The white-quilled black bustard. Animal Kingdom 73: 25-28.

139. Belton, W. 1984. Birds of Rio Grande do Sul, Brazil. part 1: Rheidae through Furnariidac. Bull. Amer. Mus Nat. Hist. 178(4).

140. Belton, W. 1985. Birds of Rio Grande do Sul, Brazil. part 2: Formicariidae through Corvidae. Bull. Amer. Mus. Nat. Hist. $180(1)$.

141. Beltran, J. 1987. El Mirlo de Agua (Cinclus schulzi). Nuestras Aves 5(13): 23-25

142. Bennun, L.A. 1987. Ringing and recapture of Spotted Ground Thrushes Turdus fischeri fischeri at Gede, Kenya coast: indications of site fidelity and population size stability. Scopus 11: 1-5

143. Benson, C.W. 1960. The birds of the Comoro Islands: results of the British Ornithologists' Union Centenary Expedition 1958. Ibis 103h: 5-106.

144. Benson, C.W. and Benson, F.M. 1977. The birds of Malawi.

145. Benson, R.H. and Benson, K.L.P. 1990 . Estimated size of Black-capped Vireo population in northern Coahuila, Mexico. Condor 92: 777-779.

146. Benson, R.H. and Benson, K.L.P. 1991. Reply to Scott and Garton. Condor $93: 470-472$.

147. Benson, C.W., Brooke, R.K., Dowsett, R.J. and Irwin, M.P.S. 1971. The birds of Zambia. Collins, London.

148. Benussi, E. 1986. Observations on the presence of Pygmy Cormorant (Phalacrocorax pygmaeus) in Italy. Ric. Biol. Selvaggina Suppl. 10: 29-31.

149. Bereszynski, A. and Kaczmarkowski, M. 1983. Bustard (Otis tarda L.) in Poland. Pp. $118-120$ ln: Goriup, P.D. and Vardhan, H. (eds.) Bustards in decline. Tourism and Wildlife Society of India, Jaipur.

150. Berg, A.B., van den and Bosman, C.A.W. 1986. Suppleme ntary notes on some birds of Lore Lindu Reserve, central Sulawesi. Forktail 1: 7-13

151. Bergier, P. and Bergier, F. 1990. A birdwatcher's guide ta Morocco. Prior, Huntingdon, U.K.

152. Berlepsch, H.G. von 1908. On the birds of Cayenne. Novitates Znologicae 15: 103-164, 261-324.

153. Berlioz, J. 1962. Etude d'une collection d'oiseaux de Guyane francaise. Bul. Mis. Nat. Hist. Nat. 34: 131-143

154. Bertonatti, C. and Gonzalez, F. 1992. Lista de vertebrados argentinos en peligro de extincion. Fundacion Vida Silvestre Argentina, Boletin Tecnico No. 8.

155. Best, H.A. 1984. The fonds of Kakapo on Stewart Island as determined from their feeding sign. New Zealand J. Ecol. 7: 71-83.
156. Best, H. and Powlesland, R. 1985. Kakapo. John McIndoe and New Zealand Wildlife Service. Dunedin, New Zealand.

157. Bhushan, B. 1986. Rediscovery of the Jerdon's or Douhle-handed Courser Cursarius bitorquatus (Blyth). J. Bombay Nat. Hist. Soc. 83: 1-14.

158. Bhushan, B. 1987. Photogaphic record of the Jerdon's or Douhle-banded Courser Cursorius bitorquatus. J. Bambay Nat. Hist. Sac. 83 (Suppl.): 159-162.

159. Bhushan, B. 1992. Red Data hird: Jerdon's Courser. Warld Birdwatch 14(4): 12.

160. Bibby, C.J. and Hill, D.A. 1987. Status of the Fuerteventura Stonechat Saxicola dacotiae. Ibis 129: 491-498.

161. Biber, J.-P. 1992. Red Data hird: Lesser Kestrel. World Birdwatch 14(2): 14.

162. Bierregaard, R.O. 1984. Observations of the nesting biology of the Guiana Crested Eagle (Marphnus guianensis). Wilson Bull. 96: 1-5

163. Bishop, K.D. 1983. Some notes on non-passerine birds of west New Britain. Emu 83: 235-241.

164. Bissor, A.J. 1983. Checklist of the hirds of the Channel Islands. Stapels Press.

165. Blake, E.R. 1953. Birds of Mexico: a guide for field identification. Chicago University Press, Chicago.

166. Blake, E.R. 1977. Manual of Neotropical hirds. University of Chicago Press, Chicago.

167. Blakers, M., Davies, S.J.J.F. and Reilly, P.N. 1984 The allas of Australian birds. Royal Australian Ornithologists' Union and Melbourne University Press, Melbourne.

168. Blancou, L. 1948. Contrihution à l'étude des oiseaux de l'Oubangui-Chari oriental. L'Oisean et la Revue Français d'Ormithologie 18: 33-77.

169. Blasius, W. 1884. Über die neusten Ergehnisse von Herm. F.J. Grahowsky's Ornithologisehen Forschungen in Sïd-Ost Borneo, J. Om. 32: 210-227.

170. Bleiweiss, R. and Olalia, P.M. 1983. Notes on the ecology of the Black-breasted Puffleg on Volcan Pinchincha, Ecuador. Wilsan Bulletin 94: 656-661.

171. Bloch, D. and Sorensen, S. 1984. Check list of Faroes birds. Torshaven.

172. Blockstein. D.E. 1983. Population and taxonomic status of the endangered Grenada Dove (Leptotila wellsi). Ant. Zool. 28(4): 135A. Abstract only

173. Blockstein, D.E. 1987. Endangered hirds of Grenada, West Indies. Progress report. Unpublished.

174. Blockstein, D.E. 1991. Population declines of the endangered endemic birds on Grenada, West Indies. Bird Conserv. Intematn. 1:83-91.

175. Blondel, J. and Blondel, C. 1964. Remarques sur l'hivernage des limicoles et autres oiseaux aquatiques au Maroc. Alauda 32: 250-279.

176. Blot, J. 1985. Contribution de la connaissance de la biologie et de l'ecologie de Francolinus ochropectus Dorst et Jouanin. Alatudo 53: 244-256.

177. Bocke, J.D. 1978. A food source of the Marvellous Spatuletail. Ibis 120: 551 . 
178. Bond, J. 1956. Check-list of birds of the West Indies. The Academy of Natural Sciences of Philadelphia.

179. Bond, J. 1985. Birds of the West Indies. 5th edition. Collins, London.

180. Bond, J. and Meyer de Schauensee, R. 1942-1943. The birds of Bolivia. Proc. Acad, Nat. Sci. Philadelphia 94: 307-391, 95: 167-221.

181. Bonde, K. 1981. An annatated checklist to the birds of Lesotho (preliminary edition). Privately published, Maseru.

182. Boonsong Lekagul and Round, P.D. 1991. A guide to the birds of Thailand. Saha Kam Bhaet Co. Lid.. Bangkok.

183. Booth, D.T. 1987. Home range and hatching success of Malleefowl, Leipaa acellara Gould (Megapodiidae), in Murray Mallee near Renmark, S.A. Aust. Wildl. Res. 14: 95-104.

184. Borello, W. and Borello, R. 1987. The Cape Vulture colonies in Botswana in 1985 and 1986. Vuluure News 17: 37-42.

185. Borodin, A.M. (ed.). 1984. Red data book of the USSR: rare and endangered species of onimals and planks. Vol. I: animals [tn Russian]. 2nd edition. Promyshiennost, Moscow.

186. Bosch, K. 1991. Welche Gelbkopfamazone hahen Sie? Gefied. Welt 115: 342-344.

187. Boshoff, A.F. and Robertson, A.S. 1985. A conservation plan for the Cape Vulture colony at Potberg, de Hoop Nature Reserve, southwestern Cape Province. Bontebok 4: 25-31.

188. Boshoff, A.F. and Vernon, C.I. 1987. The Cape Vulture colonies at Karnmelkspruir 1984-1986 and Balloch 1978-1986, north-eastern Cape Province. Vulture News 17: 31-36.

189. Boume, W.R.P. 1971. The birds of the Chagos group, Indian Ocean. Atoll Res. Bull. 149: 175-207.

190. Bourne, W.R.P. 1976. On Subfossil bones of Abbotl's Booby Sula ahhotii from the Mascarene Islands, with a note on the proportions and distribution of the Sulidae. lbis 118: 119-123.

191. Bourne, W.R.P. and Davis, A.C.F. 1983. Henderson Island, central South Pacific, and its birds. Notomis 30: 233-243.

192. Boussekey, M., Saint-Pie, J. and Morvan, O. 1992. Beobachtungen an einer Population des Rotohraras (Ara rubrogenys) im Rio-Caine-Tal, Zentral Bolivien. Papageien 5: 54-57, 95-99.

193. Bowden, C.G.R. 1987. The Yemen Thrush in North Yemen. Sandgrouse 9: 87-89.

194. Bradley, P.E. 1985. A guide to the birds of the Cayman Islands. P.E. Bradley, George Town. Grand Cayman.

195. Brandis, C. 1989. Sighting of a Northem Shoveler at Lake Cowal, central New South Wales. Aust. Birds 23(2): 37-38.

196. Brandt, A. and Machado, R.B. 1990. Área de alimentaçāo e comportamento alimentar de Anodorhynchus leari. Ararajuba 1: 57-63.
197. Braun. M.J., Braun, D.D. and Terrill, S.B. 1986 Winter records of the Golden-cheeked Wartler (Dendroica chrysoparia) from Mexico. Amer. Birds 40(3): 564-566.

198. Brazil, M. 1984. Observations on the behaviour and vocalizations of the Okinawa Rail Rallus okinawae.J. Jap. College of Dairying (Ebetsu, Hokkaido) 10: 437-449.

199. Brazil, M. 1984. One on tape is worth three in the bush. $B B C$ Wildlife 2: 574-575.

200. Brazil, M. 1985. The endemic hirds of the Nansei Shoto. Pp. 10-35 In: World Wildlife Fund Japan, Conservation of the Nansei Shoto, 2. Wnrld Wildlife Fund, Tokyo, Japan.

201. Brazil, M. 1985. Owl of the selling sun. BBC Wildife 3: 110-115.

202. Brazil, M. 1985. [Notes on the Okinawa Rail Rallus akinawae: observations at night and at dawn.] Tori 33: 125-126. (In Japanese.)

203. Brazil, M. 1986. Sea ea 'le sunrise. BBC Wildlife 4: 588-592.

204. Brazil, M. 1987. Where have all the Baikal Teals gone? Oriental Bird Cluh Bull. 5: 29.

205. Brazil, M. 1991. The birds of Japan. Christopher Helm, London.

206. Brazil. M. and Yamamoto, S. 1989. The status and distrihution of owls in Japan. Pp. 389-401 in Meyburg, B.-U. and Chancellor, R.D. (eds.) Raptors in the modern world. Proceedings of the III World Conference on Birds of Prey and Owls, Eilat, Israel, 22-27 March 1987. World Working Group on Birds of Prey and Owls, Berlin.

207. Bregulla, H. 1987. Zur Biologie des Kagu, Rhynochetos jubatus. Zool. Garten N.F. 57: 349-365

208. Bregulla, H.L. 1992. Birds of Vanuotu. Anthony Nelsnn. Shropshire, UK.

209. Breuil, M. 1989. Les oiseaux d'Islonde. Le Chevalier Paris.

210. Brichelti, P. and Massa, B. 1984. Check-list degli uccelli italiani. Riv. ital. Omithol. 54: 3-37.

211. British Ornithologists' Union 1971. The status of birds in Britain and lreland. British Ornithnlogists' Union, London.

212. Britton, P.L. (ed.). 1980. Birds of East Africa. East Africa Natural History Society, Nairnbi.

213. Brock. M.K. 1991. Genetic assessment of the captive breeding programs for the Puerto Rican Parrot Amazora vituata, and other Carihhean parrots. Ph.D thesis, Department of Biology, Queen's University, Kingsion, Ontario.

214. Broni, S.C. 1985. Social and spatial foraging patterns of the Jackass Penguin Spheniscius demersus. S. Afr. J. Zool. 20: 241-245.

215. Brooke, R.K. 1981. The feral pigeon: a 'new' bird for the South Afric an list. Bokmakierie 33: 37-40.

216. Brooke, R.K. 1984. South African Red Data Book: birds. South African National Scientific Programmes Report No. 97. 
217. Brooke, M. de L. 1985. The annual cycle of the Toc toc Foudia sechellanum on Cousin Island, Seychelles. Ibis 127: 7-15

218. Brooke, M. de L. 1987. The birds of the Juan Femandez Islands, Chile. (Study Report 16). International Council for Bird Preservation, Cambridge, U.K.

219. Brooke, M. de L. 1988. Distribution and numbers of the Masafuera Rayadito Aphrasura masafuerae on Isla Alejandro Selkirk, Juan Fernandez archipelago, Chile. Bull. Brit. Om. Club 108: 4-9.

220. Brooks, D.J., Evans, M.1., Martins, R.P. and Porter. R.F. 1987. The stalus of birds in North Yemen and the records of the OSME Expedition in autumn 1985 Sandgrouse 9: 4-66.

221. Brosset, A. and Erard, C. 1977. New faunistic records from Gabon. Bull. Brit. Om. Club 97: 125-132.

222. Brosset, A. and Erard, C. 1986. Les oiseaux des regions forestières du nord-est du Gahon, 1. Société Nationale de Protection de la Nature, Paris.

223. Brown, C.J. 1985. The status and conservation of the Cape Vulture in SWA/Namihia. Vulture News 14: 4-15.

224. Brown, C.J. and Hines, C.J.H. 1987. Western Banded Snake Eagles in Namihia. Gabar 2(2): 40-42.

225. Brown, L.H. 1976. Observations on Pel's Fishing Owl Scotopelia peli. Bull. Brit. Om. Club 96: 49-53.

226. Brown, L.H. and Amadon. D. 1968. Eagles, hawks and falcons of the world. 2 volumes. Country Life Books, Feltham, Middlesex.

227. Brown, L.H., Urban, E.K. and Newman, K. 1982. The birds of Africa 1. Academic Press, London and New York.

228. Brown, P.B. and Wilson, R.1. 1984. Orange-bellied Parrot recovery plan. National Parks and Wildlife Service. Tasmania.

229. Brown, P.B., Wilson. R., Loyn, R., Murray, N. and Lane, B. 1985. The Orange-hellied Parrot - an RAOU conservation statement. Royal Australasian Ornithologists' Union, Melhourne.

230. Browne, P.W.P. 1981. New hird species in Mauritania. Malimbus 3(2): 63-72.

231. Bruce, M.D. 1982. Occurrence of the Lesser Adjutant Stork Leptoptilos javanicus on Bali. Indonesia. Bull. Brit. Om. Club 102: 39-40.

232. Brudenell-Bruce, P.G.C. 1975. The birds of New Providence and the Bahamia Islands. Collins, London.

233. Brüggemann, J. 1877. Über eine Vögelsammlung aus Süd-Ost Borneo. Abhandl. Notum. Vereins Bremen 5: 453-466.

234. Bruner, P.L. 1972. Field guide to the birds of French Polynesia. Pacific Scientific Information Center, Bernice P. Bishop Museum, Honolulu, Hawaii.

235. Buckley, P.A. 1988. The world's first known juvenile Cox's Sandpiper. British Birds 81: 253-257.

236. Buden. D.W. 1987. The birds of the southem Bahamas. British Ornithologists' Union Check-list Na. 8. BOU, London.
237. Buden, D.W. 1990. The birds of Rum Cay, Bahama Islands. Wilson Bull. 102: 451-468.

238. Bugalho, F.F. 1987. Great Bustard in Portugal. Pp 65-76 ln: Farago, S. (ed.) The Great Bustard Otis tarda $L$.). Proceedings of the symposium in Budapest on June 2nd 1987. International Council for Game and Wildlife Conservation, Budapest.

239. Buhl, K. 1983. Trouble in paradise...the Hawaiian Crow. AFA Watchbird 10(3): 26-30.

240. Bullock, 1. 1990. Birds of the Republic of Seychelles. Seychelles Ministry of Education and the International Council for Bird Presevation, Cambridge, U.K.

241. Bundy, G. 1976. The birds of Libya. British Ornithologists' Union, London.

242. Bundy, G., Connor, R.J. and Harrison, C.J.O. 1989. Birds of the eastem province of Saudi Arahia. H.F. \& G. Witherby, London.

243. Bundy, G. and Warr, E. 1980. A checklist of the birds of the Arahian Gulf States. Sandgrouse 1: 4-49.

244. Bunn, D.S., Warhurton, A.B. and Wilson, R.D. 1982. The Barn Owl. Poyser, Calton, U.K

245. Burbridge, A.A. and Jenkins, R.W.G. (eds.) 1984 Endangered vertebrates of Australio and its island territories. Australian National Parks and Wildlife Service, Canherra.

246. Burkt, W. 1992. Report on partial census of Ravine la Chaloupe for White-breasted Thrasher (Ramphocinclus brachyunus) on March 8, 1992. Unpuhlished.

247. Burton, J.A. (ed.). 1973. Owis of the world, their evolution, stnucture and ecology. Peter Lowe, London.

248. Butler, L.Y. 1979. The hirds of Ecuador and the Galopagos Archipelago. The Ramphastos Agency, Portsmouth, N.H

249. Butler, P.J. 1981. The St. Lucia Amazon (Amazana versicolor): its changing status and conservation. Pp. 171-180 In: Pasquier, R.F. (ed.) Conservation of New World parrots. Smithsonian Institution Press for the International Council for Bird Preservation. Washington D.C.

250. Butler, P.J. 1990. Some ohservations on polymorphism in the St Vincent Parrot Amazona guildingii. Dodo 26 [1989]: 98-107.

251. Butler, P.J. 1991. Saint Lucia Parrot Amazona versicolor: consolidating a conservation campaign. RARE Center for Tropical Conservation, Philadelphia.

252. Butler, P.J. 1992. Parrots, pressures, people, and pride. Pr. 25-46 ln: Beissinger, S.R. and Snyder, N.F.R. (eds.) New World parrats in crisis: solutions from conservotion biology. Smithsonian Institution Press, Washington, D.C.

253. Cade, T.J. 1982. The falcons of the world. Collins, London.

254. Caleda, M., Lanante, R. and Viloria, E. 1986. Preliminary studies of the Palawan Peacock Pheasant Polyplectron emphanum. In: Ridley, M. (ed.) Pheasants in Asia 1986. World Pheasant Association. Basildon, U.K. 
255. Camargo, H.F. de A. and Camargo, E.A. de. 1964 Occorencia de Jodopleura p. pipra no Estado de Sao Paulo, Brasil, e algumas notas sobre Jadopleura isabellae. Pap. Dep. Zool. Sao Paulo 16: 45-55

256. Cameron, A.W. 1976. Birds of St. Pierre et Miquelon Archipelago. Naturaliste can. 94: 389-420.

257. Cardiff, S.W. and Remsen, J.V. Jr. 1981. Three bird species new to Bolivia. Bull. Bril. Om. Club 101: 304-305.

258. Carey, C.C. 1961. A list of the birds of the Channel islands. Trans Soc. Guemseaise.

259. Carroll, R.W. 1988. Birds of the Central African Republic. Molimbus 10(2): 177-200.

260. Carswell, M. 1984 Checklist of the birds of Uganda. Compiled from Brition. P.L. (ed.) and Backhurst, G.C. Birds of East Africa: their habitat, status and distrbution. East A frican Natural History Society, Nairobi.

261. Casanova, F.D. 1989. The Houbara Bustard in the Canary Islands: towards a recovery plan. Bustard Studies 4.

262. Castelino, M. 1990. Un ave nueva para la República Argentina y segunda mención para otra. Nótulas Faunisticas 21.

263. Calaneaunu, I. 1978. Aves. Fanra Respublicii Socialiste Romania. Vol. XV. Fasc. 1. Editura Academiei RSR, Bucarest.

264. Cave, F.O. and Macdonald, J.D. 1955 The birds of Srdan. Edinghurgh, Oliver and Boyd, Scotland.

265. Chalmers, M.L. 1986. Annolated checklist of the birds of Hong Kong. Fourth edition. The Hong Kong Bird Watching Society, Hong Kong.

266. Chang, W.F. and Severinghaus, S.R. 1979. Notes on the Yellow Tit Parts holsti of Taiwan with discovery of its nest. Bull. Brit. Om. Club 99: 54-56.

267. Chasen, F.N. 1935. A handlist of Malaysian birds. Bull. Raffles Mus. 11:1-389.

268. Chasen, F.N. and Hoogerwerf, A. 1941. Birds of the Netherlands Indian Mt. Leuser expedition 1937 to North Sumatra. Treubia 18 (Suppl.): 1-125.

269. Chebez, J.C. 1986. El Guacamayo Violaceo (Anadorhynchus glnucus). Nuestras Aves 4(9): 17-20.

270. Chebez, J.C. 1986. Nuestras aves amenazadas. 13. Carpintero caracanela (Dryocopus galeatus). Nuestras Aves 4(10): 16-18.

271. Cheke, A.S. 1987. Observations on the surviving endemic birds of Rodrigues. Pp. 364-402 In: Diamond, A.W. (ed.) Studies of Mascarene island birds. Camhridge University Press, Cambridge, U.K.

272. Cheke, A.S. 1987. The ecology of the smaller landbirds of Mauritius. Pp. 151-207 In: Diamond. A.W. (ed.) Studies of Mascarenc island birds. Cambridge University Press, Cambridge, U.K.

273. Chele, A.S. 1987. The ecology of the surviving native land-birds of Réunion. Pp. 301-358 In: Diamond, A.W. (ed.) Studies of Mascarene island birds. Cambridge University Press, Cambridge, U.K.
274. Cheke, A.S. 1987. The legacy of the Dodoconservation in Mauritius. Oryx 21 : 29-36.

275. Cheke, R.A. 1982. More bird records from the Republic of Togo. Malimbiss 4: 55-56.

276. Cheke, R.A. 1986. The supposed occurrence of the White-necked Picathartes Picathartes gymnocephalus in Togo. Bull. Brit. Orn. Club 106: 152.

277. Cheke, R.A. and Walsh, J.F. 1980. Bird records from the Republic of Togo. Malimbus 2(2): 112-120.

278. Cheke, R.A. and Walsh, J.F. 1984. Further bird records from the Republic of Togo. Malimbus 6(1/2): 15-22.

279. Cheke, R.A., Walsh, J.F. and Fishpool, D.C. 1985. Bird records from the Republic of Niger. Malimbus 7: 73-90.

280. Cheke, R.A., Walsh, J.F. and Sowah. S.A. 1986. Records of hirds seen in the Repuhlic of Togn during 1984-1986. Malimhus 8(2): 51-72.

281. Cheng Tso-hsin 1987. A synopsis of the avifauna of China. Science Press, Beijing-

282. Cheshire, N.G. 1990. Notes on seabirds - reports received in 1989. Sea Swallow 39: 18-37.

283. Child, P. 1986. Black-fronted Tern hreeding at high allitude. Notomis 33: 193-194.

284. Christensen, S. and Sorensen, U.G. 1989. A review of the migration and wintering of Aquila pomarina and Aquiln nipalensis orienralis. $\mathrm{Pp}$. 139-150 In: Meyburg, B.-U. and Chancellor, R.D. (eds.) Raptors in the mndern world. Proceedings of the III World Conference on Birds of Prey and Owls, Eilat. Israel, 22-27 March 1987. World Working Group on Birds of Prey and Owls, Berlin.

285. Clancey, P.A. (ed.). 1980. Checklist of southem African birds. Southern Afric an Omithnlogical Society, Pretoria.

286. Clancey, P.A., Brooke, R.K., Crowe, T.M. and Mendelsohn, J.M. 1987. S.A.O.S. checklist of sawthem African birds. First updating report. Southern African Ornithological Society, Johanneshurg.

287. Clapham, C.S. 1964. The hirds of the Dahlac Archipelago. Jbis 106: 376-388.

288. Clark, A. 1977. Review of the records of three Palearctic ducks in southem Africa. Bull. Brit. Om. Club 97: 107-114.

289. Clark. R.J. and Mikkola. H. 1989. A preliminary revision of threatened and near-threatened nnclurnal birds of prey of the world. Pp. 371-388 In: Meyburg, B.-U. and Chancellor, R.D. (eds.) Raptors in the madem world. Proceedings of the Ill World Conference on Birds of Prey and Owls, Eilat, Israel, 22-27 March 1987. World Working Group on Birds of Prey and Owls, Berlin.

290. Clark, W.D. 1991. Hyacinth Macaws: nesting habits in the wild. AFA Watchhird 18(3): 8-10.

291. Clark, W.S. 1986. What is Buten ventralis? Birds of Prey Bull. 3: 115-118.

292. Clark, W.S. and Parslow, R. 1991. A specimen record of Shikra Accipiter badius for Saudi Arabia. Sandgrouse 13: 44-46. 
293. Clarke, R.O.S. and Duran Patiño, E. 1991. The Redfronted Macaw (Ara mubrogenys) in Bolivia: distribution, abundance, biology and conservation. Buena Vista: unpublished repon to Wildlife Conservation International and the International Council for Bird Preservation.

294. Claut, M.N. 1981. South Island Kokako (Callaeas cinerea cinerea) in Nothofagus forest. Notomis 28: 256-259.

295. Clements, F.A. and Bradbear, N.J. 1986. Status of wintering Black-necked Cranes Grus nigricollis in Bhutan. Forkail 2: 103-107.

296. Clinton-Eitnjear, J. 1986. Status of the Green-cheeked Amazon in north-eastern Mexico. AFA Warchbird 12(6): 22-24.

297. Clinton-Eitniear, J. 1990. Amazona oratrix belizensis. AFA Watchbird 17(2): 25-26

298. Clubb, K.J. and Clubh, S.L. 1991. Status of macaws in aviculture. AFA Watchbird 18(3): 21-23

299. Coates, B.J. 1985-1990. Birds of Papua New Guinea. 2 vols. Dove Publications. Alderley, Australia

300. Coats, S. and Phelps, W.H. 1985. The Venezuelan Red SiskIn: case history of an endangered species. Pp. 977-985 in Buckley, P.A., Foster, M.S., Morton. E.S., Ridgely. R.S. and Buckley, F.G. (eds.) Nearropical amithology. American Omithologists Union Ornithological Monograph No. 36. AOU, Washington D.C.

301. Colebrook-Robjent, J.F.R. and Osborne, T.O. 1973. Nesting of Verreaux's Eagle Aquila verreaici in Zambia. Bull. Brit. Om. Club 93: 166.

302. Colebrook-Robjent, J.F.R. and Osborne. T.O. 1974. High density hreeding of the Red-necked Falcon Falco chicquera in Zambia. Bull. Brit. Om. Club 94: 172176.

303. Collar. N.J. 1979. The world status of the Houbara: a preliminary review. $12 \mathrm{pp}$. (unpaginated) In: Coles. C.L. and Collar, N.J. (eds.) Symposium papers: the Great Busiard (Otis tarda), Sofia, Bulgaria, May 26ih, 1978 [and] the Houbara Bustard (Chlamydotis undulata). Athens, Greece, May 24 th 1979 [Fordinghridge. U.K.: The Game Conservancy.]

304. Collar, N.J. 1985. Red Data hird: The Eskino Curlew. World Birdwatch 7(1): 5 .

305. Collar. N.J. 1985. Red Data hird: The Juan Fernandaz Firccrown. World Birdwatch 7(2): 5.

306. Collar. N.J. 1985. Red Data bird: The Freckled Duck. World Birdwatch 7(3): 5.

307. Collar. N.J. 1985. The world slatus of the Great Bustard. Bicstard Siudies 2: 1-20

308. Collar, N.J. 1986. Red Data bird: The Tomba Bowerbird. World Birdwatch 8(1): 5 .

309. Collar. N.J. 1986. Red Data hird: Black-capped Vireo. World Birdwatch 8(2): 5 .

310. Collar. N.J. 1986. The best kept secret in Brazil. World Birdwatch 8(2): 14-15.

311. Collar, N.J. 1987. Red Data bird: Hook-billed Hermit. World Birdwatch $9(1): 5$.
312. Collar, N.J. 1987. Rising sun, falling trees. World Birdwatch 9(1): 6-7.

313. Collar, N.J. 1989. Red Data birds: the cockatoos. World Bindwatch 11(1): 5.

314. Collar, N.J. 1989. Red Data birds: the African additions. World Birdwatch 11(4): 5.

315. Collar, N.J. 1991. Red Data bird: Great Bustard. World Bindwatch 13(1): 13.

316. Collar, N. 1992. Red Data hird: Red Siskin. World Birdwatch 14(1): 13 .

317. Collar, N.J. and Andrew. P. 1987. Red Data birds: the cochoas. World Birdwatch 9(4): 5 .

318. Collar, N.J and Andrew, P. 1988. Birds to watch: the ICBP world check-list of threatened birds. International Council for Bird Preservation, Cambridge, U.K.

319. Collar. N.J. and Gonzaga, L.P. 1988. Cracids at risk: the global perspective. Paper presented at the Second International Cracid Symposium. Caracas. Venezuela, 1988

320. Collar. N.J., Gonzaga. L.P. Jones. P.J. and Scott, D. A. 1987. Avifauna da Mata Allantica. Pp. 73-84 In: Seminario sobre desenvolvimento economico e impacto ambiental em areas do iropico umido brasileiro, 1 , Bclem. 1986. Companhia Vale do Rio Doce, Rio de Janeiro.

321. Collar. N.J., Gonzaga, L.P., Krabhe, N., Madroño Nieto. A., Naranjo, L.G.. Parker, T.A. 111 and Wege, D.C. 1992. Threatened hirds of the Americas: the ICBP/IUCN Red Dala Book, 2. 3rd edition Cambridge, U.K.

322. Collar, N.J. and Goriup. P.D. (eds.). 1983. Report of the 1CBP Fuerteventura Houbara Expedition. 1979. Bustard Studies 1.

323. Collar, N.J., Round, P.D. and Wells, D.R. 1986. The past and future of Gumey's Pitta Pinta gumeyi. Forktail 1: 29-51.

324. Collar, N.J. and Stuart, S.N. 1985. Threatened birds of Africa and related islands: The ICBP/IUCN Red Data Bonk. Part 1. 3rd edition. Intemational Council for Bird Preservation and International Union for Conservation of Nature and Natural Resources, Cambridge, U.K.

325. Collar, N.J. and Tattersall, I. 1987. J.T. Last and the type-locality of Benson's Rockthrush Monticola bensoni. Bull. Brit. Om. Club 107: 55-59.

326. Collar. N.J. and Sluart. S.N. 1988. Threatened birds and bindiversity: seventy-five forests important for bird species at risk in the Afrotropical and Malagasy realm. International Council for Bird Preservation. Cambridge, U.K.

327. Colston. P.R. and Curry-Lindahl, K. 1986. The birds of Mount Ninba, Liberia. British Museum (Natural History). London.

328. Commissie voor de Nederlandse Avifauna 1970. Avifaunn van Nederland. Brill. Leiden.

329. Commission pour l'avifaune helge 1967. Avifaune de Belgique. Gerfout 57: 273-465.

330. Conder. P.J. 1979. A list of the hirds of Aldemey. 
Contreras, J.R., Berry, L.M., Contreras, A.O. Bertonati, C.C. and Utges, E.E. 1990. Allas amilogeográfico de la provincia del ChacoRepública Argentina. Corrientes: FVSA Capítulo Corrientes (Quadernos Técnicos Félix de Azara 1).

332. Contreras-Balderas, A.J. 1988. New records of birds from Nuevo Leon, Mexico. Southwest. Nat. 33: 251. 252.

333. Cooper, J. 1985. New breeding locality data for southern African seabirds: Jackass Penguin Spheniscus demersus. Cormorant 13: $8 \mathrm{I}$

334. Cortes, J.E., Finlayson, J.C., Mosquera, M.A. and Garcia, E.F.J. 1980. The birds of Gibraliar.

335. Cooper, W.T. and Forshaw, J.M. 1977. The birds of paradise and bower birds. Collins, Sydney.

336. Coulter, M.C. 1984. Seabird conservation in the Galapagos Islands, Ecuador. Pp. 237-244 In: Croxall. J.P., Evans, P.G.H. and Schreiber, R.W. (eds.) Status and conservation of the world's seabirds. (Techn. Publ. 2). International Council for Bird Preservation, Cambridge, U.K

337. Coulter, M.C., Cruz, F. and Cruz, J. 1985. A programme to save the Dark-rumped Petrel, Pterodroma phaeopygia, on Floreana Island Galapagos, Ecuador. Pp. 177-180 In: Moors, P.J (ed.) Conservalion of island birds. (Techn. Publ. 3). International Council for Bird Preservation. Cambridge, U.K.

338. Counsell, D. 1988. The RAFOS expedition to Belize Feb-Mar 1986. Royal Air Force Omitholagical Society J. 18: 17-63.

339. Cowling, S.J. 1978. The status of endangered waterfowl and wetlands in Australia. Pp. 123-131 In: Tyler, M.J. (ed.) The status of endangered Atustralasian wildlife. Royal Zoological Society of Australia, Adelaide.

340. Cowling, S.J. and Davies, S.J.J.F. 1983. Sialus of Austratian birds with special refrence to captive breeding. Melbourne: Department of Conservation.

341. Cox, G. 1990. The unicorn's nest. WPA News 29: $8-10$.

342. Cramp, S. (ed.). 1985. Handbook of the birds of Europe, the Middle East and North Africa: the birds of the Western Palearctic. Volume 4. Oxford University Press, Oxford.

343. Cramp, S. (ed). 1988. Handbook of the birds of Europe, the Middle East and North Africa: the birds of the Westem Palearctic. Volume 5. Oxford University Press, Oxford.

344. Cramp, S. and Simmons, K.E.L. (eds.). 1977 Handbook of the birds of Europe, the Middle East and North Africa: the hirds of the Westem Palearctic. Volume 1. Oxford University Press, Oxford.

345. Cramp, S. and Simmons, K.E.L. (eds.). 1980. Handbook of the birds of Europe, the Middle East and North Africa: the birds of the Westem Palearctic Volume 2. Oxford University Press, Oxford.

346. Cramp, S. and Simmons, K.E.L. (eds.). 1983. Handbook of the birds of Europe, the Middle East and North Africa: the birds of the Westem Palearctic. Volume 3. Oxford University Press, Oxford.
347. Cranbrook, Lord 1980. Birds recorded within Gunung Mulu National Park. Pp. 157-164 In: Hanbury Tenison, R. Mulu: the rain forest. Weidenfeld and Nicolson, London.

348. Crivelli, A.J. 1987. The ecology and behaviour of the Dalmatian Pelican, Pelecanus crispus Bruch. A world endangered species. Commission of the European Communities and Station Biologique de la Tour de Valat. Unpublished report.

349. Crivelli, A.J. and Schreiber, R.W. 1984. Status of the Pelecanidac. Biol. Conserv. 30: 147-156.

350. Crivelli, A.J. and Vizi, O. 1981. The Dalmatian Pelican Pelecanus crispus Bruch 1832, a recently world endangered bird species. Biol. Conserv. 20 297-310.

351. Crockett, D.E. 1979. Reöiscovery of the Chatham Island taiko solved century-old mystery. Forest and Bird 13(4): 8-13

352. Crockett, D.E. 1986. Taiko research project. 1985. 1986 expedition report. Unpublished.

353. Cruz, J.B. and Cruz, F. 1987. Conservation of the Dark-rumped Petrel Plerodroma phaeopygia in the Galapagos Islands. Ecuador. Biol. Conserv. 42: 303-311.

354. Cuello, J.P. 1985. Lisıa de referencia y bibliografia de las aves Uruguayas. Museo Damaso Antonio Larranaga.

355. Curry, R.L. and Grant, P.R. 1991. The Floreana Mockingbird: demography and viahility of a relict population. Abstract 245 in the Program of the 109th stated meeting of the American Ornithologists' Union, 14-17 August 1991. Committee on Local Arrangements (etc.), Montreal. Unpublished.

356. Curry, P.J. and Sayer, J.A. 1979. The inundation zone of the Niger as an environment for Palearctic migrants. Ibis 121: 20-40.

357. Danforth, S.T. 1934. The birds of Antigua. Auk $51: 350-364$

358. Danforth. S.T. 1935. The birds of Saint Lucia Monographs of the University of Puerto Rico, Physi_al and Biological Sciences, series B, no. 3.

359. Danforth, S.T. 1936. The birds of St Kitts and Nevis. Tropical Agriculiure 13(8): 213-217.

360. Danforth, S.T. 1939. The birds of Guadeloupe and adjacent islands. J. Agric. Univ. Puerto Rico 23(1): $9-46$.

361. Danforth, S.T. 1939. The birds of Montserrat. $J$ Agric. Univ. Puerto Rico 23(1): 47-66.

362. Danks, A. 1990. Red Data bird: Noisy Scrub-bird. World Birdwatch 12(4): 13.

363. Darby, J. 1985. The great Yellow-eyed Penguin count. Forest \& Bird 16(2): 16-18.

364. Davies, A.G. 1987. The Gola Forest Reserves, Sierra Leone: wildlife conservation and forest management. International Union for Conservation of Nature and Natural Resources, Gland, Switzerland and Cambridge, U.K 
365. Davis, F.W., Hilgartner, W.B. and Steadman, D.W. 1985. Notes on the diets of Geolrygon montana and Calumba caribaea in Jamaica. Bull. Brit. Om. Club 105: 130-133.

366. Davis, 1. 1979. Additions to the Birds of Suriname. Cantinental Birdlife 1(6): 136-146.

367. Davis, T.J. and O'Neill, J.P. 1986. A new species of antwren (Formicariidae: Herpsilochmus) from Peru, with comments on the systematics of other members of the genus. Wilson Bull. 98: 337-352.

368. Davison, G.W.H. 1981. A survey of terrestrial hirds in the Gunung Mulu National Park, Sarawak. Sarawak Mus. J. 27: 283-293.

369. Davison, G.W.H. 1982. Systematics within the genus Arborophila Hodgson. Federation Mus. J. 27: 125-134.

370. Dean, W.R.J. and Tarboton, W.R. 1983. Osprey breeding records in South A frica. Ostrich 54: 241-242.

371. DeDios [sic]. A. and Hill, P. 1990. Breeding the Spix Macaw. Bird World 13(4) (November/December): $16,18$.

372. Dee, T.J. 1985. Red Data bird: The Seychelles Warbler. World Birdwatch 7(4): 3.

373. Dee, T.J. 1987. The status and distribution of the endemic birds of Madagascar. International Council for Bird Preservation, Cambridge, U.K.

374. Dekker, R.W.R.J. 1990. The distribution and status of nesting grounds of the Maleo Macracephalon maleo in Sulawesi, Indonesia. Biol. Conserv. 5: 139-150.

375. Dekker, R.W.R.J. and Wattel, J. 1987. Egg and image: new and traditional uses for the Maleo (Macrocephalon malea). Pp. 83-87 In: Diamond, A.W. and Finion, F.L. (eds.) The value of birds. International Council for Bird Preservation. Cambridge, U.K.

376. Delacour, J. 1927. Arborophila davidi sp. nov. Bull Brit. Om. Club 47: 169

377. Delacour, J. 1977. The pheasants of the world. 2nd edition. World Pheasant Association and Spur Publications, Hindhead, Surrey.

378. Delacour, J. and Amadon, D. 1973. Curassous and related birds. American Museum of Natural History, New York.

379. Delacour, J. and Jahouille, P. 1931. Les oiseaux de I'Indachine francaise, 4 vols. Exposition Coloniale Internationale, Paris.

380. Delacour, J. and Jahouille, P. 1940. Listes des oiseaux de l'Indochine française, completée et mise a jour. L'Oiseak et R.f.O. 10: 89-220.

381. Delacour, J. and Mayr, E. 1946. Birds of the Philippines. Macmillan, New York.

382. Delibes, M. 1978. Ecologia alimenticia del aguila imperial iberica (Aquila adalberti) en el Colo Donaña durante la crianza de los pollos. Donaña, Acıa Venebrata 5 : $35-60$

383. Dementiev, G.P. and Gladkov, N.A. (eds.). 1951 1954. Birds of the Saviet Union. 6 vols. Israel Program for Scientific Translation, Jerusalem
384. Derenne, P., Lufbery, J.X. and Tollu, B. 1974. L'avifaune de l'Archipel Kerguelen. CNFRA 40: $107-148$

385. Desenne, P. and Strahl, S.D. 1991. Trade and the conservation status of the family Psittacidae in Venezuela. Bird Conservation Intemational 1: 153-169

386. Desfayes, M. 1987. Evidence for the ancient presence of the Bald Ibis Geranticus eremita in Greece. Bull. Brit. Orn. Clisb 107: 93-94.

387. Devas, R.P. 1970 Birds of Grenada and St Vincent and the Grenadines. [3rd ed.] St. George'a Grenada, Carenaga Press XV, 88pp. Grenada

388. Devillers, P. 1977. Observations at a hreeding colony of Lanws (belcheri) atlanticus. Gerfaut 67: 22-43.

389. Diamond, A.W. 1987. Studies of Mascarene island birds. Academic Press, London.

390. Diamond, J.M. 1976. Recommendations for minimising environmental casts of mining on Rennell. Report to the Central Planing Office, Government of the Solomon Islands.

391. Diamond, J.M. 1982. Rediscovery of the Yellowfronted Gardener Bowerbird. Science $216(4544): 431-434$.

392. Diamond, J.M. 1984. Back from the hrink of extinction. Nature 309:308.

393. Diamond, J.M. 1985. New distributional records and taxa from the outlying mountain ranges of New Guinea. Emu 85: 65-91.

394. Diamond, J.M. 1987. Extant unless proven extinct? Or, extinct unless proven extant? Conserv. Biol. 1: $77-79$.

395. Diaz Montes, V.R. 1991. Status of the highly endangered White-winged Guan. Crocid Newsletter 1(1): 1,6

396. Dick, J.A., McGillivray, W.B. and Brooks. D.J. 1984. A list of birds and their weights from Saul, French Guiana. Wilson Bulletin 96(3): 347-365.

397. Dickey, D.R. and van Rossem, A.J. 1938. The birds of El Salvador. Publication of the Field Museum Natural History. Zoology series 23: 1-609.

398. Dickinson. E.C. and Eck, S. 1984. Notes on Philippine hirds, 2. A second Philippine record of Sterna bemsteini. Bull. Brit. Om. Club 104: 72.

399. Dickinson, E.C., Kennedy, R.S. and Parkes, K.C. 1991. The birds of the Philippines. B.O.U. Check-list No.12. British Ornithologists' Union, Tring, Herts.

400. Disi, A.M. and Bouran, A.H. 1987. A check-list of the Hashemite Kingdom of Jordan: an ecological outlook. Publication of the University of Jordan.

401. Dobson, R. 1952. The birds af the Channel Islands. Staples Press, Newton Abbott, London.

402. Donahue, P.K. and Pierson J.E. 1982. Birds of Suriname: an annatated checklist. Privately published.

403. Dornhusch, M. 1983. Status, ecology and conservation of Great Bustard in the German Democratic Republic. Pp. 89-90 in Goriup, P.D. and Vardhan, H. (eds) Bustards in decline. Tourism and Wildlife Society of India, Jaipur. 
404. Dornbusch, M. 1985. Die gegenwartige Situation vom Aussterben bedrohter Tierarten in der DDR. Hercynia (N.F.) 22: 221-227

405. Doughty, R.W. 1989. The retum of the Whooping Crane. University of Texas Press, Austin, Texas.

406. Downer, A. and Sutton, R. 1990. Birds of Jamaica: a photographic field guide. Cambridge University Press, Cambridge.

407. Dowsett, R.J. 1979. Recent additions to the Zambian list. Bull. Brit. Om. Cluh 99: 94-98

408. Dowsett-Lemaire, F. 1989. Ecological and biogeographical aspects of forest bird communities in Malawi. Scopus 13: 1-80.

409. Draulans, D. 1986. On the distribution and foraging behaviour of the Malagasy Heron Ardea humbloti. Ostrich 57: 249-251.

410. Dreux, P. and Milon, P. 1967. Premiëres observations sur l'avifaune de l'lle aux Cochons (Archipel Crozet). Alauda 35: 27-32.

411. Duckworth, F. 1974. The Whale-headed Stork in Ethiopia. Bull. Brit. Orn. Club 94: 3-4.

412. Duffy, D.C., Hays, C. and Plenge, M.A. 1984. The conservation status of Peruvian seabirds. Pp. 245-259 In: Croxall, J.P., Evans, P.G.H. and Schreiber, R.W. (eds.) Status and conservation of the world's seabirds. (Techn. Publ. 2). International Council for Bird Preservation, Camhridge, U.K.

413. Duffy, D.C., Wilson, R.P., Ricklefs, R.E. Brnni, S.C. and Veldbuis, H. 1987. Penguins and purseseiners: competition or co-existence? Natn. Geogr. Res. 3: 480-488.

414. Dupuy, A.R. 1976. Première observation d'un Vautour fauve Gyps fulvis au Sẻnégal. Ardea 44: 33-334.

415. Dybbro. T. 1978. Oversigi over Danmorks fugle. Dansk Ornithologisk Forening, Denmark.

416. Ebenhard, N.T. 1979. First record in the Seychelles of Northem Pintail. Bull. Brit. Om. Club 99: 39-40.

417. Eccles, S.D. 1988. The birds of Sao Tome - record of a visit, April 1987 with notes on the rediscovery of Bocage's Longhill. Malimbus 10(2): 207-217.

418. Eck, S. 1986. Eutriorchis astur im Dresden Tierkunde-Museum. J. Om. 127: 93-94.

419. Edwards, E.P. and Lea, R.B. 1955. Birds of the Monserrate area, Chiapas, Mexico. Condor 57: 31-54.

420. Ehrlich, P.R., Dobkin, D.S. and Wheye, D. 1992. Birds in jeopardy: the imperiled and extinct birds of the United States and Canada, including Hawaii and Puerta Rico. Stanford University Press, Stanford

421. Eichacker, X. 1990. First breeding records of Verreaux's Eagle Aquila verreauxii in Saudi Arabia. Sandgrouse 12: 53-54.

422. Eisentraut, M. 1973. Die Wirheltierfauna von Fernando Poo und Westkamerun. Bonn. zool. Monogr. No.3, 428 pp.

423. Eley. J.W. 1982. Systematic relationships and zoogeography of the White-winged Guan (Penelope albipennis) and related forms. Wilson Bull. 94: 241-259.
424. Elgood, J.H. 1982. The birds of Nigeria. B.O.U. Check-list No.4, British Ornithologists' Union. Tring, Herts.

425. Elliott, H.F.I. 1957. A contribution to the ornithology of the Tristan da Cunha group. Ibis 99: 545-586.

426. Ellis, D.H. and Peres, G. 1983. The Pallid Falcon Falca kreyenborgi is a color phase of the Austral Peregrine Falcon (Falco peregrinus cassini). Auk 100: 269-271, 442 .

427. Ellis-Joseph, S., Hewston, N. and Green, A. 1992. Global waterfowl conservation assessment and management plan. Captive Breeding Specialist Group and The Wildfowl and Wetland Trust. First review draft, 15 March 1992.

428. Elwell, N. 1970. Marabous in winter - by the hundred. Bokmakierie 22: 69-71.

429. Emlen, 1.T. 1977. Land bird communities of Grand Bahama Island: the stnucture and dynamics of an avifauna. American Ornitholngists' Union (Orn. Monogr. 24).

430. Emmerson, K.W., Martin. A., Delgado, G. and Quilis. V. 1986. Distrihution and some aspects of the breeding biology of Bolle's Pigeon (Columha bollit) on Tenerife. Vogelwelt 107: 52-65.

431. Engbring, J. and Pratl, H.D. 1985. Endangered birds in Micronesia: their history, status and future prospects. Bird Conserv. 2: 71-105.

432. Erickson, K.R. and Heideman, P.D. 1983. Nntes on the avifauna of the Balinsasayo rainforest region, Negros Oriental, Philippines. Silliman J. 30: 63-72.

433. Erickson, R. 1977. First record of Knot Calidris canutus, and other records, from Belize (British Honduras). Bull. Brit. Orm. Club 97: 78-81.

434. Escalante, R. 1980. Notas sobre algunas aves de la lavertiente atlantica de Sud America (Rallidae, Laridae). Resumenes, I Jomados de Ciencias Naturales, Montevideo 33-34.

435. Escalante-Fliego, P. and Peterson, A.T. 1992. Geographic variation and species limits in middle American wondnymphs (Tholurania). Wilson Bull. 104: 205-219.

436. Escott, C.J, and Holmes, D.A. 1980. The avifauna of Sulawesi, Indonesia: faunistic nntes and additions. Bull. Brit. Om. Cluh 100: 189-194.

437. Estudilio Lopez, J. 1983. Considerations in regard to rare and endangered species of cracids bnth in nature and captivity. Pp. 45-61 In: Proceedings fof thel Jean Delacour/IFCB Symposium on hreeding hirds in coptivity. International Foundation for the Conservation of Birds. North Hollywood. California.

438. Etcheberry, R. 1982. Les oiseaux de Saint-Pierre et Miquelon. ONC, Paris.

439. Evans, D.L. 1982. Status repnrts on twelve raptors U.S. Fish and Wildlife Service, Special Scientific Report, Wildlife No. 238

440. Evans, 1.M. and Pienkowski, M.W. 1991. World status of the Red Kite: a background to the experimental reintroduction to England and Scolland. Brit. Biirds 84: 171-187.

441. Evans. P. 1990. Birds of the eastem Caribbean. Macmillan, New York. 
442. Evans, P.G.H. 1986. Dominica, West Indies. World Birdwatch 8(1): 8-9.

443. Evans, P.G.H. 1986. Monitoring seabirds in the North Allantic. Pp. 179-206 In: Medmaravis and Monbaillu, V. (eds.) Mediterranean Marine Avifauna.

Medmaravis, Springer-verlag. Berlin.

444. Evans, P.G.H. 1991. Status and conservation of Imperial and Red-necked Parrots Amazona imperialis and A. arausiaca on Dominica. Bird Conserv. Intematn. 1: 11-32.

445. Eve, R. and Guigue, A.M. 1989. Sunvey of the Mahakam River delta, East Kalimantan, with special reference 10 its waterbirds. (Puhl. no. 45). Asian Wetland Bureau, Kuala Lumpur.

446. Everett, W.T. 1988. Notes from Clarion Island. Condar $90: 512-513$.

447. Everett, W.T. and Anderson, D.W. 1991. Status and conservation of the breeding seabirds on offshore Pacific islands of Baja California and the Gulf of California. Pp. 115-139 ln: Croxall, J.P. (ed.) Seabird status and conservation: a supplement. (Techn. Publ. 11). International Council for Bird Preservation. Cambridge. U.K.

448. Fa, J.E. and Bell, D.J. 1990. The Volcano Rabbit Romeralagus diazi. Pp. 143-146 ln: Chapman, J.A. and Flux, J.E. (eds.) Rahhits, hares and pikas: stalus survey and conservation action plan. International Union for Conservation of Nature and Natural Resources, Gland, Switzerland.

449. Faaborg, J. 1983. Why share a mate? Living Bird Quarterly 2(2): 14-17.

450. Faaborg, J. 1984. Potential for restocking Galapagos Hawks on islands where they have been extirpated. Noticias de Galapagas 39: 28-30.

451. Faaborg. J. and Arendt. W.J. 1985. Wildlife assessments in the Caribbean. San Juan. Puerto Rico: Institute of Tropical Forestry.

452. Faaborg, J. 1985. Reproductive success and survivorship of the Galapagos Hawk Butco galapagoensis: potential costs and henefits of conperative polyandry. Ibis $128: 337-347$.

453. Faanes, C.A. and Senner, S.E. 1991. Status and conservation of the Eskimo Curlew. Amer. Birds 45: $237-239$.

454. Falla, R.A., Sibson, R.B. and Turbott. E.S. 1978. The new guide to the birds of New Zealand and outlying islands.

455. FAO 1981. Checklist of the hirds of Afghanistan. In: Afghanistan, a contriburion to a conservation strategy.

456. Farago, S., Ena, V. and Martinez, A. 1987. Comparison of the state of Great Bustard stocks in Hungary and Spain. Pp. 51-63 In: Farago. S. (ed.) The Great Bustard fOtis tarda L.). Pmceedings of the symposium in Budapest on June 2nd 1987. International Council for Game and Wildlife Conservation. Budapest.

457. Farkas, T. 1967. Omilhogeographie Ungams. Duncker \& Humblot. Berlin.
458. Farronato, 1. and Fracasso, G. 1989. [Nesting of Comcrake, Crex crex, in the Province of Vicenza (NE Italy).| Riv. Ital. Om. 59: 196-200. (In ltalian.)

459. Feare, C.J. 1973. Numenius minutus, Falco subbuteo and Caprimulgus eurapaeus in the Seychelles. Bull. Brit. Om. Club 93: 99-101.

460. Feare. C.J. 1975. Further migrant hirds in the Seychelles. Bull. Brit. Om. Club 95: 48-50.

461. Feare, C.J. and High, J. 1977. Migrant shorebirds in the Seychelles. Ibis 119: 323-338.

462. Fei Dian-jin 1989. [An observation and investigation on the hreeding situation of the Oriental Stork in Qiqihar Suburhan District.] Zool. Res. 10: 263-270.

463. Ferianc. O. 1977/79, Vtâky Slovenska 1, 2 (2nd ed.) Veda: Bratislava

464. Fernandez-Cruz, M. and Arauio, J. (eds.). 1985. Sisuacion de la avifauna de la Peninsula lherica, Baleares y Macaranesia. Sociedad Espanola de Ornitologia, Madrid

465. ffrench, R.P. 1991. A guide to the hirds of Trinidad and Tohago. 4th edition. Livingston Publishing, Wynnewood, Pennsylvania.

466. Fiebig, J. and Jander, G, 1985. Der Steppenschlammlaufer, Limnodromus semipalmatus, als mongolischer Brutvogel. Ann. Om. 9: 107-111.

467. Fisher, D.J. 1985. Observations on Relict Gull in Mnngolia. Dutch Birding 7: 117-120.

468. Fitzpatrick, J.W. and O'Neill, J.P. 1979. A new todytyrant from northern Peru. Auk 96: 443-447.

469. Fitzpatrick. J.W. and O'Neill, J.P. 1986. Oths petersoni, a new screech-owl from the eastern Andes, with systematic notes on $O$. colombianis and $O$. ingens. Wilson Bull. 98: 1-14.

470. Fitzpatrick, J.W. and Willard, D.E. 1982. Twenty-one bird species new or little known from the Republic of Colombia. Bull. Brit. Om. Club 102: 153-158.

471. Fitzpatrick, J.W., Willard, D.E. and Terhorgh, J.W. 1979. A new species of humminghird from Peru. Wilson Bull. 91: 177-186.

472. Fjeldsà, J. 1981. Podiceps toczanowskii (Aves, Podicipedidae), the endemic grebe of Lake Junin, Peru. A review. Stecnstrupia 7: 237-259.

473. Fjeldsá, J. 1983. A Black Rail from Junin, central Peru: Laterallus jamaicensis tuerosi ssp. nov. (Aves, Rallidae). Steenstrupia 8(13): 277-282.

474. Fjeldsa, J. 1984. Three endangered South American grebes (Podiceps): case histories and the ethics of saving species by human interventinn. Ann. Zool. Fennici 21: 411-416.

475. Fjeldsai, J. 1987. Relict forests in the High Andes of Peru and Bolivia. Technical report from the Polylepis forest expedition of the Zoological Museum (University of Copenhagen). 1987, with some preliminary suggestinns for habitat conservation. Unpublished.

476. Fjeldsa, J. 1991. The aclivity of birds during snowstorms in high-level woodlands in Peru. Bull. Brit. Om. Cluh 111: 4-11. 
477. Fjeldsà, $J$. and Krabbe, N. 1986. Some range extensions and other unusual records of Andean birds. Bull. Brit. Om. Club 106: 115-124.

478. Fjeldsã, J. and Krabbe, N. 1990. Birds of the high Andes. Zoological Museum, University of Copenhagen and Svendborg, Copenhagen.

479. Fjeldsȧ, J., Krabbe, N. and Parker, T.A. 1987. Rediscovery of Cinclodes excelsior aricamae and notes on the nominate race. Bull. Brit. Om. Club 107: 112-114.

480. Flint, P.R. and Stewart, P.F. 1991. The birds of Cyprus. 2nd edition. British Ornithologists ${ }^{\circ}$ Union Checklist No. 6. London.

481. Flint, V.E., Boehme, R.L., Kostin, Y.V. and Kuznetzov, A.A. 1984. A field guide to birds of the USSR. Princeton University Press, Princeton, New Jersey.

482. Folkestad, A.O. 1984. Situasjonen for havorna Haliaeetus alhicilla in Norge. Vàr Fuglefauna 7: 209-216.

483. Forbes-Watson. A. 1969. Notes on birds observed in the Comoros on behalf of the Sinithsonian Institution. Atoll Res. Bull. 128: 1-23.

484. Forshaw, J.M. and Cooper, W.T. 1981. Australian parrats. Lansdowne, Melbourne.

485. Forshaw, J.M. and Conper, W.T. 1989. Parrots af the world. 3rd edition. Bland ford.

486. Fosherg, F.R., Sachet, M.-H. and Stoddart, D.R. 1983. Henderson Island (southeastern Polynesia): summary of current knowledge. Atoll Res. Bull. 272.

487. Frade, F. 1951. Catálogo das aves de Moçambique. Ministerio das Colonias, Lisbon.

488. Frade, F. and Bacelar. A. 1955. Catalogo das aves da Guiné Pontuguesa - Non passeres. Annl Junta lnvest. Ulitam. 10:7-173.

489. Fraga, R.M. 1990. El Tordo Amarillo al borde de la extinción. Nuestras Aves 23: 13-15.

490. Frey, H. 1985. The Black-hooded Red Siskin, an endangered species; an interview with Pat Dempko. AFA Watchbird 12(4): 40-42.

491. Friedmann. H. 1950. The birds of North and Middle America, I1. Bull. U.S. Nat. Mus. 50

492. Frisch, J.D. and Frisch, S. 1981. Aves Brasilciras Vol. 1. Dalgas-Ecollec Ecologica Tecrica. San Pauln.

493. Frith, C.B. 1974. New observations of migrants and vagrants from Aldabra, Farquhar and Astove Alolks, Indian Ocean. Bull. Brit. Om. Club 94: 12-19.

494. Frumkin, R. and Adar, M. 1989. First breeding records of Sparrowhawk in 1srael. Om. Soc. Middle East Bull. 23: 20-22.

495. Fry, C.H., Hosken, J.H. and Skinner, D. 1986. Further observations on the breeding of Slaty Egrets Egretta vinaceigula and Rufoushellied Herons Ardeola nufiventris. Ostrich 57: 61-64.

496. Fry, C.H., Keith, S. and Urban, E. 1988. The birds of Africa. Volume 3. Academic Press, London and San Diego.

497. Fry, C.H. and Smith, D.A. 1985. A new swallow from the Red Sea. 1bis 127: 1-6.
498. Fuehrer, L. von 1934. A supplement to the omis of Montenegro and Albania. Ibis (13)4: 172.

499. Fujimaki, Y. 1987. Joint survey report of Japan and USSR on Steller's Sea-Eagle. P. 49 In: The Third Japan-USSR Bird Protection Sympasium, 2I November 1986, summary. Wild Bird Society of Japan [Tokyo].

500. Fullager, P.J. and Disney, H.J. de S. 1975. The birds of Lord Howe Islands: a report on the rare and endangered species. Bull. ICBP 12: 187-202.

501. Gallagher, M. 1982. Nesting of the Lappet-faced Vulture Torgos tracheliotus in Oman. Bull. Brit. Om. Club 102: 135-139.

502. Gallagher, M. and Brown, M.R. 1982. Golden Eagle Aquila chrysaetos breeding in Oman, eastern Arabia. Bull. Brit. Om. Club 102: 41-42.

503. Gallagher. M.D., Scoll, D.A., Ormond, R.F.G. Connor, R.J. and Jennings, M.C. 1984. The distribution and conservation of seahirds breeding on the coasts and isiands of Iran and Arabia. Pp. 421-456 In: Croxall, J.P., Evans, P.G.H. and Schreiher, R.W. (eds.) Status and conservation of the world's seabirds. (Techn. Puhi. 2). International Council for Bird Preservation, Cambridge, U.K.

504. Gallagher. M. and Woodcock, M.W. 1980. The birds of Oman. Quartet Books, London.

505. Garcia, F. (undated) Las aves de Cuba, I: especies endenicas. La Habana: Gente Nueva.

506. Garget1, V. 1990. The Black Eagle: a study. Acom Books, Randburg, South Africa.

507. Garnell, M.C. 1983. A management plan for nature conservation in the Line and Phnenix Islands, Part 1: descriptinn. Unpublished.

508. Gamett, S. 1987. An Australian record of Gumey's Eagle Aquila gumeyi. Aust. Bird Watcher 12(4): 134-135

509. Garrido, O.H. 1985. Cuhan endangered hirds. Pp. 992-999 in Buckley, P.A., Foster, M.S., Morion, E.S., Ridgely, R.S. and Buckley, F.G. (eds.) Neotrapical amitholngy. American Ornithologists ${ }^{\circ}$ Union. Ornitholngical Mnnograph 36. AOU, Washington D.C.

510. Garrido, O.H. 1986. Las palomas. Editorial Cientifico-Tecnica, La Hahana.

511. Garrido, O.H and Montaña Garcia, F. 1975. Carílogo de las aves de Cuha. Academia de Ciencias de Cuba. La Hahana.

512. Garson, P.J. 1983. The Cheer Pheasant Catreus wallichii in Himachal Pradesh, western Himalayas: an update. World Pheasant Assoc. J. 8: 29-39.

513. Gast. S.E. and King. B. 1985. Notes on Philippine birds. 7. Recent records of the Chinese Egret Egretta eulophotes from Luzon. Mindoro and Palawan, Philippines. Bull. Brit. Om. Club 105: 139-141.

5J4. Gaston. A.J. 1984. Is hahitat destruction in India and Pakistan beginning to affect the stalus of endemic passerine hirds? J. Bomhay Nat. Hist. Soc. 81: 636-641.

515. Gaston, A.J., Garson, P.J. and Hunter, M.L. $198 \mathrm{I}$. Present distribution and status of pheasants in Hinachal Pradesh, western Himalayas. World Pheasant Assoc. J. 6: 10-30. 
516. Gaston, A.J., Islam, K. and Crawford, J.A. 1983. The current status of the Western Tragopan Tragopan melanocephalus. Warld Pheasant Assoc. J. 8: 40-49.

517. Gaston, A.J. and Macrell, J. 1980. Green Munia (Estrilda formosa) at Dethi, and other interesting records for 1978. J. Bambay Nat. Hist. Soc. 77: 144-145

518. Gaston, A.J. and Singh, J. 1980. The status of the Cheer Pheasant Catreus wallichii in the Chail Wildlife Sanctuary, Himachal Pradesh. Warld Pheasant Assac. J. $5: 68-73$.

519. Gatter, W. 1985. Ein neuer Bulbul aus Westafrika (Aves, Pycnonotidae).J. Om. 126: 155-161.

520. Gee, J.P. 1984. The birds of Mauritania. Malimbus 6: $31-66$.

521. Geeson, J. and Geeson, J. 1990. First Red Kite record for the Gambia. Malimbus 11: 144.

522. Genot, J.-C. 1989. Athene nocula, world hibliography.

523. Gensbol, B. 1986. Collins guide to the birds of prey of Britain and Europe, North Africa and the Middle East. Collins, London.

524. Gibson-Hill, C.A. 1947. Notes on the birds of Christmas Island. Bull. Raffles Mus. 18: 87-165.

525. Gibson-Hill, C.A. 1949. The birds of the CocosKeeling Islands (Indian Ocean). Ibis 91: 221-243.

526. Gibson-Hill, C.A. 1950. A checklist of the birds of Singapore Island. Bull. Raffles Mus. 21: 132-183.

527. Gibson-Hill. C.A. 1956. Ornithological notes from the Raffles Museum. 23. Notices of four birds new to, or rare in, the Malay Peninsula. Bull. Raffes Muts. 27: 180

528. Gill, B.J. 1987. Notes of the birds, reptiles and mammals of Tangatapu and Eua (Tonga). Notomis $34(3): 217-223$

529. Gill, R.E. and Handel, C.M. compilers. 1990. Summary of the proceedings from the workshop on Bristle-thighed Curlews, February 14-16, 1990 Anchorage, Alaska. U.S. Fish and Wildlife Service, Anchorage, Alaska. Un rublished.

530. Gill, R.E., Lanctot, R.B., Mason, J.D. and Handel, C.M. 1991. Observations on habitat use, breeding chronology and parental care in Bristle-thighed Curlews on the Seward Peninsula, Alaska. Wader Sudy Group Bull. 61: 28-36.

531. Gill, R.E. and Redmond. R.L. 1992. Distribution, numbers and habitat of Bristle-thighed Curlews (Numenius tahitiensis) on Rangiroa Atoll. Notomis 39: 17-26.

532. Gilliard, E.T. 1950. Notes on a collection of birds from Bataan, Luzon, Philippine lslands. Bull. Amer. Mus. Nat. Hist. 94: 457-504.

533. Gilliard, E.T. and LeCroy, M. 1967. Results of the 1958-1959 Gilliard New Britain Expedition, 4: annotated list of birds of the Whiteman Mountains. New Britain. Bull. Amer. Mus. Nat. Hist. 135: 173-216.

534. Girard-Audine, M. and Pineau, J. 1972. Emberiza striolata et Vanellus gregarius dans le Tangerois. Alaida $41: 317$.
535. Giraudoux, P., Degauquier, R., Jones, P.J., Weigel, J. and Isenmann, P. 1988. Avifaune du Niger: etat des conaissances en 1986. Malimbus 10: 1-140.

536. Glade. A.A. (ed.). 1988. Red list of Chilean terrestrial vertebrates. Impresimas Comerciales, for CONAF, Santiago.

537. Glutz von Blotheim, U.N. and Bauer, K. 1966-1991. Handbuch der Vogel Mitteleuropas. Bands 1, 2, 3, 4, $5.6,7,8 / 1,8 / 11,9,10 / 1,10 / 11,11 / 1,11 / 11,12 / 1$, $12 / 11$.

538. Gochfeld, M. 1978. Social facilitation of singing: group size and flight song rates in the Pampas Meadowlark Stumella defilippii. Ihis 120: 338-339.

539. Godfrey, E. 1986. The birds of Canada. 2nd ed. National Museum of Natural Sciences, Ottawa, Canada.

540. Gollop, J.B.. Barry, T.W. and Iversen. E.H. 1986. The Eskimo Curlew. A vanishing species. Regina, Saskatchewan: Saskatchewan Natural History Society (Spec. Publ. 17).

541. Golovushkin. M.1. and Osipova, M.A. 1988. [Nesting of Imperial Eagle (Aquila heliaca) in the region south to Baikal.] Omitologiya 23: 205-206.

542. Gonzaga, L.P. 1983. Notas sobre Dacnis nigripes Pelzeln, 1856 (Aves, Corebidae). Iheringia Ser. Zool. 63: 45-58.

543. Gonzaga, L.P. 1984. Voa araponga, voa macuco, que o homem vem ai...Ciencia Hoje 2(11): 18-24.

544. Gonzaga. L.P. and Pacheco, J.F. 1990. Two new suhspecies of Formicivora serrana (Hellmayr) from south-eastern Brazil, and notes on the type-locality of Fomicivora deluzae Ménétriès. Bull. Brit. Om. Club 110: 187-193.

545. González Alonso, H., Sirois, J., McNicholl, M.K., Hamel, P.B., Godinez, E., McRae, R.D., Acosts, M., Rodriguez, D., Marcos, C. and Hemández, J. 1990. Preliminary results of a cooperative bird-handing projecl in the Zapata Swamp, Cuha, January 1988. Canad. Wildl. Serv. Progr. Notes no. 187.

546. Gonzalez, L.M., Gonzalez, J.L., Garzon, J. and Heredia, B. 1987. Censo y distribucion del Aguila Imperial Iherica. Aquila (heliaca) adalherti Brehm, 1861, en España durante el periodo 1981-1986. Bol. Est. Cent. Ecol. 16(31): 99-109.

547. Gonzảlez-Garcia, F. 1984. Aspectos biologicos del Pavon Oreophasis derhianus G.R. Gray (Aves: Cracidae) en la Reserva Natural "El Triunfo", Municipio del Angel Albion Corzo, Chiapas, Mexico. Tesis profesional. Facultad de Ciencias Biologicas, Universidad Veracruzana, Xalapa, Veracruz. Mexico.

548. González-Garcia, F. 1991. Observaciones sohre la ecología y hiologia reproductiva del Pavón Oreophasis derbianus en la Reserva de la Biosfera "El Triunfo", Chiapas, México. Paper presented at IV Congreso de Ornitología Neotropical, Quito, Exuador, November 1991. Unpublished.

549. Gonzalez-Grande, J.L. 1981. Spain's Imperial Eagle. Nat. Hist. 90: 40-43.

550. Gonzales, Z.J. 1977. Sobre la presencia en Chile de Sappho sparganura sappho (Lesson) (Aves: Trochilidae). Bolerin Omitologico 9(1/2): 11-12. 
551. Goodman, S.M. and Meininger, P.L. 1989. The birds af Egypt. Oxford University Press, Oxford.

552. Goodwin, D. 1976. Crows of the world. British Museum (Natural History), London.

553. Goodwin, D. 1982. Estrildid finches of the world British Museum (Natural History) and Oxford University Press, London and Oxford.

554. Goodwin, D. 1983. Pigeons and doves of the world. 3rd edition. British Museum (Natural Histnry) and Comell University Press, London and New York

555. Gore, M.E.J. 1968. A checklist of the birds of Sabah. Borneo. Ibis 110: 165-196.

556. Gore, M.E.J. 1990. The birds of the Gompia. 2nd ed British Omithologists" Union Check List No. 3. BOU. London.

557. Gore, M.E.J. and Gepp, G.R.M. 1978 Las aves del Uruguay. International Council for Bird Preservation. (Pan-American Section), Montevideo.

558. Gore, M.E.J. and Won, P-O. 1971. The bircls of Karea. Royal Asiatic Society, Korea Branch, in conjunction with Taiwan Puhlishing Co., Seoul

559. Goriup, P.D. (ed.). 1983. The Howbara Bustard in Maracco: a report of the Al-Areen//CBP March 1982 preliminary survey. [International Council for Bird Preservation, Cambridge, U.K.. on behalf of Al-Areen Wildlife Park, Bahrain.]

560. Goriup, P.D. and Kapowicz, Z.J. 1985. A review of the past and recent status of the Lesser Florican. Bustard Studies 3: 163-182.

561. Goriup, P.D., Osborne, P.E. and Everett, S.J. 1989. Bustards in Meru National Park, Kenya: a preliminary survey. Bustord Studies 4.

562. Goriup, P.D. and Parr, D.F. 1985. Results of the ICBP bustard survey of Turkey, 1981. Bustard Studies 2: 77-98.

563. Goriup, P.D. and Vardhan, H. (eds.), 1983. Bustards in decline. Jaipur: Tourism and Wildlife Society of India.

564. Gorman, K.M. 1990. Breeding the endangered Venezuelan Black-hooded Red Siskin. AFA Wotchhird 17(1): 7-12.

565. Gorman, K.M. 1992. AFA Red Siskin Project. AFA Watchbird 18(6): 24-25.

566. Graham, G.L., Graves, G.R., Schulenberg, T.S. and O'Neill, J.P. 1980. Seventeen hird species new to Peru from the Pampas de Heath. Ank 97: 366-370.

567. Grant, P.R. 1986. Ecology and evolution of Damin's finches. Princeton University Press. Princetnn.

568. Grantsau, R. 1967. Sobre o genero Argastes, com a descricao de uma subespecie nova (Aves, Trochilidae). Pap. Avuls. Zno!. 21: 21-31.

569. Graves, G.R. 1980. A new species of metaltail humminghird from northern Peru. Wilson Bull. 92: 1-7.

570. Graves, G.R. 1982. First record of the Brown Wood Rail, Aranides wolfi in Pens. Gerfow 72: 237-238.
571. Graves, G.R. and Giraldo, O.J.A. 1987. Population status of the Rufous-fronted Parakeet (Bolborhynchus fermugineifrans), a Colombian endemic. Gerfout 77: 89-92

572. Graves, G.R. and Weske, J.S. 1987. Tangara phillipsi, a new species from the Cerros del Sira. eastern Peru. Wilson Bull. 99: 1-6.

573. Graves, G.R. and Zusi, R.L. 1990. Avian hody weights from the lower Rio Xingu. Bull. Brit. Om. Club 110: 20-25.

574. Green, A.A, 1984. Additional hird records from Bamingui-Bangoran National Park, Central African Republic. Molimbus 6(1/2): 14-28.

575. Green, A.A. and Sayer, J.A. 1979. The birds of Pendjari and Arli National Parks (Benin and Upper Volta). Malimbus 1(1): 14-28

576. Greenway, J.C. 1958. Extinct and vonishing hirds of the world. American Committee for International Wildilife Protection (Ser. Puhl. 13). New York.

577. Greenway, J.C. 1967. Extinct and vanishing birds of the world. Second edition. Dover Puhlications, New York.

578. Gretton, A. 1987. Gumey's Pitta: the struggle to survive. World Birdworch 9(4): 6-7.

579. Gretton. A. 1990. Red Data hird: Slender-hilled Curlew. Warld Birdwatch 12(3): 11.

580. Gretton, A., Komdeur, J. and Komdeur, M. 1991. Saving the Magpie Rnhin. World Birdwatch 13(1): 10-11.

581. Grice, D., Caughley, G. and Short, J. 1986. Density and distrihution of the Australian Bustard Ardeotis ousiralis. Biol. Conserv. 35: 259-267.

582. Grimes, L. 1987. The hirds of Ghana. B.O.U. Checklist No. 9. British Ornithologists' Union, London.

583. Grimmett, R. 1986. News and views: White-winged Ducks in Thailand. Bull. Oriental Bird Clut 3: 7.

584. Grimmett, R, 1989. Aquatic Warbler. World Birdwatch 11(2): 5 .

585. Grzybowski, J.A. I991. Black-copped Vireo Nireo atricapillus) recovery plan. U.S. Fish and Wildlife Service, Austin, Texas.

586. Grzybowski, J.A., Clapp, R.S. and Marshall, J.T. 1986. History and current populatinn status of the Black-capped Vireo in Oklahoma. Amer. Birds 40: 1151-1161.

587. Guedes. N.M.R. 1991. Ohservaçôes de ninhos de arara azul (Anodorhynchus hyocinthinus) no Pantanal. P.6 In: Resumos, 1 Congresso Brasileiro de Ornitologia. Belém: Museu Paraense Emilio Goeldi.

588. Guedes. N.M.R. and Harper, L.H. 1991. Nest site characteristics of the Hyacinth Macaw (Anodorhynchus hyacinthinus) in the Nhecolândia reginn of the Brazilian Pantanal. Ahstract 261 in the Program of the 109th Stated Meeting of the American Ornithologists' Union, 14-17 August 1991. Committee nn Local Arrangements (etc.), Montreal. Unpublished.

589. Guerriat, H. and Ittelet, M. 1982. [A survey nn the status of the Black Kite Milvus migrans in Belgium.] Aves 19: 183-191. 
590. Guichard, K.M. 1947-48. Notes on Sarothmura ayresit and three birds new to Abyssinia. Bull. Brit. Om. Club 68: 102-104.

591. Gutierrez, R.J. and Carey, A.B. (eds.). 1985. Ecology and management of the Spotted Owl in the Pacific norhiwest. United States Department of Agriculture (General Technical Report PNW-185), Oregon.

592. Haas, V., Haussler, U. and Stick, R. 1981. Turle Dove Streptopelia turtur in Kenya. Scopus 5: 128.

593. Hadden, D. 1981. Birds of the North Solomons. Papua New Guinea: Wau Ecology Institute (Handbook No. 8).

594. Haftom, S. 1971. Norges fugler. Universiteteforlaget Oslo, Norway.

595. Haig, S.M. 1992. Piping Plover, In: Poole, A., Stettenheim P. and Gill, F. (eds.) The birds of North America, no.2. Academy of Natural Sciences of Philadephia, Philadelphia and American Ornithologists Union, Washington, D.C.

596. Haig, S.M. and Oring, L.W. 1985. Distribution and status of the Piping Plover throughout the annual cycle. J. Field Om. 56: 334-345.

597. Hails, C.J. and Jarvis, F. 1987. Birds of Singapore. Times Editions, Singapore.

598. Hall, B.P. 1956. First record of the Chinese Lesser Crested Tem. Thalasseus zimmermanni, from Thailand. Bull. Brit. Om. Cluh 76:87.

599. Hall, B.P. and Moreau, R.E. 1962. A study of the rare birds of Africa. Brit. Mus. Nat. Hist. Rool) 8: $315-378$.

600. Hall, B.P. and Moreau, R.E. 1970. An allas of speciation in African passerine birds. British Museum (Natural History), London.

601. Hambler, C., Hambler, K. and Newing, J.M. 1985. Some observations on Nesillas aldabranus, the endangered brush warbler of Aldabra Atoll. with hypotheses on its distribution. Aloll Res. Bull. 290.

602. Hamel, P.B. 1986. Bachman's Warbler. A species in peril. Smithsonian Institution Press. Washington D.C

603. Hämmerli, J. 1991. Haltung und Zucht des Spix-Aras (Cyanopsilla spixii). Gefied. Freund 38: 130-145

604. Hamsch, S. 1987. Zur Biolngie der Mesitornithiformes Madagaskars. Falke 34: 160-162.

605. Hancock, J. 1989. Extinction stalks the storks of Asia. World Birdwatch 1](1): 1 .

606. Hancock, J. and Kushlan, J. 1984. The herons handbook. Croom Helm, London.

607. Hancock, J.A., Kushlan, J.A. and Kahl, M.P. 1992. Storks, ibises and spoonbills of the world. Academic Press, London.

608. Haney, J.C. 1987. First sight record of Orangebreasted Falcon for Belize. Wilson Bull. 95:314-315.

609. Haney, J.C. 1987. Aspects of the pelagic ecology and behaviour of the Black-capped Petrel (Plerodroma hasitata). Wilson Bull. 99: 153-168.

610. Hanmer, D.B. and Roseveare, M. 1989. The first record of the Shoehill (Balaeniceps rex) in Malawi. Scopus 12(3/4): 92-93.
611 Hannecan, F. 1988. Les oiseaux menacés de la Nouvelle-Caledonie et des îles proches. Pp. 143-165 In: Thibault, J.C. and Euyot, I. (eds.) Livre rouge des oiseaux menaćes des regions francaises outre-mer. Conseil International pour la Protection des Oiseaux. Saint-Cloud, France.

612. Hannecan, F. and Letocan, Y. 1980-1983. Oiseaux de Nouvelle Caledonie et des Loyautes. 2 vols. Les Editions Cardinalis, Nouméa, New Caledonia.

613. Hanson. D.A. 1982. Distribution of the Quetzal in Honduras. Auk 99: 385.

614. Harris, M. 1982. A field guide to the birds of Galapagos. Revised edition. Collins, London.

615. Harris, M.P. 1979. Population dynamics of the Flightless Cormorant Nannopterum harrisi. Ihis 122: $135-146$

616. Harrison, J.M. 1933. A contrihution to the nmithology of Bulgaria. Ibis (13) 3: 494-521, 589-611.

617. Harrison, C.S., Naughton, M.B. and Fefer, S.1. 1984. The status and conservation of seabirds in the Hawaiian archipelago and Johnston Atoll. Pp. 513-562 In: Croxall, J.P., Evans. P.G.H. and Schreiher, R.W. (eds.) Staus and consenation of the world's seabirds. (Techn. Publ. 2). Intemational Council for Bird Preservation, Cambridge, U.K.

618. Harrison, P. 1985. Seahirds an idenjification guide. Revised edition. Christopher Helm. Bromley. Kent.

619. Hart, J. 1991. Conservation of the Lear's Macaw: management of an endangered species. Pp. 48-51 In: Clinton-Eitniear, J. (ed.) Proceedings of the First Mesoamerican Workshop on the Conservation and Management of Macaws. (Misc. Publ. 1). Center for the Study of Tropical Birds, Inc.. Antonio. Texas.

620. Harvey, W.G. 1986. Twn additions to the avifauna of China, Anas angustirostris and Hippolais pallida. Bull. Brit. Om. Club 106: 15 .

621. Harvey, W.G. 1990. Birds in Bangladesh. University Press Lid., Dhaka.

622. Hasegawa. H. 1982. The breeding status of the Short tailed Albatross Diomedea albatnes, on Torishima, 1979/80 - 1980/81. J. Yamashina Inst. Ormith. 14: $16-24$.

623. Hasegawa, H. 1984. Status and conservation of seahirds in Japan. with special attention to the Shorttailed Alhatross. Pp. 487-500 ln: Croxall, J.P., Evans, P.G.H. and Schreiher R.W. (eds.) Status and conservation of the world's seabirds. (Techn. Publ. 2). International Council for Bird Preservation. Camhridge, U.K.

624. Hasegawa, H. 1991. Red Data hird: Short-tailed Albatross. World Birdwatch 13(2): 10.

625. Haverschmidt, F. 1968. Birds of Surinam. Edinburgh and London: Oliver and Boyd.

626. Haverschmidt, F. 1972. Accipiter poliogaster in Surinam. J. Om. 113: 338-339.

627. Haverschmidt, F. 1972. Bird records from Surinam. Bull. Brit. Om. Cluh 92: 49-53.

628. Haverschmidt, F. 1975. Mnre hird records from Surinam. Bull. Brit. Om. Cluh 95: 74-77. 
629. Haverschmidt, F. 1983. First record of the Litte Egret (Egretta garzella) in Suriname. Wilson Bull. 95: 315.

630. Hay, J.R., Best, H.A. and Powlesland, R.G. 1985. Kokako. John McIndoe and New Zealand Wildlife Service, Dunedin, New Zealand.

631. Hay, R. 1984. The Tuamotu Sandpiper: little known, little cared for. Farest and Bird 15(4): 17.

632. Hay, R. 1986. Bird canservation in the Pacific islands (Study Report 7). International Council for Bird Preservation, Cambridge, U.K

633. Haydock, G.L. 1954. A survey of the birds of St Helena. Osirich 25: 62-75.

634. Hayes, F.E., Goodman, S.M. and López, N.E. 1990. New or noteworthy bird records from the Matogrosense region of Paraguay. Bull. Bril. Om Club 110: 94-103

635. Hayman, P., Marchant, J. and Prater, T. 1986 Shorebirds. Croom Helm, Lnndon and Sydney.

636. Hays, C. 1984. The Humholdt Penguin in Peru. Orys 18:92-95

637. Hays, C. 1986. Effects of the 1982-83 El Niño on Humboldt Penguin colonies in Peru. Biol. Conserv. 36: $169-180$

638. Heath, M. and Long, A. 1991. Habitat, distribution and status of the Azure-rumped Tanager Tangara cabanisi in Mexico. Bird Consen. Intematn. 1: 223-254.

639. Heim de Balsac, H. and Mayaud, N. 1962 Les oiseanx du nard auest de l'Afrique, distrinhurion geagrophique. ecolagies, migratians, reproduction. Ecyclopedie ornithologique vol. 10. Paris, P. Lechevalier, 1962. France.

640. Helander, B. 1980. Fargringmarkning av havsorn - en lagesrapport. Fauna ach Flora 4: 183-187.

641. Helander, B., Olsson. M. and Reutergardh, L. 1982. Residue levels of organochlorine and mercury compunds in unhatched eggs and the relationships to breeding success in White-tailed Sea Eagles Haliaetus albicilla in Sweden. Holarctic Ecology 5: 349-366.

642. Helbig, A.J. and Wells. D.R. 1990. Steppe Eagles in Peninsular Malaysia in 1987. Dutch Birding 12: 77-79.

643. Hellenic Ornithological Society 1987. [Decrease in the population of the Lesser Kestrel.] Nature 37: 35. (In Greek.)

644. Helversen, O. von, Hodge, M. and Martens, J. 1969. Catalogus Faunae Graeciae, Pt. 2, Aves.

645. Helvoort, B. van 1987. Status and conservation needs of the Bali Starling. Bull. Orientol Bird Club 5: 9-12.

646. Henry, G.M. 1971. A guide to the birds of Ceylon. 2nd edition. Oxford University Press, London.

647. Heredia, B., Gonzalez, L.M., Gonzalez, J.L. and Alonso, J.C. 1985. La emancipacion y dispersion de los jovenes de Aguila Imperial en el Parque Nacional de Donaña. Vida Silvestre 53: 37-43.

648. Hermes, N. 1980. Endangered species. In: Haigh. C. (ed.) Endangered animals of New Sauth Wales. National Parks and Wildlife Service, Sydney.

649. Hermes, N. 1985. Birds of Norfolk Island

Wonderland Publications, Norfolk Island.
650. Hernandez-Camacho, J.1. and Rodriguez-Machecha, J.V. 1986. Status geografico y taxonomico de Malathrus armenti Cabanis 1851 (Aves: Icleridae). Caldasia 15: 655-664

651. Herremans, H., Stevens, J., Vangeluwe, D. and Soilih, A. 1990. Red Data bird: Grand Comoro Scops Owl. World Birdwatch 12(1-2): 13.

652. Hicks, J. and Yorkston, H. 1982. Notes on the breeding of the Christmas Island Imperial Pigeon Ducula whartoni. Australian Bird Watcher 9: 247-251

653. Hillgarth, N., Stewar-Cox, B. and Thouless, C. 1986 The decline of the Green Peafowl Pava numicws. In: Ridley, M. (ed.) Pheasants in Asia 1986. World Pheasant Assocjation. Basildon. U.K.

654. Hilty, S. 1985. Distrihutional changes in the Colombian avifauna: a preliminary blue list. Pp. 1000 1012 in Buckley, P.A., Foster, M.S., Morton, E.S., Ridgely, R.S. and Buckley, F.G. (eds.) Neoropical amithalagy. American Ornithologists' Union Ornithological Mnnograph No. 36. AOU, Washington D.C.

655. Hilty, S. and Brown, W.L. 1986. A guide to the birds of Colomhia. Princetnn University Press, Princeton, New Jersey.

656. Hirschfeld, E. 1991. Rare hirds in Bahrain 1989 and 1990. Om. Soc. Middle East Bull, 26: 20-25.

657. Hockey, P.A.R. 1986. The Canary Islands Expedition. Quagga 16: 22-23.

658. Hockey, P.A.R. 1987. The influence of coastal utilisation by man on the presumed extinction of the Canarian Black Oystercatcher Haemotopis meadewaldoi Bannerman. Biol. Conserv. 39: 49-62.

659. Hoffmann, T.W. 1984. National red dota list of endangered and rare birds of Sri Lanka. Ceylon Bird Club, Colombo.

660. Holland, C.S. and Williams, J.M. 1978. Observations on the birds of Antigua. American Birds 32: $1095-1105$

661. Hollom, P.A.D., Porter, R.F., Christensen, S. and Willis, 1988. Birds of the Middle East and North Africa. T. and A.D. Poyser, Calton.

662. Hoimes, D.A. 1969. Bird notes from Brunei: December 1967 - September 1968. Sarawak Mus. J. 17: 399.402 .

663. Holmes, D.A. 1977. Faunistic notes and further additions to the Sumatran avifauna. Bull. Brit. Om. Club 97: 68-71.

664. Holmes. D.A. and Burton, K. 1987. Recent notes on the avifauna of Kalimantan. Kukilo 3: 2-32.

665. Holmes, P. and Wood, H. 1980. The report of the ormithological expedition to Sulawesi. Unpublished

666. Holyoak. D.T. 1974. Undescrihed land hirds from the Cook Islands, Pacific Ocean. Bull. Brit. Om. Club 94: $145-150$.

667. Holyoak, D.T. and Seddon, M.B. 1990. Distributional notes on the birds of Benin. Malimhus 11: 128-134.

668. Holyoak, D.T. and Seddon, M.B. 1991. Notes sur la répartition des oiseaux du Niger. Alauda 59: $55-57,116-120$ 
669. Holyoak, D.T. and Thibault, J-C. 1984. Contribution à l'étude des oiseaux de Polynesie orientale. Mem. Mus. Natn. Hist. Nat. Ser. A., Zool. 127: 1-209.

670. Hoogendoom, W. and Mackrill, E.J. 1987. Audouin's Gull in southwestern Palearctic. Dutch Birding 9: $99-107$.

671. Hoogerwerf, A. 1966. Some notes on the genus Trichastomo, especially on the validity of $T$. sepiarium minus from east Java, and ahout the status of $T$. vanderbilti and $T$. liberale from northern Sumatra. Misc. Rep. Yamashine Inst. Omith. Zool. 4: 294-301.

672. Hopkinson, G. and Masterson, A.N.B. 1977. On the occurrence near Salisbury of the Whitewinged Fluftail. Haneyguide 91: 25-28.

673. Hopkinson, G. and Masterson, A.N.B. 1984. The occurrence and ecological preferences of certain Rallidae near Salibury, Zimhabwe. Pp. 425-440 ln: Ledger, J. (ed.) Proc. Fifih Pan Afr. Om. Congr. Southern African Ornithological Sociely. Johannesburg.

674. Hovel, H. 1987. Check-list of the birds of Israel with Sinai: an annolated check-list. SPNI.

675. Howell, S.N.G. 1992. The Short-crested Coquette: Mexico's least-known endemic. Birding 24: 87-91.

676. Howell. S.N.G. and Webb. S. 1990. The seabirds of Las Islas Revillagigego, Mexico. Wilson Bull. 102: $140-146$.

677. Howell, S.N.G. and Webh, S. 1992. New and noteworthy hird records from Guatemala and Honduras. Bull. Brit. Om. Cluh 112:42-49.

678. Howells, W.W. and Finn, K.J. 1979. The occurrence of Denham's Bustard at Wankie National Park and in north-west Rhodesia with notes on movements and behaviour. Honeyguide 97: 4-12.

679. Howes, J. 1988. Nordmann's Greenshank Tringa gutifer: status and threats. Asian Welland News 1: 12.

680. Howes, J. and Lamber, F. 1987. Some notes on the status, field identification and foraging characteristics of Nordmann's Greenshank. Woler Sudy Group Bull. 49: 14-17.

681. Howes, J.R. and NPWO 1986. Evaluation of Saran'ak wetlands and their intportance to waterbirds. Repon 3. Palau Bruit. Asian Wetland Bureau. Kuala Lumpur.

682. Hudec, K. 1972-1983. Fauma CSSR, Svazek 24 PlaciAves. 3 vols. Academia Nakladarelstvi Ceskoslovenské Akademie Ved, Praha.

683. Huizinga, M. 1984. Bolle's Laurel Pigeon and Laurel Pigeon on La Palma. Dutch Birding 6: 134-135.

684. Hulten, M. and Wassenich, V. 1960-1961. Die Vögelfauna Lurembourgs. Victnr Buck, Luxemhourg.

685. Hunter, L.A. 1988. Status of the endemic Altlan Grebe of Guatemala: is it extinct? Conder 90: $906-912$.

686. Hussain. S.A. 1984. Some aspects of the biology and ecology of Narcondam Homhill (Rhyliceros narcondami). J. Bombay Nal. Hist. Soc. 81: 1-18.

687. Hutchinson, C.D. 1989. Birds in Ireland. Poyser. Calton, U.K.
688. Hutson, A.M. 1975. Observations on the birds of Diego Garcia. Chagos Archipelago. Aloll Res. Bull. 175 .

689. lapichino, C. and Massa, B. 1989. The birds of Sicily: an annotated checklist. B.O.U. Check-list No. 11. British Omithologists' Union, London.

690. Jjsselstein, C. 1992 . Report on the censusing of the St Lucia White-breasted Thrasher, Ramphocinclus brachyurus sanctaeluciae. 6 August 1992. Unpuhlished.

691. Imher, M.J. 1987. Breeding ecology and conservation of the Black Petrel (Procellaria parkinsoni). Notomis 34: 19-39.

692. Imher, M.J. 1991. The Jamaican Petrel - dead or alive? Gosse Bird Club Broadsheet no. 57: 4-9.

693. Imher, M.J., Cruz, J.B., Grove, J.S., Lavenherg, R.J., Swift, C.C. and Cruz, E. 1992. Feeding ecology of the Dark-rumped Petrel in the Galápagns Islands. Condor 94: 437-447.

694. Ingels, J., Parkes, K.C. and Farrand. J. 1981. The status of the macaw generally hut incorrectly called Ara caninde (Wagler). Gerfau 71: 283-294.

695. Inskipp, C. 1989. Nepal's forest birds: their status and conservation. International Council for Bird Preservation (Monograph No, 4.). Cambridge, U.K.

696. Inskipp, C. and Collar, N.J. 1984. The Bengal Flnrican: its conservation in Nepal. On:x 18: 30-35.

697. Inskipp, C. and Inskipp, T. 1983. Results of a preliminary suney of Bengal Floricans Houbaropsis bengalensis in Nepal and India, 1982. (Study Report 2). Intemational Council for Bird Preservation. Camhridge, U.K.

698. Inskipp. C. and Inskipp. T. 1985. A survey of Bengal Floricans in Nepal and India, 1982. Bustord Sudies 3: $141-160$.

699. Inskipp, C. and Inskipp. T. 1986. Birds and forests in Nepal. Forkail 1: 53-64.

700. Inskipp, C. and Inskipp, T. 1991. A guide in the birds of Nepal. 2nd ed. Christopher Helm.

701. Irwin. M.P.S. 1982. The birds of Zimhahwe. Salisbury Quest Puhlishing. Harare.

702. Isenmann. P. 1979. Wo uherwinten der Seggenrohrsanger (Acrocepholus paludicola) Beitr. Vogelkd. 25: 366-367.

703. Ishihara, T. 1986. (The Amami Ground Thrush distinct from White's Ground Thrush.) Strix 5: 60-61. (In Japanese.)

704. Islam, K. and Crawford, J.A. 1985. Brood habilat and roost siles of Western Tragopans in northeastern Pakistan. World Pheasant Assoc. J. 10: 7-14.

705. Islam, K. and Crawford, J.A. 1986. Summary of Western Tragnpan Project in Pakistan with recommendations for conservation of the species. In: Ridley. M. (ed.) Pheasants in Asia 1986. World Pheasant Association, Basildon, U.K.

706. Isler, M.L. and Isler. P.R. 1987. The tanogers: natural hisiory, distihution and identification. Smithsnnian Institution Press, Washington D.C. 
707. Jackson, F.J. and Sclater, W.L. 1938. The birds of Kenya Colony and the Ugandan Protectorate. Gurney and Jackson, London.

708. Jackson, H.D. 1973. The Cape Eagle Owl Bubo capensis in Moçambique. Bull. Brit. Om. Cluh 93: 10.

709. Jackson, J.A. 1986. Biopolitics, management of federal lands, and the conservation of the Redcockaded Woodpecker. Amer. Birds 40: 1162-1168.

710. Jackson, J.A. 1991. Will-o'-the-Wisp. Living Bird $Q$. 10: 29-32.

711. Jacob, J-P. and Courbet, B. 1980. Oiseaux des mer nicheurs sur la côte Algerienne. Gerfaut 70: 385-401.

712. Jacob, J-P., Lafontaine, R.-M., Chiwy, B., Devillers, P., de Visscher, N.-M. and Ledant, J.-P. 1985. Fiches d'information sur les espèces enumerées dans l'annexe 1 de la directive 79/409/CEE. Unpublished report to EEC.

713. Jahncke Aparicio, J. and Riveros-Salcedo, J.C. 1991 Hábitos de anidación del Potoyunco Peruano Pelecanaides gamotii. Resúmenes del IV Congreso de Ornitologís Neotropical, 3-9 de Noviembre 1991. Quito, Ecuador (resumen 20).

714. Jahrsdoerfer, S. 1990. Endangered and threatened wildlife and plants; final rule to list the Goldencheeked Warbler as endangered. Federal Register 5.5 (no.249, December 27): 53153-53160.

715. Jaksic, F.M. and Jimenez, J.E. 1986. Conservation status of raptors in Chile. Birds of Prey Bull. No 3.

716. James, P.C. 1984. The status and cnnservation of seabirds in the Mediterranean Sea. Pp. 371-375 In Croxall, J.P., Evans, P.G.H. and Schreiter, R.W. (eds.) Status and conservation of the world's seahirds. (Techn. Puhl. 2). International Council for Bird Preservation, Cambridge, U.K.

717. Jang, H. 1989. Baer's Pochard in Pakistan. J. Bombay Nat. Hist. Soc. 86: 96-97.

718. Jaramillo, A. 1992. Eskimo Curlew: a glimmer of hope. Birders J. 1: 202.

719. Jarry, C. and Larigauderie, F. 1974. Notes faunistiques sur quelques oiseaux du Senegal. Oisean 44: $62-71$.

720. Jeggo, D.F. 1990. Preliminary international studhook: St Vincent Parrot Amazona guildingii. Unpublished.

721. Jeggo, D.F. 1991. Captive hreeding and the conservation of the S1 Lucia Parrot Amazona versicolor and St Vincent Partot A. guildingii. Pp. 18-28 In: Colley, R. (ed.) Parrots in captivity. Assnciation of British Wild Animal Keepers, Bristol.

722. Jeggo, D.F. and Anthony, D. 1991. A repont nn the 1990 field survey of the St Lucia Parrot Amazona versicolor. Dodo 27: 102-107.

723. Jeggo, D.F., Anthony, D. and John, L. 1988. A report on the 1988 survey of the St. Lucia Parmt Amazona versicolor. Dodo 25: 24-30.

724. Jehl, J.R. 1972. On the cnld trail of an extinct petrel. Pacific Discovery 25(6): 24-29.

725. Jehl, J.R. 1982. The biology and ta xonomy of Townsend's Shearwater. Gerfaut 72: 121-135.
726. JehI. J.R. 1983. Can the Socnrro Dove and Socorro Mockinghird be saved? Pp. 5-9 In: Proceedings lof the/ Jean Delacour/IFCB Symposium on breeding birds in captiviry. International Foundation for the Conservation of Birds, Nonth Hollywood, California.

727. Jehl, J.R. 1984. Comings and goings on a desert isle. Natural History 93(2): 6-12.

728. Jehl, J.R. and Everett, W.T. 1985. History and status of the avifauna of Isla Guadalupe, Mexico. Trans. San Diego Soc. Nat. Hist. 20(17): 313-336.

729. Jehl, J.R. and Parkes, K.C. 1982. The status of the avifauna of the Revillagigedns Islands, Mexico. Wilsan Bull. 94: 1-19.

730. Jehl. J.R. and Parkes, K.C. 1983. "Rcplacements" of landbird species on Socorro Island, Mexico. Auk 100: 551-559

731. Jenkins, J.M. 1983. The native forest birds of Guam. American Ornithnlngists' Union Omithnlogical Monograph 31. AOU. Washington D.C.

732. Jenkins, J.M. and Agucn, C.F. 1981. Status of candidate endangered bird species on Saipan. Tinian and Rola of the Mariana Islands. Micronesica 17: 184-186.

733. Jennings, M.C. 1981. The birds of Saudi Arabia: a check-list. Published hy the author. Whittlesford, Cambridge.

734. Jennings, M.C., Heathcote, P.C., Parr. D. and Baha el Din. S.M. 1985. Omithological suncy of the Ras Dit area and the islonds at the mouth of the Gulf of Suez, Egypt. Report prepared for B.P. Petroleum Development Co. (Egypt) Lid.

735. Jenny, 3.P. and Burnham, W.A. 1987. Preliminary observations on the hiology of the Orange-breasted Falcon (Falco deiroleucus). III Congress de Ornitolngia Neotropical: Resumenes. Unpublished.

736. Jenny. J.P. and Cade. T.J. 1986. Ohservations on the binlogy of the Orange-hreasted Falcon Falco deirolewcus. Wilson Bull. 3: 119-124.

737. Jensen, A. 1991. Forest future in the halance. World Birdwatch 13(4): 10-11.

738. Jessop, A.E. and Reid, T. 1986. Winter surveys of the Orange-hellied Parrot Neophema chrysogaster in Victoria, 1984 and 1985. Rnyal Australasian Omitholngists' Union. Victoria.

739. Ji Jia-yi and Yu Xin-jian 1990. [The numerical distribution of storks and cranes in Shandong Province.| Znol. Res. 11: 46. (In Chinese.)

740. Johnsgard, P.A. 1983. The huniminghirds of North Americo. Smithsonian Institution Press. Washington D.C.

741. Johnsgard. P.A. 1983. Cranes of the world. Croom Helm, London and Canherra.

742. Johnsgard. P.A. 1986. The pheasants of the world. Oxford University Press, Oxford.

743. Johnsgard, P.A. 1986. Plovers, sandpipers and snipes of the world. University of Nehraska Press, Lincoln and London.

744. Jnhnsgard, P.A. 1988. The quails, partridges and francolins of the world. Oxford University Press, Oxford. 
745. Johnsgard. P.A. 1991. Bustards, hemipodes and sandgrouse: birds of dry places. Oxford University Press, Oxford.

746. Johnson, A. and Chebez, J.C. 1985. Sobre la situacion de Mergus actosetaceus Vieillot (Anseriformes:

Anatidae) en la Argentina. Historia Natural Supl. no. 1: 1-16.

747. Johnson, A.W. 1967. The birds of Chile. Volume 2. Platt Estahlecimientos Graficos S.A., Buenos Aires.

748. Johnson, E.D.H. 1972. Observations on the birds of the Upper Blue Nile Basin. Bull. Bril. Om. Club 92: 42-49.

749. Johnson, G. and Smiddy, P. 1989. Black Stork in Co. Dublin - a species new to Ireland. Irish Birds 4: 69-70.

750. Johnson, T. 1987. Red Data bird: White-breasted White-eye. World Birdwatch 9(3): 5.

751. Johnson, T. 1988. Caribhean island paradises threatened. World Birdwatch 10(3/4): 6-7.

752. Johnson, T.B., Snyder, N.F.R. and Franks, M.A. 1991. Thick-billed Parrot Reintroduction Project Progress Report of Decemher 23, 1991. Arizona Game and Fish Department. Unpublished.

753. Johnstone, G.W. and Kerry, K.R. 1976. Omithological observations in the Australian sector of the Southem Ocean. Pp. 725-738 in: Proceedings of the 16th Omithological Congress. Australian Academy of Science, Canberra.

754. Jones, C.G. 1987. The larger land-birds of Mauritius. Pp. 208-300 ln: Diamond A.W. (ed.) Studies of Mascarene lsland birds. Canhridge University Press. Cambridge, U.K.

755. Jones, P.J. and Tye. A. 1988. A survey of the avifauna of Sao Tome and Principe. (Study Repor 24). International Council for Bird Preservation. Cambridge, U.K

756. Jouventin, P., Stahl, J.C., Weimerskirch, H. and Mougin, J.L. 1984. The seahirds of the French subantarctic islands and Adelie Land, their status and conservation. Pp. 609-625 In: Croxall, J.P., Evans, P.G.H. and Schreiber. R.W. (eds.) Stains and conservation of the world's seabirds. (Techn. Puhl. 2). International Council for Bird Preservation. Cambridge, U.K

757. Jözsef, P. 1980. Magyar Marlánani Bibliografia.

758. Juana, E., de, and Varela, J. 1987. Audouin's Gull, Chafarinas, 1987. Seahird Group Newsletter 50: 16.

759. Juana, E., de, Varela, J. and Wilt, H.-H. 1984. The conservation of seahirds at the Chafarinas islands. Pp. 363-370 ln: Croxall, J.P.. Evans, P.G.H. and Schreiber, R.W. (eds.) Stotus and conservation of the world's seabirds. (Techn. Puhl. 2). International Council for Bird Preservation, Cambridge, U.K.

760. Juniper, T. 1990. A very singular hird. $B B C$ Wildlife $8=674-675$.

761. Juniper, T. 1990. Spix's Macaw - a lone survivor. World Birdwatch 12(3): 13.

762. Juniper, T. 1991. Last of a kind. Birds Intemotn. 3: $10-16$.

763. Juniper, T. and Yamashita, C. 1990. The conservation of Spix's Macaw. Onyx 24: 224-228.
764. Juniper, A.T. and Yamashita, C.1991. The habitat and status of Spix's Macaw Cyanopsina spirii. Bird Conserv. Intematn. 1: 1-9.

765. Jurek, R.M. 1990. An historical review of Califomia Condor recovery programmes. Vulure New' 23: 3-7.

766. Karim. F. 1985. A note on the disappearance of the Bengal Florican from Bangladesh. Bustard Studies 3: 161-162.

767. Karl, B.J. and Best, H.A. 1982. Feral cats on Stewart Island; their foods, and their effects on Kakapo. New Zealand J. Zool. 9: 287-294.

768. Kasparek, M. 1989. Status and distrihution of the Great Bustard and the Little Bustard in Turkey. Bustard Sindies 4.

769. Kasparek. M. and van der Ven, J. 1983. Birds of Turkey. 1: Ercek Golu. Published hy the authors, Heidelhurg.

770. Kebede, E. and Hillman, J.C. 1989. First recorded breeding of Greater Flamingns Phoenicoptents nuber roseus in Ethiopia. Flamingo Res. Group Newsl. $5: 7$.

771. Keller, C. 1992. Em busca da ararinha de Spix. Atualidades Omiunlógicas 45: 5 .

772. Kelly, S.T. and DeCapita, M.E. 1982. Cowhird control and its effect on Kirtland's Warbler reproductive success. Wilsan Bull. 94: 363-365.

773. Kemp. A.C. 1980. The hinds of prey of southem Africa. Winchester Press, Johanneshurg.

774. Kemp, A.C. 1980. The importance of the Kruger National Park for hird conservation in the Republic of South Africa. Knedne 23: 99-122.

775. Kemp. A.C. 1988. The systematics and zoogeography of Oriental and Australasian hombills (Aves: Bucerotidae). Bonn. Znol. Beitr. 39: 315-345.

776. Kemp. A.C. 1989. Estimation of hinlngical indices for little-known African owls. Pp. 441-449 In: Meyburg, B.-U. and Chancellor. R.D. (eds.) Raptors in the modern workt. Proceedings of the 111 World Conference on Birds of Prey and Owls, Eilat, Israel, 22-27 March 1987. World Working Group on Birds of Prey and Owls, Berlin.

777. Kemp. A.C. and Crowe, T.M. 1985. The systematics and zoogeography of Afrotropical hornhills (Aves: Bucerotidae). Pp. 279-324 In: Schuchmann. K.L. (ed.) Proceedings of the Intemational Sympasium on African Vertehrates. Zoologisches Forschungsinstitut und Museum Alexander Konig. Bonn.

778. Kennerley, P.R. 1987. A survey of the birds of the Poyang Lake Nature Reserve, Jiangxi Province, China, 29 December 1985 - 4 January 1986. Hong Kong Bird Report 1984/1985:97-111.

779. Kennerley, P.R. and Bakewell, D.N. 1987. Nordmann's Greenshank in Hong Kong: a review of the identification and status. Hong Kong Bird Report 1986: 83-100

780. Kerley, G.1.H. 1986. Jackass Penguins harmed in South Africa oil spills. World Birdwatch 8(2); 4 .

781. Khan, M.A.R. 1982. Wildlife of Bangladesh - o checklist. 
782. Khan, M.A.R. 1986. The threatened White-winged Wood Duck Cairina scutulata in Bangladesh. Forksail 2: 97-10].

783. Kibler, L.F. 1950. Notes on the birds of Guam. Auk 67: $400-403$

784. Kiff, L. 1990. To the brink and back: the battle to save the California Condor. Terra 28(4): 6-18.

785. King, B. 1985. Wild sighting of Hume's Pheasant in Burma. World Pheasant Association News 8: 21 .

786. King, B. 1986. Repon on pheasants at Jiuzhaignu and Baihe Panda Reserves in north-west Sichuan, snuthern China. World Pheasant Association News 11: 20-22.

787. King, B. 1987. Some notes on the birds of the Yi Shan area of NW Jiangxi Province, China. Hang Kang Bird Repart 1984-1985: 115-119.

788. King, B. 1987. Wild sighting of Cahot's Tragopan. Warld Pheasant Associotion News 18: 21-23.

789. King. B. 1987. The Waterfall Swift Hydrachous gigas. Bull. Brit. Om. Club 107: 36-37.

790. King, B. 1987. Some ohservations at Pangquanguo Reserve in west central Shanxi Province in NE China Hang Kong Bird Repon 1984/1985: 112-114

79]. King, B. 1990. Hieraeetus morphnoides on Halmahera. Kukila 5: 77

792. King, B.F., Dickinson, E.C. and Woodcock, M W. 1975. A field guide to the hirds of Somih-East Asia Collins, London.

793. King. W.B. 1974. Conservation status of hirds of central Pacific islands. Elepoio 34: 70-74.

794. King, W.B. 1981. Endangered hirds of the world: the ICBP Bird Red Dato Book. Smithsonian Institution Press and International Council for Bird Preservation. Washington D.C.

795. Kitson, A.R. 1980. Lanis relictus - a review. Bull. Brit. Om. Club 100(3): 178-185.

796. Klapste, J. 1982. Bat Hawk nn Celehes in Decemher 1981. Dutch Birding 4: 29-30.

797. Knoder, C.E. 1983. Elliot's Pheasant conservation. World Pheasant Assoc. J. 8: 11-28

798. Kohalmy, T. 1987. Data on the conditins of the eastern subspecies of Great Bustard (Otis tardo dybowskii Tacz. 1874) In: Farago, S. (ed.) The Gireat Bustard (Otis tarda L.). Proceedings of the syanosium in Budapest on June 2nd 1987. International Council for Game and Wildlife Conservation. Budapest.

799. Kolosov, A.M. (ed.). 1983. |Red data baok of the RSFSR: animals.] Rossel 'khozizdat', Mosenw. (In Russian.)

800. Konrad, P.M. 1981. Status and ecology of the Wattled Crane in Africa. Pp. 220-237 ln: Lewis, J.C. and Masatomi, H. (eds.) Crane research around the world. Robinson Press, Fon Collins, Colorado.

801. Kooiman. J.G. 1940. Mededeelingen over het voorkomen in Oostjava voor dit gewsel nog niet in de literatuur genoemde vogels. Ardea 29: 98-108.

802. Koschmann, J.R. and Price, P.L. 1987. The Thickbilled Parrot. AFA Wotchbird 14(1): 48-53.
803. Koster, S.H. and Gretlenherger J.F. 1983. A preliminary survey of the birds of Park W, Niger. Malimhus 5(2): 62-72.

804. Krahbe, N. 1984. An additional specimen of the Swallow-tailed Colinga Phibalura Jlavirostris boliviana. Bull. Brit. Om. Cluh 104: 68-69.

805. Krupa, R.E. 1989. Social and biological implications for endangered species management: the Philippine Eagle Pithecophaga jefferyi. Pp. 301-314 In: Meyhurg, B.-U. and Chancellor, R.D. (eds.) Raptors in the modem world. Proceedings of the III World Conference on Birds of Prey and Owls, Eilat, Israel, 22-27 March 1987. World Working Group on Birds of Prey and Owls, Berlin.

806. Kulkarni, B.S. 1981. Ecology and hehaviour of Great Indian Bustard (Family Otididae). J. Bambay Nat. Hist. Soc. 78: 375-377.

807. Kumerlneve, H. 1962. Zur Kenntnis der Avifauna Kleinasiens. Bonner Znologische Beiträge 12: 1-318.

808. Kumerloeve, H. 1962. Notes on the hirds of the Lebanese Republic. Iraq Natural History Museum Puhlications 20 and 21.

809. Kumerloeve. H. 1967-1969. Recherches sur l'avifaune de la République arabe syrienne. Alanda 35: 243-266: 36: 1-26, 190-207; 37: 43-58, 114-134, 188-205.

810. Kumerloeve, H. 1984. The Waldrapp, Geronticus eremira (Linnaeus. 1758): historical review, taxonomic history, and present status. Biol. Conserv. 30: $363-373$.

811. Kuroda. N. 1933-1936. Birds of the island of Java. Published by the author. Tokyo.

812. Kyllingstad, H.C. 1986. A record of Bald this from the Sinai Mountains. O.S.M.E. Bull. 17: 1-2.

813. LaBastille. A. 1990. And now they are gone. Intematn. Wildlife 20(4): 18-23.

814. Labinger, Z., Gorney, E. and Parslow, R. 1991. First record of Shikra Accipiter hadius in Israel. Sondgrouse 13: $46-49$

815. La Cock. G.D. 1986. The Southern Oscillation. environmental anomalies. and mortality of two southern African seabirds. Climotic Change 8: $173-184$

816. La Cock, G.D., Duffy. D.C. and Conper. J. 1987. Population dynamics of the African Penguin Spheiscus demersis at Marcus Island in the Benguela Upwelling ecosystem: 1979-85. Biol. Conserv. 40: 117-126.

817. La Touche, J.D.D. 1925-1934. A handbonk of the birds of eastem China. Taylor and Francis, London.

818. Lamanı, F. and Puzanov, V. 1962-1963. [Checklist of the birds of Albania.| Bull. U. Sh. Tirane Ser. Shk. Nat. 16(3): 87-102; 16(4): 100-117; 17 (1): 96-99; 17(2): 122-125.

819. Lamarche, B. 1980-1981. Liste commentée des oiseaux du Mali. Malimhus 2: 121-158:3(2): 73-102.

820. Lamarche, B. 1987. Liste conmentée des oiseaux de Mauritanie. Privately puhlished, Nouakchout.

821. Lamarche, B. 1988. Liste commentée des oisearux de Mauritanie. Supplément No. I. Privately puhlished, Nouakchott. 
822. Lamber, F. 1983. Report of an expedition to survey the status of the St Vincent Parrot Amazona guildingii. (Sudy Report 3). International Council for Bird Preservation, Cambridge, U.K.

823. Lambert. F. 1985. The St Vincent parrot, an endangered Caribbean bird. Oryx 19:34-37.

824. Land, H.C. 1970. Birds of Guatemala. Livingston Publishing, Wynnewood, Pennsylvania.

825. Langrand, O. 1987. Distribution, status and conservation of the Madagascar Fish-eagle Haliaeelus vociferoides Desmurs, 1845. Biol. Conserv. 42: 73-77.

826. Langrand, O. 1991. Guide to the birds of Madagascar Yale University Press. New Haven and London.

827. Lane, B. 1987. Shorebirds in Australia. Nelson Publishers, Melhoume.

828. Langrand, O. and Meyhurg, B.-U. 1984. Range, status and biology nf the Madagascar Sea Eagle Halioeelus vociferoides. Pp. 269-278 In: Meyburg, B.-U. and Chancellor, R.D. (eds.) Roptors in the modem world. Proceedings of the III World Conference on Birds of Prey and Owls, Eilat, Israel, 22-27 March 1987. World Working Group on Birds of Prey and Owls. Berlin.

829. Langrand, O. and Meyburg, B-U. 1984. Birds of prey and owls in Madagascar: thair distribution. status and conservation. In: Mendelsohn. J.M. and Sapsford. C.W. 1984. Proceedings of the Second Symposium on African Predatory Birds, 22-26 August 1983. Natal Bird Club, Durhan, South Africa.

830. Lanning, D.V. 1982. Survey of the Red-fronted Macaw (Ara nubrogenys) and Caninde Macaw (Ara caninde) in Bolivis, December 1981 - March 1982. Unpublished.

831. Lanning, D.V. 1991. Distribution and breeding biology of the Red-fronted Macaw. Wilson Bull. 103: $357-365$.

832. Lanning, D.V. and Shiflell, J.T. 1981. Status and nesting ecology of the Thick-hilled Parrot (Rhynchopsiua pachyrhyncha). Pp. 393-401 In: Pasquier, R.F. (ed.) Conservation of New World parrots. (Techn. Puhl. 1). International Council for Bird Preservation, Smithsnnian Institution Press, Washington D.C.

833. Lanning, D.V. and Shiflett, J.T. 1983. Nesting ecology of Thick-billed Parrots. Condor 85: 66-73

834. Lansdown, R. 1990. Little-known Oriental bird: Chinese Egret. Oriental Bird Club Bull. 11: 27-30.

835. Lanyon, S.M., Stolz, D.F. and Willard, D.E. 1990. Clytoctantes atrogularis, a new species of anthird from western Brazil. Wilson Bull. 102: 571-580.

836. Lara, O.F. 1987. Notas reproductivas de Jabint mycteria, una cigueña en peligro de extinction. Flora, Fawaa y Areas Silvesires 4(2): 35.

837. Laubmann. A. 1939-1940. Die Vögel von Paraguay. Wissenschaftiche Ergebnisse der Deutschen GranChaco Expedition. 2 vols. Strecker and Schroder, Stuttgar.

838. Lavers, R. and Mills, J, 1984. Tokahe. John McIndoe and New Zealand Wildlife Service. Duncdin. New Zealand.
839. Lawson, P.W. and Lanning, D.V. 1981. Nesting and status of the Maroon-fronted Parrot (Rhyrchopsina terrisi) Pp.385-392 In: Pasquier, R.F. (ed.) Consenation of New World parrots. (Techn. Publ. 1). International Council for Bird Preservation, Smithsonian Institution Press, Washington D.C.

840. LeCroy, M., Peckover, W.S., Kulupi, A. and Manseima, J. 1984. Bird observations on Nomanby and Fergusson, D'Entrecasteaux Islands, Papua New Guinea. Baroko, PNG: Division of Wildlife (Wildlife in Papua New Guinea 83/1).

841. Ledant, J.-P. Jacob, J.-P., Jacobs, P., Malher, F., Ochando, B. and Roché, J. 1981. Mise à jour de l'avifaune algérienne. Gerfout 71 : 295-398.

842. Ledger, J. 1985. Egyptian extinction. Quagga 12:10-12.

843. Le Grand, G. and Funado, D. 1982. Omithological hiblingraphy of the Azores. Bol. Mus. Munic. Funchal 34: 142-190.

844. Lelliott, A.D. 1981. Cheer Pheasants in west-central Nepal. World Pheasant Assoc. J. 6:89-95.

845. Lever, C. 1987. Naluralized birds of the world. Longman Scientific and Technical. Harlow, U.K.

846. Lewis, R.E. 1986. A rain-forest raptor in danger. Oryx 20: 170-175.

847. Lewis, A. and Pomeroy, D.A. 1988. A hird allas of Kenya.

848. Liedel, K. 1982. Verhreitung und Ockoingie des Steppenschlammlaufers, Limnodronuts semipalmalts (Blyth). Ann. Om. 6: 147-162.

849. Liefde, M. van. 1992. Status of the West Indian Whistling Duck in the Cayman Islands. IWRB Threatened Waterfowl Research Group Newsletter 2: $11-12$.

850. Ligon, J.D., Stacey. P.B., Connor, R.N., Bock, C.E. and Adkisson, C.S. 1986. Report of the American Onithologists' Union Committee for the conservation of the Red-cockaded Woodpecker. Auk 103: 848-855.

851. Lindsey, G.D. and Arend, W.J. 1991. Radin-tracking Puerto Rican Parrots: assessing triangulation accuracy in an insular rain forest. Carih. J. Sci. 27: 46-53.

852. Lindsey, G.D., Arendt, W.J., Kalina, J. and Pendleton, G.W. 1991. Home range and movements of juvenile Puerto Rican Parrots. J. Willt. Mgmt. 55: 318-322.

853. Lippens, L. and Wille, H. 1972. Aulas des oiseaur de Belgique el d'Europe occidentale.

854. Lippens. L. and Wille. H. 1976. Les oiseoux du Zaire. Lannoo, Tielt, Belgium.

855. Lisle, M. de. 1956. Sur une observation de Balaeniceps rex au Cameroun. L'Oiseau 26: 1-3.

856. Lobkov, E.G. and Neufeldt, 1.A. 1986. Distrihution and hiolngy of the Steller's Sea Eagle - Haliaeetus pelagicus pelagicus (Pallas). Proc. Zool. Inst. Acad. Sci. USSR 150: 107-146.

857. Long, J.L. 1981 . Introduced birds of the warld. David and Charles. Newton Ahhot.

858. Longmore, N.W. 1985. A Sydney specimen of Neodrepanis huypoxantha (Philepittidae). Bull. Brit. Om. Cluh 108: 43-50. 
859. Lowette, M. 1981. The birds of Cameroon: an annotated checklist. Verhandelingen Koninklijke Academie. Brussel (kl. Wet) 43(163): 1-295.

860. Louette, M. 1987. A new weaver from Uganda? Ibis 129: 405-406.

861. Lowette, M. 1988. Additions and corrections to the avifauna of Zaire (2). Bull. Brit. Om. Club 108: 43-50.

862. Louette, M., Stevens, J., Bijnens, L. and Janssens, L. 1986. Comoro Islands endemic bird survey. International Council for Bird Preservation, final report (30 April 1986).

863. Love, J.A. 1983. The return of the Sea Eagle. Cambridge University Press, Cambridge, U.K

864. Lovel, T.W.I. 1976. The present status of the Congo Peacock. J. World Pheasant Assoc. I: 48-57.

865. Lovenskold, H.L. 1964. Avifauna Svalbardiensis. Norsk Polarinstituut Shrifter 129:1-460.

866. Low, R. 1986. Parrots: their care and breeding. 2nd edition. Blandford Press, Dorset.

867. Lowe, D.W., Matthews, J.R. and Moseley, C.J.. (eds.). 1990. Puerto Rican Parrot. Amazona vilsasa. Pp. 565-567 In: The official World Wildlife Fund guide to endangered species of North America.

868. Lowe, D.W., Matthews, J.R. and Moseley, C.J., (eds.). 1990. Piping Plover, Charadrius melodss. $\mathrm{Pp}$. 590-593 in The official World Willije Fund guide to endangered species of North America.

869. Lowe, D.W., Mathews, J.R. and Mnseley, C.J., (eds.). 1990. Thick-billed Parrot, Rhynchopsisa pachyrhyncha. Pp. 692-693 In: The afficial World Wildlife Fund guide to endangered species of North America

870. Loyn, R.H., Lane, B.A., Chandler, C. and Carr. G.W. 1986. Ecology of Orange-bellied Parrots Neophema chrysogaster at their main remnant wintering site. Emu 86: 195-206.

871. Lu Tai-chun, He Fen-qi and Liu Ru-sun 1986. The Chinese Monal Lophophores thrysii: studies of its ecology and biology. In: Ridley, M. (ed.) Pheasants in Asia 1986. World Phasant Association, Basildon, U.K.

872. Lucca, E.R de. 1992. El Aguila Coronada Harpyhaliaerus coronaus en San Juan. Nuestras Aves 26: 25 .

873. Ludlow, F. 1937. The birds of Bhutan and adjacent territories of Sikkim and Tibet. Ibis (14) I: 1-46, 249-293, 467-505.

874. Ludlow, F, and Kinnear, N. 1944. The birds of Southeastern Tibet. Ibis 86: 43-86, 176-208, 348-389.

875. Luthin, C.S. 1987. Rare visit of Oriental Crested Ibis. Flying Free 5(2): 4-5.

876. Luthin, C.S. 1987. Status and conservation priorities for the world's stork species. Colonial Waterbirds 10: 181-202.

877. Luthin, C., Frederick, P. and Morales, G. 1988. The Scarlet Ibis (Eudocimus ruber): status, conservation and recent research.

878. Machado, R.B. and Brand1, A. 1990. Projeto ararinha azul: relatório final. Belo Horiznnte: Fundaçãn Biodiversitas, unpublished.
879. Mackinnon, J. 1983. Methods for the conservation of Oku Mauntain Forest, Cameroon. (Study Report 15). International Council for Bird Preservation, Camhridge, U.K.

880. MacKinnon, J. 1988. Field guide to the birds of Java and Bali. Gadjah Maja University Press, Yogyakarta, Indonesia.

881. Mackinnon, J. and Wind, J. 1980. Birds of Indonesia UNDP/FAO National Parks Develnpment Project, Bogar, Indonesia.

882. Mackworth-Praed, C.W. and Grant, C.H.B. 1957. Birds of eastern and north-easterm Africa. Volume 1. Longmans, London.

883. Mackworth-Praed, C.W. and Grant, C.H.B. 1962 Birds of the southem third of Africa. Volume 1. Longmans, London.

884. Mackworth-Praed, C.W. and Grant, C.H.B. 1973. Birds of central and western Africa. Series 3. Volume 2. Longman, Jpswich.

885. Maclean, G.L. 1985. Roherts' birds of southem Africa. Trustees of the John Voelcker Bird Book Fund, Cape Town.

886. Macleod, H. 1985. Cameronn. World Birdwalch $7(4): 4-5$

887. Macleod, H.L. 1987. The conservation of Oku Mountain Forest, Cameroon. (Study Report 15). International Council for Bird Preservation. Cambridge, U.K.

888. Macleod, H. and Parrntt, J, 1991. Red Data bird: Bannerman's Turaco. World Birdwatch 13(4): 12.

889. Madge, S. and Burn, H. 1988. Wildfowl. Christopher Helm. London.

890. Magalhaes, J.C.R. de, 1978. Especies cinegelicas e protecao a fauna na regiao sudeste, com especial referencia ao estade de Sao Paulo. Pp. 62-67 In: Seminario sobre caca amadorista. Fundacao Brasileira para a Conservaçān da Natureza. Rio de Janeiro.

891. Magnin, G. 1991. A record nf Brown Fish Ow! Ketupa zeylonensis from Turkey. Sandgrouse 13: $42-44$.

892. Magrath, R.D., Ridley, M.W. and Woinarski, J.Z. 1985. Status and hahitat requirements of Lesser Floricans in Kathiawar, western India. Bustard Sudies 3: 185-193.

893. Makatsch. W. 1981. Verzeichnis der Vogel der Deutschen Demakratischen Repuhlik. Neumann Verlag, Leiprig-Radebeul.

894. Malhrant, R. 1952. Faune du centre africoin français. 2nd edition. Lechevalier, Paris

895. Malhrant, R, and Maclatchy, A. 1949. Faune de l'equateur africain français. 1. Oiseaux. Lochevalier, Paris.

896. Mann, C.F. 1986. Christmas Island Frigatehirds Fregara andrewsi on the Kenya cnast. Bull. Brit. Om. Cluh 106: 89-90.

897. Mann. C.F. 1987. A checklist of the birds nf Brunei Darussalam. Bnunei Museum Joumal 6: 170-212.

898. Mann, C.F. 1988. Bird teport for Brunei Darussalam, July 1986 to June 1988. Brinei Mus. J. 6: 88-111. 
899. Manry, D.E. 1985. Distribution, abundance and conservaation of the Bald Ibis Geronticus calvus in southern Africa. Biol. Conserv. 33: 351-362.

900. Manry, D.E. 1985. Reproductive performance of the Bald Ibis Geronticus calvus in relation to rainfall and grass-buming. Ibis 127: 159-173.

901. Mansoori, J. 1985. The status of the Houbara Bustard in Iran. Bustard Studies 3: 97-100.

902. Marchant, S. and Higgins, P. 1991. The handbook of Australian, New Zealand and Antarctic birds. Volume 1: Emus to ducks. Royal Australasian Omithologists Union.

903. Marcus, M.J. 1983. Additions to the avifauna of Honduras. Auk 100: 621-629.

904. Marion, L. and Marion, P. 1982. [The Spoonhill Platalea leucorodia nests at the Lac de Grand-Lieu, France.] Alauda 50: 241-249.

905. Marle, J.G. van and Voous, K.H. 1988. The birds of Sumatra. British Ornithologists Union Check-list 10. $\mathrm{BOU}$, London.

906. Marquiss, M. and Newton, I. 1982. The Goshawk in Britain. Brit. Birds 75: 243-260.

907. Marshall, J.T. 1978. Systematics of smaller Asian night birds based on voice. (Orn. Monogr. 25). American Ornithologists' Union, Kansas.

908. Marsden, A. 1979. A list of the birds of Sark.

909. Marin, A. 1985. Première conservation du Pigeon Trocaz (Columba trocaz bollii) à l'île de Hierro (lles Canaries). Alauda 53: 137-140.

910. Martin, A. 1987. Allas de las aves nidificantes en la isla de Tenerife. Instituo de Estudios Canarios. La Laguna.

911. Martin, R. 1981. An extension of range of the Mountain Buzzard. Bokmakierie 33: 93.

912. Marindale, J. 1986. The Freckled Duck - an RAOU conservation statement. (Repnrt 22). Royal Australasian Ornithologists' Union, Melbourne.

913. Martinez García, O. and Martínez Garcia, A. 199! Primer reporte de nidificación y observaciones de Ferminia cerveroi (Aves: Trnglodytidac) para Cuba. Pitire 4(2): 10.

914. Martuscelli, P. 1991. Predaçāo de Megalobulimus paranaguensis Pelsbry \& Thering, 1900

(Megalobulimidae, Mollusca) por Leucopternis lacemulata. P.29 In: Resumos, I Congreso Brasileiro de Omitologia. Belém: Museu Paraense Emilio Goeldi.

915. Mason, V. 1989. The birds of Bali. Periplus Editions, Berkeley, California and Singapore.

916. Massey-Stewart, J, 1987. The "Lily of birds": the success story of the Siberian White Crane. Oryx 21:6 10.

917. Masterson. A. 1986. Wattled Cranes - hot seating? Honeyguide 32: 154-155.

918. Matthews, G.V.T. and Evans, M.E. 1974. On the behaviour of the White-headed Duck with special reference to breeding. Wildfowl 25: 56-66.

919. Matthews, L.H. 1929. The birds of South Georgia
920 Mathews, N.J.C. and Scott, L.B. 1980. A new distribution record for the Cape Eagle Owl. Bokmakierie 32: 99-100.

921. Matvejev, S.D. and Vasic, V.F. 1973. Catalogus Faunae Jugoslaviae, IV/3, Aves. Yugoslavia.

922. Mauersberger, G. 1981. Anmerkungen zur Avifauna Nordkoreas. Min. Zool. Mus. Berlin, Suppl Ann. Om 5: $15-62$.

923. Mauersberger, G., Wagner, S.. Wallschlager. D. and Warthold, R. 1982. Neue Daten zur Avifauna mongolica. Mitr. Zool. Mus. Berlin 58: $11-74$.

924. Mayaud, N. 1953. Liste des oiseaux de France. Alauda $21: 1-63$.

925. Mayaud, N. 1982-84. Les oiseax du nord-ouest de I'Afrique. Notes complementaires. Alauda 50: 45-67, 116-145, 286-309. 51: 271-301. 52: 266-284.

926. Mayol, J. 1978. Els ancells de Is Balears. Manuals d'Introducćio a les Naturalesa, 2. Edit. Moll, Mallorca. 150pp.

927. Mayol, 3. 1981. [Evaluation of the Majorcan colonies of Eleonora's Falcon. Falco eleonorae, during summer 1981.] Bol. Est. Cent. Ecol. 20: 21-25. (In Spanish.)

928. Mayr, E. 1944. The birds of Timor and Sumba. Bull. Amer. Mus. Nat. Hist. 83: 123-194.

929. Mayr. E. 1945. Birds of the Southwest Pacific. Macmillan. New York.

930. Mayr, E. and Coltrell, G.W. (eds.). 1978. Check-list of the birds of the world. Volume 1,2 nd edition. Museum of Comparative Zoology, Cambridge, Massachusetis.

931. Mayr, E. and Vuilleumier, F. 1983. New species of birds described from 1966 to 1975. J. Om. 124: $217-232$.

932. McFarlane, R.W. 1992. A stillness in the pines: the ecology of the Red-cockaded Woodpecker. W.N Norton, New York.

933. MicGuigan. C. 1987. Omithology report. Pp. 10-27 In: Tellow, S.L. (ed.) Combridge Conservation Study 1985: Taita Hills, Kenya. (Study Report 19). International Council for Bird Preservation. Cambridge, U.K.

934. McKean, J.L. 1987. A first record of Christmas Island Frigatebird Fregata andrewsi on Timor. Kukila 3(1-2): 47

935. McNee, S. 1986. Sun'eys of the Western Whipbird and Westem Bristlebird in western Australia, 1985. Royal Australasian Omithologists' Union, Victoria.

936. McNeely, J. 1992. 1992 Ivory-billed Woodpecker expedition report. Unpuhlished.

937. McNicholl, M.K. 1985. Profiles on risk status of Canadian hirds: 2. Piping Plover. Alherta Naturalist 15(4): 135-138

938. McNutt, J.W. 1984. A peregrine falcon polymorph. Observations on the reproductive behaviour of Falco kreyenborgi. Condor 86: 378-382.

939. Medway, Lord and Wells, D.R. 1976. The birds of the Malay Peninsula. Volume 5: conclusions and survey of every species. H.F. and G. Witherby and Penerbit Universiti Malaya, London and Kuala Lumpur. 
940. Mees, G.F. 1961. A systematic review of the IndoAustralian Zosteropidae (Par I). Zool. Verh. 50: $1-168$.

941. Mees, G.F. 1969. A systematic review of the IndoAustralian Zosteropidae (Pan IID). Zool. Verh. 102: $1-390$.

942. Mees, G.F. 1971. Systematic and faunistic remarks on birds from Bomeo and Java, with new records. Znol. Meded. 45: 225-244.

943. Mees, G.F. 1977. Zur Verbreitung von Phaethomis malaris (Nordmann) (Aves, Trochilidae). Znologische Mededelingen 52: 209-211.

944. Mees, G.F. 1977. Additional records of birds from Formosa (Taiwan). Zool. Meded. 51: 243-264

945. Mees, G.F. 1980. The sparrow-hawks (Accipiser) of the Andaman Islands. Joumal of the Bombay Noturol History Society 77: $371-412$.

946. Mees, G.F. 1985. Nomenclature and systematics of birds from Suriname. Proc. Koninklijke Nederlandse Akademie Series C. 88(1): 75-91.

947. Meinertzhagen, R. 1954. The birds of Arobia. Oliver and Boyd, Edinburgh and London.

948. Meininger, P.L., Duiven, P., Marteijn, E.C.L. and Spanje, T.M. van. 1990. Notable bird observations from Mauritania. Malimbus 12: 19-24.

949. Meininger, P.L. and Mullie, W.C. 1981 . Preliminary list of the birds of Egypt.

950. Mellink, E. and Palacios, E. 1990. Observations on Isla Guadalupe in Novemher 1989. Westem Birts 2]: $177-180$.

951. Melville, D.S. 1984. Seahirds of China and the surrounding seas. Pp. 501-51 I In: Croxall, J.P., Evans, P.G.H. and Schreiber, R.W. (eds.) Status and conservotion of the world's seabirds. (Techn. Puhl. 2). International Council for Bird Preservation, Cambridge, U.K.

952. Melville, D.S. and Round, P.D. 1982. Further records of the Asian Dowitcher Limnodromus semipalmans from Thailand, with notes on its distribution and identification. Nol. Hist. Bull. Siam. Soc. 30(2): 119-204.

953. Ménégaux, M.A. 1904. Catalogue des oiseaux rapportés par $M$. Geay de la Guyane française et du Conteste France-Bresilien. Bulletin du Muséum Notional d'Histoire Naturelle 10: 107-119, 174-186.

954. Ménégaux, M.A. 1907. Oiseaux de la Guyane française donnés au Museum par M. Rey, gouvemeur des Colonies. Bulletin du Muséum Notional d'Histoire Naturelle 13: 493-499.

955. Menkhorst, P.W. and Isles, A.C. 1981. The Night Parrot Geopsittacus occidentalis: evidence of its occurrence in north-western Victoria during the $1950 \mathrm{~s}$. Emu 81: 239-240.

956. Mercado, N.K. 1985. Aves de Bolivio. Editorial Gisber, La Paz.

957. Meretsky, V.J. and Snyder, N.F.R. 1992. Range use and movements of California Condors. Condor 94: 313-335.
958. Merton, D.V., Morris, R.B. and Alkinson, I.A.E. 1984. Lek behaviour in a parrot: the Kakapo Strigops habroptilus of New Zealand. Ibis 126: 277-283.

959. Merwe, F. van der. 1981. Review of the status and biology of the Black Harrier. Ostrich 52: 193-207.

960. Meyburg, B.-U. 1982. Seltene und vom Aussterben bed-typogramylerohte Greifvogel (III): der Spanische Ksiseradler Aquila (heliaca) adolberti. Der Falkner 31/32: $21-30$.

961. Meyburg, B.-U. 1986. Threatened and near-threatened diumal birds of prey of the world. Birds of Prey Bull. 3: 1-12.

962. Meyhurg, B.-U. 1987. Clutch size, nestling aggression and breeding success of the Spanish Imperial Eagle. Brit. Birds 80: 308-320.

963. Meyburg, B.-U. 1989. The Spanish Imperial Eagle Aquilo (helioco) adalherti: its hiology, status and conservation. Pp. 255-268 In: Meyhurg, B.-U. and Chancellor, R.D. (eds.) Raptors in the modem world Proceedings of the III World Conference on Birds of Prey and Owls. Eilat, Jsrael. 22-27 March 1987. World Working Group on Birds of Prey and Owls, Berlin.

964. Meyburg, B.-U., Balen, S. van, Thiollay, J.-M. and Chancellor, R.D. 1989. Observations on the endangered Java Hawk Eagle Spizaetus bortelsi. Pp. 279-285 In: Meyburg. B.-U. and Chancellor, R.D. (eds.) Roptors in the modern world. Proceedings of the II] World Conference on Birds of Prey and Ow/s, Eilat, Israel, 22-27 March 1987. World Working Group on Birds of Prey and Owls, Berlin.

965. Meyburg, B.-U. and Meyburg, C. 1983. Distribution and present status of the Black Vulture Aegypius monochics. Bull. World Working Group on Birds of Prey 1: 172.

966. Meyer de Schauensee, R. 1966. The species of birds of South Americo and their distrihution. Livingston Publishing Company for the Academy of Natural Science-typogramylea, Narberth, Penn.

967. Meyer de Schauensee, R. 1982. A guide to the birds of South America. Academy of Natural Sciences, Philadelphia.

968. Meyer de Schauensee, R. 1984. The birds of China. Oxford University Press, Oxford.

969. Meyer de Schauensee, R. and Phelps, W.H. 1978. A guide to the birds of Venezuelo. Princeton University Press, Princeton, New Jersey.

970. Meyers, J.M. and Barrow, W.C. 1992. Hahitat of breeding and nonhreeding pairs of Puerto Rican Parrots (Amazona vittata) afler Hurricane Hugo. Program abstract, Society of Conservation Biology, June 27-July 1 1992. Virginia Polytech Institute and State University, Blackshurg, VA.

971. Mian, A. 1986. Ecological impact of Arah falconry on Houhara Bustard in Baluchistan. Environ. Conserv. 13: $41-46$.

972. Michael, G.A. 1987. Notes on the breeding hiology and ecology of the Mariana or Guam Crow. Avicult. Mog. 93(2): 73-82.

973. Miller, B.M. and Miller, C.M. 1992. Distrihutional notes and new species records for hirds in Belize. Occas. Pap. Belize Nat. Hist. Soc. 1(1): 6-25. 
974. Miller, C.M. 1991. Keel-billed Motmot: Belize"s rarest bird. Belize Audubon Soc. Newslener 23(1): 12.

975. Mills, J.A., Lavers, R.B. and Lee, W.G. 1984. The Takahe - a relict of the Pleistocene grassland avifauna of New Zealand. New Zealand J. of Ecology 7: 57-70.

976. Mills, J.A. and Williams, G.R. 1978. The status of endangered New Zealand birds. Pp. 147-168 In: Tyler, M.J. (ed.) The status of endangered Australasian wildlife. Royal Zoological Society of Australia. Adelaide.

977. Milton, G.R. 1985. Notes on the distribution of the Masked Finfoot Heliopais personata in Indonesia. Kukila 2: 41-43.

978. Mitchell, 1. 1991. Yeliow-faced Parrotlets (Forpus xamthops). Mag. Parrat Soc. 15: 56-57.

979. Mitchell, P. 1988. Unusual sighting reports. Bird Obs. $678: 87-88$

980. Modinger, B.A., Holman, G.M. and Morales, M.B. 1986. Guia de campo de las aves de Chile. Editorial Universitaria, Santiago.

981. Monroe, B.L. Jr. 1968. A distributional survey of the birds of Honduras. Omithological Monographs 7: $1-457$.

982. Moreau. G. 1990. [A new breeding species for France: the Common Crane.] Alauda 58: 244. (In French.)

983. Morel, G. 1972. Liste commentée des oiseaur du Sénégal el de la Gambie. ORSTOM. Dakar.

984. Morel, G.J. and Morel, M.-Y. 1988. Liste des oiseaux de Guinee. Malimbus 10(2): 143-176.

985. More1, G.J. and Morel. M.-Y. 1988. Nouvelles données sur l'hivernage de la tourterelle des bois. Streptopelia turtur en afrique de l'ouest: nord de la Guinee. Alauda 56(2): 85-91.

986. Morel, G.J. and Morel, M.-Y. 1990. Les oiseanc de Senegambie, notices et cartes de distribution. Editions de $1^{*}$ Orstom. Paris.

987. Morel, M-Y. 1987. La tourterelle des bois Streptopelia turtur dans l'ouest africaln: mouvements migratoire et regime alimentaire. Malimbus $9(1): 23-42$

988. Morel, R. 1989. A note on the status of bustards in Mauritania, Mali and Senegal. Bustard Stuties 4.

989. Moreno, J.M. 1988. Guia de las aves de las Islas Canarias. Editorial Interinsular Canaria, Santa Cruz do Tenerife. Canaries Inst.

990. Morgan-Davis, A.M. 1965. On the Kori Bustard Ardeoris kori (Burcheli) in north-western Tamzania. Bull. Brit. Orn. Club 85: $145-147$

991. Morris, G.E. 1987. News of Nam Cat Tien. Garmlax 2: 3-5.

992. Mosimann, P. and Juillard, M. 1988. [Breeding status and winter distribution of the Red Kite Milvis milvis in Swizerland.] Om. Beob. 85: 199-206.

993. Mottley, J. 1863. Observations on the birds of southeastern Borneo. Proc. Znol. Soc. London 1863: 206-224.
994. Muller, H.H. 1988 . Erster Brutnachweis des vom Aussterben bedrohten Taiko-Sturmvogels (Pterodroma mageniae) auf den Chatham islands (Neuseeland). Seevogel $9(1):$ 9-11.

995. Müller-Bierl, M. and Cordier. C. 1991. Auf den Spuren des Rotohraras Ara ruhrogenys. Gefied. Welt 115: $413 \cdot 416$.

996. Munn, C.A., Silva, C.P. da, Renton, K. and Valqui, M. 1991. Eine kurze Studie ūher nistende Hyazintharas im Pantanal. Papageien 5/91: 145-148; 6/91: 178-181.

997. Munn, C.A., Thomsen. J.B. and Yamashita, C. 1987. Survey and status of the Hyacinth Macaw (Anondorhynchus hyacinthinus) in Brazil, Bolivia, and Paraguay. Unpublished.

998. Muntaner, J., Ferreri, X. and Martinez-Vilalta, A. 1983. Allas dels ocells nidificants de Catalunya $y$ Andorra. Ketrés Editora, Barcelona.

999. Myers, P. and Hansen, R.L. 1980. Rediscovery of the Rufous-faced Crake (Laterallis renoptenus). Auk 97: $901-902$.

1000. Nagy, K.A., Siegfried, W.R. and Wilson, R.P. 1984. Energy utilization by free-ranging Jackass Penguins, Spheniscus demersus. Ecolngy 65: 1648-1655.

1001. Nakhasathien, S. 1987. The discovery of Storm's Stork Ciconia stormi in Thailand. Forktail 3: 43-49

1002. Narang. M.L. 1989. Occurrence of the Western Horned Pheasant Tragopan melanocephalus east of $R$. Sutlej in Himachal Pradesh. Ann. Biol. (Ludhiana) 5: $153-154$.

1003. Narosky, S. 1977. Una nueva especie del genera Sporophila (Emberizidae). Homero 11: 345-348.

1004. Narosky. T. and Yzurieta, D. 1987. Giva para la idenificacion de las aves de Argentina y Uniguay. Vazquez Mazzini Editnres, Buenos Aires.

1005. Nash, S.V. and Nash, A.D. 1986. Records of the White-winged Duck Cairina scululata in Sumatran peatswamp forest. Oriental Bird Club Bull. 3: 17.

1006. Naurais. R. de. 1986. Une reproduction dr Fringilla . leydea (Wehb. Berthelot et Mnquin-Tandnn) dans un biotope inattendu. Cyanopica 3: 533-538.

1007. Navarrn, A.G., Peterson, A.T., Escalante, B.P. and Benilez, H. 1992. Cypseloides storeri, a new species of swif from Mexico. Wilson Bull. 104: 55-64.

1008. Navarro. A.G., Torres, M.G. and Escalante, B.P. 1991. Catálogo de aves, Museo de Zoología, Facultad de Ciencias, Universidad Nacional Autónoma de Méxica. México [City]: Museo de Zoología “Alfnnso L. Hertera*. Facultad de Ciencias, UNAM.

1009. Navas, J.R. 1991. Gruiformes. Fanna de agua dulce de la República Argentina 43(3): 1-80.

1010. Negret, A.J. 1991. Reportes recientes en el Parque Nacional Munchique de aves consideradas raras o amenazadas de extincińn. Noved. Colombianas (nueva epoca) $3: 39-45$

1011. Negret, A.J. and Acevedo, C.1. 1990. Reportes recientes de Leptosittaca branickii, ave colombiana amenazada de extincińn. Noved. Colombianas (nueva epoca) 2 : 70-71. 
1012. Nelson, J.B. 1974. The distribution of Abbolt's Booby Sula abbatti. Ibis 116: 368-369.

1013. Nelson, J.B. and Powell. D. 1986. The breeding ecology of Ahbott's Booby Sula abbouti. Emus 86(1): 33-46.

1014. Neumann, J. and Kolbe, U. 1990. [The birds of East Germany - an annotated species list.| Falke 37(7): 229-232.

1015. Newby, J.. Grettenberger, J. and Watkins, J. 1987 The birds of northern Air. Niger. Malimbus 9(1): 4-16.

1016. Newman, K. 1989. Birds of Botswana. Southern Book Publishers (Phy)

1017. Newman, K., Johnston-Stewart, N. and Medland, B. 1992. The birds of Malawi. A supplement to Newman's Birds of Southern Africa. Southern Book Publishers (Piy), Cape Town.

1018. Niethammer, G., Kramer, H. and Wolters, H.E. 1964 Die Vögel Dertschlands, Arenliste. Frankfurt A.M

1019. Nightingale, T. and Hill, M. 1992. The birds of Bahrain. Immel.

1020. Nikolaus, G. 1987. Distribulional atlas of Sudan's birds, with notes on hahitat ander Zoologische Monographien 25

1021. Norderhaug. A. and Norderhaug. M. 1984. Status of the Lesser White-fronted Gonse Anser erythropus in Fennoscandia. Swedish Willlife Reseorch 13 (1): $171-185$.

1022. Nores, M. and Yzuriela, D. 1984. Distribucion y situacion actual de las parahas y parabachis en Bolivia (Aves: Psittacidae). Consejo Internacional para la Preservacion de las Aves. Unpublished.

1023. Nores, M.. Yzurieta, D. and Salvador, S.A. 1987. Distribucion y situacion actual del Mirlo de Agua Pecho Rojo (Cinclus schulzi). 111 Congreso de Ornitologia Neotropical: Resumenes. Unpublished.

1024. Norton, R.L. and Clarke, N.V. 1989. Additions to the birds of the Turks and Caicos Islands. Florida Field Nat. 17: 32-39.

1025. Norton, R.L. and Hohbs, A. 1987. First record of Purple-throaled Carib for the Greater Antilles and notes on vagrants in the Virgin Islands. Carihhean $J$. Sci. 23: 462-463.

1026. Nowak, E. 1983. Die Scopfkasarka, Tadama cristata (Kuroda, 1917) - eine vom Aussterben bedrohte Tierart (Wissenstand und Vorschlage zum Schutz.). Bonn. Zool. Beitr. 34: 235-271.

1027. Nowak, E. 1984. Ueber das vermutliche Brut-und Ueberwinterungsgebiet der Schopfkasarka Tadnmn cristata. J. Om. 125: 103-105.

1028. Nowak, E. 1984. Ueber einige Farbungs- und Verhalrensmermale der Schopkasarka. Falke 31: 150-155.

1029. O Myong Sok 1984. Wiederentdeckung der Schopfkasarka, Tadoma cristata, in der Koreanischen Demokratischen Volksrepuhlik. J. Om. 125: 102-103.

1030. O'Donnell, C.F.J. 1984. The North Island Kokako (Callaeas cinerea wilsoni) in the Westem King Country and Taranaki. Notomis 31(2): 131-144.
1031. O'Neill, J.P., Solar R.G. del, Ortiz. T.E., Eley, J.W. and Williams, M.D. 1981. The White-winged Guan Penelape albipennis; its rediscovery, status, nesting. systematics, and recommendations for its continued survival. In: Primer Simposio Internacional de la Familia Cracidae: Memorias. Universidad Nacional Autonoma de Mexico [Mexico City].

1032. Ogilvie, M. and Ogilvie, C. 1986. Flamingos. Alan Sullon, Gloucester. U.K.

1033. Ogle. D. 1986. The status and seasonality of birds in Nakhon Sawan Province, Thailand. Nat. Hist. Bull. Siam Sac. 34(2): 115-143.

1034. Olrog, C.C. 1984. Las aves Argentinas. Administracion de Parques Nacionales.

1035. Olrog, C.C. 1985. Status of wet forest raptors in northern Argentina. Pp. 191-197 In: Newton. 1. and Chancellor. R.D. (eds.) Conservation studies on raptors. (Techn. Puhl. 5). International Council for Bird Preservation, Camhridge, U.K

1036. Olsen, P.D., Mooney, N.J. and Olsen, J. 1989. Status and conservation of the Norfolk Island Bontook Ninox novaeseelandiae undulata. Pp. 415-422 In: Meyburg. B.-U. and Chancellor, R.D. (eds.) Raptors in the modem world. Proceedings of the III World Conference on Birds of Prey and Owls, Eilat, Israel. 22-27 March 1987. World Working Group on Birds of Prey and Owls, Berlin.

1037. Olsen, P.D. and Olsen. J. 1986. Distrihution, status, movements and breeding of the Grey Falcon Falco hypoleucas. Emu 86: 47-5I.

1038. Olsen, P.D. and Stokes, T. 1989. State of knowledge of the Christmas Island Hawk-Owl Ninor squampila natalis. Pp. 411-414 In: Meyburg. B.-U. and Chancellor. R.D. (eds.) Raptors in the modem warld. Proceedings of the 111 World Conference on Birds of Prey and Owls, Eilat, Israel, 22-27 March 1987. World Working Group on Birds of Prey and Owls, Berlin.

1039. Oren, D.C. and Novaes, F.C. 1986. Ohservations on the Golde: Parakeel Aratinga guornutha in northern Brazil. Biol. Cons. 36: 329-337.

1040. Omithological Society of Turkey 1971. Check list of the birds of Turkey.

1041. Ortiz-Crespo, F.1. 1984. First twentieth-century specimen of the Violet-throated Metaltail Metallura baroni. Bull. Brit. Om. Club 104: 95-97.

1042. Ortiz-Crespo, F., Greenfield, P.J. and Matheus, J.C. 1990. Birds of Ecuador, locational checklist with English and Spanish common names. Ecuador Ornith. Corp.

1043. Oriz Tejada, E. and Purisaca Puicon, J. 1981. Estudio preliminar sobre la Pava alihlanca (Penelope albipennis) Taczannwski 1877. Pp. 192-202 in Primer Simposia Intemacional de la Familia Cradidae: Memorias. [Mexico City] Universidad Nacional Autonoma de Mexico.

1044. Osborne. P.E. 1986. Survey of the hirds of Fuerteventura, Canary Islands, with special reference to the stalus of the Canarian Houbara Bustard Chlamydotis undulata. (Study Report 10). International Council for Bird Preservation. Cambridge, U.K. 
1045. Osborne, P.E. and Tigar, B.J. 1990. Distribution and slatus of rare birds in Lesotho. Br. Ecol. Soc. Bulletin 21: 121-128

1046. Osborne, P.E. and Tigar, B.J. 1992. Priorities for bird conservation in Lesotho, southern Africa. Biol. Conserv. 61: 159-169.

1047. Ostapenko, W.A. and Zewenmjadag, N. 1983. Uher Verbreitung. Anzahl und Biologie der Kraniche im Ostteil der Mongolischen Volksrepublik. Beirr. Vogelkd. 29: 274-278.

1048. Otero, C. 1987. The Great Bustard (Otis tarda) in Spain. Pp. 43-50 In: Farago, S. (ed.) The Great Bustard (Otis tarda L.). Proceedings of the symposium in Budapest on June 2nd 1987. Intemational Council for Game and Wildlife Conservation, Budapest

1049. Ottenwalder, J.A. 1988. Flamingos in Haiti. World Birdwatch 10(3/4): 8 .

1050. Owen, R.P. 1977. A checklist of the birds of Micronesia. Micronesica 13:65-81

1051. Owre, O.T. 1966. The Crowned Crane at Lake Rudolf. Bull. Brit. Om. Club 86(3): 54-56.

1052. Oyarzo, H. and Cisternas, G. 1990. Proyecto conservación de la Tagua Cornuda (Fulica comuda) en la Laguna Santa Rosa. Informe no.2. Corporación Nacional Forestal, Ministerio de Agricultura. Unpublished.

1053. Oyarzo, H. and Cisternas, G. 1991. Proyecto - Conservación de la Tagua Cornuda (Fulica comua) en la Laguna Santa Rosa, III Región de Atacama. Chile". Primer informe semestral 1991. Corporación Nacional Forestal, Ministerio de Agricultura. Unpublished.

1054. Padron, F.P. 1983. Las aves de Canarias.

1055. Palma, L. 1985. The present situation of birds of prey in Portugal. Pp. 3-14 In: Newton. 1. and Chancellor, R.D. (eds.) Conservation studies on raptors.

1056. Parker. S.A and Reid. N. 1978. Remarks on the status of some Australian passerines. Pp. 109-115 In: Tyler. M.J. (ed.) The status of endangered Australasian wildlife. Royal Zoological Society of Australia. Adelaide.

1057. Parker, S.A. and Reid. N. 1979. Remarks on the status of some Australian passerines. In: Tyler, M.J (ed.) The status of endangered Australasian wildlife. Royal Zoological Society of South Australia. Adelaide.

1058. Parker, T.A. 1981. Distribution and biology of the White-cheeked Cotinga Zaratomis stresemanni, a high Andean frugivore. Bull. Brit. Om. Club 101: 256-265

1059. Parker, T.A. 1982. First record of the Chilean Woodstar Eulidia yarrellii in Pers. Bull. Brit. Om. Club 102: 86 .

1060. Parker, T.A. 1983. Rediscovery of the Rufous-fronted Antthrush (Formicarius nufifrons) in southeastern Peru Gerfaut 73: 287-289.

1061. Parker, T.A., Bates, J. and Cox, G. 1992. Rediscovery of the Bolivian Recurvehill with notes on other litte-known species of the Bolivian Andes. Wilson Bull. 104: 173-177.

1062. Parker, T.A. and O'Neill. J.P. 1980. Notes on little known birds of the upper Uruhamha Valley, southern Peru. Auk 97: 167-176.
1063. Parker. T.A. and Parker, S.A. 1982. Behavioural and distributional notes on some unusual hirds of a lower montane cloud forest in Peru. Bull. Brit. Om. Club 102: $63-70$.

1064. Parker, T.A., Parker. S.A. and Plenge, M.A. 1982. An annotated checklist of Peruvian hirds. Buteo Books. Vermillion, South Dakota.

1065. Parker, T.A. and Remsen, J.V. 1987. Fifty-two Amazonian bird species new to Bolivia. Bull. Brit. Orn. Club 107: 94-107.

1066. Parker, T.A. and Remsen. J.V. Jr. 1987. Fifty-two Amazonian bird species new to Bolivia. Bull. Brit. Om. Club 107(3): 94-107.

1067. Parker, T.A., Schulenherg, T.S., Graves, G.R. and Braun, M.J. 1985. The avifauna of the Huancabamha region, northern Peru. Pp. 169-197 In: Buckley, P.A., Foster. M.S.. Morton, E.S., Ridgely, R.S. and Buckley, F.G. (eds.) Neotropical omithology. American Ornithologists' Union Ornithological Monograph No. 36. AOU, Washington D.C.

1068. Parker, V. 1988. Birds of Swaziland. Bokmakierie 40: 77-79

1069. Parker, V. 1992. Swaziland bird checklist. The Conservation Trust of Swaziland. Swaziland.

1070. Parkes, K.C. 1990. Was the Socorro Mockingbird (Mimodes graysani) a predator on small birds? Wilson Bull. 102: 317-320

1071. Parkes, K.C. and Dickinson. E.C. 1991. Types, type localities, and variation in some races of the Colasisi or Philippine Hanging Parrot Loriculus philippinensis. Bull. Bris. Om. Club 111: 104-110.

1072. Parrot-Holden, J. 1987. The Socorro Dove - its destiny. AFA Watchhird 14(5): 48-49.

1073. Pasquier, R.F. ed. 1981. Consenvation of New World parrots. (Techn. Publ. 1). International Council for Bird Preservation. Smithsonian Institution Press and International Council for Bird Presevation. Washington D.C. and Cambridge, U.K.

1074. Pateff, P. 1950. Ptitzite W bulgarija [The birds of Bulgaria.] Sofia.

1075. Patzwahl, S. 1991. Vogelpark Walsrode places Spix's Macaw in breeding program. AFA Watchbird $18(3): 4,7$

1076. Payne, R.B. 1982. Species limits in the indigobirds (Ploceidae, Vidua) of West Africa: mouth mimicry, song mimicry and description of new species. Misc. Publ. Mus. Zool., Univ. Michigan 162: 96pp.

1077. Payne, R.B. 1985. The species of parasitic finches in West Africa. Malimbus 7(2): 103-113

1078. Paynter, R.A. and Traylnr, M.A. 1977. Omithalogical gazetteer of Ecuador. Museum of Comparative Zoology, Cambridge, Massachusetts.

1079. Pearman. M. 1990. Behaviour and vocalizations of an undescribed canastero Asthenes sp. from Brazil. Bull. Brit. Om. Club 110: 145-153.

1080. Penny, M. 1974. The birds of Seychelles and the outlying islands. Collins. London.

1081. Penry, E.H. 1979. Sight records of the Sooty Falcon Falco concolor in Zamhia. Bull. Brit. Om. Club 99: 63-65. 
1082. Perennou, C. 1991. African waterfowl census 1991 . TWRB.

1083. Perennou, C. and Mundkur, T. 1991. Asian waterfowl census 1991. IWRB, Slimhridge, U.K.

1084. Pérez-Rivera, R.A. 1980. Confirmation of two new species from Puerto Rico. American Birds 34: 935.

1085. Pérez-Rivera, R.A. 1990. [Status of the Plain Pigeon in the Greater Antilles.] Sci.-Cienc. Bial. Cient. Sur 17: 21-25. (In Spanish.)

1086. Pérez-Rivera, R.A. and Ruiz-Lebrón, C.R. 1992. Situación actual de la población de palomas sabaneras (Calumba inamata wetmarei) en Cidra, Puerto Rico. Universidad de Puerto Rico. Colegio Universitario de Humacao. Departamento de Biología Memorias del Décimo Simposio sobre la Flore y Fauna de Puerto Rico y el Caribe), Humacao, Puento Rico.

1087. Peters, J.L. 1929. Birds of the island of Anguilla, W. Indies. Auk 44: 532-538.

1088. Peters, J.L. 1934-1987. Checklist of the birds of the warld. Harvard University Press, Cambridge, Massachusells.

1089. Peterson, R.T. and Chalif, E.L. 1973. A field guide to Mexican birds. Houghton Mifnin Company, Boston

1090. Petretti, F. 1985. Preliminary data on the status of the Little Bustard in Italy. Bustord Sudies 2: 165-172.

1091. Pfeffer, P.L. 1960-1961. Etude d'une collection d'oiseaux de Borneo. L'Oisean 30: 154-168, 191-218; 31: 1-29.

1092. Phelps, W.H. and Phelps, W.H. 1958. Lista de las aves de Venezuela con su distribucion, 2. Parte 1. No passeriformes. Boln. Sac. venez. Cienc. Nat. 19(90): 1-317

1093. Phillips, W.W.A. 1963. The hirds of the Maldive Islands, Indian Ocean. Joumnl of the Bombay Notural Histary Saciety 60: 546-584.

1094. Phillips, W.W.A. 1978. Annotated checklist of the birds of Ceylon (Sri Lanka). Wildlife and Nature Protection Socicty, Colomho.

1095. Pickering, R. 1981. An annototed checklist of the birds of Vanuatu. Unpuhlished.

1096. Pickering, R.H. (ed.). 1985. Santo Mountains Expedition 1985, preliminary report. Vanuatu Natural Science Society, Pon Vila. Unpuhlished.

1097. Piechocki, R 1968. Beitrāge zur avifauna der mongolei teil I. non-passeriformes. Ergehnisse der MongolischDeutschen Biologischen Expedition seit 1962. Mint Zool. Mus. Berlin, Bd, 44, Hefi 2. 1968 Pp. 149-292.

1098. Piechocki, R., Stubbe, M.. Uhlenhaut, K. and Sumjaa, D. 1981. Beitrage zur Ávifauna der Mongolei. Mitt. Zool. Mus. Berlin 57, Suppl. Ann. Om. 5: 71-128.

1099. Pierce, R.J. 1986. Differences in susceptibility to predation during nesting hetween Pied and and Black Stilts (Himantopus spp.) Auk 103: 273-280.

1100. Piersma, T. 1986. Coastal waders on three Canary Islands in March-April 1986. Wader Sudy Group Bull. 48: $19-20$.

1101. Pilcher, C.W.T., Gregory, G., Tye. A. and Ahmed. M.S. 1990. Additions to the country list produced by the Kuwait Avifaunal Survey, 1985-7. Sandgrouse 12: $31-36$.
1102. Pineschi, R.B. 1990. Aves como dispersores de sete espécies de Rapanea (Myrsinaceae) no maciço do Itatiaia, estados do Rio de Janeiro e Minas Gerais. Ararajuba 1: 73-78.

1)03. Pinto, O. 1937. Arolinha Oxypelia cyanopis Pelzeln, so conhecida do Brasil, e uma das aves mais raras que existem. Bol. Bioogico (N.S.) 3(5): 17-18.

1104. Pinto, O. 1944. Calologo das aves do Brasil. Segunda parte. Sao Paulo: Departamento de Zoologia.

1105. Pinto, O. 1945. Cinquenta anos de investigacao omitologica. Arc. Zool. Est. Saa Paulo 4: 261-340.

1106. Pinto, O. 1954. Aves do ttatiaia. Bol. Parq. Nac. Itatiaia no. 3

1107. Pinto, O. 1978. Novo calalogo das aves do Brasil. Departmento de Zoologia, Sao Paulo.

1108. Pinto. A.A. de R. 1983. Omitologia de Angola. Instituto de Investigacao Cientifica Tropical, Lisbon.

1109. Piper, S.E. and Ruddle, P. 1986. An initial evaluation of the Cape Vulture colonies at Mkamhati, Transkei. Vullure News 15: 7-12.

1110. Pizzey, G. 1980. A field guide to the hirds of Australia. Collins, Sydney.

1111. Podolsky, R. and Kress, S.W. 1992. Altraction of the endangered Dark-rumped Petrel in recorded vocalizations in the Galápagos Islands. Condor $94: 448-453$.

1112. Pokorny, F. and Pikula. J. 1987. Artificial breeding, rearing and release of Reeves Pheasant (Symalicus reevesi) (Gray 1829) in Czechoslovakia. World Pheasant Assoc. J. 12: 75-80.

1113. Pont, J.E. du. 1971. Philippine birds. Delaware Museum of Natural History, Greenville, Delaware.

1114. Poole. A.F. 1989. Ospreys: a natural and unnatural history. Camhridge University Press, Camhridge, U.K.

1115. Potapov, R.L. and Flint, V.E. (eds.). 1987. The birds of the USSR: Gallifomes, Gruiformes. Nauka. Leningrad. (In Russian.)

1116. Poulsen, M.K., Bloch, H., Rahhek, C. and Rasmussen, J.F. 1989. Loja - garden of Ecuador. World Birdwatch 11(3): 10.

1117. Pratt. H.D., Enghring, J., Bruner, P.L. and Berret, D.G. 1980. Notes on the taxonomy, natural history, and status of the resident hurds of Palau. Condor $82: 117-131$.

1118. Pralt, J.D., Bruner, P.L. and Berrett, D.G. 1987. A field guide to the hirds of Hawaii and the tropical Pacific. Princeton University Press. Princeton. New Jersey.

1119. Pratt. T.K. 1987. Recent ohservations March through May 1987. Elepaio 47: 93-95.

1120. Priednieks, J.. Strazds. M., Strazds, A. and Petrins, A. 1989. Latvian hreeding bird atlas 1980/1984.

1121. Prigogine, A. and Louette. M. 1984. A new race of the Spotted Ground-thrush. Znothera guttata, from Upemha, Zaire. Gerfant 74: 185-186.

1122. Prince, P.A. and Payne. M.R. 1979. Current status of birds at South Georgia. British Antarctic Survey Bulletin 48: 103-118. 
1123. Probst, J.R. 1986. A review of factors limiling the Kirtand's Warbler on its breeding grounds. Amer. Midland Nat. 116 (1): 87-100.

1124. Probst, J.R. and Hayes, J.P. 1987. Pairing success of Kirtland's Warblers in marginal vs. suitable hahitat. Ask 104: 234-241.

1125. Pruett-Jones, S.G., Pruett-Jnnes, M.A. and Knight, R.L. 1980. The White-tailed Kite in North and Middle America: current status and recent population changes. American Birds 34: 682-688.

1126. Rabor, D.S. 1962. The impact of deforestation on birds of Cebu. Philippines, with new records for that island. VIII Bull. Intemarional Council far Bird Presevation: $79-85$

1127. Radtke, G.A. 1991. Haltung und Zucht des Kapuzenzeisigs (Spinus cucullatus). Voliere 14: 59-61.

1128. Raffaele, H.A. 1983. The raising of a ghost - Spinus cucullatus in Puerto Rico. Auk 100 (3): 737-739.

1129. Raffaele, H.A. 1989. A guide to the birds of Puerto Rico and the Virgin Islands. Revised edition. Princeton University Press, Princelon, New Jersey.

1130. Rahmani, A.R. 1987. Protection for the Great Indian Bustard. Oryx 21: 174-179.

1131. Rahmani, A.R. and Manakadam, R. 1985. Presen status of the Great Indian Bustard. Bustard Sudies 3: 123-132.

1132. Ramyle.J. 1988. [Confirmation of the presence of Spheniscus humboldi Meyen (Aves: Spheniscidac) in Colombia.] Trianea (Bogota) 1: 141-143.

1133. Rand, A.L. and Gilliard, E.T. 1967. Handhook of New Guinea birds. Weidenfeld and Nicolsnn, London.

1134. Rand, A.L. and Traylor, M.A. 1954. Manual de las aves de El Salvador. Universidad de El Salvador. El Salvador.

1135. Randall. R.M. and Randall, B.M. 1986. The diet of Jackass Penguins Spheniscus demenus in Algna Bay, South Africa, and its bearing on population declines elsewhere. Biol. Conserv. 37: 119-134.

1136. Rands, M., Rands, G. and Porter, R. 1988. Birds in the Yemen Arah Republic. Report of the expedition of the Omithological Sociery of the Middle East. OSME, Sandy, U.K.

1137. Rauzon, M.J., Harrison, C.S. and Conant, S. 1985 The status of the Sooty Storm-petrel in Hawaii. Wilson Bull 97(3): 390-392.

1138. Razdan, T. and Mansoori, J. 1989. A review of the bustard situation in the Islamic Republic of Iran. Bustard Sudies 4.

1139. Redford, K.H. 1987. Parque das Emas. Ciencin Hoje $7(38): 42-48$

1140. Remsen, J.V. Jr. 1984. Natural hisinry notes on some poorly known Bolivian hirds. Part 2. Gerfaut 74: 163179.

1141. Remsen, J.V. Jr. 1986. Was Bachman's Warbler a hamboo specialist? Auk 103: 216-219

1142. Remsen, J.V. Jr. and Parker, T.A 1983. The contribution of river-created hahitats to bird species richness in Amazonia. Bionropica 15: 223-231.
1143. Remsen, I.V. Jr., Parker, T.A. and Ridgely, R.S. 1982. Natural history notes on snme poorly known Bolivian hirds. Gerfart 72: 77-87.

1144. Remsen, J.V. Jr. and Traylor, M.A. 1983. Additions to the avifauna of Bolivia, part 2. Condar 85: 95-98.

1145. Remsen. J.V. Jr. and Traylor, M.A. 1989. An annotated list of the hirds of Bolivia. Buteo Books, Vermillion, South Daknta.

1146. Remsen, J.V., Traylor, M.A. and Parkes, K.C. 1986. Range extensions of some Bolivian hirds, 2 (Columbidae to Rhinocryptidae). Bull Bril. Om. Club 106: 22-32.

1147. Reville, R., Tranter, J. and Yorkston, H. 1987. Maniloring the endangered Abholt's Boohy an Christmas Island 1983-1986. Australian National Parks and Wildlife Service (Occasional Paper No. 11), Canberra

1148. Rheinwald, G., Hill, A. and Ringlehen, H. 1981. Die Vögel der Bundesrepuhlik Deutschland und Berlin (West), Artenliste. Dachverhand Deutscher Avifaunisten, Verlag.

1149. Richards, D.K. 1982. The birds of Conakry and Kakulima, Democratic Republic of Guinea. Malimbus $4(2)$ : 93-103.

1150. Richardson, C. 1990. The birds of the Unired Arab Enirates. Hobby Puhlicatinns. Duhai and Werrington, U.K.

1151. Ridgely, R.S. 1980. Notes on some rare or previously unrecorded hirds in Ecuador. Amer. Birds 34: 242-248.

1152. Ridgely, R.S. 1981. The current distrihution and status of mainland Neotropical parrots. Pp. 233-384 ln Pasquier, R.F. (ed.) Conservation of New World parrots. (Techn. Puhl. 1). International Council for Bird Preservation. Smithsnnian Institution Press, Washington D.C.

1 153. Ridgely, R.S. 1989. A guide to the birds of Panama, with Costa Rica, Nicaragua and Honduras. Princeton University Press, Princeton, New Jersey.

1154. Ridgely, R.S. and Tudnr. G. 1989. The birds of Sauth America. Volume 1: the oscine passerines. University of Texas Press, Austin. Texas.

1155. Riley, J.H. 1938. Birds from Siam and the Malay Peninsula in the U.S. National Museum collected by Drs. Hugh M. Smith and William L. Abhntt. Bull. U.S. Natn. Mus. 172: 1-581.

1156. Rinke, D. 1986. The status of wildlife in Tonga. Oryx 20(3): 146-151.

1157. Riper, C. van. 1980. Ohservations on the breeding of the Palila Psintrostra hailleui of Hawaii. Ihis 122: 462-475.

1158. Ripley, S.D. 1977. Rails of the world. M.F. Feheley Publishers, Toronto.

1159. Ripley, S.D. 1982. A synopsis of the birds of India and Pakistan. 2nd edition. Bombay Natural History Society. Bombay.

1160. Ripley. S.D. and Bnnd. G.M. 1966. The hirds of Socotra and Abd-el-Kuri. Smithsonian Miscellaneaus Collections 151(7): 1-37. 
1161. Ripley, S.D. and Rabor, D.S. 1958. Notes on a collection of hirds from Mindoro Island, Philippines. Bull. Peabady Mus. Nat. Hist. 13: 1-83.

1162. Risberg, L. 1990. Sveriges Faglar. Serviges Ornit. For., Stockholm.

1163. Riveros, G.G. 1980. Nuevos datos sobre distribucion de Oreatrachilus leucopleurus Gould 1847. Anales Mus. Nat. Hist. nat. Valparaiso 13: 299-300.

1164. Riveros Salcedo, J.C., Sánchez S.R. and Ascensios V.D. 1991. Distribución y status del Pachaloro Farpus xanthaps. Resúmenes del IV Congreso de Ornitología Neotropical, 3-9 de Noviembre 1991, Quito, Ecuador (resumen 160).

1165. Robbins, M.B., Parker, T.A. and Allen, S.E. 1985. The avifauna of Cerro Pirte, Darien, eastern Panama Omithalogical Monagraphs 36: 198-232.

1166. Robbins, M.B. and Ridgely, R.S. 1990. The avifauna of an upper tropical cloud forest in southwestern Ecuador. Prac. Acad. Nat. Sci. Philadelphia 142: $59-71$.

1167. Roberson, D. 1980. Rare birds of the west coast of Narth America. Woodcock Publications, California

1168. Roberts. J.L. 1979. Observation of the migration of raptors and other large soaring birds in Bulgaria. Ibis $121: 301-312$.

1169. Roberts, P. 1987. Is the Aldabra brush warbler extinct? Oryx 21: 209-210

1170. Roberts. T. 1991-1992. The hirls of Pakiston. 2 vols Oxford University Press, Karachi.

1171. Robers, T.J. 1985. Zangi Nawar-portrait of a unique lake in the desert, J. Bomhay Nal. Hist. Soc. 82: $540-547$.

1172. Robertson, A. and Febnuary, E. 1986. Towards an historical perspective of Cape Vulures and domestic stock: a view from the southwestern Cape. Vuluure News 15: 4-6.

1173. Robertson, A.S. 1986. Noles on the breeding cycle of Cape Vultures (Gyps copratheres). Raptor Research 20: $51-60$

1174. Robertson. A.S. and Boshoff, A.S. 1986. The feeding ecology of Cape Vultures Gyps coprotheres in a stockfarming area. Bial. Conserv. 35: 63-86.

1175. Robertson, C.J.R. (ed.). 1985. Complete book of New Zealand birds. Readers' Digest, Sydney.

1176. Robertson, C.J.R. and Bell, B.D. 1984. Seabird status and conservation in the New Zealand region. Pp. 573586 In: Croxall, J.P., Evans. P.G.H. and Schreiber, R.W. (eds.) Status and conservation of the warld's seabirds. (Techn. Publ. 2) International Council for Bird Preservation. Cambridge, U.K.

1177. Robinson, H.C. and Kloss, C.B. 1918. Results of an expedition to Korinchi Peak. Sumatra. Part 2: birds. $J$. Fed. Malay Siates Mus. 8: 81-284.

1178. Robson, C.R. 1986. Recent observations of hirds in Xizang and Qinghai provinces. China. Forktail 2: 67-82.

1179. Robson, C.R. 1987. Pheasants in Vietnam. Garrular 3: 2-3.
1180. Rockingham-Gill, D.V. 1983. On the distribution of the Kori Bustard (Otis kori) in Zimbabwe. Pp. 97-103 In: Goriup, P.D. and Vardhan, H. (eds.) Bustards in decline. Tourism and Wildlife Society of India, Jaipu-

1181. Rockingham-Gill, D.V. 1989. The status of bustards in Zimbabwe. Bustard Studies 4.

1182. Röder, J. 1990. Beobachtungen bei der Haltung und Zucht des Grünkardinals. Voliere 13: 184-186.

1183. Rodríguez, D. and Sánchez, B. 1991. Ecología de las palomas terrestres cubanas (géneros: Georrygan y' Siamoenas). Unpublished.

1184. Rodriguez-Estrella, R., Mata, E. and Rivera, L. 1992. Ecological notes on the Green Parakeet of isla Socorro, Mexico. Condor 94: 523-525.

1185. Rojas, F. 1991. Biología reproductiva de la cotorra: Amazona barbadensis (Aves: Psittaciformes) en la Península de Macanao, Edo. Nueva Esparta. Lic. Biol. thesis, Universidad Central de Venezuela.

1186. Rokitansky, G. 1964. Casalogus Faunae Austriae. XXIb Aves.

1187. Romero-Zambrano, H. 1983. Revision del status zoogeografico y redescripcion de Odontopharus strophium (Gould) (Aves: Phasianidae). Callasia 13(65): 777-786.

1188. Rooke, 1. 1986. Survey of the White-breasted Whiteeye and the Norfolk Island Boobook Owl on Norfolk Istand, Octaber-November 1985. Royal Australasian Ornithologists" Union, Victoria.

1189. Rooks, D. 1989. Bird observations in Tobago, December 1987 to November 1989. J. Trinidad and Tabago Field Nat. Cluh 1989: 15.

1190. Rosenberg, D.K. and Harcourt, S.A. 1987. Population sizes and potential conservation problems of the endemic Galpagos Penguin and Flightless Cormorant. Noricias de Galapagas 45: 24-25.

1191. Rosenberg, D.K., Valle, C.A., Coulter, M.C. and Harcourt, S.A. 1990. Monitoring Galápag ss Penguins and Flightless Cormorants in the Galápagos Islands. Wilsan Bull. 102: 525-532.

1192. Roth, P. 1990. Spix-Ara Cyanopsitta spixii: was wissen wir heute üher diese seltenen Vögel? Berichı über ein 1985-1988 durchgefürtes Projekt. Papageien 3/90: $86-88 ; 4 / 90: 121-125$.

1193. Round, P.D. 1985. Records of the Asian Dowitcher Limnodromus semipalmatus in Thailand. Oriental Bird Club Bull. 1: 5-7.

1194. Round. P.D. 1988. Resident forest hirds in Thailand: their status and conservation. International Council for Birú Preservation (Monograph No. 2). Cambridge, U.K.

1195. Round, P.D. and Treesucon. U. 1986. The rediscovery of Gurney's Pitta Pilla gumeyi. Forkail 2: $53-66$.

1196. Rowan, M.K. 1983. The doves, parrots, lories and cuckoos of southem Africa. David Philip. Cape Town.

1197. Rowley, J.S. and OrT, R. 1964. A new bummingbird from southern Mexico. Condor 66: 81-83.

1198. Roy, J.R.G. 1980. British Virgin Islands avifauna. Unpublished report. 
1199. Rumboll, M.A.E. 1990. Tres aves nuevas para la Argentina. Nuestras Aves 22(8): 28.

1200. Ruschi, O. 1973. Uma nova espécie de Threnetes (Aves, Trochilidae). Bol. Mis. Biol. Prof. Mello Leitao 37: 1-6.

1201. Ruschi, O. 1975. Threnetes christinae sp. nov. Bol. Mus. Bial. Praf. Mello Leinao 83: 1-3.

1202. Russell, S.M. 1964. A distributional study of the birds of British Honduras. Omithological Managraphs 1: 1-95.

1203. Ruttledge, R.F. 1975. A list of the birds of /relond.

1204. Sacarrao, G.F. and Soares, A.A. 1979. Names Portugueses pard as aves da Europa com anotaçōes. Arquives da Museu Bacage Series 2. Vol. 6 No. 23 395-480.

1205. Sakai, H.F., Ralph, C.J. and Jenkins, C.D. 1986. Foraging ecology of the Hawaiian Crow, an endangered generalist. Condor 88: 211-219.

1206. Saleh, M. 1989. The status of the Houbara Bustard in Egypt. Bustard Studies 4.

1207. Salomonsen, F. 1950-1951. Gnonlands Fugle. The birds of Greenland. Ejnar Munksgaard, Kohenhavn.

1208. Salter, R.E. 1983. Summary of currently available information on internationally threatened wildlife species in Burma. Rangoon: Food and Agriculture Organization of the United Nations (Working People's Settlement Board: Nature Conservation and National Parks Project, Burma, FO: Bur $/ 80 / 006$ Field Document 7/83).

1209. Salvador, S., Narosky, S. and Fraga, R. 1986. First description of the nest and eggs of the Rufous-throated Dipper (Cinclus schulzi) in northwestern Argentina Gerfaut 76: 63-66.

1210. Salvan, J. 1967-1969. Contrihution à l"étude des oiseaux du Tchad. Oiseau 37: 255-284; 38: 53-85, $127-150,249-273 ; 39: 38-69$.

1211. Sanderson. J. 1982. Birds of the Turks and Caicos Islands. Turks and Caicas Current Nov./Dec. 1982.

1212. Sane, S.R., Kannan, P., Rajendran, C.G., Ingle, S.T. and Bhagwat, A.M. 1986. On the taxonomic status of Psittacula intermedia (Rothschild). J. Bambay Nat. Hist. Sac, 83: 26-37.

1213. Sanft, K. 1960. Aves/Upupae: Bucerotidae. Das Tierreich 76: 174.

1214. Sankaran, R. 1987. The Lesser Florican. Sanctuary 7: 26-37.

1215. Sankaran, R. and Rahmani. A.R. 1986. Study of ecology of certain endangered species of wildlife and their habitats: the Lesser Florican, annual report? 1985-86. Bombay Natural History Society. Bombay.

1216. Santaella, L. and Sada, A.M. 1991. The avifauna of the Revillagigedo Islands. Mexico: additional data and observations. Wilson Bull. 103; 668-675.

1217. Sarker, S.U. and Iqbal, M. 1985. Observations on Pallas's Fish Eagle Hatiaeetus leuconphus in Bangladesh. Bull. Warld Working Group on Birds of Prey 2: 100-102.

1218. Saxena, V.S. and Meena, B.L. 1985. Occurrence of Lesser Floricans in forest plantations in Rajasthan. India, Bustard Suties 3: 183-184
1219. Schade, F. and Masi Pallarés, R. 1969. Las aves del Paraguay. IIla parte (Psittacidae-Picidae). Revista Parag. Microbiol. 4: 77-96.

1220. Schenck, H. and Aresu, M. 1985. On the distribution number and conservation of the Little Bustard in Sardinia (Italy). 1971-1982. Bustard Sindies 2: $161-164$

1221. Schenk, J. 1940. Namen verzeichnis der Vögel des Historischen Ungarns. Aquila 42-45, 1935-1938, pp. 9-79.

1222. Scherer Neto, P. and Martuscelli, P. 1992. Conservação e biologia do Papagaio-da-cara-roxa, Amazona brasiliensis, nos estados de São Paulo e Paraná. Segundo relatório parcial. Unpublished repon to The Nature Conservancy and Fundaçāo SOS-Mata Atlântica.

1223. Schemazarov, E. 1988. [New breeding site of Dalmatian Pelican Pelecanus crispus in Uzbekistan.] Ornitologiya 23: 225. (In Russian.)

1224. Schlatter, R.P. 1984. The status and conservation of seabirds in Chile. Pp. 261-269 In: Croxall. J.P., Evans, P.G.H. and Schreiber, R.W. (eds.) Sratus and conservation of the world's seahirds. (Techn. Publ. 2). International Council for Bird Preservation. Cambridge, U.K.

1225. Schmulz, E. 1977. Die Vögel der Manggarai (Flores), Ruteng, Flores.

1226. Schodde, R. 1978. The status of endangered Papuasian birds, and Appendix. Pp. 133-145 and 185-206 respectively In: Tyler, M.J. (ed.) The stauss of endangered Australasian wildlife. Royal Zoological Society of South Australia, Adelaide.

1227. Schodde, R., Fullagar, P. and Hermes. N. 1983. A review of Norfolk Island birds past and present. Special publication no. 8. Australian National Parks and Wildlife Service, Canherra.

1228. Schodde, R., and Mathews, S.J. 1977. Cantributions to Papuasian amithology 5: Survey of the birds of Taam Island, Kai group. Division of Wildlife Research Technical Paper no. 33. CSIRO. Australia.

1229. Schouteden, H. 1966. La faune omithologique du Rwanda. Doc. zool. Mus. Roy. Afr. centr. 10:1-130.

1230. Schouteden, H. 1966. La faune ornithologique du Burundi. Doc. zool. Miks. Roy. Afr. centr. 11: 1-81.

1231. Schuchmann K-L. 1978. Allopatrische Artbildung bei der Kolighrigattung Trochilus. Ardea 66: 156-172.

1232. Schuchmann, K-L. 1978. Notes on the Rufous-capped Thombill Chalcastigma nuficeps, a new hummingbird species for Colomhia. Bull. Brit. Orn. Club 98: 115-116.

1233. Schuchmann. K-L. 1980. Okologie und evolution der Trochilidenfauna auf den ozeanischen Inseln der Karihischen See. Bonner Zool. Beilr. 31: 289-309.

1234. Schuchmann. K-L. 1984. Two humminghird species, one a new suhspecies, new to Bolivia. Bull. Brit. Orm. Club 104: 6 .

1235. Schulenherg, T.S. 1989. What is Cox's Sandpiper? Bitish Birds 82: 622-623. 
1236. Schulenberg. T.S., Allen, S.E., Stoz, D.F. and Wiedenfeld, D.A. 1984. Distributional records frnm the Cordillera Yanachaga, central Peru. Gerfaut 74: $57-70$.

1237. Schulenberg. T.S. and Binford, L.C. 1985. A new species of tanager (Emberizidae. Thraupinae, Tangara) from southem Peru. Wilson Bull. 97: 413-420.

1238. Schulz, H. 1985. A review of the world status and breeding distribution of the Little Bustard. Bustard Studies 2: 131-152.

1239. Scoll, D.A. and Brooke, M. de L. 1985. The endangered avifauna of southeastern Brazil: a repnrt on the BOU/WWF expedition of 1980/81 and 1981/82. Pp. 115-139 ln: Diamond, A.W. and Lovejoy, T.E. (eds) Conservation of tropical forest birds. (Techn. Publ. 4). International Council for Bird Preservation. Cambridge. U.K.

1240. Scoll, D.A., and Carp, E. 1982. A midwinter survey of wetlands in Mesopotamia, Iraq: 1979. Sandgrouse 4: 60-76.

1241. Scoll, D.A., Hamadani. H.M. and Mirhosseyni. A.A. 1975. The birds of Iran. Department of Envirnnment. Tehran, Iran.

1242. Scott, J.M. and Garton, E.O. 1991. Population estimates of the Black-capped Vireo. Condor 93: 469-470.

1243. Scolt, J.M., Mountainspring, S., Riper, C. van, Kepler, C.B., Jacobi, J.D., Burr, T.A. and Giffin, J.G. 1984. Ansual variatinn in the distribution, abundance, and habitat response of the Palila (Loxioides baillemi). Auk 101: 647-664.

1244. Segonzac, M. 1972. Données récentes sur la faune des lles Saint-Paul et Nouvelle Amsterdam. L'Oisen 42 No. Spec.: 3-68.

1245. Serle, W. 1965. A third contrihution to the ornithology of the British Cameroons. Ihis 107: 60-94.

1246. Serle, W. 1981. The breeding seasons of hirds in the lowland rain forest and in the montane forest of West Cameroon. Ibis 123: 62-74.

1247. Serle, W. and Morel, G.J. 1977. A field guide to the birds of West Africa. Collins, London.

1248. Serpell, J., Collar, N., Davis, S. and Wells, S. 1983. Submission to the Foreign and Commonwealth Office on the future conservation of Henderson Island in the Pitcaim Group. Unpublished.

1249. Servat, G. and Pearson, D.L. 1991. Natural history notes and records for seven poorly known bird species from Amazonian Peru. Bull. Brit. Om. Club 111: 92-95.

1250. Severinghaus, L.L. 1989. The status and conservation of Lanyu Scops Owl Ous elegans batelensis. Pp. 423 431 In: Meyburg, B.-U. and Chancellor, R.D. (eds.) Raptors in the modem world. Proceedings of the III World Conference on Birds of Prey and Owls. Eilat, Israel, 22-27 March 1987. World Working Group on Birds of Prey and Ow/s, Berlin.

1251. Severinghaus, S. 1986. The adaptability of Mikado and Swinhoe's Pheasants to disturhed habitats in Taiwan. Unpaginated abstract In: Ridley, M. (ed.) Pheasants in Asia 1986. World Pheasant Assnciation.

Basildon, U.K.
1252. Severinghaus, S.R. 1978. Recommendations for the conservation of the Swinhoe's and Mikado Pheasants in Taiwan. World Pheasant Assac. J. 3: 79-89.

1253. Severinghaus, S.R. and Blackshaw, K.T. 1976. A new guide to the birds of Taiwan. Me Ya Publications, Taipei.

1254. Shaw, D. and Maxwell, T.C. 1988. First recond of the Mississippi Kite for Bolivia. J. Raptor Res. 22: 90.

1255. Sheldon, B.C.K. and Duckworth, J.W. 1990. Rediscovery of the Madagascar Serpent-eagle Euriorchis astur. Bull. Brit. Om. Cluh 110: 126-130.

1256. Shelton, P.C. 1976. The natural history of Johnston Atoll, central Pacific Ocean. Atoll Research Bulletin (192). 479pp.

1257. Shi Dong-chou, Yu Xiao-ping, Chang Xiu-yun and Lu Bao-zhong 1989. [The breeding hahits of the Crested Jois (Nipponia nippon). I Zool. Res. 10: 327-332.

1258. Shihaev, Yu.A. 1987a. Distrihutional survey of Japanese Crested Ibis by questionnaire using colour postcards in Far East USSR. Summary, p. $48 \mathrm{ln}$ : The Third Japan-USSR Bird Protection Symposium, $2 I$ November 1986. Wild Bird Society of Japan, Tokyo.

1259. Shibaev, Yu.A. 1987h. Interim report on population survey of Steller's Sea-Eagle in winter in USSR. Summary, p. 48 In: The Third Japan-USSR Bird Protection Symposium, 21 Novemher 1986. [Tokyo]: Wild Bird Society of Japan

1260. Shnbrak, M. and Rahmani, A. 1991. Notes on the Arahian Bustard Ardeotis arabs in Saudi Arabia. Sandgrause 13: 14-23.

1261. Shor, L.L. 1975. A znogengraphic analysis of the South American chaco avifauna. Bull. Amer. Mus. Nat. Hist. 154(3): 163-352.

1262. Short, L.L. 1976. Notes on a collection of hirds from the Paraguayan chaco. Amer. Mus. Novit. 2597.

1263. Short, L.L. 1977. Wild life along the Nile, 28 Octaber 10 17 November, 1977. Repon on the American Museum of Natural History's Nile Tour to Egypt. American Museum of Nalural History, New York.

1264. Shor, L.L. 1982. Woodpeckers of the world. Delaware Museum of Natural History, Greenville, Delaware.

1265. Short, L.L. and Cartonell, M. 1987. Cuha. World Birdwatch 9(3): 8-9.

1266. Shor, L.L., Home, J.F.M. and Muringo-Gichuki, C. 1990. An annotated checklist of the birds of East Africa. Praceedings of the Westem Foundation of Vertehrate Znology 4(3).

1267. Sick, H. 1969. Aves brasileiras ameaçadas de extinçāo e nocoes gerais de conservaçãn de aves dn Brasil. Anales Acad. Bras. Cienc. 41 (supl.): 205-229.

1268. Sick, H. 1979. Notes on some Brazilian hirds. Bull. Brit. Om. Club 99: 115-120.

1269. Sick, H. 1979. Decouverte de la patrie de l'Ara de Lear Anodorhynchus leari. Alauda 47: 59-60.

1270. Sick, H. 1984. Omitologia Brasileira. Volume 1. Editora Universidade de Brasilia, Brasilia.

1271. Sick, H. 1985. Omitongia brasileira, ıma introdı̧̧āo. Editora Universidade de Brasilia, Brasilia. 
1272. Sick. H. 1990. Notes on the taxonomy of Brazilian parrots. Ararajuba 1: 111-112.

1273. Sick, H. and Teixeira, D.M. 1979. Notas sobre aves brasileiras raras ou ameaçadas de extinçāo. Publ. avuls. Mus. Nac. 62.

1274. Siegel, A. 1983. Birds of Montserrat. Montserrat National Trust, Montserrat.

1275. Silva, C.P. da, Munn, C.A., Cintra, R., Renton, K., Valqui, M. and Yamashita, C. 1991. Breeding ecology of Hyacinth Macaws. Psittascene 3(3): 1-3.

1276. Silva, F. 1981. Contribuicao ao conhecimento da papagaio charao, Amazona pretrei (Temminck, 1830) (Psittacidae, Aves). Inheringia, Ser. Zool. 58. 79-85.

1277. Silva, G.L. da and Nacinovic, J.B. 1991. Birds as indicator [sic] for the conservation of Atlantic Forests in Bahia, Brazil. Interim project report to WWF for the period July 1990-July 1991.

1278. Silvius, K. M. 1991. Ecology and conservation of Amazona barbadensis on Margarita Island, Venezuela . Summary report of activities and results, 1989-1991. Unpublished.

1279. Silvius, M.J. 1988. On the importance of Sumatra's east coast for waterbirds, with notes on the Asian Dowitcher Limnodromus semipalmatus. Kukila 3: 117 137.

1280. Silvius, M.J., Verheugt, W.J.M. and Iskandar, J 1986. Coastal wetlands inventory of southeast Sumatra. (Study Report 9). International Council for Bird Preservation, Cambridge, U.K.

1281. Simons, T.R. 1985. Biology and behaviour of the endangered Hawaiian Dark-rumped Petrel. Condor 87: 229-245.

1282. Sinclair, J.C. 1983. S.A.O.S. Rarities Committee report. Bolonakierie 35: 35-40.

1283. Sinclair, J.C. 1987. S.A.O.S. Rarities Committee's annual report. Bokmakierie 39: 12-14

1284. Skokova, N.N. and Vinogradov, V.G. 1986. (Waterfawl habitat consenvation.) Agropromizdat, Moscow. (In Russian.)

1285. Slud, P. 1964. The birds of Costa Rica, distribution and ecology. Bull. Amer. Mus. Nat. Hist. 128: 1-430.

1286. Slud, P. 1967. The birds of Cocos 1sland. Bull. Amer. Mus. Nat. Hist. 134(4): 261-296.

1287. Smeenk, C. 1985. Whales and seabirds. Pp. 6-10, 2426 In: Postma, H. and Rommets, J. (eds.) Progress repart Snellius II Expedition. Theme II. Ventilation of deep sea basins. Cnuise MV Tyra, January 4 February 6 1985. Royal Netherlands Academy of Arts and Sciences/Indonesia Institute of Sciences. Netherlands.

1288. Smet, K. de. 1989. The Houbara Bustard in Algeria: a preliminary report. Bustard Sudies 4.

1289. Smiet, A.C. 1985. Notes on the field status and irade in Moluccan parrots. Biol. Conserv. 34: 181-194.

1290. Smith, E.F.G.. Arctander. P., Fjeldsa, J. and Amir, O.G. 1991. A new species of shrike (Laniidae: Laniarius) from Somalia, verified by DNA sequence data from the only known individual. Ibis 133: 227 235.
1291. Smith, G.T. 1978. The Noisy Scruh-bird. Pp. 117-121 In: Tyler, M.J. (ed.) The staitus of endangered Australasian wildlife. Royal Zoological Society of Australia, Adelaide.

1292. Smith, G.T. 1978..The status of Australian parrots. Pp. 101-108 In: Tyler, M.J. (ed.) The status of endangered Australasian wildlife. Royal Zoological Society of Australia, Adelaide.

1293. Smithers, R.H.N. 1964. A checklist of the birds of the Bechuanaland Pratectorate and the Caprivi Strip. National Museums of Southern Rhodesia.

1294. Smythies, B.E. 1953. The birds of Burma. 2nd edition Oliver and Boyd, Edinburgh.

1295. Smythies, B.E. 1981. The birds of Bameo. 3rd edition. The Sabah Society and the Malayan Nature Society, Malaysia.

1296. Smythies, B.E. 1986. The birds of Burma. 3rd edition. Nimrod Press, Liss, Hampshire, U.K. and Silvio Mattacchione and Co., Pickering, Ontario.

1297. Snow, D. 1950. The birds of São Tomé and Principe in the Gulf of Guinea. Ibis $92: 579-595$.

1298. Snow, D.W. (ed.). 1978. An allas of speciation in African non-passerine birds. British Museum (Natural History), London.

1299. Snow, D.W. 1979. Atlas of speciation in African nonpasserine birds - addenda and corrigenda. Bull. Brit. Om. Club 99: 66-68.

1300. Snow, D.W. 1980. A new species of cotinga from southeastern Brazil. Bull. Brit. Om. Club 100: 213-215.

1301. Snow, D.W. 1982. The cotingas. British Museum (Natural History) and Oxford University Press, London and Oxford.

1302. Snow, D.W. (in prep). Handbook of the birds of Europe, the Middle East and North Africa: the birds of the Western Palearctic. Volume 6. Oxford University Press, Oxford.

1303. Snyder. D.E. 1966. The birds of Gilyana. Peabody Museum, Salem.

1304. Snyder, N.F.R., King, W.B. and Kepler, C.B. 1982. Biology and conservation of the Bahama Parrot. Living Bird 19:91-114.

1305. Snyder, N.F.R., Wiley, J.W. and Kepler, C.B. 1987 The parrots of Luquillo: naural history and conservation of the Puerto Rican Parrot. Western Foundation of Vertebrate Zoology, Los Angeles.

1306. Sociedad Española de Omitologia 1981 . [First general survey of Griffon Vulture breeding colonies in the Iberian peninsula, 1979.] Ardeola 26-27: 165-312.

1307. Solonen, T. 1985. Suamen linnussa. Lintutieto, Helsinki.

1308. Somadikarta, S. 1968. The Giant Swiftet Collacalia gigas Hartert and Butler. Auk 85: 549-559.

1309. Soneghet, M. 1991. Sobre a biologia reprodutiva do "com-com" Fomicivora serrana linoralis (Formicariidae) na itha do Cabo Frio-Arraial do Cabo, RJ. P.13 In: Resumos, I Congresso Brasileiro de Ornitologia. Belém: Museu Paraense Emilio Goeldi. 
1310. Sonobe, K. and Izawa, N. 1987. Endangered bird species in the Karean Peninsula. Museum of Korean Nature (Kores University in Tokyo) and Wild Bird Society of Japan, Tokyo.

1311. Sophasan, S. and Dobias, R. 1984. The fate of the "princess bird", or White-eyed River Martin (Pseudochelidon sirintarae). Natural History Bulletin of the Siam Society 32(1): 1-10.

1312. Squire, J.E. 1990. Some southern records and other observations of the Buff-breasied Button-quail Tumir alivei. Aust. Bird Watcher 13: 149-152.

1313. Stattersfield, A. 1987. Red Data bird: The Mandarin Duck. World Birdwatch 9(2): 5.

1314. Staub, F. 1976 Birds of the Mascarenes and Saint Brandon. Orginisation Normale des Enterprises Liée, Labama House, Pon Louis, Mauritius.

1315. Steadman, D.W. and Olson. S.L. 1985. Bird remains from an archaeological site on Henderson Island, South Pacific: man-caused extinctions on an 'uninhabited' island. Proc. Nain. Acad. Sci. U.S.A. 82: 6191-6195.

1316. Steadman, D.W., Stull, J. and Eaton, S.W. 1979. Natural history of the Ocellated Turkey. World Pheasant Assoc. J. 4: 15-37.

1317. Stepanyan, L.S. 1987. TThe first record of Aviceda jerdani (Blyth 1842) (Accipitridae, Aves) in northern Viet Nam.] Biol. Nauki (Mosc.). 1987(1): 42-45.

1318. Stepanyan, L.S. 1990. IA new hypothesis of the origin of Calidris paramelanotos (Scolopacidae, Aves). I Zoal. Zh. 69: 148-151. (In Russian)

1319. Stepanyan, L.S., Bold, A. and Fomin, V.E. 1988. [Taxonomic list of birds of Mongolia.] Omilologiya 23: 26-35. (In Russian).

1320. Sterbetz, 1. 1982. Migration of Anser enythropus and Branta ruficollis in Hungary 1971-1980. Aquila 89: 107-114.

1321. Stewan, P.A. 1980. Population trends of Barn Owls in North America. American Birds 34:698-700.

1322. Stiles, F.G. 1985. Geographic variation in the Fierythroated Humminghird Panterpe insignis. Omithalogical Monographs 36: 23-30.

1323. Stiles, F.G. 1987. Observaciones sobre lo simacion actual del Picaflor Rojo de Jwan Femandez Sephanoides fernandensis, con recomendaciones para un estudio integral de su ecologia y biologia pablacional. Oficina Regional de la FAO para America Latina y el Caribe.

1324. Stiles, F.G. 1990. Un encuentro con el Mosquerito Antioqueño. Phylloscartes lanyoni Graves. Bol. SAO 1(2): 12-13.

1325. Stiles, F.G., Skutch, A.F. and Gardner, D. 1989. A guide to the birds of Cosia Rica. Christopher Helm, London.

1326. Stockton de Dod, A. 1987. Aves de la Republica Daminicana. 2nd edition. Museo Nacional de Historia Natural, Santo Domingo.

1327. Stoddart, D.R. 1981. Abhott's Booby on Assumptinn. Atoll Research Bulletin 225: 27-32.
1328. Stokes, T. 1979. On the possible existence of the New Caledonian Wood Rail Tricholimnas lafresnayanus. Bull. Brit. Om. Club 99: 47-54.

1329. Stokes, T. 1980. Notes on the landhirds of New Caledonia. Emu 80: 81-86.

1330. Stokes, T. 1988. A review of the birds of Christmas Island, Indian Ocean. Australian National Parks and Wildlife Service, Occasional Paper No. 16.

1331. Siokes, T., Sheils, W. and Dunn, K. 1984. Birds of the Cocos (Keeling) Islands, Indian Ocean. Emu 84: $23-28$

1332. Stone, C.P., Loope, L.L. and Smith, C.W. 1988 Conservation biology in the Galapagos Archipelago: perspectives from Hawaii. Elepaio 48 ; 1-8.

1333. Storer, R.W. 1981. The Rufous-faced Crake (Laterallus xenopterus) and its Paraguayan congeners. Wilson Bull. 93: 137-144.

1334. Storer, R.W. 1989. Notes on Paraguayan birds. Occasional Papers Museum Zool. University of Michigan 719, 21 pp.

1335. Strauhe, F.C. 1991. Notas sobre a distribuição de Eleothreptus anomalus (Gould, 1837) e Caprimulgus langirostris longirostris Bonaparte, 1825 no Brasil (Aves; Caprimulgidae). Acta Biol. Leopoldensia 12: 301-312.

1336. Stuan. S.N. 1986. Usambara Mountains. World Birdwalch 8(3): 8-9.

1337. Stuart, S.N. and Collar, N.J. 1985. Subspeciation in the Karamnja Apalis Apalis karamojae. Bull. Brit. Om. Club. 105: 86-89.

1338. Suarez. O. 1986. Lista de especies en peligro de extinction. Unpublished.

1339. Sukhinin, A.N., Bel'skaya, G.C. and Zhernov, 1.V. 1972. The distribution of the Litlle Owl Athene nocma in Turkmenistan.] Omilologiya 10: 216-227. (In Russian).

1340. Sultana, J. and Gauci, C. 1982. A new guide to the birds of M:alta. The Omithological Society, Valletta.

1341. Sultanov, G.S. 1980. The red hook of T/ahekskoi S.S.R.] Lizbekskii Biol. Zh. 1980(5): 49-52.

1342. Summers, R.W. 1985. Demographic variations in the movements of Upland Geese Chloephaga picla and Ruddy-headed Geese Chloephaga nubidiceps in the Falkland Islands. J. Zool, Lond. (A) 206: 1-15.

1343. Summers, R.W., Underhill, L.G., Middleton, D. and Buckland, S.T. 1985. Tumover in the proulation of Ruddy-headed Geese (Chloephaga nubidiceps) at Goose Green, Folkland Islands. J. Appl. Ecol. 22: 635-643.

1344. Sutton, R. 1981. Hunting the Jamaican Pauraque. Goase Bird Club Broadsheet 37: 4-5.

1345. Sveriges Ornitologiska Fōrening 1978. Sveriges fäglar, aktuell översikt över deras uthredning, numerär ach flytning samt nàgot om svensk omitologi. Sveriges Ornitnlngiska Förening. Stockholm.

1346. Swales, M.K. 1965. The sea-birds of Gough Island. Ibis 107: 17-42, 215-229. 
1347. Swanbeck, A.B. and Seiler, C.F. 1987. First sighting of a Short-eared Owl in the US Virgin Islands. Florida Field Naturalist 15: 52-53

1348. Swennen, C. and Marteijn, E.C.L. 1988. Foraging behaviour of Spoon-billed Sandpiper Eurynorhynchus pygmeus on a mudflat in Peninsular Thailand. Nat Hist. Bull. Siam Sac. 36: 85-88.

1349. Taczanowski, L. and Stolzmann, J. 1881. Notice sur la Loddigesia mirabilis Bourc. Proc. Zool. Soc. Londan: 827-834.

1350. Tarboton, W. 1984. The status and conservation of the Wattled Crane in the Transvaal. Proceedings of the $V$ Pan African Omithological Congress 665-678.

1351. Taylor, K. 1990. A birder's guide to Costa Rica. Revised second edition. Privately published.

1352. Taylor, R.H. 1985. Status, habits and conservation of Cyanoramphus parakeets in the New Zealand region. Pp. 195-211 In: Moors, P.J. (ed.) Consenvation of island birds. (Techn. Publ. 3). International Council for Bird Preservation, Cambridge, U.K.

1353. Taylor, R.H., Heatherbell, E.G., and Heatherbell, E.M. 1986. The Orange-fronted Parakeet (Cyanaramphus malherbi) is a color morph of the Yellow-crowned Parakeet C. auriceps. Natomis 33: 17-22.

1354. Taylor, P.B. and Taylor, C.A. 1988. The status movements and breeding of some birds in the Kikuyu Escarpment Forest, central Kenya highlands. Tauraco 1: 72-89.

1355. Teixeira, D.M. 1987. Notas sobre o "gravatazeiro". Rhopomis ardesiaca (Wied, 1831) (Aves, Formicariidae). Rev. Brasil. Biol. 47: 409-414.

1356. Teixeira, D.M. 1987. A new tyrannulet (Phylloscornes) from northeastern Brazil. Bull. Brit. Om. Club $107(1): 37-41$.

1357. Teixeira, D.M. 1987. Notas sohre Terenura sicki Tejxeira \& Gonzaga. 1983 (Aves, Formicariidae). Bol. Miss. Paraense Emilio Goeldi, Ser. Zool. 3(2): 241-251

1358. Teixeira, D.M. and Carnevalli, N. 1989. Nova espécies de Scyralopus Gould, 1837, do nordeste do Brasil (Passeriformes, Rhinocryptidae). Bol. Mus. Nac. Rio de Janeira n.s. Zool. no.331

1359. Teixeira, D.M. and Gonzaga, L.P. 1983. Um novo Furnariidae do nordeste do Brasil: Philydor novaesi sp. nov. (Aves, Passeriformes). Bol. Mus. Paraense Emilio Goeldi NS Zool. 124: 1-22.

1360. Teixeira, D.M. and Gonzaga, L.P. 1983. A new antwren from northeastern Brazil. Bulletin of the British Omithologists Club 103: 133-135.

1361. Teixeira, D.M., Luigi, G. and Almeida, A.C.C. 1990. A redescoberta de lodopleura pipra leucopygia (Salvin, 1885) no nordeste do Brasil (Passeriformes, Cotingidae). P. 179 In: Resumos, XVII Congresso Brasileira de Zoologia. Universidade Estadual de Londrina, Londrina.

1362. Teixeira, D.M., Nacinovic, J.B. and Pontual, F.B. 1987. Notes on some birds of northeastem Brazil. Bulletin of the British Omithologists Club 107: 151157.
1363. Teixeira, D.M., Nacinovic, J.B. and Tavares, M.S 1986. Notes on some birds of northeastern Brazil. Bulletin of the British Omithologists Club 106: 70-74.

1364. Teixeira, D.M. and Nacinovic, J.B. 1990. A plumagem natal de Taoniscus nanus. Ararajuba 1: $113-114$

1365. Teixeira, D.M. and Negret, A. 1984. The Dwarf Tinamou (Taoniscus nanus) of Central Brazil. Auk 101: 188-189.

1366. Teixeira, D.M. and Snow, D.W. 1982. Notes on the nesting of the Red-billed Curassow Crar blumenbachii. Bull. Brit. Om. Club 102: 83-84.

1367. Temple, S.A. and Wallace, M.P. 1989. Survivorship patterns in a population of Andean Condors Vulur gryphus. P. 247-251 In: Meyburg, B.-U. and Chancellor. R.D. (eds.) Raptors in the madern warld. Proceedings of the III World Conference on Birds of Prey and Owls, Eilat. Israel, 22-27 March 1987. World Working Group on Birds of Prey and Owls, Berlin.

1368. Tets, G.F van and Fullager, P.J. 1984. Status of seabirds breeding in Australia. Pp. 559-571 in Croxall, J.P.. Evans, P.G.H. and Schreiber, R.W. (eds.) Status and conservation of the world's seabirds. (Techn. Publ. 2). Isternational Council for Bird Preservation. Cambridge, U.K.

1369. Turbott, E.G. (Ed) 1990. Checklist of the birds of New Zealand and the Rass Dependency, Antarctica. 3rd edition.

1370. Thelen, K.D. and Faizool, S. 1980. Plan for a system of national parks and other pratected areas in Trinidad and Tabaga. Forestry Division, Ministry of Agriculture Land and Fisheries, Trinidad.

1371. Themido, A.A. 1952. Aves de Portugal. Memariase Estudas do Museu Zoologica de Universidade de Caimbra, no. 213 .

1372. Thibault, J.-C. 1983. Les aiseaur de la Carse.

1373. Thibault, J.-C. 1988. Menaces et conservation des oiseaux de Polynesie française. Pp. 87-124 In: Thibault. J.-C. and Guyot, I. (eds.) Livre rouge des oiseaur menacés des regions francaises d'outre mer. Conseil International pour la Protection des Oiseaux.

1374. Thibault, J.-C. and Rives, C. 1975. Birds of Tahiti.

1375. Thiede, U. 1982. "Yambaru Kuina" (Rallus akinawae), eine neue entdecke Rallenart in Japan. Vagelwell 103: 143-150.

1376. Thiollay, J.-M. 1984. Raptor community structure of a primary rain forest in French Guiana and effect of human hunting pressure. Raptar Research 18: $117-122$

1377. Thiollay, J.-M. 1985. Birds of prey in French Guiana - a preliminary survey. Bull. Warld Warking Group on Birds of Prey 2: 11-15.

1378. Thiollay, J.-M. 1985. Birds of Jvory Coast: status and distribution. Malimbus 7(1): 1-59. 
1379. Thiollay, J.-M. 1989. Distribution and ecology of Palearctic birds of prey wintering in West and Central Africa. Pp. 95-107 ln: Meyburg, B.-U. and Chancellor, R.D. (eds.) Raptars in the modem world. Proceedings of the III World Conference on Birds of Prey and Owls, Eilat, Israel, 22-27 March 1987. World Working Group on Birds of Prey and Owls, Berlin.

1380. Thiollay, J.-M. 1989. Area requirements for the conservation of rain forest raptors and game birds in French Guiana. Conservation Biology 3(2): 128-137.

1381. Thomas, W.W. 1964. A preliminary list of the hirds of Cambodia. Unpublished.

1382. Thomé, G. and Thomé, H. 1986. [The birds of Lebanon.] Publ. Univ. libanaise. (In Arabic.)

1383. Thompson, H. 1992. Red Data bird: White-necked Picatharnes. World Birdwatch 14(3): 13.

1384. Thompson. M.C. and Hackman, C.D. 1968. Birds of the Tokelau islands. Notomis 15: 109-117.

1385. Thompson, P.M. 1987. Zahamena Forest (Madagascar) Expedition 1985. (Study Repon 20) International Council for Bird Preservation. Cambridge, U.K.

1386. Thomsen, J.M. and Munn, C.A. 1988. Cyanopsitua spixii: a non-recovery report. Parrotletter 1(1): 6-7.

1387. Thomsen, P. and Jacobsen, P. 1979. The birds of Tunisia. Peder Jacobsen, Copenhagen.

1388. Thonnerieux, Y. 1988. Etat des connaissances sur la reproduction de l'avifaune du Burkina Faso (ex HauteVolta). Oiseau Rev. Francaise Omithol. 58: 120-145.

1389. Tilson, R.L. and Kok, O.B. 1980. Habitat ecology of Black Storks in the Kuiseh River. Madoqua $11: 347-349$.

1390. Timmermann, D. 1938-1949. Die Vögel Islands. Islendingà Rit. 28:239-524.

1391. Todd, D. 1983. Pritchard's Megapode on Niuafo'ou Island, Kingdom of Tonga. World Pheasant Association Joumal 8: 59-68.

1392. Todd, D. 1984. The Tahili Flycatcher Pomarea nigra tabuensis in Tonga; rejection of an "extinct" subspecies. Bull. Brit. Om. Cluh. 104: 72.

1393. Tomhack, D.F. 1986. Ohservations on the hehaviour and ecology of the Mariana Crow. Condor 88: 398-401.

1394. Tomialojc, L. 1990. Ptaki Polski rozmieszczenie i liczebnosc. Pantstwowe Wydawnictwo Naukowe. Warszawa.

1395. Trail, P.W. 1978. Sight records of two species new for Surinam. Ardea 66: 184-185.

1396. Traylor, M.A. 1952. Notes on hirds from the Marcapata Valley, Cuzco, Peru. Fieldiana, Zool. 34(3): 17-23.

1397. Tuck, L.M. and Borotra, M.J. 1972. Additions to the avifauna of St. Pierre and Miquelon. Can. Fld. Nat. 86: 279-284.

1398. Tumer, D.A. 1974. Cape Grass Owl in Ethinpia. Bull. Brit. Om. Club 94: 38-39.
1399. Tumer, D.A. 1982. The status and distribution of the Arahian Bustard Otis arabs in northeastem Africa and its possible occurrence in northern Kenya. Scopus 6: 20-21.

1400. Tumer, D.A. 1989. The status of Denham's Bustard in Kenya. Bustard Suudies 4.

1401. Tumer, D.A. and Dowsell, R.J. 1988. Additions and corrections to Afrotmpical and Malagasy avifaunas, 1. Westem Indian Ocean islands. Tauraca 1: 130-138.

1402. Turner, D.A. and Forhes-Watson, A.D. 1976 Additional migrant recnrds from Seychelles. Bull. Brit. Om. Club 96: 57-58.

1403. Tumer, D.A. and Goriup, P.D. 1989. The status of Denham's Bustard in Kenya. Bustard Suties 4: 170-173.

1404. Tumer. A. and Rose, C. 1989. Swallows and martins of the world. Christopher Helm. Kent.

1405. Tye, A. and Tye, H. 1991. Bird species on S1 Andrew and Old Providence islands, west Carihbean. Wilson Bull. 103: 493-497.

1406. Tyrrell. E.Q. 1990. Humminghirds of the Caribbean.

1407. UNDP/FAO 1982. National conservation plan for indonesia. 4: Nusa Tenggara. Field repon of UNDP/FAO National Parks Development Project INS/78/061. Bogor: Food and Agriculture Organizalion of the Uniled Nations (Field Report 44).

1408. U.S. Fish and Wildlife Service 1983. Yellowshouldered Blackbird recovery plan. U.S. Fish and Wildlife Service, Allanta, Genrgia.

1409. U.S. Fish and Wildlife Service 1985. Red-cockaded Woodpecker recovery plan. U.S. Fish and Wildife Service, Atlanta, Genrgia.

1410. Uthan, E.K. and Brown, L.H. 1971 A checklist of the birds of Ethiopia. Dept. of Biolngy, Haile Sellassie 1 University, Addis Ahaha, Ethiopia.

1411. Urban. E.K., Fry, C.H. and Keith, S. 1986. The birds af Africa. Volume 2. Academic Press, London and Florida.

1412. Valverde, J.A. 1957. Aves del Sahara Español. Instituto de estudins africanns. Madrid.

1413. Van Ee, C.A. 1981. Status of the Blue Crane in south and southwest Africa. P. 259 in: Lewis, J.C. and Masatomi. $\mathrm{H}$ (eds.) Crane research around the world. Rohinson Press, For Collins, Colorado.

1414. Vardhan, H. 1985. A report on the status of bustards in India. Bustard Studies 3: 113-118.

1415. Varty, N. 1991. The status and conservation of Janaica's threatened and endemic forest avifauna and their hahitats following Hurricane Gilhert. Bird Conserv. Intemain. 1: 135-151.

1416. Vany, N., Adams, J., Espin, P. and Hambler, C. (eds.). 1986. An omitholngical survey of Lake Tota, Colombia. 1982. (Study Report 12). International Council for Bird Preservation. Cambridge, U.K.

1417. Vasiliú. G.D. 1968. Systema Avium Romaniae. Alauda Supp.

1418. Vatev, 1.T. 1991. Ohservations de Flamant rose Phoenicopterus ruher en Bulgarie. Alouda 59: 53-54. 
1419. Vaurie, C. 1964. A survey of the birds of Mongolia. Bulletin of the American Museum of Natural Histony 127: 103-143.

1420. Vaurie, C. 1980. Taxonomy and geographical distribution of the Furnariidae (Aves, Passeriformes). Bulletin of the American Museum of Natural Histony 166(1): 1-357.

1421. Vázquez, M.A. and Maldonado Rodriguez, D.M.A. 1990. Loro Cabeza Amarilla (Amazona ochrocephala) en el estado de Tamaulipas. Unpublished.

1422. Veitch, C.R. 1985. Methods of eradicating feral cats from offshore islands in New Zealand. Pp. 125-141 ls: Moors, P.J. (ed.) Conservarion of island birds. (Techn. Publ. 3). International Council for Bird Preservation, Cambridge, U.K.

1423. Ven, J. van der. 1984. 81 species in need of special protection in council of Europe countries. Unpuhlished report to Council of Europe.

1424. Verheugt, W. 1987. Conservation status and action program for the Milky Stork Mycteria cinera. Calonial Waterbirds 10(2): 211-220.

1425. Verheugt. W. 1988. Red Data bird: Asian Dowitcher. World Birdwatch $10(1): 5$

1426. Vernon, C.J. and Piper, S.E. 1986. The Cape Vulture colony at Colleywobbles. Transkei, in 1984 and 1985. Vulture New's 15: 27-28.

1427. Verrill. G.E. 1892. Notes on the fauna of the island of Dominica: lists of birds obtained and observed, with notes on their habitats, nests and eggs.

1429. Verrill, A.H. 1905. Additions to the avifauna of Dominica: notes on species hitherto unrecorded with descriptions of three new species and a list of all birds now known to occur on the island. Privately published. Barbados.

1431. Vides-Almonacid, R. 1990. Observaciones sobre la utilización del hábitat y la diversidad de especies de aves en una laguna de la puna argentina. Homero 13: $117-28$

1432. Viellard, J. 1971. Données biogeographiques sur l'avifaune d'Afrique centrale. 1. Alauda 39: 227-248.

1433. Vieillard, J. 1971-1972. Données biogéographiques sur l'avifaune d'Afrique centrale. Alauda 39: 227-248; 40: $63-92$.

1434. Vielliard, J. 1990. Uma nova espécie de Asthenes da serra do Cipó, Minas Gerais, Brasil. Ararajuba I: $121-122$.

1435. Vierheilig, M.B. and Vierheilig. H. 1988. Beiträge zu Status und Biologie von Papageien der Insel Margarita, Venezuela. Gefied. Welt 112: 50-52, 91.

1436. Viksne, J. (ed.). 1983. [Birds of Latvia: territorial distribution and number.] Zinatne, Riga .

1437. Vilella, F.J. and Zwank, P.J. 1988. Red Data hird: Puerto Rican Nightjar. World Birdwatch 10(3/4): 9.

1438. Vilina, Y.A. and Capella, J.J. 1991. Estado de conservación del Pato Yunco, Pelecanaides gamoti, en Chile. Resúmenes del IV Congreso de Ornitolngía Neotcopical, 3-9 de Noviembre 1991, Quito. Ecuador (resumen 136)
1439. Viljoen, P.J. 1983. Distribution, numbers and group size of the Karoo Korhaan in Kaokoland, South West Africa. Ostrich 54: 50-51.

1440. Village, A. 1990. The Kestrel. Poyser, Calton, U.K.

1441. Vinokurov, A.A. 1982. Present status of the Branta ruficollis population and measures for its conservation. Aquila 89: 115-122.

1442. Vo Quy 1983. [A catalogue of the birds of Vietnam.] Pp. 12-43 In: Medvedev, L.N. (ed.) [Fauna and ecalagy of the animals of Viemam.I Nauka. Moskva.

1443. Vooren, C.M. and Chiaradia, A. 1990. Seasonal abundance and behaviour of coastal birds on Cassino Beach, Brazil. Omitología Neotropical 1: 9-24.

1444. Voous, K.H. 1948. Notes on a collection of Javanese birds. Limosa $21: 85-100$.

1445. Voous, K.H. 1983. Birds af the Netherlands Antilles. De Walburg Pers, Zutphen.

1446. Vries, T. de. 1984. Problems of reintroducing native animals on islands where they have heen exterminated. Noticias de Galapagos 40: 12.

1447. Vries. T. de. 1989. Conservation, status and ecological importance of the Galapagos Hawk Butea galapagaensis. Pp. 327-330 In: Meyburg, B.-U. and Chancellor, R.D. (eds.) Raptors in the modem world. Proceedings of the III World Conference on Birds of Prey and Owls, Eilat, Israel, 22-27 March 1987. World Working Group on Birds of Prey and Owls, Berlin.

1448. Walker. F.J. 1981. Notes on the birds of northern Oman. Sand grouse 2: 33-55

1449. Walkinshaw, L. 1973. Cranes of the world. Winchester Press, New York.

1450. Wall, J.W. 1992. Worldtwitch. Winging it 4(4): 53

1451. Wallace, D.I.M. 1982. Ohservations on migrant birds at Azraq and north-east Jordan, up to April 1967. Sandgrouse 4: 77-99.

1452. Wallace, D.I.M. 1984. Selected observations from Lebanon, Syria and Jordan in the springs of 1963 and 1966. Sandgrause 6: 24-47.

1453. Walsh, J.F. 1977. Nesting of the Jahiru Stork Ephippiorhynchus senegalensis in West Africa. Bull. Brit. Om. Club 97: 136 .

1454. Walsh, J.F. 1987. Records of birds seen in northeastern Guinea in 1984-85. Malimhus $9(2)$ : 105-122.

1455. Walsh, J.F., Cheke, R.A. and Sowah, S.A. 1990. Additional species and breeding records of birds in the Republic of Togo. Malimbus 12: 2-18.

1456. Walter, A., Walter, J.P. and Brown, C.J. 1986. Breeding record for the Cape Eagle Owl in Namibia. Madaqua 14: 429-431.

1457. Waltrer, H. 1979. Eleonora's Falcon, adaptotions to prey and habitat in a social raptor.

1458. Warham, J. 1990. The petrels: their ecology and breeding systems. Academic Press. London.

1459. Warr, F.E. 1986. A list of Qatar's birds. Qatar Natural History Group Joumal 1983-86:38-51. 
1460. Watkins, B.P. and Furness, R.W. 1986. Population status, breeding and conservation of the Gough Moorhen. Ostrich 57: 32-36.

1461. Watling, D. 1982. Birds of Fiji, Tonga and Samna. Millwood Press, Wellington.

1462. Watling, D. 1983. Sandbox incubator. Animal Kingdom 86(3): 30-35.

1463. Watling, D. 1983. Omithological notes from Sulawesi Emu 83: 247-261

1464. Watling, D. 1986. Rediscovery of a petrel and new faunal records on Gau Island. Oryx 20(1): 31-34.

1465. Watling, D. and Lewanavanua, R.F. 1986. A note to record the continuing survival of the Fiji (MacGillvray's) Petrel Pseudobulweria macgillivrayi Ibis 127: 230-233.

1466. Watson, G.E. 1975. Birds of the Antorctic and SubAntarctic. American Geophysical Union, Washington D.C.

1467. Weaver, C.M. 1982. Breeding habits and status of the Golden-shouldered Parrot Psephouss chrysoplerygius in Queensland. Emu 82: 2-6.

1468. Weinrich, J. 1987. The Kirtland's Warbler in 1987. Michigan Department of Natural Resources (Wildlife Division Report No. 3074)

1469. Weinrich, J. A. 1988. Status of the Kirtland's Warbler, 1987. Jack-Pine Warhler 66: 155-158.

1470. Weiss, J. 1986. Tatigke itshericht 1977-84 der Arbreitsgruppe Feldornithologie. Regulus 4: 167-306.

1471. Welch, G.R. and Welch, H.J. 1984. Djibouti expedition March 1984. A preliminary survey of Francolinus ochropectus and the birdlife of the country. Privately published, Knotingley.

1472. Welch, G.R and Welch, H.J 1984. Birds seen on an expedition to Djibouti. Sandgrouse 6: 1-23.

1473. Welch, G.R and Welch, H.J 1986. Djibouti 11 autumn '85. Unpublished.

1474. Welch, G.R and Welch, H.J 1988. Djibouti III preliminary report. Om. Snc. Middle East Bull. 20: 12.

1475. Welch, G.R and Welch, H.J 1989. A preliminary survey of the Arabian Bustard in Djibouti. Bustard Studies 4.

1476. Welch, G., Welch, H., Denton, M. and Cogilan. S. 1986. Djibouti II preliminary report. World Pheasant Assaciation News 12: 24-27.

1477. Wells, D.R. 1985. The forest avifauna of western Malesia and its conservation. Pp. 213-232 ln: Diamond, A.W. and Lovejoy, T.E. (eds.) Conservation of tropical forest birds.

1478. Wells, J.G. 1887. A catalogue of the birds of Grenada, W. Indies, with observations thereon. Proceedings of the United States National Museum 9: 609-633.

1479. Wells, S. 1988. Red Data bird: Philippine Eagle. World Birdwatch 10(2): 5 .

1480. Weske, J.S. and Terborgh, J.W. 1977. Phaethomis koepckeae a new species of hummingbird from Peru. Condor 79: 143-147.

1481. Weske, J.S. and Terborgh, J.W. 1981. Otus marshalli a new species of screech owl in Peru. Auk 98: 1-7.
1482. Wetmore, A. and Swales, B.H. 1931. The birds of Haiti and the Dominican Republic. Bulletin of the United States National Museum 155.

1483. Wheeler, W.R. 1975. Report on rare and endangered bird species from the Australian mainland. Bull. I.C.B.P. 12: 159-264.

1484. Wheelwright, N.T. 1983. Fruits and the ecology of Respendent Quetzals. Auk 100: 286-301.

1485. White, C.N.M. 1939. A contribution to the ornithology of Crete. Ibis (14) 3: 106-136.

1486. White, C.M.N. and Bruce, M.D. 1986. The birds of Wallacea (Sulawesi, the Moluccas and Lesser Sunda Islands, Indonesia): an annotated checklist. British Ornithologists' Union Checklist No. 7. BOU, London.

1487. White, D.M. 1985. A report on the captive breeding of Australian Bustards at Serendip Wildife Research Station. Bustard Studies 3: 195-212.

1488. Whitney, B.M. 1992. Observations on the systematics, behavior, and vocalizations of "Thamnamanes" accidentalis (Formicriidae). Auk 109: 302-308.

1489. Whitten, A.J., Bishop. K.D., Nash, S.V. and Clayton, L. 1987. One or more extinctions from Sulawesi, Indonesia? Conservation Biology 1: 42-48.

1490. Wiedenfeld, D.A., Schulenberg, T.S. and Robbins, M.B. 1985. Birds of a tropical deciduous forest in extreme northwestern Pen. Pp. 305-315 In: Buckley, P.A., Foster, M.S., Morton, E.S., Ridgely, R.S. and Buckley, F.G. (eds.) Neotropical omithology. American Ornıthologists' Union Ornithological Monograph No. 36. AOU, Washington D.C.

1491. Wilhur, S.R. and Kiff, L.F. 1980. The Califomia Condor in Baja Califomia, Mexico. American Birds 34: $856-859$

1492. Wilcove, D.S. 1987. Public lands management and the fate of the Spotted Owl. Amer. Birds 41(3): 361-367.

1493. Wilcove, D.S. 1992. In praise of ohscurity. Living Bird 11: 36-37.

1494. Wild Bird Society of Japan. 1982. A field guide to the birds of Japan. Tokyo.

1495. Wiley, J.W. 1985. The Puerto Rican Parrot and competition for its nest sites. Pp. 213-223 ln: Moors, P.J. (ed.) Conservation of island birds. (Techn. Publ. 3). International Council for Bird Preservation, Cambridge. U.K.

1496. Wiley, J.W. 1986. Status and conservation of raptors in the West Indies. Birds of Prey Bull. 3: 57-70.

1497. Wiley, J.W. 1991. The status and conservation of parrots and parakeets in the Greater Antilles, Bahama Islands, and Cayman Islands. Bird Conserv. Intematn. 1: $187-214$.

1498. Wiley, J.W. and Ottenwalder, J.A. 1990. Birds of islas Beata and Alto Velo. Dominican Repuhlic. Studies on Neotropical Fauna and Environment 25(2): 65-88.

1499. Wiley, J.W., Post, W. and Cruz, A. 1991. Conservation of the Yellow-shouldered Blackbird Agelaiks xanthomus, an endangered West Indian species. Biol. Conserv. 55: 119-138. 
1500. Wiley, J.W. and Wiley, B.N. 1981. Breeding season ecology and behaviour of Ridgway's Hawk Buteo ridgwayi. Condor 83: 132-151.

1501. Wilkinson, R.J. 1991. Systematic list of bird species recorded at Barito Ulu. Pp. 30-54 in: Anon. The avifauna of Barito Ulu, central Bomeo. (Study Report 48). International Council for Bird Preservation, Cambridge, U.K.

1502. Willgohs, J.F. 1984. Havom i Norge. Trondheim: Direktorat for Vilt og Ferskvannsfisk.

1503. Willi, G. and Broggi, M.F. 1983-1986. Die Vogelwel des Fürstentums Liechtenstein unter Berücksichtigung der benachbarten Gebiete. Ber. Bot.-Zool. Ges. Liechtenstein-Sargans-Werdenberg 12: 61-117; 14 $103-143 ; 15: 37-82$.

1504. Williams, A.J. 1984. The status and conservation of seabirds on some islands in the African sector of the Southem Ocean. Pp. 627-635 In: Croxall, J.P., Evans P.G.H. and Schreiber, R.W. (eds.) Status and conservation of the world's seabirds. (Techn. Publ. 2). International Council for Bird Preservation, Cambridge, U.K.

1505. Williams, A.J. n.d. 1987 Popular checklist of the birds of South West Africa/Namibia. Department of Agriculture and Nature Conservation, Windhoek

1506. Williams, G.R. 1960. The birds of the Pitcairn Islands, central South Pacific Ocean. Ibis 102: 58-70.

1507. Williams, G.R. and Given, D.R. 1981. The red data baak of New Zealand. Nature Conservation Council, Wellington.

1508. Williams, J. 1987. Wattled Crane survey in Caprivi. Quagga 18: 22-23.

1509. Williams, M.D. 1986. Preliminary report on the China Cranewatch 1986. Unpublished.

1510. Williams, M.D., Bakewell, D.N., Carey, G.J. and Holloway, S.J. 1986. On the bird migration at Beidaihe, Hebei Province, China, during spring 1985. Forkaail 2: 3-20.

1511. Williams, M.J. 1986. The number of Auckland Island Teal. Wildfowl 37: 63-70.

1512. Willis, E.O. 1972. Taxonomy, ecology and behaviour of the Sooty Ant-tanager (Habia guturalis) and other ant-tanagers (Aves). Amer. Mus. Navit. 2480.

1513. Willis, E.O. and Oniki, Y. 1981. Levantamento preliminar de aves em treze areas do Estado de Sao Paulo. Rev. Brasil. bial. 41: 121-135.

1514. Willis, E.O. and Oniki, Y. 1985. Bird specimens new for the state of Sao Paulo, Brazil. Rev. Brasil. Biol. 45: 105-108.

1515. Willis, E.O. and Oniki, Y. 1987. Winter nesting of Idopleura pipra (Cotingidae) in southeastern Brazil. III Congress de Omitologia Neotropical: Resumenes. Unpublished.

1516. Willis, E.O. and Oniki, Y. 1990. Levantamento preliminar das aves de inverno $\mathrm{em} \mathrm{dez}$ áreas do sudoeste de Mato Grosso, Brasil. Ararajuba 1: 19-38.

1517. Willis, E.O. and Weinberg. L.F. 1990. Terenura sicki em Pernambuco. $O$ Charāa 16: 14.
1518. Wilson, R.P. 1985. The Jackass Penguin (Spheniscus demersus) as a pelagic predator. Mar. Ecol. Prog. Ser. 25: $219-227$.

1519. Wilson, R.P. 1985. Seasonality in diet and breeding success of the Jackass Penguin Spheniscus demersus. J. Om. 126: 53-62.

1520. Wilson, R.T. 1976. The Ostrich Struthio camelus in Darfur, Republic of Sudan. Bull. Brit. Om. Club 96: 123-125.

1521. Wilson, V.J. 1972. Notes on Otis denhami jacksoni from the Nyika plateau. Bulletin of the British Omithologists Club 92: 77-81.

1522. Wingate, D.B. 1973. A checklist and guide to the birds of Bermuda. Wingate, Bermuda.

1523. Wingate, D.B. 1985. The restoration of Nonsuch Island as a living museum of Bermuda's precolonial terrestrial biome. Pp. 225-238 ln: Moors, P.J.(ed.) Conservation of island birds.

1524. Winkler, R. 1984-1987 Avifauna der Schweiz, eine kommentierte Artenliste. Der Om. Beohachter 81 (56). Ala, Schweizerische Gesellschaft fur Vogelkinde und Vogelschutz, Switzerland.

1525. Winterbottom, J.M. 1971. A preliminary check list of the birds of South West Africa

1526. Winterbottom. J.M. 1974. Turtle Dove Streptopelio turur in South West Africa. Bull. Brit. Om. Club 94: 19.

1527. Wodzicki, K. 1971. The birds of Niue Island, South Pacific: an annotated checklist. Notomis 18: 291-304.

1528. Woinarski, J.C.Z., Eckert, H.J. and Menkhorst, P.W. 1988. A review of the distrihution, hahitat and conservation status of the Western Whipbird Psophodes nigrogularis lencogaster in the Murray mallee. S. Aust. Om. 30: 146-153.

1529. Wolff, S.W. and Milstein, P. le S. 1976. A guide to the terrestrial gamebirds of the Transvaal. Transvaal Provincial Administration, Nature Conservation Division, Pretoria

1530. Wolff, S.W. and Milstein, P. le S. 1976. Rediscovery of the Whitewinged Fluffail in South Africa. Bokmakierie 28: 33-36.

1531. Won Pyong-oh 1976. Checklist of the binds of the Republic of Korea.

1532. Wood, D.S., Leberman, R.C. and Weyer, D. 1986. Checklist of the birds of Belize. Carnegie Museum of Natural History, Pittsburgh.

1533. Wood, P. 1987. Report of the 1986 University of East Anglia Martinique Oriole Expedition. (Study Repon 23). International Council for Bird Preservation, Cambridge, U.K.

1534. Woods, C.A. and Ottenwalder, J.A. (undated) Binds of the national parks of Haiti. University of Florida, Gainesville.

1535. Woods, R.W. 1988. A guide to the birds of the Falkland Islands. Anthony Nelson.

1536. Wotzkow, C. 1985. Status and distribution of Falconiformes in Cuba. Bull. World Working Group on Birds of Prey 2: 1-10. 
1537. Wotzkow, C. 1986. Ecological observations of Gundlach's Hawk Accipiter gundlachii in Cuba. Birds of Prey 2: 1-10.

1538. Wotzkow, C. 1991. New subspecies of Gundlach's Hawk, Accipiter gundlachi (Lawrence). Birds of Prey Bull. 4: 271-292.

1539. Wu Sen-Hsiong and Yang Hsiouying 1991. A guide to the wild birds of Taiwan. Taiwan Wild Bird Information Centre and Wild Bird Society of Japan. $274 \mathrm{pp}$.

1540. Wu Zhi-kang and Hsu Wei-shu 1986. The distribution and abundance of White-crowned Long-tailed

Pheasants Symaticus reevesi in Guizhou province, China. In: Ridley, M. (ed.) Pheasants in Asia 1986. World Pheasant Association, Basildon, U.K.

1541. Yamashina, Y. and Mano, T. 1981. A new species of rail from Okinawa Island. $J$. Yomashino Inst. Om. $13(3): 1-6$

1542. Yamashits, C. 1987. Field observations and comments on the Indigo Macaw (Anndorhynchus leari), a highly endangered species from north-eastern Brazil. Wilson Bulletin 99: 280-282.

1543. Yamashita, C. and Valle, M. de P. 1990. Ocorrência de duas aves raras no Brasil central: Mergus octoselaceus and Tigrisoma fasciatum fascialum. Ararajuba 1: 107-109.

1544. Yang Lan 1989. [A new subspecies of the Sported Owlet Athene brama-A. b. poikila.] Zool. Res. 10: 303-308.

1545. Young, H.G. and Smith, J.G. 1989. The search for the Madagascar Pochard. Dodo 26: 17-34.

1546. Young, L.. Garson, P.J. and Kaul, R. 1987. Calling behaviour and social organization in the Cheer Pheasant: implications for survey technique. World Pheasant Assoc. J. 12: 30-43.

1547. Young, L., Hussain, M. and Asker, G. 1986. Margala Hills Cheer Pheasant reintroduction project progress report - 1985. In: Ridley, M. (ed.) Pheasants in Asia 1986. World Pheasant Association, Basildon. U.K.
1548. Yurlov, A.K. 1981. [Asian Dowitcher Limnodramus semipalmatus in the Lake Chany region (western Siberia).] Pp. 102-109 ln: Yurlov, K.T. (ed.) Ekologiya i biotsenoticheskie svyazi pereletnykh ptits zapadnoi sibiri. Novosibirsk. (In Russian).

1549. Zheng Guang-mei, Zhao Xin-ru, Song Jie, Liu Zongxing and Zhou Hongqing 1985. [On the breeding ecology of Tragopan cabori.] Acta Ecol. Sin. 5: 379 . 385. (In Chinese).

1550. Zheng Guang-mei, Zhao Xin-ru, Song Jie, Liu Zongxing and Zhou Hongqing 1986. [Feeding ecology of the Cabot's Tragopan Tragapan caboti.] Acta Ecal. Sin. 6: 283-288. (In Chinese).

1551. Zheng Guang-mei, Zhao Xin-ru and Song Jie 1986. On the breeding ecology of the Cabot's Tragopan Tragopan caboti. In: Ridley, M. (ed.) Pheasants in Asia 1986. World Pheasant Association. Basildon, U.K.

1552. Zimmer, J.T. 1937. Studies of Peruvian birds. No. XV1l. American Museum Novilates 785.

1553. Zimmer, J.T. 1937. Studies of Peruvian hirds. No. XXV. American Museum Novitates 917.

1554. Zimmer, J.T. 1951. Studies of Peruvian hirds. No. 61. American Museum Novitales 1540.

1555. Zimmerman, D.A. 1978. Eared Trogon - immigrant or visitor? Americon Birds 32: 135-139.

1556. Zino, F. 1991. The Madeira Freira conservation project. World Birdwatch 13(2): 8-9.

1557. Zino, F. and Zino, P.A. 1986. An account of the habitat, feeding habits, density, hreeding and need of protection of the Long-toed Wood Pigeon, Columba trocaz. Bncogiana 97.

1558. Zonfrillo, B. 1977. Re-discovery of the Andean Condor Vulur gryphus in Venezuela. Bult. Brit. Om. Club 97: 17-18.

1559. Zubakin. W.A. and Flint, W.E. 1980. Oekologia und Verhalten der Reliktmöwe (Larus relictus Lonnb.). Beitr. Vcgelkd. 26: 253-275. 



\section{INDEX}

abbotti, Papasula 7

(abbotti, Sula) 7

abeillei, Abeillia 144

Abeillia 144

(Aburria) 67

abyssinicus, Asio 126

(abyssinicus, Asio otus) 126

abyssinicus, Turtur 92

acadicus, Aegalius 125

(Acanthis) 214

Accipiter 24

accipitrinus, Deroptyus 104

Aceros 172

Acestrura 145

Acracephalus 190

Actenaides 171

acunhae, Nesospiza 208

acula, Anas 16

acuticauda, Apus 144

acuticaudata, Aratinga 97

adalberti, Aquila 29

(adalberti, Aquila heliaca) 29

adela, Oreatrochilus 164

Adelomyia 145

Adjutant, Greater 12

Adjutsnt, Lesser 12

adorabilis, Lophomis 161

(adorabilis, Paphosia) 161

adscitus, Planycercus 111

Aegolius 125

Aegypius 29

aegyptiacus, Alopochen 15

aenea, Glaucis 157

aeneocauda, Metallura 163

Aepypodius 65

aequatorialis, Androdon 148

aequinactialis, Buteogallus 36

(Aerodramus) 144

aeruginosus, Circus 38

aestiva, Amazona 93

aethiapicus, Threskiamis 14

Aethopyga 204

afer, Euplectes 220

afer, Turtur 92

affinis, Ninax 132

affinis, Penelopides 174

(affinis, Penelopides panini) 174

afra, Eupodotis 79

afraoides, Eupodoris 79

(afracides, Eupodotis afra) 79

africanus, Bubo 127

africanus, Gyps 42

africanus, Spizaetus 53

Afropavo 67

(Afrotis) 79

Agapamis 92

Agelaiks 213

Agelastes 67

agilis, Amazona 93

Aglaeactis 145

Aglaiacercus 145

Agriacharis 67

Agriomis 183

(Agriamis, albicauda) 183

Aix 15
Akalat, East Coast 200

Akalat, Gabela 200

Akalat, Gunning's 200

Akalat, lringa 200

Akalat. Usambara 200

Akepa, 212

Akialoa, 212

Akiapolaau, 211

Akikiki, 212

Akohekohe, 212

Alauda 186

alba, Cacatua 100

(alba, Egretta) 9

alba, Tyta 123

Albatross, Amsterdam 4

Albatross, Short-tsiled 4

Albatross, Steller's 4

albatrus. Diomedea 4

alberni, Crax 65

alberti, Pueridophora 230

albertinae, Streptocitta 226

albertinum, Glaucidium 129

(albertisi, Drepanamis) 228

alberisi. Epimachus 228

albicaudatus, Buteo 33

albicilla, Haliaeetus 43

albicollis, Leucachloris 161

albicallis, Leucaptemis 48

albidinuchus, Lorius 107

albifacies, Sceloglaur 140

albifrons, Amazona 93

albifrons, Amblyospiza 219

albigula, Buteo 33

(albigula, Butea brachyuns) 33

albigularis, Rhinomyias 199

(albigularis, Rhinomyias gularis) 199

albinucha, Ploceus 222

albipectus, Pyrrhura 117

albipennis, Penelope 66

albirostris, Anthracoceras 173

albirastris, Bubalomis 220

(albitarsus, Ciccaba) 140

albilarsus, Strix 140

albocoronata, Microchera 163

albogularis, Accipiter 24

albagularis, Otus 134

albagularis, Phalcobaenus 64

(albogularis, Phalcoboenus megalopterus) 64

albogularis, Zosterops 205

albalimbatus, Megalurus 196

alboniger, Spizaetus 53

albonotalus, Butea 33

albostriatus, Chlidonias 84

(albostriatus, Chlidonias hybridus) 84

albus, Casmerodius 9

Alcedo 171

(alcinus, Machaerhamphus) 49

alcinus, Macheiramphus 49

Alcippe 191

aldabrana, Nesillas 197

aldabranus, Falca newtoni 59

Alectrurus 183

Alecthe 191

Alethe, Cholo 191
Alethe, Cholo Mountain 191 alexandrae, Pohtelis 112 alexandri, Archilochus 149 alexandri, Psittacula 115 alfredi, Otus 134 alice, Chlarostilbon 152 aliciae, Aglaeactis 145 alinae, Eriocnemis 155 Alisterus 93 alius, Malaconotus 189 alixii, Clytoctantes 179 alleni, Grallaria 180 alopex, Falco 56

Alopachen 15

alpinus, Anairetes 183

Alseonax, Chapin's 196 aleaicus, Falco 56

alticola. Poospiza 209

alrirostre Chrysomma 192

(altirostris, Moupinia) 192

aluco, Strix 140

amabilis, Amazilia 145

amabilis, Charmosyna 102

amabilis, Loriculus 106

Amadina 215

(amami, Turdus) 202

Amandava 215

Amaurocichla 191

amauropteryx, Vidua 225

Amauromis 75

amazilia. Amazilia 146

Amazilia 145

Amazon, Black-billed 93

Amazon, Blue-cheeked 94

Amazon, Blue-fronted 93

Amazon, Caribbean 95

Amazon, Cuban 95

Amazon. Festive 94

Amazon, Green-cheeked 95

Amazon, Hispaniolan 95

Amazon, lmperial 94

Amazon, Kawall's 95

Amazon, Lilac-crowned 94

Amazon, Mealy 94

Amazon, Orange-winged 94

Amazon, Puerto Rican 96

Amazon, Red-browed 95

Amazon, Red-crowned 95

Amazon, Red-lored 94

Amazon, Red-necked 94

Amazon, Red-spectacled 95

Amazon, Red-tailed 94

Amazon, Red-topped 95

Amazon, Saint Lucia 95

Amazon, Saint Vincent 94

Amazon, Scaly-naped 95

Amazon. Tucuman 95

Amazon, Vinaceous 95

Amazon, White-fronted 93

Amazon, Yellow-billed 94

Amszon, Yellow-crowned 95

Amazon, Yellow-faced 96

Amazon, Yellow-fronted 95

Amazon, Yellow-headed 95

Amazon, Yellow-lored 96

Amazon, Yellow-naped 94 
Amazon, Yellow-shouldered 94 Amozona 93

amazonica, Amazona 94

amazonina, Hapalopsittaca 106

ambigua, Aro 96

Amblyomis 227

Amblyospiza 219

amboinensis, Alisterus 93

americano, Grus 73

americana, Rhea 1

americanus, Daptrius 55

americanus, Siphonarhis 143

amethysticollis, Heliangelus 157

amethystina, Calliphlax 150

amethystinus, Lampomis 160

(Ammodramks) 210

amoenus, Phylloscopus 198

(Ampelion) 183

amsterdamensis, Diomedea 4

amurensis, Falco 56

Amytornis 191

Anairetes 183

(anambrae, Estrilda) 216

Anaplectes 219

Anas 16

andicola, Agriomis 183

andinus, Phoenicopterus 14

andinus, Podiceps 3

(andinus, Podiceps nigricollis) 3

andrewsi, Fregata 8

Androdon 148

anerythra, Pitta 185

angelinae, Otus 134

(angelinae, Otus spilocepholus) 134

angolensis, Gypohierax 42

angustirostris, Marmaronetza 21

ankoberensis, Serinus 214

anno, Calypte 150

annamorulae, Melaenomis 196

Anodorhynchus 96

Anomalospize 220

anomalus, Eleothreptus 143

anomalus, Zosterops 205

Anorthinus 173

Anser 19

antorcticus, Rallus 77

Anlbind, Grey-headed 18

Antbird, Rio Branco 179

Antbird, Scalloped 181

Antbird, Slender 181

Antbind, White-masked 181

Anthocephola 148

anthoides, Asthenes 178

(anthoides, Thripophoga) 178

anthopeplus, Polycelis 112

anthophilus, Phaethomis 165

anthracinus, Buteogallus 37

Anthracoceros 173

Anthrocothorax 148

Anthreples 204

(Anthropoides) 74

Anthus 187

antigone, Grus 73

antipodes, Megadyples 3

antoniae, Carpodectes 182

Antpecker, Jameson's 218

Antpecker, Red-fronted 218

Antpitta, Bicolored 180

Antpitta, Brown-banded 180
Antpitta, Giant 180

Antpitta, Hooded 180

Antpitta, Moustached 180

Antpitta, Tachira 180

Antshrike, Plumbeous 180

Antshrike, Speckled 181

Antshrike, White-bearded 179

Anthrush, Rufous-fronted 180

Antvireo, Bicolored 180

Antvireo, Plumbeous 180

Antvireo, Western 180

Antwren, Alagoas 181

Antwren, Ash-throated 181

Antwren, Ashy 181

Antwren, Black-hooded 180

Antwren, Narrow-billed 180

Antwren, Orange-bellied 181

Antwren, Pectoral 181

Antwren, Restinga 180

Antwren, Rio de Janeiro $18 \mathrm{I}$

Antwren, Yellow-numped 181

Apalis $19 !$

Apalis, Kabobo 191

Apalis, Karamoja 192

Apalis, Kungwe 191

Apalis, Long-billed 197

Apalis, White-winged 191

Apalopteron 206

Aphantochroa 149

Aphelacephala 192

Aphrasura 177

opivorus, Pernis 51

Aplonis 226

opoda, Paradisaea 229

opolinari, Cistothorus 190

opperti, Phyllastrephus 188

opproximans, Cincus 39

(approximans, Circus aeruginosus) 39

Aprosmictus 96

Apleryx 1

Apus 144

oquila, Eutoxeres 157

aquilo, Fregata 8

Aquila 29

Ara 96

orabs, Ardeotis 78

(arabs, Choriotis) 78

oracori, Pteroglossus 176

Aracari, Black-necked 176

Aracari, Chestnut-eared 176

Aracari, Green 176

araea, Falco 56

Aramidopsis 75

orarauna, Aro 96

Aratingo 97

oraksioca, Amazona 94

arborea, Dendrocygna 20

Arborophila 67

Arcanator 192

arcanus, Prilinapus 89

archeri, Buteo 33

(archeri, Buteo augur) 33

archeri, Heteromirafro 186

(archeri, Heteromirafra ruddi) 186

Archilachus 149

Ardea 8

andens, Arborophila 67

(andens, Coliuspasser) 220

ardens, Euplectes 220 andens, Selasphorus 168

(Ardeola) 8

Ardeotis 78

andesiaca, Rhopomis 181

ardosiaceus, Falco 56

orfaki, Oreopsinacks 110

argentea, Apalis 191

(orgentea, Apalis rufogularis) 191

argentina, Columba 86

argus, Argusianus 68

Argus, Crested 70

Argus, Great 68

Argusianus 68

aricomae, Cinclades excelsior 178

(aricomae, Cinclodes) 178

(aricomae, Geopsittacus occidentalis) 110

arremonops, Oreothraupis 208

ascalaphus, Bubo 128

(ascolophus, Bubo bubo) 128

ashi, Mirafra 187

asio, Otus 134

Asio 126

Asity, Small-billed 186

assimilis, Chlarostilbon 152

(assimilis, Chlorostilbon canivetii) 152

assimilis, Circus 39

astec, Arotinga 98

Asthenes 178

Astropio 227

Astrapia, Arfak 227

Astrapia, Huon 227

Astrapia, Ribbon-tailed 227

Astrapia, Splendid 228

Astrapia, Stephanie's 228

astreans, Acestrura 145

(astreans, Acestrura heliodor) 145

astrild, Estrilda 216

astur, Eutriorchis 42

Asturina 32

Atelomis 172

ater, Daptrius 56

(ater, Nesophylax) 77

oterrima, Pseudobulwerio 4

(aterrimo, Pterodroma) 4

alerrimus, Probosciger 113

Athene 127

atlanticus, Larus 84

(atlanticus, Larus belcheri) 84

Atlantisio 75

Atlapetes 207

(atro, Afrotis) 79

atra, Chalcopsina 101

atro, Manucodia 229

atro, Myiogra 197

atro, Porzana 77

atra, Pyriglena $18 \mathrm{I}$

atricapillus, Otus 134

atricapillus, Vireo 213

Arrichomis 186

atricollis, Ortygospiza 218

atrogularis, Clytoctantes 180

(atrogularis, Textu) 223

atropurpurea, Xipholena 183

Atthis 149

Actila 184

Attila, Ochraceous 184

attwoteri, Tympanuchus cupido 72 aucklandica, Anas aucklandica 16 aucklandica, Caenocorypha 83 
audax, Aquila 30 audouinit, Larus 84 Augastes 149

augur, Buteo 33

auguralis, Buteo 34

augusti, Phaethomis 165

Aulacorhynchus 176

aurantia, Tyto 124

aurantiffrons, Loriculus 106

aurantius, Ploceus 222

auratus, Chlorostilbon 152

aurea, Aratinga 97

aureliae, Haplophaedia 157

aureocincta, Bangsia 207

(aureocincta, Buthraupis) 207

(aureodorsalis, Bangsia) 207

aureodorsalis, Buthraupis 207

aureonucha, Ploceus 222

aureoventris, Chlorostilbon 152

aurescens, Heliodoxa 158

(aurescens, Potyplancta) 158

auricapilla, Aratinga 97

auriceps, Cyanoramphus 103

auricollis, Ara 96

auricularis, Puffinus 6

(auricularis, Puffinus puffinus) 6

aurifrons, Bolborhynchus 99

aurita, Heliothryx 159

auritus, Nettapus 22

auropalliata, Amazona 94

aurorae, Ducula 88

(australasia, Halcyon) 171

australasia, Todirhamphus 171

australis, Ardeotis 78

(australis, Choriotis) 78

australis, Phalcoboenus 64

australis, Vini 120

Automolus 178

aurumnalis, Amazona 94

autumnalis, Dendrocygna 20

Avadavat, Green 215

Aviceda 32

Avocetbill, Mountain 164

Avocertula 149

Awlbill, Fiery-tailed 149

axillaris, Elanus 41

arillaris, Pterodroma 4

(axillaris, Pzeradroma hypoleuca) 4

aymara, Bolborhynchus 99

ayresi, Sarothrura 77

ayresii, Hieraaeus 46

Aythya 19

azurea, Cochoa 193

(azurea, Cochoa azurea) 193

Babbler, Austen's 201

Babbler, Bagobo 201

Babbler, Black-browed 196

Babbler, Deignan'a 201

Babbler, Flame-lempled 201

Babbler, Jerdon's 192

Babbler, Marsh 198

Babbler, Snowy-throated 201

Babbler, Sooty 201

Babbler, Vanderbilt'a 196

Babbler, White-breasted 200

bachmanii, Vermivora 211

badeigularis, Spelacomis 200

badius, Accipiter 24

badius, Phodilus 123 baen, Aythya 19

baeri, Leucippus 161

baeri, Poospiza 209

(baileyi, Ammodramus) 210

baileyi, Xenospiza 210

baillewi, Larioides 212

(baillewi, Psittirostra) 212

bailloni, Baillonius 176

Baillonius 176

bairdi, Oreomystis 212

bakeri, Sericulus 227

bakkamoeno, Otus 134

balaenarum, Siema 85

Balaeniceps 11

Balearica 73

balli, Otus 134

ballmanni, Malimbus 221

Bangsia 207

banksit, Calyptorhynchus 101

bannemani, Ploceus 222

bannemani, Tauraco 121

barbadensis, Amazona 94

barbarus, Otus 134

barbata, Penelope 66

barbalus, Amytomis 191

barbalus, Dendrortyx 68

barbarus, Gypaetus 42

Barbet, Black-banded 175

Barbet, Toucan 175

Barbet, White-mantled 175

Barbtail, White-throated 178

Barbthmat, Band-tailed 170

Barbthroat, Bronze-tailed 170

Barbthroat, Pale-tailed 170

Barbthroat, Sooty 170

Barbthroat, Sparkling-tailed 170

Bam-Owl, Common 123

Bam-Owl, Minahassa 124

Bam-Owl, Sula 125

(bamandi, Bamardius) 111

bamardi, Platycercus 111

(Bamardius) 111

baroni, Metallura 163

barrabandi, Pionopsilta 110

barroti, Heliothryx 159

bartelsi, Spizaetus 53

Basileuterus 211

Basilomis 226

batavica, Touit 119

Bateleur, 55

batesi, Ploceus 222

(Bathmocercus) 200

Batis 192

Batis, Forest 192

Batis, Gabon 192

Batis, Verreaux's 192

Batrachostomus 143

baudinii, Calyptorhynchus 101

Bay-Owl, Congo 123

Bay-Owl, Oriental 123

Baza, African 32

Baza, Asian 32

Baza, Black 32

Baza, Crested 32

Baza, Jerdon's 32

Baza, Madagascar 32

Baza, Pacific 32

beaudouini, Cincaetus 38

Bebromis 192
Becard, Slaty 183

beccarii, Cochoa 193

(beccarii, Cochoa azurea) 193

beccarii, Otus 136

becki, Psendobulweria 4

(becki, Puerodroma rostrala) 4

(becki, Pheradroma) 4

bello, Goethalsia 157

(bellicosus, Hieraactus) 52

bellicosus, Polemaetus 52

(bengala, Estrilda) 219

bengalensis, Buba 128

(bengalensis, Bubo bubo) 128

bengalensis, Eupodotis 79

bengalensis, Gyps 43

(bengalensis, Houbaropsis) 79

bengalus, Uraeginthus 219

benjamini, Urosticte 170

benschi, Monias 72

(bensoni, Monticola) 199

bensani, Pseudocossyphus 199

berigora, Falco 56

berlepschi, Acestrura 145

berlepschi, Asthenes 178

berlepschi, Dacnis 208

berlepschi, Thripophaga 179

bemieri, Anas 16

bernsteini, Stema 85

bernsteinit, Megapodius 65

Berryeater, Black-headed 182

beryllina, Amazilia 146

beryllinus, Loriculus 106

Besra, 29

biamicus, Falco 56

Biatas 179

bicalcaralum, Polyplectron 70

bicolor, Accipiter 24

bicolor, Cyanophaia 154

bicalor, Dendrocygna 21

bicolor, Lonchura 217

bicolor, Nigrita 218

bicolor, Rhyacomis 199

(bicolor, Spermestes) 217

bicomis, Buceros 174

bidentatus, Harpagus 45

bievi, Gamulax 194

(bilineatus, Pogoniulus) 175

(bilophum, Heliactin) 157

Bird-of-paradise, Blue 230

Bird-of-paradise, Count Raggi's 230

Bird-of-paradise, Crested 228

Bird-of-paradise, Emperor of Germany 230

Bird-of-paradise, Emperor 230

Bird-of-paradise, Greater 229

Bird-of-paradise, King-of-Saxony $\mathbf{2 3 0}$

Bind-of-paradise, King 228

Bird-of-paradise, Lesser 230

Bird-of-paradise, Loria's 228

Bird-of-paradise, MacGregor's 229

Bird-of-paradise, Magnificent 228

Bird-of-paradise, Raggiana 230

Bird-of-paradise, Red 230

Bind-of-paradise, Ribbon-tailed 227

Bind-of-paradise, Standard-wing 231

Bird-of-paradise, Superb 228

Bird-of-paradise, Twelve-wired 231

Bird-of-paradise, Wanle-billed 228

Bird-of-paradise, Wilson's 228 
Bird-of-paradise, Yellow-breasted 228

Bird-of-paradise, Goldie's 229

Bird-of-paradise, Princess Stephanie's 228

Bishop, Black-winged 220

Bishop, Fire-crowned 220

Bishop, Golden 220

Bishop, Orange 220

Bishop, Yellow-crowned 220

bishopi, Moho 206

bistrialus, Burhinus 81

(bitorquatus, Cursorius) 82

bitorquatus, Rhinoptilus 82

Black-Cockatoo, Glossy 101

Black-Cockatoo, Red-tailed 10]

Black-Cockatoo, Slender-billed 101

Black-Cockatoo, White-tailed 10]

Black-Cockatoo, Yellow-tailed 101

Black-Flycatcher, Liberian 196

Black-Hawk, Common 37

Black-Hawk, Lesser 37

Black-Hawk, Mang rove 37

Blackbird, Forbes's 213

Blackbird, Saffron-cowled 213

Blackbird, Yellow-shouldered 213

(blakistoni, Bubo) 131

blakistoni, Ketupa 131

Bleeding-heart, Luzon 88

Bleeding-heart, Mindoro 88

Bleeding-heart, Negros 88

Bleeding-heart, Sulu 88

Bleeding-hear, Tawitawi 88

blewitri, Athene 127

blighi, Myiophonus 197

Blossomerown, 148

Blue-Flycatcher, Rueck's 193

Bluebill, Western 219

Bluebonnet, 109

blumenbachii, Crax 65

blythii, Tragopan 71

boanersis, Monarcha 196

(boanensis, Monarcha trivirgatus) 196

Bobwhite, Masked 68

bocogei, Bostrychia olivacea 13

(bocagei, Lompribis olivacea) 13

bocagii, Amaurocichla 191

Bois, Coq de 201

Boissonneaua 149

Bolbopsinacus 99

Bolborhynchus 99

bollii, Columba 86

bombus, Acestrura 145

bonana, Icterus 213

bonapanei, Coeligena 153

bonthaina, Ficedula 194

boobook, Ninox 132

(boobook, Ninox novaeseelandiae) 132

Boobook, Norfolk 132

Boobook, Southem 132

Boobook, Sumba 133

Booby, Abbott's 7

borealis, Numenius 83

borealis, Picoides 177

bomea, Eos 104

Bostrychia 12

(Bostrychiabocagei) 13

(Bostrychiarara, bocagei) 13

Boubou, Bulo Burti 189

boucardi, Amazilia 146

bougainvillei, Actenoides 171 (bougainvillei, Monachalcyon) 171

bougueri, Urochroa 170

(bourbonnensis, Tchilrea) 201

bourbonnensis, Terpsiphone 201

bourcieri, Phaethomis 165

(bounki, Neophema) 109

bourki, Neopsephorus 109

bouvieri, Scotopelia 140

Bowerbird, Adelber 227

Bowerbird, Adelbert Regent 227

Bowerbird, Fire-maned 227

Bowerbird, Golden-fronted 227

Bowerbird, Yellow-fronted 227

boyciana, Ciconia 11

(boyciana, Ciconia ciconia) 11

broccatus, Moho 206

bracei, Chlorostilbon 152

(bracei, Chlorostilbon maugeus) 152

brachylopha, Lophomis delattrei 162

(brachylopha, Lophomis) 162

Brachypteracias 172

brachyplenis, Buteo 34

brachypterus, Dasyomis 194

Brachypteryx 192

(brachyura, Pitta nympha) 185

brachyunus, Accipiter 24

brochyurus, Buteo 34

brachyurus, Graydidascalus 106

brachyurus, Ramphocinclus 190

Bradypterus 192

brama, Athene 127

branicki, Heliodoxa 158

branicki, Leptosiltoca 106

Branta 19

brasilianum, Glaucidium 129

brasiliensis, Amazono 94

brassi, Philemon 206

brehmeri, Turtur 92

brehmii, Monarcha 196

brehmii, Psittacella 114

brevicaudo, Paradigalla 229

brevipes, Accipiter 24

brevipes, Aratinga holochlora 98

(brevipes, Aratinga) 98

Brilliant, Black-throated 159

Brilliant, Empress 158

Brilliant, Fawn-breasted 158

Brilliant, Green-crowned 158

Brilliant, Pink-throated 158

Brilliant, Rufous-webbed 158

Brilliant, Velvet-browed 159

Brilliant, Velvet-crowned 159

Brilliant, Violet-fronted 158

Bristle-Tyrant, Antioquia 184

Bristlebird, Eastern 194

Bristlebird, Westem 194

Bristlebird, Western Rufous 194

Bristlefront, Stresemann's 182

Broadbill, African Green 177

Broadbill, Grauer's 177

Bruadbill, Guam 197

brodiei, Glaucidium 129

Brolga, 74

Bronze-Cuckoo, Green-cheeked 123

brookii, Ous 134

Brotogeris 99

brucei, Orus 135

briijnit, Aepypodius 65

(bruijnii, Drepanomis) 228 bruijnii, Epimachus 228

bruijnii, Micropsina 108

brunneata, Rhinomyias 199

brunneus, Speirops 205

Brush-Finch, Olive-headed 207

Brush-Finch, Pale-headed 207

Brush-Finch, Yellow-headed 207

Brush-turkey, Bruijn's 65

Brush-turkey, Gray's 65

Brush-turkey, Waigeo 65

Brush-warbler, Aldabra 197

Brush-Warbler, Rodriguez 192

Brush-Wabler, Seychelles 192

(bryaniae, Calliphlox) 166

bryantae, Philodice 166

Bubalomis 220

bubo, Bubo 128

Bubo 127

Bubulcus 8

Buceros 174

buckleyi, Micrastur 62

(buergersi, Accipiter) 41

buergersi, Erythrotriorchis 41

buettikoferi, Nectorinia 204

buffoni, Circus 39

buffonii, Chalybura 151

(Bugeranus) 74

Bulbul, Blue-wattled 189

Bulbul, Mauritius 188

Bulbul, Mottle-breasted 188

Bulbul, Olivaceous 188

Bulbul, Streak-breasted 188

Bulbul, Yellow-bearded 188

bulweri, Lophura 69

Bunting, Big-billed 208

Bunting, Gough 209

Bunting, Grosbeak 208

Bunting, Japanese Yellow 208

Bunting, Tristan 208

Bunting, Yellow 208

burchellii, Neotis 80

Burhinus 81

bumeisteri, Microstilbon 163

bumesii, Prinia 199

Busarellus 32

Bush-Crow, Ethiopian 231

Bush-Crow, Stresemann's 231

Bush-Quail, Manipur 70

Bush-Tanager, Yellow-green 207

Bush-Wartler, Long-billed 192

Bushbird, Recurve-billed 179

Bushbird, Rondônia 180

Bushchat, Hodgson's 200

Bushchat, Stoliczka's 200

Bushchat, Tímor 199

Bushchat, White-bellied 199

Bushchat, White-browed 200

Bushchat, White-throated 200

Bushlark, Sidamo 187

Bushshrike, Cameroon Mountain 189

Bushshrike, Green-breasted 189

Bushshrike, Kupé 189

Bushshrike, Monteiro's 189

Bushshrike, Serle's 189

Bushshrike, Uluguru 189

Bustard, Arabian 78

Bustard, Australian 78

Bustard, Black 79

Bustard, Black-bellied 79 
Bustand, Blue 79

Bustard, Buff-crested 79

Bustard, Denham's 80

Bustard, Great 80

Bustard, Great Indian 78

Bustard, Hartlaub's 79

Bustard, Heuglin's 80

Bustard, Houbara 78

Bustard, Indian 78

Bustard, Karoo 80

Bustand, Kori 78

Bustard, Little 81

Bustard, Little Brown 79

Bustard, Ludwig's 80

Bustard, Nubian 80

Bustard, Red-created 80

Bustard, Rueppell's 79

Bustard, Senegal 80

Bustard, Stanley 80

Bustard, Vigors's 80

Bustard, White-bellied 80

Bustard, White-quilled 79

Butastur 33

buteo, Buteo 34

Buteo 33

Buteogallus 36

Buthraupis 207

butleri, Accipiter 25

butleri, Strix 140

Buttonquail, Black-breasted 72

Buttonquail, Buff-breasted 72

Buttonquail, Sumba 72

Buttonquail, Worcester's 72

Buzzand, African Red-tailed 34

Buzzard, Archer's 33

Buzzard, Augur 33

Buzzard, Black-breasted 45

Buzzard, Common 34

Buzzard-Eagle, Black-chested 42

Buzzard-Eagle, Grasshopper 33

Buzzard-Eagle, Grey 42

Buzzard-Eagle, Grey-faced 33

Buzzard-Eagle, Rufous-winged 33

Buzzard, Eurasian 34

Buzzard, Forest 35

Buzzard, Grasshopper 33

Buzzard, Grey-faced 33

Buzzard, Jackat 36

Buzzard, Lizard 47

Buzzard, Long-legged 36

Buzzard, Madagascar 34

Buzzard, Mountain 35

Buzzard, Red-necked 34

Buzzard, Red-tailed 34

Buzzard, Rough-legged 35

Buzzard, Rufous-winged 33

Buzzard, Upland 34

Buzzard, White-eyed 33

cabanisi, Tangara 210

caboti, Tragopan 71

Cacatua 100

cachinnans, Herpetotheres 62

Cacicus 213

Cacique, Selva 213

cactorum, Aratinga 98

caerulescens, Estrilda 216

caerulescens, Eupodotis 79

caerulescens, Geranospiza 42

caerulescens, Microhierax 63 caenuleus, Elanus 41

(caenuleus, Elanus Leucurus) 41

(cafra, Neoris) 80

cahow, Pierodroma 4

Cahow, 4

caica, Pionopsitta 110

Caique, Black-headed 110

Caique, White-bellied 110

Cairina 20

(Calandrella) 186

caledonicus, Platycercus 111

Calidris 83

califomianus, Gymnogyps 23

californicum, Glaucidium 129

(californicum, Glaucidium gnoma) 129

Callaeas 227

calliope, Stellula 169

Calliphlox 150

callipiera, Pyrrhura 117

Callocephalon 101

Caloenas 85

calolaema, Lampornis 160

Caloperdix 68

Calothorax 150

calthropae, Psittacula 115

calva, Treron 91

(calva, Treron australis) 91

(calvus, Aegypius) 53

calvus, Geronticus 13

calvus, Sarcogyps 53

(calvus, Torgos) 53

Calypte 150

Calyptophilus 207

Calyptorhynchus 101

Calypura 182

Calyptura, Kinglet 182

Comarhynchus 207

cambadiana, Arborophila 67

camelus, Struthio 1

camerunensis, Francolinus 68

camerunensis, Vidua 226

Campephaga 187

Campephilus 176

Campylopterus 150

canadensis, Grus 73

Canary, Sao Tome 214

Canary, Yellow-fronted 215

Canastero, Austral 178

Canastero, Berlepsch's 178

Canastero, Cipó 178

Canastero, Pale-tailed 178

Canastero, White-tailed 178

cancellatus, Prosobonia 84

candicans, Caprimulgus 143

candida, Amazilia 146

canicapilla, Nigrita 218

canicapillus, Serinus 214

(canicapillus, Serinus gularis) 214

caniceps, Psinacula 115

canicularis, Aratinga 98

caninde, Aro 96

canivenii, Chlorostilbon 152

canorus, Melierax 49

(cantans, Euodice) 217

cantans, Lonchura 217

(cantans, Psittirostra) 212

cantons, Telespiza 212

canus, Agapomis 92

capellei, Treron 91 capense, Glaucidium 129

capensis, Anas 16

capensis, Asio 126

capensis, Bubo 128

capensis, Dena 89

capensis, Tyto 124

capistrata, Nesocharis 218

capitalis, Ploceus 223

capilola, Paroaria 209

Capilo 175

capnodes, Otus 138

Caprimulgus 143

Caracara, Black 56

Caracara, Carunculated 64

Caracara, Chimango 64

Caracara, Common 64

Caracara, Crested 64

Caracara, Forater'a 64

Caracara, Guadalupe 64

Caracara, Mountain 64

Caracara, Red-throated 55

Caracara, Striated 64

Caracara, White-throaled 64

Caracara, Yellow-headed 63

Caracara, Yellow-throated 56 carbonaria, Cercomacra 179

Cardinal, Red-crested 209

Cardinal, Yellow 208

Cardinal, Yellow-billed 209

cardinalis, Chalcopsitta 101

Carduelis 214

Carib, Green-throated 156

Carib, Purple-throated 156

caribaea, Columba 86

caribaeus, Vireo 213

(caribboea, Pherodroma) 5

carinatum, Electron 172

carolae, Parotia 230

caroli, Polyonymus 167

Carpococcyx 122

Carpodectes 182

Carpornis 182

carunculata. Paradigalla 229

(carunculatus, Bugeranus) 74

carunculatus, Creadion 227

carunculatus, Grus 74

(carunculatus, Leucocarbo) 7

carunculatus, Phalacrocorax 7

carunculatus, Phalcoboenus 64

(carunculatus, Philestumus) 227

caryophyllacea, Rhodonessa 22

Casmerodius 9

caspius, Tetraogallus 71

cassidix, Aceros 172

cassidix, Lichenostomus melanops 206

(cassidix, Lichenostomus) 206

(cassidix, Meliphaga melanops) 206

(cassidix, Meliphaga) 206

(cassidix, Rhyticeros) 172

cassini, Gymnostinops 213

cassini, Malimbus 221

(cassini, Psarocolius) 213

castonea, Pithys 181

castaneiventris, Amazilia 146

castaneoventris, Lampormis 160

castaneum, Glaucidium 129

(castaneum, Glaucidium capense) 129

castanilius, Accipiter 25

castanonotus, Glaucidium 130 
(cestanonotus, Glaucidium radiatum) 130 (castanops, Tyto novachollandiae) 124 castanopterum, Glaucidium 130 caslanotis, Pheroglossus 176 castelnaudii, Aglaeactis 145 catamene, Loriculus 107 (catamene, Loriculus amabilis) 107 Catherpes 190

Catreus 68

caudatus, Spelacamis 200

cayanensis, Leptodon 48

cebuensis, Copsychus 193

(cebuensis, Copsychus niger) 193

ceciliae, Phylloscartes 184

celebensis, Pemis 52

celebensis, Scolopax 84

cenchraides, Falco 57

centralasicus, Caprimulgus 143

centralis, Vidua 225

Centropus 122

Cephalopterus 182

(Ceratotriccus) 184

Cercamacra 179

cerverai, Cyanolimnas 76

cerverai, Ferminia 190

Chachalaca, Plain 66

Chaetocercus 151

Chaelomis 192

Chaffinch, Blue 214

Chaffinch, Canary Islands 214

(Chalcites) 123

chalconotus, Phalacracorax 7

Chalcopsina 101

chalcopterus, Pionus 110

Chalcostigma 151

chalybata, Manucadia 229

(chalybeata, Hypochera) 225

chalybeata, Vidua 225

chalybeus, Lophomis 162

Chalybura 151

Chanting-Goshawk, Dark 49

Chanting-Goshawk, Eastem 50

Chanting-Goshawk, Pale 49

Charadrius 82

chariessa, Apalis 191

charltonit, Arbaraphila 67

(charlianii, Tropicopendix) 67

Chamosyna 102

Chat, Canary 199

Chat, Canary Islands 199

chathamensis, Haematopus unicalar 81

(chathamensis, Haematopus) 81

cheela, Spilomis 53

Chelictinia 37

cherriei, Synallaxis 179

cherriei, Thripophaga 179

cherrug, Falco 57

(Chertusia) 82

chicquera, Falco 57

chilensis, Phoenicopterus 14

chimachima, Milvago 63

chimaera, Uratelomis 172

chimango, Milvago 64

chimboraza, Oreatrochilus 164

(chimboraza, Oreatrochilus estella) 164

chionagaster, Accipiter 25

chionogaster, Amazilia 146

chionopectus, Amazilia 146

chianura, Elvira 155 chirivi, Brologeris 99

(chiriri, Brotogeris versicolurus) 99

Chlamydotis 78

Chlidonias 84

Chloebia 215

Chlorestes 152

chloris, Anthus 187

chlorocercus, Leucippus 161

chlorocencus, Lorius 107

Chlorochrysa 207

Chlorocichla 188

chlorolepidotus, Trichaglassus 120

chloronothos, Zosterops 205

Chloropeta 192

chloroptera, Aratinga 98

chloropterus, Alisterus 93

chloropterus, Ara 96

chloromynchus, Centropus 122

Chlorospingus 207

Chlorastilban 152

chlorotis, Anas aucklandica 16

chaliba, Otus 135

cholaensis, Alethe 191

Chondrohierax 37

(Chariatis) 78

chrysaetas, Aquila 30

Chrysacoccyx 123

chrysagaster, Neaphema 108

Chrysalampis 153

Chrysammo 192

chrysoparia, Dendraica 211

chrysapterus, Brotogeris 99

chrysopterygius, Psephatus 114

chrysostoma, Neophema 109

chrysura, Hylocharis 159

Chrysuronia 153

chthonia, Grallaria 180

Cicadabird, Sula 188

(Ciccaba) 140

Cicinnurus 228

Ciconia 11

Cinclodes 178

Cinclodes, Royal 178

Cinclodes, White-bellied 178

Cinclus 190

cincto, Notiamystis 206

cincta, Poephila cincla 219

cinerascens, Aplonis 226

cinerascens, Circaetus 37

cinerascens, Prinia 199

cinerea, Callacas 227

cinerea. Mycteria 12

cinerea, Paaspiza 209

cinereicauda, Lampomis 160

cinereiceps, Phyllastrephus 188

cinereifrons, Gamulax 194

cinereus, Circaetus 38

cinerews, Circus 39

cinnamomeipectus, Hemitriccus 184

Cinnyricinclus 226

Circaenus 37

circumcinctus, Spiziapteryx 64

Circus 38

cirthatus, Spizaetus 54

cirnocephalus, Accipiter 25

cirrochlaris, Aphantachroa 149

Cisticala 193

Cisticols, Island 193

Cisticola, Socotra 193
Cisticola, Tana River 193

Cistothorus 190

clamasor, Asio 126

(clamator, Rhinoptynx) 126

clamosus, Atrichomis 186

clanga, Aquila 30

Claravis 85

clarkii, Otus 135

clemenciae, Lampormis 160

Cliff-Swallow, Red Sea 187

clypeara, Anas 17

(clypeata, Spatula) 17

Chytoctantes 179

Chrolaema 153

Chyrothynchus 193

Cnemophilus 228

Cocatoo, Sulphur-crested 100

coccineus, Laxaps 212

Cochaa 193

Cochos, Javan 193

Cochoa, Sumstran 193

Cock-of-the-rock, Guianan 183

Cock-of-the-rock, Andean 183

Cockatoo, Bare-eyed 101

Cockstoo, Blue-eyed 101

Cockatoo, Ducorps's 100

Cockatoo, Gang-gang 101

Cockatoo, Goffin's 100

Cockatoo, Greater Sulphur-crested 100

Cockatoo, Leadbeater's 100

Cockatoo, Lesser Sulphur-crested 101

Cockatoo, Major Mitchell's 100

Cockatoo, Moluccan 100

Cockatoo, Palm 113

Cockatoo, Philippine 100

Cockatoo, Pink 100

Cockatoo, Red-vented 100

Cockatoo, Roseate 104

Cockatoo, Salmon-crested 100

Cockatoo, Tanimbar 100

Cockatoo, Umbrella 100

Cockatoo, White 100

Cockstoo, Yellow-crested 101

coelestis, Aglaiocercus 145

coelestis, Forpus 105

coelestis, Hypothymis 195

caeligena, Coeligena 153

Coeligena 153

Caenacorypha 83

caeruleogularis, Lepidopyga 161

coenulescens, Coracina 187

Coloptes 177

Colasisi, 107

Calibri 154

Colinus 68

(Coliuspasser) 220

Collared-Dove, African 90

Collared-Dove, Mouming 90

collaria, Amazona 94

callaris, Accipiter 25

Collacalia 144

colombianus, Otus 135

calombica, Thalurania 169

(colombica, Thalurania furcara) 169

colonus, Rhinomyias 199

coloria, Enthrura 215

colubris, Archilochus 149

Columba 86

columbarius, Falco 57 
Columbina 87

columboides, Psittacula 115 comatus, Aceros 172

(comatus, Rhyticeros) 172

Combassou, Senegal 225

comeri, Gallinula nesiotis 76

(comeri, Gallinula) 76

Comet, Bronze-tailed 167

Comet, Grey-bellied 169

Comet, Red-tsiled 168

comrii, Manucodia 229

concinna, Glossopsina 105

concolor, Falco 57

concolor, Neospiza 214

concolor, Xenospingus 210

condamini, Eutareres 157

condita, Tijuca 183

Condor, Andean 23

Condor, California 23

Conebill, Tamarugo 211

congensis, Afropavo 67

Conirostrum 211

connivens, Ninox 132

Conothraupis 208

conoveri, Leptorila 89

conspicillatus, Forpus 105

constantii, Heliomaster 159

Conur, Olive-throated 98

Conure, Austral 104

Conure, Black-capped 118

Conure, Black-headed 108

Conure, Blaze-winged 117

Conure, Blue-crowned 97

Conure, Brown-throated 99

Conure, Cactus 98

Conure, Crimson-bellied 118

Conure, Crimson-fronted 98

Conure, Cuban 98

Conure, Dusky-headed 99

Conure, El Oro 118

Conure, Fiery-shouldered 117

Conure, Finsch's 98

Conure, Flame-winged 117

Conure, Golden 98

Conure, Golden-capped 97

Conure, Golden-crowned 97

Conure, Golden-plumed 106

Conure, Green 98

Conure, Green-cheeked 118

Conure, Hispaniolan 98

Conure, Hoffmann's 117

Conure, Jandaya 98

Conure, Maroon-tailed 118

Conure, Mitred 98

Conure, Nanday 108

Conure, Orange-fronted 98

Conure, Painted 118

Conure, Patagonian 103

Conure, Peach-fronted 97

Conure, Pearly 118

Conure, Queen of Bavaria's 98

Conure, Red-bellied 117

Conure, Red-breasted 117

Conure, Red-eared 117

Conure, Red-fronted 99

Conure, Red-masked 98

Conure, Rose-crowned 118

Conure, Rose-headed 118

Conure, Santa Marta 118
Conure, Slender-billed 104

Conure, Sun 99

Conure, White-eared 117

Conure, White-eyed 98

Conure, White-necked 117

Conure, Yellow-eared 109

(conversii, Discosura) 167

conversii, Popelairia 167

convexus, Anthracoceros 173

cookii, Cyanoramphus 103

(cookii, Cyanoramphus novaezelandiae) 103

cookii, Pieradroma 5

cooperi, Otus 135

cooperii, Accipiter 25

Coot, Gough lsland 76

Coot, Horned 76

coprotheres, Gyps 43

Copsychus 193

Coquette, Adorable 161

Coquette, Black-crested 162

Coquette, Dot-eared 162

Coquette, Festive 162

Coquette, Frilled 162

Coquette, Peacock 162

Coquette, Racket-tailed 155

Coquette, Rufous-crested 162

Coquette, Short-crested 162

Coquette, Spangled 162

Coquette, Tufted 162

Coquette, White-crested 161

cora, Thaumastura 169

Coracina 187

Coracopsis 103

Cordonbleu, 219

Cordonbleu, Red-cheeked 219

Corella, Little 101

Corella, Long-billed 101

Corella, Tanimbar 100

Corella, Western 101

Cormorent, Flightless 7

Cormorant, Galapagos 7

Cormorant, Pygmy 7

comuta, Fulica 76

comuta, Heliactin 157

comutus, Eunymphicus 105

coromandus, Bubo 128

coronata, Paroaria 209

coronatus, Anthracoceros 174

coronarus, Harpyhaliaetus 45

coronatus, Malurus 196

(coronatus, Spizaetus) 54

coronatus, Stephanoaetus 54

Coronet, Buff-tailed 149

Coronet, Chestnut-breasted 150

Coronet, Velvet-purple 149

corrugatus, Aceros 172

(corrugatus, Rhyticeros) 172

coruscans, Colibri 154

conina, Terpsiphone 201

Corvus 231

Corythaeola 121

corythaix, Tauraco 121

(corythair, Tauraco persa) 121

coscoroba, Coscoroba 20

Coscoraba 20

Cossypha 193

costae, Calypte 150

costaricensis, Touit 119 (costaricensis, Touit dilecrissima) 119 Cotinga 182

Cotinga, Banded 182

Cotinga, Grey-winged 183

Cotinga, Kinglet 182

Cotinga, Shrike-like 182

Cotinga, White-cheeked 183

Cotinga, White-winged 183

Cotinga, Yellow-billed 182

Cotumicops 75

Coua 123

Coua, Delalande'a 123

Coua, Snsil-eating 123

Coucal, Black-hooded 122

Coucal, Green-billed 122

Coucal, Javan 122

Coucal, Short-toed 122

Coucal, Stere's 122

Coucal, Sunda 122

couloni, Ara 97

courseni, Synallaxis 179

Courser, Jerdon's 82

caxeni, Cyclopsitta diophthalma 104

(coxeni, Opopsitta diophthalma) 104

Crab-Hawk, Rufous 36

Crake, Com 75

Crake, Dot-winged 77

Crake, Henderson 77

Crake, Olivier's 75

Crake, Rufous-faced 77

Crake, Rusty-flanked 76

Crake, Siberian 75

Crake, Speckled 75

Crake, Waters's 78

Crake, White-winged 77

Crane, Black Crowned 73

Crane, Black-necked 74

Crane, Blue 74

Crane, Common 74

Crane, Cuban Sandhill 73

Crane, Demoiselle 75

Crane, Grey Crowned 73

Crane, Hooded $\mathbf{7 4}$

Crane, Mississippi Sandhill 73

Crane, Red-crowned 74

Crane, Sandhill 73

Crane, Sarus 73

Crane, Siberian 74

Crane, Siberian White 74

Crane, Stanley 74

Crane, Wattled 74

Crane, White-naped 75

Crane, Whooping 73

crassus, Poicephalus 112

Crar 65

Creadion 227

creatopus, Puffinus 6

crecca, Anas 17

Creeper, Kauai 212

Creeper, Molokai 212

Creeper, Oahu 212

crex, Crex 75

Crex 75

Crinifer 121

Criniger 188

crispus, Pelecanus 6

cristata, Calyptura 182

cristata, Conthaeola 121

cristata, Goura 89 
cristata, Gubematrix 208

cristata, Lophostrix 131

cristala, Tadoma 23

cristaus, Orthorhynchus 164

cristinae, Threnetes 170

Cracias 193

Crocias, Grey-crowned 193

crossleyi, Alelomis 172

Crossleyia 193

crossoptilon, Crassoptilon 68

Crossoptilon 68

Crow, Banggai 231

Crow, Florea 231

Crow, Guam 231

Crow, Hawaiian 231

Crow, Mariana 231

Crowned-Pigeon, Blue 89

Crowned-Pigeon, Maroon-breasted 89

Crowned-Pigeon, Scheepmaker's 89

Crowned-Pigeon, Solomon Islands 89

Crowned-Pigeon, Southem 89

Crowned-Pigeon, Victoria 89

Crowned-Pigeon, Western 89

cruensasa, Pyrthura 117

cruentus, Ihaginis 69

crumeniferus, Leptoptilos 12

Crypsirina 231

cryptoxanthus, Poicephalus 112

Crypturellus 2

cubensis, Tyrannus 185

Cuckoo, Bay-breasted 123

Cuckoo-falcon, African 32

Cuckoo-falcon, Madagascar 32

Cuckoo-hawk, A frican 32

Cuckoo-hawk, Madagascar 32

Cuckoo, Rufnus-bressted 123

Cuckoo-shrike, Blackish 187

Cuckoo-shrike, Ghans 187

Cuckoo-shrike, Mauritius 188

Cuckoo-shrike, Réunion 188

Cuckoo-shrike, Slaty 188

Cuckoo-shrike, Sula 188

Cuckoo-shrike, Western Wattled 187

Cuckoo-shrike, White-winged 188

cucullata, Carduelis 214

cucullata, Crypsirina 231

cucullata, Grallaricula 180

cucullata, Lonchura 217

cucullatus, Ploceus 223

(cucullatus, Spemestes) 217

(cucullaws, Spinus) 214

cuculoides, Aviceda 32

cuculoides, Glaucidium 130

(cunicularia, Athene) 140

cunicularia, Speotyto 140

cupreiceps, Elvira 155

cupreoventris, Eriocnemis 155

cupripennis, Aglaeactis 145

Curaeus 213

Curassow, Alagoas 66

Curassow, Blue-billed 65

Curassow, Blue-knobbed 65

Curassow, Great 66

Curssow, Helmeted 66

Curassow, Homed 66

Curassow, Red-billed 65

Curassow, Wattled 66

Curassow, Yellow-knobbed 65

Curlew, Bristle-thighed 83
Curlew, Eskimo 83

Curlew, Slender-billed 84

(Cursorius) 82

curvipennis, Campylopterus 150

Cut-thraat, 215

cunieri, Falco 58

cuvierii, Phaeachroa 164

cyaneus, Circus 39

cyanifrons, Amazilia 146

cyanocephala, Amazilia 146

cyanocephala, Psitracula 115

cyanocephala, Stamoenas 90

cyanogenia, Eos 104

Cyanolimnas 76

Cyanoliseus 103

Cyanolyca 231

cyanopectus, Stemochyta 169

Cyanophaie 154

cyanophrys, Euphenusa 156

cyanopis, Columbina 87

Cyanopsitta 103

cyanoptera, Brotogeris 100

cyanopterus, Pterophanes 167

cyanopygius, Forpus 105

Cyanaramphus 103

cyanura, Amazilia 146

cyanuncs, Psitrinus 117

cyanus, Hylocharis 159

Cyclopsitta 103

Cygnus 20

Cynanthus 154

Cyomis 193

Cypseloides 144

Dabchick, Alaotra 3

Dabchick, Madagascar 3

dachilleae, Nannopsittaca 108

Dacnis 208

Dacnis, Black-legged 208

Dacnis, Scarlet-breasted 208

Dacnis-Tanager, Turquoise 209

Dacnis, Turquoise 209

dacotiae, Saricola 199

damii, Xenopirostris 189

Damophila 154

danjoui, Jabouilleia 195

Dapple-throat, 192

Daptrius 55

Dasyomis 194

daubenioni, Crax 65

davidi, Arborophila 67

davidi, Strix 140

(davidi, Strix uralensis) 140

davidianus, Paradoromis 197

(davisoni, Phoenicoparnus papillosa) 14

davisoni, Pseudibis 14

decipiens, Streptopelia 90

decora, Amazilia 146

(decora, Amazilia amabilis) 146

decora, Paradisaea 229

defilippiana, Pterodroma 5

(defilippiana, Pherodroma cookii) 5

(defilippii, Stumella) 213

degodiensis, Mirafra 187

deiroleucus, Falco 58

delalandei, Coua 123

delatrei, Lophomis 162

delphinae, Colibri 154

demersus, Spheniscus 3

Dendrocygna 20
Dendroice 211

Dendrortyx 68

denhani, Neotis 80

dentasa, Petronia 222

derbiano, Psinacula 115

derbianus, Oreophasis 66

derbyi, Eriocnemis 155

Deroptyus 104

desmarestii, Psittaculirostris 116

devillei, Pyrhura 117

diabolicus, Eurostopodus 143

diadema, Chamosyna 102

diandi, Lophura 69

Dicaeum 203

dickinsoni, Falco 58

Dicrurus 227

Didunculus 87

Diglossa 208

dilectissima, Touit 119

dimidiata, Pomarea 198

dimorpha, Uroglaux 142

dinellianus, Pseudocolopteryx 185

Dioch, Red-headed 224

diadon, Harpagus 45

Diomedea 4

diophthalma, Cyclopsira 103

(diophthalma, Opopsitua) 103

(Diphyllodes) 228

Dipper, Rufous-throated 190

discolor, Lothamus 106

Discosura 155

discuns, Prioniturus 113

dissimilis, Psephotus 114

(dissimilis, Psephorus chrysopterygius) 114

distans, Amazilia 146

Diving-Petrel, Peruvian 6

dohertyi, Ptilinopus 89

(dohmii, Glaucis) 167

dahmii, Ramphodon 167

dolei, Palmeria 212

domicella, Lorius 107

dominicus, Anthracothorax 148

Dorsdito, Dinelli's 185

(doriae, Accipiler) 49

dariae. Megatriorchis 49

Doricha 155

dorotheae, Amylomis 191

dorsale, Ramphomicron 168

Doryfera 155

Dove, African Mourning 90

Dove, Blue-headed 92

Dove, Clnven-feathered 87

Dove, Grenada 89

Dove, Laughing 90

Dove, Long-tailed 89

Dove, Masked 89

Dove, Namaqua 89

Dove, Ochre-bellied 89

Dove, Palm 90

Dove, Pink-headed 90

Dove, Red-eyed 90

Dove, Rock 86

Dove, Rnse-grey 90

Dove, Senegal 90

Dove, Socurro 92

Dove, Somali Stock 87

Dove, Tambourine 92

Dove, Tolims 89 
Dove, Turtle 91

Dove, Vinaceoua 91

Dowitcher, Asian 83

Dowitcher, Asiatic 83

Drepanopita 87

(Drepanomis) 228

(Dreptes) 204

Drongo, Comoro 227

Drongo, Grand Comoro 227

Drongo, Mayotte 227

(Dryocichloides) 200

Dryocopus 177

(Dryolimnas) 77

Dryotriorchis 41

dubius, Leptoptilos 12

Duck, Comb 22

Duck, Ferruginous 19

Duck, Freckled 23

Duck, Hartlaub's 22

Duck, Hawaiian 19

Duck, Laysan 18

Duck, Mandarin 15

Duck, Marbled 21

Duck, Muscovy 20

Duck, Pink-headed 22

Duck, White-headed 22

Duck, White-winged 20

Duck, White-winged Wood 20 ducorpsii, Cacalua 100

Duculo 88

dufresniana, Amazona 94

duidae, Campylopterus 150

duivenbodei, Chalcopsitta 102

dupontii, Tilmatura 170

duyvenbodei, Aethopyga 204

Dysithamnus 180

Eagle, Adalbert's 29

Eagle, African Black 31

Eagle, African Crowned 54

Eagle, American Harpy 45

Eagle, Asian Black 47

Eagle, Ayres's 46

Eagle, Bald 44

Eagle, Bateleur 55

Eagle, Black 31,47

Eagle, Black-and-chestnut 51

Eagle, Black-chested 42

Eagle, Black Solitary 45

Eagle, Bonelli's 46

Eagle, Booted 46

Eagle, Crested 50

Eagle, Crowned 45

Eagle, Crowned Solitary 45

Eagle, Golden 30

Eagle, Great Philippine 52

Eagle, Greater Sponed 30

Eagle, Grey-headed Fishing 47

Eagle, Grey Sea 43

Eagle, Guianan Crested 50

Eagle, Gumey's 30

Eagle, Harpy 45

Eagle, Imperial 30

Eagle, Indian Black 47

Esgle, Isidor'a 51

Eagle, Lesser Fishing 47

Eagle, Lesser Spotted 31

Eagle, Litule 46

Eagle, Long-crested 49

Eagle, Martial 52
Eagle, Monkey-eating $\mathbf{5 2}$

Eagle, New Guinea 45

Eagle, New Guinea Harpy 45

Eagle-Owl, African 127

Eagle-Owl, Akun 128

Eagle-Owl, Banded 129

Eagle-Owl, Barred 129

Eagle-Owl, Cape 128

Eagle-Owl, Dusky 128

Eagle-Owl, Foreat 128

Eagle-Owl, Fraser'a 129

Eagle-Ow1, Giant 128

Eagle-Ow1, Lesser 132

Eagle-Owl, Mackinder'a 128

Eagle-Owl, Milky 128

Eagle-Owl, Pharaoh 128

Eagle-Ow1, Philippine 128

Eagle-Owl, Rock 128

Eagle-Owl, Shelley's 129

Eagle-Owl, Sooty 128

Eagle-Ow!, Spot-bellied 128

Eagle-Owl, Spolled 127

Eagle-Owl, Verreaux's 128

Eagle, Philippine 52

Eagle, River 44

Eagle, Rufous-bellied 46

Eagle, Short-loed 38

Eagle, Solitary 45

Eagle, Spanish Imperial 29

Eagle, Spotted 30

Eagle, Steppe 31

Eagle, Verreaux's 31

Eagle, Wahlberg's 32

Eagle, Wedge-tailed 30

Eagle, White-headed 44

Eagle, White-tailed 43

Eagle-Owl, Eurasian 128

Eagle-Owl, Mountain 128

Eagle-Oww, Usambara 129

Eared-Nightjar, Satanic 143

Eared-Pheasant, Brown 68

Eared-Pheasant, Manchurian 68

Eared-Pheasant, Tibetan 68

Eared-Pheasant, White 68

ecaudatus, Terathopius 55

echo, Psintacula 115

(echo, Psittacula eques) 115

Eclectus 104

(Edithomis) 76

edward, Amazilia 147

edwardsi, Lophura 69

edwardsii, Psittaculirostris 116

egregia, Pyrthura 117

Egret, Cattle 8

Egret, Chinese 10

Egret, Great 9

Egret, Great White 9

Egret, Litule 10

Egret, Slaty 10

Egretta 10

eisentrauti, Melignomon 175

Elanoides 41

Elanus 41

elophra, Collocalia 144

(elaphrus, Aerodramus) 144

Electron 172

elegans, Laniisoma elegans 182

(elegans, Laniisoma) 182

elegans, Neopheme 109 elegans, Otus 135

(elegans, Otus scops) 135

elegans, Platycercus 111

eleonorae, Falco 58

Eleothreptus 143

elgini, Spilomis 53

eliciae, Hylocharis 159

eliza, Doricha 155

elliati, Athis 149

ellioti, Symaticus 71

elliotii, Pitta 185

elphinstonii, Columba 86

Elvira 155

Emberiza 208

Emerald, Andean 147

Emerald, Blue-tailed 152

Emerald, Brace's 152

Emerald, Coppery 153

Emerald, Coppery-headed 155

Emerald, Cuban 152

Emerald, Fork-tailed 152

Emerald, Garden 152

Emerald, Glittering-throaled 147

Emerald, Green-tailed 152

Emerald, Hispaniolan 153

Emerald, Honduran 147

Emerald, Nartow-tailed 153

Emerald, Plain-bellied 147

Emerald, Puerto Rican 152

Emerald, Red-billed 152

Emerald, Sapphire-spangled 147

Emerald, Short-tailed 152

Emerald, Tachira 146

Emerald, Versicolored 147

Emerald, White-bellied 146

Emerald, White-chested 146

Emerald, White-tailed 155

emphanum, Polyplectron 70

Empidonar 184

enganensis, Ous 135

(enganensis, Otus umbra) 135

Enicognathus 104

enicura, Doricha 155

ensifera, Ensifera 155

Ensifera 155

ensipennis, Campylopterus 150

Eolophus 104

Eos 104

Ephippiorhynchus 12

Epimachus 228

eremila, Geronticus 13

Eremomela 194

Eremomela, Turner's 194

Eriocnemis 155

erithacus, Psillacus 116

(Erithacus) 195

erythrauchen, Accipiser 25

(erythrocephalus, Automolus) 178 erythrocephalus, Hylocryptus 178

erythrogenys, Aratinga 98 erythrogenys, Microhierax 63 enthronemius, Accipiter 25 erythronotos, Formicivora 180 (erythranolos, Mymotherula) 180 erythrophthalma, Lophura 69 enythrops, Quelea 224

enythroptera, Gallicolumba 88 erythropterus, Aprosmictus 96 erythropus, Accipiter 25 
erythropus, Anser 19

Erythrotriorchis 41

Erythrura 215

estella, Oreotrochilus 164

Estrilda 216

Eudocimus 13

Eugenes 156

Eulampis 156

Eulidia 156

(Eulipaa) 65

eulophores, Egrena 10

Eunymphicus 105

(Euodice) 217

euops, Aratinga 98

eupatria, Psitzacula 115

Eupetomena 156

Eupherusa 156

Euplectes 220

Eupodotis 79

eupogon, Metallura 163

Euprilatis 171

Eurostopodus 143

eurynome, Phaethomis 165

Eurynorhynchus 83

euryplera, Opisthoprora 164

Euscarthmus 184

euteles, Trichoglossus 120

Eutoxeres 157

Eutrichomyias 194

Eurriorchis 42

evelynae, Calliphlox 150

(evelynae, Philodice) 150

evereti, Aceros 172

everetti, Dicaeum 203

everetti, Monarcha 196

(everetti, Monarcha frater) 196

(everetti, Rhyticeros) 172

everetti, Tumix 72

(everetti, Turnix sylvatica) 72

everetti, Zoothera 202

exarhatus, Penelopides 174

excellens, Campylopterus 150

(excellens, Campyloptenus curvipennis) 150

(excelsior, Geosina) 178

exilis, Loriculus 107

eximia. Eupherusa 156

eximius, Platycercus 111

exortis, Heliangelus 158

exquisitus, Cotumicops 75

exsul, Psinacula 115

Fairy, Black-eared 159

Fairy, Purple-crowned 159

Fairy-wren, Purple-crowned 196

falcatus, Campylopterus 150

falcirostris, Sporophila 209

falcirostris, Xiphocolaptes 177

Falco 56

Falcon, African Pygmy 64

Falcon, Alaj 56

Falcon, Amur 56

Falcon, Aplomado 58

Falcon, Barbary 59

Falcon, Bat 60

Falcon, Black 61

Falcon, Brown 56

Falcon, Eastern Red-footed 56

Falcon, Eleonora's 58

Falcon, Grey 58
Falcon, Gyr 61

Falcon, Laggar 58

Falcon, Lanner 56

Falcon, Laughing 62

Falcon, Litule 58

Falcon, Manchurian Red-footed 56

Falcon, New Zealand $\mathbf{5 9}$

Falcon, Orange-breasted 58

Falcon, Peregrine 60

Falcon, Prairie 59

Falcon, Pygmy 64

Falcon, Red-footed 62

Falcon, Red-headed 57

Falcon, Red-necked 57

Falcon, Saker 57

Falcon, Sooty 57

Falcon, Spot-winged 64

Falcon, Teita 58

Falcon, Westem Red-footed 62

Falcon, White-rumped 64

Falconet, Black-legged 63

Falconet, Black-sided 63

Falconet, Black-thighed 63

Falconet, Bornean 63

Falconet, Collared 63

Falconet, Fielden's 64

Falconet, Philippine 63

Falconet, Pied 63

Falconet, Red-legged 63

Falconet, Red-thighed 63

Falconet, Spot-winged 64

Falconet, White-fronted 63

Falconet, White-rumped 64

fallax, Leucippus 161

familiare, Apalopteron 206

familiaris, Acrocephalus 190

fanny, Myrtis 163

fannyi, Thalurania 169

fanovanae, Newtonia 197

Fantail, Malaita 199

farinosa, Amazona 94

fasciata, Amadina 215

fasciatus, Accipiter 25

fasciatus, Hieraaetus 46

fasciinucha, Falco 58

fasciolatus, Circaetus 38

fastuosa, Tangara 210

fastuosus, Epimachus 228

feae, Plerodroma 5

(feae, Pterodroma mollis) 5

feae, Turdus 201

femoralis, Cinnyricinclus 226

femoralis, Falco 58

Ferminia 190

femandensis, Sephanoides 168

femandinae, Colaptes 177

fermugineifrons, Bolbortynchus 99

ferrugineus, Enicognathus 104

festiva, Amazona 94

Ficedula 194

ficedulinus, Zosterops 205

Fig-Parrot, Coxen's Double-eyed 104

Fig-Parrot, Desmarest's 116

Fig-Parrot, Double-eyed 103

Fig-Parrot, Dwarf 103

Fig-Partot, Edwards's 116

Fig-Parrot, Golden-headed 116

Fig-Parrot, Large 116

Fig-Parrot, Orange-breasted 104
Fig-Parrot, Salvadori's 116

fimbriasa, Amazilia 147

fimbriatum, Callocephalon 101

Finch, Aurora 219

Finch, Gough 209

Finch, Gouldian 215

Finch, Green Singing 215

Finch, Grey Singing 214

Finch, Laysan 212

Finch, Mangrove 207

Finch, Nightingale 208

Finch, Nihos 212

Finch, Ribbon 215

Finch, Slender-billed 210

Finch, Southern Black-throated 219

Finch, Tanager 208

Finch, Tristan 208

Finch, Wilkins's 208

Finfoot, Asian 78

Finfoot, Masked 78

finschi, Amazona 94

finschi, Aratinga 98

finschii, Micropsitta 108

finschii, Psinacula 115

(finschii, Psinacula himalayana) 115

Fire-eye, Fringe-backed 181

Fireback, Crested 69

Fireback, Crestless 69

Fireback, Diard's 69

Fireback, Siamese 69

Fireback, Vieillot's 69

Firecrown, Green-backed 168

Firecrown, Juan Fernandez 168

Firefinch, African 216

Firefinch, Bar-breasted 216

Firefinch, Black-bellied 216

Firefinch, Black-faced 217

Firefinch, Blue-billed 216

Firefinch, Brown-backed 216

Firefinch, Lavender 216

Firefinch, Red-billed 217

Firefinch, Senegal 217

Firefinch, Vinaceous 217

Fiscal, Newton's 189

fischeri, Agapomis 92

fischeri, Tauraco 121

(fischeri, Tauraco corythaix) 12]

fischeri, Turdus 202

Fish-Eagle, African 44

Fish-Eagle, Band-tailed 44

Fish-Eagle, Madagascar 44

Fish-Eagle, Pallas's 44

Fish-Eagle, Solomon 44

Fish-Eagle, Vulturine 42

Fish-Eagle, White-bellied 44

Fish-Owl, Blakiston's 131

Fish-Owl, Brown 131

Fish-Owl, Buffy 131

Fish-Owl, Malay 131

Fish-Owl, Tawny 131

Fishing-Owl, Pel's 140

Fishing-Owl, Rufous 140

Fishing-Owl, Vermiculated 140

Flamingo, American 15

Flamingo, Andean 14

Flamingo, Caribbean 15

Flamingo, Chilean 14

Flamingo, Greater 15

Flamingo, James' 14 
Flamingo, Lesser 14

Flamingo, Puna 14

(lammea, Laxops) 212

flammea, Paroreomyza 212

flammeolus, Otus 135

Rammeus, Asio 126

flammula, Selasphorus 168

flaveolus, Platycercus 111

Ravescens, Boissonreaua 149

Ravicans, Foudio 221

Mavicans, Prionitunus 113

Javiceps, Allaperes 207

flavifrons, Amblyomis 227

favifrons, Poicephalus 112

Ravigula, Serinus 214

(Ravipes, Bubo) 131

favipes, Ketupa 131

Ravipes, Malimbus 221

(Ravipes, Ploceus) 221

Alavirostris, Humblotio 195

flavirostris, Paradoxomis 197

favovirens, Chlorospingus 207

Alavoviridis, Trichoglossus 120

Ravus, Agelaius 213

(lavus, Xanthopsar) 213

flavis, Zosterops 205

Flicker, Cuban 177

Flicker, Femandina's 177

flarensis, Corvus 231

Florican, Bengal 79

Florican, Lesser 79

fariceps, Anthocephala 148

Florisuga 157

fosculus, Loriculus 107

Flowerpecker, Brown-backed 203

Flowerpiercer, Venezuelan 208

Flufftail, Slender-billed 78

Flufftail, Waters'a 78

Fluffail, White-winged 77

fuminensis, Mymatherula 181

fuviatilis, Prinia 199

Flycatcher, Biak 197

Flycatcher, Black-banded 194

Flycatcher, Blue-breasted 193

Flycatcher, Brown-chested 199

Flycatcher, Chapin's 196

Flycatcher, Cook Islands 198

Flycatcher, Cuban 185

Flycatcher, Damar 194

Flycatcher, Damar Blue 194

Flycatcher, Fatu Hiva 199

Flycatcher, Grand Comoro 195

Flycatcher, Grey-breasted 184

Flycatcher, Guam 197

Flycatcher, Humblot's 195

Flycatcher, Lompobattang 194

Flycatcher, Marquesan 198

Flycatcher, Matinan 194

Flycatcher, Nimba 196

Flycatcher, Pacific Royal 184

Flycatcher, Rarotonga 198

Flycatcher, Rowley's 194

Flycatcher, Sumba 194

Flycatcher, Sumbs Blue 194

Flycatcher, Tahiti 198

Flycatcher, Timor Blue 194

Flycatcher, Ua Huka 198

Flycatcher-Wartler, Thin-billed 192

Fody, Msuritius 221
Fody, Rodrigues 221

Fody, Seychelles 221

Fody, Yellow 221

Foliage-gleaner, Alagoas 178

Foliage-gleaner, Henna-hooded 178

Foliage-gleaner, Rufous-necked 178

forbesi, Curaeus 213

forbesi, Cyanoramphus auriceps 103

forbesi, Leptodon 48

Forest-Falcon, Barred 63

Foreat-Falco $n$, Buckley's 62

Forest-Falcon, Collared 63

Forest-Falcon, Leaser Collared 62

Forest-Falcon, Lined 63

Forest-Falcon, Plumbeous 63

Forest-Falcon, Slaty-backed 63

Forest-Falcon, Traylor's 62

farficaus, Elanoides 41

Formicarius 180

Farmicivora 180

formosa, Amandava 215

formosa, Anas 18

(Vormosa, Estrilda) 215

formosa, Sitta 203

Forpus 105

Foudia 221

francesii, Accipiter 25

franciae, Amazilia 147

franciscanus, Euplectes 220

(franciscanus, Euplectes orix) 220

franciscanus, Xiphocolaptes 177

Francolin, Cameroon 68

Francolin, Djibouti 68

Francolin, Mount Cameroon 68

Francolin, Nahan's 68

Francolin, Ochre-breasted 68

Francolin, Swamp 68

Francolin, Swierstra's 68

Francolinus 68

Fregata 8

Freirs, 5

freycineti, Myiagra 197

Friarbird, Brass's 206

Friartird, Dusky 207

Friarbird, Morotai 207

Frigatebird, Andrews' 8

Frigatebird, Ascension 8

Frigatebird, Christmas 8

Fringilla 214

(fringillaris, Calandrella) 186

fringillaris, Spizocarys 186

fringillarius, Microhierax 63

fringilloides, Lonchura 217

(ringilloides, Spermestes) 217

Frogmouth, Dulit 143

frommi, Pyrenestes 219

frontalis, Pynhura 117

frontalis, Sporophila 209

frontalis, Sparopipes 225

frugivonu, Calyptophilus 207

Fruit-Dove, Canuculated 89

Fruit-Dove, Marianas 90

Fruit-Dove, Negros 89

Fruit-Dove, Rapa 90

Fruit-Dove, Red-moustached 90

Fruit-Dove, Red-naped 89

fuertesi, Hapalopsinaca 106

(fuertesi, Hapalopsittaca amazonina) 106

fulgens, Eugenes 156 fulgidus, Psitrichas 117

Fulica 76

fuliginosus, Otus 135

(fuliginosus, Otus bakkamoena) 135

fulvescens, Strix 141

(fulvescens, Strix varia) 141

Fulvetta, Gold-fronted 191

Fulvetta, Yellow-fronted 191

fulvus, Gyps 43

funereus, Aegolius 125

funereus, Calyptorhynchus 101

funcata, Thalurania 169

(furcatus, Ceratotriccus) 184

furcatus, Hemitriccus 184

funcifer, Heliomaster 159

fuscata, Pseudeos 114

fuscicapillus, Philemon 207

fuscipennis, Dicrurus 227

fusconota, Nigrita 218

fuscus, Melanotrochilus 162

fuscus, Pionus 110

gabar, Melierar 49

(gabar, Micronisus) 49

(gabela, Erithacus) 200

gabela, Prionops 189

gabela, Sheppardia 200

Galah, 104

galapagoensis, Buteo 34

Galbula 175

galeata, Ducula 88

galeatus, Basilomis 226

galeatus, Dryocopus 177

galericulata, Aix 15

galerita, Cacatua 100

galeritus, Anorrhinus 173

galgulus, Loriculus 107

Gallicolumba 88

gallicus, Circaetus 38

Gallinago 83

Gallinula 76

Gallirallus 76

Gallus 69

gambensis, Plectropterus 22

(gambieri, Halcyon) 171

gambieri, Todirhamphus 171

Gampsonyx 42

Garganey, 18

garleppi, Poospiza 209

gamotii, Pelecanoides 6

Garrular 194

garrulus, Lorius 107

garzetta, Egretta 10

geelvinkiana, Micropsitta 108

gentilis, Accipiter 26

geoffroyi, Augastes 149

geoffroyi, Geoffroyus 105

(geoffroyi, Schistes) 149

Geoffroyus 105

(Geopsittacus) 110

(Geositla) 178

Geathlypis 211

Geranoaetus 42

Geranospiza 42

germaini, Polyplectron 70

Geronticus 13

Gerygone 195

Gerygone, Biak 195

Gerygone, Salvadori's 195

gibsoni, Chlorostilbon 152 
gigantea, Grallaria 180 gigantea, Melampitta 229 gigantea, Pseudibis 14 (gigantea, Thaumatibis) 14 (gigas, Collacalia) 144 gigas, Hydrochous 144 gigas, Patagana 164 gigas, Podilymbus 3 gilberti, Kupeomis 195 (gilberti, Lioptilus) 195 gilvicollis, Micrastur 63 (gilvicallis, Micrastur ruficollis) 63 gindiana, Eupodotis 79 (gindiana, Eupodonis ruficrisıa) 79 gingica, Arborophila 67 glabricollis, Cephalopterus 182 gladiator, Malaconous 189 Glaucidium 129

Glaucis 157

glaucogularis, Ara 97 glaucopis, Thalurania 169 glaucopoides, Eriacnemis 155 glaucops, Tyı 124 (glaucops, Tyta alba) 124 glaucus, Anodorhynchus 96 globulosa, Crax 66 Glassopsinta 105 gnama, Glaucidium 130 (godeffroyi, Halcyon) 171 godeffroyi, Todirhamphus 171 gadefrida, Claravis 85 godini, Eriocnemis 155 goeringi, Hemispingus 208 Goethalsia 157 gaffini, Cacarua 100 gaisagi, Gorsachius 10 galandi, Ploceus 223 Goldentail, Blue-throated 159 Goldenthroat, Green-tailed 167 Goldenthrost, Tepui 167 Goldenthroat, White-tailed 167 Goldfinch, Sao Tome 214 goldiei, Psinteuteles 116 (goldiei, Trichoglossus) 116 goldii, Ninox 133

Goldmania 157 goliath, Ardea 8 goliath, Ducula 88 Gon-gon, 5

Goose, Aleutian 19

Goose, Egyptian 15

Goose, Hawaiian 20

Goose, Knob-billed 22

Goose, Lesser White-fronted 19

Goose, Red-breasted 20

Goose, Spur-winged 22

Gorsachius 10

Goshawk, African 29

Goshawk, Asian Crested 29

Goshawk, Australasian 25

Goshawk, Black 27

Goshawk, Black-mantled 27

Goshawk, Brown 25

Goshawk, Chestnut-mantled 41

Goshawk, Chinese 28

Goshawk, Crested 29

Goshawk, Doria's 49

Goshawk, Fiji 28

Goshawk, Frances's 25
Goshawk, Gabar 49

Goshawk, Gray's 26

Goshawk, Grey 27

Goshawk, Grey-bellied 28

Goshawk, Grey-headed 28

Goshawk, Grey-throated 27

Goshawk, Henst's 26

Goshawk, Meyer's 27

Goshawk, Moluccan 26

Goshawk, New Britain 28

Goshawk, New Caledonia 26

Goshawk, New Guinea Grey-headed 28

Goshawk, Northern 26

Goshawk, Papuan 27

Goshawk, Pied 24

Goshawk, Red 41

Goshawk, Red-chested 29

Goshawk, Slaty-backed 26

Goshawk, Spot-tailed 29

Goshawk, Sulawesi 26

Goshawk, Variable 27

Goshawk, White 27

Goshawk, White-bellied 26

goudori, Lepidopyga 161

goughensis, Rowettia 209

gouldiae, Chloebia 215

(gouldiae, Erythrura) 215

gouldii, Lophornis 162

gounellei, Phaethornis 165

Goura 89

goyderi, Amytomis 191

gracilirostris, Chlorapeta 192

Grackle, Red-bellied 213

Gracula 226

Grallaria 180

Grallaricula 180

gramineus, Tanygnathus 118

grammiceps, Stachyris 200

grammicus, Pseudoscops 139

grandis, Bradypterus 192

grandis, Rhabdomis 203

(grandis, Rhabdomis inomatus) 203

granulifrons, Prilinopus 89

Grass-Owl, African 124

Grass-Owl, Eastem 124

Grass-Parakeet, Blue-winged 109

Grass-Parakeet, Elegant 109

Grass-Parakeet, Splendid 109

Grass-Parakeet, Turquoise 109

Grass-Warbler, Bristled 192

Grass-wren, Westem 191

Grassbird, Bristled 192

Grassbird, Fly River 196

Grassbird, Marsh 196

Grasswren, Carpentarian 191

Grasswren, Eyrean 191

Grasswren, Grey 191

Grasswren, Red-winged 191

Grasswren, Thick-billed 191

graueri, Bradypterus 192

graueri, Pseudocalyptomena 177

Graybird, Luzon 187

Graybird, Moluccan 188

Graybird, White-winged 188

Graydidascalus 106

grayi, Hylocharis 159

graysoni, Mimodes 190

graysoni, Zenaida 92

Grebe, Alaotra 3
Grebe, Atitlsn 3

Grebe, Colombisn 3

Grebe, Delscour'a Litule 3

Grebe, Giant 3

Grebe, Junin 3

Grebe, Madagascar 3

Grebe, Madagascar Little 3

Grebe, Puna 3

Green-Pigeon, African 91

Green-Pigeon, Bruce'\& 91

Green-Pigeon, Large 91

Green-Pigeon, Sumba 91

Green-Pigeon, Timor 91

Green-Pigeon, Yellow-bellied 91

Greenbul, Appert's 188

Greenbul, Dusky 189

Greenbul, Grey-crowned 188

Greenbul, Liberian 188

Greenbul, Prigogine's 188

Greenbul, Spot-winged 188

Greenshank, Nordmann's 84

Greenshank, Spotted 84

(gregaria, Chetrusia) 82

gregarius, Vanellus 82

Griffon, Cape 43

Griffon, Eurasian 43

Griffon, Himalayan 43

Griffon, Long-billed 43

Griffon, Rueppell's 43

grisea, Mymatherula 181

griseiceps, Accipiter 26

griseiceps, Basileuterus 211

griseiceps, Myrmeciza 181

griseipecus, Empidonax 184

(griseipectus, Lathrotriccus) 184

griseiventris, Taphralesbia 169

griseogularis, Accipiter 27

griseogularis, Phaethamis 165

griseus, Passer 222

Grosbeak, Sao Tome 214

Grosbeak, White-fronted 219

Ground-Cuckoo, Banded 123

Ground-Cuckoo, Malay 122

Ground-Cuckoo, Sunda 122

Ground-Dove, Blue-eyed 87

Ground-Dove, Marquesan 88

Ground-Dove, Polynesian 88

Ground-Dove, Purple-barred 85

Ground-Dove, Purple-winged 85

Ground-Dove, Santa Cruz 89

Ground-Dove, Society 1slands 88

Ground-Dove, Thick-billed 88

Ground-Dove, Wetar 88

Ground-Dove, White-collared 88

Ground-Roller, Crossley's 172

Ground-Roller, Long-tailed 172

Ground-Roller, Rufous-headed 172

Ground-Roller, Scaled 172

Ground-Roller, Short-legged 172

Ground-Thrush, Everett's 202

Ground-Thrush, Forest 202

Ground-Thrush, Kibale 202

Ground-Thrush, Oberlaender's 202

Ground-Thrush, Spotted 202

Ground-Thrush, Timor 202

Ground-Warbler, White-winged 211

Grouse, Caucasian 71

grus, Grus 74

Grus 73 
sryphus, Vultur 23 grimeki, Threnetes 157 Guaiabero, 99 guainumbi, Polytmus 167 guajana, Pitta 185 Guan, Bearded 66 Guan, Cauca 66 Guan, Chestnut-bellied 66 Guan, Crested 66 Guan, Highland 66 Guan, Horned 66 Guan, White-winged 66 guarouba, Araringa 98 (guarouba, Guaruba) 98 (Guaruba) 98 guatemalae, Otus 134 Gubermatrix 208 guerinii, Oxypagon 164 guianensis, Morphnus 50 guildingii, Amazona 94 guilielmi, Poradisaea 230 guimeti, Klais 160 guinea, Columba 86 Guinea fowl, White-breasted 67 gularis, Accipiter 26 (gularis, Accipiter virgatus) 26 gularis, Francolinus 68 gularis, Heliodoxa 158 gulielmi, Poicephalus 112 gulielmiterii, Cyclopsina 104 (gulielmitertii, Opopsitta) 104 Gull, Audouin's 84

Gull, Chinese Black-headed 85 Gull, Olrog's 84

Gull, Relict 85

Gull, Saunders' 85

Gull, White-eyed 84 gundlachi, Accipiter 26 (gunningi, Erithacus) 200 gunningi, Sheppardia 200 gumeyi, Aquila 30 gumeyi, Mimizuku 132 (gumeyi, Otus) 132 gumeyi, Pitro 185 guttata, Zoothera 202 guttifer, Tringo 84 gutruralis, Saxicola 199 gury, Phaethomis 165 gymnacepholus, Picotharies 198 Gymnocrex 76

Gymnogene, African 52

Gymnogene, Madagascat 52

(Gymnogloux) 136

Gymnogyps 23

Gymnostinops 213

Gypaerus 42

Gypohierax $\mathbf{4 2}$

Gypopsitta 106

Gyps 42

Gyrfalcon, 61

Habroptila 76

habroptilus, Strigops 118

haematina, Spermophaga 219

haematodus, Trichoglossus 120 haematogaster, Northiella 109 (haematogaster, Psephorus) 109 hoemotonotus, Psephotus 114 Haemotopus 81

haemototis, Pionopsina 110 haematuropygia, Cacatuo 100 haesitatus, Cisticola 193

hagedash, Bostrychio 12

(hagedash, Hagedashia) 12

(Hagedashia) 12

(Halcyon) 171

Haliaeetus 43

haliaetus, Pandion 23

Haliastur 45

(Halietor) 7

(hamatus, Helicolestes) 52

hamatus, Rostrhamus 52

Hamirostro 45

hamlini, Clytorhynchus 193

handleyi, Amazilia 147

Hanging-Parrot, Blue-crowned 107

Hanging-Parrot, Ceylon 106

Hanging-Parrot, Green 107

Hanging-Parrot, Green-fronted 107

Hanging-Parrot, Orange-fronted 106

Hanging-Parrot, Papuan 106

Hanging-Parrot, Philippine 107

Hanging-Parrot, Red-billed 107

Hanging-Parrot, Sangihe 107

Hanging-Parrot, Vernal 107

Hanging-Parrot, Wallace's 107

Hanging-Parrot, Yellow-throated 107

Hanging-Parrot, Moluccan 106

Hanging-Parrot, Sulawesi 107

Hapalopsinaco 106

haplochrous, Accipiter 26

haplochrous, Turdus 201

Haplophaedia 157

homani, Crossoprilon 68

(harmani, Crossoptilon crossoprilon) 68

Horpagus 45

Horpia 45

Harpyhaliaeus 45

harpyja, Harpia 45

Harpyopsis 45

Harrier, Black 40

Harrier, Cinereous 39

Harrier-Eagle, Brown 38

Harrier-Hawk, African 52

Harrier-Hawk, Madagascar 52

Harrier, Hen 39

Harrier, Long-winged 39

Harrier, Montagu's 40

Harrier, Northem 39

Harrier, Pale 39

Harrier, Pallid 39

Harrier, Pied 40

Harrier, Spotted 39

Harrier, Swamp 39

(harrisi, Nannopterum) 7

harrisi, Phalacrocorax 7

harrisii, Aegolius 125

harterti, Batrachostomus 143

hanerti, Ficedulo 194

harterti. Phlogophilus 167

(hartlaubi, Dacnis) 209

hartlaubi, Otus 135

hartlaubi, Pseudodacnis 209

(hartlaubii, Cairina) 22

hartlaubii, Eupodotis 79

(hartloubii, Lissonis) 79

hartlaubii, Pteronetto 22

hasitata, Pteradroma 5

harinhensis, Lophura 69 hawailensis, Corvus 231

Hawk, African Long-tailed 55

Hawk, Barred 48

Hawk, Bat 49

Hawk, Bay-winged 51

Hawk, Bicolored 24

Hawk, Black-chested 48

Hawk, Black-collared 32

Hawk, Black-faced 48

Hawk, Broad-winged 35

Hawk, Chestnut-shouldered 41

Hawk, Collared Fishing 32

Hawk, Cooper's 25

Hawk, Crane 42

Hawk, Duck 60

Hawk-Eagle, African 47

Hawk-Eagle, Ayres's 46

Hawk-Eagle, Black 54

Hawk-Eagle, Black-and-white 54

Hawk-Eagle, Blyth's 53

Hawk-Eagle, Cassin's 53

Hawk-Eagle, Changeable 54

Hawk-Eagle, Chestnut-bellied 46

Hawk-Lagle, Crested 54

Hawk-Eagle, Crowned 54

Hawk-Eagle, Feather-toed 54

Hawk-Eagle, Hodgson's 54

Hawk-Eagle, Javan 53

Hawk-Eagle, Mountain 54

Hawk-Eagle, Ornate 54

Hawk-Eagle, Philippine 54

Hawk-Eagle, Tyrant 54

Hawk-Eagle, Wallace's 54

Hawk, Ferruginous 36

Hawk, Galapagos 34

Hawk, Great Black 37

Hawk, Grey 32

Hawk, Grey-backed 48

Hawk, Grey-bellied 28

Hawk, Grey Frog 28

Hawk, Grey-lined 32

Hawk, Gundlach's 26

Hawk, Gurney's 35

Hawk, Harris's 5]

Hawk, Hawaiian 36

Hawk, Hispaniolan 36

Hawk, Long-tailed 55

Hawk, Mantled 48

Hawk, Marsh 39

Hawk, Masked 48

Hawk-Owl, Admiralty 132

Hawk-Ow1, Andaman 132

Hawk-Owl, Bismarck 133

Hawk-Owl, Brown 133

Hawk-Owl, Chestnut 133

Hawk-Owl, Christmas 133

Hawk-Owl, Christmas Island 133

Hawk-Owl, Jungle 133

Hawk-Owl, Madagascar 133

Hawk-Owl, Manus 132

Hawk-Owl, New Britain 132

Hawk-Owl, New Ireland 133

Hawk-Owl, Ochre-bellied 132

Hawk-Owl, Papuan 142

Hawk-Owl, Philippine 133

Hawk-Owl, Russet 132

Hawk-Owl, Solomon 132

Hawk-Owl, Sooty-backed 133

Hawk, Pigeon 57 
Hawk, Plain-breasted 29

Hawk, Plumbeous 48

Hawk, Puns 35

Hawk, Red-backed 36

Hawk, Red-shouldered 35

Hawk, Red-tailed 35,36

Hawk, Ridgway'a 36

Hawk, Roadaide 35

Hawk, Rough-legged 35

Hawk, Rufous-tailed 36

Hawk, Rufous-thighed 25

Hawk, Savanna 37

Hawk, Semi-collared 25

Hawk, Semiplumbeous 48

Hawk, Sharp-shinned 28

Hawk, Short-tailed 34

Hawk, Slate-colored 48

Hawk, Swainson'a 36

Hawk, Tiny 28

Hawk, Variable 35

Hawk, White 48

Hawk, White-breasted 25

Hawk, White-browed 48

Hawk, White-necked 48

Hawk, White-rumped 35

Hawk, White-tailed 33

Hawk, White-throated 33

Hawk, Zone-tailed 33

Hawk-Eagle, Sulaweri 54

Hawk-Owl, Moluccan 133

Hawk-Owl, Speckled 133

hectori, Gallirallus australis 76

heinrichi, Cossypha 193

heinrothi, Puffinus 6

(heinrathi, Puffinus therminieri) 6

Heleia 204

(helenae, Calypte) 162

helenae, Hypothymis 195

helenae, Lophomis 162

helenae, Mellisuga 162

(helenae, Paphosia) 162

helenae, Parotia 230

(helenae, Paratia Lawesii) 230

heliaca, Aquila 30

Heliactin 157

Heliangelus 157

helianthea, Coeligena 153

(Helicolestes) 52

heliobates, Camarhynchus 207

heliodor, Acestrura 145

Heliodoxa 158

Heliomaster 159

Heliopais 78

Heliothryx 159

helleri, Turdus alivaceus 202

(helleri, Turdus) 202

Helmetcrest, Bearded 164

Helmetshrike, Angola 189

Helmetshrike, Gabela 189

heloisa, Athis 149

Hemignathus 211

hemilasius, Buseo 34

hemileucurus, Campylopterus 151

hemileucurus, Phlogophilus 167

hemileucus, Lampomis 160

Hemispingus 208

Hemispingus, Slaty-backed 208

Hemitriceus 184

henicagrammus, Accipiter 26
Henicopemis 45

henrici, Ficedula 194

henstii, Accipiter 26

herberri, Slachyris 201

hercules, Alcedo 171

herioti, Cyomis 193

(herioti, Nillava) 193

Hermit, Broad-tipped 165

Hermit, Bronzy 157

Hermit, Buff-bellied 166

Hermit, Cinnamon-throated 166

Hermit, Dusky-throated 166

Hermit, Great-billed 165

Hermit, Green 165

Hermit, Grey-chinned 165

Hermit, Hook-billed 167

Hermit, Koepcke'a 165

Hermit, Little 165

Hermit, Long-tailed 166

Hermit, Margaretta's 165

Hermit, Minute 165

Hermit, Needle-billed 166

Hermit, Pale-bellied 165

Hermit, Planalto 166

Hermit, Porculla 165

Hermit, Reddish 166

Hermit, Rufous-breasted 157

Hermit, Saw-billed 168

Hermit, Scale-throated 165

Hermit, Sooty-capped 165

Hermit, Straight-billed 165

Hermit, Tawny-bellied 166

Hermit, White-bearded 165

Hermit, White-browed 166

Hermit, White-whiskered 166

Heron, Goliath 8

Heron, Imperial 8

Heron, Madagascar 8

Heron, White-bellied 8

Herpetotheres 62

Herpsilochmus 181

herrani, Chalcastigma 151

heteraclitus, Geaffroyus 105

Heteromirafra 186

heteropagan. Chalcastigma 151

(Heteraspizias) 37

heterurus, Tanygnathus 119

heuglini, Ploceus 223

heuglinii, Neotis 80

Hieraaetus 46

Hill-Partridge, Rickett's 67

Hill-Partridge, White-eared 67

Hillstar, Andean 164

Hillstar, Black-breasted 164

Hillstar, Ecuadorian 164

Hillstar, Wedge-tailed 164

Hillatar, White-sided 164

Hillstar, White-tailed 170

himalayana, Psittacula 115

himalayensis, Gyps 43

Himantopus 81

hindei, Turdoides 201

hirsula, Glaucis 157

Hirundo 187

hispidus, Phaethomis 165

histrio, Eos 104

Hobby, African 58

Hobby, Australian 58

Hobby, Eurasian 61
Hobby, Northem 61

Hobby, Orienta] 61

hoedtii, Gallicolumba 88

hoematotis, Pyrrhuro 117

hoffmanni, Pyrthura 117

holochlora, Aratinga 98

holosericea, Drepanopilo 87

holosericeus, Eulampis 156

(holosericeus, Sericotes) 156

holospilus, Spilomis 53

(holospilus, Spilomis cheela) 53

holsti, Parus 202

hombroni, Actenoides 171

(hombroni, Halcyan lindsayi) 171

(hombroni, Halcyon) 171

Honey-buzzard, Barred 52

Honey-buzzard, Black 45

Honey-buzzand, European 51

Honey-buzzard, Long-tailed 45

Honey-buzzard, New Britain 45

Honey-buzzard, Papuan 45

Honey-buzzard, Orienta] 52

Honeycreeper, Black-faced 212

Honeycreeper, Crested 212

Honeyeater, Black-chested 206

Honeyester, Bonin 206

Honeyeater, Helmeted 206

Honeyeater, Long-bearded 206

Honeyeater, Regent 207

Honeyeater, Tagula 206

Honeyeater, Wetar 206

Honeyguide, Yellow-footed 175

(Hoplopterus) 82

hordeaceus, Euplectes 220

Hombill, Black 174

Hombill, Blyth's 173

Hombill, Brown 173

Hombill, Bushy-crested 173

Hombill, Great 174

Hombill, Helmeted 174

Hombill, Knobbed 172

Hombill, Luzon 174

Hombill, Mindanao 174

Hombill, Mindoro 174

Hombill, Narcondam 173

Hombill, Palawan 174

Hombill, Papuan 173

Hombill, Plain-pouched 173

Hombill, Rhinoceros 174

Hombill, Rufous 174

Hombill, Rufous-cheeked 173

Hombill, Rufous-necked 173

Hombill, Samar 175

Hombill, Sulawesi 174

Hombill, Sulu 174

Hornbill, Sumba 172

Hombill, Tarictic 175

Hombill, White-crowned 172

Hombill, Wreathed 173

Hombill, Wrinkled 172

Hombill, Writhed 173

Hombill, Writhed-billed 173

Horned-Pheasant, Crimson 71

Homed-Pheasant, Westem 71

(Houbaropsis) 79

hoyi, Onus 134

huallagae, Aulacorhynchus 176

huancavelicae, Asthenes dorbignyi 178

(huancavelicae, Asthenes) 178 
hueti, Touit 119

(huhula, Ciccaba) 141

huhula, Strix 141

humbloti, Ardea 8

Humblotia 195

humboldti, Spheniscus 3

humei, Sphenocichla 200

humiae, Symaticus 71

humilis, Eupodotis 79

humilis, Ichthyophaga 47

Hummingbird, Allen's 168

Hummingbird, Amazilia 146

Hummingbird, Amethyst-throsied 160

Hummingbird, Anns's 150

Hummingbird, Antillean Crested 164

Hummingbird, Azure-crowned 146

Hummingbird, Beautiful 150

Hummingbird, Bee 162

Hummingbird, Berylline 146

Hummingbird, Black-bellied 156

Hummingbird, Black-chinned 149

Hummingbird, Black-fronted 160

Hummingbird, Blue-capped 156

Hummingbird, Blue-chested 145

Hummingbird, Blue-headed 154

Hummingbird, Blue-tailed 146

Hummingbird, Blue-throated 160

Hummingbird, Blue-vented 147

Hummingbird, Broad-billed 154

Hummingbird, Broad-tailed 168

Hummingbird, Buff-bellied 148

Hummingbird, Buffy 161

Hummingbird, Bumblebee 149

Hummingbird, Calliope 169

Hummingbird, Cerise-throated 168

Hummingbird, Charming 146

Hummingbind, Chestnut-bellied 146

Hummingbird, Cinnamon 147

Hummingbird, Copper-numped 147

Hummingbird, Costa's 150

Hummingbird, Dusky 154

Hummingbird, Emerald-chinned 144

Hummingbird, Fawn-breasted 148

Hummingbird, Fiery-throated 164

Hummingbird, Garnet-lihroated 161

Hummingbird, Giant 164

Hummingbird, Gilded 159

Hummingbird, Glow-throated 168

Hummingbird, Green-and-white 148

Hummingbird, Green-bellied 148

Hummingbird, Green-fronted 148

Hummingbird, Heliotrope-throated 168

Hummingbird, Indigo-capped 146

Hummingbird, Lucifer 150

Hummingbird, Magnificent 156

Hummingbird, Mangrove 146

Hummingbird, Many-spotted 169

Hummingbird, Oasis 168

Hummingbird, Oaxaca 156

Hummingbird, Olive-sponted 161

Hummingbird, Purple-chested 147

Hummingbird, Rivoli's 156

Hummingbird, Ruby-throsted 149

Hummingbird, Ruby-topaz 153

Hummingbird, Rufous 168

Hummingbird, Rufous-cheeked 157

Hummingbird, Rufour-tailed 147

Hummingbird, Sapphire-bellied 161

Hummingbird, Sapphire-throated 161
Hummingbird, Scaly-breasted 164

Hummingbird, Scintillant 168

Hummingbird, Scissor-tailed 160

Hummingbird, Shining-green 161

Hummingbird, Snowy-breasted 147

Hummingbird, Sombre 149

Hummingbird, Sparkling-tailed 170

Hummingbird, Speckled 145

Hummingbird, Spot-throated 161

Hummingbird, Steely-vented 147

Hummingbird, Stripe-tailed 156

Hummingbind, Swallow-tailed 156

Hummingbind, Swond-billed 155

Hummingbird, Tooth-billed 148

Hummingbird, Tumbes 161

Hummingbird, Vervain 162

Hummingbird, Violed-crowned 148

Hummingbird, Violet-bellied 154

Hummingbird, Violet-capped 157

Hummingbird, Violet-chested 169

Hummingbird, Violet-headed 160

Hummingbird, Volcano 168

Hummingbird, Wedge-billed 149

Hummingbird, White-bellied 146

Hummingbird, White-eared 160

Hummingbird, White-tailed 157

Hummingbird, White-throated 161

Hummingbird, Wine-throated 149

Hummingbird, Xantus's 160

huttoni, Ptilinopus 90

hyacinthinus, Anodorhynchus 96

(Hydranassa) 10

Hydrachous 144

hydrocorax, Buceros 174

Hyetomis 123

Hylocharis 159

Hylocryptus 178

Hylonympha 160

hylophila, Strix 141

(Hylorchilus) 190

(Hypargos) 218

hyperythra, Brachypteryx 192

hyperythrus, Campylopterus 151

(Hypochera) 225

hypochroma, Sporophila 209

hypogrammica, Pyrilia 219

hypoinachrous, Lorius 108

hypoleucos, Falco 58

hypoleucus, Capino 175

Hypopyrhus 213

hyposrictus, Taphrospilus 169

Hypothymis 195

hypoxantha, Gerygone magnirostris 195

(hypoxantha, Gerygone) 195

hypoxantha, Pyrthura 118

hypoxanthus, Neodrepanis 186

Hypsipetes 188

ibadanensis, Malimbus 221

(ibis, Ardeola) 8

ibis, Bubulcus 8

Ibis, Bald 13

Ibis, Crested 13

Ibis, Dwarf Olive 13

Ibis, Giant 14

Ibis, Hadada 12

Ibis, Hermit 13

Ibis, Japanese Crested 13

Ibis, Northern Bald 13

Ibis, Sacred 14
Ibis, Scarlet 13

Ibis, Southern Bald 13

Ib is, Spot-breasted 13

Ibis, White-shouldered 14

ichthyaetus, Ichthyophaga 47

Ichthyophaga 47

(Ichthyophaga nana) 47

icterorhynchus, Otus 135

icterotis, Ognorhynchus 109

icteroris, Platycercus 111

Icterus 213

Ictinaetus 47

Ictinia 47

idaliae, Phaethomis 165

(Idioptilon) 184

ignita, Lophura 69

iheringi, Fomicivara 180

imizator, Accipizer 26

impejanus, Lophophorus 69

imperatrix, Heliodaxa 158

tmperia!-Pigeon, Christmas 88

Imperial-Pigeon, Giant 88

Impericl-Pigeon, Grey 88

Imperial-Pigeon, Marquesan 88

Imperial-Pigeon, Mindoro 88

Imperial-Pigeon, New Caledonian 88

Imperial-Pigeon, Nukuhiva 88

Imperial-Pigeon, Polynesian 88

imperialis, Amazona 94

(imperialis, Ardea) 8

imperialis, Campephilus 176

imperialis, Lophura 69

Impeyan, Chinese 69

inca, Coeligena 154

Inca, Black 154

Inca, Bronzy 153

Inca, Brown 154

Inca, Collared 154

Inca-Finch, Grey-winged 208

Incaspiza 208

incognita, Vidua 226

indica, Eupodoris 79

(indica, Sypheorides) 79

indicus, Butastur 33

indicus, Gyps 43

Indigobind, Baka 225

Indigobird, Green 225

Indigobird, Jambandu 225

Indigobird, Village 225

indus, Haliastur 45

inexpectata, Torreamis 210

inexpectatus, Chlorosrilbon 152

inexspectata, Tyto 124

infuscara, Synallaxis 179

infuscalus, Henicapemis 45

ingens, Otus 135

innotata, Aythya 19

inopinorum, Polyplectron 70

inomata, Columba 86

inomata, Lophura 69

insignis, Ardea 8

insignis, Panterpe 164

insignis, Polihierax 64

insignis, Saricala 200

insularis, Junco 208

(insularis, Junco hyemalis) 208

insularis, Otus magicus 136

(insularis, Otus) 136

insulata, Sporophila 209 
interjecta, Vidua 225

(inferjecta, Vidua orientalis) 225

intermedia, Psittacula 115

intemigrans, Perisoreus 231

Iodopleura 182

iphis, Pomarea 198

iracunda, Metallura 163

ireneae, Otus 136

iriditarques, Columba 86

iris, Coeligena 153

iris, Psitteuteles 116

(iris, Trichoglossus) 116

isabellae, Oriolus 226

isidori, Oroaetus 51

(isidori, Spizaetus) 51

isura, Lophoictinia 49

Thaginis 69

Loos 188

Jabiru 12

Jabiru, 12

Jabauilleia 195

Jacamar, Coppery-chested 175

Jacamar, Three-toed 175

Jacamaralcyon 175

Jacobin, Black 162

Jacobin, White-necked 157

jacquinoti, Ninax 132

jacula, Heliodoxa 158

(jacutinga, Aburria) 67

jacutinga, Pipile 67

jamoicensis, Butea 35

(jamesi, Phoenicoparrus) 14

jamesi, Phaenicapterus 14

jandaya, Aratinga 98

japonensis, Grus 74

jardini, Baissanneaua 149

jardinii, Glaucidium 130

javanicus, Leptoptilos 12

javensis, Megalaima 175

Jay, Dwarf 23]

Jay, Sichuan 231

Jay, Sichuan Grey 231

Jay, Sooly 231

Jay, White-throated 231

jefferyi, Pithecophaga 52

jerdoni, Aviceda 32

Jewelfront, Gould's 158

jabiensis, Manucadia 229

johannae, Doryfera 155

(johonnis, Acanthis) 214

johannis, Carduelis 214

johnstoniae, Trichoglossus 120

jonquillaceus, Aprosmictus 96

josefinae, Chamosyna 102

jourdanii, Chaetocercus 151

jubatus, Rhynachetos 78

Jubula 13)

jugger, Falco 58

jugularis, Brotogeris 100

jugularis, Eulampis 156

julie, Damophila 154

Junca 208

Junco, Guadalupe 208

Jungle-Babbler, Black-browed 196

Jungle-Flycatcher, Brown-chested 199

Jungle-Flycatcher, Henna-tailed 199

Jungle-Flycatcher, Sulawesi 199

Jungle-Flycatcher, White-throsted 199

Jungle fowl, Grey 69
Jungle fowl, Sonnerat's 69

junoniae, Columba 86

kaboboensis, Apalis 191

(kaboboensis, Apalis porphyrolaema) 191

kaempferi, Hemitriccus 184

(kaempferi, Idioprilan) 184

Kagu, 78

Kaka, 109

Kaka, Norfolk 109

Kakapo, 118

Kakariki, Yellow-fronted 103

Kakawahie, 212

kalinowskii, Nothoprocta 2

Kamao, 197

karamojae, Apalis 192

Kaupifalca 47

kawalli, Amazona 95

Kes, 109

keayi, Gallicolumba 88

keiensis, Micropsitta 108

kennicotrii, Otus 136

(kennicortii, Otus asio) 136

keraudrenii, Manucodia 229

(keraudrenii, Phonygammus) 229

kerriae, Crypturellus 2

Kestrel, Aldabra 59

Kestrel, American 61

Kestrel, Australian 57

Kestrel, Banded 62

Kestrel, Barted 62

Kestrel, Common 62

Kestrel, Dickinson's 58

Kestrel, Eurasian 62

Kestrel, Fox 56

Kestrel, Greater 60

Kestrel, Grey 56

Kestrel, Lesser 59

Kestrel, Madagascar 59

Kestrel, Mauritius 60

Kestrel, Moluccan 59

Kestrel, Nankeen 57

Kesirel, Neuton's 59

Kesirel, Seychelles 56

Kestrel, Spotted 59

Kestrel, White-eyed 60

Kestrel, White-rumped $\mathbf{5 8}$

Ketupa 131

(ketupu, Bubo) 131

kerupu, Kerupa 131

(kibalensis, Turdus) 202

kibalensis, Zooshera 202

kienerii, Hieraaesus 46

kinabaluensis, Spilomis 53

(kinabaluensis, Spilamis cheela) 53

King-Parrol, Ambon 93

King-Parrot, Australian 93

King-Parrot, Green-winged 93

King-Parrot, Moluccsn 93

King-Parrot, Papuan 93

Kingbird, Giant 185

King fisher, Blue-capped 171

Kingfisher, Blyth's 171

Kingfisher, Cinnamon-backed 171

Kingfisher, Cook lslands 172

Kingfisher, Hombron's 171

Kingfisher, Lesser Sunda 171

Kingfisher, Mangaia 172

Kingfisher, Mangareva 171

Kingfisher, Marquesan 171
Kingfisher, Moustached 171

Kingfisher, Tuamou 171

kingi, Acrocephalus 190

kingi, Agleiocercus 145

kirtlandii, Dendroica 211

Kite, African Swallow-tailed 37

Kite, American Swallow-tailed 41

Kite, Australian Black-shouldered 41

Kite, Bat 49

Kite, Black 50

Kite, Black-breasted 45

Kite, Black-eared 50

Kite, Black-shouldered 4]

Kite, Black-winged 41

Kite, Brahminy 45

Kite, Cayenne 48

Kite, Cuban Hook-billed 37

Kite, Double-toothed 45

Kite, Everglade $\mathbf{5 2}$

Kite, Grey-headed 48

Kite, Hook-billed 37

Kite, Letter-winged 41

Kite, Mississippi 47

Kite, Pariah 50

Kite, Pearl 42

Kite, Plumbeous 47

Kite, Red 50

Kite, Rufous-thighed 45

Kite, Scissor-tailed 37

Kite, Slender-billed 52

Kite, Snail 52

Kite, Square-tailed 49

Kite, Swallow-tailed 41

Kite, Whistling 45

Kite, White-collared 48

Kite, White-tailed 41

Kite, Yellow-billed 50

Kiwi, Litule Spotted 1

Klais 160

kleinschmidri, Erythnura 215

kochi, Piua 185

koeniswaldiana, Pulsatrix 139

koepckeae, Cocicus 213

koepckeae, Ous 136

koepckeae, Phaethamis 165

Kokako, 227

kollori, Synallaxis 179

Koloa, 19

Korhaan, Black 79

Korhaan, Black-bellied 79

Korhaan, Blue 79

Korhaan, Crested 80

Korhaan, Damara 79

Korhaan, Karoo 80

Korhaan, White-bellied 80

kori, Ardeolis 78

(kori, Choriotis) 78

krameri, Psinaculo 116

kreyenborgi, Falco 60

kubani, Corvus 231

kuehni, Myzomela 206

kuehni, Zosterops 205

kuhli, Leucopternis 48

kuhlii, Vini 120

(kupeensis, Malaconotus) 189

kupeensis, Telopharus 189

Kupeomis 195

lacemulata, Leucoptemis 48

laceriosa, Woodfordia 205 
Lactea, Amazilia 147

Laczeus, Bubo 128

Lafresnaya 160

Lafresnayanus, Gallirallus 76

(lafresnayanus, Tricholimnas) 76

Lafresnayi, Lafresnaya 160

Lagonasticta 216

lagopus, Buteo 35

lalandi, Stephanaris 169

Lammergejer, 42

Lampornis 160

(Lampribis) 13

Lamprolaima 161

Lanaiensis, Myadestes 196

(lanaiensis, Phaeomis obscurus) 196

Lancebill, Blue-fronted 155

Lancebill, Green-fronted 155

Lanceolatus, Spizaetus 54

langbianis, Crocias 193

(langsdorfi, Discosura) 167

langsdorff, Popelairia 167

Laniisomo 182

lanioides, Lipaugus 183

Lanius 189

Lanner, 56

lanyoni, Phylloscortes 184

laperouse, Megapodius 65

Lapwing, Javsnese 82

Lapwing, Sociable 82

largipennis, Campylopterus 151

Lark, Archer's 186

Lark, Ash's 187

Lark, Botha's 186

Lark, Degodi 187

Lark, Raso 186

Lark, Raza Island 186

Lark, Rudd's 186

Lark, Sidamo 187

Lark, Sidamo Long-clawed 187

Lark, Somali Long-clawed 186

Lark, South African Long-clawed 186

Larus 84

Larvaricola, Vidua 225

Laterallus 76

lathomi, Colyptorhynchus 101

Lathamus 106

(Lathrotriccus) 184

laticincta, Playsieira 198

(laricincta, Platysteira pelrata) 198

latifrons, Microhierax 63

Latirostris, Calyptorhynchus 101

latirosiris, Cynanthus 154

Laughingthrush, Ashy-headed 194

Laughingthrush, Biet's 194

Laughingthrush, Black-hooded 194

Laughingthrush, Collared 195

Laughingthrush, Snowy-cheeked 195

Laughingthrush, Sukatschev's 195

Laughingthrush, White-speckled 194

lawesii, Parotia 230

(lawrencii, Gymnoglaux) 136

Lawrencii, Otus 136

Laysanensis, Anas 18

(laysanensis, Anas platyrhynchos) 18

leadbeateri, Cacatua 100

leadbeateri, Heliadaxa 158

Leaf-Warbler, Sombre 198

Leari, Anodorhynchus 96

ledanti, Sitza 203
Leipoo 65

lemosi, Cypseloides 144

lempiji, Otus 136

(lempiji, Otus bakkamoena) 136

lendu. Muscicapa 196

(Leanardina) 201

Lepidopyga 161

Leplasthenura 178

Leprodon 48

leptogrammica, Srrix 141

Leploptilos 12

leprorhynchus, Enicognathus 104

Leptositzaca 106

leptosomus, Brachypleracias 172

Leptotila 89

Lesbia 161

(letitiae, Discosura) 167

leritiae, Popelairia 167

lertii, Jubulo 131

(lettii, Lophosirix) 131

Leucippus 161

(Leucocarbo) 7

leucocephala, Amazona 95

leucocephala, Oryura 22

leucacepholus, Aceras 173

leucocephalus, Holioeerus 44

(leucocephalus, Rhyticeros) 173

Leucochtoris 161

leucagaster, Amazilia 147

leucogaster, Holiaeetus 44

leucogaster. Pionites 110

leucogeranus, Grus 74

leucolepis, Phyllastrephus 188

leucopareia, Bronsa canadensis 19

Leucopeza 211

(leucophaeus, Aerodramus) 144

leucophreus, Collocalia 144

leucophaeus, Speirops 205

leucophtholmus, Aratinga 98

leucophthalmus, Larus $\mathbf{8 4}$

leucopleurus, Oreotrochilus 164

Leucopsar 226

Leucoptemis 48

leucopygius, Serinus 214

leucorodia, Platalea 13

leucorthous, Buteo 35

leucoryphus, Haliacelus 44

leucoryphus, Playrinchus 184

Leucosticius, Bubo 128

leucoris, Hylacharis 160

leucotis, Ous 136

leucotis, Pyrrhura 117

leucurus, Elanus 41

leucurus, Threnetes 170

leuphores, Aviceda 32

levraudi, Laterollus 76

Lewinia 77

Lhuysii, Lophophorus 69

liberatus, Lanius 189

Uichenostomus 206

Lichmera 206

Likh, 79

Lilianae, Agapomis 92

(Lilianae, Agapomis lilianae) 92

lilliae, Lepidopyga 161

Limnodromus 83

Lineatus, Buteo 35

lineatus, Milvus 50

lineola, Bolborhynchus 99
Linnet, Warsangli 214

Liocichla 195

Liocichla, Mount Omei 195

Liocichla, Omei Shan 195

(Lioptilus) 195

Lipangus 183

(Lissotis) 79

likoralis, Dasyomis broadbenti 194

Lituralis, Fomicivora 180

liventer, Buzastur 33

livia, Columba 86

Livingstonii, Tauraco 122

(livingstonii, Tauraco corythaix) 122

Lobata, Campephogo 187

Laboparadisea 228

Laddigesia 161

Lachkeni, Threneies 170

Lanchuro 217

Longbill, Bocage's 191

Longbill, Pulitzer's 195

longicauda, Discosura 155

longicauda, Henicopernis 45

langicarda, Psittacula 116

longicaudatus, Spelaeomis 200

longicomis, Otus 136

(longicomis, Otus scops) 136

longimembris, Tylo 124

(longimembris, Tyto capensis) 124

longipennis, Falco 58

longipes, Xenicus 186

longirostra, Rukio 204

longirostris, Dasyamis 194

(Iongirostris, Dasyomis brachypterus) 194

longirostris, Heliomaster 159

longirostris, Rhizothera 71

longuemareus, Phaethomis 165

Laphaetus 49

Lophoictinia 49

Lophophorus 69

Lophorina 228

Lophamis 161

Lophostrix 131

(Lophotis) 80

Lophura 69

lorenzi, Vidua 226

(Loria) 228

loriae, Cnemophilus 228

(lorioe, Loria) 228

Loriculus 106

Lorikeet, Blue 120

Lorikeet, Blue-crowned 120

Lorikeet, Blue-fronted 103

Lorikeet, Duchess 102

Lorikeet, Emerald 109

Lorikeet, Fairy 102

Lorikeet, Goldie's 116

Lorikeet, Green-naped 120

Lorikeet, Henderson 121

Lorikeet, Iris 116

Lorikeet, Johnstone's 120

Lorikeet, Josephine's 102

Lorikeet, Kuhl's 120

Lorikeet, Little 106

Lorikeet, Little Red 102

Lorikeet, Meek's 102

Lorikeet, Meyer's 120

Lorikeet, Mindanao 120

Lorikeet, Musk 105

Lorikeet, Musschenbroek's 109 
Lorikeet, New Caledonian 102

Lorikeet, Olive-headed 120

Lorikeet, Orange-billed 109

Lorikeet, Omate 120

Lorikeet, Palm 102

Lorikeet, Papuan 102

Lorikeet, Perfect 120

Lorikeet, Plum-faced 110

Lorikeet, Pohnpei 120

Lorikeet, Purple-crowned 106

Lorikeet, Pygmy 103

Larikeet, Rainbow 120

Lorikeet, Red-chinned 102

Lorikeet, Red-collared 120

Lorikeet, Red-flanked 102

Lorikeet, Red-fronted 102

Lorikeet, Red-spotted 102

Lorikeet, Red-throated 102

Lorikeet, Scaly-breasted 120

Lorikeet, Scarlet-breasted 120

Lorikeet, Stephen's 121

Lorikeet, Streaked 102

Lorikeet, Striated 102

Lorikeet, Tahitian 120

Lorikeet, Ultramsrine 121

Lorikeet, Varied 117

Lorikeet, Whiskered 110

Lorikeet, Wilhelmins's 103

Lorikeet, Yellow-and-green 120

Lorikeet, Yellow-billed 109

Lorius 107

lory, Lorius 108

Lory, Biak Red 104

Lory, Black 101

Lory, Black-capped 108

Lory, Black-winged 104

Lory, Blue-eared 104

Lory, Blue-streaked 104

Lory, Brown 102

Lory, Cardinal 101

Lory, Chattering 107

Lory, Collared 110

Lory, Dusky 114

Lory, Duyveribode's 102

Lory, Eastern Black-capped 108

Lory, Greater Streaked 102

Lory, Iris 116

Lory, Josephine's 102

Lory, Molucean 104

Lory, Moluccan Red 105

Lory, Omate 120

Lory, Pohnpei 120

Lory, Purple-bellied 108

Lory, Purple-capped 107

Lory, Purple-naped 107

Lory, Rainbow 120

Lory, Red 104

Lory, Red-and-blue 104

Lory, Ultramarine 121

Lory, Violet-necked 105

Lory, Westem Black-capped 108

Lory, White-naped 107

Lory, Yellow-backed 107

Lory, Yellow-bibbed 107

Lory, Yellow-streaked 102

Lovebird, Abyssinian 93

Lovebird, Black-cheeked 93

Lovebird, Black-collared 93

Lovebird, Black-masked 93
Lovebird, Black-winged 93

Lovebird, Fischer'\& 92

Lovebird, Grey-headed 92

Lovebird, Lilian's 92

Lovebird, Madagascar 92

Lovebird, Masked 93

Lovebird, Nyasa 92

Lovebird, Peach-faced 93

Lovebird, Red-faced 93

Lovebird, Red-headed 93

Lovebird, Rosy-faced 93

Lovebird, Yellow-collared 93

(lowei, Alethe) 200

(lowei, Dryocichloides) 200

lowei, Sheppardia 200

loweryi, Xenoglaux 142

Loxioides 212

Loxops 212

luciae, Amazilio 147

Uuciani, Eriocnemis 155

lucidus, Hemignathus 211

lucifer, Calothorax 150

lucionensis, Tanygnathus 119

ucconensis, Prioniturus 113

ludovicae, Doryfero 155

ludwigii, Neoris 80

lugens, Haplophaedia 157

ungubris, Melampitta 229

luizae, Asthenes 178

lumachellus, Augastes 149

lunulatus, Bolbopsinacus 99

Luscinia 195

luteifrons, Nigrita 218

luteirostris, Zosterops 205

luteolus, Ploceus 223

luseoschistaceus, Accipiter 26

Luterioe, Coeligena 153

lutosus, Polybonus 64

luzonico, Gollicolumba 88

Lycocorax 229

macao, Aro 97

Macaw, Blu-winged 97

Macaw, Blue-and-gold 96

Macaw, Blue-and-yellow 96

Macaw, Blue-headed 97

Macaw, Blue-throated 97

Macaw, Buffon's 96

Macaw, Chestnut-fronted 97

Macaw, Glaucous 96

Macaw, Golden-collared 96

Macaw, Great Green 96

Macaw, Green-winged 96

Macaw, Hyacinth 96

Macaw, Illiger's 97

Macaw, Indigo 96

Macaw, Lear's 96

Macaw, Litule Blue 103

Macaw, Military 97

Macaw, Noble 97

Macaw, Red-and-green 96

Macaw, Red-bellied 97

Macaw, Red-fronted 97

Macaw, Red-shouldered 97

Macaw, Scarlet 97

Macaw, Severe 97

Macaw, Spix's 103

Macaw, Yellow-collared 96

macgillivrayi, Pseudobulweria 4

(macgillivrayi, Pierodroma) 4
Macgregaria 229

macgregorii, Cnemophilus 228

(Mochaerhamphus) 49

Macheiramphus 49

mochiki, Zoothera 202

Macrocephalon 65

macrocenco, Hylonympha 160

macrodacryla, Oceanodroma 6

(macropterus, Hoplopterus) 82

macropterus, Vanellus 82

macrorhyncha, Saricola 200

macrorhynchus, Tauraco 122

Macrosphenus 195

mocroura, Eupetomena 156

macroura. Thripophoga 179

macroura, Vidua 225

macrourus, Circus 39

(macrourus, Coliuspasser) 220

macrourus, Euplectes 220

macrourus, Urotriorchis 55

maculato, Coringo 182

(moculata, Loxops) 212

moculota, Paroreomyza 212

maculicollis, Rhynchotus rufescens 2

maculirosiris, Selenidera 176

maculosus, Caprimulgus 143

mada. Prionilurus 113

madogascariensis, Accipiter 26

madagascariensis, Asio 126

madagascariensis, Aviceda 32

Madanga 204

madaraszi. Psiltacella 114

madeira. Pherodroma 5

(madeiro, Pterodroma mollis) 5

magentae, Pterodroma 5

magicus, Otus 136

(magicus, Olus manadensis) 136

magna, Sina 203

(mognificus, Colyptarhynchus) 101

magnificus, Cicinnurus 228

(mognificus, Diphyllodes) 228

magnificus, Gorsachius 11

magnificus, Lophomis 162

magnificus, Priloris 230

magnirostris, Buteo 35

Magpie, Ceylon 231

Magpie, Ceylon Blue 231

Magpie-Robin, Seychelles 193

maillordi, Circus 40

(maillandi, Circus aeruginosus) 40

major, Bradypterus 192

major, Zoothera 202

(major, Zoothera dauma) 202

makawai, Pogoniulus 175

(malaborica, Lonchura cantans) 217

(malabaricus, Anihracoceros) 173

malacense, Polyplectron 70

malachitacea, Triclaria 120

Malococincla 196

Malaconotus 189

malogae, Metallura 163

malaitae, Rhipidura 199

malaris, Phoethomis 165

malayanus, Anchrococeros 174

malayensis, Ictinaetus 47

maleo, Macrocephalon 65

Maleo, 65

malherbi, Cyanoramphus 103

Malimbe, Ballmann's 221 
Malimbe, Black-throated 221

Malimbe, Blue-billed 221

Malimbe, Cassin'a 221

Malimbe, Crested 221

Malimbe, Gray's 221

Malimbe, Ibadan 22I

Malimbe, Red-headed 22 I

Malimbe, Red-vented 221

Malimbe, Yellow-legged 221

malimbicus, Malimbus 221

Malimbus 221

Malkoha, Red-faced 123

Mallard, Marianas 18

Malleefowl, 65

Malurus 196

manadensis, Otws 136

Manakin, Black-capped 183

Manakin, Golden-crowned 183

Mandingoo 218

mango, Anthracothorax 148

Mango, Antillean 148

Mango, Black-throated 148

Mango, Dominican 148

Mango, Green 149

Mango, Green-breasted 148

Mango, Green-throated 148

Mango, Jamaican 148

Mango, Veraguas 148

manilata, Ara 97

manillae, Penelopides 174

(manillae, Penelopides panini) 174

manipurensis, Perdicula 70

Mannikin, Black-and-white 217

Mannikin, Blue-billed 217

Mannikin, Bronze 217

Mannikin, Bronze-winged 217

Mannikin, Magpie 217

Mannikin, Pied 217

Manorina 206

mantananensis, Otus 137

(mantananensis, Otus scaps) 137

mantchuricum, Crossoptilon 68

(mantelli, Notamis) 77

mantelli, Porphyrio 77

Manucode, Crinkle-collared 229

Manucode, Curl-crested 229

Manucode, Glossy-mantled 229

Manucode, Jobi 229

Manucode, Trumpet 229

Manucodio 229

manusi, Tyto 124

(manusi, Tyto novaehollandiae) 124

maracano, Ara 97

maranhooensis, Phaethomis 166

morchei, Anthracoceros 174

margarethae, Charmosyna 102

margarettae, Phaethomis 165

(Margaromis) 178

Marmaronetta 21

Marsh-Harrier, African 40

Marsh-Harrier, Eastern 40

Marsh-Harrier, Madagascar 40

Marsh-Harrier, Western 38

Marsh-Wren, Apolinar's 190

marshalli, Otus 137

masafuerae, Aphrastura 177

Masked-Owl, Australian 125

Masked-Owl, Lesser 125

Masked-Owl, Manus 124
Masked-Owl, Minahassa 124

Masked-Owl, New Britain 124

Masked-Owl, Taljabu 125

Masked-Owl, Tasmanian 124

Masked-Weaver, Heuglin'a 223

Masked-Weaver, Ruwet's 224

Masked-Weaver, Vitelline 224

Masked-Owl, Bismarck 124

Maaked-Weaver, Victoria 224

mathewsii, Boissonneaua 150

maugaeus, Chlorostilbon 152

maurus, Circus 40

mavors, Heliangelus 158

maximiliani, Pionus 111

maximus, Pyrenestes 219

mayeri, Astrapia 227

mayeri, Columba 87

(mayeri, Nesoenas) 87

meadewaldoi, Haematopus moquini 81

(meadewaldoi, Haematopus) 81

Meadowlark, Lesser Red-breasted 213

Meadowlark, Pampas 213

meeki, Chamosyna 102

meeki, Microgoura 89

meeki, Micropsitta 108

meeki, Ninax 132

meeki, Zosteraps 205

Megadyptes 3

megaensis, Hirunda 187

Megalaima 175

megalopterus. Phalcoboenus 64

megalorynchos, Tanygnothus 119

megaloris, Orus 137

(megalotis, Otus bakkamoena) 137

Megalurus 196

Megapodius 65

megarhynchus, Ploceus 223

Megairiorchis 49

Megaxenops 178

Melaenomis 196

Melampina 229

Melampitta, Greater 229

Melampitta, Lesser 229

Melamprosops 212

melanocephala. Pionites 110

(melanocephala, Sitogra) 223

melanacephalus, Carpomis 182

melanocephalus, Ploceus 223

melanocephalus. Tragopan 71

melanochlamys, Accipiter 27

melanochlomys, Bangsia 207

(melanochlamys, Buchraupis) 207

melanocorypha, Cygnus 20

melanogaster, Eupodotis 79

(melanogaster, Lissotis) 79

melanogaster, Oreoirochilus 164

melanogaster, Tumix 72

melanogenys, Adelomyia 145

melanoleuca, Seleucidis 231

melanoleucas, Circus 40

melanoleucus, Accipiler 27

melanoleucus, Geranoaetus 42

melanoleucus, Microhierax 63

melanoleucus, Spizastur 54

melanonatus, Touit 119

Melanoperdix 70

melanops, Leucopiemis 48

melanops, Sporophila 210

melanastemon, Hamirostra 45 melanota, Pulsatrix 139

(melanotis, Anoplectes) 219

melanotis, Hapalopsituaca 106

melanoris, Manorina favigula 206

(melanotis, Manorina) 206

melanotas, Sortidiomis 22

Melanotrochilus 162

melanura, Pyrthura 118

meleagrides, Agelastes 67

Melidectes 206

Melidectes, Long-bearded 206

Melierax 49

Melignomon 175

Meliphaga 206

Meliphaga, Louisiades 206

Meliphaga, Sudest 206

mellianus, Oriolus 227

Mellisuga 162

mellisugus, Chlorostilbon 152

mellivara, Florisuga 157

melodus, Charadrius 82

melpoda, Estrilda 216

menach-nsis, Turdus 202

menagei, Gallicolumba 88

mendazae, Pomarea 198

menstruus, Pionus 111

mentawi, Otus 137

(meniawi, Ocus bakkamoena) 137

mercenaria, Amazona 95

mercierii, Prilinopus 90

Merganser, Brazilian 21

Merganser, Chinese 21

Merganser, Scaly-sided 21

Mergus 21

meridionolis, Buteogallus 37

(meridionalis. Heterospizias) 37

meridionalis, Nestor 109

Merlin, 57

Merlin, Red-headed 57

Merulaxis 182

Mesite, Brown 72

Mesite, Subdesert 72

Mesite, White-breasted 72

Mesitomis 72

mesoleuca, Canothraupis 208

metabates, Melierax 49

Metabolus 196

Metallura 163

Metaltail, Black 163

Metaltail, Coppery 163

Metaltail, Fire-throated 163

Metaltail, Neblina 163

Metaltail, Perija 163

Metaltail, Scaled 163

Melatuail, Tyrian 163

Metaltail. Violet-throated 163

Metaltail, Viridian 163

mexicanus, Falco 59

meyerdeschauenseei, Tangara 210

meyeri, Epimachus 228

meyeri, Pachycephala 197

meyeri, Poicephalus 112

meyerianus, Accipiter 27

micraster, Heliangelus 158

Micrastur 62

Micrathene 132

Microchera 163

Micragoura 89

Microhierax 63 
(Micronisus) 49

Micropsitta 108

microrhynchwm, Ramphomicron 168

Microstilban 163

micrura, Mymia 163

migrans, Milvus 50

mikado, Symaticus 71

militaris, Ara 97

milizaris, Stumella 213

Millerbird, 190

Millerbird, Nihoa 190

milleri, Grallaria 180

milleri, Polytmus 167

milleti, Garrulax 194

Milvago 63

milvus, Milvus 50

Milvus 50

Mimizuku 132

Mimades 190

mindorensis, Ducula 88

mindorensis, Otus 137

(mindorensis, Otus scops) 137

mindorensis, Penelopides 174

Miner, Black-eared 206

Miner, Dusky 206

minima, Baris 192

minima, Mellisuga 162

minimus, Spilormis 53

minar, Nothura 2

minar, Paradisaea 230

(minor, Phoeniconaias) 14

minor, Phoenicopterus 14

minor, Platalea 13

minullus, Accipiler 27

minutissimum, Glaucidium 130

mirabilis, Cyanolyca 231

mirabilis, Eriocnemis 156

mirabilis, Laddigesia 161

Mirafra 187

mirandae, Hemitriccus 184

mirandallei, Micrastur 63

(mirificus, Dryolimnas) 77

mirificus, Lewinia 77

(mirificus, Rallus) 77

mirus, Otws 137

(mirus, Otus scops) 137

(misapplied, Ara caninde) 97

mississippiensis, Ictinia 47

(mitchellii, Calliphlox) 166

mitchellit, Philadice 166

mitrata, Aratinga 98

(milu, Crax mitu) 66

mitu, Mitu 66

Mitu 66

mlokosiewiczi, Tetrao 71

mocinno, Pharomachrus 171

Mockingbird, Charles 190

Mockingbird, Floreana 190

Mockingbird, Socorro 190

modesta, Psitnacella 114

madestus, Zasterops 205

(Modulatrix) 192

Moho 206

molinae, Byrthura 118

moluccensis, Cacarua 100

moluccensis, Falco 59

monacha, Grus 74

(Monachalcyon) 171

monachus, Aegypius 29 monachus, Myiopsitza 108

monachus, Necrosyrtes 51

Monal, Chinese 69

Monal, Crestless 69

Monal, Himalayan 69

Monal, Sclater's 69

Monarch, Biak 196

Monarch, Black-chinned 196

Monarch, Celestial 195

Monarch, Celestial Blue 195

Monarch, Fatuhiva 199

Monarch, Iphis 198

Monarch, Marquesan 198

Monarch, Rarolonga 198

Monarch, Short-crested 195

Monarch, Short-crested Blue 195

Monarch, Tahiti 198

Monarch, Truk 196

Monarch, White-tipped 196

Monarcha 196

Monias 72

Monias, 72

monogrammicus, Kaupifolco 47

(montana, Alethe) 200

montana, Sheppardia 200

montana, Xenoligea 211

montani, Anthracoceros 174

(montanus, Dryocichloides) 200

montanus, Prioniturus 113

monteiri, Malaconotus 189

(monteiri, Malaconotus blanchori) 189

(Monticola) 199

Moorhen, Gough 76

Moomen, San Cristobal 76

(mareaui, Apalis) 197

moreaui, Ortholomus 197

Morepork, 132

morphnoides, Hieraaetus 46

Morphnus 50

maschata, Cairina 20

masquera, Eriacnemis 156

mosquitus, Chrysolampis 153

Motmot, Keel-billed 172

Mountain-Babbler, White-throated 195

Mountain-Finch, Cochabamba 209

Mountain-Finch, Tucuman 209

Mountain-gem, Green-breasted 160

Mountain-gem, Green-throated 160

Mountain-gem, White-bellied 160

Mountain-Tanager, Golden-backed 207

Mountain-Tanager, Masked 207

Mountaineer, Bearded 164

Mountaingem, Purple-throated 160

Mountaingem, Variable 160

Mountaingem, White-throated 160

(Moupinia) 192

mouroniensis, Zosterops 205

mozambicus, Serinus 215

muelleri, Heleia 204

mulsan, Acestrura 145

multipunctata, Tyta 125

(multipunctata, Tyto tenebricosa) 125

mulristriata, Chamasyna 102

Munia, Black-and-white 217

Munia, Bronze 217

Munia, Magpie 217

(munroi, Hemignathus) 211

Murrelet, Crested 85

Murrelet, Japanese 85
Muscicapa 196

Musaphaga 121

musschenbroekii, Neopsituacus 109

muticus, Pavo 70

Myadestes 196

myadestinus, Myadestes 197

(myadestinus, Phacomis obscurus) 197

mycteria, Jabiru 12

Mycueria 12

Myiagra 197

Myiagra, Black 197

Myioborus 211

Myiophonus 197

Myiopsitza 108

Myna, Bali 226

Myna, Bare-eyed 226

Myna, Helmeted 226

Myna, Hill 226

Myna, Sula 226

Mynah, Rothschild's 226

Mymeciza 181

Mymia 163

Mymotherula 181

Myrtis 163

Myzomela 206

Myzomela, Crimson-hooded 206

Myzomela, Wetar 206

naevius, Ramphodon 168

naevosa, Stictonetra 23

nahani, Francolinus 68

nana. Aratinga 98

nana, Cyanolyca 231

Nandayus 108

Nannopsitlaca 108

(Nannopterum) 7

nanus, Accipiter 27

nanus, Spizaetus 54

nanus, Taoniscus 2

Napothera 197

narcandami, Aceras 173

(narcondami, Rhyriceros) 173

natalis, Ninax squamipila 133

nattereri, Anthus 187

nottereri, Phaethomis 166

naumanni, Falca 59

nebulasa, Strix 141

Necrosyrtes 51

Nectarinia 204

Negrofinch, Chestnut-breasted 218

Negrofinch, Grey-crowned $\mathbf{2 1 8}$

Negrofinch, Grey-headed 218

Negrofinch, Pale-fronted 218

Negrofinch, While-breasted 218

nemoricola, Gallinago 83

Nemosia 208

nenday, Nandayus 108

Nene, 20

Neodrepanis 186

Neomorphus 123

Neophema 108

Neophron 51

Neopsephotus 109

Neopsinacus 109

Neospiza 214

Neotis 80

neoxenus, Euptilotis 171

Nesasio 132

Nesillas 197

nesiotes, Grus canadensis 73 
nesiotis, Anas aucklandica 16

Nesocharis 218

(Nesochen) 20

Nesoclopeus 77

(Nesoenas) 87

Nesomimus 190

(Nesophylax) 77

Nesospiza 208

Nestor 109

Netrapus 22

neumanni, Vidua 225

newelli, Puffinus auricularis 6

(newelli, Puffinus) 6

newtani, Coracina 188

newroni, Falco 59

newtoni, Lanius 189

Newtonia 197

Newtonia, Fanovans 197

Newtonia, Red-tailed 197

ngamiense, Glaucidium 130

(ngamiense, Glaucidium capense) 130

nicefori, Thryothorus 190

nicobarica, Caloenas 85

nicobariensis, Megapodius 65

(nicobariensis, Megapodius freycinet) 65

nicolli, Ploceus 223

(nicolli, Ploceus olivaceiceps) 223

nieuwenhuisti, Pycnonotus 189

niger, Threnetes 170

nigerrimus, Ploceus 223

Night-Heron, Japanese 10

Night-Heron, White-eared 11

Nightjar, Cayenne 143

Nightjar, Puerto Rican 143

Nightjar, Salvsdori's 143

Nightjar, Satanic 143

Nightjar, Sickle-winged 143

Nightjar, Vaurie's 143

Nightjar, White-winged 143

nigra, Astrapia 227

nigra, Cicania 11

nigra, Corocopsis 103

nigra, Melanoperdix 70

nigra, Penelopina 66

nigra, Pomarea 198

nigrescens, Rheinardia 70

nigriceps, Ardeotis 78

(nigriceps, Choriotis) 78

nigriceps, Ploceus 223

nigricollis, Anthracothorax 148

nigricollis, Busarellus 32

nigricollis, Grus 74

nigricollis, Ploceus 224

nigrigenis, Agapomis 93

(nigrigenis, Agapomis lilianae) 93

nigriloris, Estrilda 216

nigrimentum, Ploceus 224

nigripes, Dacnis 208

nigrirostris, Phaethomis 165

Nigrita 218

nigriventris, Eupherusa 156

nigrivestis, Eriocnemis 156

nigrobrunnea, Tyto 125

nigrogularis, Psophodes 199

(nigrolineata, Ciccaba) 141

nigrolineata, Strix 141

nigropectus, Biatas 179

nigrorufa, Sporophila 210

nigrorufius, Centropus 122 nigronum, Siachyris 201

Niluva, Rueck's 193

Niltava, Sanford's 194

(Nileava) 193

Ninax 132

nipalensis, Aceros 173

nipalensis, Aquila 31

nipalensis, Bubo 128

nipalersis, Spizaetus 54

nippon, Nipprnia 13

Nipponia 13

nisus, Accipiter 27

nitens, Malimbus 221

nirida, Asturina 32

nitidissima, Chlorochrysa 207

(nitidula, Hypargos) 218

niridula, Mandingoo 218

(nitidus, Buteo) 32

nobilis, Ara 97

nobilis, Oreonympha 164

nocrithenus, Caprimulgus 143

(nocticherus, Caprimulgus vociferus) 143

noctua, Athene 127

noguchii, Sapheopipo 177

Nonhiella 109

notabilis, Lichmera 206

nolabilis, Nestor 109

notatus, Chlorestes 152

notatus, Cotumicops 75

(notatus, Elanus) 41

Nothoprocta 2

Nothura 2

Nothura, Lesser 2

Notiomystis 206

(Notomis) 77

novacapitalis, Scytalopus 182

novaeguineae, Harpyopsis 45

novachollandiae, Accipiter 27

novachollandiae, Tyıo 125

novaeseelandiae, Charadrius 82

navaeseelandiae, Ninax 132

(novaeseelandiae, Ninox royano) 132

(novaeseelandiae, Thinomis) 82

novaesi, Philydor 178

novaezelandiae, Falco 59

novaczelandiae, Cyanoramphus 103

novaezelandiae, Himantopus 81

nuba, Neotis 80

nuchalis, Panus 203

nudipes, Otus 137

Nukupuu, 211

Numenius 83

nuna, Lesbia 161

Nuthatch, Algerian 203

Nuthatch, Beautiful 203

Nuthatch, Black-masked 203

Nuthatch, Giant 203

Nuthatch, Kabylian 203

Nuthatch, White-browed 203

Nuthatch, Yellow-billed 203

Nuthatch, Yunnan 203

Nyctea 134

nympho, Pitta 185

nyroca, Aythya 19

(nyroca, Nyroca) 19

(Nyroca) 19

(oberlaenderi, Turdus) 202

oberlaenderi, Zoothera 202

obscura, Luscinia 195 (obscurus, Erithacus) 195

obscurus, Hemignathus 212

occidentalis, Dysithamnus 180

occidentalis, Leucoptemis 48

occidentalis, Onychorhynchus coronarus 184

(occidentalis, Onychorhynchus) 184

occidentalis, Pezoporus 110

occidentalis, Strix 141

(occipitalis, Aegypius) 55

occipitalis, Lophaerus 49

(occipitalis, Spizaerus) 49

occipitalis. Trigonoceps 55

Oceanadroma 6

ocellata, Agriocharis 67

ocellata, Leipaa 65

ocellata, Rheinondio 70

ocellata, Strix 141

ochracea, Ninor 132

ochraceiventris, Leptotila 89

ochrocephala, Amazona 95

ochrogasier, Penelope 66

ochropectus, Francolinus 68

Ocreaus 163

octosetaceus, Mergus 21

oculea, Caloperdix 68

odiosa, Ninox 132

adomae, Metallura 163

Odontopharus 70

Oena 89

oenone, Chrysuronia 153

oenops, Columba 87

oglei, Stachyris 201

Ognorthynchus 109

okavangoensis, Vidua 225

(akavangoensis, Vidua) 225

okinawae. Gallirallus 76

(okinawae, Rallus) 76

olivaceum. Chalcostigmo I51

olivaceus, Criniger 188

olivaceus, Hypsipetes borbonicus 188

(olivaceus, Hypsipetes) 188

Olive-back, Grey-headed ? 18

oliviae, Columba 87

olivieri, Amauromis 75

(olivieri, Porzana) 75

olivii, Tumix 72

(olivii, Tumix castanota) 72

Olomao, 196

omeiensis, Liocichla 195

onslowi, Phalacrocorax 7

Onychorhynchus 184

Oo, Bishop's 206

Oo, Kauai 206

Oo, Molokai 206

Ophrysia 70

ophthalmica, Cacatua 101

Opisthoprora 164

(Opopsitua) 103

oratrix, Amazona 95

orbygnesius, Bolborhynchus 99

orcesi, Byrhura 118

oreas, Picathartes 198

Oreomystis 212

Oreonympha 164

Oreophasis 66

oreophilus, Buteo 35

Oreopsittacus 110

Oreothraupis 208 
Oreorrochilus 164

orientalis, Aquila 31

orientalis, Arborophila 67

orientalis, Vidua 225

(oriensalis, Vidua paradisaea) 225

orina, Coeligena 153

Oriole, Isabela 226

Oriole, Martinique 213

Oriole, Silver 227

Oriolus 226

omata, Urocissa 231

omatus, Cephalopterus 182

omatus, Lophornis 162

omatus, Spizaenus 54

omatus, Trichoglossus 120

Oroaetus 51

Oropendola, Baudó 213

Oropeodola, Chestnut-mantled 213

orostruthus, Arcanator 192

(orostruthus, Modulatrix) 192

(orostruthus, Phyllastrephus) 192

Oralis 66

Orthorhynchus 164

Orhotomus 197

ortizi, Incaspiza 208

(Ortygacichla) 201

Ortygospiza 218

osgoadi, Tinamus 2

Osprey, 23

ostenta, Coracina 188

Ostrich, 1

ostrinus, Pyrenestes 219

Oris 80

otus, Asio 127

Onus 134

Ou, 212

oustaleti, Anas 18

("oustaleti", Anas platythychos) 18

ovampensis, Accipiter 28

owenii, Apteryx 1

Owl. Abyssinian 126

Owl, African Marsh 126

Owl, Ashy-faced 124

Owl, Band-bellied 139

Owl, Band-tailed 139

Owl, Bare-legged 136

Owl, Barking 132

Ow1, Barn 123

Owl, Barred 142

Owl, Bay 123

Ow1, Black-and-white 141

Owl, Black-banded [4]

Owl, Boreal 125

Owl, Buff-fronted 125

Owl, Burrowing 140

Owl, Crested 131

Owl, David's 140

Owl, Desert 140

Owl, Elf I32

Owl, Fearful 132

Owl, Flammulated 135

Owl, Forest Little 127

Owl, Fulvous 141

Owl, Golden 124

Owl. Great Grey 141

Owl, Great Homed 129

Owl, Hawk 142

Owl, Hume's 140

Owl, Hume's Tawny 140
Owl, ltombwe 123

Owl, Jamaican 139

Owl, Lapp 141

Owl, Laughing 140

Owl, Litule 127

Ow1, Long-eared 127

Owl, Long-tufted Screech 134

Ow], Madagascar 125

Owl, Madagascar Red 125

Owl, Maned 131

Owl, Marsh 126

Owl, Minahsssa 124

Owl, Mouled 142

Owl, Northero Hawk 142

Owl, Northern Litule 127

Owl, Northera Saw-whet 125

Ow], Powerful 133

Owl, Rufous 133

Owl, Rufous-banded 140

Owl, Rufous-legged 14]

Owl, Rusty-barred 139

Owl, Saw-whet 125

Owl, Short-eared 126

Owl, Snowy 134

Ow], Soumagne's 125

Ow], Spectacled 139

Owl, Spotted 141

Owl, Spotted Litule 127

Ow1, Striped 126

Owl, Stygian 127

Owl, Sulawesi 125

Owi, Taliabu 125

Owl, Tanimbar 125

Owl, Tawny 140

Ow1, Tawny-browed 139

Owl, Tengmalm's 125

Owl, Unspotted Saw-whet 125

Owl, Ural 142

Ow1, White-browed 133

Owl, White-chinned 139

Owl, White-faced 140

Owl, Winking 132

Ow1, Woodford's 142

Owlet, African Bsrred 129

Owlet, Albertine 129

Owlet, Asian Barred 130

Owlet, Barred 129

Owlet, Barred Jungle 130

Owlet, Chestnut 129

Owlet, Chestnut-backed 130

Owlet, Collared 129

Owlet, Cuckoo 130

Owlet, Forest 127

Owlet, Javan 130

Owlet, Jungle 130

Owlet, Long-whiskered 142

Owlet, Pearl-spotted 130

Ow/et, Prigogine's 129

Owlet, Red-chested 131

Owlet, Scheffler's 131

Owlet, Sjostedi's 13 I

Owlet, Spotted 127

Owlet, Tibet 127

Owlet, Yellow-legged 131

owstoni, Gallirallus 76

(owstoni, Rallus) 76

Oxylabes, Yellow-browed 193

Oxypogon 164

Oxyura 22
Oystercatcher, Canarian Black 81

Oystercatcher, Canary 81

Oystercatcher, Chatham 81

Pachycephalo 197

Pachyphantes 222

Pachyramphus 183

pachyłyncha, Rhynchopsiata 118

(pachyrhyncha, Rhynchopsiua pachyrhyncho) 118

Palila, 212

pallescens, Rhynchotus rufescens 2

palliarus, Cinclodes 178

pallidiceps, Allaperes 207

pallidiceps, Columba 87

pallidigaster, Anthreptes 204

palmarum, Chamosyna 102

palmeri, Myadestes 197

(polmeri, Phaeamis) 197

Palmeria 212

paludicola, Acrocephalus 190

palustre, Pellornewm 198

palustris, Sporophila 210

pamela, Aglaeactis 145

(panayensis, Coracina) 188

Pandion 23

panini, Penelapides 175

(panini, Penelopides panini) 175

Panterpe 164

pamychlora, Nannopsittaca 108

papa, Sarcoramphus 23

Papasula 7

(Paphosia) 16I

papou, Charmosyna 102

Parabukeo 51

Paradigalla 229

Paradigalla, Long-tailed 229

Paradigalla, Short-tailed 229

Paradisaea 229

Paradise-crow, 229

Paradise-Flycatcher, Cerulean 194

Paradise-Flycatcher, Mascarene 201

Paradise-Flycatcher, Seychelles 201

Paradise-Flycatcher, Seychelles Black 201

Paradise-Kingfisher, Biak 171

Paradise-Whydah, Long-tailed 225

Paradise-Whydah, Togo 225

Paradise-Whydah, Uelle 225

Paradise-Whydah. Northern 225

(paradisea, Anthropoides) 74

paradisea, Grus 74

paradiseus, Prilaris 230

Paradoxomis 197

Parakeet, Alexandrine 115

Parakeet, Amazonian 108

Parakeet, Andean 99

Parakeet, Antipodes 103

Parakeet, Antipodes Green 103

Parakeet, Austral 104

Parakeet, Aztec 98

Parakeet, Barnard's 111

Parakeet, Barraband 113

Parakeet, Barred 99

Parakeet, Black-capped 118

Parakeet, Black-headed 108

Parakeet, Blaze-winged 117

Parakeet, Blood-ared 117

Parakeet, Blossom-headed 116

Parakeet, Blue-chested 117

Parakeet, Blue-crowned 97 
Parakeet, Blue-throated 117

Parakeet, Blyth's 115

Parakeet, Brown-breasted 117

Parakeet, Brown-throated 99

Parakeet, Burrowing 103

Parakeet, Caatinga 98

Parakeet, Cactua 98

Parakeet, Canary-winged 100

Parakeet, Chatham Island Yellow-fronted 103

Parakeet, Cobalt-winged 100

Parakeet, Crimson-bellied 118

Parakeet, Crimson-fronted 98

Parakeet, Cuban 98

Parakeet, Derbyan 115

Parakeet, Dusky-headed 99

Parakeet, El Oro 118

Parakeet, Emerald-collared 115

Parakeet, Fiery-shouldered 117

Parakeet, Flame-winged 117

Parakeet, Golden 98

Parakeet, Golden-capped 97

Parakeet, Golden-franted 99

Parakeet, Golden-plumed 106

Parakeet, Golden-winged 99

Parakeet, Green 98

Parakeet, Green-cheeked 118

Parakeet, Grey-breasted 108

Parakeet, Grey-cheeked 100

Parakeet, Grey-headed 115

Parakeet, Grey-hooded 99

Parakeet, Hispaniolan 98

Parakeet, Horned 105

Parakeet, Intermediate 115

Parakeet, Jandaya 98

Parakeet, Layard'a 115

Parakeet, Long-tailed 116

Parakeet, Malabar 115

Parakeet, Many-colourad 114

Parakeet, Maroon-bellied 117

Parakeet, Maroon-faced 117

Parakeet, Maroon-tailed 118

Parakeet, Mauritius 115

Parakeet, Mitred 98

Parakeet, Monk 108

Parakeet, Mountain 99

Parakeet, Moustached 115

Parakeet, Nanday 108

Parakeet, Newton'a 115

Parakeet, Nicobar 115

Parakeet, Norfolk 103

Parakeet, Norfolk Ialand 103

Parakeet, Ochre-marked 117

Parakeet, Olive-throated 98

Parakeet, Orange-chinned 100

Parakeet, Orange-fronted 98

Parakeet, Pacific 99

Parakeet, Painted 118

Parakeet, Peach-fronted 97

Parakeet, Pearly 118

Parakeet, Pennsnt's 111

Parakeet, Pla in 100

Parakeet, Plum-headed 115

Parakeet, Princess of Wales 112

Parakeet, Quaker 108

Parakeet, Red-breasted 115

Parakeet, Red-eared 117

Parakeet, Red-fronted 103

Parakeet, Red-masked 98
Parakeet, Reddish-bellied 117

Parakeet, Ring-necked 116

Parakeet, Rock 118

Parakeet, Rose-hesded 118

Parakeet, Rose-ringed 116

Parakeet, Rothschild'a 115

Parakeet, Rufous-fronted 99

Parakeet, Santa Marta 118

Parakeet, Scarlet-fronted 99

Parakeet, Sierra 99

Parakeet, Slaty-headed 115

Parakeet, Slender-billed 104

Parakeet, Socorro 98

Parakeet, Stanley 111

Parakeet, Sulphur-winged 117

Parakeet, Sun 99

Paraket, Tepui 108

Parakeet, Tui 100

Parakeet, Twenty-eight 112

Parakeet, White-breasted 117

Parakeet, White-eared 117

Parakeet, White-eyed 98

Parakeet, White-necked 117

Parakeet, Yellow-chevroned 99

Parakeet, Yellow-crowned 103

Parakeet, Yellow-fronted 103

Parakeet, Yellow-headed 98

paramelanotos, Calidris 83

Paraque, Jamaican 143

Pardalote, Forty-spotted 203

Pardalotus 203

(Pareudiastes) 76

pariue, Myiobonus 211

parkeri, Herpsilochmus 181

parkinsoni, Procellaria 4

Parmoptila 218

pamaguae, Megaxenops 178

Paroaria 209

Paroreomyza 212

Parotia 230

Parotia, Arfak 230

Paratia, Carola's 230

Parotia, Eastem 230

Parotia, Lawes'a 230

Paratia, Queen Carola's 230

Parotia, Six-plumed 230

Parotia, Wahnes's 230

Paratia, Western 230

Parrot, African Orange-bellied 112

Parrot, Alder 95

Partot. Alexandra's 112

Parrot, Barraband's 110

Partot, Beautiful 110

Parrot, Black 103

Parrot, Black-billed 93

Parrot, Black-eared 106

Parrot, Black-headed 110

Parrot, Black-lored 118

Parrot, Black-winged 106

Parrot, Blu-fronted 93

Parrot, Blue-backed 119

Parrot, Blue-bellied 120

Parrot, Blue-cheeked 94

Parrot, Blue-collaned 105

Parrol, Blue-headed 111

Parrot, Blue-naped 119

Parrot, Blue-rumped 117

Parrot, Blue-winged 109

Parrot, Bourke's 109
Parrol, Bronze-winged 110

Parrol, Brown 112

Parrol, Brown-headed 112

Parrot, Brown-hooded 110

Parrot, Brown-necked 112

Parrol, Caica 110

Parrot, Cape 112

Parrot, Cuban 95

Parrot, Dusky 110

Parrot, Eclectus 104

Parrot, Elegant 109

Parrol, Festive 94

Parrot, Fuertes's 106

Parrot, Golden-shouldered 114

Parrot, Great-billed 119

Parrot, Green-crowned Racket-tailed 113

Parrot, Green-headed Racket-tailed 113

Parrol, Green Leek 113

Parrot, Gray 116

Parrot, Ground 110

Parrot, Hawk-headed 104

Parrot, Hispaniolan 95

Parrot, Hooded 114

Parrot, lmperial 94

Parrot, Indigo-winged 106

Parrot, Jardine's 112

Parrot, Kawall's 95

Parrot, Lilac-crowned 94

Parrot, Maroon-fronted 118

Parrol, Mealy 94

Parrot, Meyer's 112

Parrot, Mulga 114

Parrot, Müller's 119

Parrot, Niam-niam 112

Parrot, Night 110

Parrot, Olive-shouldered 96

Parrot, Orange-bellied 108

Parrot, Orange-cheeked 110

Partot, Orange-winged 94

Partot, Palawan Racket-tailed 113

Partot, Paradise 114

Parrot, Pesquet's 117

Parrot, Pileated 110

Partot, Plum-crowned 111

Parrot, Princess 112

Parrot, Príncipe Grey 116

Partot, Puerto Rican 96

Partot, Purple-bellied 120

Parrot, Red-bellied 112

Parrot, Red-billed 111

Parrot, Red-browed 95

Parrot, Red-capped 110

Parrot, Red-cheeked 105

Parrot, Red-crowned 95

Parrot, Red-faced 106

Parrot, Red-fan 104

Parrot, Red-fronted 112

Parrot, Red-lored 94

Parrot, Red-necked 94

Parrot, Red-rumped 114

Parrot, Red-spectacled 95

Partot, Red-tailed 94

Patrot, Red-topped 95

Parrot, Red-vented 11]

Parrot, Red-winged 96

Parrot, Regent 112

Parrot, Rock 109

Parrol, Rose-faced 110

Parrot, Rueppell's 112 
Parrot, Rusty-faced 106

Parrol, Saffron-headed 110

Parrot, Saint Lucia 95

Parrol, Saint Vincent 94

Parrot, Scaly-headed 111

Parrot, Scaly-naped 95

Parrot, Scarlet-chested 109

Parrot, Senegal 112

Parrot, Short-tailed 106

Parrot, Singing 105

Parrot, Supert 113

Parrol, Swift 106

Parrot, Thick-billed 118

Parrol, Timor Red-winged 96

Parrot, Tucuman 95

Parrot, Turquoise 109

Parrot, Turquoise-fronted 93

Parrot, Vasa 103

Parrot, Vinaceous 95

Parrot, Vulturine 106

Parrot, White-bellied 110

Parrot, White-capped 111

Parrot, White-crowned 111

Parrot, White-fronted 93

Parrot, Yellow-billed 94

Parrot, Yellow-crowned 95

Parrot, Yellow-eared 109

Parrot, Yellow-faced 96

Parrot, Yellow-fronted 112

Parrot, Yellow-hesded 95

Parrot, Yellow-lored 96

Parrot, Yellow-naped 94

Parrot, Yellow-shouldered 94

Parrotbill, Black-breasted 197

Parrotbill, Crested 198

Parrotbill, Dusky 198

Parrotbill, Greater Rufous-headed 198

Parrotbill, Grey-hooded 198

Parrotbill, Maui 212

Parrotbill, Przewalski's 198

Parrotbill, Rufous-headed 198

Parrotbill, Rusty-throated 198

Parrotbill, Shor-tailed 197

Parrotfinch, Green-faced 215

Parrotfinch, Mindanao 215

Parrotfinch, Pink-billed 215

Parrotfinch, Red-eared 215

Parrotlet, Black-eared 119

Parrotlet, Blue-fronted 119

Parrotlet, Blue-rumped 105

Parrotlet, Blue-winged 105

Parrotlet, Brown-backed 119

Parrotlet, Celestial 105

Parrotlet, Dusky-billed 105

Parrotlet, Golden-tailed 119

Parrotlet, Green-rumped 105

Parrotlet, Lilac-tailed 119

Parrotlet, Mexican 105

Parrotlet, Pacific 105

Parrotlet, Red-fronted 119

Parrotlet, Sapphire-rumped 119

Parrotlet, Scarlet-shouldered 119

Parrotlet, Sclater's 105

Parrotlet, Seven-colored 119

Parrotlet, Spectacled 105

Parrotlet, Spot-winged 119

Parrotlet, Yellow-faced 105

Partridge, Bar-backed 67

Partridge, Black 70
Partridge, Cheatnut-headed 67

Partridge, Chestnut-necklaced 67

Partridge, Crested 71

Partridge, Ferruginoua 68

Partridge, Grey-breasted 67

Partridge, Hainan 67

Partridge, Long-billed 71

Partridge, Orange-necked 67

Partridge, Sichuan 67

Partridge, White-eared 67

Partridge, White-necllaced 67

Parus 202

Passer 222

passerinum, Glaucidium 130

passerinus, Forpus 105

pastazae, Galbula 175

pastinator, Cacatua 101

Patagona 164

pataganus, Cyanoliseus 103

pauliani, Ous rurilus 138

(pauliani, Otus) 138

paulistus, Phylloscartes 184

(pauxi, Crax) 66

pauri, Pauri 66

Pauxi 66

Pavo 70

pavonina, Balearica 73

pavoninus, Lophomis 162

Peacock-Pheasant, Bornean 70

Peacock-Pheasant, Crested 70

Peacock-Pheasant, Germain's 70

Peacock-Pheasant, Grey 70

Peacock-Pheasant, Iris 70

Peacock-Pheasant, Malayan 70

Peacock-Pheasant, Mountain 70

Peacock-Pheasant, Mountain 70

Peacock-Pheasant, Napoleon's 70

Peacock-Pheasant, Palawan 70

Peacock-Pheasant, Rothschild's 70

Peafowl, Congo 67

Peafowl, Green 70

Peafowl, Green-necked 70

pectoralis, Aphelocephala 192

pectoralis, Circus 38

pectoralis, Herpsilochmus 181

Pedionomus 73

pelagicus, Haliaeetus 44

Pelecanoides 6

Pelecanus 6

pelegrinoides, Falco 59

peli, Scoropelia 140

Pelican, Dalmatian 6

Pelican, Grey 7

Pelican, Spot-billed 7

pella, Topaza 170

Pellomeum 198

pelzelni, Aplonis 226

pelzelni, Ploceus 224

pelzelnii, Tachybaptus 3

pembaensis, Otus 138

penduliger, Cephaloptents 182

penelope, Anas 18

Penelope 66

Penelopides 174

Penelopina 66

Penguin, Humboldt 3

Penguin, Jackass 3

Penguin, Peruvian 3

Penguin, Yellow-eyed 3 (pennata, Puerocnemia) I

pennala, Rhea 1

pennatus, Hieraaerus 46

Peppler, Rock 112

percnoptenus, Neophron 51

Perdicula 70

perdita, Hinundo 187

Peregrine, 60

peregrinus, Falco 60

Perisoreus 231

perlata, Pyrhura 118

perlatum, Glaucidium 130

Pernis 51

peronii, Zoochera 202

persa, Tauraco 122

(persa, Tauraco corythaix) 122

personata, Heliopais 78

persanata, Prosopeia 113

personatus, Agapormis 93

perspicax, Penelope 66

perspicillata, Malacocincla 196

perspicillata, Pulsatrix 139

(perspicillatum, Trichastoma) 196

pertinax, Araringa 99

peruanum, Glaucidium 130

peruviana, Rupicala 183

peruviana, Tangara 210

periviana, Vini 120

(perversa, Ninax) 132

petersoni, Otus 137

Petrel, Beck's 4

Petrel, Bermuda 4

Petrel, Black 4

Petrel, Black-capped 5

Petrel, Cape Verde 5

Petrel, Chatham 4

Petrel, Cook's 5

Petrel, Dark-rumped 5

Petrel, Defilippe's 5

Petrel, Fiji 4

Petrel, Hawaiian 5

Petrel, Jamaica 5

Petrel, MacGillivray's 4

Petrel, Madeira 5

Petrel, Magenta 5

Petrel, Mas Atierra 5

Petrel, Mascarene 4

Petrel, Mascarene Black 4

Petrel, Parkinson's 4

Petrel, Pycroft's 5

Petrel, Réunion 4

Petrel. Westland 4

Petrel, Westland Black 4

Petroica 198

Petronia 222

Petronia, Bush 222

petrophila. Neophema 109

Pezoporus 110

Phaenicophaeus 123

Phaeochroa 164

phaeopygia, Pterodroma 5

(Phaeomis) 196

phaeosoma, Melamprosops 212

Phaethomis 165

phainopeplus, Campylopterus IS1

Phalacrocorax 7

Phalcoboenus 64

phalerata, Coeligena 153

Pharomachrus 171 
Pheasant, Argua 68

Pheasant, Blood 69

Pheasant, Bulwer'a 69

Pheasant, Cheer 68

Pheasant, Chinese Barred-backed 71

Phessant, Chir 68

Pheasant, Edwarda'a 69

Pheasant, Elliot's 71

Pheasant, Hume'。 71

Pheasant, Hume'a Bar-tailed 71

Pheasant, Imperial 69

Pheasant, Impeyan 69

Pheasant, Mikado 71

Pheasant, Ocellated 70

Pheasant, Reeves'a 71

Pheasant, Rufous-tailed 69

Pheasant, Salvadori's 69

Pheasant, Swinhoe's 70

Pheasant, Vietnamese 69

Pheasant, Vo Quy's 69

Pheasant, Wallich's 68

Pheasant, White-tailed Wattled 69

Phigys 110

Philemon 206

(Philestumus) 227

philippensis, Bubo 128

philippensis, Loriculus 107

philippensis, Ninox 133

philippensis, Pelecanus 7

(philippensis, Pseudoprynx) 128

philippensis, Spizaeus 54

philippii, Phoethomis 166

phillipsi, Tangara 210

Philodice 166

Philydor 178

Phlogophilus 167

Phadilus 123

phoebe, Metollura 163

(Phoeniconaias) 14

phoenicoptera, Pyrilia 219

Phoenicoplenus 14

Pholidornis 218

(Phonygammus) 229

phrygia, Xanthomyza 207

Phyllastrephus 188

(Phyllastrephus) 192

Phylloscartes 184

Phylloscopus 198

Phytotoma 185

Picathartes 198

Picathartes, Grey-necked 198

Picathartes, White-necked 198

pickeringii, Ducula 88

Picoides 177

picla, Psittacella 114

picta, Pyrthura 118

Picus 177

Pied-Babbler, Hinde's 201

Pied-Hombill, Malabar 174

Pied-Hombill, Malaysian 173

Pied-Hombill, Oriental 173

Piedtail, Ecuadorian 167

Piedtail, Peruvian 167

Pigeon, Afep 87

Pigeon, Bolle'a 86

Pigeon, Bolle'a Laurel 86

Pigeon, Choiseul 89

Pigeon, Dark-tailed Laurel 86

Pigeon, Laurel 86
Pigeon, Madeira Laurel 87

Pigeon, Maroon 87

Pigeon, Nicobar 85

Pigeon, Pale-capped 87

Pigeon, Penvian 87

Pigeon, Pink 87

Pigeon, Plain 86

Pigeon, Ring-tailed 86

Pigeon, Rock 86

Pigeon, Sao Tome Olive 87

Pigeon, Society lslands 88

Pigeon, Somali 87

Pigeon, Speckled $\mathbf{8 6}$

Pigeon, Speckled Rock 86

Pigeon, Tooth-billed 87

Pigeon, Triangular-spotted 86

Pigeon, Trocaz 87

Pigeon, Westem Bronze-naped 86

Pigeon, White-tailed Laurel 86

Pigeon, Yellow-legged 87

Piha, Cinnamon-vented 183

pileata, Pionopsitta 110

pilealus, Pipriles 183

Pintail, Common 16

Pintail, Northem 16

Pionites 110

Pionopsitto 110

Pionus 110

(pipile, Aburria pipile) 67

pipile, Pipile 67

Pipile 67

Piping-Guan, Black-fronted 67

Piping-Guan, Trinidad 67

Pipit, Ochre-breasted 187

Pipit, Sokoke 187

Pipit, Yellow-treasted 187

pipra, Iodopleura 182

Pipra 183

Piprites 183

Piprites, Black-capped 183

piscalor, Crinifer 121

Pithecophaga 52

Pithys 181

Pina 185

Pitta, Azure-breasted 186

Pitta, Banded 185

Pitta, Bar-bellied 185

Pitta, Black-backed 186

Pitta, Black-faced 185

Pitta, Blue-tailed 185

Pita, Fairy 185

Pitta, Gumey's 185

Pitta, Koch's 185

Pitta, Schneider's 185

Pitta, Solomons 185

Pitta, Steere's 186

Pitu, Supert 186

Pitta, Whiskered 185

placentis, Charmosyna 102

plagiata, Asturina 32

Plains-wanderer, 73

Plaintain-eater, Blue 121

Plaintain-eater, Western Grey 121

plancus, Polyborus 64

Plantain-eater, Grey 121

Plantain-eater, Violet 121

Plantcutter, Peruvian 185

Platalea 13

platenae, Gallicolumba 88 platenae, Prioniturus 113

(platenae, Prioniturus discunus) 113

plateni, Aramidopsis 75

platurus, Prioniturus 113

plarycercus, Selasphorus 168

Platycercus 111

platypterus, Buseo 35

Platyrinchus 184

Platysteira 198

Plectropterus 22

plicatus, Aceros 173

(plicalus, Rhyticeros) 173

Plocepasser 222

Ploceus 222

(Ploceus velatus vitellinus) 224

Plover, Black-banded 82

Plover, Hooded 82

Plover, Madagascar 82

Plover, New Zealand Shore 82

Plover, Piping 82

Plover, Saint Helena 82

Plover, Shore 82

Plover, Sociable 82

Plover, Sunda 82

Plovercrest, 169

plumbea, Icrinia 47

plumbea, Leucoplemis 48

plumbeus, Dysithamnus 180

plumbeus, Micrastur 63

(plumbeus, Thamnomanes) 180

Plumeleteer, Bronze-tailed 152

Plumeleteer, White-vented 151

Pochard, Baer's 19

Pochard, Ferruginous 19

Pochard, Madagascar 19

Pochard, White-eyed 19

(podargina, Pyrrogloux) 137

podorginus, Otus 137

Podiceps 3

Podilymbus 3

poecilochrous, Butea 35

poeciloptenus, Nesoclopeus 77

poensis, Bubo 129

Poephila 219

Pogoniulus 175

(Pogonocichla) 201

Poicephalus 112

Polemaetus 52

Polihierax 64

poliocepholus, Accipiter 28

poliocerca, Eupherusa 157

poliogaster, Accipiter 28

polionoto, Leucoptemis 48

poliopareia, Estrilda 216

(poltopareia, Estrilda poludicola) 216

poliopienus, Melierax 50

(polioptenus, Melierax canonus) 50

polleni, Xenopirostris 189

Polyboroides 52

Polyborus 64

Polyonymus 167

polyosoma, Bureo 36

(Polyplancta) 158

Polyplectron 70

Polyzelis 112

polymus, Trochilus 170

Polymus 167

Pomarea 198

pomarina, Aquila 31 
Poo-uli, 212

poortmani, Chlorostilbon 152

Poorwill, Jamaic an 143

Poospiza 209

Popelairia 167

(popelairii, Discasura) 167

popelairii, Popelairia 167

porcullae, Phaethomis 165

porphyrealopha, Musophaga 121

(porphyreolophus, Tauraca) 121

Porphyrio 77

porphyrocephala, Glossopsitta 106

Porzana 77

Prairie-Chicken, Attwater's 72

Premnoplex 178

pretrei, Amazona 95

pretrei, Phaethornis 166

preussi, Ploceus 224

prevosti, Anthracothorax 148

prigoginei, Chlorocichla 188

prigoginei, Nectarinia 204

(prigoginei, Nectarinia afra) 204

prigoginei, Phodilus 123

princeps, Accipiter 28

princeps, Leucoptemis 48

princeps, Melidectes 206

princeps, Psittacus erithacis 116

principalis, Campephilus 176

Prinia 199

Prinia, Long-tailed 199

Prinia, River 199

Prinia, Rufous-vented 199

Prioniturus 113

Prionops 189

pritchardii, Megapodius 65

Probosciger 113

Procellaria 4

procerus, Hemignathus 212

productus, Nestor 109

Prosepeia 114

Prosobonia 84

Prosopeia 113

prunellei, Coeligena 154

pryeri, Megalurus 196

przewalskii, Paradoxomis 198

(Psaracolius) 213

Psephotus 114

Pseudeos 114

Pseudibis 14

Pseudobulweria 4

Pseudacalyptomena 177

Pseudochelidon 187

Pseudocolopteryx 185

Pseudocossyphus 199

Pseudodacnis 209

Pseudonestor 212

(Pseudoptynx) 128

Pseudascops 139

psittacea, Psittirostra 212

psiltacea, Treron 91

Psituacella 114

Psittacula 115

Psinaculirostris 116

Psillacus 116

Psitteuteles 116

Psittinus 117

Psittirostra 212

Psittirastra 212

Psiturichas 117
Psophodes 199

psychopompus, Scytalopus 182

Pteridophora 230

Pterodrama 4

Pteroglossus 176

Preronetta 22

Prerophanes 167

Prilinopus 89

(Ptilolaemus) 173

ptilorhyncus, Pernis 52

Priloris 230

Puaiohi, 197

Puffinus 6

Puffleg, Black-breasted 156

Puffleg, Black-thighed 155

Puffleg, Blue-capped 155

Puffleg, Colourful 156

Puffleg, Coppery-bellied 155

Puffleg, Emerald-bellied 155

Puffleg, Glowing 156

Puffleg, Golden-breasted 156

Puffleg, Greenish 157

Puffleg, Hoary 157

Puffleg, Sapphire-vented 155

Puffleg, Turquoise-throated 155

pulchella, Charmosyna 102

pulchella, Neophema 109

pulchellus, Caprimulgus 143

pulcher, Calotharax 150

pulcherrimus, Psephotus 114

pulchra, Macgregoria 229

pulchra, Pionopsilla 110

pulizeri, Mocrosphenus 195

pulla, Grus canadensis 73

pullarius, Agaparnis 93

pullicauda, Neopsitlocus 109

Pulsarix 139

punctotus, Falco 60

punctulata, Ninox 133

punicea, Columba 87

Purpletuft, Buff-throated 182

purpurascens, Penelope 66

purpurala, Touit 119

Purpureicephalus 117

pusilla, Coenocorypha 83

pusilla, Glossapsiara 106

pusillus, Loriculus 107

pusio, Micropsilla 108

Pycnonotus 189

pycrofii, Pterodroma 5

(pycrofii, Plerodroma langirostris) 5

pygargus, Circus 40

(pygmaeus, Halietor) 7

pygmaeus, Phalacrocorax 7

pygmeus, Eurynorhynchus 83

Pygmy-goose, African 22

Pygmy-Ow1, Andean 130

Pygmy-Owl, Cuban 131

Pygmy-Owl, Fernginous 129

Pygmy-Owl, Least 130

Pygmy-Parrot, Buff-faced 108

Pygmy-Parrot, Finsch's 108

Pygmy-Partot, Geelvink 108

Pygmy-Parrot, Meek's 108

Pygmy-Parrot, Red-breasted 108

Pygmy-Parrot, Yellow-ca.ped 108

Pygmy-Tyrant, Rufous-sided 184

Pygmy-Owl, Collared 129

Pygmy-Owl, Eurasian 130
Pygmy-Owl, Mountain 130

Pygmy-Owl, Northern 129

pyra, Topaza 170

Pyrenestes 219

Pyriglena 181

pyrilia, Pionopsina 110

pyrohypogaster, Hypopyrthus 213

pyropygia, Hylocharis 160

pyrthocephalus, Phaenicophaeus 123

pyrrhops, Hapalopsittaca 106

(pyrthops, Hapalopsittaca amazonina) 106

pyrthopterus, Brotogeris 100

pyrthopterus, Lycocorax 229

Pyrthura 117

(Pyrroglaux) 137

Pytilia 219

Pytilia, Crimson-winged 219

Pytilia, Red-faced 219

Pytilia, Red-winged 219

Pytilia, Yellow-winged 219

quadragintus, Pardalotus 203

Quail-Dove, Blue-headed 90

Quail, Himalayan 70

Quailfinch, African 218

Quelea 224

Quelea, Red-headed 224

querquedula, Anas 18

Quetzal, Eared 171

Quetzal, Resplendent 171

rabieri, Picus 177

rabori, Napothera 197

Racket-tail, Booted 163

Racquet-tail, Blue-crowned 113

Racquet-tail, Blue-headed 113

Racquet-tail, Blue-winged 113

Racquet-tail, Buru 113

Racquet-tail, Golden-mantled 113

Racquet-tail, Green 113

Racquet-tail, Montane 113

Racquet-tail, Yellow-breasted 113

Racquet-tail, Mindanao 113

radiaum, Glaucidium 130

(radiatus, Accipiter) 4041

radialus, Erythrotriorchis 41

radiatus, Polyboroides 52

radiceus, Carpacoccyx 122

radiolosus, Neamarphus 123

raggiana, Paradisaea 230

Rail, Asian Yellow 75

Rail, Austral 77

Rail, Bald-faced 76

Rail, Bar-winged 77

Rail, Bogotá 77

Rail, Brown-banded 77

Rail, Guam 76

Rail, Henderson Island 77

Rail, Inaccessible 75

Rail, Invisible 76

Rail, Junin 77

Rail, Lord Howe 76

Rail, New Caledonian 76

Rail, New Caledonian Wood 76

Rail, Okinswa 76

Rail, Plain-flanked 77

Rail, Platen's 75

Rail, Sakalava 75

Rail, San Cristobal Mountain 76

Rail, Schlegel's 76 
Rail, Snoring 75

Rail, Speckled 75

Rail, Swinhoe's 75

Rail, Wallace'a 76

Rail, Woodford's 77

Rail, Zapata 76

raimondii, Phytotomo 185

Rallus 77

ramphastinus, Semnomis 175

Ramphastos 176

Ramphocinclus 190

Ramphodon 167

Ramphomicron 168

ranivorus, Circus 40

ropar, Aquila 31

rara, Bostrychia 13

rara, Lagonosticta 216

raricola, Vidua 225

Rayadito, Mas Afuera 177

razae, Alauda 186

(razae, Calandrella) 186

rectunguis, Ceniropus 122

Recurvebill, Bolivian 179

recurvirostris, Avocettula 149

Redstar, Luzon 199

Redatart, Paria 211

Redstart, Yellow-faced 211

Reed-Warbler, Nsuru 191

Reed-Warbler, Finsch'a 191

Reed-Warbler, Speckled 191

Reed-Warbler, Streaked 191

reevesii, Symaticus 71

regalis, Buteo 36

regalis, Heliangelus 158

regius, Cicinnurus 228

regulorum, Balearica 73

rehsei, Acrocephalus 191

relictus, Larus 85

religiosa, Gracula 226

respublico, Cicinnurus 228

(respublica, Diphyllodes) 228

restrictus, Cisticola 193

reticulata, Eos 104

rex, Boloeniceps 11

Rhabdomis 203

Rhabdomis, Long-billed 203

mami, Lamprolaima 161

Rhea I

Rhea, Common 1

Rhea, Danwin's I

Rhea, Greater I

Rhea, Lesser I

Rheinardia 70

rhinoceros, Buceros 174

Rhinomyias 199

(Rhinoplax) 174

Rhinoptilus 82

(Rhinoptynx) 126

Rhipidure 199

Rhizothera 71

rhadocephala, Pyrrhura 118

rhodocorytha, Amazona 95

rhadogaster, Pymthura 118

Rhadonessa 22

Rhadopis 168

Rhopomis 181

Rhyacomis 199

Rhynchapsitta 118

Rhynchotus 2
Rhynochetos 78

(Rhyticenos) 172

richandsi, Dryocopus javensis 177

ricordii, Chlorostilbon 152

ridgwayi, Aegolius 125

ridgwayi, Buteo 36

ridgwayi, Colinus virginianus 68

ridgwayi, Thalurania colombica 169

(ridgwayi, Thalurania) 169

riedelii, Tanysipuera 171

Riflebird, Magnificent 230

Riflebird, Paradise 230

Riflebird, Queen Vicloria'a 230

Riflebird, Victoria's 230

Ringneck, Mallee 111

Ringneck, Port Lincoln 112

riocourii, Chelictinia 37

(riocourii, Elanus) 37

risora, Alectrurus 183

(risora, Yesapa) 183

River-Martin, White-eyed 187

Roatelo, Brown 72

Roatelo, White-breasted 72

Robin, Black-throsled 195

Robin, Black-throated Blue 195

Robin-Chat, Angolan 193

Robin-Chat, White-headed 193

Robin, Chatham 198

Robin, Chatham lsland Black 198

Robin, Dappled Mountain 192

Robin, lrings Ground 200

Robin, Rufous-headed 195

Robin, Swynnerton's 201

Robin, Swynnerton's Forest 20

Robin, Usambsra Ground 200

Robinchat, Farkss's 199

roborotus, Otus 137

robustus, Poicephalus 112

rochussenii, Scolopax 84

Rock-Thrush, Benson's 199

rockefelleri, Nectarinia 204

Rockfowl, Grey-necked 198

Rockfowl, Red-headed 198

Rockfowl, White-necked 198

Rockfowl, Yellow-headed 198

(rodericanus, Acrocephaliss) 192

rodericanus, Bebromis 192

rodolphei, Stachyris 201

rogersi, Aslantisia 75

Rollulus 7

roquettei, Phylloscones 184

rorotus, Eclectus 104

roseata, Psittocula 116

(roseicapilla, Cacatma) 104

roseicapilla, Ptilinopus 90

roseicapillus, Eolophus 104

roseicollis, Agapomis 93

Rosella, Crimson 11]

Rosella, Eastern 111

Rosella, Golden-mantled 111

Rosella, Green 111

Rosella, Mealy 11

Rosella, Norhem 111

Rosella, Psle-headed 111

Rosella, Tasmanian 111

Rosella, Westem 111

Rosella, Yellow 111

rosenbergi, Amazilia 147

rosenbergii, Gymnocrex 76 rosenbergit, Tyto 125

roseogrisea, Streptopelia 90

roseus, Phoenicopterus is

Rostrhamus 52

rothschildi, Astrapia 227

rothschildi, Leucopsor 226

rothschildi, Pyrenestes 219

rouloul, Rollulus 71

Roulroul, 71

rourei, Nemosia 208

Rowettia 209

rowleyi, Eurrichomyias 194

Roysl-Flycatcher, Western 184

rubecula, Poospiza 209

ruber. Eudocimus 13

ruber. Phaethomis 166

ruber, Phoenicopterus 15

rubescens;, Gallicolumba 88

nubicunda, Grus 74

rubiginosus, Trichoglossus 120

rubinoides, Heliodoxa 158

rubra, Crax 66

rubra, Foudia 221

rubro, Paradisaea 230

rubricala, Lagonosticla 216

rubricauda, Clytolaema 153

rubriceps, Anaplecles 219

(rubriceps, Malimbus) 219

rubricollis, Charadrius 82

rubricollis, Molimbus 221

rubrifrons, Parmoptila 218

(nubrifrons, Pamoptila woodhousei) 218

rubrigularis, Charmosyna 102

rubritorques, Anthreptes 204

(nubritorques, Anthreptes rectirostris) 204

mubritorquis, Trichoglossus 120

(nubrilorquis, Trichoglossus

haematodus) 120

rubrogenys, Ara 97

rubronolata, Chamosyna 102

Ruby, Brazilian 153

ruckeri, Threnetes 170

ruckii, Cyomis 193

nuddi, Heleromirafro 186

(nuddi, Mirafra nuddi) 186

nadolfi. Ninox 133

(nudolfi, Ninox novaeseelandioe) 133

rudolphi, Paradisaea 230

(ruecki, Muscicapa) 193

(ruecki, Nillava) 193

rueppellii, Eupodotis 79

rueppellii, Gyps 43

rueppellii, Poicepholius 112

rufa, Ninox 133

(rufo, Ortygocichlo) 201

rufa, Trichocichla 201

nufescens, Atrichomis 186

rufescens, Otus 137

rufescens, Rhynchotus rufescens 2

ruficouda, Myrmeciza 181

ruficeps, Cholcostigma 151

(nuficeps, Erithacus) 195

ruficeps, Luscinio 195

ruficeps, Paradoxomis 198

(nuficollaris, Halcyon) 172

ruficollaris, Todirhamphus 172

ruficollis, Automolus 178

ruficollis, Brünto 20

ruficollis, Madango 204 
ruficollis, Micrastur 63 (ruficollis, Syndacryla) 178 ruficrissa, Urosticte 170 (ruficrissa, Urosticte benjamini) 170 ruficrista, Eupodotis 80 (ruficrista, Lophotis) 80 rufifrons, Fomicarius 180 rufigularis, Falco 60 rufigularis, Hyetomis 123 rufinus, Buteo 36 rufipectus, Arborophila 67 rufipectus, Spilamis 53 rufipennis, Butastur 33 rufipennis, Nectarinia 204 rufipes, Strix 141 rufitorques, Accipiter 28 rufiventris, Accipiter 28 rufiventris, Poicephalus 112 rufiventris, Saltator 209 rufocinerea, Grallaria 180 rufofuscus, Buteo 36 rufogularis, Pachycephala 197 rufolavatus, Tachybaptus ruficollis 3 (rufolavatus, Tachybaptus) 3 nffomarginatus, Euscarthmus 184 (nufomerus, Chalcites malayanus) 123 rufomerus, Chrysococcyx 123 rufopicta, Lagonosticta 216 rufus, Campylapienus 151 rufus, Selasphanus 168 nugensis, Metabolus 196 ruki, Rukia 204

Rukia 204

rupicola, Pyrthura 118 rupicola, Rupicola 183 Rupicola 183 rupicoloides, Falco 60 Rush-Warbler, Grauer's 192 rushiae, Pholidomis 218 nuspalii, Tauraco 122 russarus, Chlorostilbon 153 rusticolus, Falco 61 rutila, Amazilia 147 rutilus, Otus 138

ruweti, Ploceus 224

Sabrewing, Buff-breasted 150 Sabrewing, Grey-breasted 151 Sabrewing, Lazuline 150 Sabrewing, Long-tailed 150 Sabrewing, Napo 151 Sabrewing, Rufous 151 Sabrewing, Rufous-breasted 151 Sabrewing, Santa Marta 151 Sabrewing, Violet 151

Sabrewing, Wedge-tailed 150 Sabrewing, White-tailed 150 Saddleback, 227

Sagittarius 55 sagittatus, Otus 138

Saker, 57

salamonis, Gallicolumbo 88 Saltator 209

Saltator, Rufous-bellied 209 salusarius, Crypurellus enythropus 2 (saltuarius, Crypturellus) 2 salvadorii, Psinaculirostris 116 samarensis, Penelopides 175 (samarensis, Penelopides panini) 175 sanctaecatarinae, Otus 134 sanclaecrucis, Zosterops 205 sanctachelenae, Charadrius 82 sanctithomae, Brotogeris 100 Sandpiper, Cox's 83 Sandpiper, Sharp-billed 84 Sandpiper, Spoonbill 83 Sandpiper, Tuamotu 84 sandvicensis, Branta 20 (sandvicensis, Nesochen) 20 sanfondi, Cyomis 194 sanfondi, Haliacerus 44 (sanfordi, Niltava) 194 sanguinea, Cacalua 101 santaecrucis, Gallicolumba 89 santavestris, Aplanis 226 Sapheopipo 177

Sapphire, Blue-chinned 152 Sapphire, Blue-headed 159 Sapphire, Flame-rumped 160 Sapphire, Golden-tailed 153 Sapphire, Rufous-throsted 160 Sapphire, White-chinned 159 Sapphirewing, Great 167 sapphirina, Hylocharis 160 Sappho 168

Sarcogyps 53

Sarcoramphus 23

Sarkidiomis 22

Sarothrura 77

Sarothrura 78

sasin, Selasphorus 168

satyra, Tragopan 71

saucerroutei, Amazilia 147

saundersi, Lonus 85

savilei, Eupodotis 80

(savilei, Lophotis) 80 (sawtelli, Aerodramus) 144 sawtelli, Collacalia 144 Saricolo 199

scapularis, Alistenus 93

Scelaglaux 140

Scepomycter 200

schalowi, Tauraca 122

(schalowi, Tauraco corythaix) 122

scheepmakeri, Goura 89

schefferi, Glaucidium 131

(schefleri, Glaucidium capense) 131

schistacea, Coracina 188

schistacea, Leucoptemis 48

schistacea, Zoothera 202

(Schistes) 149

schleiermacheri, Polyplectron 70

(schleiermacheri, Polyplectron malacense) 70

schneideri, Pitta 185

Schoutedenapus 144

schoutedeni, Schoutedenapus 144

schreibersil, Heliodoxa 159

schuetni, Tauraco 122

(schuettii, Tauraco corythaix) 122

schulzi, Cinclus 190

Scimitar-Babbler, Short-tailed 195

scintilla, Selasphorus 168

scitulus, Trochilus 170

sclateri, Forpus 105

sclateri, Lophophorus 69

Scolopax 84

scops, Otus 138

Scops-Owl, African 138
Scops-Owl, Andaman 134

Scops-Owl, Elegant 135

Scops-Owl, Enggano 135

Scops-Owl, Flores 134

Scopa-Owl, Giant 132

Scops-Owl, Grand Comoro 138

Scops-Owl, Indian 134

Scops-Owl, Javan 134

Scops-Owl, Lesser Sunda 138

Scops-Owl, Luzon 136

Scopa-Owl, Madagascar 138

Scops-Owl, Mantanani 137

Scops-Owl, Mentaur 139

Scops-Owl, Mentawai 137

Scops-Owl, Mindoro 137

Scops-Owl, Morden'g 136

Scops-Owl, Palau 137

Scopa-Owl, Palawan 135

Scops-Owl, Pallid 135

Scops-Owl, Papuan 136

Scops-Owl. Pemba 138

Scops-Owl, Philippine 137

Scops-Owl, Rajah 134

Scops-Owl, Reddish 137

Scops-Owl, Sandy 135

Scops-Owl, Senegal 138

Scops-Owl, Seychelles 136

Scops-Owl, Simeulue 139

Scops-Owl, Sokoke 136

Scops-Owl, Sported 138

Scops-Ow1, Wallace's 138

Scops-Owl, White-faced 136

Scops-Owl, White-fronted 138

Scops-OwI, Collared 136

Scops-Owl, Eurasisn 138

Scops-Owl, Mantanani 137

Scops-Owl, Mindanso 137

Scops-Owl, Moluccan 136

Scops-Owl, Mountain 138

Scops-Owl, Oriental 139

Scops-Owl, Sao Tome 135

Scops-Ow], Striated 135

Scops-Owl, Sulawesi 136

Scops-Owl, Sumatran 139

Scotopelia 140

Screech-Owl, Austral 139

Screech-Ow], Balsas 138

Screech-Owl, Bare-shanked 135

Screech-Ow], Bearded 134

Screech-Owl, Black-capped 134

Screech-Owl, Cloud-forest 137

Screech-Owl, Colombian 135

Screech-Ow], Eastern 134

Screech-Owl, Koepcke's 136

Screech-Owl, Pacific 135

Screech-Ow1, Puerto Rican 137

Screech-Owl, Rufescent 135

Screech-Owl, Tawny-bellied 139

Screech-Owl, Tropical 135

Screech-Ow], Vermiculated 139

Screech-Owl, West Peruvian 137

Screech-Owl, Western 136

Screech-Ow!, Whiskered 139

Screech-Owl, White-throated 134

Screech-Owl, Cinnamon 137

Screech-Owl, Variable 134

scriptus, Elanus 41

Scrub-bird, Noisy 186

Scrub-bird, Rufous 186 
Scrub-Warbler, Ja River 192

Scrub-Warbler, Grauer'a 192

Scrubfowl, Marianaa 65

Scrubfowl, Micronesian 65

Scrubfowl, Moluccan 65

Serubfowl, Nicobar 65

Scrubfowl, Sula 65

scutatus, Augastes 149

scutatus, Malimbus 221

scutulata, Cairina 20

scutulata, Ninox 133

Scytalopus 182

Sea-Eagle, Sanford'a 44

Sea-Eagle, Solomon 44

Sea-Eagle, Steller's 44

Sea-Eagle, White-bellied 44

Sea-Eagle, Pallas'в 44

sechellarum, Copsychus 193

sechellarum, Foudia 221

(sechellensis, Acracephalus) 192

sechellensis, Bebramis 192

Secretary-bird, 55

seductus, Otus 138

(seductus, Otus asia) 138

Seedcracker, Black-bellied 219

Seedeater, Black-and-tawny 210

Seedeater, Buffy-fronted 209

Seedeater, Buffy-throated 209

Seedeater, Entre Rios 210

Seedeater, Grey-and-chestnut 209

Seedeater, Hooded 210

Seedeater, Marsh 210

Seedeater, Narosky'a 210

Seedeater, Rufous-rumped 209

Seedester, Temminck's 209

Seedeater, Tumaco 209

Seedeater, West Afric an 214

Seedeater, White-rumped 214

Scedeater, Yellow-throated 214

sefilata, Parotia 230

Selaspharus 168

Selenidera 176

Seleucidis 231

selopulo, Strix 141

semilarvata, Eos 104

Semioptera 231

semipalmatus, Limnodramus 83

semiplumbea, Leucaptemis 48

semiplumbeus, Rallus 77

semitorquata, Streptapelia 90

semitorquatus, Micrastur 63

semitorquatus, Polihierax 64

Semnomis 175

semperi, Leucopeza 211

senegala, Laganosticia 217

senegalensis, Ephippiarhynchus 12

senegalensis, Eupodotis 80

senegalensis, Otus 138

senegalensis, Streptopelia 90

senegalus, Poicephalus 112

senex, Todirosinum 185

senilis, Pianus 111

senilaides, Pionus 111

sephaniodes, Sephanoides 168

Sephanoides 168

sericea, Lobaparadisea 228

(Sericates) 156

Sericulus 227

Serin, Ankober 214
Serin, Yellow-throated 214

Serinus 214

Serpent-Eagle, African 41

Serpent-Eagle, Andaman 53

Serpent-Eagle, Congo 41

Serpent-Eagle, Crested 53

Serpent-Eagle, Dark 53

Serpent-Eagle, Kinabalu 53

Serpent-Eagle, Madagascar 42

Serpent-Eagle, Nicobar 53

Serpent-Eagle, Philippine 53

Serpent-Esgle, Mountain 53

Serpent-Eagle, Sulaweai 53

serpentarius, Sagituarius 55

(semana. Formicivoro litworalis) 180

serrirastris, Colibri 154

setifrons, Xenomis 181

severa, Ara 97

severus, Falca 61

Shag, Bronze 7

Shag, Chatham 7

Shag, Rough-faced 7

Shame, Black 193

(Shape, Ninox salomonis) 133

sharpei, Terenura 181

Shearail, Mexican 155

Sheartail, Peruvian 169

Sheartail, Slender 155

Shearwater, Heinroth's 6

Shearwater, Newell's 6

Shearwater, Pink-footed 6

Shearwater, Townsend's 6

Shelduck, Crested 23

shelleyi, Bubo 129

Sheppardia 200

Shikra, 24

Shikra, Nicobar 25

Shining-Parrot, Crimson 114

Shining-Parrot, Masked 113

Shining-Parrot, Red 114

Shoebill, 11

Short-tail, Sao Tome 19]

Shortwing, Rusty-bellied 192

Shortwing, Rusty-breasted 192

Shoveler, Northern 17

Shrike, Sao Tome Fiscal 189

Shrike-Tyrant, White-tailed 183

Shrikebill, Rennell 193

sicki, Terenura 181

Sicklebill, Black 228

Sicklebill, Black-billed 228

Sicklebill, Brown 228

Sicklebill, Buff-tailed 157

Sicklebill, Pale-billed 228

Sicklebill, White-billed 228

Sicklebill, White-tipped 157

sidamoensis, Heteramirafra 187

(sidamaensis, Mirafra) 187

siemiradzlii, Carduelis 214

siju, Glaucidium 131

Silky-crow, 229

Silverbill, African 217

(silvestris, Edithomis) 76

silvestris, Gallinula 76

(silvestris, Pareudiastes) 76

silvicala, Otus 138

simani, Selasphorus 168

Simorenops 179

simplex, Geoffrayus 105 sintillata, Chalcopsitua 102

Siphanorhis 143

(siquijarensis, Hypsipeles) 188

siquijarensis, Lxos 188

sirintarae, Pseudachelidon 187

Siakin, Red 214

Siskin, Saffron 214

Siakin, Yellow-faced 2]4

(Sitagra) 223

Sitta 203

sjastedti, Glaucidium 131

sladeniae, Apus barbolus 144

(sladeniae, Apus) 144

Snake-Eagle, Banded 37

Snake-Eagle, Beaudouin's 38

Snake-Eagle, Black-chested 38

Snake-Eagle, Brown 38

Snake-Eagle, Fasciated 38

Snake-Eagle, Short-toed 38

Snake-Eagle, Smaller Banded 37

Snake-Eagle, Southern Banded 38

Snipe, Chatham Islands 83

Snipe, New Zealand 83

Snipe, Subantarctic 83

Snipe, Wood 83

Snowcap, 163

Snowcock. Caspian 71

Snowcock, Tibetan 71

snowi, Mymothenula 181

sociabilis, Rostrhamus 52

Softtail, Orinoco 179

Softrail, Russet-mantled 179

Softuil, Striated 179

sokokensis, Anthus 187

solangiae, Silla 203

solitarius, Butea 36

solitarius, Harpyhaliaetus 45

solivarius, Phigys 110

salitarius, Tinamus 2

solaensis, Accipiter 28

solamonensis, Nesasio 132

solsritialis, Aratinga 99

sonneratii, Gallus 69

Sooty-Owl, Greater 125

Sooty-Ow], Lesser 125

sardidus, Cynanthus 154

sardidus, Pianus 111

sorghaphilus, Acrocephalus 191

sararcula, Tyta 125

(sororcula, Tyla novachallandioe) 125

saumagnei, Tyta 125

Spadebill, Russet-winged 184

sparganura, Sappho 168

Sparrow, Bush 222

Sparrow, Cuban 210

Sparrow, Grey-headed 222

Sparrow, Sierra Madre 210

Sparrow-Weaver, Chestnut-crowned 222

Sparrow, Zapata 210

Sparrowhawk, African Litule 27

Sparrowhawk, Besra 29

Sparrowhawk, Bicolored 24

Sparrowhawk, Black 27

Spartowhawk, Burger's 41

Sparrowhawk, Chestnut-bellied 25

Sparrowhawk, Chestnut-flanked 25

Sparrowhawk, Chinese 28

Sparrowhawk. Collared 25

Sparrowhawk, Eurasian 27 
Sparrowhawk, Greal 27

Sparrowhawk, Imitator 26

Sparrowhawk, Japanese 26

Spartowhawk, Levant 24

Sparrowhawk, Litule 27

Sparrowhawk, Little Banded 24

Sparrowhawk, Madagascar 26

Sparrowhawk, Moluccan 25

Sparrowhawk, Moluccan Barred 26

Sparrowhawk, New Britain 24

Sparrowhawk, Nicobar 25

Sparrowhawk, Ovampo 28

Sparrowhawk, Pied 24

Sparrowhawk, Red-thighed 25

Sparrowhawk, Rufous-chested 28

Sparrowhawk, Rufoug-necked 25

Sparrowhawk, Semi-collared 25

Sparrowhawk, Slaty-mantled 26

Sparrowhawk, Small 27

Sparrowhawk, Spot-tailed 29

Sparrowhawk, Tiny 28

Sparrowhawk, Vinous-breasted 28

sparverius, Falco 61

(Sparula) 17

Spatuletail, Marvelous 161

speciosa, Geothlypis 21

speciasa, Stachyris 201

spectabilis, Dryatriarchis 41

Speirops 205

Speirops, Fernando Po 205

Speirops, Príncipe 205

Spelaearnis 200

spencei, Heliangelus 158

Speotyto 140

(Spermestes) 217

Spermophaga 219

Spheniscus 3

Sphenocichla 200

sphenunus, Haliastur 45

spilocephalus, Otus 138

spilogaster, Hieraaetus 47

(spilogaster, Hieraaetus fasciatus) 47

spilonotus, Circus 40

(spilonotus, Circus aeruginosus) 40

spiloptera, Parzana 77

Spilomis 53

Spinetail, Apurimac 179

Spinetail, Blackish-headed 179

Spinetsil, Chestnut-throsted 179

Spinetail, Hoary-throated 179

Spinetail, Pernambuco 179

Spinetail, Pinto's 179

Spinetail, Plain 179

Spinetail, Russet-bellied 179

(Spinus) 214

spixii, Cyanopsirta 103

Spizaetus 53

Spizastur 54

Spiziapieryx 64

Spizocorys 186

splendens, Prosepeia 114

(splendens, Prosepeia tabuensis) 114

splendida, Neaphema 109

splendidissima, Astrapia 228

spodiurus, Pachyramphus 183

Spoonbill, Black-faced 13

Spoonbill, Eurasian 13

Spoonbill, White 13

(Sparaeginthus) 215
Sparophila 209

Sparopipes 225

spurius, Purpureicephalus 117

squalidus, Phaethomis 166

squamasa, Eos 105

squamatus, Mergus 21

squamigera, Brachypteracias 172

squamipila, Ninar 133

squamosus, Heliomaster 159

Stachyris 200

Standardwing, Wallace's 231

stanleyi, Chalcostigma 151

Starfrontlet, Blue-throated 153

Starfrontlet, Buff-winged 153

Starfrontlet, Dusky 153

Starfrontlet, Golden-bellied 153

Starfrontlet, Rainbow 153

Starfrontlet, Violet-throsted 154

Starfrontlet, White-tailed 153

Starling, Abbott'a 226

Starling, Bali 226

Starling, Mountain 226

Starling, Pohnpei 226

Starling, Rarotonga 226

Starling, Santo Mountsin 226

Starling, Sula 226

Stamoenas 90

Starthroat, Blue-tufted 159

Starthroat, Long-billed 159

Starthroat, Plain-capped 159

Starthroat, Stripe-breasted 159

steerii, Centropus 122

steerii, Pita 186

Stellula 169

stenura, Chlarostilbon 153

stephaniae, Astrapia 228

Stephanoaetus 54

Stephanoxis 169

stepheni, Vini 121

Stema 85

(Stema albostriata) 84

Stemoclyta 169

sterhopteron, Wetmorethraupis 210

stictolophus, Lophomis 162

Srictonema 23

stictoplera, Touit 119

stigmalus, Loriculus 107

Stilt, Black 81

Stitchbird, 206

Stonechat, Fuerteventura 199

Stork, Black 11

Stork, Marabou 12

Stork, Milky 12

Stork, Oriental 11

Stork, Oriental White 11

Stork, Saddle-billed 12

Stork, Saddlebill 12

Stork, Storm's 11

Stork, Whale-headed 11

Storm-Petrel, Guadalupe 6

Storm-Petrel, Tristram's 6

stormi, Cicania 11

(stomi, Ciconia episcopus) 11

Streamertail, 170

strenua, Aratinga 99

strenua, Ninox 133

Streptacitta 226

Streptopelia 90

(stresemanni, Ampelion) 183 stresemanni, Merulaxis 182

stresemanni, Otus spilocephalus 139

(stresemanni, Otus) 139

stresemanni, Zaratomis 183

stresemanni, Zavattariomis $\mathbf{2 3 1}$

striatus, Accipiter $\mathbf{2 8}$

strianus, Chactomis 192

striatus, Simaxenops 179

strigirostris, Didunculus 87

Strigops 118

Striped-Babbler, Negros 201

Strix 140

strophianus, Heliangelus 158

strophizm, Odontophorus 70

Struthia 1

stuarti, Phaethomis 166

Stumella 213

stygius, Asio 127

subbutea, Falca 61

subcristata, Aviceda 32

subflava, Amandava 215

(subflavus, Sporaeginthus) 215

subniger, Falco 61

subachraceus, Phaethomis 166

subpersonatus, Placeus 224

subruficollis, Aceros 173

(subruficollis, Aceros plicatus) 173

(subruficollis, Rhyliceros) 173

subrilis, Buteogallus 37

sukatschewi, Gamulax 195

sula, Coracina 188

(sula, Coracina morio) 188

(Sula) 7

sulfuratus, Ramphastos 176

sulphurata, Emberiza 208

sulphurea, Cacatua 101

sumatranus, Bubo 129

sumatranus, Tanygnathus 119

sumichrasti, Catherpes 190

(sumichrasri, Hylorchilus) 190

Sunangel, Amethyst-throated 157

Sunangel, Gorgeted 158

Sunangel, Little 158

Sunangel, Merida 158

Sunangel, Orange-throated 158

Sunangel, Purple-throated 158

Sunangel, Royal 158

Sunangel, Tourmaline 158

Sunbeam, Black-hooded 145

Sunbeam, Purple-backed 145

Sunbeam, Shining 145

Sunbeam, White-tufted 145

Sunbird, Amani 204

Sunbird, Apricot-breasted 204

Sunbird-asity, Yellow-bellied 186

Sunbird, Banded 204

Sunbird, Elegant 204

Sunbird, Giant 204

Sunbird, Grey-chinned 204

Sunbird, Marungu 204

Sunbird, Prigogine's Double-collared 204

Sunbird, Rockefeller's 204

Sunbird, Rufous-winged 204

Sunbird, Sanghir 204

Sunbird, Sao Tome 204

Sunbird, Sumba 204

Sungem, Horned 157

sunia, Otus 139

(sunia, Otus scops) 139 
superba, Lophorina 228 superba, Pitta 186

superciliaris, Ninox 133 superciliasa, Ophrysia 70 superciliasus, Accipiter 28 superciliasus, Pachyphantes 222 superciliasus, Phacthamis 166 superciliasus, Plocepasser 222 (superciliasus, Ploceus) 222 surda, Touit 119

Sumia 142

swainsoni, Butea 36 swainsonii, Chlorostilbon 153 swainsanii, Gampsanyx 42 swainsanit, Palytelis 113 swalesi, Turdus 202

Swallow, Red Sea 187

Swallow, White-tailed 187

Swamp-Warbler, Giant 192

Swamp-Warbler, Grauer's 192

Swamp-Warbler, Yellow 192

Swan, Black-necked 20

Swan, Coscoroba 20

swierstrai, Francalinus 68

Swift, Congo 144

Switt, Dark-rumped 144

Switt, Fermando Po 144

Swift, Schoutederia 144

Swift, Waterfall 144

Swift, White-chested 144

Swiftlet, Aitu 144

Swiftlet, Giant 144

Swiflet, Polynesian 144

Swiflet, Sawtell'a 144

Swiflet, Seychelles 144

Swiftlet, Tahiti 144

swindemianus, Agapornis 93

swinhoii, Lophura 70

(swynzertoni, Paganocichla) 201

swynnerioni, Swynnertonia 201

Swynnertonia 201

sybillae, Lampornis 160

(sybillae, Lampamis viripallens) 160

Sylph, Long-tailed 145

Sylph, Violet-railed 145

sylvestris, Gallirallus 76

(sylvestris, Tricholimnas) 76

Synallaxis 179

(Syndactyla) 178

Synthlibaramphus 85

(Sypheatides) 79

Syrmaricus 71

symatophorus, Phaethomis 166

tabuensis, Prosapeia 114

tachiro, Accipiter 29

Tachybaptus 3

taczanowskii, Leucippus 161

saczanowskii, Nathoprocta 2

laczanowski, Podiceps 3

Tadorna 23

tahitiensis, Numenius 83

Taiko, Chatham Island 5

Tailorbird, Long-billed 197

Takahe, 77

tamaragense, Conirostrum 211

Tanager, Azure-rumped 210

Tanager, Black-and-gold 207

Tanager, Black-backed 210

Tanager, Black-cheeked 210
Tanager, Chat 207

Tanager, Cherry-throated 208

Tanager, Cone-billed 208

Tanager, Gold-ringed 207

Tanager, Green-capped 210

Tanager, Multicolored 207

Tanager, Orange-throated 210

Tanager, Seven-colored 210

Tanager, Sira 210

Tangara 210

Tanygnathus 118

Tanysiptera 171

Taoniscus 2

Tapaculo, Bahia 182

Tapaculo, Brasilia 182

Taphralesbia 169

Taphraspilus 169

taranta, Agapomis 93

tarda, Otis 80

(Latei, Margaromis) 178

totei, Premnoplex 178

Tauraco 121

Tawny-Eagle, African 31

Tawny-Eagle, Eurasian 31

(Tchitrea) 201

Teal, Auckland Island Brown 16

Teal, Baikal 18

Teal, Campbell Island Brown 16

Teal, Cape 16

Teal, Common 17

Teal, Garganey 18

Teal, Green-winged 17

Teal, Laysan 18

Teal, Madagascar 16

Teal, Marbled 21

Teal, New Zealand Brown 16

teesa, Butastur 33

Telespiza 212

Telophorus 189

tenebricosa, Tyta 125

uenebrosus, Phyllastrephus 189

tener, Loriculus 107

(tener, Loriculus stigmatus) 107

tenuirastris, Cacatua 101

tenuirastris, Numenius 84

tephranotum, Glaucidium 131

Terathopius 55

Terenura 181

Tern, Black-fronted 84

Tem, Chinese Crested 85

Tern, Damara 85

Tern, Kerguelen 85

Terpsiphone 201

terrisi, Rhynchopsitua 118

(terrisi, Rhynchapsina pachyrhyncha) 118

Tetraka, Appert's 188

Tetraka, Dusky 189

Tetraka, Grey-crowned 188

Tetraa 71

Tetraagallus 71

(tetrax, Otis) 81

letrax, Tetrax 81

Tetrax 81

texrilis, Amylomis 191

(Textu) 223

teydea, Fringilla 214

Teydefinch, 214

teysmannii, Treron 91

(Thalasseus) 85 thalassinus, Colibri 154

Thalurania 169

(Thamnomanes) 180

Thawmastura 169

(Thaumatibis) 14

theomacha, Ninor 133

theresiae, Metallura 163

cheresiae, Polyomus 167

Thick-knee, Double-atriped 81

Thicketbird, Long-legged 201

(Thinomis) 82

thomensis, Columba 87

(thomensis, Dreptes) 204

thamensis, Nectarinia 204

tharacicus, Charadrius 82

Thombill, Black-backed 168

Thombill, Blue-mantled 151

Thombill, Bronze-tailed 151

Thombill, Olivaceous 151

Thombill, Purple-backed 168

Thombill, Rainbow-bearded 151

Thombill, Rufous-capped 151

Thorntail, Black-bellied 167

Thorntail, Coppery 167

Thomtail, Green 167

Thomtail, Wire-crested 167

Thrasher, Socorro 190

Thrasher, White-breasted 190

Threneles 170

Threskiamis 14

Thripaphaga 179

Thrush, Amami 202

Thrush, Everett's 202

Thrush, Fawn-breasted 202

Thrush, Grey-sided 201

Thrush, Hawaiian 196

Thrush, La Selle 202

Thrush, Natal 202

Thrush, Orange-banded 202

Thrush, Slaty-backed 202

Thrush, Small Kauai 197

Thrush, Spotted Forest 202

Thrush, Tanimbar 202

Thrush, Teits 202

Thrush, Unicolored 201

Thrush, Yemen 202

Thryotharus 190

ribetanus, Tetrangallus 71

tickelli, Anorrhinus 173

(tickelli, Ptiloloemus) 173

Tiger-Parrot, Brehm's 114

Tiger-Parrot, Madarasz's 114

Tiger-Parrot, Modest 114

Tiger-Parrot, Painted 114

Tijuca 183

Tilmatura 170

timorensis, Ficedula 194

Tinamou, Argentinian Red-winged 2

Tinamou, Black 2

Tinamou, Bolivian Red-winged 2

Tinamou, Brazilian Red-winged 2

Tinamou, Dwarf 2

Tinamou, Kalinowski's 2

Tinamou, Magdalena 2

Tinamou, Solitary 2

Tinamou, Taczanowski's 2

Tinamou:, Choco 2

Tinamus 2

Tinkerbird, White-chested 175 
tinnunculus, Folco 62

tirica, Brotogeris 100

Tit-hylia, 218

Tit-Spinetail, White-browed 178

Tit-Tyrant, Ash-breasted 183

Tit, White-winged 203

Tit, White-winged Black 203

Tit, Yellow 202

tithys, Synallaxis 179

toboci, Amazilia 147

toco, Ramphastos 176

Todimamphus 171

Todirostrum 185

Tody-Flycatcher, Buff-cheeked 185

Tody-Tyrant, Buff-breasted 184

Tody-Tyrant, Cinnamon-breasted 184

Tody-Tyrant, Fork-tailed 184

Tody-Tyrant, Kaempfer's 184

togoensis, Vidua 225

(Logoensis, Viduo orientalis) 225

Topaz, Crimson 170

Topaz, Fiery 170

Topaza 170

Torgos $\mathbf{5 5}$

torquata, Coeligena 154

torquatus, Pedionomus 73

Torreomis 210

comidus, Arrila 184

torridus, Selaphorus 168

torridus, Selasphorus 168

corringtoni, Columba 87

Toucan, Channel-billed 176

Toucan, Keel-billed 176

Toucan, Red-billed 176

Toucan, Toco 176

Toucanet, Saffron 176

Toucanet, Spot-billed 176

Toucanet, Yellow-browed 176

Touit 119

toussenelii, Accipiter 29

taxapei, Charmosyna 103

(trachelioutus, Aegypius) 55

tracheliotus, Torgos 55

Tragopan 71

Tragopan, Black-headed 71

Tragopan, Blyth's 71

Tragopan, Cabot's 71

Tragopan, Grey-bellied 71

Tragopan, Indian 71

Tragopan, Satyr 71

Tragopan, Western 71

Tragopan, Yellow-billed 71

Trainbearer, Black-tailed 161

Trainbearer, Green-tailed 161

traversi, Petroica 198

Tree-Babbler, Negros 201

Tree-Babbler, Rough-templed 201

Tree-Babbler, White-breasted 200

Tree-Duck, Cuban 20

Tree-Duck, Fulvous 21

Tree-Duck, West Indian 20

Tree-Duck, White-faced 21

Tree-Partridge, Chestnut-breasted 67

Treepie, Hooded 231

Treepie, Hooded Racket-tailed 231

Treron 91

Trichastoma 201

Trichocichla 201

Trichoglossus 120
(Tricholimnas) 76

trichopsis, Otus 139

Triclaria 120

inicolor, Ploceus 224

(tricolor, Xiphidiopterus) 82

inidactyla, Jacamaralcyon 175

trifasciarus, Nesomimus 190

Trigonoceps 55

Tringa 84

trinatatus, Accipiter 29

tristrami, Oceanodroma 6

trivirgatus, Accipiter 29

trocaz, Columba 87

Trochilus 170

mogladytes, Estrilda 216

Trogon, Eared 17!

(Tropicoperdix) 67

(tropicus, Corvus) 231

Trumpetbird, 229

Tsikirity, Aldabra 197

tucanus, Ramphastos 176

iucumano, Amazona 95

tuerosi, Laterallus 77

(tuerosi, Laterallus jamaicensis) 77

tumultuosus, Pionus 111

Turaco, Bannerman's 121

Turaco, Black-billed 122

Turaco, Black-tip Crested 122

Turaco, Crested 122

Turaco, Fischer's 121

Turaco, Great Blue 121

Turaco, Guinea 122

Turaco, Knysna 121

Turaco, Livingstone's 122

Turaco, Purple-crested 121

Turaco, Ruspoli's 122

Turaco, Schalow's 122

Turaco, Verreaux's 122

Turaco, Violet 121

Turaco, Violet-crested 121

Turaco, Yellow-billed 122

Turdoides 201

Turdus 201

Turkey, Ocellated 67

umeri, Eremamela 194

Turnix 72

Turtle-Dove, Western 91

urtur, Streptopelia 91

Turtur 92

Twinspot, Green 218

Twinspot, Green-backed 218

tympanistria, Turtur 92

Tympanuchus 72

typica, Coracina 188

rypus, Polyboroides 52

Tyrannulet, Alagoas 184

Tyrannulet, Long-tailed 184

Tyrannulet, Minas Gerais 184

Tyrannulet, Sāo Paulo 184

tyrannus, Spizaetus 54

Tyrannus 185

Tyrant, Strange-tailed 183

tyrianthina, Metallura 163

Tyto 123

tzacal, Amazilia 147

(ultima, Psitrirostra) 212

ulrima, Telespiza 212

ultramarina, Vidua 225

ultramorina, Vini 121
(Uliramarina, Vidua) 225

ulula, Surnia 142

umbra, Otus 139

Umbrellabird, Amazonian 182

Umbrellabird, Bare-necked 182

Umbrellabird, Long-watuled 182

Umbrellabird, Ornate 182

uncinatus, Chondrohierax 37

underwoodii, Ocreatus 163

undulata, Chlamydotis 78

undulata, Ninox navaeseelandiae 132

undularus, Aceros 173

(undulatus, Rhyticeros) 173

unicincla, Columba 87

unicinctus, Parabuteo 51

unicolor, Corvus 231

unicolor, Cyanoramphus 103

unicolor, Mesitomis 72

(unicamis, Crax) 66

unicornis, Pauxi 66

Uraeginthus 219

uralensis, Strix 142

Uralelomis 172

Urochroa 170

urochrysia, Chalybura 152

Urocissa 231

Uroglaux 142

uropygialis, Zosterops 206

Urosticte 170

Urotriorchis 55

unubitunga, Buteogallus 37

usheri, Asthenes 178

ussheri, Scotopelia 140

usta, Otus 139

(usto, Otus watsonii) 139

vanderbilti, Malacocincla 196

(vanderbilti, Trichastoma) 196

vandewateri, Otus 139

Vanellus 82

Vanga, Pollen's 189

Vanga, Van Dam's 189

varia, Strix 142

variegata, Mesitomis 72

variegata, Ninox 133

variegaticeps, Alcippe 191

varius, Psephotus 114

vasa, Coracopsis 103

Velvetbreast, Mountain 160

venezuelensis, Diglossa 208

ventralis, Accipiter 29

ventralis, Amazona 95

ventralis, Buteo 36

venustus, Plarycercus 111

veraguensis, Anthracothorax 148

vermiculatus, Otus 139

(vermiculatus, Otus guatemalae) 139

Vermivora 211

vernalis, Loriculus 107

verreaurii, Aquila 31

versicolor, Amazilia 147

versicolor, Amazona 95

versicolor, Psitteuteles 117

(versicolor, Trichoglossus) 117

versicolurus, Brologeris 100

verticalis, Prioniturus 113

(verticalis, Prioniturus discurus) 113

vesper, Rhodopis 168

vespertinus, Falco 62

vestilus, Eriocnemis 156 
verula, Orralis 66

vicina, Meliphaga 206

victoria, Goura 89

victoriae, Lesbia 161

victoriae, Ploceus 224

victoriae, Paloris 230

victoriae, Sina 203

Vidua 225

viduata, Dendrocygna 21

vigil, Buceros 174

(vigil, Rhinoplax) 174

vigorsii, Eupodotis 80

vilasboasi, Pipra 183

villaviscensia, Campylopterus 151

vinacea, Amazona 95

vinacea, Lagonosticta 217

(vinacea, Laganosticta larvata) 217

vinacea, Streptopelia 91

vinaceigula, Egretia 10

(vinaceigula, Hydranassa) 10

vindhiana, Aquila 31

(vindhiana, Aquila rapax) 31

Vini 120

viola, Heliangelus 158

violacea, Musophaga 121

Violet-ear, Brown 154

Violet-ear, Green 154

Violet-ear, Sparkling 154

Violet-ear, White-vented 154

violiceps, Amazilia 148

violiceps, Goldmania 157

violifer, Coeligena 154

vipia, Grus 75

Virea 213

Vireo, Black-capped 213

Vireo, Saint Andrew 213

Vireo, San Andrés 213

(virgata, Ciccaba) 142

virgata, Stema 85

virgata, Strix 142

virgatus, Accipiter 29

virginianus, Bubo 129

(virga, Anthropoides) 75

virgo, Grus 75

viridicata, Pymhura 118

viridicauda, Amazilia 148

viridifacies, Enthrura 215

viridifrons, Amazilia 148

viridigaster, Amazilia 148

viridigenalis, Amazona 95

viridigula, Anthracothorax 148

viridipallens, Lampomis 160

viridis, Anthracothorax 149

viridis, Pteroglossus 176

Visorbearer, Hooded 149

Visomearer, Hyacinth 149

vitellinus, Ploceus 224

vitellinus, Ramphastos 176

vittata, Amazona 96

vocifer, Haliacetus 44

vociferoides, Haliaeetus 44

vosseleri, Bubo 129

Vultur 23

Vulture, African White-backed 42

Vulture, Asian White-backed 43

Vulture, Bearded 42

Vulture, Black 29

Vulture, Cape 43

Vulture, Cinereous 29
Vulture, Egyplian 51

Vulture, Eurasian Griffon 43

Vulture, Himalayan Griffon 43

Vulture, Hooded 51

Vulture, Indian Black 53

Vulture, King 23

Vulture, Lappet-faced 55

Vulture, Long-billed 43

Vulture, Nubian 55

Vulture, Oriental White-backed 43

Vulture, Palm-nut 42

Vulture, Pondicherry 53

Vulture, Red-headed 53

Vulture, Rueppell's 43

Vulture, Rueppell's Griffon 43

Vulture, Scavenger 51

Vulture, White-backed 42

Vulture, White-headed 55

Vulture, White-rumped 43

vulturina, Gypopsitua 106

waalia, Treron 91

wagleri, Aratinga 99

wahlbergi, Aquila 32

wahnesi, Paratia 230

waldeni, Aceros 173

(waldeni, Aceros leucocephalus) 173

(waldeni, Rhyticeros leucocephalus) 173

waldenii, Dicrurus 227

Waldrapp, 13

(wallacei, Eulipoa) 65

wallacei, Megapodius 65

wallacii, Habroptila 76

wallacii, Semioptera 231

wallichii, Catreus 68

wallicus, Pezoparus 110

Warbler, Aldabra 197

Warbler, Aquatic 190

Warbler, Bachman's 211

Wamler, Fly River Grass 196

Warbler, Golden-cheeked 211

Warbler, Grey-headed 211

Warbler, Japanese Marsh 196

Warbler, Kirtland'a 211

Warbler, Kulambangra 198

Warbler, Long-legged 201

Warbler, Mrs Moreau's 200

Warbler, Nihoa Reed 190

Warbler, Papyns Yellow 192

Warbler, Rodrigues 192

Warbler, Semper's 211

Warbler, Seychelles 192

Warbler, White-winged 211

Warbling-Finch, Cinereous 209

Warbling-Finch, Plain-tailed 209

Warbling-Finch, Rufous-breasted 209

Water-Redstart, Luzon 199

Water-Redstart, Philippine 199

watersi, Sarothrura 78

waterstradti, Prioniturus 113

(waterstradti, Prioniturus montanus) 113

watertonii, Thalurania 169

watsonii, Otus 139

Watle-eye, Banded 198

Watlle-eye, Black-throated 198

Waxbill, Anambra 216

Waxbill, Bar-breasted 216

Waxbill, Black-bellied 216

Waxbill, Black-faced 216

Waxbill, Black-lored 216
Waxbill, Black-rumped 216

Waxbill, Common 216

Waxbill, Fawn-breasted 216

Waxbill, Golden-breasted 215

Waxbill, Kiabo 216

Waxbill, Lavender 216

Waxbill, Orange-breated 215

Waxbill, Orange-cheeked 216

Waxbill, Pink-cheeked 216

Waxbill, Red-eared 216

Waxbill, Saint Helena 216

Waxbill, Vinaceous 217

Waxbill, Zebra 215

Weaver, Bannerman's 222

Weaver, Bates's 222

Weaver, Black-chinned 224

Weaver, Black-headed 223

Weaver, Black-necked 224

Weaver, Blue-billed 219

Weaver, Clarke's 223

Weaver, Compact 222

Weaver, Cuckoo 220

Weaver, Entebbe 224

Weaver, Finn's Baya 223

Weaver, Golden-backed 224

Weaver, Golden-naped 222

Weaver, Grosheak 219

Weaver, Himalayan 223

Weaver, Lake Lufira 224

Weaver, Layard's Black-headed 223

Weaver, Little 223

Weaver, Loango 224

Weaver, Loango Slender-billed 224

Weaver, Maxwell's Black 222

Weaver, Napoleon 220

Weaver, Orange 220

Weaver, Parasitic 220

Weaver, Preuss's 224

Weaver, Red-headed 219

Weaver, Scaly-fronted 225

Weaver. Slender-billed 224

Weaver, Speckle-fronted 225

Weaver, Spot-backed 223

Weaver, Tanzanian Mountain 223

Weaver, Thick-billed 219

Weaver, Usambara 223

Weaver, Vieillot's Black 223

Weaver, Village 223

Weaver, White-billed Buffalo 220

Weaver, White-cheeked Olive 218

Weaver, White-naped 222

Weaver, Yellow 223

Weaver, Yellow-backed 223

Weaver, Yellow-capped 224

Weaver, Yellow-legged 221

Weaver, Yellow-mantled 224

weddellii, Aratinga 99

Weka, Buff 76

wellsi, Lepionila 89

westlandica, Procellaria 4

wetmorei, Buthraupis 207

wetmorei, Rallus 77

Wetmarethraupis 210

wharioni, Ducula 88

Whip-poor-will, Puerto Rican 143

Whipbird, Western 199

Whistler, Red-lored 197

Whistler. Vogelkop 197

Whistling-Duck, Black-bellied 20 
Whistling-Duck, Fulvous 21

Whistling-Duck, Red-billed 20

Whistling-Duck, West Indian 20

Whistling-Duck, White-faced 21

Whistling-Thrush, Ceylon 197

White-eye, Ambon Yellow 205

White-eye, Buru Mountain 204

White-eye, Comoro 205

White-eye, Ganonga 205

White-eye, Gizo 205

White-eye, Golden-bellied 206

White-ye, Great Truk 204

White-eye, Javan 205

White-eye, Large Ponhpei 204

White-eye, Large Truk 204

White-eye, Lemon-throated 205

White-eye, Little Kai 206

White-eye, Long-billed 204

White-eye, Mauritius Olive 205

White-eye, Mount Karthala 205

White-eye, Nendo 205

White-eye, Principe 205

White-eye, Rufous-throated 204

White-eye, Sanford's 205

White-eye, Santa Cnuz 205

White-eye, Sao Tome 205

White-eye, Seychelles Grey 205

White-eye, Spot-breasted 204

White-eye, Sudeat 205

White-eye, Sulawesi 205

White-eye, Tagula 205

White-eye, Timor 204

White-eye, Truk 204

White-eye, White-breasted 205

White-eye, White-chested 205

White-eye, White-throated 205

White-eye, Splendid 205

Whiteface, Chestnut-breasted 192

Whitetip, Purple-bibbed 170

Whitetip, Rufous-vented 170

whimeyi, Micrathene 132

whimeyi, Pomarea 199

Whydah, Pin-tailed 225

Whydah, Red-collared 220

Whydah, Yellow-backed 220

Whydah, Yellow-mantled 220

Widowbird, Red-collared 220

Widowbird, Yellow-mantled 220

Wigeon, Cape 16

Wigeon, Eurasian 18

withelminae, Chamosyna 103

wilkinsi, Nesospiza 208

williami, Metallura 163

wilsoni, Coeligena 154

wilsoni, Hemignathus 211

(wilsoni, Hypochero) 226

wilsani, Vidua 226

wilsonit, Chondrohierax uncinatus 37

(wilsonii, Chondrohierax) 37

(winifredae, Bathmocercus) 200

winifredae, Scepomycter 200

Wirebird, 82

Wood-Dove, Black-billed 92

Wood-Dove, Blue-headed 92

Wood-Dove, Blue-spotted 92

Wood-Dove, Red-billed 92
Wood-Duck, Black-billed 20

Wood-Owl, African 142

Wood-Owl, Brown 141

Wood-Owl, Hume's 140

Wood-Owl, Mottled 141

Wood-Owl, Sichuan 140

Wood-Owl, Spotted 141

Wood-Owl, Tawny 140

Wood-Partridge, Bearded 68

Wood-Partridge, Black 70

Wood-Partridge, Fernuginous 68

Wood-Pigeon, African 87

Wood-Pigeon, Ceylon 87

Wood-Pigeon, Grey 86

Wood-Pigeon, Nilgiri 86

Wood-Pigeon, Purple 87

Wood-Pigeon, Silvery $\mathbf{8 6}$

Wood-Quail, Gorgeted 70

Woodcock, Moluccan 84

Woodcock, Obi 84

Woodcock, Sulawesi 84

Woodcreeper, Moustached 177

Woodcreeper, Snethlage's 177

woodfordi, Nesoclopeus 77

Woodfordia 205

(woodfordii, Ciccaba) 142

woodfordii, Sirix 142

Woodhen, Lord Howe 1sland 76

(woodi, Leonardina) 201

woodi, Trichastoma 201

Woodnymph, Crowned 169

Woodnymph, Fork-tailed 169

Woodnymph, Green-crowned 169

Woodnymph, Long-tailed 169

Woodnymph, Mexican 169

Woodnymph, Violet-capped 169

Woodpecker, Helmeted 177

Woodpecker, Imperial 176

Woodpecker, lvory-billed 176

Woodpecker, Noguchi's 177

Woodpecker, Okinawa 177

Woodpecker, Pryer's 177

Woodpecker, Red-cockaded 177

Woodpecker, Red-collared 177

Woodpecker, Tristram's 177

Woodstar, Amethyst 150

Woodstar, Bahama 150

Woodstar, Chilean 156

Woodstar, Esmeraldas 145

Woodstar, Gorgeted 145

Woodstar, Little 145

Woodstar, Magenta-throated 166

Woodstar, Purple-collared 163

Woodstar, Purple-throated 166

Woodstar, Rufous-shafted 151

Woodstar, Santa Marta 145

Woodstar, Short-tailed 163

Woodstar, Slender-tailed 163

Woodstar, White-bellied 145

warcesteri, Tumix 72

Wren, Apolinar's 190

Wren-babbler, Short-tailed 200

Wren-Babbler, Assam 200

Wren-Babbler, Luzon 197

Wren-Babbler, Mishmi 200

Wren-Babbler, Rabor's 197
Wren-Babbler, Rufous-throated 200

Wren-Babbler, Rusty-throated 200

Wren-Babbler, Tailed 200

Wren-Babbler, Tawny-breasted 200

Wren-Babbler, Wedge-billed 200

Wren, Bush 186

Wren, Lilac-crowned 196

Wren, New Zealand Bush 186

Wren, Niceforo's 190

Wren, Slender-billed 190

Wren, Sumichrast's 190

Wren, Zapata 190

wumizusume, Synthliboramphus 85

wyvilliana, Anas 19

(wyvilliana, Anas platyrhynchos) 19

xanthogonys, Heliodoxe 159

xantholara, Amazona 96

xanthomus, Agelaius 213

Xanthamyza 207

xanthophrys, Crossleyio 193

(xonthophrys, Phyllastrephus) 193

xanthophrys, Pseudonestor 212

xanthops, Amazona 96

xanthops, Forpus 105

(Xanthopsar) 213

xanthopterygius, Forpus 105

xantusii, Hylocharis 160

Xenicus 186

Xenoglaur 142

Xenoligea 211

Xenopirostris 189

Xenops, Great 178

xenopterus, Laterallis 77

Xenomis 181

Xenospingus 210

Xenospiza 210

xenothorax, Leptasthenure 178

(xenothorar, Leptasthenura pileata) 178

(Xiphidiopterus) 82

Xiphocolaptes 177

Xiphalena 183

yarrellii, Carduelis 214

yarrellii, Eulidia 156

(yarrellii, Spinus) 214

yanuqui, Phaethomis 166

Yellowbrow, Madagascar 193

Yellowthroat, Black-polled 211

yersini, Garrulax 195

(Yetapa) 183

yucotanensis, Amazilia 148

yunnanensis, Sitta 203

zappeyi, Paradoxomis 198

Zaratomis 183

Zavaltariomis 231

zelichi, Sporophila 210

Zenaida 92

(zeylonensis, Bubo) 131

zeylonensis, Ketupa 131

zimmeri, Synallaxis 179

(zimmermanni, Thalasseus) 85

(zonarius, Bamardius) 112

zonarius, Platycercus 112

zoniventris, Falco 62

Zoothera 202

Zasterops 205 



The UK Joint Nature Conservation Committee was established by the Environmental Protection Act 1990 "for the purposes of nature conservation, and fostering the understanding thereof" in Great Britain as a whole and outside Great Britain. It is a committee of the three country agencies (the Countryside Council for Wales, English Nature and Scottish Natural Heritage), together with independent members and representatives from Northem Ireland and the Countryside Commission, and is supported by a specialist staff. JNCC and the three country agencies carry forward duties previously undertaken by the Nature Conservancy Council.

JNCC's statutory responsibilities include:

- the establishment of common scientific standards;

- the undertaking and commissioning of research;

- advising Ministers on the development and implementation of policies for or affecting nature conservation for Great Britain as a whole or nature conservation outside Great Britain;

- the provision of advice and dissemination of knowledge to any persons about nature conservation.

JNCC also has the UK responsibility for European and international matters affecting nature conservation.

Details of publications produced by JNCC are available from JNCC, Monkstone House, City Road, Peterborough PEl 1JY. 